\title{
Phytosociological analysis of European larch forests in the Southeastern Alps
}

\author{
I. Dakskobler ${ }^{1}$, A. Seliškar ${ }^{2}$ \& A. Rozman ${ }^{3}$
}

Keywords: phytosociology, synsystematics, hierarhical classification, UPGMA, Rhodothamno-Laricetum, Julian Alps, Karavanke, Kamnik-Savinja Alps, Triglav National Park, Slovenia, Italy, Natura 2000.

Ključne besede: fitocenologija, sinsistematika, hierarhična klasifikacija, UPGMA, Rhodothamno-Laricetum, Julijske Alpe, Karavanke, KamniškoSavinjske Alpe, Triglavski narodni park, Slovenija, Italija, Natura 2000.

Received: 10. 4. 2018

Revision received: 28. 5. 2018

Accepted: 11. 6. 2018

\begin{abstract}
Using the (unweighted) average linkage clustering (UPGMA) method we classified 458 phytosociological relevés of Larix decidua forests in the Southeastern Alps into 25 clusters. Based on their analysis we described the following new subassociations: Rhodothamno-Laricetum deciduae geetosum rivalis, sorbetosum chamaemespili, piceetosum abietis, adoxetosum moschatellinae, cystopteridetosum fragilis, cyclaminetosum purpurascentis, dryadetosum octopetalae and sorbetosum ariae. The selected method proved adequate in identifying the differences between larch stands on potential subalpine spruce and beech sites, and larch forests on the upper forest line, as well as the differences between initial larch stages on the upper forest line and more stable development stages on better developed soils on promontories and ledges above the upper beech forest line. Larch forests occur mainly in the altitudinal belt between $(1,500) 1,600$ and 1,800 $(1,900) \mathrm{m}$, on shady aspects and slopes that are steeper than $30^{\circ}$. They are some of the best preserved forest types in the Southeastern Alps, on smaller surface areas (Macesnje above the Beli Potok valley in the Julian Alps) even virgin forests, and their role as biotopes is exceptional.
\end{abstract}

\begin{abstract}
Izvleček
485 fitocenoloških popisov macesnovih gozdov iz Jugovzhodnih Alp smo s hierarhično klasifikacijo z metodo kopičenja na podlagi povezovanja (netehtanih) srednjih razdalj (UPGMA) razdelili v 25 skupin. Na podlagi njihove analize smo opisali naslednje nove subasociacije: Rhodothamno-Laricetum deciduae geetosum rivalis, sorbetosum chamaemespili, piceetosum abietis, adoxetosum moschatellinae, cystopteridestosum fragilis, cyclaminetosum purpurascentis, dryadetosum octopetalae in sorbetosum ariae. Izbrana metoda je zadovoljivo zaznala razlike med macesnovimi stadiji na potencialnih rastiščih subalpinskih smrekovih in bukovih združb ter macesnovimi gozdovi na zgornji gozdni meji, prav tako razlike med začetnimi (inicialnimi) stopnjami macesnovja na zgornji meji uspevanja gozda in bolj stabilnimi razvojnimi stopnjami na bolj razvitih tleh na pomolih in policah nad zgornjo mejo uspevanja bukve. Macesnovi gozdovi so najbolj pogosti v višinskem pasu med (1500) $1600 \mathrm{~m}$ in 1800 (1900) $\mathrm{m}$, na osojnih legah in na strminah nad $30^{\circ}$. So eden izmed najbolj ohranjenih gozdnih tipov v Jugovzhodnih Alpah, na manjših površinah (Macesnje nad dolino Belega potoka v Julijskih Alpah) celo pragozdovi in imajo izjemno biotopsko vlogo.
\end{abstract}

1 Scientific Research Centre of the Slovenian Academy of Sciences and Arts, Institute of Biology, Regional Unit Tolmin, Brunov drevored 13, SI-5220 Tolmin. E-mail: igor.dakskobler@zrc-sazu.si

2 Grobeljska cesta 6 b, 1234 Mengeš, Slovenia. E-mail: ase@siol.com

3 Biotechnical Faculty of the University in Ljubljana, Department of Forestry and Renewable Forest Resources, Večna pot 83 , 1000 Ljubljana. E-mail: andrej.rozman@bf.uni-lj.si 


\section{Introduction}

European larch (Larix decidua) and larch forests in Slovenia have received a lot of attention in the last decade, but the first phytosociological table was published much earlier (Dakskobler 1996). In an extensive paper (Dakskobler 2006) we reported, based on an analysis of 110 relevés, that larch forests in the Julian Alps unquestionably belong to the association Rhodothamno-Laricetum deciduae Willner et Zukrigl 1999 and that their existing syntaxonomic classification at the rank of subassociation of Alpine dwarf pine stands (Rhodothamno-Pinetum mugo laricetosum) was inappropriate. This was subsequently confirmed also by Zupančič \& Žagar (2007). The description and syntaxonomical divison of the association RhodothamnoLaricetum deciduae in Austria, which differentiated two subassociations: typicum and festucetosum rubrae (Karner 2007a,b), did not consider Slovenian sources (Dakskobler 2006, Zupančič \& Žagar 2007). Karner (2007a: 215) only mentioned "Gebietsausbildung" from the Southern Alps with differential species Homogyne sylvestris, Saxifraga cuneifolia, Senecio cacaliaster, Anemone trifolia and Knautia drymeia. Further research into larch forests was the result of the targeted research project that took place between 2008 and 2010 (Dakskobler et al. 2010a) and was followed by several papers in which we determined their surface area in Slovenia (Dakskobler et al. 2010b) as well as the distribution of a rare tree fungus Laricifomes officinalis and a rare epiphytic lichenized fungus Letharia vulpina, which both grow on old larch trees (Dakskobler et al. 2011a,b,c). We also published new relevés of this community from the Julian Alps and the Karavanke Mts. Phytosociological tables of the association RhodothamnoLaricetum were published also in articles discussing green alder communities in Slovenia (Dakskobler et al. 2013a) and the distribution of Peucedanum ostruthium in the Peca Mts. (Dakskobler et al. 2013b). Larix decidua and its communities were discussed also in two monographs (Dakskobler et al. 2012, 2016), in several professional and scientific papers (Dakskobler et al. 2011d, Kutnar \& Dakskobler 2014, Dakskobler 2015a,b,c).

According to our findings, the origin of larch forests in Slovenia is various. In part they occur on primary sites on very steep to perpendicular, usually shady rocky slopes in the belt of montane beech and fir-beech forests as well as on ledges and prominences in rock walls of mountain ridges at the altitude of 1,650 to $1,850(1,950) \mathrm{m}$, where beech cannot grow. The main characteristic of these sites is that larch occurs in all stand layers and regenerates very well, while other tree species (spruce, mountain ash, in places silver fir, beech and sycamore maple) occur only sporadically and obviously lack the strength to replace larch in the succession. Examples of such primary larch stands are under Čisti vrh, Velika and Mala Tičarica above Spodnja Trenta, in Apica above the mountain pasture Zapotok, Sleme and Robičje above the Mala Pišnica valley, Prednja Glava above Suha Pišnica, Macesnje above the Beli Potok valley, Na Pragu under Šplevta, Kališče, Macesence and Požgana Mlinarica above the Vrata valley, Macesnovec above the Kot valley, Brda above the Krma valley and in some places in the Kamnik-Savinja Alps (e.g. under Veliki Vrh and on the ledges of Kočna in Jezersko and under Raduha in the Solčava region).

Extensive larch stands that surround pastures on highmountain plateaus (e.g. a part of Komna and the Triglav Lakes Valley, Velo Polje and Fužina pasturelands in Bohinj, the northern part of Pokljuka in the Julian Alps and Veža - the Dleskovec Plateau in the Savinja Alps) and larch forests in the eastern part of the Karavanke Mountains (Mts. Peca and Olševa) are probably of a different origin. Primary forests here (that very likely used to be at least partly beech or spruce forests) must have been cleared or burnt for pasture at one time and the pasture area there was much larger than it is today. In the secondary succession larch established itself as a pioneer species that regenerates naturally, so there is very little possibility that it could soon be naturally replaced by beech or spruce.

As a rule, primary larch forests are not managed forests and have an explicitly protective role. In terms of nature conservation they are defined as a habitat type of European conservation concern, designated as "Alpine Larix decidua forests (9420)" in the Habitat Directive. Their distribution in Slovenia and criteria for monitoring their preservation were presented in a special report (Šilc et al. 2017).

Based on the relevé material collected for this analysis (a total of 458 relevés) we extended the phytosociological analysis from 2006, which mainly focused on the western and southern parts of the Julian Alps, to the larger part of the Southeastern Alps in the narrow sense (without the mountains in the Italian regions of Veneto and Trentino Alto Adige), i.e. the Italian and Slovenian parts of the Julian Alps, as well as to the Slovenian part of the eastern and western Karavanke Mts. and the Kamnik-Savinja Alps. We were primarily interested in the following:

- Is the floristic composition and structure of primary larch stands on ledges and prominences above the upper beech forest line distinctly different from the floristic composition and structure of pioneer (secondary) larch forests that are presumably a long successional stage on former pastures on high-mountain plateaus, still in the belt of subalpine beech or spruce forests?

- Is the floristic composition of larch stands in the Julian Alps different from that of larch stands in the KamnikSavinja Alps and the Karavanke Mountains? Can there 
be more than one geographical variant of the association Rhodothamno-Laricetum in the Southeastern Alps?

- Are larch stands that occur on extreme sites in the belt of montane-altimontane beech and fir-beech forests floristically distinctly different from larch stands on the upper forest line?

- Is the characteristic species combination based on 458 relevés different from the combination obtained based on 110 relevés? Should the diagnostic species selected in 2006 be evaluated also in view of the analysis of 458 relevés?

\section{Methods}

A total of 458 relevés of larch stands are stored in the FloVegSi database (Seliškar et al. 2003). The analysis focused on our relevés exclusively, ignoring the eight relevés of the successional stage under Črni Vrh above Batava, which is classified into the subassociation RhodothamnoLaricetum saxifragetosum paniculatae - (Dakskobler 1996, 2006), and Tregubov's relevés (1962), which had only recently been found in a manuscript, but are clearly more species-poor than ours and will be used in the analysis of successional processes during the past 60 years. All relevés were initially arranged in one table, in which we grouped the stand layers recorded on site (the upper tree layer, lower tree layer, upper shrub layer, lower shrub layer, herb and moss layer) into four main layers: the tree layer (E3), the shrub layer (E2), the herb layer (E1) and the moss layer (E0). For the purpose of subsequent processing we labelled epiphytic lichenised fungi and some of the wood decay fungi on larch trees as layer E3c.

We transformed Braun-Blanquet's scale $(r,+, 1,2,3,4,5)$

- Braun-Blanquet (1964) - into cover percentages (0$100 \%$ ) and calculated, for different layers (two shrub layers and two tree layers), the total coverage of the main layers using the below equation (Jennings et al. 2009, Maarel van der \& Franklin 2013),

$$
C_{i}=\left[1-\prod_{j=1}^{n}\left(1-\frac{\% \operatorname{cov} j}{100}\right)\right] \times 100
$$

where $\operatorname{cov} j$ is species cover in layer $j$. In the phytosociological table we converted the calculated total covers back to the original Braun-Blanquet scale.

The relevés were compared by means of hierarchical classification using the Unweighted average linkage clustering method (UPGMA) and Wishart's similarity ratio. Percentage covers $(0-100 \%)$ were modified by square root $(\sqrt[2]{\operatorname{cov}})$. Based on the results, we arranged the relevés into partial tables.

The gradients of the main ecological factors were determined using distance based redundancy analysis (db-RDA, Legendre \& Anderson 1999). We used a set of canonical (constrained) climatic and several other environmental variables and added, through regression, other environmental variables and mean Landolt indicator values (LIV) (Landolt et al. 2010) to the ordination graph - Appendix Table 1.

In identifying the indicator species of the syntaxa we used the Indicator Value Index (Legendre \& Anderson 1999, De Caceres \& Legendre 2009) and $\phi-$ phi value (Chytrý et al. 2002). The permutation test was used to eliminate the species with a non-significant occurrence optimum in a particular cluster. Species with frequency $\geq 15 \%$, a phi coefficient $\geq 0.25$ and a difference in frequencies among clusters $\geq 10 \%$, were considered to be good candidates for differential species (Slezak et al. 2016).

Numerical comparisons were made with the software package SYN-TAX (Podani 2001) and R (R Core Team 2017), using the package vegan (Oksanen et al. 2017) and indicspecies (De Caceres \& Legendre 2009).

In describing new subassociations and variants we use the concept of relative differential species. It refers to a species that is usually abundant in the stands of the association Rhodothamno-Laricetum, but has an obviously higher frequency or medium coverage in a certain group of relevés and thus distinctly characterises them. Some of the syntaxa could only be named after such species, because we could not identify differential species that do not occur in stands of other similar syntaxa. Based on our experience and more than 2,000 relevés stored in the FloVegSi database, most beech associations in Slovenia lack good character species, which are ideally confined to the sites of one or very few associations. Diagnostic species are only relative character species, but on the whole, beech associations are still well differentiated from one another.

Geoelemental, ecological and phytosociological designation of plant species follows the Flora alpina (Aeschimann et al. 2004a,b,c). For the diagnostic species of the class Vaccinio-Piceetea we also rely on the experience of our older colleague M. Zupančič and on our own experience with diagnostic species of the syntaxa Erico-Pinetea, Quercetalia pubescenti-petraeae, Fagetalia sylvaticae, Querco-Fagetea, Elyno-Seslerietea, Festuco-Brometea, Asplenietea trichomanis. There are several species that could be assigned to more than one syntaxonomical unit. Classification of such species depends on our own experience with sites and plant communities in Slovenia. On the whole, by analysing the species structure according to phytosociological groups we can explain the differences in researched stands and described syntaxa.

The nomenclatural source for the names of vascular plants is the Mala flora Slovenije (Martinčič et al. 2007). The nomenclature of Flora alpina - Sesleria caerulea (Aeschimann et al. 2004) was used for the taxon Sesleria caerulea subsp. calcaria (MFS). According to Rottensteiner (per- 
sonal communication), taxon Aconitum lycoctonum subsp. ranunculifolium, which is reported in MFS, is in fact Aconitum lupicida. Martinčič $(2003,2011)$ is the nomenclatural source for the names of mosses and Suppan et al. (2000) is the nomenclatural source for the names of lichenized fungi. The determination of some less frequent mosses and lichenized fungi is not always reliable. The nomenclatural sources for the names of syntaxa are Theurillat (2004) and Šilc \& Čarni (2012). Buser (2009) is the source of data on the geological bedrock, and the source for the nomenclature of soil types is Urbančič et al. (2005). Climate data (precipitation volume, mean temperature, mean moisture and snow cover duration) were obtained from high resolution raster maps provided by the Environmental Agency of the Republic of Slovenia, Ministry of the Environment and Spatial Planning (http://www.arso.gov.si/).

\section{Results and discussion}

\subsection{Ecological conditions in the studied larch stands}

The average annual daily temperature in the study area is between 2 and $4{ }^{\circ} \mathrm{C}$ and the annual precipitation level ranges between 2,000 and $3,500 \mathrm{~mm}$. The snow cover persists for 140 to 180 days (Figures 1,2). The vertical range of the localities of the relevés ranges between 500 to $1,905 \mathrm{~m}$ a.s.l. (with the highest located larch stands observed at $1,950 \mathrm{~m}$ a.s.l.), the highest density of relevés is at the elevations of 1,500 to $1,800 \mathrm{~m}$. Further to the west, in the Dolomites, stands of the association RhodothamnoLaricetum occur also much higher than 2,000 m a.s.l. (anonymous reviewer, in litt.). The amount of annual precipitation, which always exceeds $2,000 \mathrm{~mm}$, as well as the size and height of mountain chains are definitely a decisive factor for such distribution of larch stands in the SE Alps. The ratio between shady (N, NE, E, NW) and sunny aspects (S, SE, SW) is $75: 25$. About $70 \%$ of the relevés were made on slopes of 30 degrees or steeper and about $40 \%$ of the relevés on slopes of 40 degrees or steeper. On average, shady aspects on moderately steep, 30-degree slopes prevail (Figure 3). Limestone bedrock prevails at around $38 \%$ of the plots, and a very similar percentage of plots have dolomite limestone bedrock. Geological bedrock in about $8 \%$ of the relevés is admixed with marlstone, claystone or chert, and in about $6 \%$ of the plots the parent material is talus or rockfall. The soil type of more than $97 \%$ of the relevés is rendzina. The phytosociological relevés were made at the peak of the vegetation period, which lasts from July to September, mainly in the period between 2009 and 2017. Larch stands are generally open and receive a lot of light, the tree layer cover is only 50 to $70 \%$, the shrub layer covers between 20 and
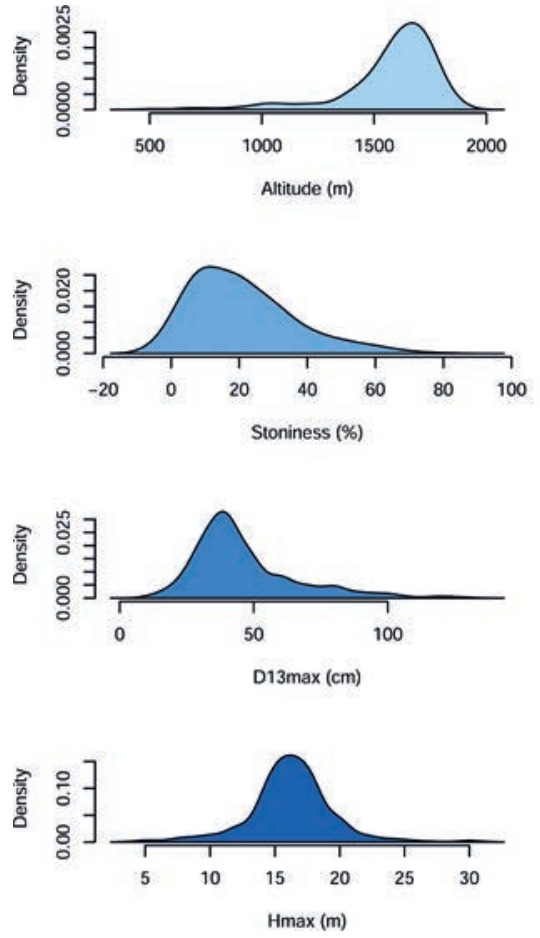
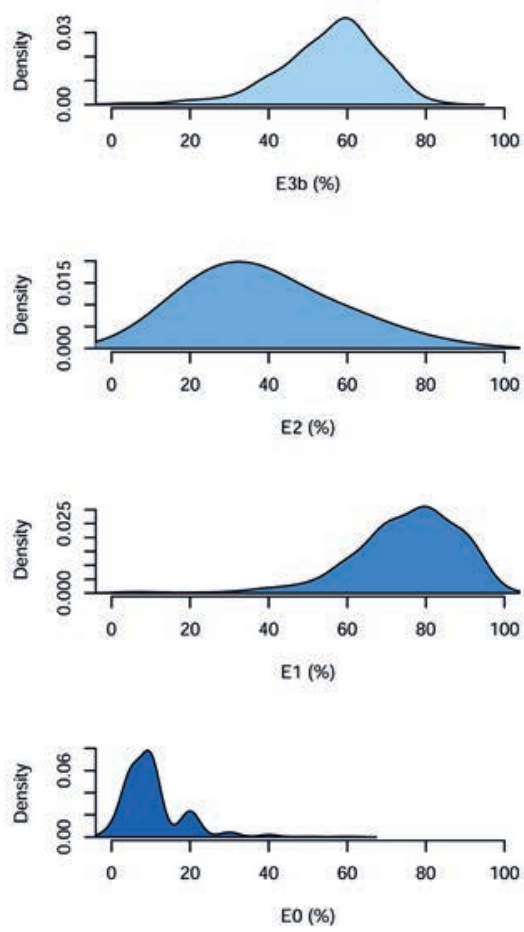

Figure 1: Density plots of some ecological variables and stand parameters in European larch forests. Slika 1: Gostote porazdelitve nekaterih ekoloških spremenljivk in sestojnih parametrov v macesnovju.
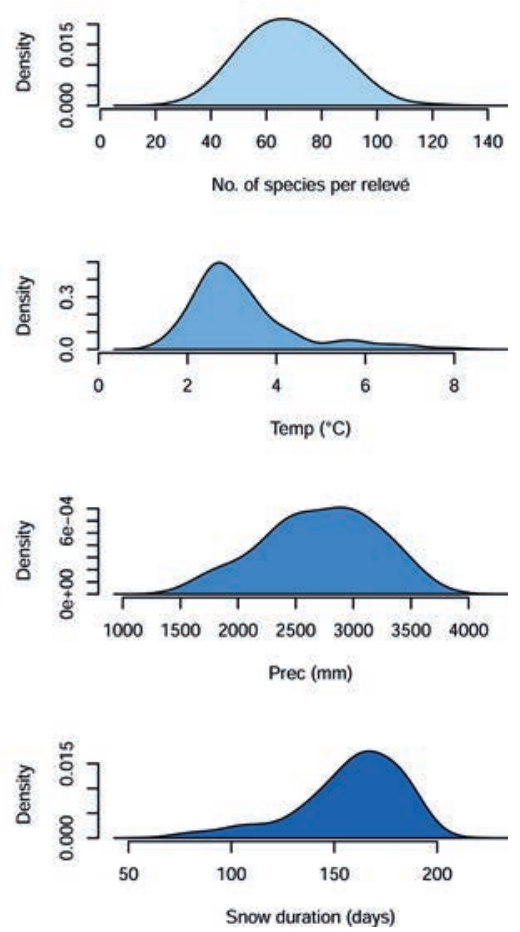

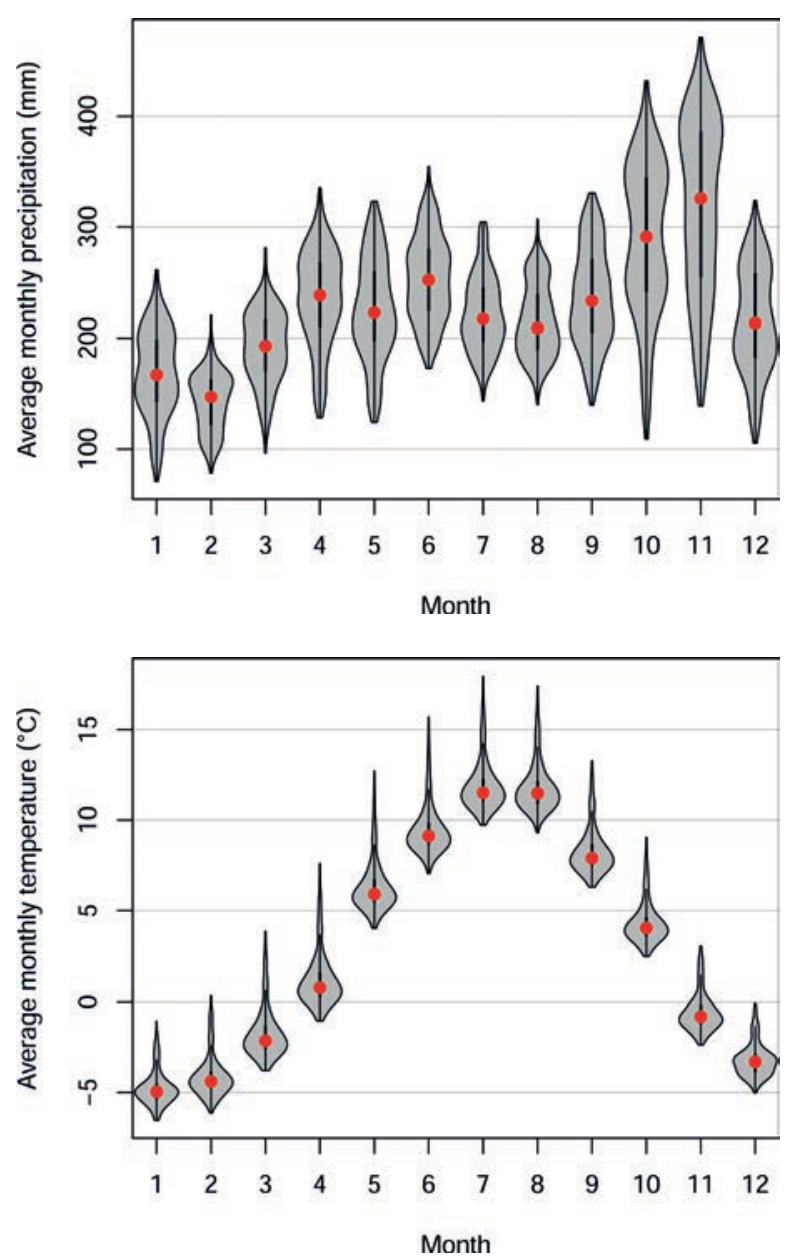

Figure 2: Density plots of average monthly temperatures and precipitation in European larch forests. Red dots represent medians. Slika 2: Gostote porazdelitve povprečnih mesečnih temperatur in padavin $\mathrm{v}$ macesnovju. Rdeče točke so mediane.

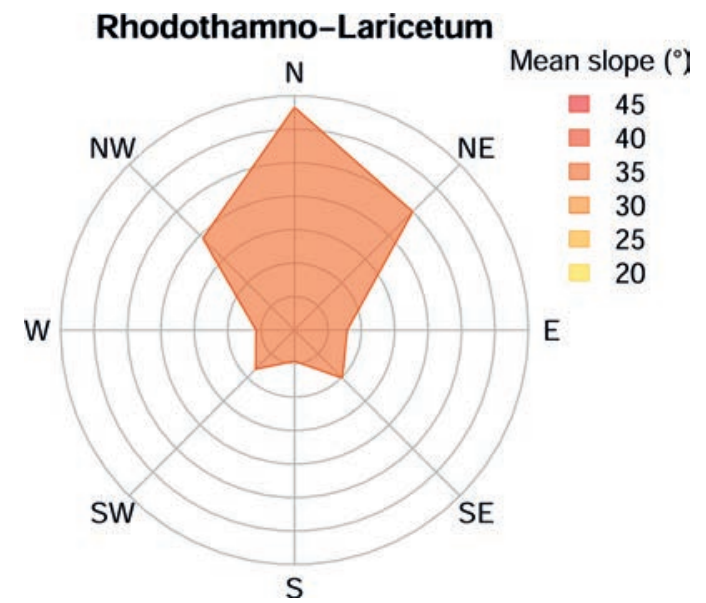

Figure 3: Rose diagram of aspects and mean slope in European larch forests.

Slika 3: Roža nebesnih leg s prikazom povprečne strmine macesnovih sestojev.
$40 \%$, the species-rich herb layer cover is 70 to $90 \%$ and the moss ground cover is about $10 \%$. Most of the plots, which were mainly $400 \mathrm{~m}^{2}$ in size, comprised between 60 and 80 plant taxa; the highest number of taxa per plot was 122. In most of the stands, the maximum diameter at breast height is $40 \mathrm{~cm}$ and the upper tree height less than $20 \mathrm{~m}$, even though the trees can be substantially larger in older (virgin) stands (e.g. Macesnje above Beli Potok), with diameters at breast height of up to $120 \mathrm{~cm}$ and tree height reaching up to $32 \mathrm{~m}$.

\subsection{Overview of established syntaxa and their nomenclatural types}

In hierarchical classification, 458 relevés from the Southeastern Alps (Figure 4) formed 24 large groups, whereas several relevés were distinctly different from others. These were arranged in Table 1. Similarity between 24 groups that can be classified into a specific syntaxonomic rank was determined with hierarchical classification in the synoptic table (Figure 5). In their classification into the syntaxonomic system we took into account the already described and typified subassociations (Dakskobler 2006, Karner 2007a, b): anemonetosum trifoliae, ostryetosum carpinifoliae, linnaeetosum borealis, saxifragetosum paniculatae, typicum and festucetosum rubrae. Comparison with the subassociations described in Austria showed that the subassociations typicum and festucetosum rubrae are floristicaly clearly different from most of our syntaxa and that larch forests in the Southeastern Alps cannot be classified into these two subassociations.

Since the number of relevés analysed in this paper is much higher than in 2006 and because we used a different

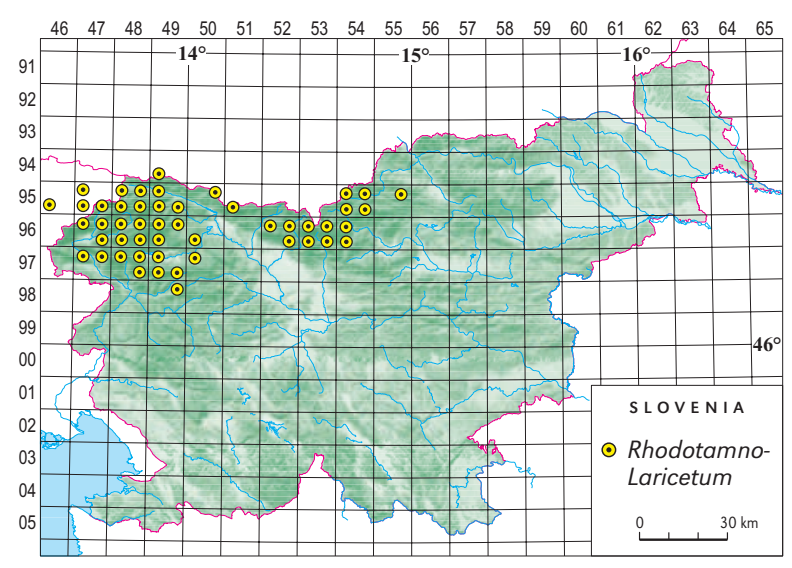

Figure 4: Approximate localities of recorded European larch stands in the Southeastern Alps (Slovenia, NE Italy).

Slika 4: Približna lokacija popisanih sestojev v Jugovzhodnih Alpah (Slovenija, NE Italija). 


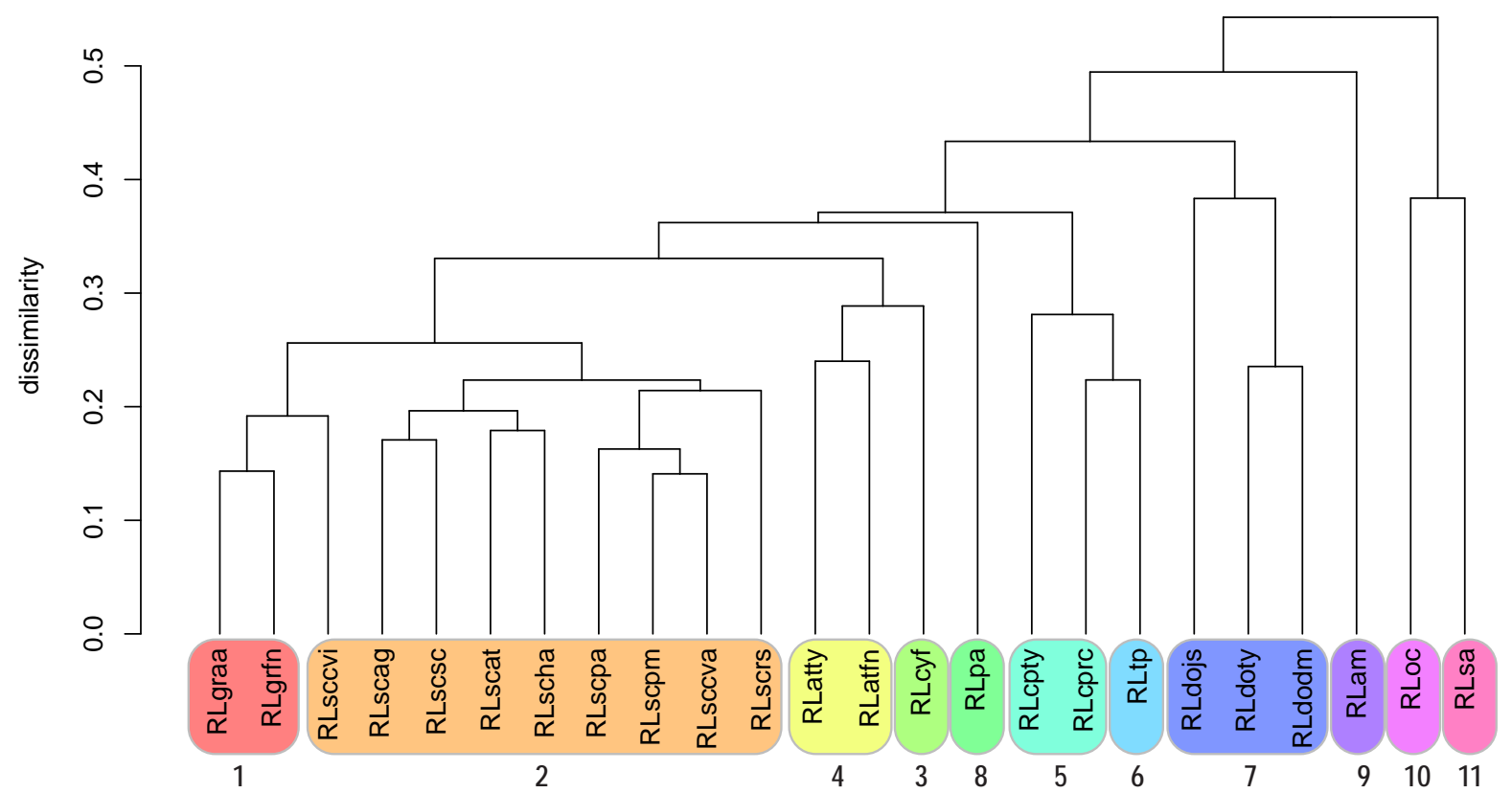

Figure 5: Dendrogram of 24 groups of Larix decidua stands in the Southeastern Alps, UPGMA, 1 - similarity ratio. The subasociations are numbered (see legend below).

Slika 5: Dendrogram 24 skupin macesnovja v Jugovzhodnih Alpah, UPGMA, komplement Wishartovega koeficienta podobnosti. Subasociacije so oštevilčene (glej legendo spodaj).

method this time (UPGMA instead of MISSQ), the relevés that were published in 2006 grouped somewhat differently. As a result, the subassociation anemonetosum trifoliae now comprises only the relevés of larch stands on very steep shady slopes, mostly in the fir-beech forest belt, as well as some relevés which were classified into the subassociation ostryetosum in 2006. The stands classified within the variant anemonetosum trifoliae var. Sorbus chamaemespilus in 2006 and other similar groups of relevés in the left part of the dendrogram in Figure 5 are now classified as a new subassociation sorbetosum chamaemespili and are considered to be the central stands of natural larch forests in the subalpine zone along the upper forest line in the Southeastern Alps. The differential species of the new subassociation are geographical differential species of the syntaxon Rhodothamno-Laricetum var. geogr. Anemone trifolia.

The established groups are syntaxonomically evaluated as follows:

1. Rhodothamno-Laricetum geetosum rivalis subass. nova, nomenclatural type, holotypus, is relevé 7 in Table 2

var. Adenostyles alliariae (RLgraa)

subvar. Luzula luzuloides subvar. Laserpitium peucedanoides

var. Festuca nitida (RLgrfn)

subvar. Luzula luzuloides subvar. Campanula scheuchzeri
2. Rhodothamno-Laricetum sorbetosum chamaemespili subass. nova (syn. anemonetosum trifoliae Dakskobler 2006, typus excluded, var. Sorbus chamaemespilus pro parte), nomenclatural type, holotypus, is relevé 19 in Table 10

var. Calamagrostis villosa (RLsccvi)

subvar. Alnus viridis

subvar. Luzula luzuloides

var. Adenostyles glabra (RLscag)

subvar. typica

subvar. Aconitum ranunculifolium

var. Saxifraga cuneifolia (RLscsc)

subvar. typica

subvar. Festuca nitida

var. Anemone trifolia (RLscat)

var. Homogyne alpina (RLscha)

var. Poa alpina (RLscpa)

var. Pinus mugo (RLscpm)

var. Calamagrostis varia (RLsccva)

var. Rubus saxatilis (RLscrs)

3. Rhodothamno-Laricetum cystopteridetosum fragilis subass. nova, nomenclatural type, holotypus, is relevé 4 in Table 15 (RLcyf)

4. Rhodothamno-Laricetum anemonetosum trifoliae Dakskobler 2006 (incl. ostryetosum carpinifoliae Dakskobler 2006, typus excluded, pro parte minor)

var. typica (RLatty)

subvar. Sorbus aria 
var. Festuca nitida (RLatfn)

subvar. Heliosperma alpestre

subvar. Anemone trifolia

5. Rhodothamno-Laricetum cyclaminetosum purpurascentis subass. nova, nomenclatural type, holotypus, is relevé 9 in Table 17

var. typica (RLcpty)

var. Rhodothamnus chamaecistus (RLcprc)

6. Rhodothamno-Laricetum var. Thymus polytrichus (RLtp)

7. Rhodothamno-Laricetum dryadetosum octopetalae subass. nova, nomenclatural type, holotypus, is relevé 4 in Table 20

var. typica (RLdoty)

subvar. Carex firma

subvar. Ranunculus carinthiacus

var. Juniperus sibirica (RLdojs)

subvar. Aconitum ranunculifolium

subvar. Parnassia palustris

var. Daphne mezereum (RLdodm)

8. Rhodothamno-Laricetum piceetosum abietis subass. nova, nomenclatural type, holotypus, is relevé 6 in Table 16 (RLpa)

var. Adoxa moschatellina

var. Knautia longifolia

9. Rhodothamno-Laricetum adoxetosum moschatellinae subass. nova, nomenclatural type, holotypus, is relevé 6 in Table 23 (RLam)

10. Rhodothamno-Laricetum ostryetosum carpinifoliae

Dakskobler 2006 (RLoc)

var. Buphthalmum salicifolium

var. Melampyrum vulgatum

11. Rhodothamno-Laricetum sorbetosum ariae subass. nova, nomenclatural type, holotypus, is relevé 7 in Table 25 (RLsa)

12. Rhodothamno-Laricetum linnaeetosum borealis Dakskobler 2006 (relevés 6 and 7 in Table 1)

13. Rhodothamno-Laricetum saxifragetosum paniculatae Dakskobler 2006 (Dakskobler 1996, Table 1, relevés 9-16).

\subsection{Ecological characteristics of the established syntaxa}

To help us explain the ecological and floristic differences between the established syntaxa we made a synoptic table (Appendix Table 2) with 27 columns, without taking into account the relevés from Table 1 (which are included in Column 25 that shows the total floristic composition based on 458 relevés). For the sake of comparison we added two columns (26 and 27) with syntaxa from Austria, typicum and festucetosum rubrae (Karner 2007b), which were not taken into account in other analyses, because they do not belong to the studied forest community in the Southeastern Alps. We also made a table with the analysis of proportions of groups of diagnostic species in the syntaxa (Table 27). Another valuable tool was the analysis of gradients of the main ecological factors and indicator values.

\subsubsection{Analysis of the main gradients}

The ordination diagram (Figure 6) shows two main ecological gradients. The first is the altitude or temperature gradient, which is positively correlated with variability of seasonal precipitation (BIO15) and negatively correlated with the duration of the snow cover and air humidity. The second gradient indicates the total precipitation volume, which is negatively correlated with variability in temperature seasonality (BIO4). The precipitation gradient also separates the relevés from the Kamnik-Savinja Alps from the relevés in the Julian Alps. The variability of larch forest vegetation increases with altitude (lower temperatures). The volume of precipitation does not correspond to LIV for humidity, which is probably due to

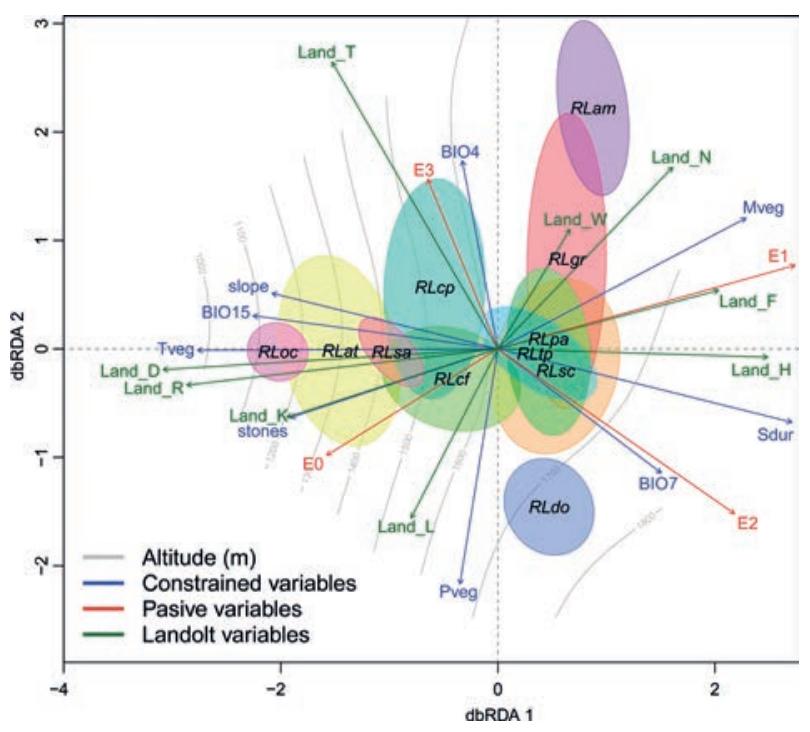

Figure 6: Distance based redundancy analysis (db-RDA) ordination plot. The ellipses represent the standard deviation of the relevés of individual European larch syntaxa. The first two canonical axes explain $9.1 \%$ of the variability in data. On the first axis, syntaxa are distributed according to mean temperature conditions and altitude, while the sum of precipitation during the vegetation season has the greatest influence on the second axis.

Slika 6: Slika ordinacije db-RDA. Elipse predstavljajo standardni odklon popisov posameznih skupin macesnovja. Prvi dve kanonični osi pojasnita $9,1 \%$ variabilnosti. Na prvi osi se skupine popisov porazdeljujejo po temperaturnih razmerah in po nadmorski višini, na drugi osi pa ima največji vpliv količina padavin $\mathrm{v}$ času vegetacijske sezone. 
the fact that the areas where larch stands occur receive sufficient precipitation and LIV tend to indicate soil moisture retention capacity. This tendency is reflected also in LIV for moisture, which is inversely proportional to the LIV for rockiness, and is partly related also to the number of snow cover days.

Figure 7: Ecograms of mean Landolt indicator values (LIV) for moisture, reaction and nutrients. The ellipses represent the standard deviation of LIV for each individual syntaxon

Slika 7: Ekogrami Landoltovih povprečnih indikatorskih vrednosti (LIV). Elipse predstavljajo standardni odklon LIV za vsak posamezen sintakson.

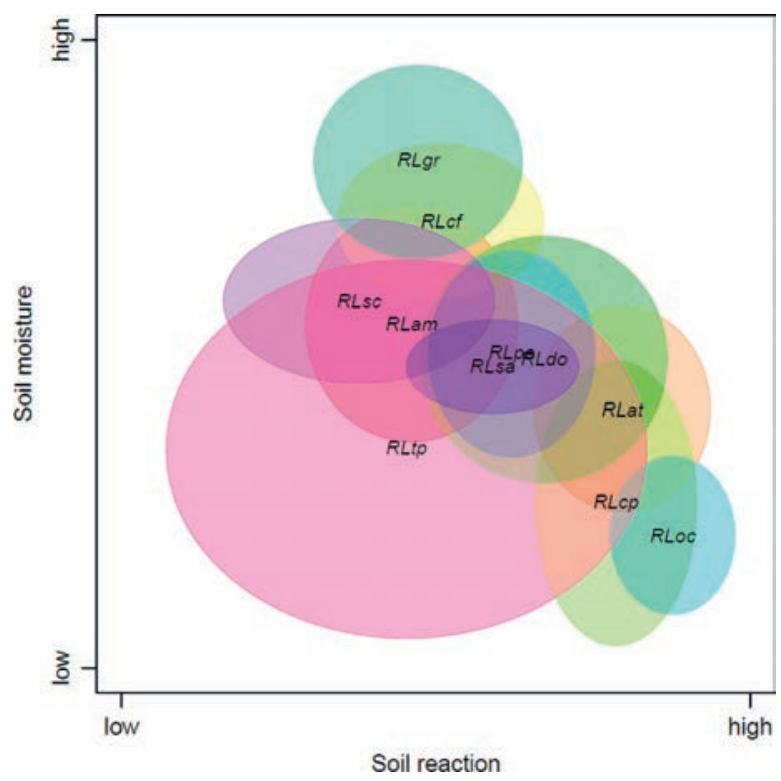

\subsubsection{Short description of syntaxa - commentary to Tables 1-28}

Table 1 incorporates very diverse relevés that did not group into any of the 24 groups of relevés. Relevés 1 to 3 (made under Črni Vrh above the Batava gorge, in the foothills of Mt. Porezen) belong to the subassociation Rhodothamno-Laricetum ostryetosum carpinifoliae, but are very poor in species, especially those from the class Erico-Pinetea; two of the diagnostic species, Rhododendron hirsutum and Rhodothamnus chamaecistus, are absent. Relevés 4 and 5 in this table, one is from the slopes of
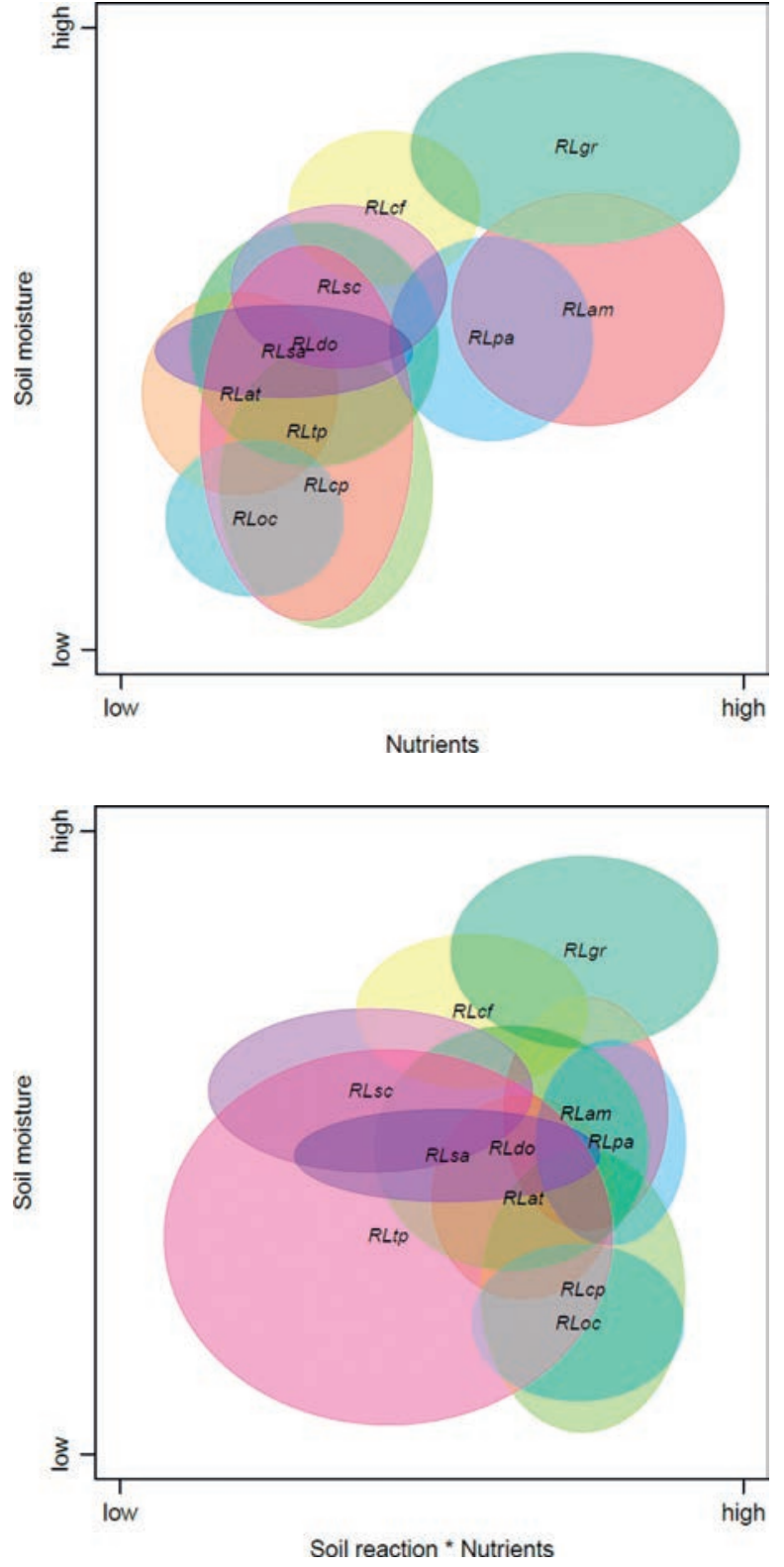

the Ponce ridge above the Tamar valley, the other from the shady slopes of Mt. Olševa, characterise a developing larch forest, a pole stage stand in a torrential gully or a gravelly hollow - i.e. a pioneer form on sites that are extreme for forest growth. Relevés 6 and 7 are from Soteska in Bohinj. This is the lowest-lying natural larch forest in Slovenia, growing at the altitude of 500 to $520 \mathrm{~m}$ a.s.l. and classified into the subassociation RhodothamnoLaricetum linnaeetosum borealis (Dakskobler 2006: 134, 143). Its differential species are Linnaea borealis, Cypripedium calceolus and Betula pubescens subsp. carpatica. Flo- 
ristically the most similar to the relevés of this subassociation is the relevé of pioneer larch stands on a fan at the bottom of Macesnov Graben gorge in the valley of Beli Potok under Mt. Kukova Špica. Betula pubescens subsp. carpatica occurs in this stand as well. Relevés 9 to 13 also indicate non-typical forms of larch forest, relevé 9 a pioneer stand on a gravelly, avalanche prone slope under Sleme above the valley of Mala Pišnica, relevé 10 represents the fragments of the larch forest on a steep to perpendicular shady slope of the promontory under Šoštar above the Kacenpoh gorge in the upper Bača Valley, parent material is claystone; relevé 11 is an open larch stand on a very rocky site under Debeljak in the Polovnik ridge (in the area of the WWI frontline), relevés 12 and 13 are open larch stands on very rocky terrain in Konte under Mt. Šoštar (on the Gorenjska side of the mountain). These stands are treated at the rank of the association Rhodothamno-Laricetum.

\subsubsection{Rhodothamno-Laricetum geetosum rivalis (Column 1 in Table 26)}

Tables 2 and 3 (Columns 1 and 2 in Appendix Table 2 and Table 27) show groups of larch forest relevés on nutrient-rich sites. This is corroborated also by the analysis of LIV (Figure 8) and is reflected in the vegetation with a relatively high proportion of tall herbs, character species of the class Mulgedio-Aconitetea, and a relatively small proportion of character species of the class Erico-Pinetea. These two tables comprise the relevés that could be treated, in the wider sense, under the central subassociation sorbetosum chamaemespili, but are the most mesophilous compared to other forms of this subassociation, which is confirmed also by the analysis of LIV. These stands occur on slopes with persistent snow cover that lasts long into the spring. The soil is mainly nitrogen- and humus-rich
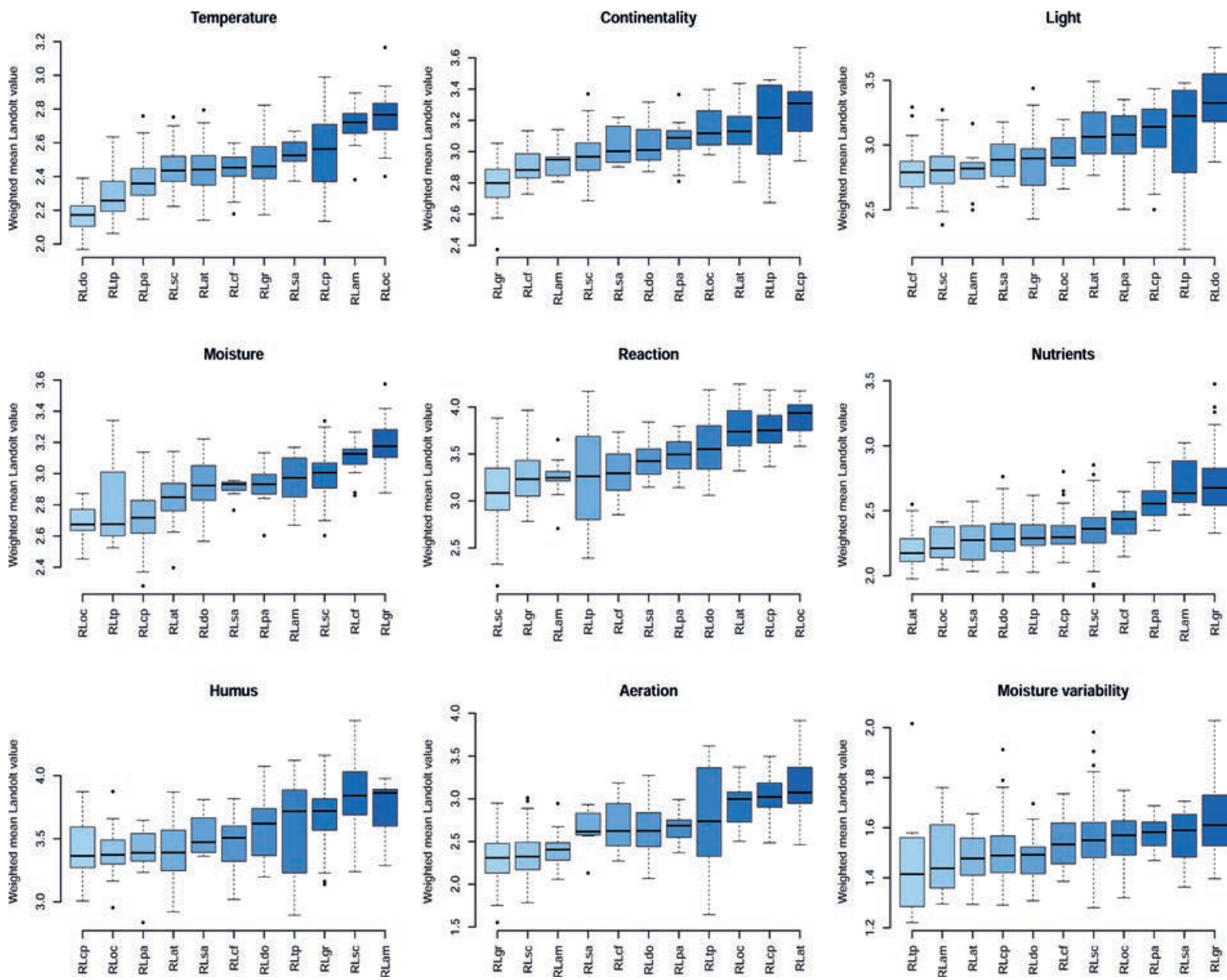

Figure 8: Boxplots of LIV by identified syntaxa in European larch stands. Syntaxa are ordered by median for each LIV.

Slika 8: Okvirji z ročaji LIV po ugotovljenih sintaksonih macesnovja, sintaksoni so urejeni po mediani za vsako LIV. 

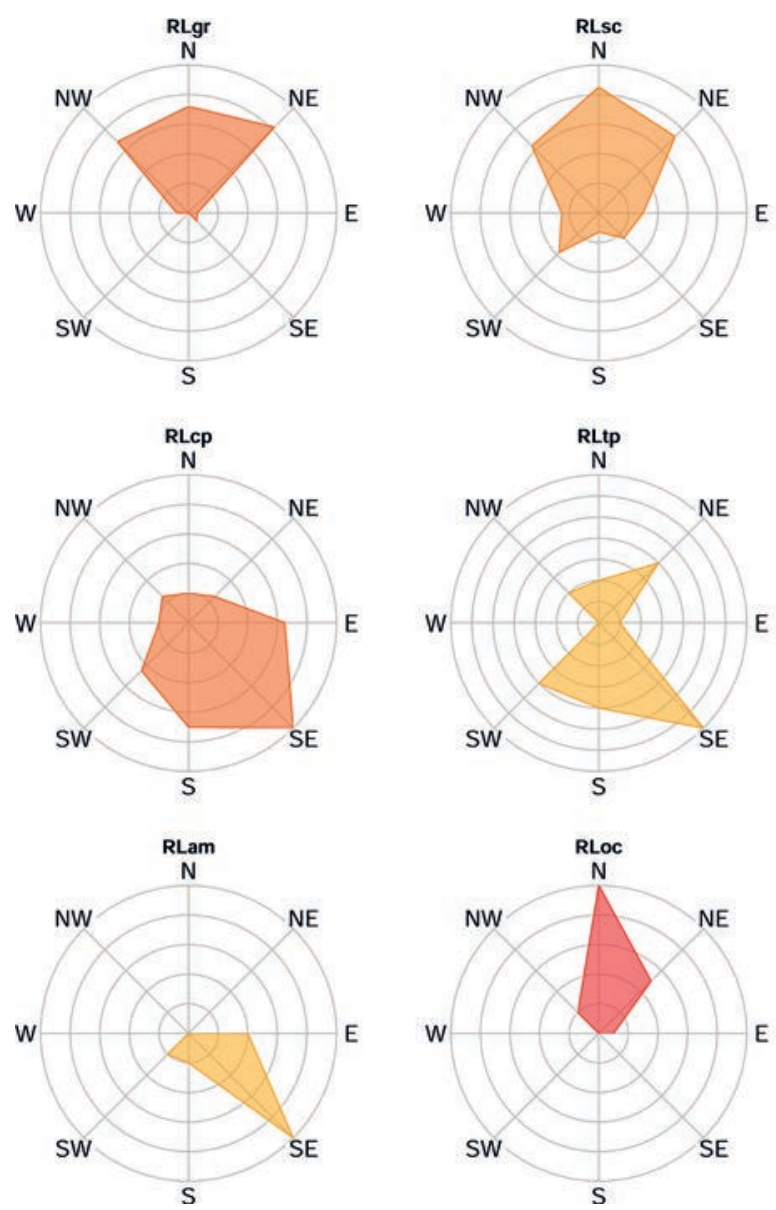

Figure 9: Rose diagrams of aspects with mean slope by syntaxa.

Slika 9: Rože nebesnih leg s prikazom povprečne strmine po sintaksonih.

rendzina. These stands are therefore classified into the new subassociation Rhodothamno-Laricetum geetosum rivalis. The relative differential species of the subassociation are Geum rivale, Chaerophyllum hirsutum, Rumex arifolius and Senecio cacaliaster. At $\mathrm{p}$-value $\mathrm{p}<0.01$, the indicator species analysis (Dufrene \& Legendre 1997) shows the following differential species: Adenostyles alliariae, Alnus viridis, Chaerophyllum hirsutum, Chrysosplenium alternifolium, Doronicum austriacum, Geum rivale, Poa hybrida, Primula elatior, Rumex arifolius, Saxifraga rotundifolia, Senecio cacaliaster and Stellaria nemorum. Significant phi values ( $\mathrm{p} \leq 0.05)$ were indicated also for Deschampsia cespitosa and Oxalis acetosella.

We distinguish two variants - one on deeper soils (brown rendzinas with transitions to brown soil on limestone) - var. Adenostyles alliariae, and another on more shallow and stony soils, var. Festuca nitida. Stands of the variant var. Adenostyles alliariae can be further subdivided into two subvariants, a more acidophilous subvariant with Luzula luzuloides (parent material is mainly dolo-
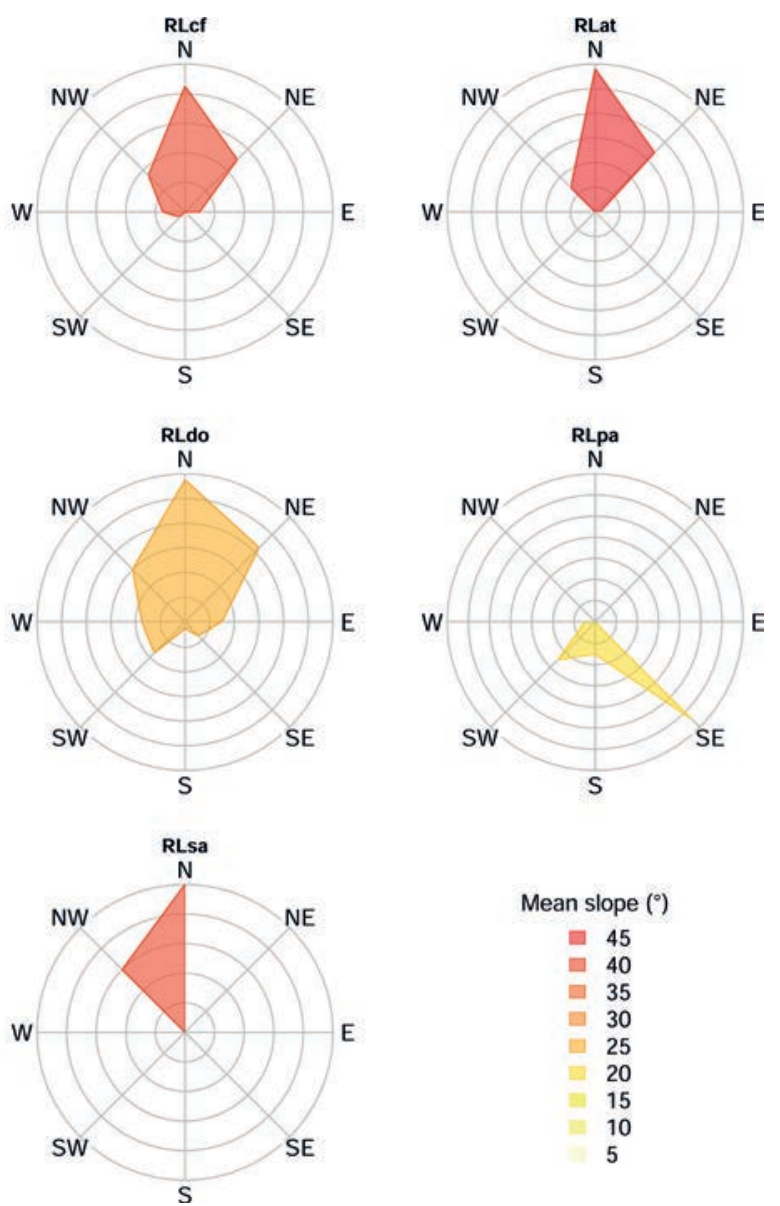

mite with an admixture of chert or marlstone, differential species are also Stellaria nemorum, Senecio ovatus and Helleborus niger) and a more basophilous var. Laserpitium peucedanoides (the differential species is also Pulsatilla alpina subsp. austroalpina). The relevés of the first subvariant are mainly from the Karavanke Mts. (Mala Peca, Raduha, Olševa, Uršlja Gora, Završnica, Begunjščica) and are, at least in part, also secondary; some of them may have developed on sites of the subalpine spruce forest - e.g. the stands under Mt. Mala Peca (Zupančič 1999, Dakskobler et al. 2013). The relevés of the second subvariant are from the Julian Alps (the valleys of Krma and Lopučnica, Stara Fužina pasturelands). The differential species of var. Festuca nitida include Carex ferruginea and Campanula cochleariifolia, which also indicate rockier, but still moist sites, and partly also Deschampsia cespitosa, which indicates a probable impact of small ruminants grazing in the past. The variant can be subdivided into two subvariants. Characteristically, the parent material on sites of the stands of the subvariant with Luzula lu- 
zuloides, which prevail in the Kamnik-Savinja Alps and in the Karavanke Mts. (Raduha, Olševa, Strelovec, Mala Peca), is interlayered with marlstone or chert, whereas the stands of the subvariant with Campanula scheuchzeri (the differential species is also Aconitum lycoctonum subsp. ranunculifolium $=$ A. lupicida) predominate in the Julian Alps (especially Macesnovec above the Kot valley and Sleme above Mala Pišnica valley).

\subsubsection{Rhodothamno-Laricetum sorbetosum chamaemespili (Column 2 in Table 26)}

Table 4 (Column 3 in Appendix Table 2 and Table 27) comprises the relevés that are, in terms of floristic composition, transitional between the stands of the subassociation geetosum rivalis and stands of the central subassociation sorbetosum chamaemespili. They mainly occur on promontories and slopes on or above the upper beech line, on slopes that are difficult to access and therefore hardly or not at all disturbed by man, predominantly on shady, steep and, due to the persistent snow cover, rather moist sites, on rendzinas with poorly decomposed organic matter. They still comprise a relatively large proportion of species of the class Mulgedio-Aconitetea, but compared with the stands of the subassociation geetosum rivalis also a substantially larger proportion of species of the class Vaccinio-Piceetea. Fidelity analysis suggests Calamagrostis villosa, Lycopodium annotinum, Vaccinium vitis-idaea and V. myrtillus as relative differential species, although they are frequent also in some other subasociations. These stands, with most of the relevés made in the Karavanke and the Kamnik-Savinja Alps, several also in the Julian Alps, are classified into the subassociation sorbetosum chamaemespili, the variant with Calamagrostis villosa. Similarly to Luzula sylvatica, which is indicated by the indicator species analysis, Calamagrostis villosa is also only a relative differential species of the variant because of its significant cover, and both these species are present in most of the recorded larch stands. Pulmonaria stiriaca, which occurs in only two relevés, also has negligible differential value. There are some slight differences among the relevés of this variant. Conditionally, we could distinguish between two subvariants: subvar. Alnus viridis and subvar. Luzula luzuloides, where the stands of the latter are characterised by a distinctly larger proportion of acidophilous species of spruce forests. Relevés 16 and 17 are atypical for this variant, but were assigned to it through the classification method.

Table 5 (Column 4 in Appendix Table 2 and Table 27) comprises the relevés classified into the syntaxon Rhodothamno-Laricetum sorbetosum chamaemespili var. Adenostyles glabra. Adenostyles glabra is also only a relative differential species of the variant as it is widely distributed in larch forests. With its considerable constancy and cov- erage it characterises, compared to the stands of the previous variant, rockier sites with shallow rendzina, partly still in the belt of subalpine beech and spruce forests, mainly in the Julian Alps and in areas where past human impact was more profound. In addition to the typical subvariant (subvar. typica) we also distinguish the subvariant with Aconitum lycoctonum subsp. ranunculifolium (A. lupicida); another differential species is also Carex ferruginea, which occurs on slightly moister sites.

Relevés in Table 6 (Column 5 in Appendix Table 2 and Table 27) are classified into the syntaxon RhodothamnoLaricetum sorbetosum chamaemespili var. Saxifraga cuneifolia. Most of them were made on rocky, high-Karst terrain between the Soča Valley and the Komna plateau or the Triglav Lakes Valley (plateaus between the pasture of Za Skalo and Komna, and in Dol za Bajarjem under Mt. $\mathrm{Kal}$ ), in the Trenta Valley above the Zapotok pasture and on shady slopes under the ridge of Mt. Srebrnjak (Apica forest reserve), all of them in the Julian Alps. Most of these stands are located in grazing areas on or above former pastures, which means that their physiognomy was largely influenced by man; however, in the last fifty years the human impact has been negligible and very natural stands are gradually developing there. They are situated at or already above the upper beech forest line. Saxifraga cuneifolia is a relative differential species, because it has higher constancy here than in other forms. The indicators of these cold and relatively moist sites with poorly decomposed organic matter in the soil are also Calamagrostis villosa, Dryopteris dilatata, Hieracium murorum and Polystichum lonchitis. In addition to the typical subvariant (subvar. typica) we distinguish also the subvariant with Festuca nitida (the differential species include Soldanella alpina and Athyrium filix-femina), which indicates comparatively moister sites.

Table 7 (Column 6 in Appendix Table 2 and Table 27) comprises eight relevés that are typical for the subassociation Rhodothamno-Laricetum sorbetosum chamaemespili and are classified into the variant with Anemone trifolia. The indicator species analysis indicates the relative differential species Daphne mezereum, Melampyrum pratense subsp. vulgatum, Prenanthes purpurea, Vaccinium vitisidaea. This variant comprises larch stands above or just along the upper beech line that are either natural or have not been exposed to human impact for a long time, in the Julian Alps, including the Italian side, on the slopes of Cima del Cacciatore / Kamniti Lovec at Monte Santo di Lussari / Svete Višarje. Two of the stands from this mountain range stand out, because Rhododendron ferrugineum occurs there on raw humus, despite the limestone bedrock.

Table 8 (Columns 7 in Appendix Table 2 and Table 27) comprises relevés classified into the variant RhodothamnoLaricetum sorbetosum chamaemespili var. Homogyne alpina. 
Homogyne alpina is a relative differential species as it has $100 \%$ constancy. Some of the relevés were made in the areas of former high mountains pastures, most of them on very steep, shady, slightly moist slopes on extremely nutrient-poor soil (LIV analysis), where the impact of grazing on the vegetation was evident only on their edges, if at all. These relevés were made in the Julian Alps and the western Karavanke Mts. (the valley of Železnica), one relevé in the Kamnik-Savinja Alps. Site conditions in the stands of this syntaxon are indicated also by higher constancy or medium cover of Lycopodium annotinum, Pinus mugo and Rhytidiadelphus triquetrus. The listed species indicate distinct dominance of spruce species in these stands and slow decomposition of soil organic matter. The group of relevés from the Železnica valley is differentiated also by the taxon Sorbus aucuparia subsp. glabrata.

The stands in Table 9 (Column 8 in Appendix Table 2 and Table 27) are similar in terms of floristic composition, but were made mainly on high-mountain plateaus (the Lopučnica valley, pasture Klek on Pokljuka Plateau, Dleskovec Plateau / Veža in the Kamnik-Savinja Alps), on more nutrient-rich soil (LIV analysis), where the impact of grazing is still at least partly felt. At least some of these stands are secondary; they have developed at the contact with or even on sites of subalpine spruce, and in part even beech forests. This is not evident in the species composition at present, except in a few relevés. In his in-depth silvicultural analyses, Firm (2016) nevertheless determined gradually increasing recruitment of spruce from lower to higher stand layers in these stands. They are classified into the variant with Poa alpina (a relative differential species that indicates a greater impact of grazing; the indicator value analysis reveals only the differential species Sorbus chamaemespilus and Alchemilla vulgaris agg.) and distinguished from the stands in Table 8 by a higher spruce cover and slightly more frequent occurrence of beech (which also confirms that they are likely to be secondary) as well as by very rare occurrence of Anemone trifolia.

Table 10 (Column 9 in Appendix Table 2 and Table 27) comprises relevés which are differentiated from the stan$\mathrm{ds}$ of the syntaxon Rhodothamno-Laricetum sorbetosum chamaemespili var. Anemone trifolia by a substantially higher cover of Pinus mugo in the shrub layer. These relevés characterize a typical form of this subassociation, which is classified into the variant Rhodothamno-Laricetum sorbetosum chamaemespili var. Pinus mugo. The indicator value analysis shows average ecological conditions in all of the recorded larch stands. We identified the largest and the tallest larch trees here as well as the highest percentage of the shrub layer cover from among all determined syntaxa. The indicator species analysis indicates two relative differential species, Aposeris foetida and Erica carnea. Even though these stands slightly differ from one another and some of them indicate the contact of larch stands with subalpine beech forests (subvar. Fagus sylvatica), they are similar (in particular relevés 8 to 35) in that they were made in areas where human impact, if any, is very low. We estimate that on some of the sites (Macesnje above the Beli Potok valley, Prednja Glava under Prisojnik, Votlo Sleme, perhaps also Robičje and Požgana Mlinarica) these are primary forests which, at least in the case of Macesnje, could be considered virgin forest. Some of the relevés are also from the Karavanke Mts. and the Kamnik-Savinja Alps. This is the characteristic vegetation structure of natural larch forests on steep and very difficult to access promontories above Alpine valleys that is currently subject only to indirect human impact, such as air pollution.

Table 11 (Column 10 in Appendix Table 2 and Table 27) comprises a group of slightly heterogeneous relevés from the Julian Alps, the Karavanke and the Kamnik-Savinja Alps that belong to the central subassociation, but are slightly different from its typical variant. They show evident human impact, although long past (forest reserve Kalce in the Kamnik-Savinja Alps), or occur as slightly pioneer forms in high-mountain cirques (e.g. in Carnizza di Rio Zapraha / Carnizza di Camporosso / Žabniška Krnica cirque above Zajzera in the Italian part of the Julian Alps). These stands are classified into the variant with Calamagrostis varia; with its high coverage, this is only a relative differential species, but is widely distributed in larch forests.

Table 12 (Column 11 in Appendix Table 2 and Table 27) comprises fewer relevés, which in hierarchical classification grouped separately from the relevés of the typical variant of the central subassociation. They were made in the Julian and Kamnik-Savinja Alps and in the Karavanke Mts., in areas where organic matter and raw humus accumulate, which indicates a high blueberry and cranberry cover. They are treated as the variant with Rubus saxatilis, which is only a relative differential species owing to its high constancy (100\%). The indicator species analysis indicates humus-rich and moist sites characterised also by Athyrium filix-femina, Cardamine enneaphyllos, Centaurea montana, Cystopteris montana, Dryopteris expansa, Gymnocarpium dryopteris, Maianthemum bifolium, Melica nutans, Oxalis acetosella and Vaccinium myrtillus.

\subsubsection{Rhodothamno-Laricetum cystopteridetosum fragilis (Column 3 in Table 26)}

Syntaxonomic classification of larch stands in Table 15 (Column 14 in Appendix Table 2 and Table 27) is slightly problematic. In terms of floristic similarity these stands evidently group with relevés of the subassociation anemonetosum trifoliae, but Sesleria caerulea no longer has such large cover and abundance. These are very rocky, 
steep and rather moist sites in the subalpine belt of the Julian Alps, characterised by higher abundance and medium cover of Saxifraga cuneifolia, Luzula nivea, Alnus viridis, Cerastium subtriflorum, Poa alpina, Campanula scheuchzeri, Saxifraga aizoides, Cystopteris fragilis, Festuca calva, Heliosperma pusillum and Paederota lutea than other comparable larch forest forms, whereas the indicator species analysis (IndVal and phi value) identified also $G a-$ lium pumilum, Bartramia halleriana, Polypodium vulgare, Conocephalum conicum, Ranunculus platanifolius, Saxifraga tenella and several others. LIV indicate that these are, in addition to the sites of the variant geetosum rivalis var. Adenostyles alliariae, the moistest sites in the studied larch forest types. These stands are classified into the new subassociation Rhodothamno-Laricetum cystopteridetosum fragilis. Cystopteris fragilis is a relative differential species because of a higher constancy than it has in other forms of larch forests. It is a character species of chasmophytic communities on moist rock fissures.

\subsubsection{Rhodothamno-Laricetum anemonetosum trifoliae (Column 4 in Table 26)}

The dendrogram in Figure 5 shows a small cluster of relevés demonstrating higher dissimilarity with the stands of the central subassociation. There are no species specifically indicating this, at least not in that they are not present in other forms of the association Rhodothamno-Laricetum. Nevertheless, ecological differences between the sites allow classification into two subassociations: RhodothamnoLaricetum cystopteridetosum fragilis and RhodothamnoLaricetum anemonetosum trifoliae. These comprise larch forests on very steep and mainly shady, rocky slopes with shallow rendzina, in places almost lithosol, on sites that are extreme in terms of forest growth, mainly still in the belt of the altimontane and subalpine beech or fir-beech forests. Tables 13 and 14 (Columns 12 and 13 in Appendix Table 2 and Table 27) comprise stands of the subassociation anemonetosum trifoliae, where the herb layer is characterised by a high cover of Sesleria caerulea, which is a relative differential species of the subassociation, as are also Primula auricula, Carex brachystachys and Saxifraga crustata. The name of the subassociation anemonetosum trifoliae is merely a result of the fact that the releve which we selected in 2006 as the nomenclatural type of the subassociation anemonetosum trifoliae was also included in this group, and according to the rules of the Code (Weber et al. 2000), we must retain the name associated with it (a more appropriate name would be seslerietosum caeruleae).

Compared to the central subassociation (sorbetosum chamaemespili) the proportion of species of spruce forests is considerably smaller on account of a higher proportion of the species of classes Elyno-Seslerietea, Thlaspietea rotun- difolii and Asplenietea trichomanis. These stands are partly secondary, as they are distributed also in areas where the surrounding beech and fir-beech forests used to be heavily cut. Due to their distinctly protective role there have not been any recent human interventions into these forests. Relevés of the typical variant (var. typica) are mainly from the Julian and Kamnik-Savinja Alps (Table 13). Among the four main groups of syntaxa the stands of this variant occur on the steepest and distinctly shady (N-NE) slopes, on the most nutrient-poor sites and the airiest and most alkaline (or the least acidic) soils. They are characterised by a low shrub layer cover and average elevation of around $1,400 \mathrm{~m}$. The species that have a certain differential value for this syntaxon are also Hedysarum hedysaroides, Paederota lutea and Valeriana saxatilis. A group of relevés in the left part of Table 13 (subvar. Sorbus aria) are slightly similar also to the stands of the subassociations ostryetosum carpinifoliae and sorbetosum ariae (differential species are diagnostic species of the order Quercetalia pubescenti-petraeae and taxon Hedysarum hedysaroides subsp. exaltatum).

The relevés in Table 14 are classified into the same subassociation, the variant with the species Festuca nitida, indicating rocky and slightly moist sites in the Karavanke Mts. and the Julian Alps, mainly in the subalpine belt, on the upper beech line. Compared to the relevés of the stands of the typical variant they occur on nutrient-richer soils. Differential species of the variant include Carex ferruginea and Saxifraga rotundifolia. The indicator species analysis indicates also the following relative differential species: Adenostyles glabra, Asplenium viride, Erysimum sylvestre, Galium laevigatum, Gymnocarpium robertianum, Homogyne sylvestris, Moneses uniflora, Pseudorchis albida and Rumex scutatus. We distinguish two subvariants: subvar. Heliosperma alpestre on gravelly sites on dolomite and subvar. Anemone trifolia on limestone sites.

\subsubsection{Rhodothamno-Laricetum cyclaminetosum purpurascentis (Column 5 in Table 26)}

Table 17 (Column 16 in Appendix Table 2 and Table 27) comprises relevés of larch stands from the Julian and Kamnik-Savinja Alps and the eastern Karavanke Mts. They typically occur on sunny steep slopes on dolomite limestone and dolomite, in the subalpine belt, along or above the upper beech line. Landolt's indicator values indicate rather dry sites with alkaline soils and diminished species diversity. In part, these stands could also be secondary, having developed on former pastures through succession. Although human impact has not been evident in the past decades, these stands are nevertheless exposed to fires due to their sunny and dry exposition (e.g. extensive larch stands under the ridge of Monte Cimone / Strma Peč above Val Raccolana / Reklanica). In terms of 
floristic composition they differ from other larch stands described in this paper in that they have a substantially larger proportion of species of classes Erico-Pinetea and Elyno-Seslerieted and a significantly smaller proportion of species of the class Mulgedio-Aconitetea. They are classified into the new subassociation Rhodothamno-Laricetum cyclaminetosum purpurascentis. The differential species of the new subassociation are Cyclamen purpurascens, Betonica alopecuros, Erica carnea and Calamagrostis varia (the latter two especially due to their significant cover value), indicators of these sites are also Clinopodium vulgare, Euphorbia cyparissias, Galium lucidum, Genista radiata and several others. The fidelity analysis suggests also Globularia cordifolia and Valeriana montana as differentials. In addition to the typical variant we also distinguish the variant with Rhodothamnus chamaecistus (Table 18, Column 17 in Appendix Table 2 and Table 27). Some of its stands occur also on shady slopes, i.e. on comparatively colder sites; they are characterised by substantially higher abundance and medium cover of dwarf pine (Pinus mugo), in part also by Carlina acaulis and Carex mucronata. Relevés 14-16 in Table 18 are floristically and ecologically slightly different; for now, they are still discussed under this syntaxon (cyclaminetosum purpurascentis var. Rhodothamnus chamaecistus) as a special subvariant with Homogyne discolor (its diffrential species are also Juncus monanthos and Dryas octopetala, which suggests a similarity with the stands of the subassociation dryadetosum octopetalae).

\subsubsection{Rhodothamno-Laricetum var. Thymus} polytrichus (Column 6 in Table 26)

Table 19 comprises a small group of very diverse relevés. In particular relevés 1 to 4 differ significantly among each other; they are provisionally treated at the rank of the association Rhodothamno-Laricetum and were not incorporated into the synoptic table. These relevés could be classified into the syntaxon Rhodothamno-Laricetum sorbetosum chamaemespili var. Rubus saxatilis, although they did not group with its relevés. Relevés $5-12$ in the same table (Column 18 in Appendix Table 2 and Table 27) are very similar; all of them have a large cover of Erica carnea in the herb layer and relative differential species include Daphne striata and Thymus praecox subsp. polytrichus. They are slightly similar to the relevés of the syntaxon Rhodothamno-Laricetum cyclaminetosum var. Rhodothamnus chamaecistus, but are for the time being treated only at the rank of the ecological variant Rhodothamno-Laricetum var. Thymus polytrichus. They characterise larch forests in the Julian Alps on sunny slopes and shallow rendzinas in the subalpine belt (above the beech belt); one relevé was made on a very steep shady slope under Škrbina in the Kamnik-Savinja Alps, still in the altimontane belt.

\subsubsection{Rhodothamno-Laricetum dryadetosum octopetalae (Column 7 in Table 26)}

Tables 20, 21 and 22 (Columns 19-21 in Appendix Table 2 and Table 27) comprise stands of larch forests on the upper forest line, most of them in the Julian Alps, mainly on high-Karst plateaus and in glacial cirques. The soil is shallow, lithosol or rendzina. The development and structure of these stands were partly the result of past use, in particular grazing, and even more of the abundant snow cover and frequent snow avalanches. Most of the stands at present are pioneer, some may have developed as late as in the last seventy to one hundred years. Larch is at its upper line of occurrence, with relief and snow avalanches preventing it from spreading to higher elevations. Stands are very open, at the contact with subalpine grasslands. This is reflected also in the species composition, which has the lowest proportion of species from the class Vaccinio-Piceeted and the highest proportion of species of classes Elyno-Seslerietea (species with a significant phi value are Homogyne discolor, Polygonum viviparum, Bartsia alpine, Juncus monanthos) and Thlaspietea rotundifolii (significant phi value: Cirsium spinosissimum, Biscutella laevigata) compared to other types of larch stands. These stands are classified into the new subassociation Rhodothamno-Laricetum dryadetosum octopetalae, whose differential species is a character species of stony alpine swards, Dryas octopetala. The syntaxon var. typica (Table 20) comprises relevés from the Mala Pišnica valley, Carnizza di Riofreddo, Carnizza di Camporosso / Trbiška and Žabniška Krnica, Lopučnica valley and the cirque of Za Akom. In addition to the differential species of the subassociation, the following character species of alpine grasslands and screes also frequently occur in its stands: Carex firma, C. sempervirens, Juncus monanthos, Homogyne discolor, Heliosperma alpestre, Biscutella laevigata, with a lower constancy also a number of other species. We distinguish two subvariants: subvar. Carex firma (on more initial soils) and subvar. Ranunculus carinthiacus (on more developed soils differential species include Leontodon hispidus, Campanula scheuchzeri and Poa alpina).

Relevés of the stands of the variant with Juniperus sibirica (Table 21) are from the upper part of the Triglav Lakes Valley, the upper forest line. They typically occur on gentle slopes and have a high medium cover of Rhododendron hirsutum, Rhodothamnus chamaecistus and Juniperus sibirica; also abundant are the species of alpine swards and screes: Heracleum austriacum subsp. siifolium, Pulsatilla alpina subsp. austroalpina, Helianthemum nummularium subsp. grandiflorum, Astrantia bavarica, Dryopteris villarii, Festuca nigrescens (as a result of admixed marlstone), with lower constancy also many other species. Compared to the typical variant these sites are even more extreme and 
their stands are one of the most initial forms of natural larch forests. The sites of the stands of the variant with Daphne mezereum (Table 22) are slightly more mesophilous and have better developed soils, so they comprise more diagnostic species of the class Vaccinio-Piceetea. Two subvariants are distinguished in the stands of this variant. The stands of the subvariant with Aconitum lycoctonum subsp. ranunculifolium (A. lupicida) occur mainly in Dol za Bajarjem under Mt. Kal (the differential species is also Festuca nigrescens). Stands of the subvariant with Parnassia palustris are characteristic for more extreme sites on shady aspects. Its differential species include Tofieldia calyculata, Bartsia alpina and Pedicularis verticillata.

\subsubsection{Rhodothamno-Laricetum piceetosum abietis (Column 8 in Table 26)}

Table 16 (Column 15 in Appendix Table 2 and Table 27) comprises relevés of larch forests with a significant proportion of spruce. These stands were recorded in the Julian Alps: the Lopučnica valley, Zgornja Komna plateau, Stara Fužina pasturelands (Ovčarija pasture), the northern part of the Pokljuka Plateau, on relatively nutrientrich sites on limestone. The impact of past management, clear-cutting and grazing is still evident here and in part these are still the sites of subalpine spruce forest from the association Adenostylo glabrae-Piceetum. Nevertheless, their present species composition does not allow for their classification into the subassociation Adenostylo glabraePicetum laricetosum (Zupančič 1999), with the exception of relevé 1 (Karavanke Mts., Hrašenska Planina below Mt. Belšcica), so they are classified into the new subassociation Rhodothamno-Laricetum piceetosum abietis. The relative differential species of the subassociation are Picea abies, Daphne mezereum, Anemone nemorosa and Rhodiola rosea, but according to the indicator species analysis numerous other species also have a certain differential value, which indicates certain differences compared to the other described syntaxa. We distinguish two variants, a more mesophilous var. Adoxa moschatellina (its stands are somewhat similar to the stands of the subassociation adoxetosum moschatellinae) and var. Knautia longifolia (open stands on more acidophilous sites).

\subsubsection{Rhodothamno-Laricetum adoxetosum moschatellinae (Column 9 in Table 26)}

Larch stands in Table 23 (Column 22 in Appendix Table 2 and Table 27) mainly occur on the high-mountain plateau of Veža (Dleskovec Plateau) in the Kamnik-Savinja Alps, only two relevés are from the Julian Alps. They are predominantly secondary and have at least partly developed on the sites of subalpine spruce and beech forests. Soil conditions are considerably more favourable than in the stands of previously described syntaxa. They are classified into the new subassociation Rhodothamno-Laricetum adoxetosum moschatellinae. The differential species of the subassociation are Adoxa moschatellina, Myosotis sylvatica agg., Ajuga pyramidalis, Knautia drymeia, Circaea alpina and Veronica chamaedrys, but numerous other species also have certain differential value. Favourable site conditions and good nitrogen supply are reflected also in the composition by groups of diagnostic species, especially the high proportion of beech forests (from alliances Aremonio-Fagion and Tilio-Acerion and order Fagetalia sylvaticae) and species of the class Mulgedio-Aconitetea. In such site conditions larch can establish itself as a pioneer on abandoned pastures, fire sites or windbreak areas, and is subsequently gradually superseded by more demanding species.

\subsubsection{Rhodothamno-Laricetum ostryetosum carpinifoliae (Column 10 in Table 26)}

Table 24 (Column 23 in Appendix Table 2 and Table 27) comprises relevés of the previously described association Rhodothamno-Laricetum ostryetosum (Dakskobler 2006). In our new comparison, some of its relevés group also with the relevés of the subassociation anemonetosum trifolia (Table 13). Our table comprises only the relevés from the Soča Valley and shady slopes of the Jelovica Plateau above the Sava Bohinjka Valley, but similar larch stands with Ostrya carpinifolia, Fraxinus ornus and Sorbus aria on very steep rocky shady slopes in the montane and altimontane belt occur also in the Kamnik-Savinja Alps, especially in the Kamniška Bistrica valley (Tregubov 1962 treated them as syntaxon Erico-Ostryetum laricetosum). The differential species of the subassociation are above all Ostrya carpinifolia, Sorbus aria (Aria edulis), Fraxinus ornus, Laburnum alpinum, Neckera crispa, Amelanchier ovalis, Campanula cespitosa and Cotoneaster tomentosus, but there are other species with a certain differential value as well, for example Polygala chamaebuxus, Primula auricula and Carex brachystachys. We distinguish two variants, a more thermophilous var. Buphthalmum salicifolium (differential species are diagnostic species of the classes Festuco-Brometea and Trifolio-Geranietea) and a more acidophilous variant with Melampyrum pratense subsp. vulgatum (characterized by a higher proportion of diagnostic species of the class VaccinioPiceetea and more moss species).

\subsubsection{Rhodothamno-Laricetum sorbetosum ariae (Column 11 in Table 26)}

Table 25 (Column 24 in Appendix Table 2 and Table 27) comprises eight relevés of larch forests on extreme sites in the belt of pre-Alpine fir-beech forest. They are different from the stands of the subassociation ostryetosum and cannot be classified within it because they have a substantially 
higher proportion of species of spruce forests (VaccinioPiceetea) and a significantly lower proportion of species of the order Quercetalia pubescenti-petraeae, although they occur on similar sites and share several differential species (Calamagrostis arundinacea, Phegopteris connectilis, Prenanthes purpurea, Laburnum alpinum, Sorbus aria = Aria edulis, partly Ostrya carpinifolia). They are slightly similar to the stands of the subassociation Homogyno sylvestris-Fagetum laricetosum (Dakskobler 2002), which is indicated by individual beech trees occurring in almost all stand layers. Based on the entire species composition they are classified into the new subassociation Rhodothamno-Laricetum sorbetosum ariae, whose differential species are Sorbus aria, S. aucuparia and S. chamaemespilus. These sites are characterised by joint occurrence of three species from the genus Sorbus and by the occurrence of Dicranum scoparium, Calamagrostis arundinacea, Lonicera alpigena and other species.

\subsection{The characteristic combination of constant species, character and differential species of the association Rhodothamno- Laricetum}

Based on the analysis of 110 relevés we determined a total of 478 taxa in the studied community (410 vascular plants, 68 mosses and lichens). The average number of species per relevé was 71 . However, according to the most recent analysis (Column 25 in Appendix Table 2), the total number of taxa is 673 (583 vascular plants, 86 mosses and lichens and 4 fungi) and the average number of species per relevé is 69 .

The comparison between the analysis of the characteristic combination of constant species (species with a constancy of $40 \%$ or higher) based on 110 relevés from the Julian Alps alone (Dakskobler 2006) and the analysis that was based on 458 relevés (Table 28) showed several minor differences. Most of the species occur on both lists. The new list does not comprise several species from the previous list (Lonicera alpigena, Primula auricula, Huperzia selago, Cyclamen purpurascens, Luzula nivea, Maianthemum bifolium, Saxifraga cuneifolia, Selaginella selaginoides, Valeriana saxatilis, Cystopteris fragilis, Polytrichum formosum and Fissidens dubius), but comprises several new species (Aposeris foetida, Heliosperma alpestre, Lycopodium annotinum, Dryopteris dilatata, Astrantia bavarica, Veratrum album subsp. lobelianum, Festuca nitida, Gymnocarpium dryopteris, Athyrium filix-femina, Melampyrum sylvaticum, Saxifraga rotundifolia, Polygonatum verticillatum and $\mathrm{Ca}$ rex ferruginea). The difference in constancy based on the analysis that was made for a significantly higher number of relevés is only a few percent for some species, but becomes more evident in certain other species (such as Primula auricula, Valeriana saxatilis and Luzula nivea). This deviation is not so much the result of the higher number of relevés, but of the wider geographical area and site range (in the first analysis, the stands of extreme sites in the forest belt were better represented in the complete set of relevés). The new set of relevés comprises a higher proportion of relevés of larch stands on high-mountain plateaus on less extreme sites. The recently determined characteristic combination of constant species comprises 51 vascular plants ( $9 \%$ of the total number of recorded plants) and 5 mosses.

Based on a substantially higher number of relevés we examined also the original selection of character and differential species: Larix decidua, Rhododendron hirsutum, Rhodothamnus chamaecistus, Valeriana saxatilis, Primula auricula and Carex brachystachys, and geographic differential species Anemone trifolia, Paederota lutea, Laserpitium peucedanoides, Luzula nivea, Homogyne sylvestris, Astrantia carniolica and Campanula carnica. From among the listed species we eliminated Primula auricula (constancy 21\%) and Carex brachystachys (constancy 12\%), which are good indicators of more extreme forms of larch stands. Their total constancy is too low, however, so we replaced them with Heliosperma alpestre and Carex ferruginea. The character species of the association Rhodothamno-Laricetum in the Southeastern Alps comprise also Laserpitium peucedanoides and Paederota lutea, and geographic differential species with higher constancy are Homogyne sylvestris, Astrantia bavarica, Festuca nitida and Anemone trifolia. Our analysis indicated that it would be more suitable to use Paederota lutea than Anemone trifolia for the name of the southeastern-Alpine geographical variant. It has a much higher constancy and is distributed in the major part of the Southeastern Alps, whereas Anemone trifolia is very rare in certain parts of the distribution area (KamnikSavinja Alps, eastern Karavanke). Nevertheless, according to the rules of the applicable Code (Weber et al. 2000) the name does not need changing if one species characterises a syntaxon better than the already selected species.

\subsection{Species composition of the southeastern-Alpine larch stands by groups of diagnostic species}

Table 27 (Column 25) demonstrates that species of spruce forests (class Vaccinio-Piceetea) have the largest proportion in the southeastern-Alpine larch community and account for $24 \%$ of all species, character species of basophilous pine forests (Erico-Pinetea) constitute around 8\%, diagnostic 
species of beech and beech-oak forests (Aremonio-Fagion, Fagetalia sylvaticae, Querco-Fagetea) contribute around $12 \%$, diagnostic species of tall herbs and subalpine shrub communities (Mulgedio-Aconitetea, Betulo-Alnetea) and subalpine-alpine calcareous swards (Elyno-Seslerietea) more than $10 \%$ of the species. Diagnostically significant, with more than 5\% are also the groups of character species of screes (Arabidetalia caeruleae, Thlaspietea rotundifolii) and chasmophytic species (Physoplexido comosae-Saxifragion petraeae, Potentilletalia caulescentis, Asplenietea trichomanis).

\subsection{Verification of the hypotheses stated in the introduction}

In general, the floristic composition and structure of the studied larch forests does not show distinct differences between the primary larch stands on ledges and prominences above the upper beech forest line and pioneer (secondary) larch forests that are presumably a succession stage on former pastures on high-mountain plateaus, still in the belt of subalpine beech and (or) spruce forests. Nevertheless, by means of hierarchical classification we were able to identify several groups of relevés that corroborate the assumed or evident secondary origin of these stands (a stage in development in the secondary succession). This applies mainly to the relevés of the syntaxa RhodothamnoLaricetum geetosum rivalis var. Adenostyles alliaria subvar. Luzula luzuloides, Rhodothamno-Laricetum piceetosum abietis and Rhodothamno-Laricetum adoxetosum moschatellinae, to a lesser extent also to the relevés of the syntaxa Rhodothamno-Laricetum sorbetosum chamaemespili var. Poa alpina and Rhodothamno-Laricetum cyclaminetosum purpurascentis var. typica.

In terms of floristic composition the larch stands in the Julian Alps are not significantly different from larch stands in the Kamnik-Savinja Alps and the Karavanke Mts. Relevés from the Karavanke and the Kamnik-Savinja Alps grouped mainly with the relevés from similar sites in the Julian Alps. Androsace lactea, which could be the geographic differential species for the stands in the Kamnik-Savinja Alps and eastern Karavanke, occurs only in 11 relevés that usually grouped with the relevés from the Julian Alps. Relevés from the eastern Karavanke Mts. and the Kamnik-Savinja Alps grouped together in the case of syntaxa Rhodothamno-Laricetum geetosum rivalis var. Adenostyles alliariae subvar. Luzula luzuloides, RhodothamnoLaricetum geetosum rivalis var. Festuca nitida subvar. Luzula luzuloides and Rhodothamno-Laricetum adoxetosum moschatellinae.

Hierarchical classification showed evident differences in the species composition between larch stands on ex- treme sites in the belt of montane-altimontane beech and fir-beech forests (syntaxa Rhodothamno-Laricetum anemonetosum trifoliae, cystopteridetosum fragilis, ostryetosum carpinifoliae, sorbetosum ariae) and larch stands on the upper forest line (most of the other syntaxa described), as well as the differences between the stands of mature development stages (sorbetosum chamaemespili, geetosum rivalis) and initial forms (dryadetosum octopetalae).

The characteristic combination of constant species based on 458 relevés did not change considerably compared to the analysis based on 110 relevés. For the most part, the diagnostic species selected in 2006 pass the test based on the analysis of 458 relevés. Due to insufficient constancy we excluded only Primula auricula and Carex brachystachys, but added Carex ferruginea, Heliosperma alpestre, Astrantia bavarica and Festuca nitida to the diagnostic species of the association.

\section{Conclusions}

By analysing a large number of relevés (458) of larch forests in the Southeastern Alps by means of hierarchical classification (UPGMA) we determined that despite relatively insignificant differences in site conditions and similar physiognomy, they can be subdivided into many lower syntaxonomic units. Due to a different classification method compared to 2006, only the relevés of larch stands on extreme sites in the montane-altimontane belt group together in the subassociation Rhodothamno-Laricetum anemonetosum trifoliae, whereas larch stands in the subalpine belt on less extreme sites are grouped in the new subassociation Rhodothamno-Laricetum sorbetosum chamaemespili (syn. anemonetosum trifoliae var. Sorbus chamamemespilus pro parte), which comprises typical larch stands in the Southeastern Alps (var. geogr. Anemone trifolia). We also described the following new subassociations: geetosum rivalis, piceetosum abietis, adoxetosum moschatellinae, cystopteridetosum fragilis, dryadetosum octopetalae, cyclaminetosum purpurascentis and sorbetosum ariae, and within them numerous new variants and subvariants. This classification method proved to be appropriate, although some of the relevés that stood out with their species composition, could only be classified at the association level. This allowed us to relatively successfully identify the stands that are, at least in part, secondary, i.e. probable pioneer stages on sites of subalpine spruce and (or) beech communities (stands of the subassociations piceetosum, adoxetosum moschatellinae), and to effectively distinguish between larch stands on extreme sites in the belt of beech and fir-beech forests (anemonetosum trifoliae, ostryetosum carpinifoliae, sorbetosum ariae) and larch 
stands on the upper forest line (dryadetosum octopetalae). Our analysis confirmed that these forests are among the best preserved forests in Slovenia and the least subjected to direct human impact. Some of them, which is the most evident in Macesnje above the Beli Potok valley (perhaps also several stands on prominences and ledges above valleys of Tamar, Velika and Mala Pišnica, Vrata, Kot and Krma, above the cirque of $\mathrm{Za}$ Akom, on ledges below Mt. Raduha), are virgin stands and as such extremely important in the research of natural forests on the upper forest line in the Southeastern and Eastern Alps.

The eastern-Alpine larch community (RhodothamnoLaricetum) belongs to Natura 2000 habitat type 9420 Alpine Larix decidua forests. In Slovenia it populates at least 3,500 ha (Dakskobler et al. 2010, Šilc et al. 2017) as well as large surface areas in the western Julian Alps in northeast Italy (Del Favero et al. 1998: 174); it also occurs in the subalpine belt on the northern slopes of the Karavanke Mts. in Austria (Hartl et al. 2001: 46).

These are mainly protective forests and their commercial value is therefore negligible. In Slovenia, they are a constituent part of the following forest reserves: Grušnica, Pod Sopotom, Apica, Kukla, Savica-Ukanc, Trstje-Tosc, Mala Pišnica, Železnica, Za Vršičem, Belca, Smrajka, Vršič-Za Akom, Mrzle konte, Podni v Makekovi Kočni, Hude stene, Kalce, Bela-Dol-Sedelšček, Poljšak, Matkov kot-Logarska dolina (Mlinšek et al. 1980, Marenče 2003), as well as one of the most distinct forest types in the Triglav National Park (Dakskobler 2015 b, c), the Logarska dolina (Logar Valley) Landscape Park and the Topla Landscape Park. They are a habitat of the wood grouse (Tetrao urogallus) and the black grouse (Tetrao tetrix). Light-filled larch stands are also the sites of the lady's slipper orchid (Cypripedium calceolus), one of the species of European conservation interest, while certain spots at the contact of rocks and talus provide the sites for three other Natura 2000 plant species: Aquilegia iulia, Campanula zoysii and Moehringia villosa (Dakskobler et al. 2016). Larch forest species comprise also several Red List species (Anon. 2002): Betula pubescens subsp. carpatica, Cortusa matthioli, Crepis bocconi, Linnaea borealis, Ranunculus alpestris, Rhododendron ferrugineum and Veratrum nigrum, as well as several protected species (Anon. 2004): Arctostaphylos uva-ursi, Coeloglossum viride, Convallaria majalis, Cyclamen purpurascens, Dactylorhiza fuchsii, Dianthus hyssopifolius (D. monspessulanus), D. sternbergii, Epipactis atrorubens, E. helleborine, Gentiana lutea subsp. symphyandra, G. lutea subsp. vardjanii, G. pannonica, Gymnadenia odoratissima, G. conopsea, Helleborus niger, H. odorus, Huperzia selago, Iris graminea, Lilium martagon, L. carniolicum, Listera cordata, L. ovata, Leontopodium alpinum, Lycopodium annotinum, Pinguicula alpina, Platanthera bifolia, P. chlorantha, Primula auri- cula, Pulsatilla alpina subsp. austroalpina and Pseudorchis albida, rare tree fungi Laricifomes officinalis and Laetiporus huroniensis, and a lichenized fungus Letharia vulpina.

\section{Povzetek}

\section{Fitocenološka analiza macesnovih gozdov $\mathbf{v}$ Jugovzhodnih Alpah}

$\mathrm{Z}$ analizo velikega števila popisov (458) macesnovih gozdov v Jugovzhodnih Alpah (Julijskih Alpah, Karavankah, Kamniško-Savinjskih Alpah) s hierarhično klasifikacijo (UPGMA) smo dopolnili dosedanje objave o teh gozdovih v Sloveniji. Ugotovili smo, da je klub razmeroma majhnim razlikam $\mathrm{v}$ rastiščnih dejavnikih in podobnemu zunanjem videzu mogoča členitev na precej nižjih sintaksonomskih enot. Že prej opisanim subasociacijam (anemonetosum trifoliae, ostryetosum carpinifoliae, linnaeetosum borealis in saxifragetosum paniculatae) smo dodali naslednje nove subasociacije: geetosum rivalis, sorbetosum chamaemespili (sin. anemonetosum trifoliae var. Sorbus chamaemespilus pro parte), piceetosum abietis, adoxetosum moschatellinae, cystopteridetosum fragilis, dryadetosum octopetalae, cyclaminetosum purpurascentis in sorbetosum ariae, ob tem pa še številne nove variante in subvariante. Pri opisih novih subasociacij in variant smo uporabili pojem relativna razlikovalnica. Pomeni vrsto, ki je v sestojih asociacije Rhodothamno-Laricertum navadno pogosta, a ima $\mathrm{v}$ določeni skupini popisov očitno večjo stalnost ali srednje zastiranje in jih na ta način posebej označuje. Nekatere sintaksone smo lahko poimenovali le po takih vrstah, saj razlikovalnic v smislu, da se v sestojih drugih podobnih sintaksonov sploh ne pojavljajo, ni bilo moč najti, ime typicum pa je že uporabljeno za floristično precej drugačno macesnovje v Avstriji. Hierarhična klasifikacije nam je omogočila razmeroma dobro prepoznavnost sestojev, ki so vsaj deloma drugotni, torej verjetni pionirski stadiji na rastiščih subalpinskih smrekovih in (ali) bukovih združb (sestoji sintaksonov piceetosum abietis, adoxetosum moschatellinae) kot tudi dobro razlikovanje med macesnovjem na skrajnih rastiščih $\mathrm{v}$ pasu bukovih in jelovo-bukovih gozdov in macesnovjem na zgornji gozdni meji.

Višinski razpon nahajališč, kjer smo naredili popise, je od $500 \mathrm{~m}$ do $1905 \mathrm{~m} \mathrm{~nm}$. v. (pri čemer so najvišji opaženi macesnovi sestoji na nadmorski višini 1950 m), največja gostota popisov je na nadmorski višini od $1500 \mathrm{~m}$ do $1800 \mathrm{~m}$. Razmerje med osojnimi legami (N, NE, E, NW) in prisojnimi legami $(\mathrm{S}, \mathrm{SE}, \mathrm{SW})$ je $75: 25$. Okoli $70 \%$ popisov je narejeno na pobočjih s strmino $30^{\circ}$ ali več, okoli $40 \%$ popisov pa na pobočjih s strmino $40^{\circ}$ ali več. Apnenčasta podlaga prevladuje na okoli $38 \%$ popisnih ploskev, zelo podoben odstotek popisnih ploskev ima 
geološko podlago dolomitni apnenec. Na okoli $8 \%$ popisov je $\mathrm{v}$ karbonatni geološki podlagi primes laporovca, glinavca ali roženca, na okoli $6 \%$ popisnih ploskev pa je geološka podlaga pobočni grušč ali podorno skalovje. V več kot $97 \%$ popisih je talni tip rendzina.

Macesnove sestoje razlikujemo predvsem po dveh ekoloških gradientih. Prvi je gradient nadmorske višine oz. temperature, ki je pozitivno povezan $\mathrm{z}$ variabilnostjo padavin v času vegetacijske sezone (BIO15), negativno pa $s$ trajanjem snežne odeje in zračno vlažnostjo. Drugi gradient pokaže na skupno količino padavin, ki je negativno povezan $\mathrm{z}$ variabilnostjo temperature $\mathrm{v}$ času vegetacijske sezone (BIO4). Gradient količine padavin ločuje tudi popise iz Kamniško-Savinjskih Alp od popisov v Julijskih Alpah. Variabilnost vegetacije macesnovja narašča $\mathrm{z}$ nadmorsko višino (nižja temperatura). Količina padavin in povprečne vrednosti Landoltovih fitoindikacijskih ocen (LIV) za vlažnost se ne ujemata. To je verjetno posledica zadostne količine padavin povsod, kjer uspeva macesnovje in LIV bolj pokažejo na zmožnost tal, da zadržujejo vlago. LIV za vlažnost je nasprotna tej indikaciji za kamnitost, kar to potrjuje, in je delno povezano tudi s trajanjem snežne odeje.

Macesnovi gozdovi so med najbolj ohranjenimi in človek nanje najmanj neposredno vpliva med vsemi gozdovi v Sloveniji. Nekateri od njih, najbolj očitno v Macesnju nad Belim potokom (a morda tudi nekateri sestoji na pomolih in policah nad Tamarjem, Veliko in Malo Pišnico, Vrati, Kotom in Krmo in nad krnico Za Akom, na policah pod Raduho), so pragozdni in zato izjemnega pomena za preučevanje naravnega gozda na zgornji gozdni meji v Jugovzhodnih in Vzhodnih Alpah.

V 458 popisih preučene združbe smo ugotovili skupno skupno 673 taksonov (583 praprotnic in semenk, 86 mahov in lišajev in 4 glive) in povprečno število vrst na popis 69. V značilni kombinaciji stalnih vrst (vrste s stalnostjo $40 \%$ in več) je 51 praprotnic in semenk ( $9 \%$ od skupnega števila popisanih) in 5 mahov.

Na podlagi bistveno večjega števila popisov smo preverili prvotno izbiro značilnih in razlikovalnih vrst: Larix decidua, Rhododendron hirsutum, Rhodothamnus chamaecistus, Valeriana saxatilis, Primula auricula in Carex brachystachys ter geografskih razlikovalnic Anemone trifolia, Paederota lutea, Laserpitium peucedanoides, Luzula nivea, Homogyne sylvestris, Astrantia carniolica, Campanula carnica. Med naštetimi vrstami smo izločili vrsti Primula auricula (stalnost $21 \%$ ) in Carex brachystachys (stalnost $12 \%$ ), ki dobro označujeta predvsem bolj skrajne oblike macesnovja, njuna skupna stalnost pa je prenizka, zato smo ju nadomestili z vrstama Heliosperma alpestre in Carex ferruginea. Med značilnice asociacije Rhodothamno-Laricetum $\mathrm{v}$ Jugovzhodnih Alpah uvrščamo tudi vrsti Laserpitium peucedanoides in Paederota lutea, geografske razlikovalnice $\mathrm{z}$ večjo stalnostjo pa so vrste Astrantia bavarica, Homogyne sylvestris, Festuca nitida in Anemone trifolia. Ugotovili smo, da bi bila za poimenovanje jugovzhodnoalpske geografske variante vrsta Paederota lutea primernejša od vrste Anemone trifolia. Ima precej večjo stalnost in je razširjena v večjem delu Jugovzhodnih Alp, medtem ko je vrsta Anemone trifolia $\mathrm{v}$ delih areala (Kamniško-Savinjske Alpe, vzhodne Karavanke) zelo redka. Toda po pravilih zdaj veljavnega kodeksa (Weber et al. 2000) sprememba imena, če ena vrsta bolje označuje nek sintakson, kot ta ki smo jo že izbrali, ni potrebna.

$\mathrm{V}$ jugovzhodnoalpskem macesnovju z okoli $24 \%$ prevladujejo vrste smrekovih gozdov (razred VaccinioPiceetea), okoli $8 \%$ je značilnih vrst bazoljubnih borovih gozdov (Erico-Pinetea), okoli $12 \%$ je diagnostičnih vrst bukovih in bukovo-hrastovih gozdov (Aremonio-Fagion, Fagetalia sylvaticae, Querco-Fagetea), z več kot $10 \%$ so zastopane tudi diagnostične vrste visokih steblik in subalpinskih grmišč (Mulgedio-Aconitetea, Betulo-Alnetea) in subalpinsko-alpinskih travišč na karbonatni podlagi (Elyno-Seslerietea), diagnostično pomembni, z deleži nad $5 \%$, sta tudi skupini značilnih vrst melišč (Arabidetalia caeruleae, Thlaspietea rotundifolii) in skalnih razpok (Physoplexido comosae-Saxifragion petraeae, Potentilletalia caulescentis, Asplenietea trichomanis).

\section{Acknowledgements}

The research was conducted in the framework of the target research projects Natural larch stands in Slovenia (V4-0542), Updating of the vegetation system for the forest management planning purposes (V4-1141) and The design of monitoring of the conservation status of minor Natura 2000 forest habitat types in Slovenia (V4-1430) funded by the Slovenian Research Agency and Ministry of Agriculture, Forestry and Food, and the Recharge. green project funded by the European Regional Development Fund. We acknowledge also the financial support from the Slovenian Research Agency (research core funding No. P1-0236) and from the foundation Pahernikova ustanova. We would also like to thank Prof. Dr. Andrej Martinčič for the determination of Alchemilla species. Sincere thanks to Prof. Dr. Jean-Paul Theurillat for his valuable advice in the description of new syntaxa and correction of the names of subassociations, and to Branko Zupan, Dr. Aleš Poljanec, Dr. Branko Vreš, late Dr. Miran Čas, late Iztok Mlekuž, Ivan Veber, Brane Anderle, Iztok Sajko and Kostja Jerovšek for their help in the field work. Two anonymous reviewers helped us with valuable improvements and corrections. English translation by Andreja Šalamon Verbič. 


\section{References}

Aeschimann, D., Lauber, K., Moser, D. M. \& Theurillat, J.-P. 2004a: Flora alpina. Bd. 1: Lycopodiaceae-Apiaceae. Haupt Verlag, Bern, Stuttgart, Wien, 1159 pp.

Aeschimann, D., Lauber, K., Moser, D. M. \& Theurillat, J.-P. 2004b: Flora alpina. Bd. 2: Gentianaceae-Orchidaceae. Haupt Verlag, Bern, Stuttgart, Wien, 1188 pp.

Aeschimann, D., Lauber, K., Moser, D. M. \& Theurillat, J.-P. 2004c: Flora alpina. Bd. 3: Register. Haupt Verlag, Bern, Stuttgart, Wien, $322 \mathrm{pp}$.

Anonymous 2002: Pravilnik o uvrstitvi ogroženih rastlinskih in živalskih vrst v rdeči seznam. Uradni list RS 82/2002.

Anonymous 2004: Uredba o zavarovanih prosto živečih rastlinskih vrstah. Uradni list RS 46/2004.

Braun-Blanquet, J. 1964: Pflanzensoziologie. Grundzüge der Vegetationskunde. 3. Auflage. Springer, Wien - New York, 865 pp.

Buser, S. 2009: Geološka karta Slovenije 1: 250.000. Geological map of Slovenia 1.250,000. Geološki zavod Slovenije, Ljubljana.

Chytrý, M., Tichý, L., Holt, J., Botta-Dukát, Z. 2002: Determination of diagnostic species with statistical fidelity measures. Journal of Vegetation Science 13 (1): 79-90.

Dakskobler, I. 1996: Razvoj gozda na erozijskem območju na severnih pobočjih Porezna (Julijske Alpe). Razprave 4. razreda SAZU 37 (7): 147-188.

Dakskobler, I. 2002: Jelovo-bukovi gozdovi v dolinah Kneže, Zadlaščice in Tolminke (južne Julijske Alpe, zahodna Slovenija). Razprave 4. razreda SAZU 433: 111-165.

Dakskobler, I. 2006: The Association Rhodothamno-Laricetum (Zukrigl 1973) Willner \& Zukrigl 1999 in the Julian Alps. Razprave 4. razreda SAZU 471: 117-192.

Dakskobler, I. 2015a: Rastlinstvo in rastje. In: Zorn, M. \& Kladnik, D. (eds.): Dolina Triglavskih jezer. Geografija Slovenije 32, Geografski inštitut Antona Melika, Založba ZRC, Ljubljana, pp. 59-75.

Dakskobler, I. 2015b: Gozdna vegetacija Triglavskega narodnega parka. Acta Triglavensia 3: 9-39.

Dakskobler, I. 2015c: Pregled gozdnih rastišč. In: Poljanec, A. (ed.): Gozdovi v Triglavskem narodnem parku. Ekologija in upravljanje. Zavod za gozdove Slovenije, Triglavski narodni park in Oddelek za gozdarstvo in obnovljive gozdne vire, Biotehniška fakulteta Univerze v Ljubljani, Ljubljana, Bled, pp. 17-26.

Dakskobler, I., Leban, F., Rozman, A. \& Seliškar, A. 2010: Distribution of the association Rhodothamno-Laricetum in Slovenia. Razširjenost asociacije Rhodothamno-Laricetum v Sloveniji. Folia biologica et geologica 51 (4): 165-176.

Dakskobler, I., Culiberg, M., Čas, M., Čelik, T., Firm, D., Kadunc, A., Leban, F., Kobal, M., Rozman, A., Seliškar, A., Urbančič, M. \& Vreš, B. 2010: Naravni sestoji macesna v Sloveniji: zaključno poročilo projekta ciljnega raziskovalnega programa "Konkurenčnost Slovenije 20062013”, 20082010, Biološki inštitut Jovana Hadžija, ZRC SAZU, Ljubljana, 27 pp.
Dakskobler, I., Seliškar, A. \& Podgornik, G. 2011: Razširjenost in ekologija vrste Laricifomes offcinalis (Vill.) Kotl. \& Pouzar v Julijskih Alpah (Slovenija). Gozdarski vestnik 69 (3): 139-153.

Dakskobler, I., Seliškar, A. \& Batič, F. 2011: Distribution of Letharia vulpina (lichenized Ascomycetes) in the subalpine larch stands (Rhodothamno-Laricetum) in the eastern Julian Alps (Slovenia). Hacquetia 10 (1): 95-112.

Dakskobler, I. \& Seliškar, A. 2011: A rare lichen Letharia vulpina (L.) Hue also in the western Julian Alps (Italy), in the Slovenian part of the Karavanke range and in the Savinja Alps. Folia biologica et geologica 52 (12): 83-92.

Dakskobler, I., Božič, G. \& Kraigher, H. 2011: Tehnične smernice za ohranjanje in rabo genskih virov: Macesen (Larix decidua), Slovenija. Zveza gozdarskih društev Slovenije in Silva Slovenica, Ljubljana, 8 pp.

Dakskobler, I. \& Kutnar, L. 2012: Macesnovi gozdovi v Sloveniji. Vzhodnoalpsko macesnovje, združba evropskega macesna in slečnika. Gozdarski inštitut Slovenije in Zveza gozdarskih društev Slovenije, Ljubljana, $30 \mathrm{pp}$

Dakskobler, I., Rozman, A. \& Seliškar, A. 2013: Forest and scrub communities with green alder (Alnus viridis) in Slovenia. Hacquetia 12 (2): $95-185$

Dakskobler, I., Vreš, B., Seliškar, A. \& Anderle, B. 2013:

Phytosociological characteristics of sites of Peucedanum ostruthium in the Peca Mountains (eastern Karavanke, northeastern Slovenia). Folia biologica et geologica 54 (2): 5-23.

Dakskobler, I., Kutnar, L. \& Rozman, A. 2016: Macesnovje, ruševje, zelenojelševje in druge gorske grmovne združbe v Sloveniji. Silva Slovenica, Gozdarski inštitut Slovenije, 156 pp.

De Caceres, M., \& Legendre, P. 2009: Associations between species and groups of sites: indices and statistical inference. Ecology 90 (12): $3566-3574$

Del Favero, R., Poldini, L., Bortoli, P., Dreossi, G., Lasen, C. \& G. Vanone, G. 1998: La vegetazione forestale e la selvicoltura nella regione Friuli Venezia Giulia. Vol. 2. Regione autonoma FriuliVenezia Giulia. Direzione Regionale delle Foreste, Servizio delle Selvicoltura, Udine, 303 pp. + Appendix.

Dufrene, M. \& Legendre, P. 1997: Species assemblages and indicator species: the need for a flexible asymmetrical approach. Ecological Monographs 67 (3): 345-366.

Firm, D. 2016: Gozdna sukcesija in ekologija evropskega macesna (Larix decidua Mill.) v slovenskih Alpah. Doktorska naloga. Univerza v Ljubljani, Biotehniška fakulteta, Oddelek za gozdarstvo in obnovljive gozdne vire, Ljubljana, 154 pp.

Hartl, H. Stern, R. \& Seger, M. 2001: Karte der aktualen Vegetation von Kärnten. Naturwissensschaftlichen Verein für Kärnten, Klagenfurt, 80 pp. + Vegetation map.

Jennings, M.D., Faber-Langendoen, D., Loucks, O.L., Peet, R.K., Roberts, D. 2009: Standards for associations and alliances of the U.S National Vegetation Classification. Ecological Monographs 79 (2): 173-199.

Karner, P. 2007 a: Junipero-Pinetalia mugo Boscaiu 1971. In: Willner, W. \& Grabherr, G. (eds.): Die Wälder und Gebüsche Österreichs. Ein Bestimmungswerk mit Tabellen. 1. Textband. Spektrum Akademischer Verlag in Elsevier, Heidelberg, pp. 209-218. 
Karner, P. 2007 b: Pinion mugo. In: Willner, W. \& Grabherr, G. (eds.): Die Wälder und Gebüsche Österreichs. Ein Bestimmungswerk mit Tabellen. 2. Tebellenband. Spektrum Akademischer Verlag in Elsevier, Heidelberg, pp. 248-259.

Kutnar, L. \& Dakskobler, I. 2014: Ocena stanja ohranjenosti gozdnih habitatnih tipov (Natura 2000) in gospodarjenje z njimi. Gozdarski vestnik 72 (10): 419-439.

Landolt, E., Bäumler, B. Erhardt, A., Hegg, O., Klötzli, F., Lämmler, W., Nobis, M., Rudmann-Maurer, K., Schweingruber, F. H. , Theurillat, J.-P., Urmi, E., Vust, M. \& Wohlgemuth, T. 2010: Flora indicativa. 2. Auflage. Haupt Verlag, Bern-Stuttgart-Wien, 323 pp.

Legendre, P., Anderson, M. J. 1999: Distance-based redundancy analysis: testing multispecies responses in multifactorial ecological experiments. Ecological Monographs 69: 124.

Maarel, van der E. 1979: Transformation of cover-abundance values in phytosociology and its effects on community similarity. Vegetatio 39 (2): 97-114.

Maarel, van der E., Franklin, J. 2013: Vegetation Ecology. 2nd Edition. Wiley-Blackwell. 572 pp.

Marenče, M. 2003: Gozdni rezervati v Triglavskem narodnem parku. Triglavski razgledi 7 (11): 1-24.

Martinčič, A. 2003: Seznam listnatih mahov (Bryopsida) Slovenije. Hacquetia 2 (1): 91-166.

Martinčič, A. 2011: Annotated Checklist of Slovenian Liverworts (Marchanthiophyta) and Hornworts (Anthocerotophyta). Scopolia 72: $1-38$.

Martinčič, A., Wraber, T., Jogan, N., Podobnik, A., Turk, B., Vreš, B., Ravnik, V., Frajman, B., Strgulc Krajšek, S., Trčak, B., Bačič, T., Fischer, M. A., Eler, K. \& Surina, B. 2007: Mala flora Slovenije. Ključ za določanje praprotnic in semenk. Četrta, dopolnjena in spremenjena izdaja. Tehniška založba Slovenije, Ljubljana, 967 pp.

Mlinšek, D., Accetto, M., Anko, B., Piskernik, M. Robič, D., Smolej, I. \& Zupančič, M. 1980: Gozdni rezervati v Sloveniji. Inštitut za gozdno in lesno gospodarstvo pri Biotehniški fakulteti v Ljubljani, Ljubljana, 414 pp.

Oksanen, J., Blanchet, F. G., Friendly, M., Kindt, R., Legendre, P., McGlinn, D., Minchin, P. R., O’Hara, R. B., Simpson, G. L., Solymos, P., Stevens, M. H. H., Szoecs, E. \& Wagner, H. 2017: vegan: Community Ecology Package. R package version 2.4-4. https://CRAN.R-project.org/package=vegan
Podani, J. 2001: SYN-TAX 2000. Computer Programs for Data Analysis in Ecology and Systematics. User's Manual, Budapest, 53 pp.

R Core Team 2017: R: A language and environment for statistical computing. R Foundation for Statistical Computing, Vienna, Austria. URL https://www.R-project.org/.

Seliškar, T., Vreš, B. \& Seliškar, A. 2003: FloVegSi 2.0. Fauna, Flora, Vegetation and Paleovegetation of Slovenia. Computer programme for arranging and analysis of biological data. Biološki inštitut ZRC SAZU, Ljubljana.

Slezák, M., Hrivnák, R., Ujházy, K., Ujházyová, M., Máliš, F. \& Petrášová, A. 2016: Syntaxonomy and ecology of acidophilous beech forest vegetation in Slovakia. Phytocoenologia 46 (1): 69-87.

Suppan, U., Prügger, J. \& Mayrhofer, H. 2000: Catalogue of the lichenized and lichenicolous fungi of Slovenia. Bibliotheca Lichenologica 76: 1-215.

Šilc, U. \& Čarni, A. 2012: Conspectus of vegetation syntaxa in Slovenia. Hacquetia 11 (1): 113-164.

Šilc, U., Čarni, A., Dakskobler, I., Kutnar, L. Marinšek, A. Rozman, A. Sajko, I. \& Vreš, B. 2017: Zasnova monitoringa stanja ohranjenosti manjšinskih Natura 2000 gozdnih habitatnih tipov v Sloveniji. Zaključno poročilo v okviru Ciljnega raziskovalnega projekta (V4-1430). Biološki inštitut ZRC SAZU, Ljubljana, 170 pp.

Theurillat, J.-P. 2004: Pflanzensoziologisches System. In: Aeschimann, D., Lauber, K., Moser D. M. \& Theurillat J.-P.: Flora alpina 3: Register. Haupt Verlag, Bern, Stuttgart, Wien, pp. 301-313.

Tregubov, V. 1962: Naravni sestoji macesna v Sloveniji in gospodarjenje z njimi. Zbornik Inštituta za gozdno in lesno gospodarstvo Slovenije 3: 29-143.

Urbančič, M., Simončič, P., Prus, T. \& Kutnar, L. 2005: Atlas gozdnih tal. Zveza gozdarskih društev Slovenije, Gozdarski vestnik \& Gozdarski inštitut Slovenije, Ljubljana. 100 pp.

Weber, H. E., Moravec, J. \& Theurillat, J. P. 2000: International Code of Phytosociological Nomenclature. 3rd edition. Journal of Vegetation Science 11 (5): 739-766.

Zupančič, M. 1999: Smrekovi gozdovi Slovenije (Spruce forests in Slovenia). Slovenska akademija znanosti in umetnosti. Razred za naravoslovne vede, Dela (Opera) 36: 1212 + Tables, Ljubljana.

Zupančič, M. \& Žagar, V. 2007: Comparative analysis of phytocoenoses with larch (Rhodothamno-Rhododendretum var. geogr. Paederota lutea laricetosum, Rhodothamno-Laricetum). Razprave 4. razreda SAZU 48 (2): 307-335.

\section{Electronic Appendix:}

www.degruyter.com/view/j/hacq

ojs.zrc-sazu.si/hacquetia 


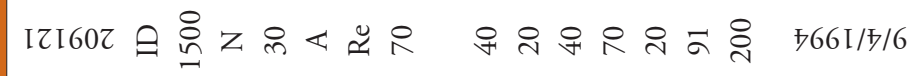

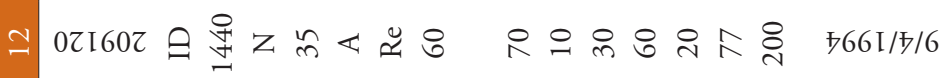

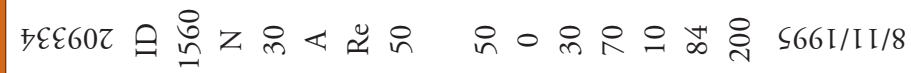

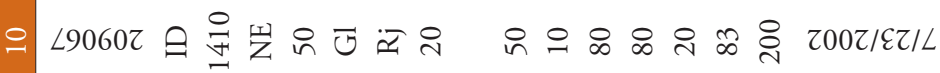

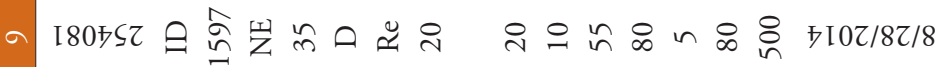

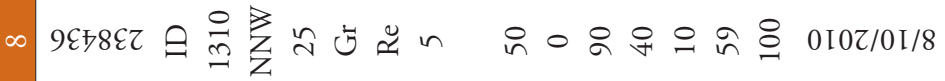

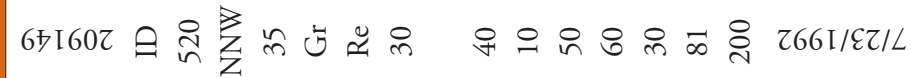

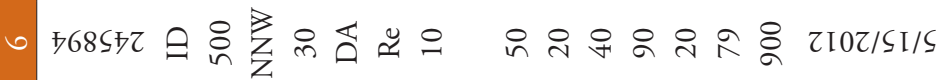

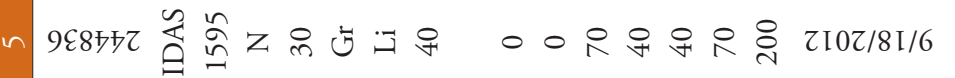

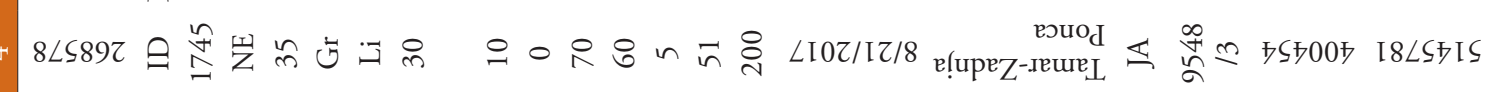

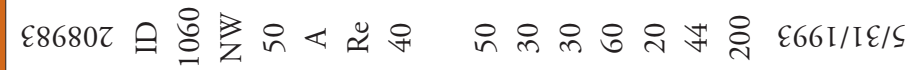

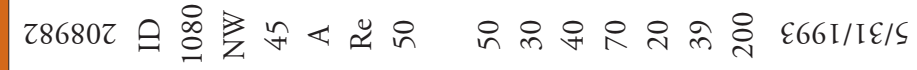

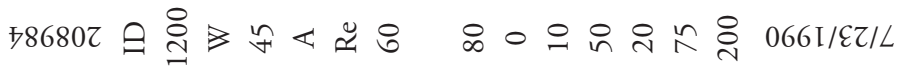

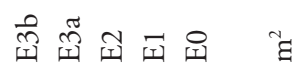

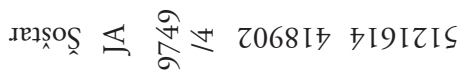

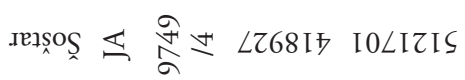

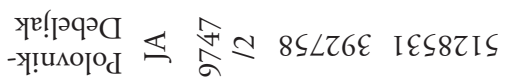

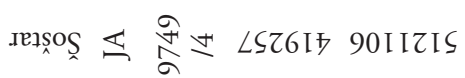

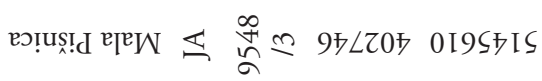

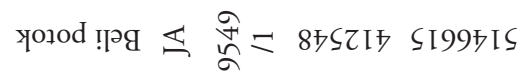

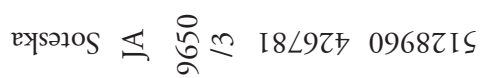

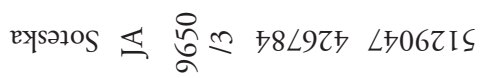

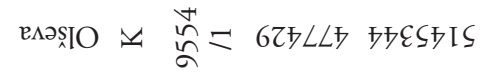

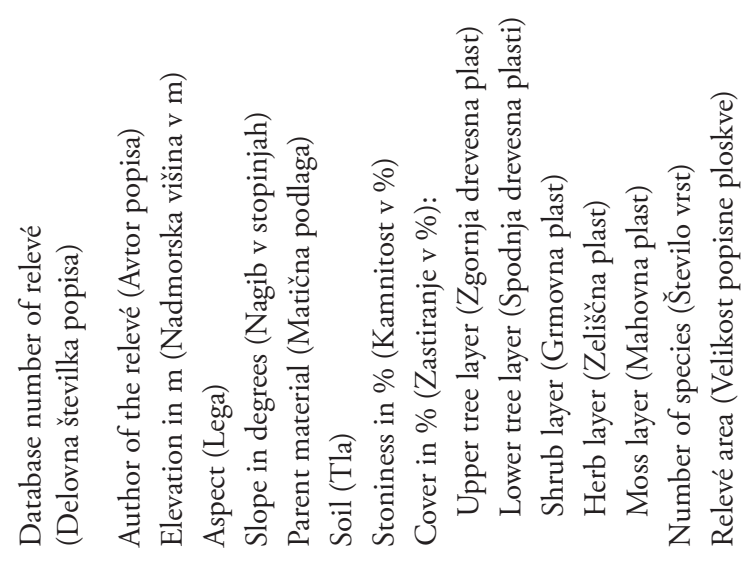




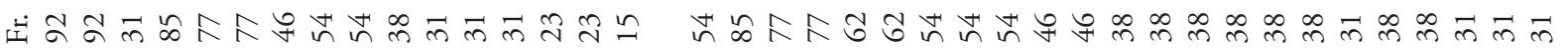

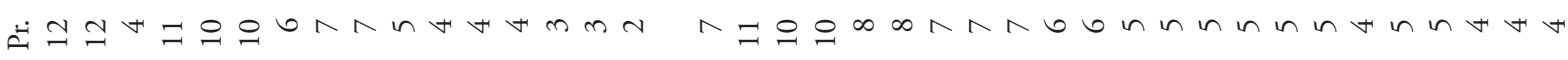
$n-+n+\cdots++\cdot+\cdot+\cdot$

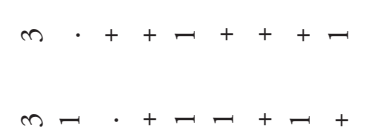

N $N+m+\cdot+\cdot+\cdot++$

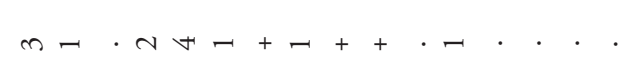

$n-\cdot+n-+\cdot$

$n-++\forall n-1$

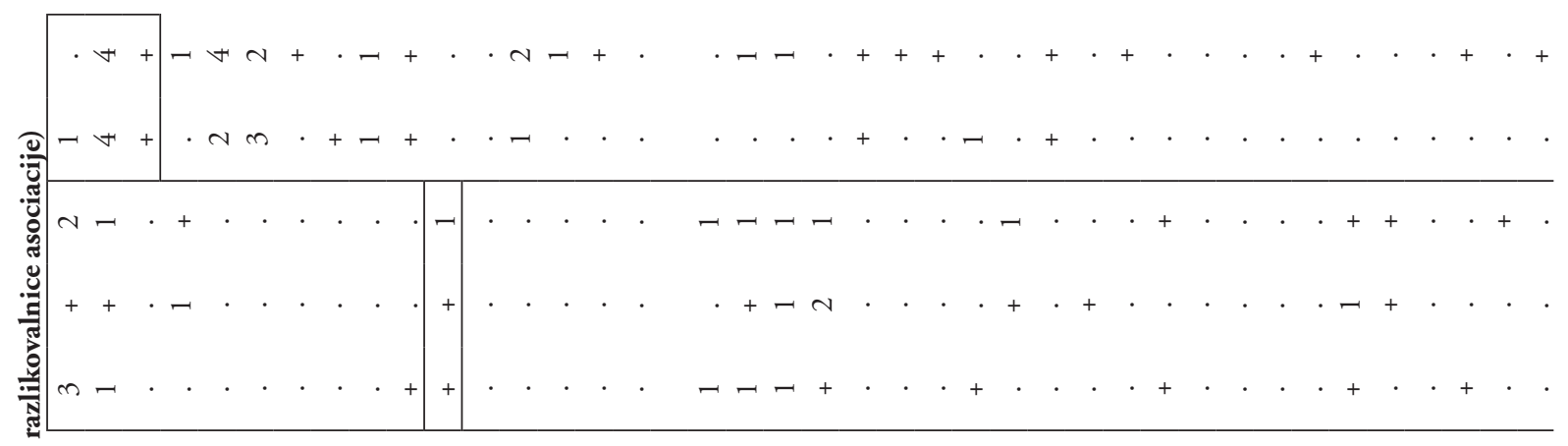

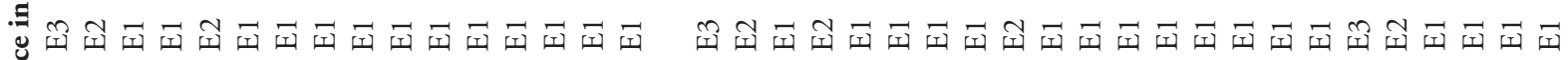




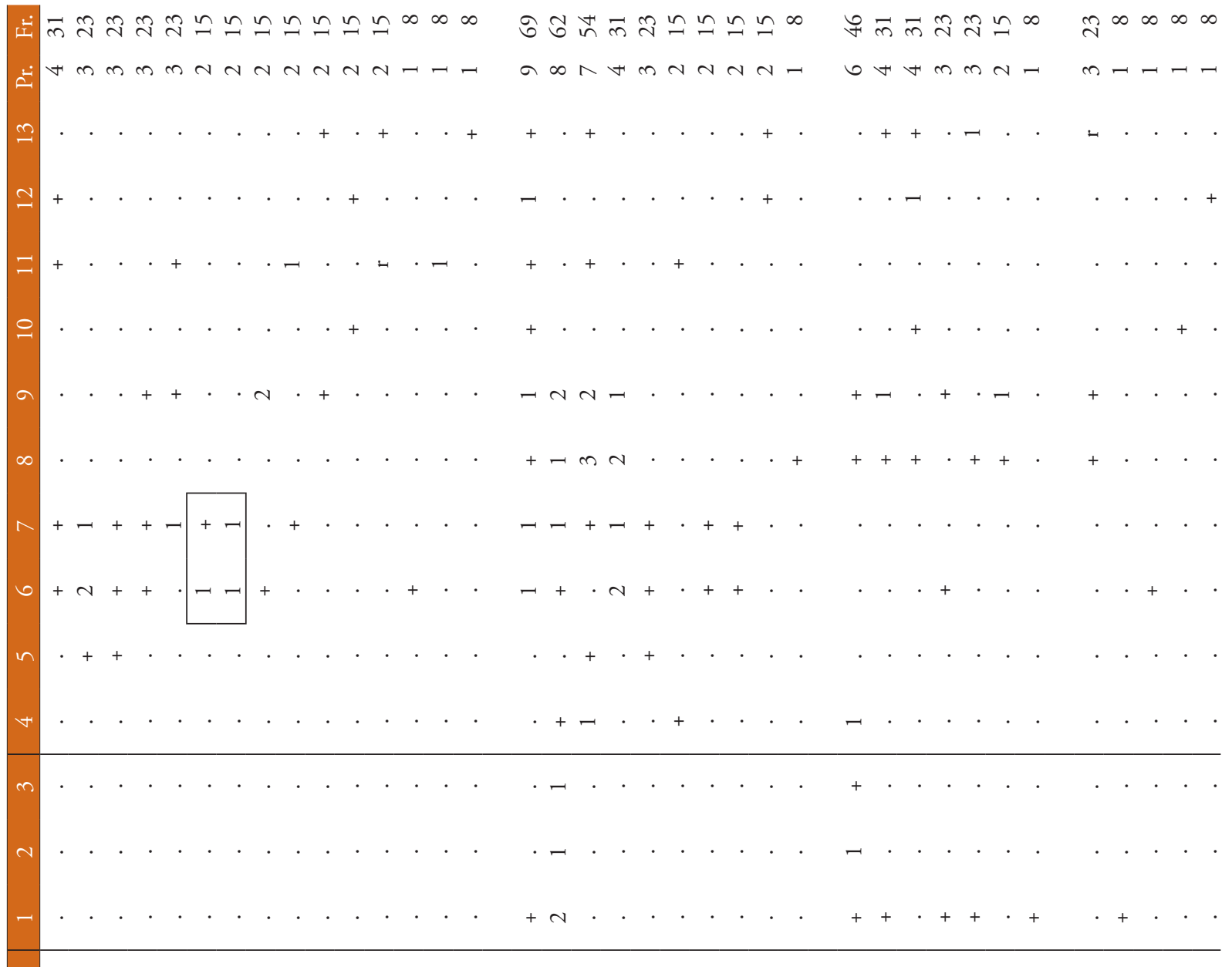

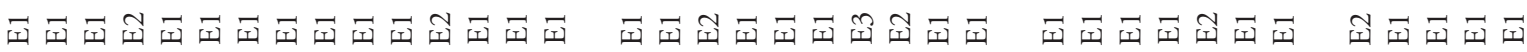

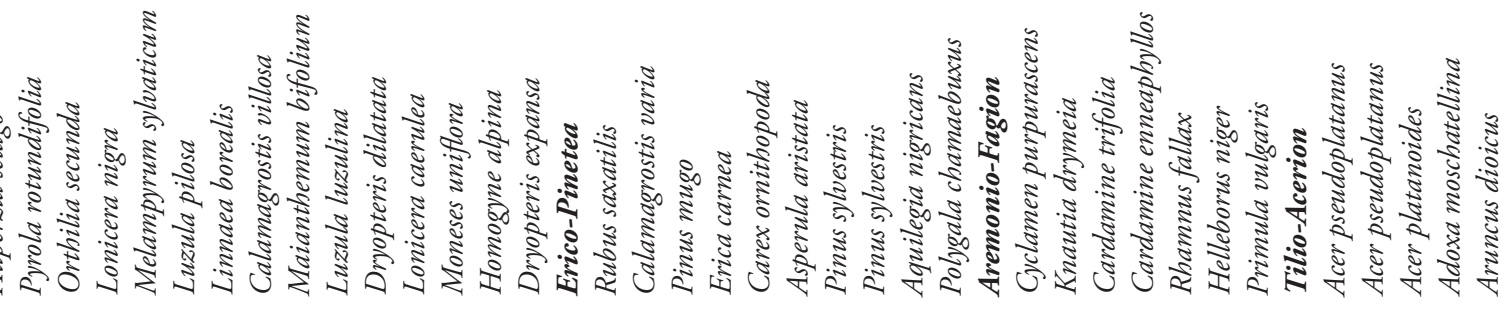




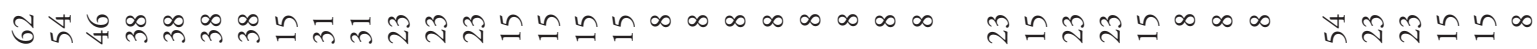

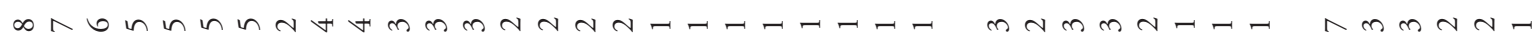

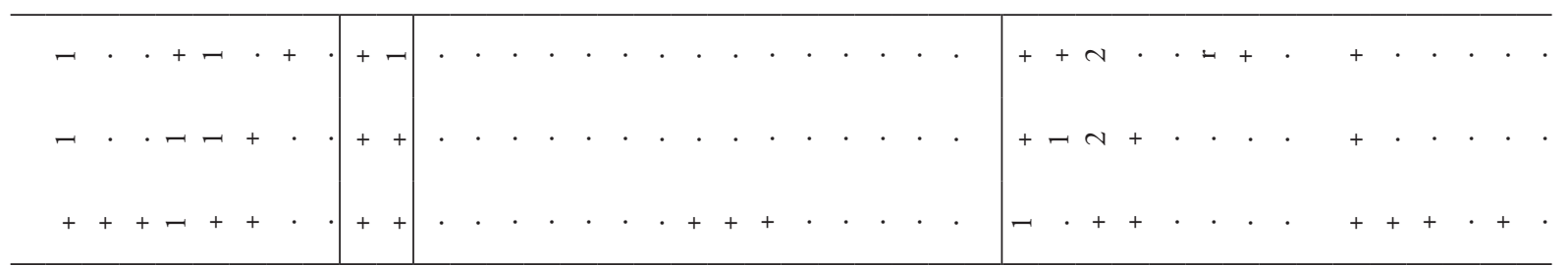

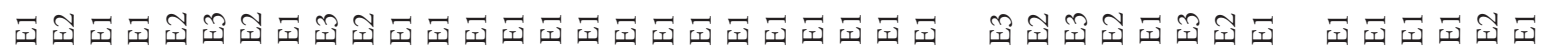

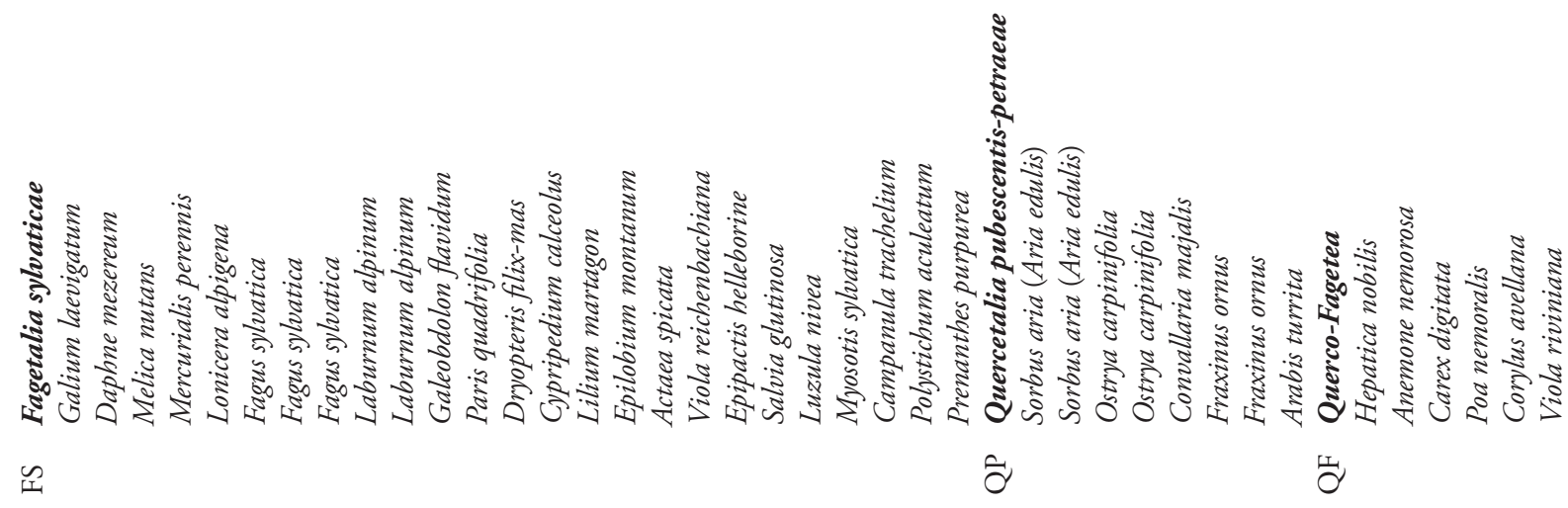




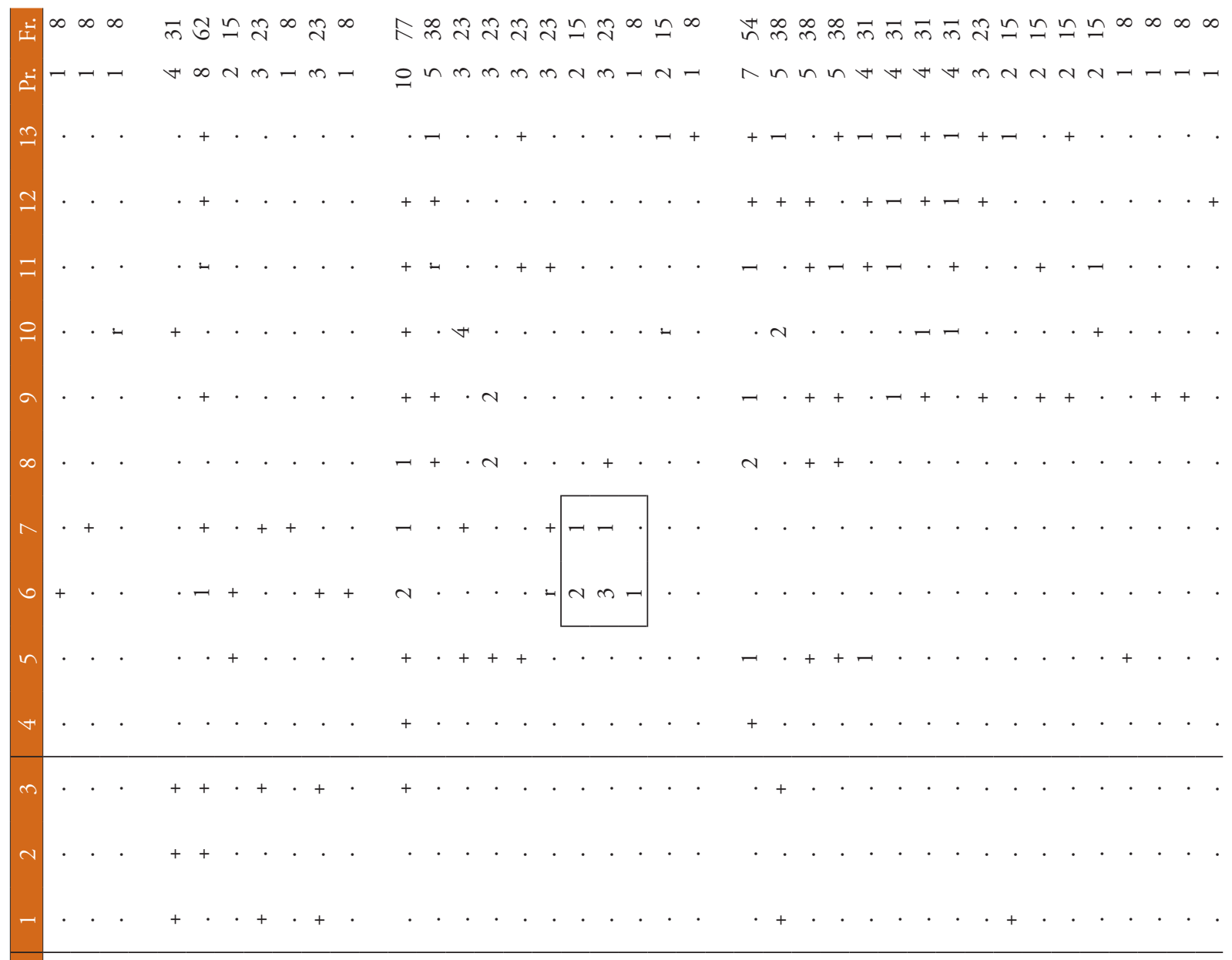

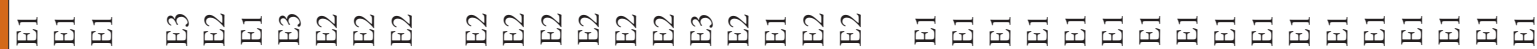

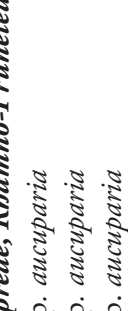

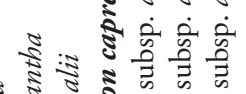




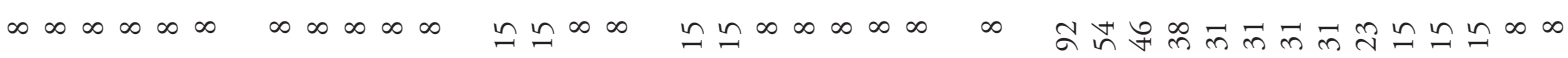

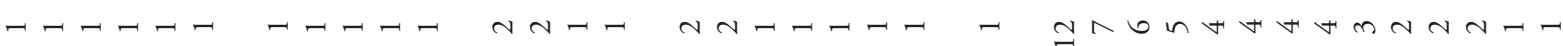
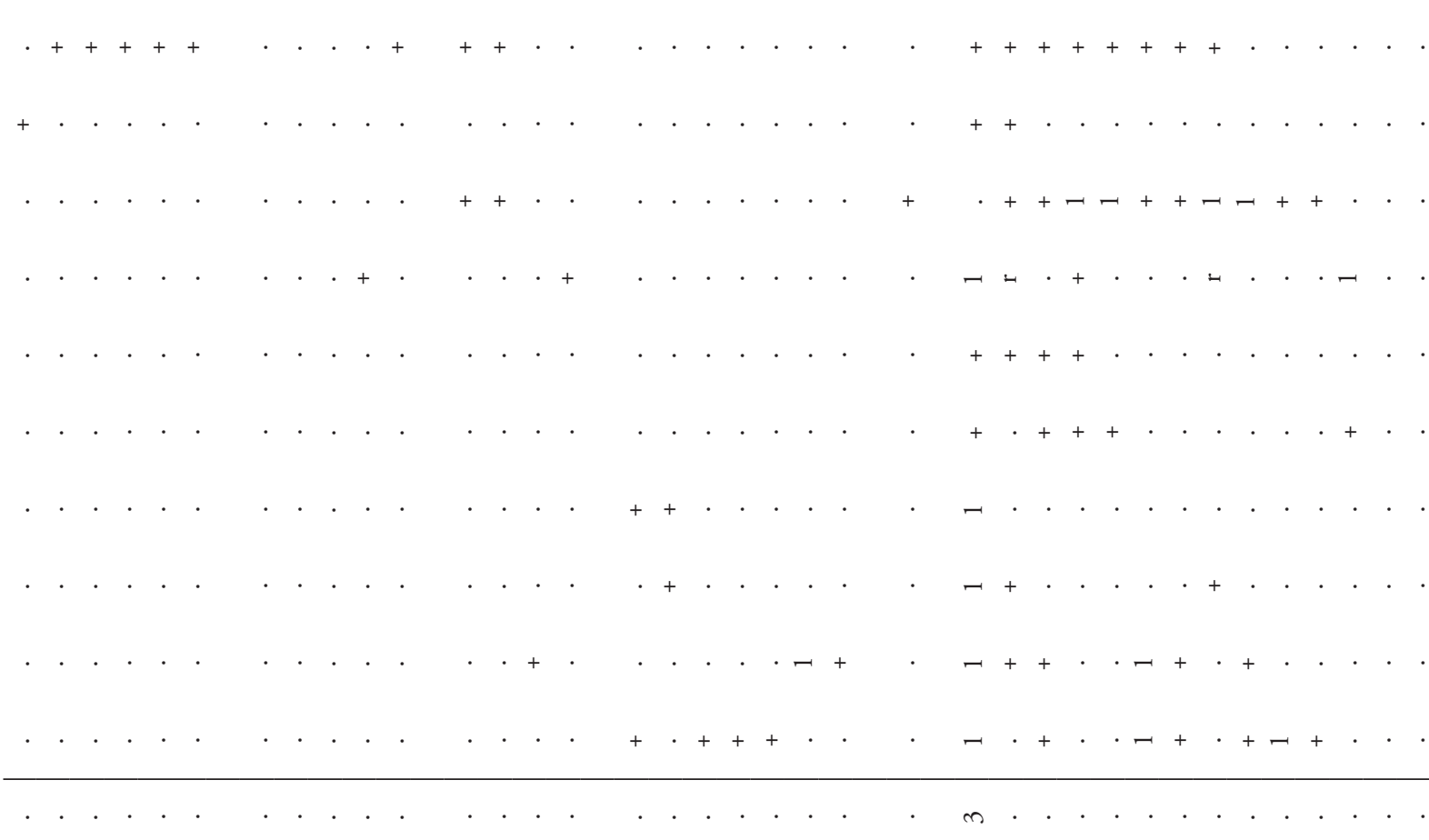

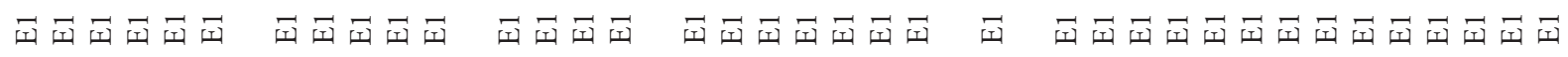

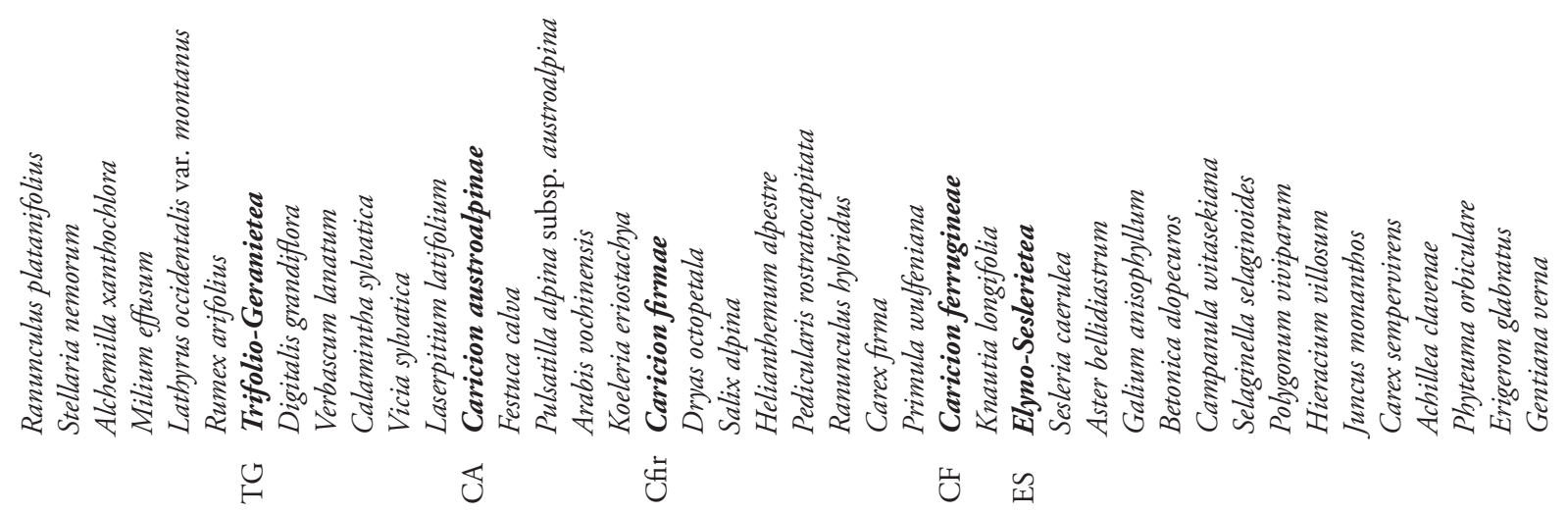




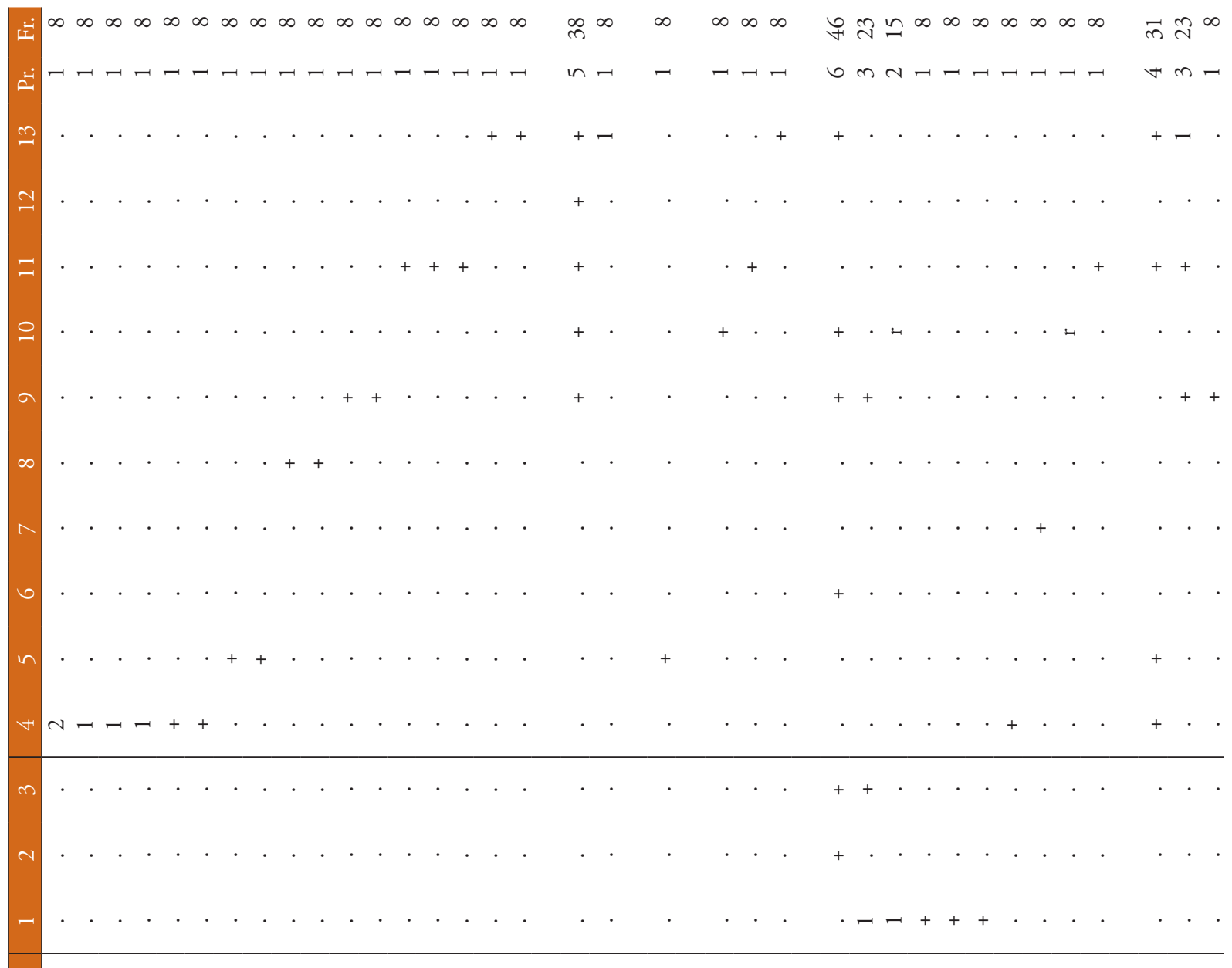

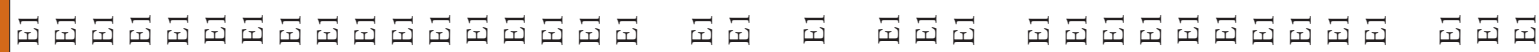

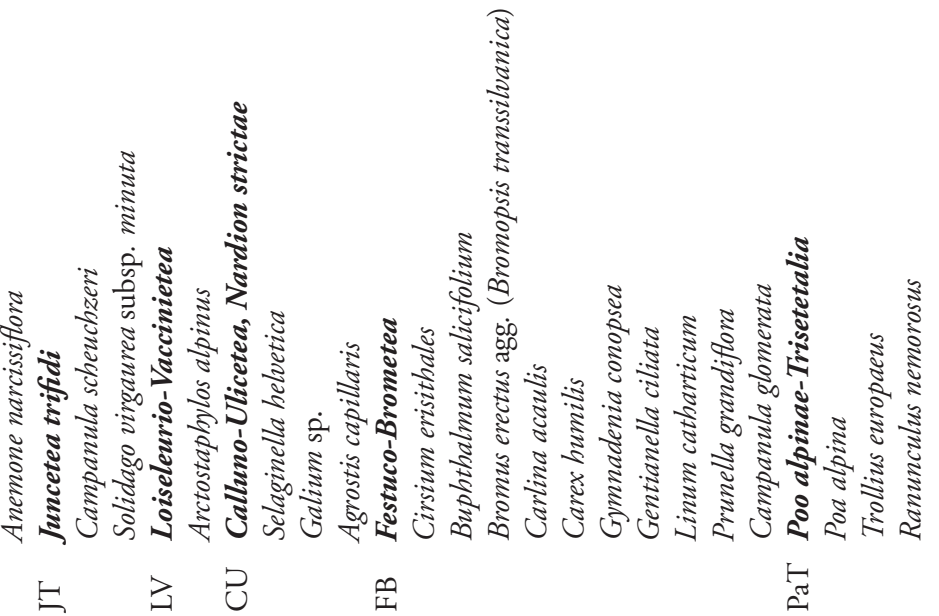




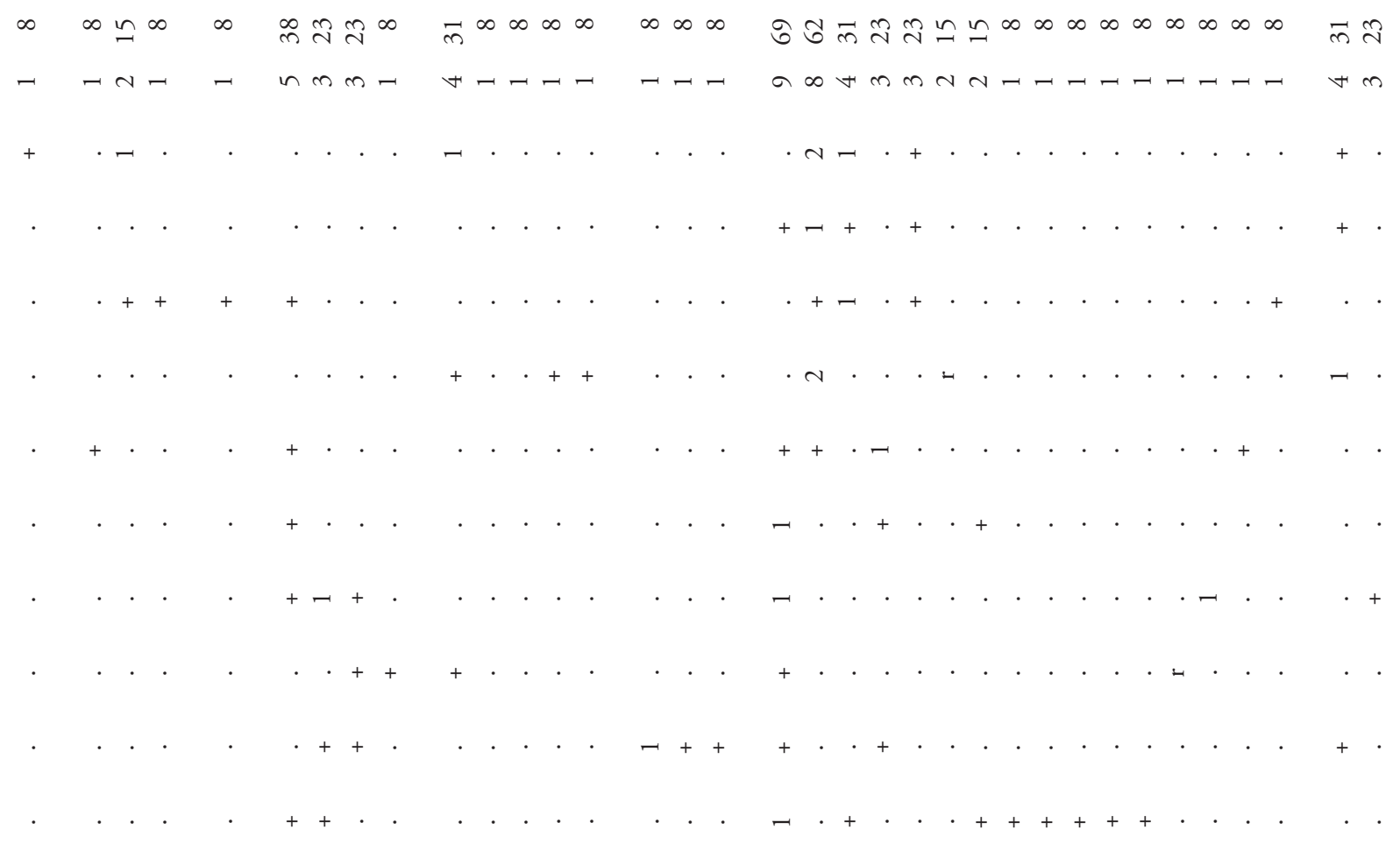

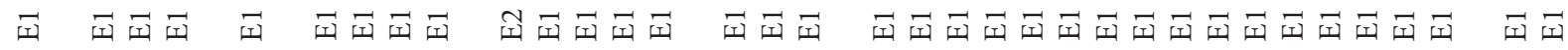

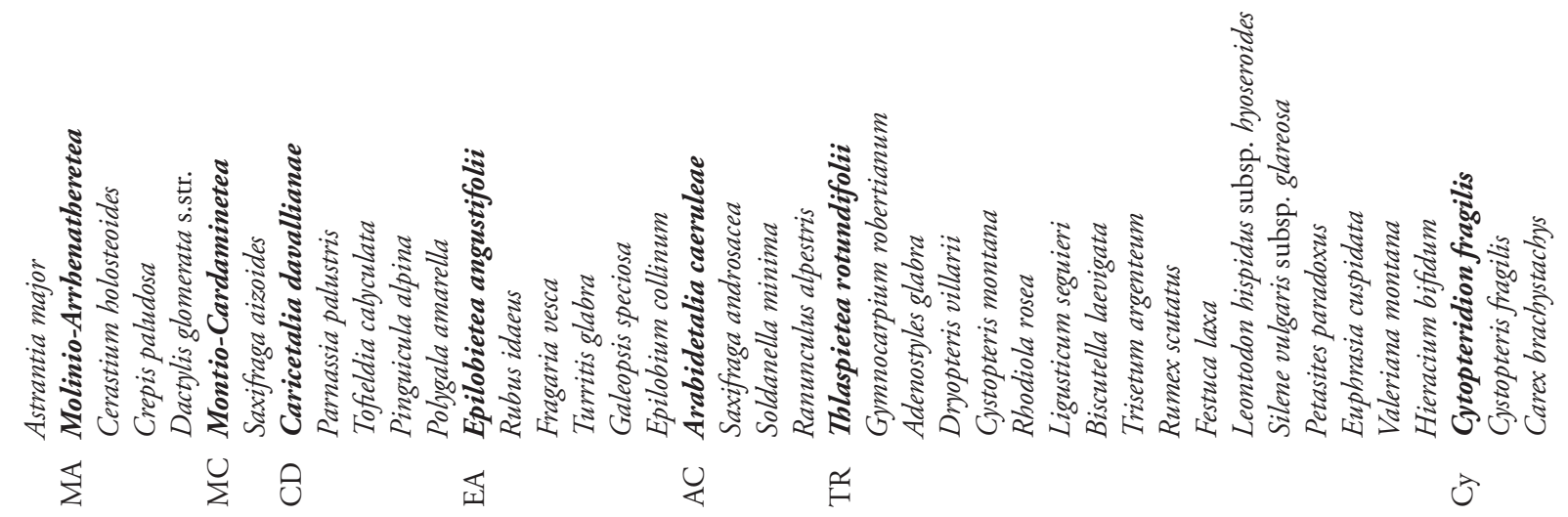




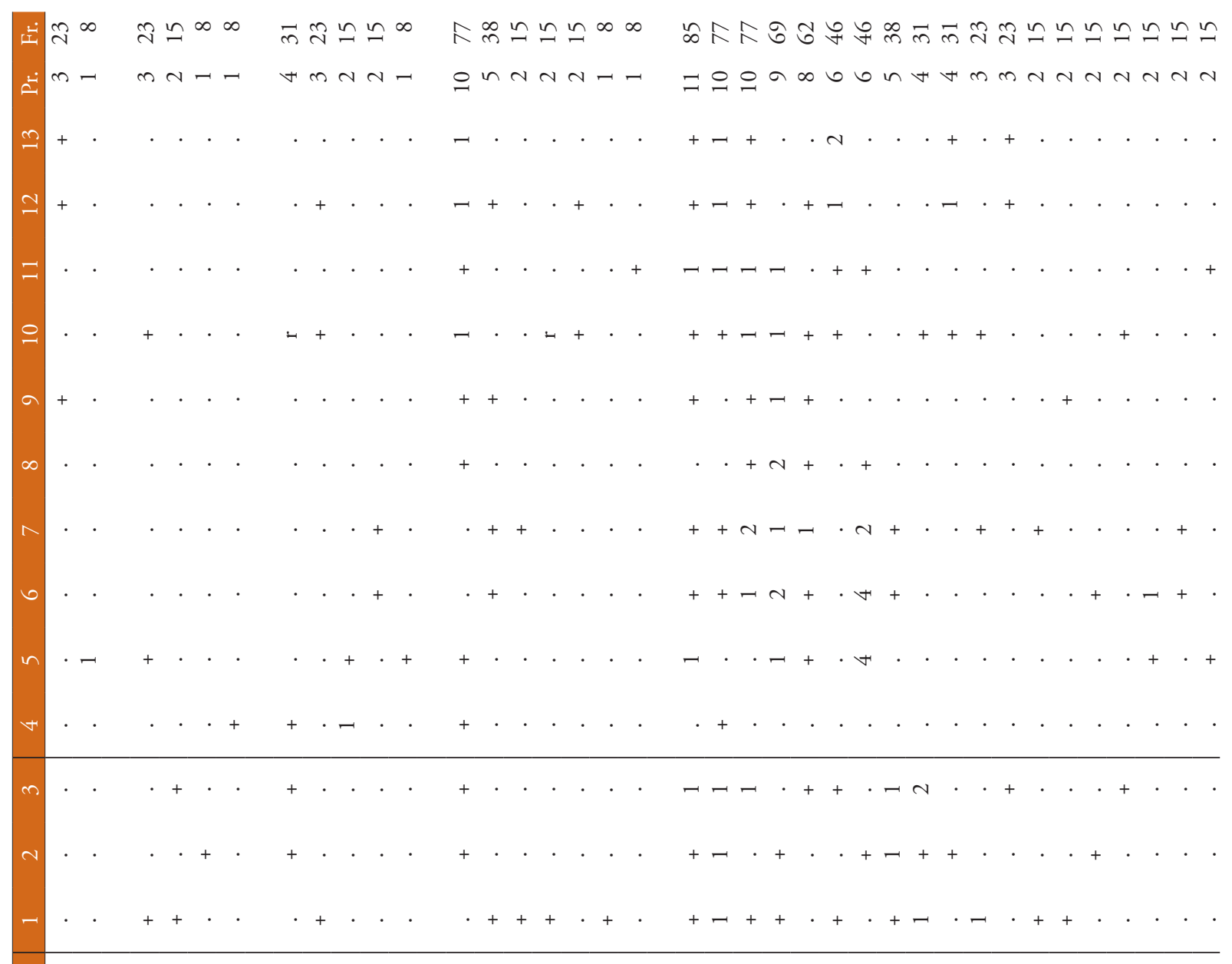

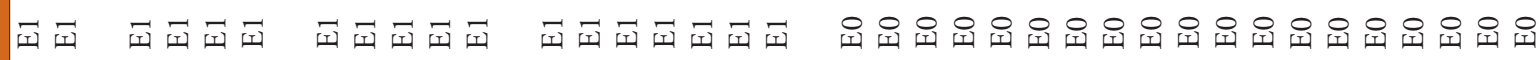
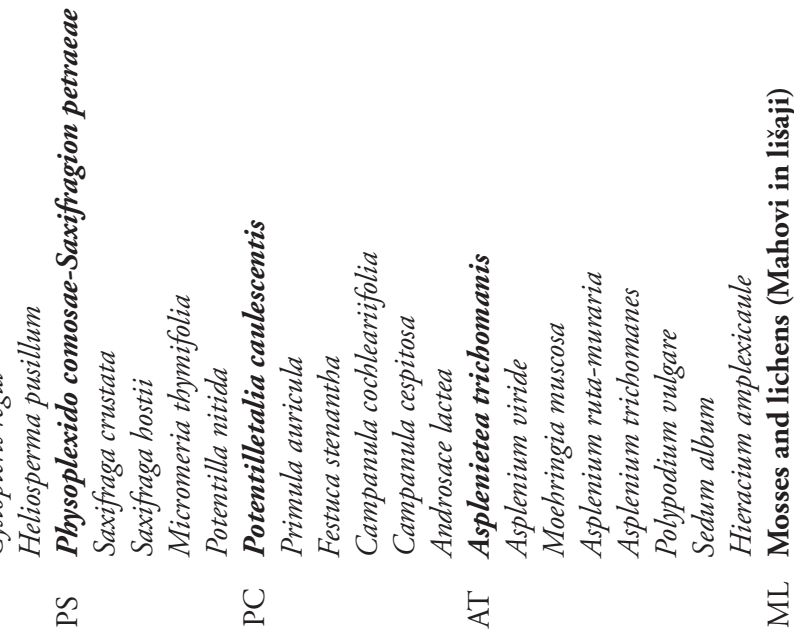


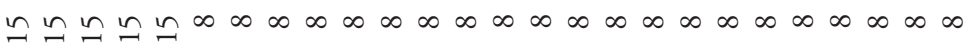

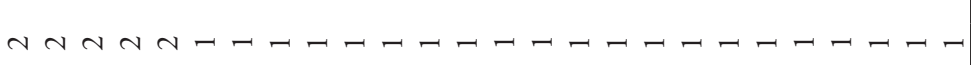

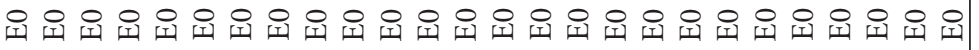

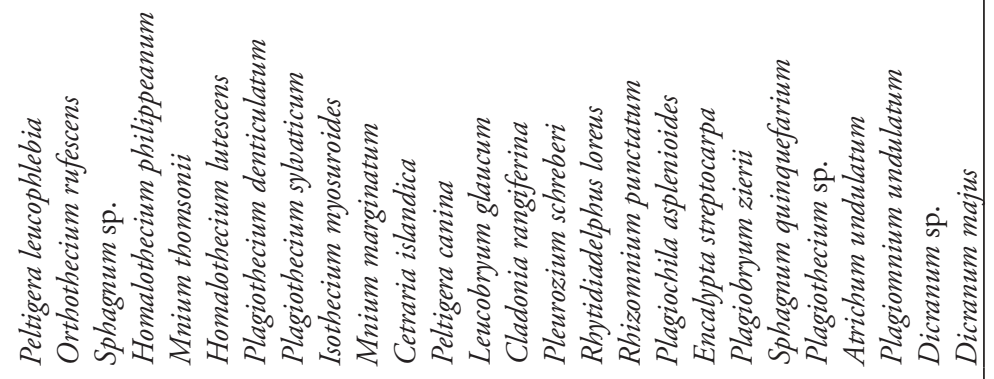

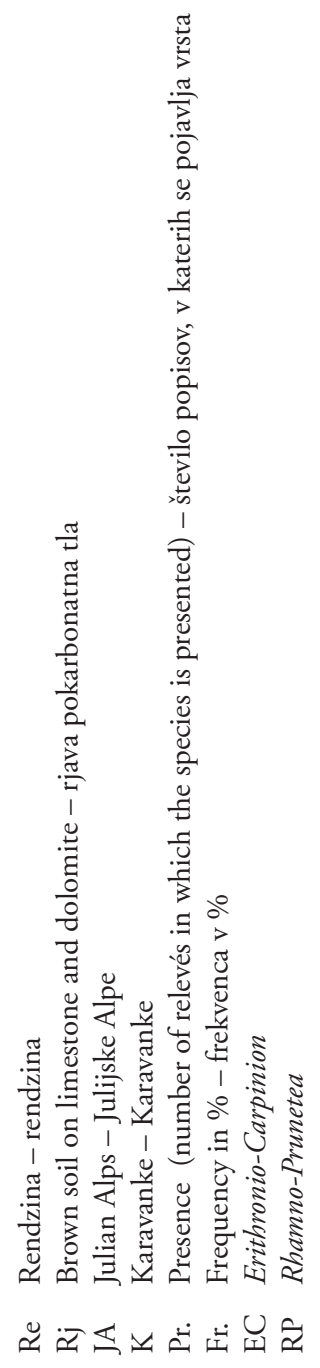

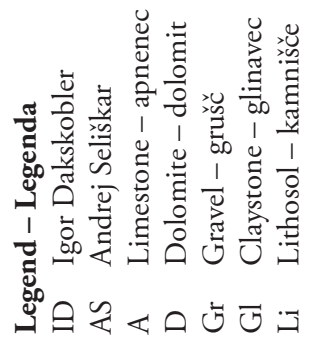


Table 2 (Tabela 2): Rhodothamno-Laricetum geetosum rivalis var. Adenostyles alliariae

Number of relevé (Zaporedna številka popisa)

Database number of relevé

(Delovna številka popisa)

Author of the relevé (Avtor popisa)

Elevation in $\mathrm{m}$ (Nadmorska višina $\mathrm{v} \mathrm{m}$ )

Aspect (Lega)

Slope in degrees (Nagib v stopinjah)

Parent material (Matična podlaga)

Soil (Tla)

Stoniness in \% (Kamnitost v \%)

Cover in \% (Zastiranje v \%):

Upper tree layer (Zgornja drevesna plast)

Lower tree layer (Spodnja drevesna plasti)

Shrub layer (Grmovna plast)

Herb layer (Zeliščna plast)

Moss layer (Mahovna plast)

Number of species (Število vrst)

Relevé area (Velikost popisne ploskve)

Date of taking relevé (Datum popisa)

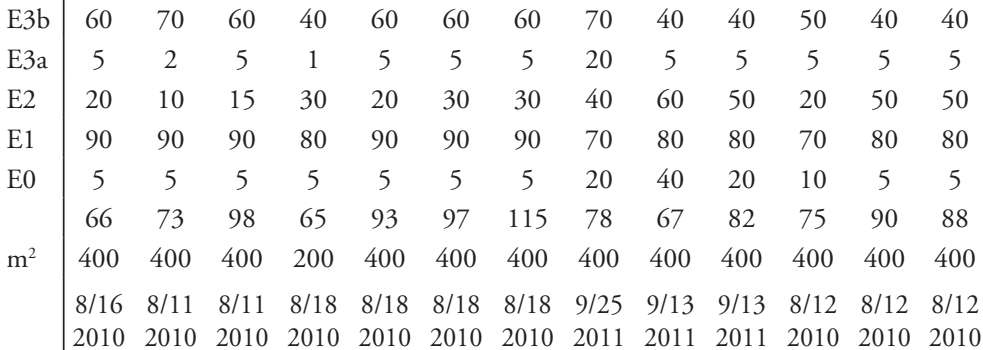

Locality (Nahajališče)

Mountain range (Pogorje)

Quadrant (Kvadrant)

Coordinate GK-Y (D-48)

$\mathrm{m}$

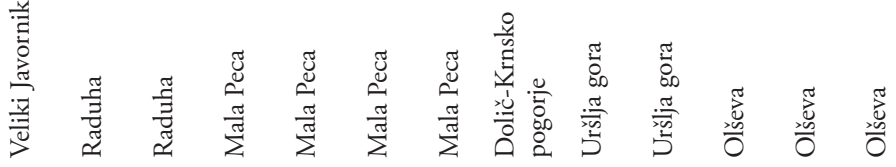

$$
\begin{aligned}
& \begin{array}{lllllllllllll}
\text { JA } & \text { KSA } & \text { KSA } & \mathrm{K} & \mathrm{K} & \mathrm{K} & \mathrm{K} & \mathrm{JA} & \mathrm{K} & \mathrm{K} & \mathrm{K} & \mathrm{K} & \mathrm{K}
\end{array} \\
& 9652955495549554955495549554964795559555955495549554 \\
& \begin{array}{lllllllllllll}
11 & 13 & 13 & 12 & 12 & 12 & 12 & 14 & 12 & 12 & 13 & 11 & 13
\end{array}
\end{aligned}
$$

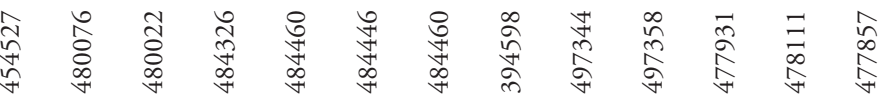

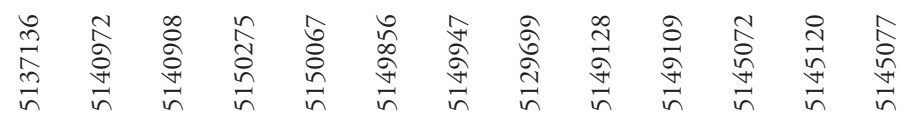

Coordinate GK-Y (D-48)

Character and differential species of the association (Značilnice in razlikovalnice asociacije)

VP

$\mathrm{VP}$

$\mathrm{VP}$

EP

ES

CF

PS

TR

VP

TR

EP

$\mathrm{CA}$

TR

$\mathrm{PC}$
Larix decidua

Larix decidua

Larix decidua

Rhododendron hirsutum

Astrantia bavarica

Carex ferrugined

Homogyne sylvestris

Heliosperma alpestre

Rhodothamnus chamaecistus

Laserpitium peucedanoides

Astrantia carniolica

Valeriana saxatilis

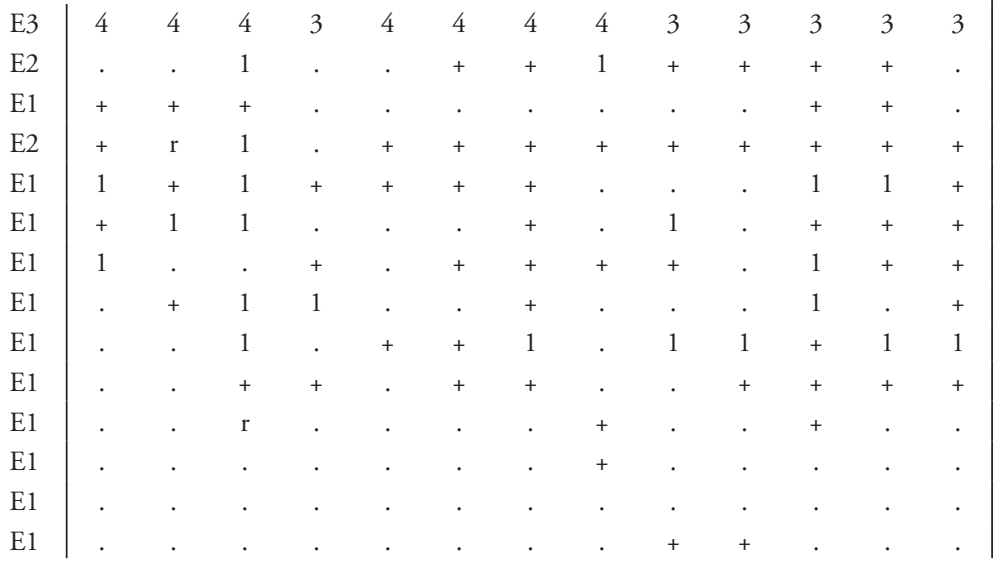


\begin{tabular}{|l|lllllllllllll} 
Number of relevé (Zaporedna številka popisa) & 1 & 2 & 3 & 4 & 5 & 6 & 7 & 8 & 9 & 10 & 11 & 12 & 13
\end{tabular}

PS Campanula carnica

E1

AF Anemone trifolia

E1

Differential species of the subassociation (Razlikovalne vrste subasociacije)

$\mathrm{MuA}$ Chaerophyllum hirsutum

MuA Geum rivale

MuA Senecio cacaliaster

MuA Rumex arifolius

\begin{tabular}{l|lllllllllllll} 
E1 & 1 & + & 1 &. & 1 & 2 & 1 & + & 1 & + & + & 1 & + \\
E1 & 1 & + & + &. & 1 & 1 & 1 &. & + & + & + & 1 & 1 \\
E1 & $\cdot$ &. &. & 2 & 3 & 2 & 2 &. &. &. & + & 1 & 1 \\
E1 &. &. &. &. & + & 1 & 1 & + &. &. &. & 1 & 1
\end{tabular}

Differential species of the variant and subvariant (Razlikovalne vrste variante in subvariante)

$\mathrm{MuA}$ Adenostyles alliariae

VP Luzula luzuloides

MuA Senecio ovatus

MuA Stellaria nemorum

AF Helleborus niger

VP Vaccinio-Piceetea

Luzula sylvatica

Vaccinium myrtillus

Polystichum lonchitis

Valeriana tripteris

Oxalis acetosella

Dryopteris dilatata

Vaccinium vitis-idaea

Picea abies

Picea abies

Picea abies

Calamagrostis villosa

Aposeris foetida

Clematis alpina

Hieracium murorum

Rosa pendulina

Gymnocarpium dryopteris

Solidago virgaurea subsp. virgaurea

Melampyrum sylvaticum

Saxifraga cuneifolia

Phegopteris connectilis

Homogyne alpina

Huperzia selago

Veronica urticifolia

Maianthemum bifolium

Dryopteris expansa

Lycopodium annotinum

Lonicera nigra

Lonicera caerulea

Gentiana asclepiadea

Calamagrostis arundinacea

Luzula luzulina

Abies alba

Abies alba

Circaea alpina

Thelypteris limbosperma

Blechnum spicant

Luzula pilosa

Moneses uniflora

Ajuga pyramidalis

\begin{tabular}{|c|c|c|c|c|c|c|c|c|c|c|c|c|c|c|}
\hline E1 & . & . & . & + & 1 & 1 & + & . & + & + & . & & + & + \\
\hline E1 & + & + & 1 & 1 & 1 & + & + & 1 & 1 & 1 & & & 1 & 1 \\
\hline E1 & + & + & . & + & + & + & + & 1 & + & + & & & + & . \\
\hline E1 & 1 & . & + & . & + & 1 & + & . & 1 & + & & & 1 & 1 \\
\hline E1 & + & 1 & 2 & + & 1 & + & 1 & . & 1 &. & . & & + & . \\
\hline E1 & 3 & 1 & 1 & 1 & 1 & 2 & 2 & 2 & 2 & 2 & & & 1 & 1 \\
\hline E1 & 1 & + & + & 1 & + & 1 & 1 & 2 & + & 1 & & & 3 & 2 \\
\hline E1 & + & + & + & . & + & + & + & 1 & 1 & + & + & & + & + \\
\hline E1 & + & . & 1 & + & + & + & + & 1 & + & 1 & & & + & 1 \\
\hline E1 & 1 & 1 & 1 & 1 & 1 & 1 & 1 & 1 & 1 & 1 & & & 1 & 1 \\
\hline E1 & 1 & + & + & 1 & + & 1 & + & 1 & + & + & & & 1 & 2 \\
\hline E1 & . & + & + & + & + & + & + & + & + & + & + & & + & + \\
\hline E3 & + & 1 & + & + & 1 & 1 & + & + & + & + & & & + & + \\
\hline E2 & 1 & . & + & + & . & + & + & + & + & + & + & & + & + \\
\hline E1 & . & + & . & . & . & . & . & . & . & . & & & . & . \\
\hline E1 & 1 & + & . & . & + & 2 & 1 & 1 & . & 1 & 2 & & 1 & 1 \\
\hline E1 & 1 & 1 & 1 & + & . & + & . & . & . & . & & & + & + \\
\hline E2 & . & . & + & . & + & . & + & + & . & + & & & . & + \\
\hline E1 & . & + & + & . & . & . & + & 1 & . & . & & & . & . \\
\hline E2 & . & . & . & + & . & + & + & + & + & + & & & 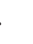 & . \\
\hline E1 & + & + & + & . & . & + & . & $\cdot$ & . & + & & & + & + \\
\hline E1 & . & . & . & . & . & . & . & + & . & . & & & + & . \\
\hline E1 & . & . & . & . & . & + & + & . & . & + & & & + & + \\
\hline E1 & 1 & . & . & . & . & + & 1 & 1 & . & . & & & + & + \\
\hline E1 & 1 & + & + & . & . & . & . & . & . & 1 & & & . & . \\
\hline E1 & + & . & . & . & . & . & . & + & . & + & & & + & . \\
\hline E1 & + & . & . & . & . & . & . & . & . & + & & & . & . \\
\hline E1 & . & . & . & . & . & . & . & 1 & . & . & & & . & . \\
\hline E1 & . & . & . & . & . & . & . & + & . & . & & & 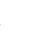 & . \\
\hline E1 & . & . & . & . & 1 & 1 & + & + & . & . & & & + & + \\
\hline E1 & . & . & . & . & . & . & . & . & + & + & & & . & . \\
\hline E2 & . & . & . & . & + & + & . & + & . & + & & & . & + \\
\hline E2 & . & . & . & . & . & . & . & + & . & . & & & . & . \\
\hline E1 & . & . & . & . & + & + & 1 & . & . & . & & & 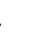 & . \\
\hline E1 & + & . & . & . & . & . & . & 1 & . & . & & & . & . \\
\hline E1 & . & + & . & + & . & . & . & . & . & . & & & . & . \\
\hline E2 & . & . & . & . & . & . & . & . & . & . & & & . & . \\
\hline E1 & . & . & . & . & . & . & . & . & . & . & & & $\cdot$ & . \\
\hline E1 & . & + & . & . & . & . & . & . & . & . & & & . & . \\
\hline E1 & . & + & . & . & . & . & . & . & . & . & & & $\cdot$ & . \\
\hline E1 & . & + & . & . & . & . & . & . & . & . & & & $\cdot$ & . \\
\hline E1 & . & . & . & . & . & . & . & . & . & . & & & . & . \\
\hline E1 & . & . & . & . & . & . & . & . & . & . & & & $\cdot$ & . \\
\hline E1 & . & . & . &. & . & . & . & . & . & . & & & . &. \\
\hline
\end{tabular}




\begin{tabular}{|c|c|c|c|c|c|c|c|c|c|c|c|c|c|c|c|}
\hline & Number of relevé (Zaporedna številka popisa) & & 1 & 2 & 3 & 4 & 5 & 6 & 7 & 8 & 9 & 10 & 11 & 12 & 13 \\
\hline \multirow[t]{8}{*}{ EP } & Erico-Pinetea & & & & & & & & & & & & & & \\
\hline & Rubus saxatilis & E1 & + & . & . & . & . & + & 1 & 1 & 1 & 1 & + & + & + \\
\hline & Pinus mugo & E2 & . & + & + & 3 & 1 & 1 & 1 & + & . & 1 & + & . & . \\
\hline & Calamagrostis varia & E1 & . & . & + & . & + & + & + & . & + & . & . & . & . \\
\hline & Aquilegia nigricans & E1 & . & . & + & . & . & . & + & . & . & . & . & . & . \\
\hline & Erica carnea & E1 & . & + & + & + & . & . & + & + & . & . & . & . & . \\
\hline & Carex ornithopoda & E1 & . & . & . & . & . & . & . & . & . & + & . & . & . \\
\hline & Galium austriacum & E1 & . & . & . & . & + & + & . & . & . & . & . & . & . \\
\hline \multirow[t]{6}{*}{$\mathrm{AF}$} & Aremonio-Fagion & & & & & & & & & & & & & & \\
\hline & Cardamine enneaphyllos & E1 & . & . & + & . & + & + & + & . & . & . & + & + & + \\
\hline & Knautia drymeia & E1 & . & + & + & . & + & + & + & . & . & . & . & + & + \\
\hline & Cardamine trifolia & E1 & 1 & . & + & . & . & . & . & . & 1 & . & . & . & . \\
\hline & Cyclamen purpurascens & E1 & . & . & . & . & . & . & . & . & . & . & . & . & . \\
\hline & Rhamnus fallax & E2 & . & . & . & . & . & . & . & . & . & . & . & . & . \\
\hline \multirow[t]{6}{*}{ TA } & Tilio-Acerion & & & & & & & & & & & & & & \\
\hline & Chrysosplenium alternifolium & E1 & . & . & . & + & + & + & + & . & . & + & + & + & . \\
\hline & Acer pseudoplatanus & E3 & . & . & . & . & + & + & . & . & . & . & . & . & . \\
\hline & Acer pseudoplatanus & E2 & . & . & . & . & . & . & + & + & . & . & . & . & + \\
\hline & Acer pseudoplatanus & E1 & . & . & . & . & . & . & . & . & . & . & . & . & . \\
\hline & Adoxa moschatellina & E1 & . & . & . & + & + & . & . & . & + & . & . & . & . \\
\hline \multirow[t]{26}{*}{ FS } & Fagetalia sylvaticae & & & & & & & & & & & & & & \\
\hline & Daphne mezereum & E2 & + & 1 & 1 & + & + & + & + & . & + & 1 & + & + & + \\
\hline & Galeobdolon flavidum & E1 & + & + & + & + & + & + & + & + & + & . & . & + & . \\
\hline & Dryopteris flix-mas & E1 & . & + & + & + & 1 & 1 & 1 & 2 & . & . & . & + & . \\
\hline & Myosotis sylvatica & E1 & + & + & 1 & 1 & + & + & + & . & + & + & + & + & + \\
\hline & Melica nutans & E1 & . & + & . & . & + & + & + & . & . & . & . & . & + \\
\hline & Mercurialis perennis & E1 & . & . & . & . & 2 & . & 1 & . & + & + & . & + & 1 \\
\hline & Paris quadrifolia & E1 & . & . & + & . & + & + & 1 & . & . & . & . & + & + \\
\hline & Lonicera alpigena & E2 & . & . & . & . & + & + & + & . & + & + & + & . & . \\
\hline & Lilium martagon & E1 & . & . & . & + & + & . & + & . & . & . & . & . & . \\
\hline & Epilobium montanum & E1 & . & + & 1 & + & + & + & + & + & . & . & . & . & . \\
\hline & Symphytum tuberosum & E1 & + & + & + & + & . & . & + & . & . & . & . & + & . \\
\hline & Actaea spicata & E1 & . & . & . & . & + & . & + & . & . & . & . & . & . \\
\hline & Euphorbia amygdaloides & E1 & . & 1 & 1 & . & + & . & 1 & . & . & . & . & . & . \\
\hline & Galium laevigatum & E1 & . & . & . & . & . & . & . & . & . & . & . & . & . \\
\hline & Polystichum aculeatum & E1 & . & . & . & . & . & . & + & . & . & . & . & . & + \\
\hline & Fagus sylvatica & E3 & . & . & . & . & . & . & . & . & . & . & . & . & . \\
\hline & Fagus sylvatica & E2 & . & . & . & . & . & . & . & $\mathrm{r}$ & . & . & . & . & . \\
\hline & Mycelis muralis & E1 & . & + & + & . & + & . & . & . & . & . & . & . & . \\
\hline & Ranunculus lanuginosus & E1 & . & . & . & . & . & . & . & . & . & . & . & . & . \\
\hline & Petasites albus & E1 & . & . & r & . & . & . & . & . & . & . & . & . & . \\
\hline & Prenanthes purpurea & E1 & . & . & . & . & . & . & . & . & . & . & . & . & . \\
\hline & Viola reichenbachiana & E1 & . & . & . & + & . & . & . & . & . & . & . & . & . \\
\hline & Epipactis helleborine & E1 & . & . & . & . & . & $\mathrm{r}$ & . & . & . & . & . & . & . \\
\hline & Luzula nivea & E1 & . & . & . & . & . & . & . & + & . & . & . & . & . \\
\hline & Phyteuma spicatum & E1 & . & . & . & . & . & . & . & . & . & . & . & . & . \\
\hline \multirow[t]{2}{*}{ QP } & Quercetalia pubescenti-petraeae & & & & & & & & & & & & & & \\
\hline & Sorbus aria (Aria edulis) & E2 & . & . & . & . & . & + & . & + & . & . & . & . & . \\
\hline \multirow[t]{5}{*}{ QF } & Querco-Fagetea & & & & & & & & & & & & & & \\
\hline & Poa nemoralis & E1 & + & 1 & 1 & + & + & . & + & + & + & . & + & + & + \\
\hline & Anemone nemorosa & E1 & + & + & + & + & . & + & + & . & . & . & . & + & . \\
\hline & Hepatica nobilis & E1 & . & . & + & + & 1 & + & + & . & 1 & + & . & . & + \\
\hline & Festuca heterophylla & E1 & . & 1 & + & + & . & . & + & . & . & . & . & + & . \\
\hline
\end{tabular}




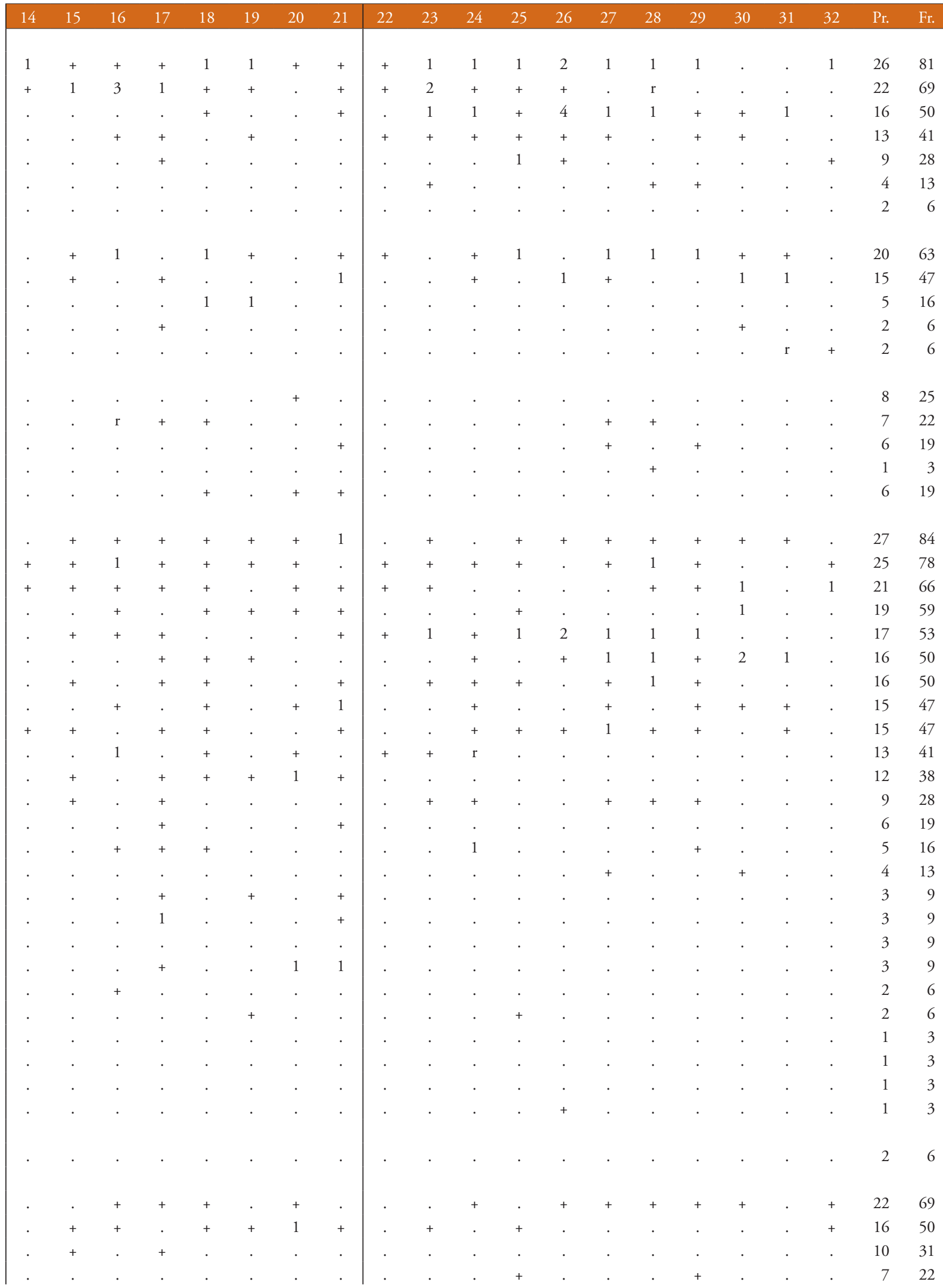


Number of relevé (Zaporedna številka popisa)

Listera ovata

Hieracium lachenalii

Carex digitata

Cruciata glabra

Moehringia trinervia

Galium sylvaticum

Pulmonaria stiriaca

SSC Sambuco-Salicion capreae, Rhamno-Prunetea

Sorbus aucuparia subsp. aucuparia

Sorbus aucuparia subsp. aucuparia

Sorbus aucuparia subsp. aucuparia

Betula pendula

BA Betulo-Alnetea

Salix appendiculata

Salix appendiculata

Alnus viridis

Sorbus chamaemespilus

Salix glabra

Ribes alpinum

Juniperus sibirica

Salix waldsteiniana

MuA Mulgedio-Aconitetea

Viola biflora

Saxifraga rotundifolia

Athyrium filix-femina

Hypericum maculatum

Veratrum album subsp. lobelianum

Geranium sylvaticum

Polygonatum verticillatum

Aconitum lycoctonum subsp. ranunculifolium

Primula elatior

Poa hybrida

Thalictrum aquilegiifolium

Ranunculus platanifolius

Doronicum austriacum

Epilobium alpestre

Peucedanum ostruthium

Alchemilla xanthochlora

Veratrum album subsp. album

Phyteuma ovatum

Cirsium carniolicum

Tephroseris crispa

Aconitum tauricum

Chaerophyllum villarsii

Scrophularia scopolii

Cicerbita alpina

Alchemilla monticola

Heracleum montanum

Silene dioica

Cortusa matthioli

Carduus carduelis

Aconitum degenii subsp. paniculatum

Aconitum angustifolium

E1

E3

E2

E1

E2

E3

E2

E2

E2

E2

E2

E2

E2

E

E1

E1

E1

E1

E1

E1

E1

E1

E1

E1

E1

E1

E1

E1

E1

E1

E1

E1

E1

E1

E1

E1

E1

E1

E1

E1

E1

E1

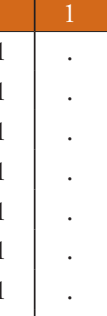




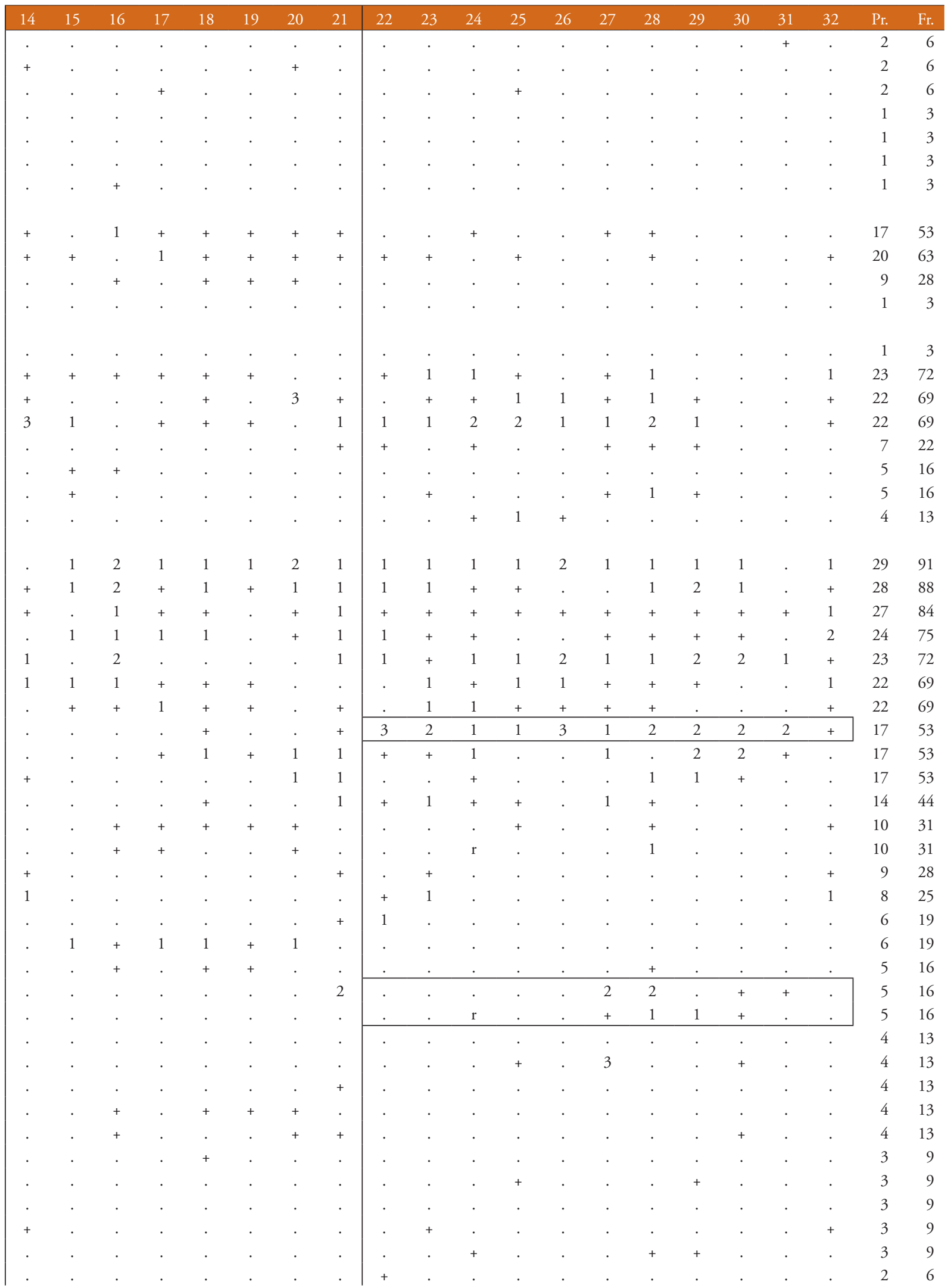


TG Trifolio-Geranietea

CA Caricion austroalpinae

Cfir Caricion firmae

CF Caricion ferrugineae

ES Elyno-Seslerietea

Athyrium distentifolium

Cirsium $x$ benacense

Milium effusum

Rumex alpinus

Aconitum napellus

Allium victorialis

Silene vulgaris subsp. antelopum

Orobanche lycoctoni

Aconitum variegatum

Myrrhis odorata

Hieracium valdepilosum

Verbascum lanatum

Pulsatilla alpina subsp. austroalpina

Heracleum austriacum subsp. siifolium

Arabis vochinensis

Festuca calva

Koeleria eriostachya

Primula wulfeniana

Carex firma

Sesleria sphaerocephala

Pedicularis rostratocapitata

Phyteuma sieberi

Knautia longifolia

Cerastium subtriflorum

Sesleria caerulea

Phyteuma orbiculare

Galium anisophyllum

Aster bellidiastrum

Campanula witasekiana

Polygonum viviparum

Betonica alopecuros

Selaginella selaginoides

Lotus alpinus

Thymus praecox subsp. polytrichus

Ranunculus montanus

Hieracium pilosum

Senecio abrotanifolius

Pedicularis verticillata

Ranunculus carinthiacus

Alchemilla vulgaris agg.

Gentianella anisodonta

Scabiosa lucida subsp. lucida

Myosotis alpestris

E1

E

E1

E1

E1

E1

E

E

E

E1

E1

E1

E1

E1

E1

E1

E1

E1

E1

E1

E1

E1

E1

E1

E1

E1

E1

E1

E1

E1

E1

E1

E1

E1

E1

E1

E1

E1

E1

E1

E

Helianthemum nummularium subsp. grandiflorum E1

Homogyne discolor

Gentiana lutea subsp. symphyandra

Bartsia alpina

$\begin{array}{llllllllllllll}1 & 2 & 3 & 4 & 5 & 6 & 7 & 8 & 9 & 10 & 11 & 12 & 13\end{array}$

E1
E1

E1
E1 1
E1

1




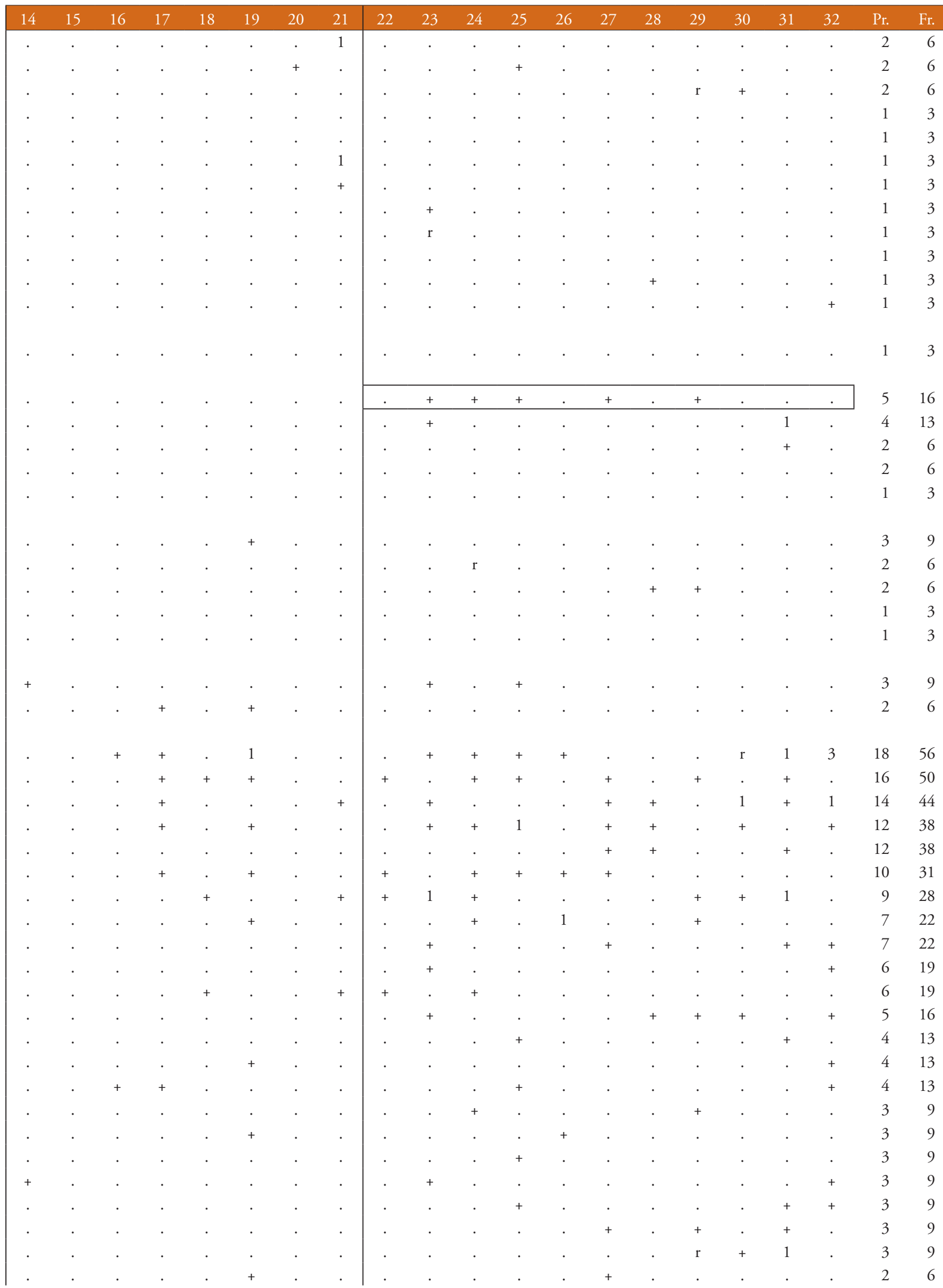




E

Juncus monanthos

Rhinanthus glacialis

Leucanthemum heterophyllum (L.maximum agg.) E1

Phleum hirsutum

Alchemilla alpigena

Cerastium strictum

Scabiosa lucida subsp. stricta

Alchemilla glaucescens

Helictotrichon parlatorei

Globularia nudicaulis

Achillea clavenae

JT Juncetea trifidi

Campanula scheuchzeri

Anthoxanthum nipponicum

LV Loiseleurio-Vaccinietea

Arctostaphylos alpinus

CU Calluno-Ulicetea

Coeloglossum viride

Gentiana pannonica

Festuca nigrescens

Potentilla aurea

Veronica officinalis

Meum athamanticum

Phyteuma zahlbruckneri

FB Festuco-Brometea

Cirsium erisithales

Buphthalmum salicifolium

Carlina acaulis

Prunella grandiflora

Euphorbia cyparissias

Gentianella ciliata

Helictotrichon praeustum

Orobanche gracilis

PaT Poo alpinae-Trisetetalia

Poa alpina

Trollius europaeus

Ranunculus nemorosus

Astrantia major

Phleum rhaeticum

Crepis aurea

Pimpinella major subsp. rubra

MA Molinio-Arrhenatheretea

Crepis paludosa

Cerastium fontanum

Deschampsia cespitosa

Veronica chamaedrys

Leontodon hispidus

Trifolium pratense

Anthoxanthum odoratum

Caltha palustris

Dactylis glomerata s.str.

Galium mollugo
E1

E1

E1

E1

E1

E

E1

E1

E1

E1

E1

E1

E1

E1

E1

E1

E1

E1

E1

E1

E1

E1

E1

E1

E1

E1

E1

E1

E1

E1

E1

E1

E1

E1

E1

E1

E1

E1

E1

E1

E1

\begin{tabular}{l|lllllll} 
& 1 & 2 & 3 & 4 & 5 & 6 & 7 \\
1 & $\cdot$ & $\cdot$ & $\cdot$ & $\cdot$ & $\cdot$ & $\cdot$ & $\cdot$ \\
1 & $\cdot$ & $\cdot$ & $\cdot$ & $\cdot$ & $\cdot$ & $\cdot$ & $\cdot$ \\
1 & $\cdot$ & $\cdot$ & $\cdot$ & $\cdot$ & $\cdot$ & $\cdot$ & $\cdot$ \\
1 & $\cdot$ & $\cdot$ & $\cdot$ & $\cdot$ & $\cdot$ & $\cdot$ & $\cdot$ \\
1 & $\cdot$ & $\cdot$ & $\cdot$ & $\cdot$ & $\cdot$ & $\cdot$ & $\cdot$ \\
1 & $\cdot$ & $\cdot$ & $\cdot$ & $\cdot$ & $\cdot$ & $\cdot$ & $\cdot$ \\
1 & $\cdot$ & $\cdot$ & $\cdot$ & $\cdot$ & $\cdot$ & $\cdot$
\end{tabular}

.

,

.

1

1

.

$\begin{array}{rrrrrr}4 & 4 & 3 & + & + & + \\ + & 1 & 1 & + & + & +\end{array}$




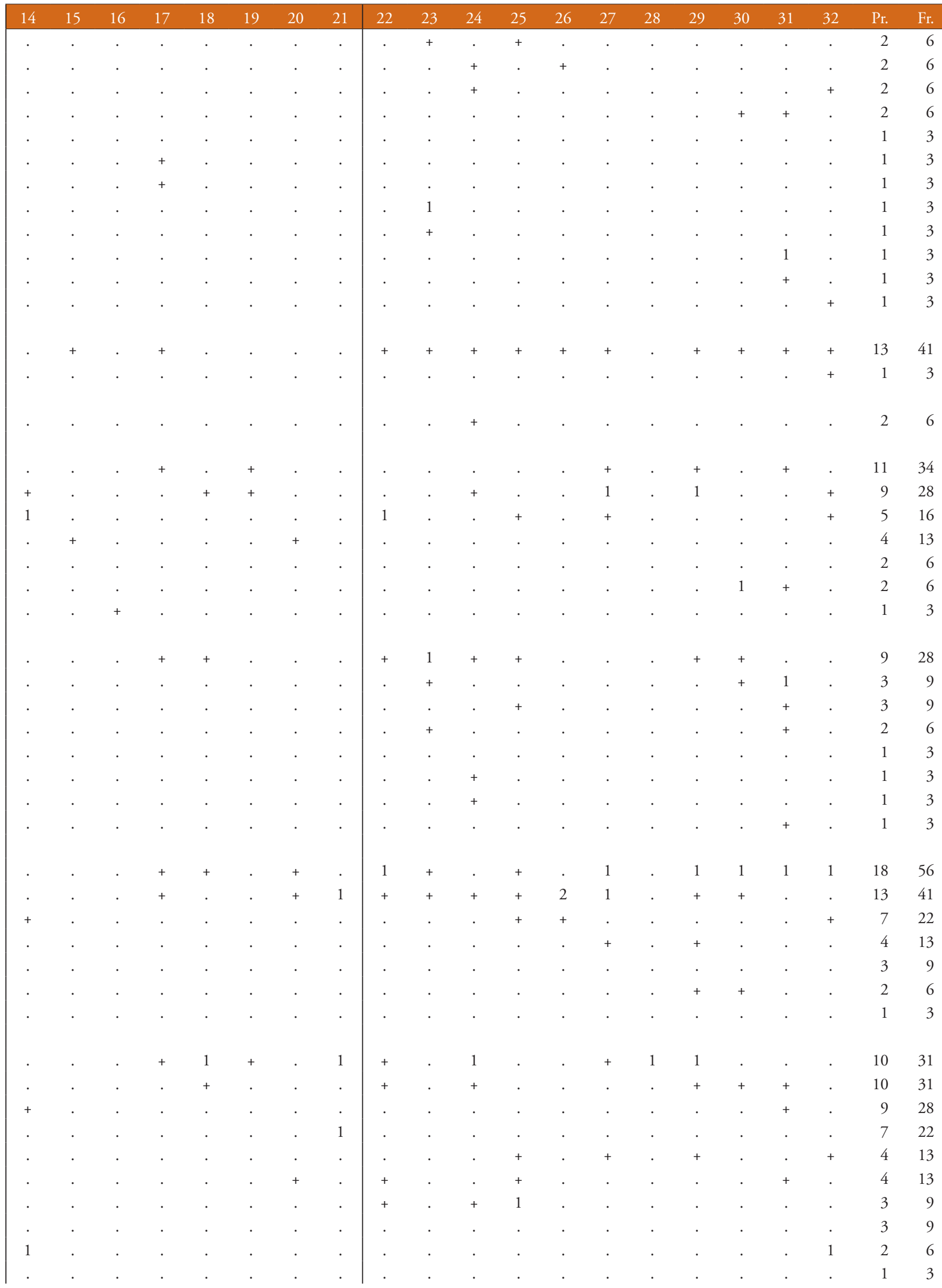


Number of relevé (Zaporedna številka popisa)

Prunella vulgaris

Ajuga reptans

Cerastium holosteoides

Trifolium repens

MC Montio-Cardaminetea

Alchemilla glabra

Saxifraga aizoides

CD Caricetalia davallianae

Parnassia palustris

Pinguicula alpina

Tofieldia calyculata

EA Epilobietea angustifoliae

Fragaria vesca

Rubus idaeus

Urtica dioica

Tussilago farfara

Chamaenerion angustifolium

AC Arabidetalia caeruleae

Soldanella alpina

Alchemilla fissa

Ranunculus alpestris

Soldanella minima

TR Thlaspietea rotundifolii

Adenostyles glabra

Cystopteris montana

Gymnocarpium robertianum

Dryopteris villarii

Arabis alpina

Valeriana montana

Rhodiola rosea

Hieracium bifidum

Trisetum argenteum

Minuartia austriaca

Scrophularia juratensis

Rumex scutatus

Saxifraga caesia

Aquilegia einseleana

Heracleum sphondylium subsp. pollinianum

Sedum atratum

Cy Cystopteridion fragilis

Cystopteris fragilis

Heliosperma pusillum

Cystopteris regia

PS Physoplexido comosae-Saxifragion petraeae

Saxifraga crustata

\begin{tabular}{llllllllllllll|}
1 & 2 & 3 & 4 & 5 & 6 & 7 & 8 & 9 & 10 & 11 & 12 & 13
\end{tabular}

PC Potentilletalia caulescentis

Campanula cochleariifolia

Androsace lactea

Primula auricula

Festuca stenantha

AT Asplenietea trichomanis

Asplenium viride

Moehringia muscosa

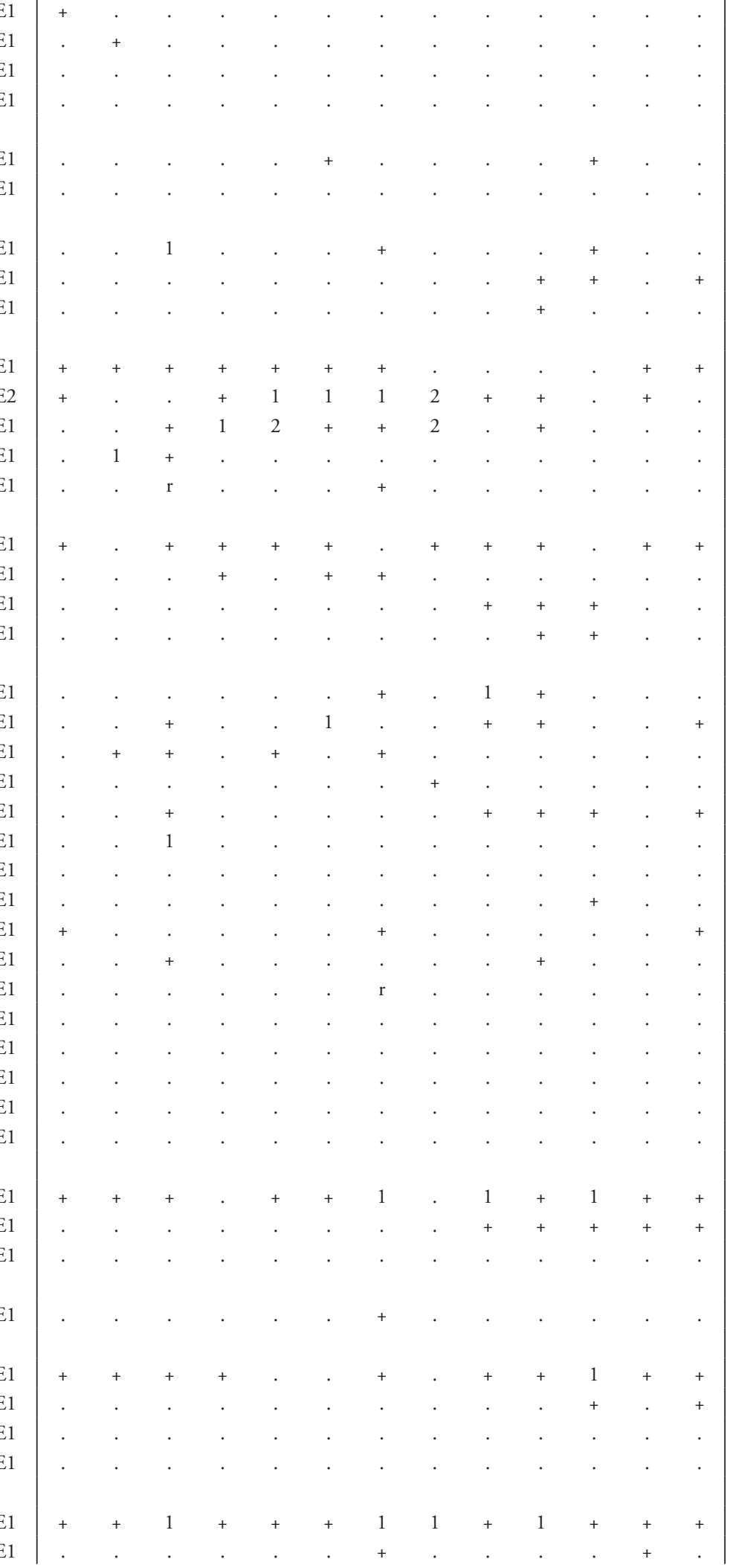




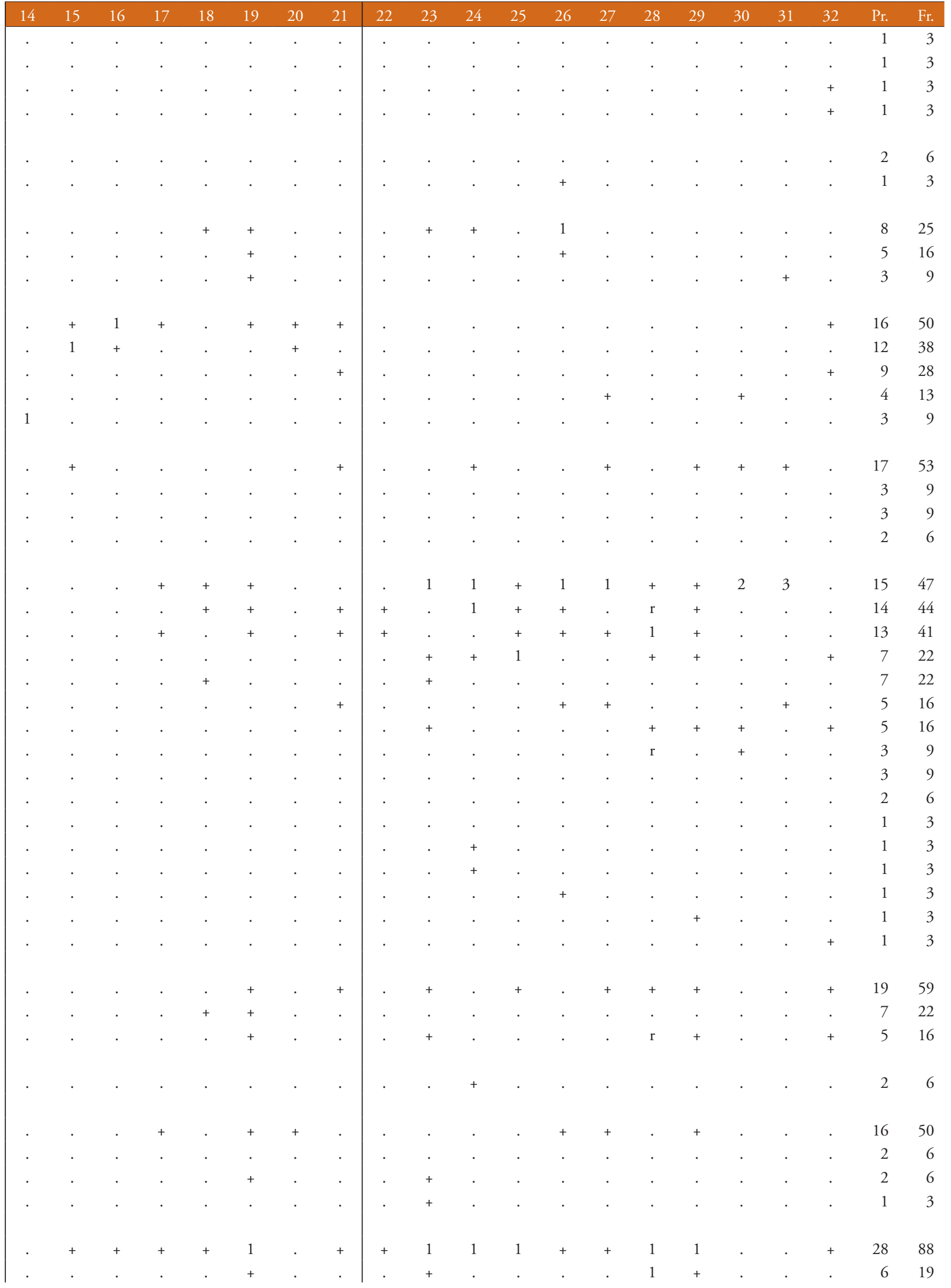


Asplenium trichomanes

Polypodium vulgare

Asplenium ruta-muraria

Fu Fungi (Glive)

Laricifomes officinalis

ML Mossees and lichens (Mahovi in lišaji)

Ctenidium molluscum

Tortella tortuosa

Dicranum scoparium

Rhytidiadelphus triquetrus

Polytrichum formosum

Hylocomium splendens

Peltigera leucophlebia

Schistidium sp.

Fissidens dubius

Plagiochila porelloides

Peltigera canina

Rhytidiadelphus loreus

Eurhynchium angustirete

Isothecium alopecuroides

Cladonia pyxidata

Orthothecium rufescens

Pseudoleskea incurvata

Marchantia polymorpha

Cetraria islandica

Mnium thomsonii

Plagiomnium cuspidatum

Plagiothecium undulatum

Conocephalum conicum

Paraleucobryum sauteri

Rhizomnium punctatum

Letharia vulpina

Mnium sp.

Thuidium tamariscinum

\begin{tabular}{|c|c|c|c|c|c|c|c|c|c|c|c|}
\hline E1 & & . & . & & + & & & & & & \\
\hline E1 & . & & . & . & . & + & & + & & . & . \\
\hline E1 & . & & . & . & . & . & & . & & . & . \\
\hline E3c & . & & . & . & . & . & & & & . & . \\
\hline E0 & + & + & + & . & + & + & + & 1 & 1 & + & + \\
\hline E0 & + & + & + & + & + & + & + & + & + & + & + \\
\hline E0 & + & . & + & + & & + & + & + & 1 & 1 & \\
\hline E0 & + & . & 1 & + & & 1 & + & . & 2 & 2 & 1 \\
\hline E0 & + & . & & & & . & + & + & 1 & 1 & + \\
\hline E0 & + & . & + & & & + & . & . & 1 & 2 & 1 \\
\hline E0 & . & . & + & & & . & . & + & . & + & \\
\hline E0 & . & & + & . & . & . & + & 1 & & . & . \\
\hline E0 & + & & . & . & . & + & + & . & + & . & . \\
\hline E0 & . & & . & . & . & + & + & . & + & . & . \\
\hline E0 & . & & . & . & + & . & & + & & . & . \\
\hline E0 & + & & . & + & . & + & & . & & . & . \\
\hline E0 & . & & + & . & . & . & . & . & + & . & . \\
\hline E0 & & & . & + & . & . & . & 1 & . & . & . \\
\hline E0 & & & . & . & . & + & . & 1 & . & . & . \\
\hline E0 & . & & . & . & . & 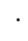 & . & . & . & + & + \\
\hline E0 & . & & . & . & . & . & . & + & . & . & . \\
\hline E0 & . & & . & . & . & . & . & & . & . & . \\
\hline E0 & . & & . & . & . & . & & & & + & . \\
\hline E0 & . & & . & . & . & . & & & & . & . \\
\hline E0 & . & & . & . & . & . & & & & . & . \\
\hline E0 & . & & . & . & . & . & & & & . & . \\
\hline E0 & . & & . & . & . & . & & & & . & . \\
\hline E0 & . & & . & . & . & . & & & & . & . \\
\hline E0 & & . & & & & & . & & . & & \\
\hline E3c & & . & & & & & . & & . & & \\
\hline E0 & & . & & & & & . & & . & & \\
\hline E0 & . & & & & & & & . & & & . \\
\hline
\end{tabular}

\section{Legend - Legenda}

ID Igor Dakskobler

AS Andrej Seliškar

AR Andrej Rozman

AP Aleš Poljanec

A Limestone - apnenec

D Dolomite-dolomit

Gr Gravel - grušč

Gl Claystone - glinavec

L Marlstone - laporovec

R Chert-roženec

Re Rendzina - rendzina

$\mathrm{Rj}$ Brown soil on limestone and dolomite - rjava pokarbonatna tla

E Eutric brown soil - evtrična rjava tla

JA Julian Alps - Julijske Alpe

KSA Kamnik-Savinja Alps - Kamniško-Savinjske Alpe

K Karavanke - Karavanke

Pr. Presence (number of relevés in which the species is presented) - število popisov, v katerih se pojavlja vrsta

Fr. Frequency in $\%$ - frekvenca v \% 


\begin{tabular}{|c|c|c|c|c|c|c|c|c|c|c|c|c|c|c|c|c|c|c|c|c|}
\hline 14 & 15 & 16 & 17 & 18 & 19 & 20 & 21 & 22 & 23 & 24 & 25 & 26 & 27 & 28 & 29 & 30 & 31 & 32 & Pr. & Fr. \\
\hline & . & & . & & & & . & . & + & . & & . & & + & & . & & + & 4 & 13 \\
\hline . & . & . & . & . & . & . & . & & . & . & . & . & . & & . & & . & & 2 & 6 \\
\hline . & . & . & . & . & . & . & . & & + & . & . & . & . & + & . & & . & r & 2 & 6 \\
\hline . & . & . & . & . & . & . & . & & . & . & . & + & . & & . & & . & . & 1 & 3 \\
\hline . & . & + & + & 1 & 1 & . & + & + & 1 & 1 & 1 & . & + & 1 & + &. & . & 1 & 24 & 75 \\
\hline . & . & . & + & 1 & . & + & . & & . & . & + & + & 1 & 1 & 1 & + & + & + & 23 & 72 \\
\hline 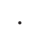 & . & . & . & + & . & + & + & + & + & + & + & + & + & + & + & + & . & 1 & 23 & 72 \\
\hline 1 & . & + & . & . & + & . & . & 1 & + & 1 & 1 & + & + & + & . & & . & + & 21 & 66 \\
\hline . & . & . & . & + & 1 & + & . & + & . & . & . & + & . & & . & & . & + & 14 & 44 \\
\hline . & . & + & . & + & . & . & . & & . & + & . & . & + & & + & & . & & 11 & 34 \\
\hline . & . & + & . & . & + & . & . & + & . & + &. & + & . & + & . & & . & + & 11 & 34 \\
\hline & . & & + & & & & . & . & & . & 1 & . & & . & & + & + & + & 8 & 25 \\
\hline & . & & . & & + & & . & . & + & . & & + & & . & & . & & . & 7 & 22 \\
\hline & . & & + & & & & . & . & & . & & . & . & . & & . & & + & 5 & 16 \\
\hline & . & . & . & & + & & . & . & & . & & . & . & . & . & . & . & . & 4 & 13 \\
\hline & . & . & . & & & & . & . & & . & . & . & . & . & . & . & . & . & 3 & 9 \\
\hline . & . & . & . & . & . & . & . & . & . & . & . & . & . & & . & . & . & . & 3 & 9 \\
\hline . & . & . & . & . & . & . & . & . & . & . & . & . & . & & . & . & . & 1 & 3 & 9 \\
\hline . & . & . & + & . & . & . & . & . & . & . & . & . & . & & . & . & . & . & 3 & 9 \\
\hline . & . & . & . & . & + & . & . & . & . & . & . & . & . & & . & . & . & . & 3 & 9 \\
\hline . & . & . & . & . & . & . & . & . & . & . & . & . & . & & . & + & . & . & 2 & 6 \\
\hline . & . & . & . & . & + & . & . & . & . & + & . & . & . & & . & . & . & . & 2 & 6 \\
\hline . & & . & . & . & . & . & . & & . & & . & & . & & . & & . & & 1 & 3 \\
\hline . & & . & . & + & . & . & . & & . & & . & & . & & . & & . & & 1 & 3 \\
\hline . & & . & & + & . & . & . & & . & & . & & . & & . & & . & & 1 & 3 \\
\hline . & & . & & . & + & . & . & & . & & . & & . & & . & & . & & 1 & 3 \\
\hline . & & . & & . & + & . & . & & . & & . & & . & & . & & . & & 1 & 3 \\
\hline . & & . & & . & + & . & . & & . & . & . & & . & & . & & . & & 1 & 3 \\
\hline . & . & . & . & . & . & . & . & . & . & . & + & . & . & . & . & . & . & . & 1 & 3 \\
\hline . & . & . & . & . & . & . & . & . & . & . & . & + & . & . & . & . & . & . & 1 & 3 \\
\hline . & . & . & . & . & . & . & . & . & . & . & . & . & . & . & . & . & . & + & 1 & 3 \\
\hline & & & & & & & & & & & & & & & & & & & 1 & \\
\hline
\end{tabular}


Table 3 (Tabela 3): Rhodothamno-Laricetum geetosum rivalis var. Festuca nitida

\section{Number of relevé (Zaporedna številka popisa)}

Database number of relevé

(Delovna številka popisa)

Author of the relevé (Avtor popisa)

Elevation in $\mathrm{m}$ (Nadmorska višina $\mathrm{v} \mathrm{m}$ )

Aspect (Lega)

Slope in degrees (Nagib v stopinjah)

Parent material (Matična podlaga)

Soil (Tla)

Stoniness in \% (Kamnitost v \%)

Cover in \% (Zastiranje v \%):

Upper tree layer (Zgornja drevesna plast)

Lower tree layer (Spodnja drevesna plasti)

Shrub layer (Grmovna plast)

Herb layer (Zeliščna plast)

Moss layer (Mahovna plast)

Number of species (Število vrst)

Relevé area (Velikost popisne ploskve)

Date of taking relevé (Datum popisa)

Locality (Nahajališče)

Mountain range (Pogorje)

Quadrant (Kvadrant)

Coordinate GK-Y (D-48)

Coordinate GK-X (D-48)

Character and differential species of the association (Značilnice in razlikovalnice asociacije)

VP

$\mathrm{VP}$

VP

EP

CF

TR

PS

ES

EP

TR

VP

CA

$\mathrm{AF}$

TR

PC
Larix decidua

Larix decidua

Larix decidua

Rhododendron hirsutum

Carex ferruginea

Festuca nitida

Paederota lutea

Astrantia bavarica

Rhodothamnus chamaecistus

Heliosperma alpestre

Homogyne sylvestris

Laserpitium peucedanoides

Anemone trifolia

Astrantia carniolica

Valeriana saxatilis

\begin{tabular}{|c|c|c|c|c|c|c|c|c|c|c|}
\hline E3 & 4 & 3 & 3 & 3 & 4 & 3 & 4 & 4 & 4 & 4 \\
\hline E2 & 1 & . & + & 1 & + & 1 & 1 & 2 & 2 & 1 \\
\hline E1 & . & + & + & . & . & + & . & . & . & + \\
\hline E2 & 2 & 2 & 3 & 2 & 3 & 1 & 3 & 2 & 2 & 3 \\
\hline E1 & + & + & 1 & 1 & 1 & 1 & + & . & 3 & 2 \\
\hline E1 & . & + & . & . & 1 & + & + & + & + & + \\
\hline E1 & . & + & . & . & + & + & + & 1 & . & + \\
\hline E1 & . & 1 & 1 & 1 & . & 1 & + & + & 1 & 1 \\
\hline E1 & . & . & $\mathrm{r}$ & . & + & . & 1 & + & . & + \\
\hline E1 & . & . & . & . & . & + & + & + & . & . \\
\hline E1 & . & . & + & + & + & + & + & . & . & + \\
\hline E1 & . & . & + & . & + & . & + & + & 1 & + \\
\hline E1 & + & . & . & . & . & . & 1 & . & . & . \\
\hline E1 & . & . & . & . & . & . & + & . & . & . \\
\hline E1 & . & . & . & . & . & . & . & + & . & + \\
\hline
\end{tabular}




\section{\begin{tabular}{lllllllll|llllllllll|ll}
11 & 12 & 13 & 14 & 15 & 16 & 17 & 18 & 19 & 20 & 21 & 22 & 23 & 24 & 25 & 26 & 27 & 28 & 29 & Pr. & Fr.
\end{tabular}}

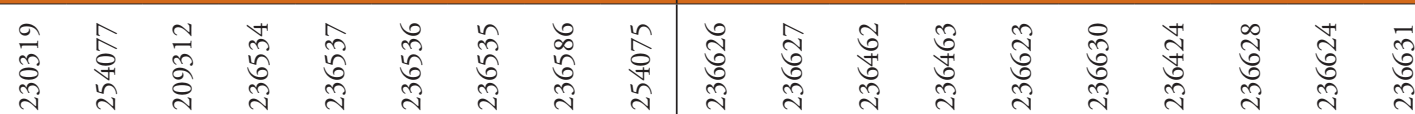
IDAR ID ID ID ID ID ID IDAS ID ID ID IDAS IDAS ID ID IDAS ID ID ID $\begin{array}{llllllllllllllllllll}1770 & 1791 & 1530 & 1770 & 1640 & 1659 & 1720 & 1760 & 1700 & 1720 & 1720 & 1710 & 1720 & 1700 & 1700 & 1680 & 1760 & 1720 & 1650\end{array}$

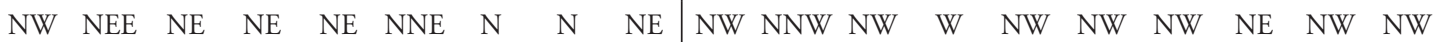
$\begin{array}{lllllllllllllllllll}35 & 30 & 35 & 35 & 35 & 35 & 35 & 40 & 30 & 40 & 45 & 35 & 40 & 40 & 45 & 40 & 35 & 45 & 40\end{array}$

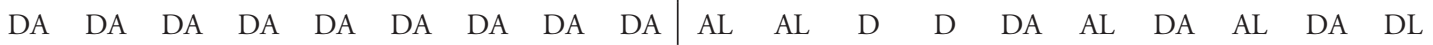
$\operatorname{Re} \operatorname{Re} \operatorname{Re} \operatorname{Re} \operatorname{Re} \operatorname{Re} \operatorname{Re} \operatorname{Re} \operatorname{Re} \quad \operatorname{Re} \operatorname{Re} \quad \operatorname{Re} \quad \operatorname{Re} \quad \operatorname{Re} \quad \operatorname{Re} \operatorname{Re} \quad \operatorname{Re} \quad \operatorname{Re} \quad \operatorname{Re}$ $\begin{array}{lllllllllllllllllll}10 & 10 & 10 & 20 & 20 & 10 & 10 & 20 & 10 & 20 & 20 & 10 & 5 & 5 & 10 & 5 & 20 & 10 & 10\end{array}$

\begin{tabular}{lllllllll|llllllllll}
50 & 40 & 60 & 40 & 40 & 60 & 40 & 40 & 60 & 70 & 60 & 70 & 70 & 60 & 60 & 70 & 60 & 60 & 50
\end{tabular} \begin{tabular}{lllllllll|llllllllll}
10 & 40 & 0 & 10 & 30 & 20 & 30 & 20 & 15 & 5 & 5 & 5 & 10 & 5 & 5 & 5 & 5 & 5 & 5
\end{tabular} $\begin{array}{lllllllllllllllllll}40 & 30 & 60 & 40 & 40 & 40 & 60 & 40 & 40 & 10 & 20 & 20 & 30 & 50 & 40 & 30 & 40 & 30 & 40\end{array}$ \begin{tabular}{lllllllll|llllllllll}
90 & 90 & 60 & 80 & 70 & 90 & 80 & 80 & 95 & 70 & 70 & 90 & 80 & 80 & 70 & 80 & 70 & 90 & 80
\end{tabular} \begin{tabular}{lllllllll|llllllllll}
10 & 5 & 10 & 5 & 5 & 5 & 5 & 5 & 5 & 5 & 10 & 5 & 5 & 5 & 10 & 5 & 10 & 5 & 10
\end{tabular} $\begin{array}{lllllllllllllllllll}78 & 84 & 94 & 87 & 73 & 99 & 88 & 91 & 65 & 93 & 81 & 84 & 65 & 87 & 101 & 68 & 86 & 82 & 91\end{array}$ $\begin{array}{lllllllllllllllllll}400 & 500 & 400 & 400 & 400 & 400 & 400 & 400 & 500 & 400 & 400 & 400 & 400 & 400 & 400 & 400 & 400 & 400 & 400\end{array}$ $\begin{array}{llllllllllllllllllll}7 / 22 & 8 / 28 & 6 / 27 & 8 / 2 & 8 / 2 & 8 / 2 & 8 / 2 & 9 / 1 & 8 / 28 & 8 / 24 & 8 / 24 & 8 / 18 & 8 / 18 & 8 / 23 & 8 / 24 & 8 / 11 & 8 / 24 & 8 / 23 & 8 / 24\end{array}$ $20092014200020102010201020102010 \quad 2014 \quad 2010201020102010 \quad 201020102010201020102010$

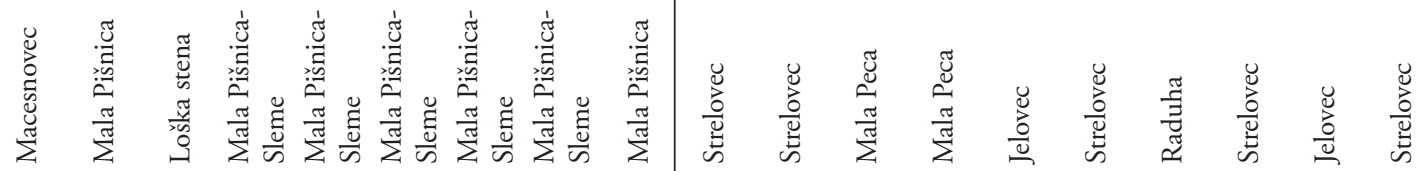
JA JA JA JA JA JA JA JA JA

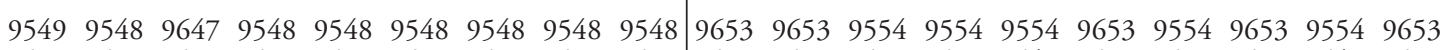

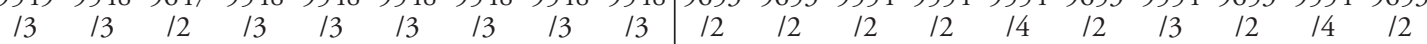

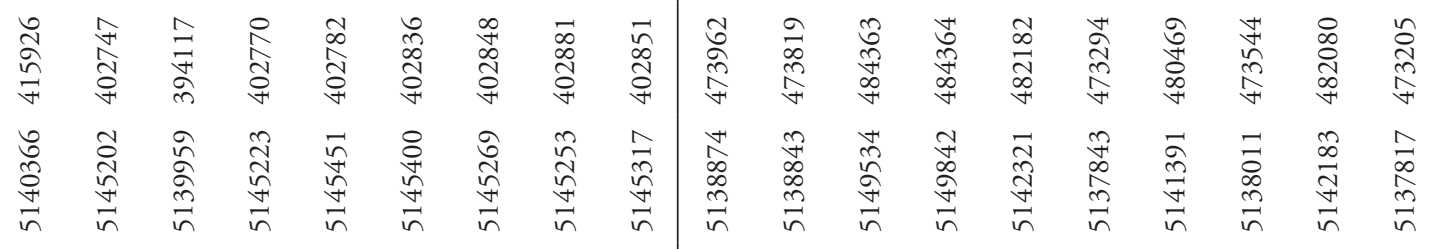

\begin{tabular}{|c|c|c|c|c|c|c|c|c|c|c|c|c|c|c|c|c|c|c|c|}
\hline 4 & 4 & 3 & 3 & 3 & 4 & 3 & 3 & 4 & 4 & 4 & 4 & 4 & 4 & 4 & 4 & 4 & 4 & 4 & 29100 \\
\hline 1 & 2 & 1 & 3 & 2 & 2 & 3 & 2 & 3 & 1 & 1 & + & . & 1 & 2 & 1 & 1 & 1 & 1 & 279 \\
\hline . & & & + & . & . & . & . & . & . & . & . & . & . & . & . & . & + & . & 6 \\
\hline 3 & 4 & 3 & 3 & 3 & 2 & 3 & 2 & 3 & 1 & 1 & 1 & 2 & 4 & 3 & 3 & 3 & 3 & 1 & 29100 \\
\hline 2 & 2 & 1 & . & 1 & 1 & 2 & 1 & 1 & + & . & + & + & 2 & 1 & 2 & . & 2 & 2 & $25 \quad 80$ \\
\hline . & + & + & + & + & + & + & 1 & 1 & + & 1 & + & . & 1 & 1 & + & + & 1 & + & 24 \\
\hline+ & 1 & . & + & + & + & + & 1 & + & + & + & + & + & . & + & + & + & . & + & 22 \\
\hline 1 & & + & . & 1 & 1 & 1 & 1 & 1 & 1 & & + & . & . & . & 1 & + & 1 & 1 & $21 \quad 72$ \\
\hline 1 & 2 & 1 & 1 & + & 1 & 1 & 1 & 1 & + & . & . & . & . & + & . & . & 1 & . & 17 \\
\hline+ & + & + & + & 1 & + & 1 & + & 1 & 1 & . & + & . & + & . & . & + & + & . & 17 \\
\hline+ & . & + & . & . & . & . & . & + & + & + & . & . & 1 & + & . & + & 1 & + & 16 \\
\hline+ & . & . & + & 1 & + & . & + & + & . & . & . & + & . & 1 & . & . & . & + & 15 \\
\hline+ & . & . & . & + & + & . & . & . & . & . & . & . & . & . & . & & . & . & 17 \\
\hline & & + & . & . & . & . & . & . & & + & . & . & . & . & . & + & . & . & 14 \\
\hline & & + & + & . & & & . & & & & . & & & & & & . & . & $\begin{array}{ll}4 & 14\end{array}$ \\
\hline
\end{tabular}


Number of relevé (Zaporedna številka popisa)

$\begin{array}{lll}1 & 2 & 3\end{array}$

34

5

Differential species of the subassociation (Razlikovalne vrste subasociacije)

MuA Chaerophyllum hirsutum

MuA Geum rivale

MuA Rumex arifolius

MuA Senecio cacaliaster

\begin{tabular}{l|l} 
E1 & 1 \\
E1 & 1 \\
E1 & + \\
E1 &.
\end{tabular}

Differential species of the variant and subvariants (Razlikovalne vrste variante in subvariant)

PC Campanula cochleariifolia

VP Luzula luzuloides

JT Campanula scheuchzeri

MuA Aconitum lycoctonum subsp. ranunculifolium

VP Vaccinio-Piceetea

Vaccinium myrtillus

Polystichum lonchitis

Luzula sylvatica

Aposeris foetida

Vaccinium vitis-idaea

Picea abies

Picea abies

Picea abies

Valeriana tripteris

Clematis alpina

Oxalis acetosella

Dryopteris dilatata

Hieracium murorum

Calamagrostis villosa

Homogyne alpina

Lycopodium annotinum

Gymnocarpium dryopteris

Melampyrum sylvaticum

Dryopteris expansa

Rosa pendulina

Huperzia selago

Saxifraga cuneifolia

Phegopteris connectilis

Abies alba

Abies alba

Abies alba

Solidago virgaurea subsp. virgaurea

Lonicera caerulea

Veronica urticifolia

Lonicera nigra

Luzula luzulina

Calamagrostis arundinacea

Thelypteris limbosperma

Gentiana asclepiadea

Maianthemum bifolium

Luzula pilosa

Avenella flexuosa

Goodyera repens

EP

Erico-Pinetea

Pinus mugo

Erica carnea

\begin{tabular}{|c|c|c|c|c|c|c|c|c|c|c|}
\hline E1 & . & . & . & + & + & + & . & + & . & . \\
\hline E1 & . & 1 & 1 & 1 & 1 & 1 & . & . & . & . \\
\hline E1 & . & . & . & . & . & + & + & + & + & + \\
\hline E1 & . & . & . & . & . & . & 1 & . & . & . \\
\hline E1 & 3 & 2 & 2 & 2 & 2 & 1 & 3 & 1 & 1 & 2 \\
\hline E1 & 1 & 1 & 1 & 1 & 1 & + & 1 & + & + & 1 \\
\hline E1 & 3 & + & 1 & . & 1 & 1 & 1 & 1 & 1 & 1 \\
\hline E1 & 1 & + & 1 & 1 & 1 & 1 & 1 & 1 & 1 & 2 \\
\hline E1 & + & + & + & 1 & 1 & 1 & 1 & 1 & 1 & 1 \\
\hline E3 & + & + & + & + & + & + & $\mathrm{r}$ & . & + & . \\
\hline E2 & + & . & + & 1 & + & + & + & + & + & + \\
\hline E1 & . & + & . & . & . & + & . & . & . & . \\
\hline E1 & + & + & 1 & 1 & 2 & 1 & 1 & 1 & + & 1 \\
\hline E2 & . & . & + & + & + & + & + & 1 & + & + \\
\hline E1 & 1 & 1 & 1 & 1 & 1 & 1 & + & 1 & 1 & 1 \\
\hline E1 & 1 & 1 & + & + & + & + & 1 & 1 & + & + \\
\hline E1 & + & . & + & + & + & + & . & + & + & + \\
\hline E1 & + & 1 & 3 & . & 2 & 3 & 2 & . & . & + \\
\hline E1 & 1 & . & . & + & + & + & + & 1 & 1 & 1 \\
\hline E1 & + & . & . & . & . & . & 1 & + & + & + \\
\hline E1 & + & . & . & + & . & + & + & . & . & . \\
\hline E1 & . & . & + & . & . & . & + & + & . & . \\
\hline E1 & . & . & . & . & . & . & 1 & . & . & + \\
\hline E2 & . & . & + & . & . & . & + & . & . & . \\
\hline E1 & . & . & . & . & . & + & + & . & + & + \\
\hline E1 & . & + & . & . & . & . & . & . & . & . \\
\hline E1 & . & + & . & . & . & + & . & . & . & . \\
\hline E3 & . & + & + & . & $\mathrm{r}$ & . & . & . & . & . \\
\hline E2 & . & + & . & . & . & . & . & . & . & + \\
\hline E1 & . & . & . & . & . & . & . & . & . & . \\
\hline E1 & . & . & . & . & . & . & . & . & . & . \\
\hline E2 & . & . & . & . & . & . & + & . & . & . \\
\hline E1 & . & . & . & . & . & . & + & . & . & + \\
\hline E2 & . & + & + & . & . & . & . & . & . & . \\
\hline E1 & . & . & . & . & . & . & . & 1 & . & . \\
\hline E1 & + & . & + & . & . & . & . & . & . & . \\
\hline E1 & . & . & . & . & . & + & + & . & . & . \\
\hline E1 & . & . & . & . & . & . & + & . & . & . \\
\hline E1 & . & . & . & . & . & . & . & . & . & . \\
\hline E1 & + & . & . & . & . & . & . & . & . & . \\
\hline E1 & . & . & . & . & . & . & . & . & . & . \\
\hline E1 & . & . & . & . & . & . & . & . & . & . \\
\hline E2 & + & 2 & 3 & + & + & + & + & 1 & 1 & + \\
\hline E1 & + & + & + & + & + & . & 1 & . & + & + \\
\hline
\end{tabular}


Number of relevé (Zaporedna številka popisa)

Calamagrostis varia

Rubus saxatilis

Aquilegia nigricans

Carex ornithopoda

Molinia arundinacea

AF Aremonio-Fagion

Knautia drymeia

Cardamine enneaphyllos

Helleborus niger

Cyclamen purpurascens

Cardamine trifolia

TA Tilio-Acerion

Acer pseudoplatanus

Acer pseudoplatanus

Acer pseudoplatanus

Adoxa moschatellina

Dryopteris affinis

FS Fagetalia sylvaticae

Daphne mezereum

Melica nutans

Paris quadrifolia

Myosotis sylvatica

Dryopteris filix-mas

Lilium martagon

Euphorbia amygdaloides

Galeobdolon flavidum

Epilobium montanum

Symphytum tuberosum

Prenanthes purpurea

Mercurialis perennis

Lonicera alpigena

Polystichum aculeatum

Mycelis muralis

Phyteuma spicatum

Fagus sylvatica

Fagus sylvatica

Ranunculus lanuginosus

Luzula nivea

Petasites albus

QP Quercetalia pubescenti-petraeae

Sorbus aria (Aria edulis)

QF Querco-Fagetea

Poa nemoralis

Anemone nemorosa

Hepatica nobilis

Festuca heterophylla

Carex digitata

Hieracium lachenalii

Dactylorhiza fuchsii

Cruciata glabra

Platanthera bifolia

\begin{tabular}{|c|c|c|c|c|c|c|c|c|c|c|}
\hline E1 & + & + & + & . & . & . & 1 & . & . & 1 \\
\hline E1 & + & + & . & . & + & . & 1 & . & . & + \\
\hline E1 & . & . & . & . & + & . & + & + & . & + \\
\hline E1 & . & . & . & . & . & . & . & . & + & . \\
\hline E1 & . & . & . & . & . & . & . & . & . & . \\
\hline E1 & 1 & + & + & . & . & 1 & + & . & 1 & + \\
\hline E1 & . & + & + & 1 & . & + & + & 1 & + & + \\
\hline $\mathrm{E} 1$ & 2 & . & + & . & . & . & 1 & . & . & . \\
\hline E1 & . & . & . & . & . & . & . & . & . & . \\
\hline E1 & . & . & . & . & . & . & . & . & . & . \\
\hline E3 & . & . & . & . & . & . & + & . & . & . \\
\hline E2 & . & . & + & . & . & . & 1 & . & . & . \\
\hline E1 & . & . & . & . & + & + & 1 & . & . & . \\
\hline E1 & . & . & . & . & . & . & . & . & . & . \\
\hline E1 & . & . & . & . & . & . & . & . & . & . \\
\hline E2 & + & + & + & + & + & + & + & . & 1 & + \\
\hline E1 & . & . & 1 & . & . & . & + & . & + & 1 \\
\hline E1 & . & . & + & + & + & . & + & . & . & . \\
\hline E1 & . & + & . & + & + & + & . & + & . & . \\
\hline E1 & . & + & . & . & + & + & . & + & . & . \\
\hline E1 & . & . & . & . & . & . & + & . & + & + \\
\hline E1 & . & . & + & . & . & . & . & . & . & . \\
\hline E1 & . & + & + & . & . & . & . & . & . & . \\
\hline E1 & . & + & . & . & . & . & . & . & . & . \\
\hline E1 & . & . & + & + & + & + & . & . & . & . \\
\hline E1 & . & . & + & . & + & . & + & . & . & . \\
\hline $\mathrm{E} 1$ & . & . & . & . & . & . & . & . & . & . \\
\hline E2 & . & . & . & . & . & . & . & . & . & . \\
\hline E1 & . & . & . & . & + & . & . & . & . & . \\
\hline E1 & . & . & + & . & . & . & . & . & . & . \\
\hline E1 & . & . & . & . & . & . & + & . & . & . \\
\hline E3 & . & . & . & . & . & . & + & . & . & . \\
\hline E2 & . & . & . & . & . & . & . & . & . & . \\
\hline $\mathrm{E} 1$ & . & . & . & . & . & . & . & . & . & . \\
\hline E1 & . & . & . & . & . & . & . & . & . & . \\
\hline E1 & . & . & . & . & . & . & . & . & . & . \\
\hline E2 & . & . & + & . & . & . & . & . & . & . \\
\hline E1 & + & + & . & . & + & + & + & + & . & . \\
\hline E1 & . & . & + & + & + & + & . & + & . & . \\
\hline E1 & 1 & + & + & . & . & . & . & . & . & . \\
\hline E1 & . & + & + & + & + & + & . & . & . & . \\
\hline E1 & . & . & . & . & . & . & + & . & . & + \\
\hline E1 & . & . & . & . & . & . & . & . & . & . \\
\hline E1 & . & . & . & + & . & . & . & . & . & . \\
\hline E1 & . & . & . & . & . & . & . & . & . & . \\
\hline E1 & . & . & . & . & . & . & . & . & . & . \\
\hline
\end{tabular}


Number of relevé (Zaporedna številka popisa)

SSC Sambuco-Salicion capreae, Rhamno-Prunetea

Sorbus aucuparia subsp. aucuparia

Sorbus aucuparia subsp. aucuparia

Sorbus aucuparia subsp. aucuparia

BA Betulo-Alnetea

Sorbus chamaemespilus

Salix appendiculata

Salix appendiculata

Alnus viridis

Salix glabra

Salix waldsteiniana

Juniperus sibirica

Ribes alpinum

MuA Mulgedio-Aconitetea

Viola biflora

Veratrum album subsp. lobelianum

Saxifraga rotundifolia

Geranium sylvaticum

Athyrium filix-femina

Hypericum maculatum

Poa hybrida

Polygonatum verticillatum

Primula elatior

Adenostyles alliariae

Epilobium alpestre

Phyteuma ovatum

Stellaria nemorum

Alchemilla xanthochlora

Chaerophyllum villarsii

Cirsium carniolicum

Senecio ovatus

Alchemilla monticola

Pedicularis recutita

Aconitum tauricum

Heracleum montanum

Veratrum album subsp. album

Lamium maculatum

Milium effusum

Aconitum napellus

Allium victorialis

Thalictrum aquilegiifolium

Peucedanum ostruthium

Ranunculus platanifolius

Aconitum variegatum

Cicerbita alpina

Agropyron caninum

TG Trifolio-Geranietea

Dianthus barbatus

Silene nutans

CA Caricion austroalpinae

Heracleum austriacum subsp. siifolium

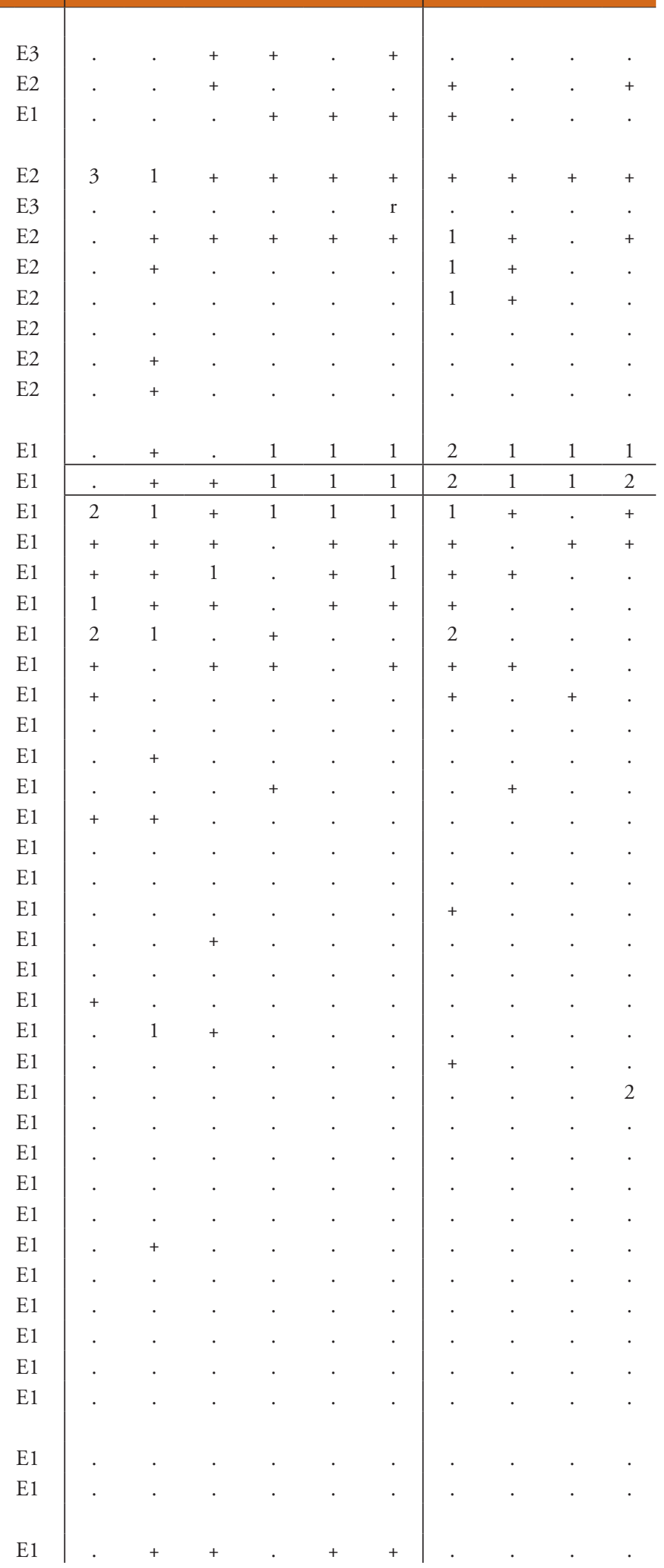


Number of relevé (Zaporedna številka popisa)

Arabis vochinensis

Pulsatilla alpina subsp. austroalpina

Festuca calva

Cfir Caricion firmae

Dryas octopetala

Carex firma

Helianthemum alpestre

Veronica aphylla

Sesleria sphaerocephala

CF Caricion ferrugineae

Cerastium subtriflorum

ES

Elyno-Seslerietea

Sesleria caerulea

Galium anisophyllum

Aster bellidiastrum

Polygonum viviparum

Selaginella selaginoides

Phyteuma orbiculare

Ranunculus montanus

Senecio abrotanifolius

Ranunculus carinthiacus

Homogyne discolor

Betonica alopecuros

Thymus praecox subsp. polytrichus

Campanula witasekiana

Juncus monanthos

Bartsia alpina

Lotus alpinus

Carex sempervirens

Alchemilla sp. (inc. A. filicaulis?)

Polygala alpestris

Helianthemum nummularium subsp. grandiflorum

Gentianella anisodonta

Scabiosa lucida subsp. lucida

Alchemilla fallax

Pedicularis verticillata

Acinos alpinus

Phleum hirsutum

Rhinanthus glacialis

Euphrasia picta

Arabis ciliata

Hieracium dentatum

Alchemilla vulgaris agg.

Cerastium strictum

Helictotrichon parlatorei

Carduus defloratus

Hieracium villosum

Alchemilla subcrenata

Anthyllis vulneraria subsp. alpestris

Alchemilla exigua

Globularia nudicaulis

\section{E1}

E1

E1

E1

E1

E1

E1

E1

E1

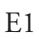

E1

E1

E1

E1

E1

E1

E1

E1

E1

E1

E1

E1

E1

E1

E1

E1

E1

E1

E1

E1

E1

E1

E1

E1

E1

E1

E1

E1

E1

E1

E1

E1

E1

E1

E1

E1

E1

E1

.

1 \begin{tabular}{llllll|llll}
1 & 2 & 3 & 4 & 5 & 6 & 7 & 8 & 9 & 10
\end{tabular}

.

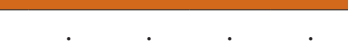

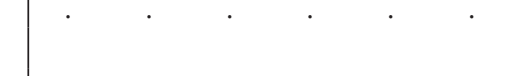

(1)

$\begin{array}{lllll}+ & 1 & 1 & 1 & 1 \\ + & + & + & + & +\end{array}$

$+++++$

(

$++++$

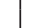

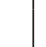

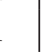

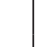

.

(1)

(1)

(1)

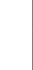

.

.

1

E1

1

1

1

E1 
Number of relevé (Zaporedna številka popisa)

1

23

\section{4}

Leontodon helveticus

pestris

Solidago virgaurea subsp. minuta

LV Loiseleurio-Vaccinietea

Arctostaphylos alpinus

Empetrum hermaphroditum

$\mathrm{CU}$ Calluno-Ulicetea

Coeloglossum viride

Festuca nigrescens

Gentiana pannonica

Veronica officinalis

Potentilla aurea

Alchemilla flabellata

FB Festuco-Brometea

Carlina acaulis

Euphorbia cyparissias

Cirsium erisithales

Prunella grandiflora

Buphthalmum salicifolium

$\mathrm{PaT} \quad$ Poo alpinae-Trisetetalia

Poa alpina

Trollius europaeus

Crepis aurea

Phleum rhaeticum

Ranunculus nemorosus

Pimpinella major subsp. rubra

MA Molinio-Arrhenatheretea

Deschampsia cespitosa

Leontodon hispidus

Veronica chamaedrys

Cerastium fontanum

Dactylis glomerata s.str.

Anthoxanthum odoratum

Crepis paludosa

Cerastium holosteoides

Trifolium repens

Galium mollugo

Prunella vulgaris

Taraxacum officinale

Trifolium pratense

MC Montio-Cardaminetea

Saxifraga aizoides

Alchemilla glabra

CD Caricetalia davallianae

Parnassia palustris

Tofieldia calyculata

Pinguicula alpina

Carex capillaris

EA Epilobietea angustifolii

Fragaria vesca

Rubus idaeus 


\section{\begin{tabular}{ll|l|llllllll}
11 &
\end{tabular}}


Number of relevé (Zaporedna številka popisa)

Urtica dioica

Tussilago farfara

Cirsium eriophorum

AC Arabidetalia caeruleae

Soldanella alpina

Ranunculus traunfellneri

Soldanella minima

TR Thlaspietea rotundifolii

Gymnocarpium robertianum

Cystopteris montana

Valeriana montana

Biscutella laevigata

Trisetum argenteum

Adenostyles glabra

Hieracium bifidum

Arabis alpina

Minuartia austriaca

Cirsium spinosissimum

Hieracium pallescens

Achillea atrata

Pritzelago alpina subsp. alpina

Hieracium glabratum

Dryopteris villarii

Leontodon hispidus subsp. hyoseroides

Cerastium carinthiacum

Crepis kerneri

Saxifraga caesia

Rumex scutatus

Cy Cystopteridion fragilis

Cystopteris fragilis

Cystopteris regia

Heliosperma pusillum

PS Physoplexido comosae-Saxifragion petraeae

Saxifraga crustata

Campanula zoysii

Saxifraga squarrosa

PC Potentilletalia caulescentis

Primula auricula

Androsace lactea

Potentilla clusiana

Festuca stenantha

AT Asplenietea trichomanis

Asplenium viride

Moehringia muscosa

Polypodium vulgare

Gypsophila repens

Kernera saxatilis

Asplenium ruta-muraria

Asplenium trichomanes

$\mathrm{Fu} \quad$ Fungi (Glive)

Laetiporus sulphureus agg. (L. horoniensis)

Laricifomes officinalis

E1

E1

E1

E1

E1

E1

E1

E1

E1

E1

E1

E1

E1

E1

E1

E1

E1

E1

E1

E1

E1

E1

E1

E1

E1

E1

E1

E1

1

$+$
10

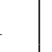

(1)

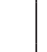

ren

.

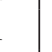

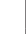

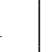

(1)


Number of relevé (Zaporedna številka popisa)

\begin{tabular}{lllll|llll}
2 & 3 & 4 & 5 & 6 & 7 & 8 & 9 & 10
\end{tabular}

ML Mosses and lichens (Mahovi in lišaji)

Tortella tortuosa

Rhytidiadelphus triquetrus

Dicranum scoparium

Ctenidium molluscum

Hylocomium splendens

Polytrichum formosum

Peltigera leucophlebia

Fissidens dubius

Orthothecium rufescens

Schistidium sp.

Letharia vulpina

Rhytidiadelphus loreus

Eurhynchium angustirete

Cetraria islandica

Sphagnum sp.

Rhizomnium punctatum

Plagiochila porelloides

Bartramia pomiformis

Hypogymnia physodes

Dermatocarpon miniatum

Dicranodontium sp.

Peltigera canina

Marchantia polymorpha

Cladonia pyxidata

Plagiothecium sylvaticum

Pseudoleskea incurvata

Mnium sp.

Ditrichum flexicaule

\begin{tabular}{|c|c|c|c|c|c|c|c|c|c|c|}
\hline E0 & + & + & + & + & + & + & + & . & + & + \\
\hline E0 & + & + & 1 & 1 & 1 & 1 & + & 1 & . & . \\
\hline E0 & + & + & + & . & + & + & . & . & + & + \\
\hline E0 &. & + & + & . & . & . & + & + & + & + \\
\hline E0 & . & + & + & 1 & + & + & . & + & . & . \\
\hline E0 & . & . & . & + & . & . & . & + & . & + \\
\hline E0 & . & . & + & + & . & + & . & . & . & . \\
\hline E0 & . & . & . & . & + & . & . & + & . & + \\
\hline E0 & . & . & . & . & . & . & + & . & . & . \\
\hline E0 & . & . & + & . & . & . & . & . & + & . \\
\hline E3c & 1 & . & . & . & . & . & . & . & . & . \\
\hline E0 & . & . & . & . & . & . & + & . & . & . \\
\hline E0 & 1 & . & . & . & . & . & . & . & . & . \\
\hline E0 & + & . & . & . & . & . & . & . & . & . \\
\hline E0 & . & . & . & + & . & . & . & . & . & . \\
\hline E0 & . & . & . & . & . & . & . & + & . & . \\
\hline E0 & . & . & . & . & . & . & . & . & . & . \\
\hline E0 & . & . & . & . & . & . & . & . & . & . \\
\hline E4 & . & . & . & . & . & . & . & . & . & . \\
\hline E0 & . & . & . & . & . & . & . & . & . & . \\
\hline E0 & . & . & . & . & . & . & . & . & . & . \\
\hline E0 & . & . & . & . & . & . & . & . & . & . \\
\hline E0 & . & . & . & . & . & . & . & . & . & . \\
\hline E0 & . & . & . & . & . & . & . & . & . & . \\
\hline E0 & . & . & . & . & . & . & . & . & . & . \\
\hline E0 & $\cdot$ & $\cdot$ & $\cdot$ & . & $\cdot$ & . & . & . & . & . \\
\hline E0 & . & . & . & . & . & . & . & . & . & . \\
\hline E0 & . & . & . & . & . & . & . & . & . & . \\
\hline
\end{tabular}

\section{Legend - Legenda}

ID Igor Dakskobler

AS Andrej Seliškar

AR Andrej Rozman

A Limestone - apnenec

D Dolomite - dolomit

L Marlstone - laporovec

Re Rendzina - rendzina

JA Julian Alps - Julijske Alpe

KSA Kamnik-Savinja Alps - Kamniško-Savinjske Alpe

K Karavanke - Karavanke

Pr. Presence (number of relevés in which the species is presented) - število popisov, v katerih se pojavlja vrsta

Fr. Frequency in $\%-$ frekvenca $\mathrm{\%}$ 


\begin{tabular}{|c|c|c|c|c|c|c|c|c|c|c|c|c|c|c|c|c|c|c|c|c|}
\hline 11 & 12 & 13 & 14 & 15 & 16 & 17 & 18 & 19 & 20 & 21 & 22 & 23 & 24 & 25 & 26 & 27 & 28 & 29 & Pr. & Fr. \\
\hline+ & + & + & + & + & + & + & + & + & + & + & + & + & + & + & 1 & + & + & + & 28 & 97 \\
\hline 1 & . & + & + & + & + & + & 1 & + & + & 1 & 1 & + & 1 & + & + & . & + & + & 25 & 86 \\
\hline+ & & + & + & . & + & + & + & + & + & 1 & + & . & . & + & . & . & + & . & 19 & 66 \\
\hline . & + & . & + & . & + & . & + & + & + & + & 1 & + & . & . & + & + & . & . & 17 & 59 \\
\hline . & . & 1 & . & . & . & . & + & . & + & 1 & . & . & + & + & + & . & + & . & 14 & 48 \\
\hline . & . & + & + & . & + & . & . & . & . & + & . & . & + & . & . & + & . & . & 9 & 31 \\
\hline . & . & . & . & . & . & . & + & . & + & + & . & . & . & + & . & . & . & . & 7 & 24 \\
\hline . & . & + & & . & + & . & + & & . & . & + & & . & . & . & & . & & 7 & 24 \\
\hline . & & + & . & . & + & + & + & + & . & & . & . & . & + & . & . & . & . & 7 & 24 \\
\hline . & . & . & . & . & . & . & + & . & . & . & + & . & . & . & + & . & . & + & 6 & 21 \\
\hline . & . & . & . & . & 1 & + & + & . & . & . & . & . & . & . & . & . & . & . & 4 & 14 \\
\hline . & . & + & . & . & . & . & . & . & . & . & . & . & + & . & . & . & . & . & 3 & 10 \\
\hline . & . & . & . & . & . & . & . & . & . & . & + & . & . & . & . & . & . & . & 2 & \\
\hline . & . & . & . & . & . & . & . & . & . & & . & . & . & . & . & . & . & . & 1 & \\
\hline . & . & . & & . & . & . & & & . & . & . & & . & . & . & & . & . & 1 & \\
\hline . & . & . & . & . & . & . & . & . & . & . & . & . & . & . & . & . & . & . & 1 & \\
\hline . & . & + & . & . & . & . & . & . & . & . & . & . & . & . & . & . & . & . & 1 & \\
\hline . & . & + & . & . & . & . & . & . & . & . & . & . & . & . & . & . & . & . & 1 & \\
\hline . & . & . & . & . & . & . & + & . & . & . & . & . & . & . & . & . & . & . & 1 & \\
\hline . & & . & . & . & . & . & + & . & & & . & . & . & . & . & . & . & . & 1 & \\
\hline . & . & . & & . & . & . & + & & . & . & . & & . & . & . & & . & & 1 & \\
\hline . & . & . & . & . & . & . & . & . & . & + & . & . & . & . & . & . & . & . & 1 & \\
\hline . & . & . & . & . & . & . & . & . & . & + & . & . & . & . & . & . & . & . & 1 & \\
\hline . & . & . & . & . & . & . & . & . & . & . & + & . & . & . & . & . & . & . & 1 & \\
\hline . & . & . & . & . & . & . & . & . & . & . & . & . & + & . & . & . & . & . & 1 & \\
\hline . & . & . & . & . & . & . & . & . & . & . & . & . & . & . & & . & . & + & 1 & 3 \\
\hline . & & . & . & . & . & . & . & . & & & . & . & . & + & . & . & . & . & 1 & 3 \\
\hline & . & . & . & . & . & . & & . & . & . & . & . & . & + & & . & . & . & 1 & \\
\hline
\end{tabular}




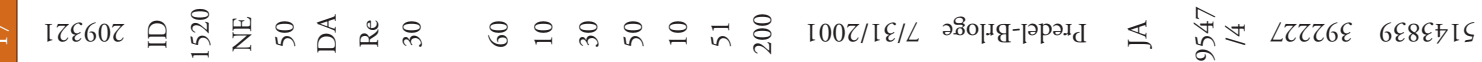

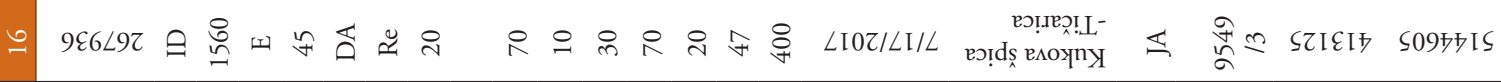

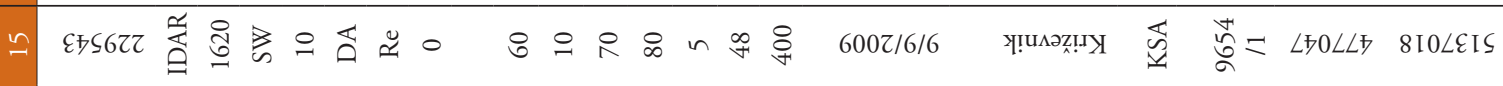

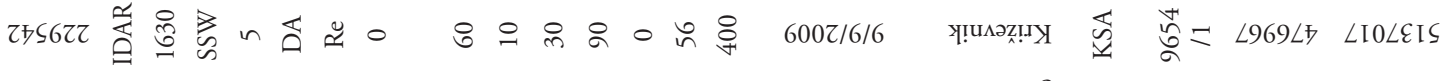

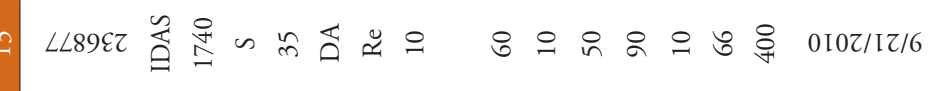

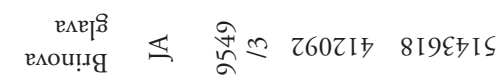

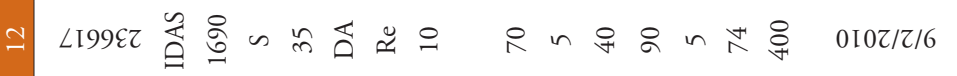

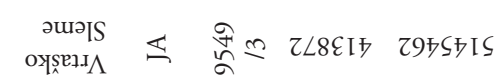
$=$

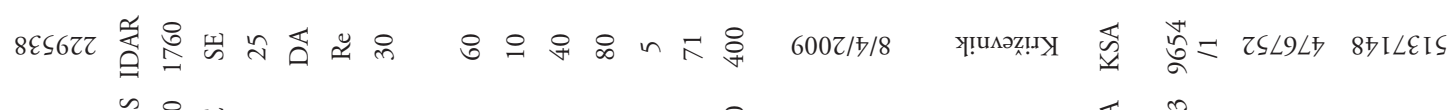

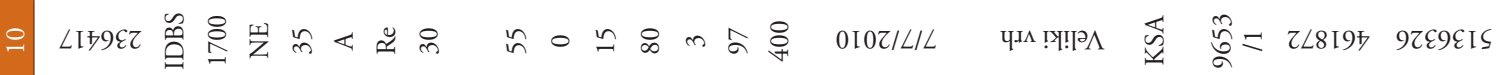

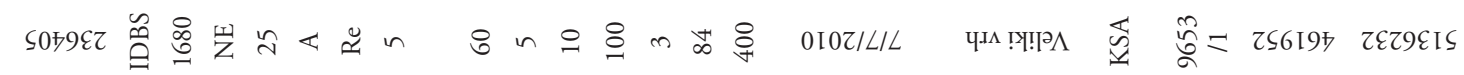

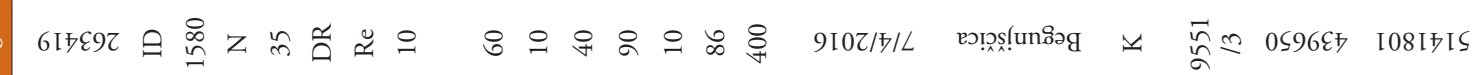

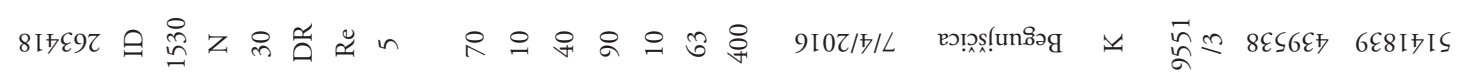

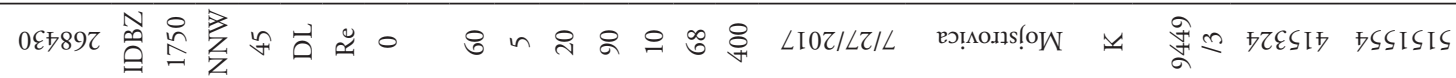

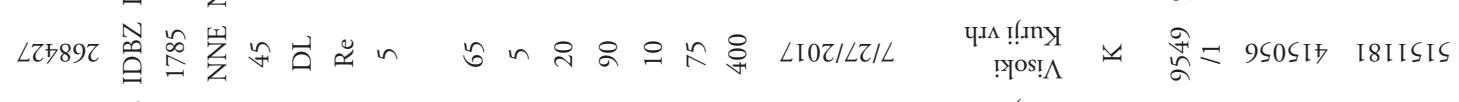

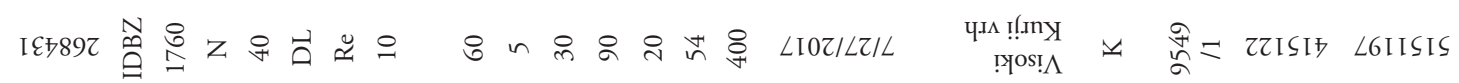

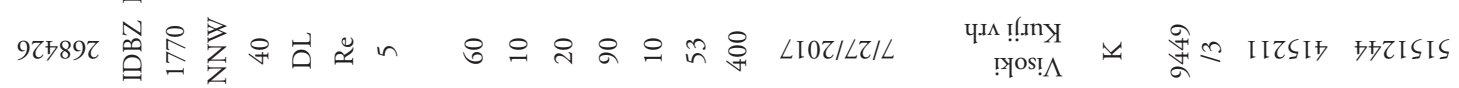

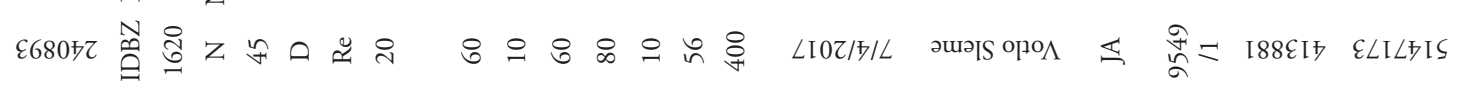

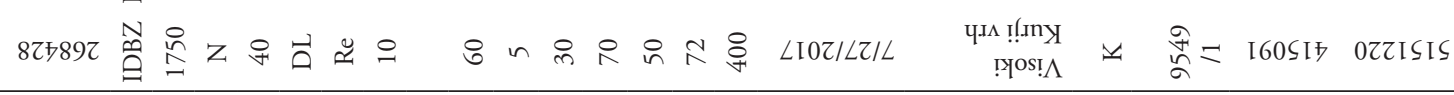

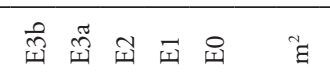
घ $\quad$ I 


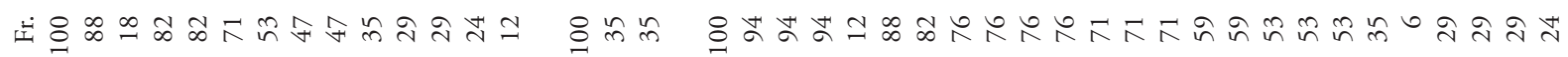

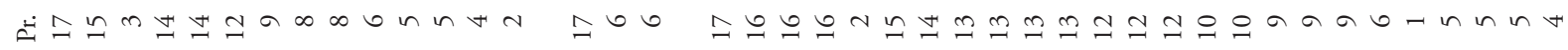

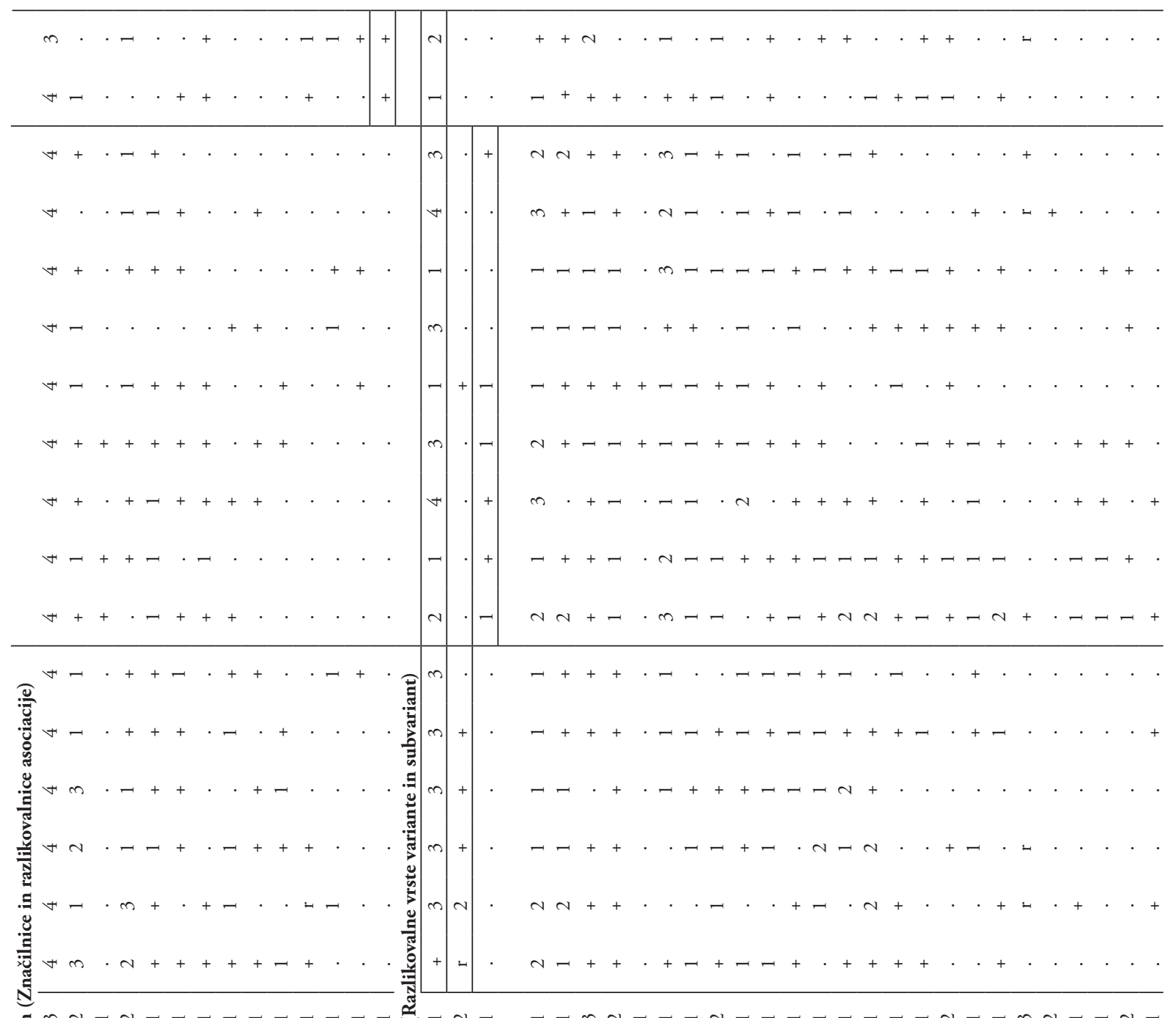

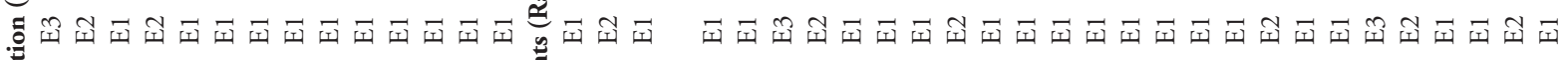

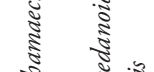




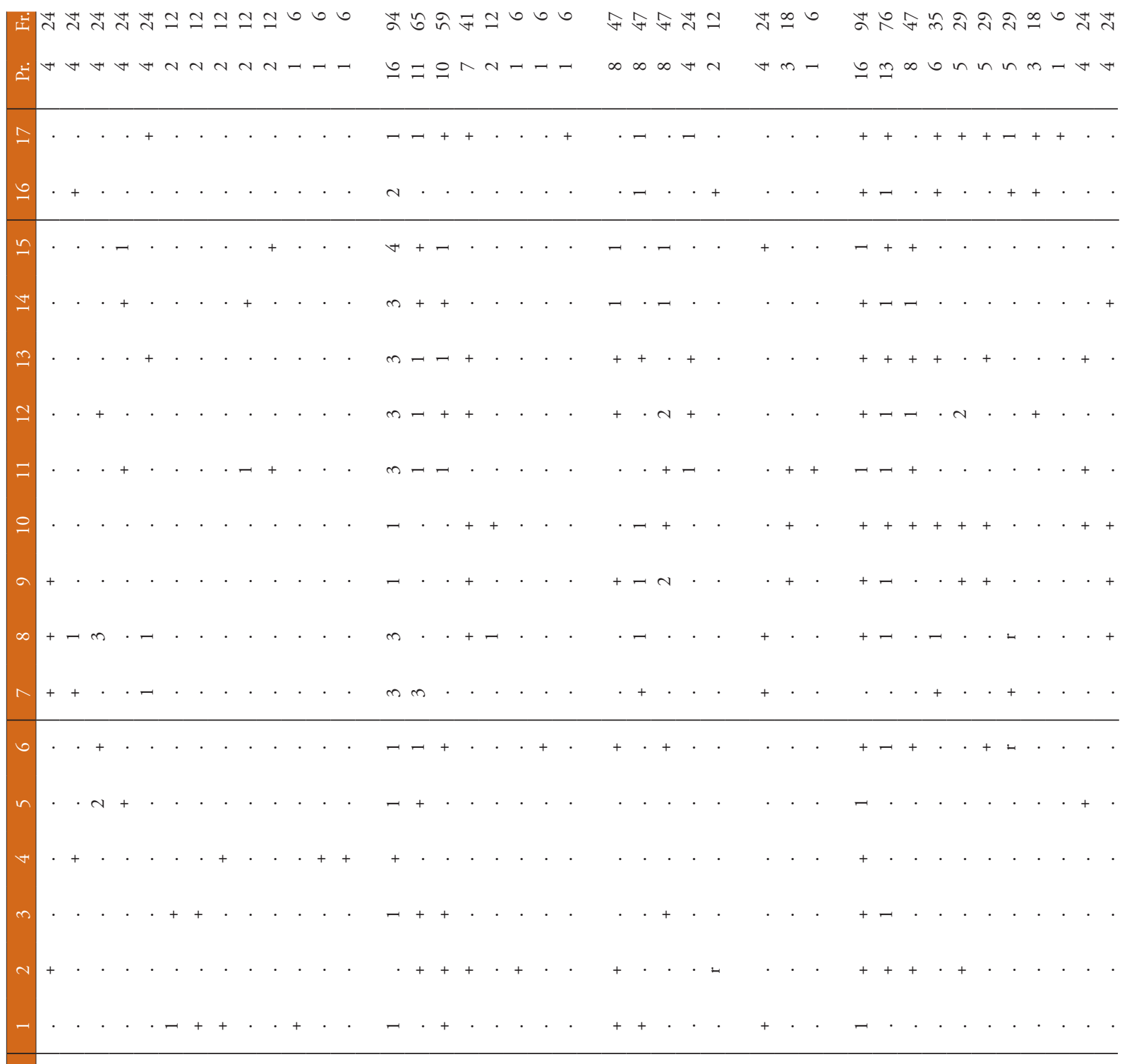

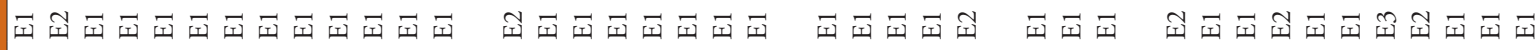


䒕

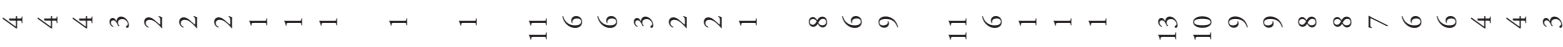

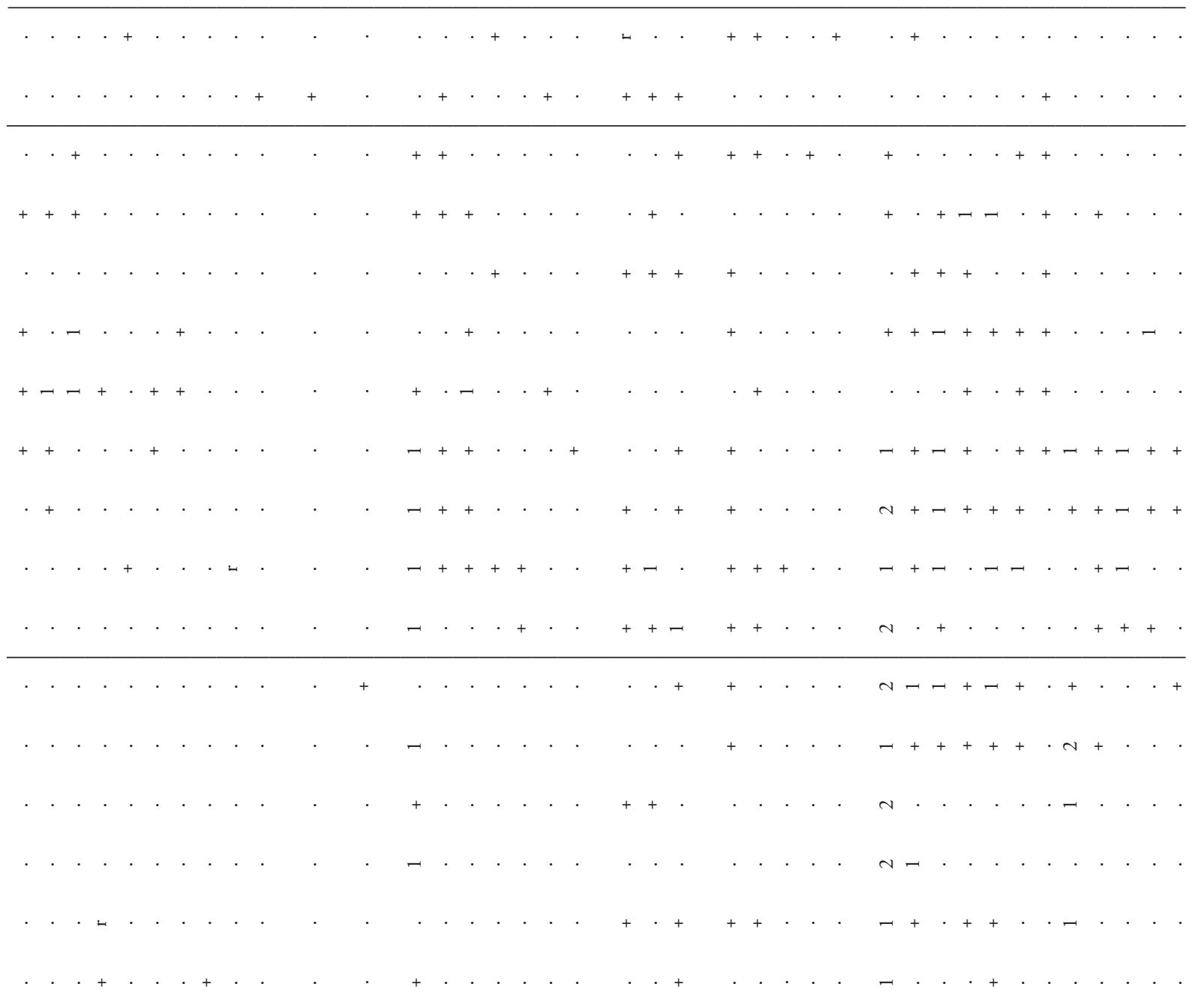

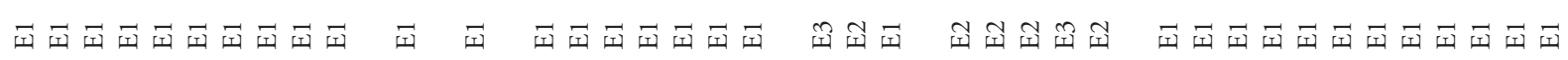

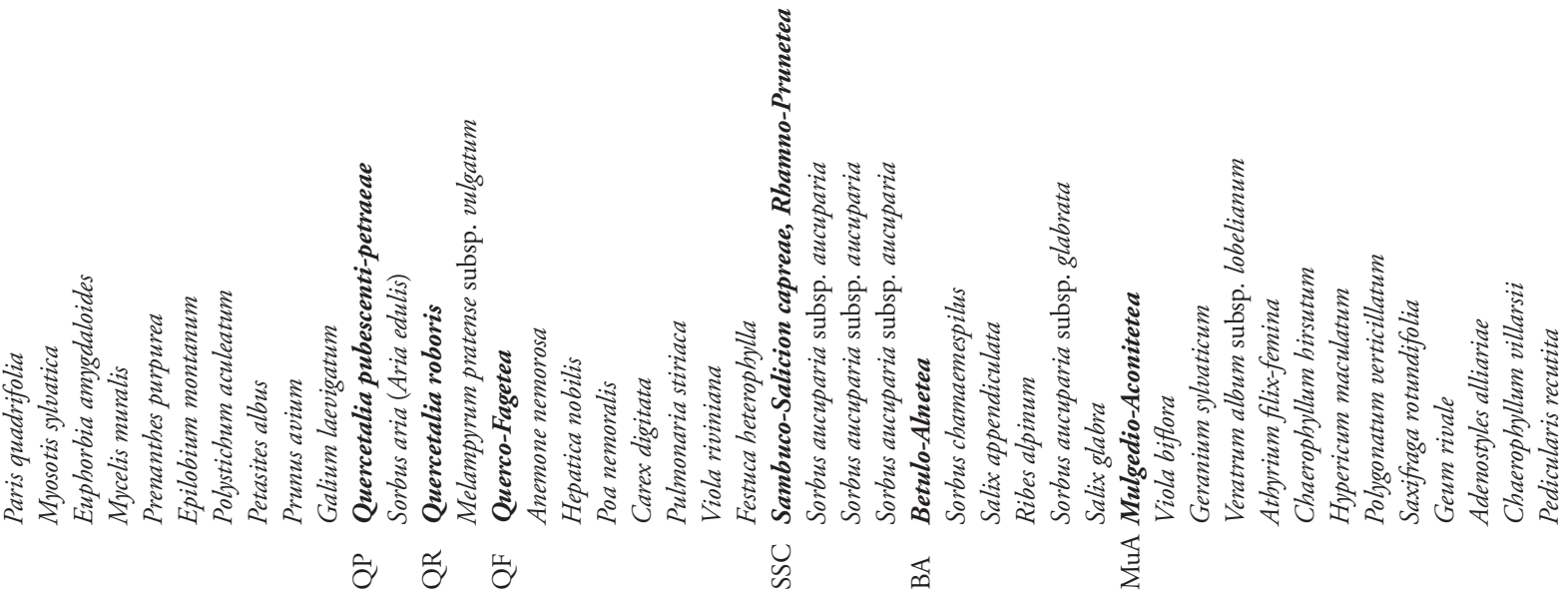




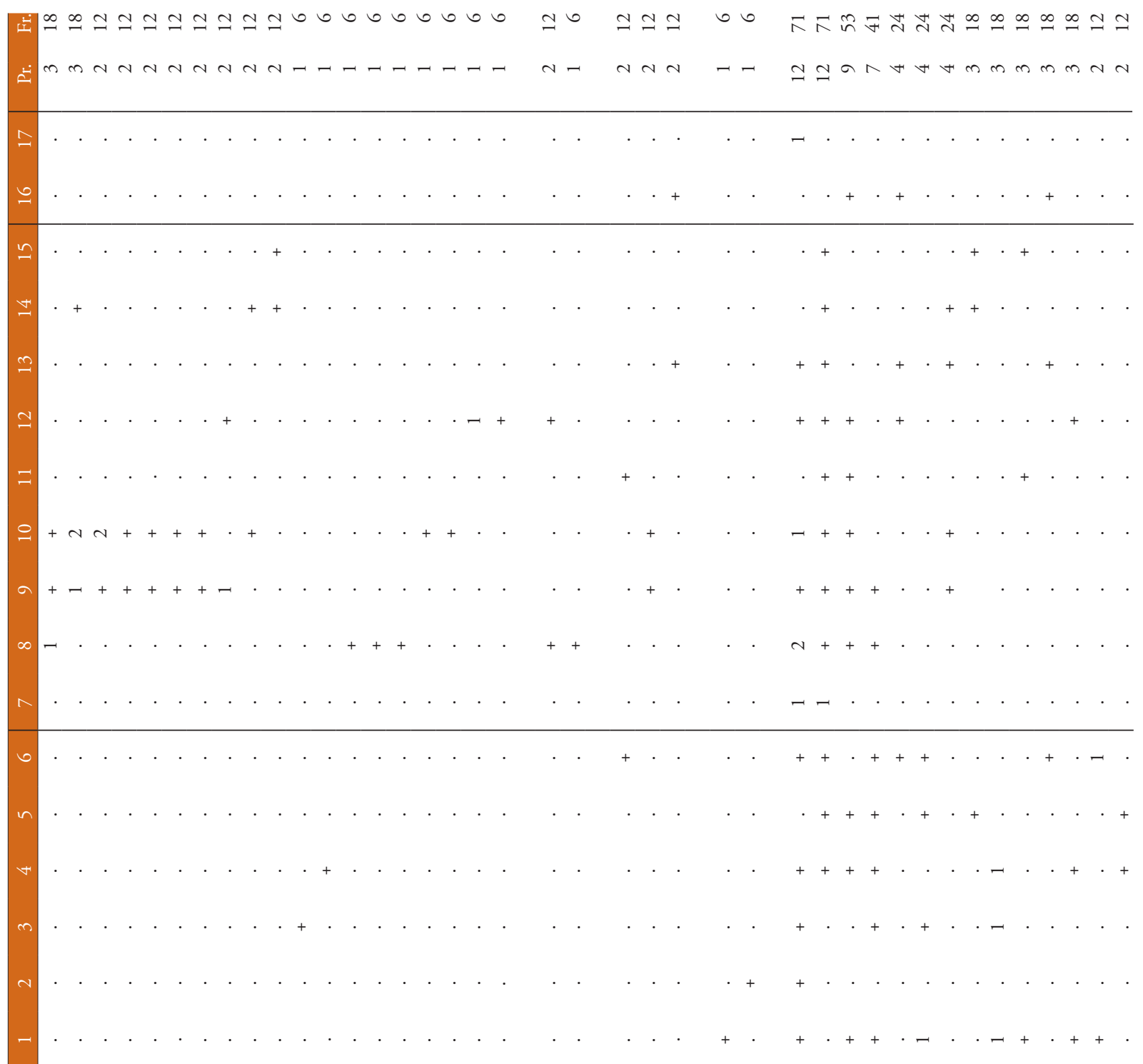

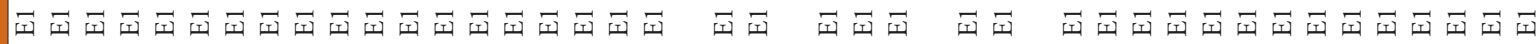




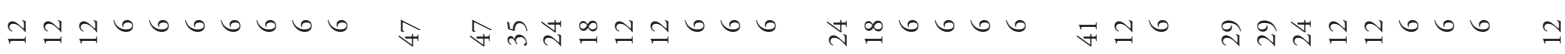

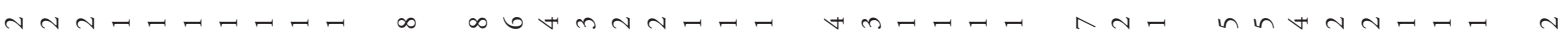

.
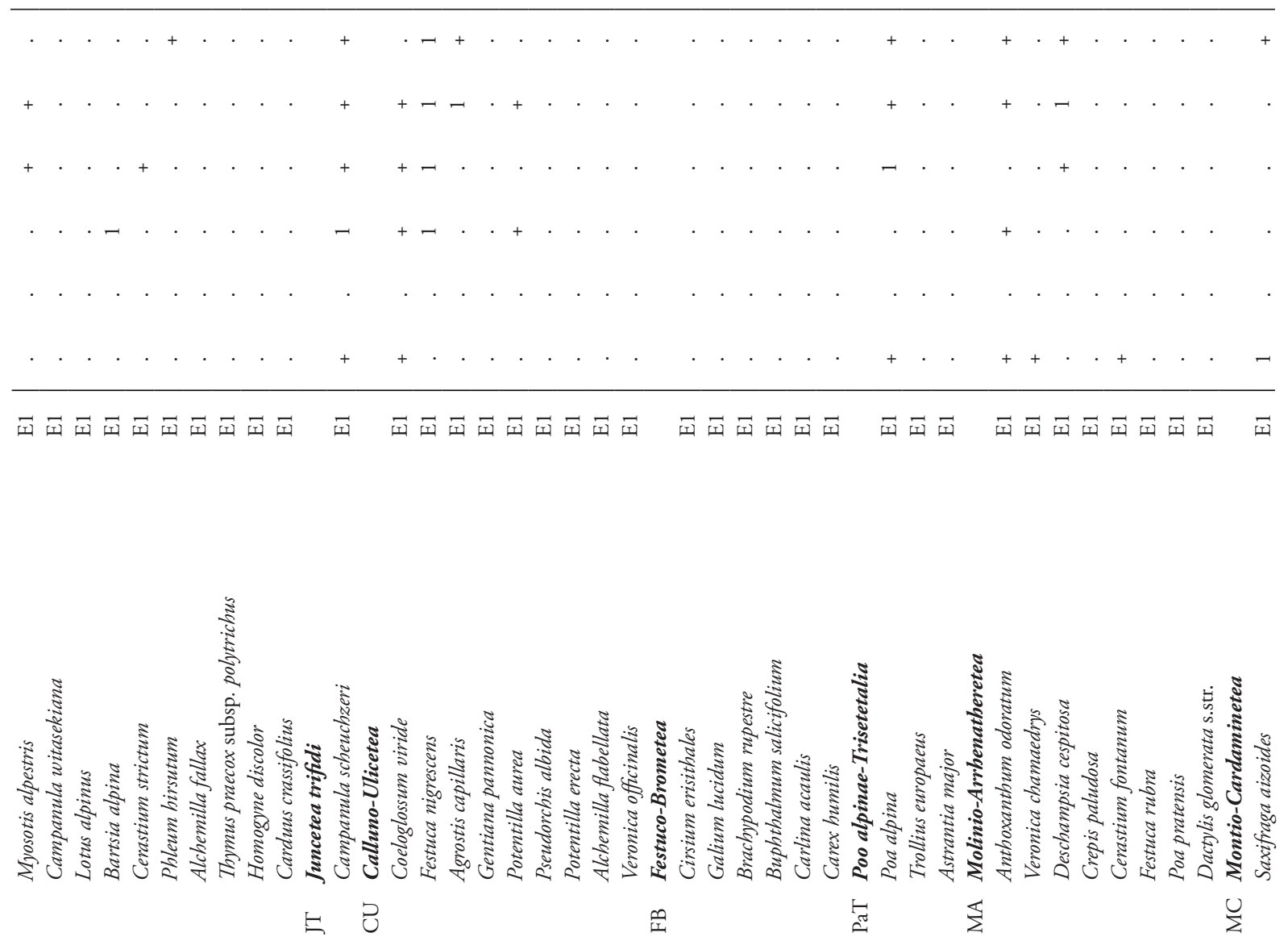


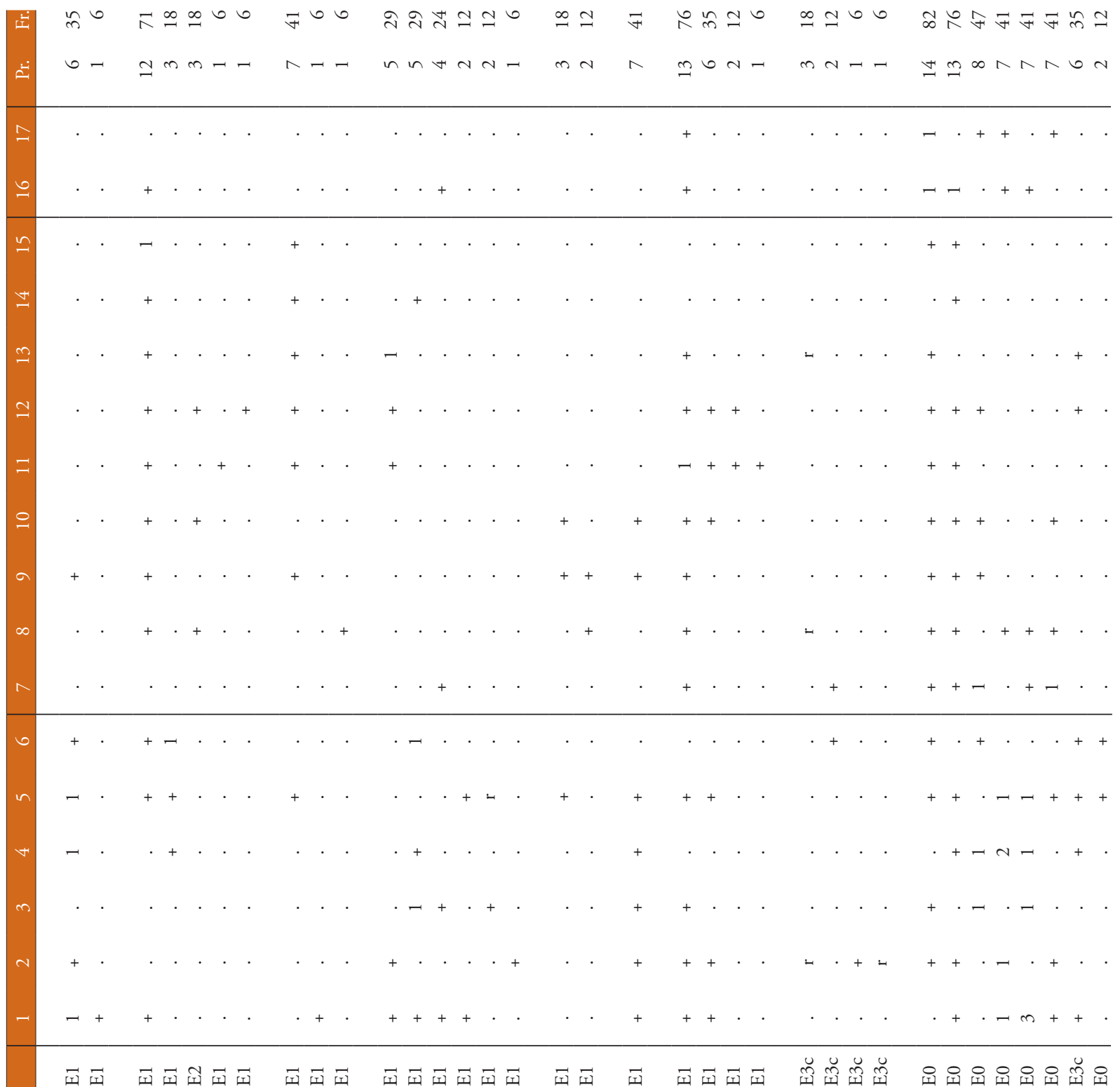

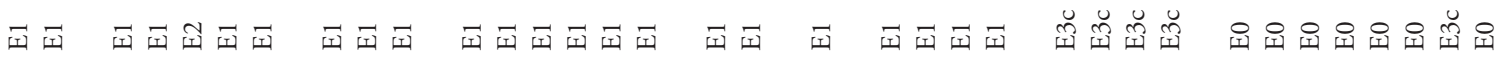

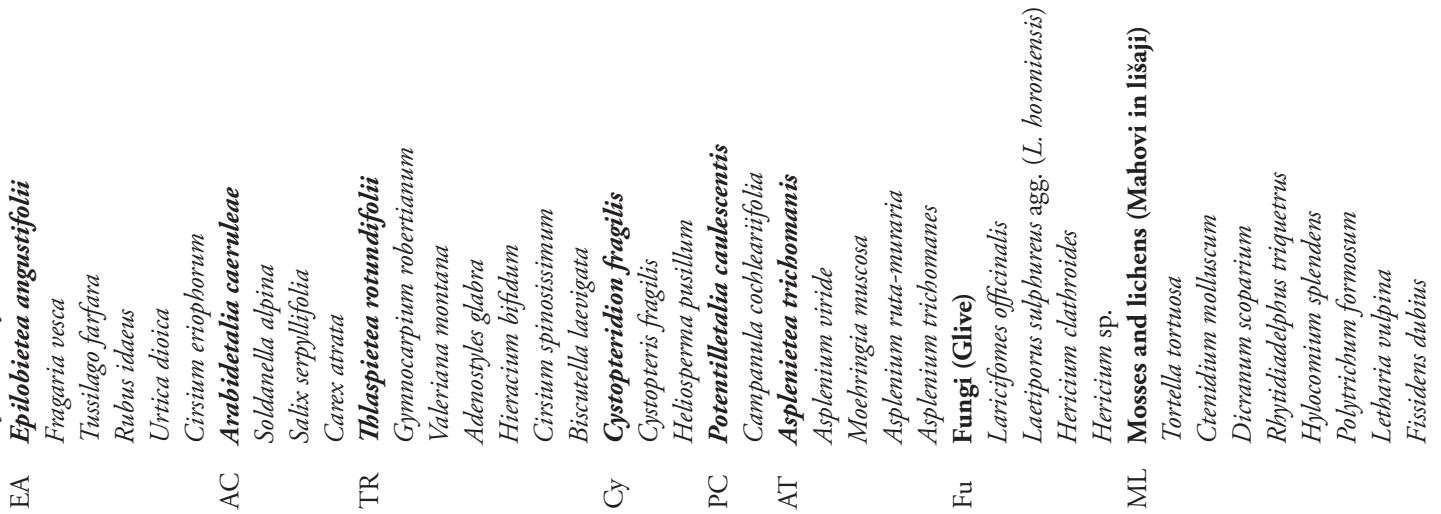




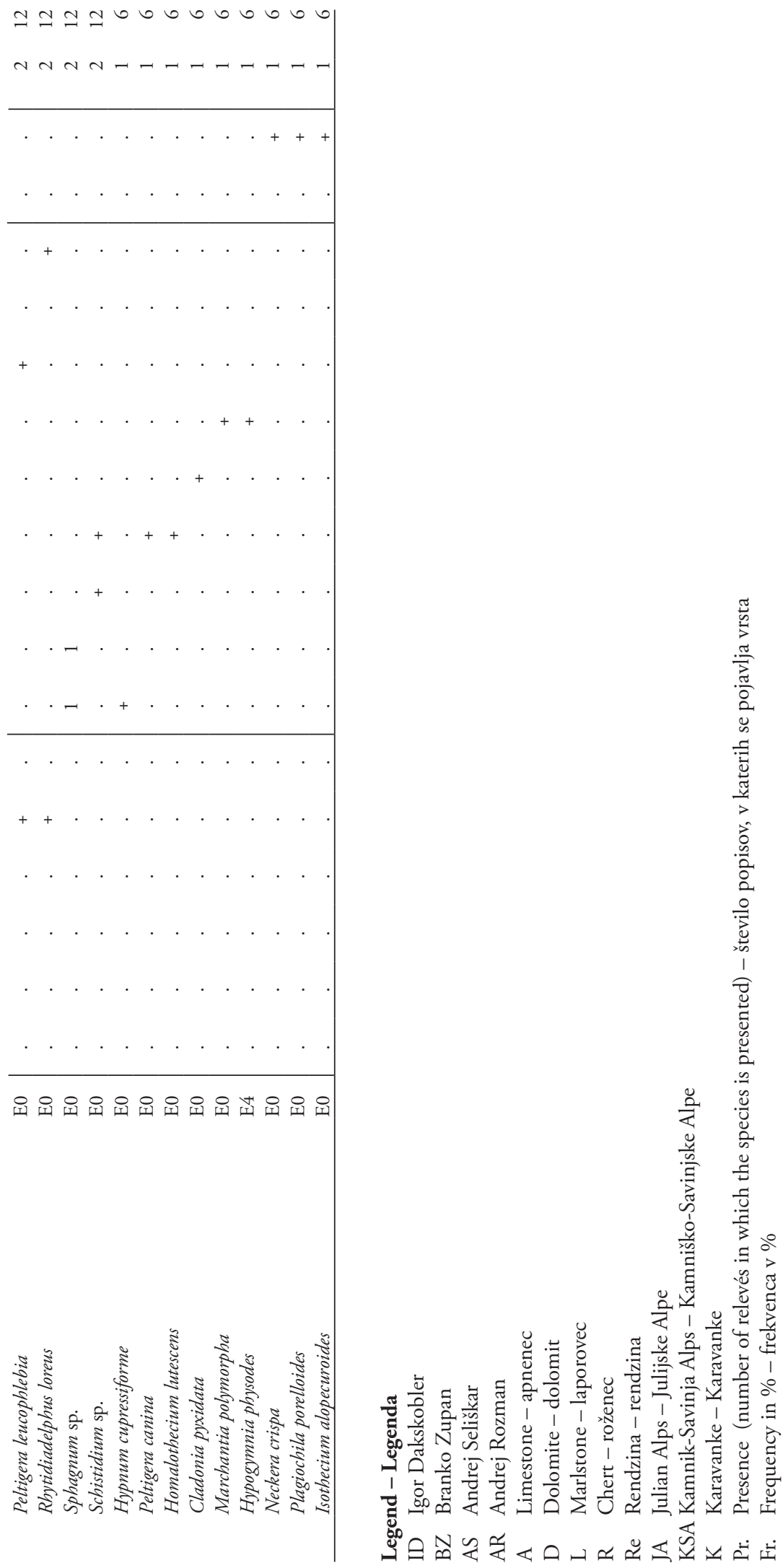


Table 5 (Tabela 5): Rhodothamno-Laricetum sorbetosum chamaemespili var. Adenostyles glabra

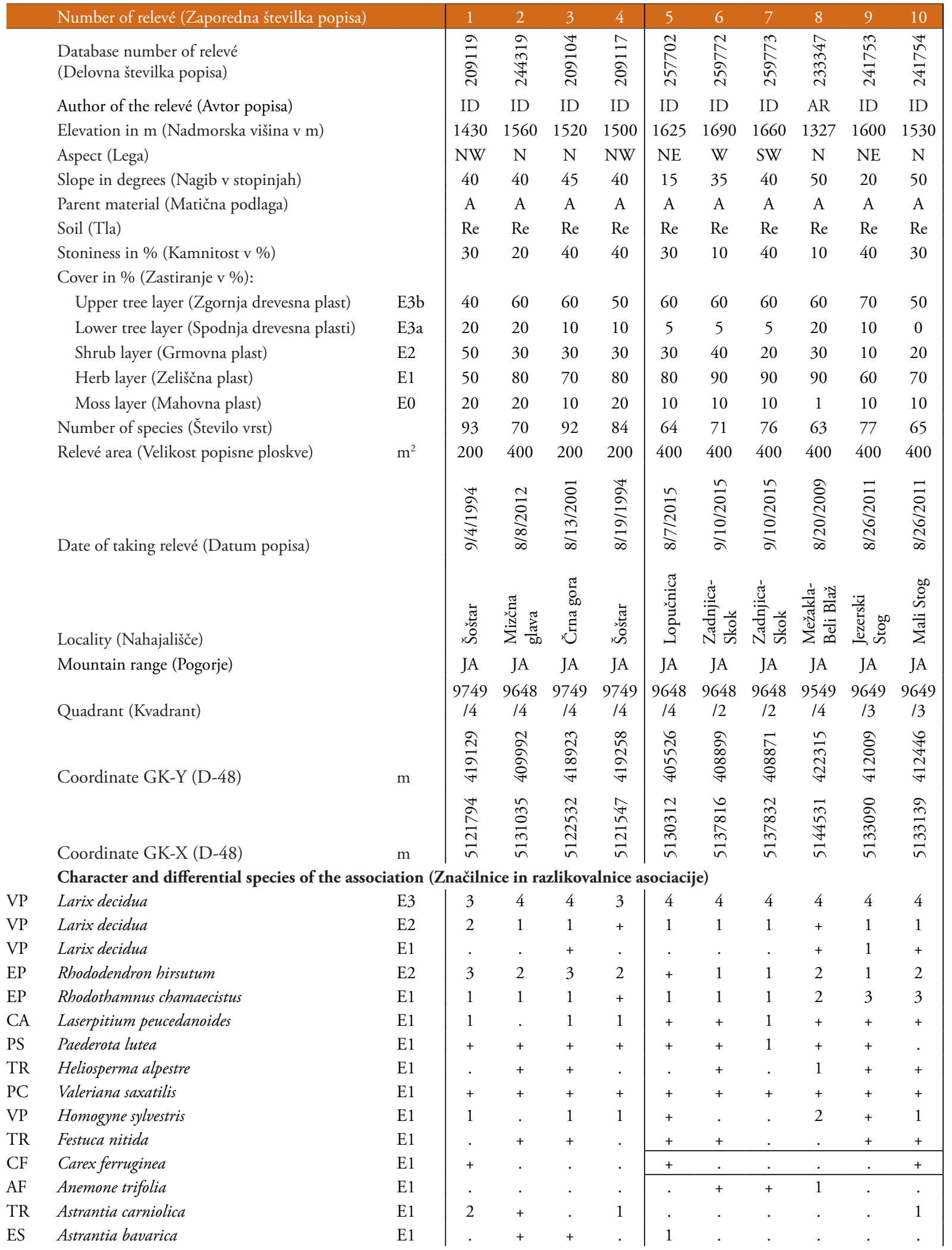




\begin{tabular}{|c|c|c|c|c|c|c|c|c|c|c|c|c|c|}
\hline 11 & 12 & 13 & 14 & 15 & 16 & 17 & 18 & 19 & 20 & 21 & 22 & Pr. & \\
\hline$\underset{\vec{N}}{\stackrel{\vec{N}}{\vec{N}}}$ & $\begin{array}{l}\hat{n} \\
\stackrel{\sigma}{\sigma}\end{array}$ & 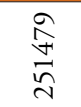 & $\begin{array}{l}\stackrel{\infty}{\infty} \\
\stackrel{+}{*} \\
\stackrel{\sim}{\sim}\end{array}$ & $\begin{array}{l}0 \\
\hat{n} \\
\stackrel{n}{n}\end{array}$ & 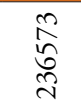 & $\begin{array}{l}\stackrel{+}{\hat{n}} \\
\hat{\sigma} \\
\stackrel{\sim}{\sim}\end{array}$ & 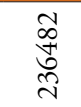 & 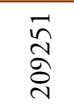 & $\begin{array}{l}\hat{\infty} \\
\hat{\hat{\sigma}}\end{array}$ & 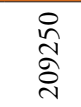 & $\begin{array}{l}\tilde{\widetilde{\alpha}} \\
\widehat{\tilde{\sigma}}\end{array}$ & & \\
\hline ID & ID & ID & ID & ID & ID & ID & IDAS & ID & ID & ID & ID & & \\
\hline 1580 & 1620 & 1660 & 1690 & 1490 & 1480 & 1400 & 1570 & 1710 & 1560 & 1700 & 1600 & & \\
\hline NNW & $\mathrm{N}$ & $\mathrm{NE}$ & $\mathrm{NE}$ & $\mathrm{N}$ & $\mathrm{NE}$ & $\mathrm{NE}$ & NW & NW & $\mathrm{N}$ & $\mathrm{N}$ & NW & & \\
\hline 35 & 35 & 35 & 15 & 40 & 35 & 30 & 40 & 35 & 35 & 45 & 35 & & \\
\hline A & A & A & A & DA & DA & Mo & $\mathrm{Gr}$ & DA & A & DA & A & & \\
\hline $\operatorname{Re}$ & $\mathrm{Re}$ & $\operatorname{Re}$ & $\operatorname{Re}$ & $\mathrm{Re}$ & $\mathrm{Re}$ & $\mathrm{Re}$ & $\operatorname{Re}$ & $\operatorname{Re}$ & $\operatorname{Re}$ & $\mathrm{Re}$ & $\mathrm{Re}$ & & \\
\hline 20 & 15 & 20 & 30 & 20 & 5 & 20 & 15 & 20 & 20 & 30 & 60 & & \\
\hline 70 & 50 & 70 & 65 & 60 & 50 & 40 & 50 & 60 & 60 & 50 & 70 & & \\
\hline 20 & 20 & 10 & 15 & 10 & 5 & 20 & 5 & 10 & 0 & 5 & 5 & & \\
\hline 20 & 20 & 60 & 40 & 20 & 40 & 50 & 20 & 30 & 40 & 30 & 20 & & \\
\hline 80 & 80 & 80 & 80 & 80 & 80 & 70 & 70 & 80 & 70 & 70 & 70 & & \\
\hline 20 & 10 & 10 & 10 & 5 & 5 & 5 & 20 & 10 & 20 & 10 & 10 & & \\
\hline 66 & 84 & 83 & 70 & 76 & 63 & 87 & 85 & 63 & 75 & 61 & 76 & & \\
\hline 400 & 400 & 400 & 400 & 400 & 400 & 400 & 400 & 200 & 200 & 200 & 200 & & \\
\hline 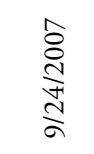 & 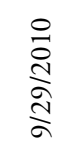 & 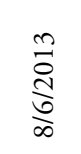 & 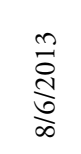 & $\begin{array}{l}\stackrel{0}{\circ} \\
\stackrel{i}{O} \\
\stackrel{ }{1}\end{array}$ & 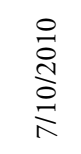 & $\begin{array}{l}\stackrel{0}{0} \\
\stackrel{ }{0} \\
\stackrel{0}{ }\end{array}$ & $\begin{array}{l}\stackrel{0}{0} \\
\stackrel{i}{0} \\
\stackrel{\infty}{\infty}\end{array}$ & 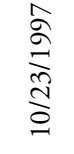 & $\underset{\stackrel{a}{a}}{\stackrel{a}{a}}$ & 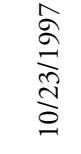 & 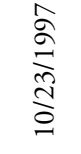 & & \\
\hline 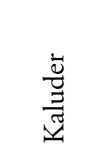 & 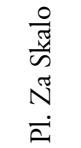 & 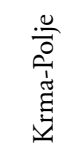 & 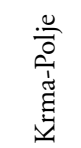 & 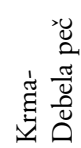 & 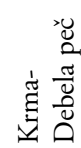 & 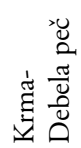 & 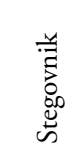 & 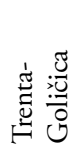 & 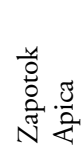 & 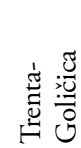 & 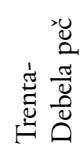 & & \\
\hline JA & JA & JA & JA & JA & JA & JA & K & JA & JA & JA & JA & & \\
\hline $\begin{array}{c}9648 \\
13\end{array}$ & $\begin{array}{c}9648 \\
13\end{array}$ & $\begin{array}{c}9649 \\
/ 1\end{array}$ & $\begin{array}{c}9649 \\
/ 1\end{array}$ & $\begin{array}{c}9649 \\
12\end{array}$ & $\begin{array}{c}9649 \\
12\end{array}$ & $\begin{array}{c}9649 \\
12\end{array}$ & $\begin{array}{c}9652 \\
12\end{array}$ & $\begin{array}{c}9648 \\
12\end{array}$ & $\begin{array}{c}9648 \\
/ 1\end{array}$ & $\begin{array}{c}9648 \\
12\end{array}$ & $\begin{array}{c}9648 \\
12\end{array}$ & & \\
\hline $\begin{array}{l}\infty \\
\infty \\
\stackrel{0}{+} \\
\stackrel{8}{+} \\
+\end{array}$ & $\begin{array}{l}\vec{\circ} \\
\stackrel{े}{0} \\
\stackrel{+}{+}\end{array}$ & $\begin{array}{l}\stackrel{0}{n} \\
\cdots \\
\approx\end{array}$ & 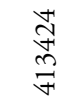 & 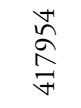 & $\begin{array}{l}\infty \\
\hat{\partial} \\
\infty \\
\forall\end{array}$ & 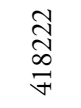 & \begin{tabular}{l}
$R$ \\
0 \\
D \\
\multirow{n}{*}{}
\end{tabular} & 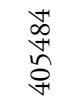 & $\begin{array}{l}\bar{\sigma} \\
\stackrel{\sigma}{\sigma}\end{array}$ & 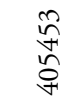 & 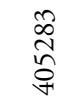 & & \\
\hline 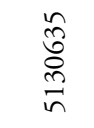 & $\begin{array}{l}\text { ఫे } \\
\text { ڤ. } \\
\text { in }\end{array}$ & 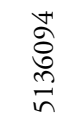 & 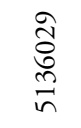 & $\begin{array}{l}\stackrel{尺}{a} \\
\widehat{\approx} \\
\stackrel{n}{n}\end{array}$ & $\frac{\infty}{\stackrel{\infty}{n}}$ & $\frac{n}{\hbar}$ & 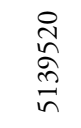 & 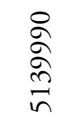 & $\begin{array}{l}\stackrel{\infty}{\hat{\alpha}} \\
\hat{n} \\
\tilde{n}\end{array}$ & 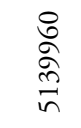 & $\begin{array}{l}\infty \\
\hat{\alpha} \\
\stackrel{n}{n}\end{array}$ & & \\
\hline 4 & 3 & 4 & 4 & 4 & 3 & 3 & 3 & 4 & 3 & 3 & 4 & Pr. & $\begin{array}{c}\text { Fr. } \\
100\end{array}$ \\
\hline 1 & 1 & 2 & 2 & 1 & 2 & 2 & 1 & 1 & 1 & 1 & + & 22 & 100 \\
\hline . & . & . & . & . & + & . & . & + & + & . & + & 8 & 36 \\
\hline 2 & 3 & 4 & 4 & 2 & 3 & 4 & 2 & 3 & 3 & 3 & 2 & 22 & 100 \\
\hline 1 & 1 & 2 & + & + & + & 1 & . & + & 1 & 1 & 1 & 21 & 95 \\
\hline+ & + & 1 & 1 & 1 & + & + & 1 & + & 1 & + & + & 21 & 95 \\
\hline . & + & + & 1 & + & + & + & 1 & + & 1 & 1 & 1 & 20 & 91 \\
\hline . & + & + & + & + & + & + & + & 1 & + & + & + & 17 & 77 \\
\hline . & + & . & & . & + & + & . & + & 1 & 1 & + & 17 & 77 \\
\hline . & 1 & 1 & 1 & 1 & 1 & 1 & 1 & 1 & . & . & + & 16 & 73 \\
\hline & 1 & 1 & 2 & + & + & + & . & + & + & . & . & 14 & 64 \\
\hline . & + & 1 & 1 & 1 & 1 & 1 & 1 & + & + & + & . & 13 & 59 \\
\hline . & + & + & r. & 1 & 1 & 1 & . & . & 1 & + & + & 11 & 50 \\
\hline . & . & + & + & 1 & 1 & 1 & . & . & . & . & . & 9 & 41 \\
\hline 1 & 1 & & & . & & & 1 & + & 1 & + & . & 9 & 41 \\
\hline
\end{tabular}




\section{Number of relevé (Zaporedna števillka popisa) \\ 6 \\ $8 \quad 9 \quad 10$}

TR Adenostyles glabra

$\mathrm{MuA}$ Aconitum lycoctonum subsp. ranunculifolium

VP

\section{Vaccinio-Piceetea}

Polystichum lonchitis

Luzula sylvatica

Vaccinium myrtillus

Vaccinium vitis-idaea

Valeriana tripteris

Picea abies

Picea abies

Picea abies

Picea abies

Clematis alpina

Aposeris foetida

Rosa pendulina

Lycopodium annotinum

Huperzia selago

Calamagrostis villosa

Oxalis acetosella

Hieracium murorum

Solidago virgaurea subsp. virgaurea

Gymnocarpium dryopteris

Homogyne alpina

Phegopteris connectilis

Melampyrum sylvaticum

Saxifraga cuneifolia

Dryopteris dilatata

Maianthemum bifolium

Dryopteris expansa

Gentiana asclepiadea

Calamagrostis arundinacea

Veronica urticifolia

Lonicera caerulea

Abies alba

Abies alba

Abies alba

Lonicera nigra

Luzula luzuloides

Luzula luzulina

Moneses uniflora

Ajuga pyramidalis

EP

Erico-Pinetea

Calamagrostis varia

Erica carnea

Rubus saxatilis

Pinus mugo

Aquilegia nigricans

Carex ornithopoda

Gymnadenia odoratissima

$\mathrm{AF}$

Aremonio-Fagion

Cyclamen purpurascens

Cardamine enneaphyllos
E

E1

E1

E1

E1

E1

E1

E3

E2

E1

E0

E2

E1

E2

E1

E1

E1

E1

E1

E1

E1

E1

E1

E1

E1

E1

E1

E1

E1

E1

E1

E2

E3

E2

E1

E2

E1

E1

E1

E1

E1

E1

E2

E1

E1

E1

E1

E1 varsta variante in subvariante)

\begin{tabular}{|llll|llllll|}
\hline 1 & 1 & 1 & 1 & 2 & + & + & 2 & 2 & 1 \\
\hline
\end{tabular}

1

.

\begin{tabular}{lllll}
1 & + & 1 & + & + \\
\hline 1 & + & 1 & + & \\
1 & 1 & + & +
\end{tabular}

$\begin{array}{llllll}1 & 1 & + & + & + & 1\end{array}$

$1++\quad 13$

$\begin{array}{lllll}1 & + & + & 1 & 3 \\ + & + & + & + & 1 \\ + & 1 & 1 & . & 1\end{array}$

(1)

(1)

(1)

1

(1)

(2)

1

1

1

1 


\section{$\begin{array}{llllllllllllll}11 & 12 & 13 & 14 & 15 & 16 & 17 & 18 & 19 & 20 & 21 & 22 & \text { Pr. } & \text { Fr. }\end{array}$}

\begin{tabular}{|c|c|c|c|c|c|c|c|c|c|c|}
\hline+ & 2 & 1 & 1 & 1 & 1 & 1 & 2 & 1 & + & 1 \\
\hline+ & 2 & + & 1 & . & . & & . & 1 & + & + \\
\hline
\end{tabular}

$$
\begin{array}{rrrrrrrrrrrrrr}
1 & + & 1 & 1 & + & + & + & + & 1 & 1 & 1 & 1 & 22 & 100 \\
2 & 2 & 2 & 2 & 1 & 1 & + & 1 & 1 & 1 & 1 & 1 & 21 & 95 \\
2 & 2 & . & + & . & . & 1 & 1 & + & 1 & + & 1 & 19 & 86 \\
+ & + & + & . & + & . & + & 1 & 1 & + & 1 & 1 & 19 & 86 \\
+ & 1 & 1 & . & . & + & + & + & 1 & + & 1 & 1 & 19 & 86 \\
1 & + & . & . & . & . & . & + & . & r & . & + & 12 & 55 \\
. & + & + & + & + & + & + & 1 & + & + & . & 1 & 18 & 82 \\
. & . & . & . & + & . & . & + & + & . & . & . & 8 & 36 \\
. & . & . & . & . & . & . & . & . & . & . & . & 1 & 5 \\
+ & + & + & . & 1 & + & + & + & . & 1 & . & + & 18 & 82 \\
1 & 1 & 1 & 1 & 1 & 1 & 1 & 1 & 1 & 1 & 1 & 1 & 18 & 82 \\
+ & . & + & + & + & + & + & + & + & . & . & . & 18 & 82 \\
1 & + & + & + & 1 & 1 & 1 & 1 & + & + & + & . & 17 & 77 \\
+ & + & + & + & + & + & + & . & + & + & + & + & 17 & 77 \\
1 & . & + & . & + & . & + & 1 & + & 1 & + & 1 & 15 & 68 \\
2 & 1 & . & + & + & + & 1 & 1 & + & . & . & + & 15 & 68 \\
1 & + & . & . & + & . & . & + & + & + & 1 & 1 & 14 & 64 \\
. & . & . & . & + & + & . & + & . & + & . & + & 14 & 64 \\
+ & 1 & + & . & 1 & . & + & 1 & . & + & . & . & 14 & 64 \\
2 & 1 & . & . & + & . & . & + & + & 1 & 1 & 1 & 13 & 59 \\
. & + & + & + & . & + & + & + & . & 1 & . & . & 13 & 59 \\
. & . & . & . & + & . & . & 1 & . & + & + & . & 12 & 55 \\
1 & 1 & . & . & . & . & . & 1 & . & + & . & + & 12 & 55 \\
1 & 1 & + & . & . & . & . & + & . & + & + & . & 9 & 41 \\
. & . & + & . & + & . & 1 & 1 & . & . & . & . & 9 & 41 \\
+ & + & . & . & . & . & + & . & + & + & . & + & 9 & 41 \\
. & . & . & . & + & . & + & + & + & . & . & . & 9 & 41 \\
. & . & + & . & . & . & . & + & . & . & + & + & 9 & 41 \\
. & . & . & . & 1 & . & + & . & . & . & . & + & 7 & 32 \\
. & . & . & . & . & . & . & . & . & . & . & . & 6 & 27 \\
1 & + & . & . & . & . & . & . & . & . & . & . & 6 & 27 \\
+ & + & . & . & . & . & . & . & . & . & . & . & 5 & 23 \\
. & . & . & . & . & . & . & . & . & . & . & + & 1 & 5 \\
+ & . & . & . & . & . & . & . & . & . & . & . & 5 & 23 \\
. & . & . & . & . & . & . & + & . & . & . & . & 2 & 9 \\
. & . & . & . & . & . & . & . & . & . & . & . & 1 & 5 \\
. & . & . & . & . & . & . & . & . & . & . & . & 1 & 5 \\
. & . & . & . & . & . & . & . & . & . & . & + & 1 & 5
\end{array}
$$$$
\begin{array}{rrrrrrrrrrrrrr}
1 & 1 & 1 & 1 & 1 & 2 & 2 & 2 & 1 & 1 & 1 & 1 & 20 & 91 \\
+ & + & 1 & 1 & + & + & + & + & + & . & 1 & 1 & 17 & 77 \\
+ & 1 & 1 & . & + & + & 1 & . & + & + & . & . & 17 & 77 \\
+ & + & . & . & + & 1 & 2 & . & + & + & + & + & 16 & 73 \\
. & 1 & + & + & . & + & + & + & . & . & . & . & 11 & 50 \\
. & . & + & . & . & . & . & . & . & . & + & + & 4 & 18 \\
. & . & . & . & + & . & . & . & . & . & . & . & 1 & 5
\end{array}
$$$$
9 \quad 41
$$$$
836
$$ 


\begin{tabular}{|c|c|c|c|c|c|c|c|c|c|c|c|c|}
\hline & \multicolumn{2}{|c|}{ Number of relevé (Zaporedna številka popisa) } & 1 & 2 & 3 & 4 & 5 & 6 & 7 & 8 & 9 & 10 \\
\hline & Knautia drymeia & E1 & . & . & $\mathrm{r}$ & . & . & . & + & . & . & . \\
\hline & Rhamnus fallax & E2 & 1 & . & . & . & . & . & . & . & . & . \\
\hline & Helleborus niger & E1 & . & . & . & . & + & . & . & . & . & . \\
\hline & Cardamine trifolia & E1 & . & . & . & . & . & . & . & & . & . \\
\hline \multirow[t]{4}{*}{ TA } & Tilio-Acerion & & & & & & & & & & & \\
\hline & Acer pseudoplatanus & E3 & . & + & . & . & . & . & . & + & . & . \\
\hline & Acerpseudoplatanus & E2 & . & . & . & . & + & . & . & - & . & . \\
\hline & Acerpseudoplatanus & E1 & . & . & . & . & . & . & . & + & . & + \\
\hline \multirow[t]{21}{*}{ FS } & Fagetalia sylvaticae & & & & & & & & & & & \\
\hline & Melica nutans & E1 & + & . & + & . & 1 & + & 1 & . & . & + \\
\hline & Daphne mezereum & E2 & . & . & $\mathrm{r}$ & + & + & + & + & + & . & + \\
\hline & Fagus sylvatica & E3 & + & $\mathrm{r}$ & + & 1 & . & . & . & . & . & + \\
\hline & Fagus sylvatica & E2 & + & + & + & + & . & . & . & 1 & $\mathrm{r}$ & + \\
\hline & Fagus sylvatica & E1 & . & . & . & . & . & . & . & + & . & . \\
\hline & Mercurialis perennis & E1 & . & . & 1 & 1 & . & . & . & . & + & . \\
\hline & Lonicera alpigena & E2 & + & 1 & . & 1 & . & + & . & $\mathrm{r}$ & . & + \\
\hline & Dryopteris filix-mas & E1 & + & . & + & . & . & . & . & & . & . \\
\hline & Lilium martagon & E1 & + & . & . & + & + & + & + & . & . & . \\
\hline & Paris quadrifolia & E1 & + & . & + & + & . & . & . & + & . & . \\
\hline & Prenanthes purpurea & E1 & . & + & + & + & . & + & . & . & . & + \\
\hline & Galium laevigatum & E1 & + & . & . & + & . & + & . & . & + & . \\
\hline & Galeobdolon flavidum & E1 & . & . & . & . & . & + & 1 & &. & . \\
\hline & Polystichum aculeatum & E1 & + & . & . & . & . & . & . & . & . & . \\
\hline & Luzula nivea & E1 & . & . & . & . & . & . & . & . & . & . \\
\hline & Laburnum alpinum & E2 & . & . & . & . & . & . & + & $\mathrm{r}$ & . & . \\
\hline & Mycelis muralis & E1 & . & . & . & . & . & . & . & $\mathrm{r}$ & . & . \\
\hline & Lathyrus vernus subsp. vernus & E1 & + & . & . & . & . & . & . & . & . & . \\
\hline & Epilobium montanum & E1 & . & . & . & . & . & . & + & . & . & . \\
\hline & Phyteuma spicatum & E1 & . & . & . & . & . & . & . & . & . & . \\
\hline \multirow[t]{4}{*}{ QP } & Quercetalia pubescenti-petraeae & & & & & & & & & & & \\
\hline & Sorbus aria (Aria edulis) & E2 & + & . & . & + & . & . & + & . & . & . \\
\hline & Sorbus aria (Aria edulis) & E1 & . & . & . & . & . & . & . & $\mathrm{r}$ & . & . \\
\hline & Convallaria majalis & E1 & . & . & . & + & . & . & . & . & . & . \\
\hline \multirow[t]{9}{*}{ QF } & Querco-Fagetea & & & & & & & & & & & \\
\hline & Anemone nemorosa & E1 & 1 & + & 1 & 1 & + & . & . & & . & . \\
\hline & Hepatica nobilis & E1 & . & . & . & . & . & . & . & 1 & . & . \\
\hline & Poa nemoralis & E1 & . & . & . & . & . & . & + & . & . & . \\
\hline & Carex digitata & E1 & . & . & . & . & . & . & . & . & + & . \\
\hline & Corylus avellana & E2 & . & . & . & . & . & . & . & $\mathrm{r}$ & . & . \\
\hline & Festuca heterophylla & E1 & . & . & . & . & . & . & . & & . & . \\
\hline & Hieracium lachenalii & E1 & . & . & . & . & . & . & . & . & . & . \\
\hline & Dactylorbiza fuchsii & E1 & . & . & . & . & . & . & . & . & . & . \\
\hline \multirow[t]{4}{*}{ SSC } & Sambuco-Salicion capreae, Rhan & & & & & & & & & & & \\
\hline & Sorbus aucuparia subsp. aucuparia & E3 & . & + & + & + & + & + & + & . & . & + \\
\hline & Sorbus aucuparia subsp. aucuparia & E2 & + & + & + & + & + & + & + & . & . & + \\
\hline & Sorbus aucuparia subsp. aucuparia & E1 & . & + & . & . & . & . & . & + & . & . \\
\hline \multirow[t]{5}{*}{ BA } & Betulo-Alnetea & & & & & & & & & & & \\
\hline & Sorbus chamaemespilus & E2 & + & 1 & + & 1 & 1 & . & . & & + & 1 \\
\hline & Salix appendiculata & E2 & + & 1 & 1 & 1 & + & + & . & + & + & + \\
\hline & Alnus viridis & E2 & . & 1 & 1 & + & + & . & . & & . & 1 \\
\hline & Salix glabra & E2 & + & . & . & . & . & . & . & & . & . \\
\hline
\end{tabular}




\begin{tabular}{|c|c|c|c|c|c|c|c|c|c|c|c|c|}
\hline \multirow[t]{4}{*}{ 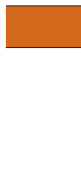 } & \multicolumn{2}{|c|}{ Number of relevé (Zaporedna številka popisa) } & \multirow{2}{*}{$\frac{1}{1}$} & \multirow{2}{*}{$\begin{array}{l}2 \\
.\end{array}$} & \multirow{2}{*}{$\begin{array}{l}3 \\
.\end{array}$} & \multirow{2}{*}{4} & \multirow{2}{*}{$\begin{array}{l}5 \\
.\end{array}$} & \multirow{2}{*}{6} & \multirow{2}{*}{$\begin{array}{l}7 \\
.\end{array}$} & \multirow{2}{*}{$\begin{array}{l}8 \\
.\end{array}$} & \multirow{2}{*}{$\begin{array}{l}9 \\
.\end{array}$} & \multirow{2}{*}{$\begin{array}{l}10 \\
.\end{array}$} \\
\hline & Juniperus sibirica & E2 & & & & & & & & & & \\
\hline & Sorbus austriaca s.lat. & E2 & + & . & . & . & . & . & . & . & . & . \\
\hline & Salix waldsteiniana & E2 & . & . & . & . & . & . & . & . & . & + \\
\hline \multirow{25}{*}{ MuA } & Mulgedio-Aconitetea & & & & & & & & & & & \\
\hline & Viola biflora & E1 & + & + & + & + & 1 & + & + & . & 1 & 1 \\
\hline & Geranium sylvaticum & E1 & + & + & + & + & . & 1 & 1 & . & + & + \\
\hline & Veratrum album subsp. lobelianum & E1 & + & 1 & + & 1 & 1 & . & + & . & + & . \\
\hline & Polygonatum verticillatum & E1 & + & . & + & 1 & 1 & . & . & . & + & + \\
\hline & Athyrium filix-femina & E1 & . & . & + & . & . & . & . & + & + & . \\
\hline & Saxifraga rotundifolia & E1 & . & + & . & . & . & . & . & . & . & . \\
\hline & Geum rivale & E1 & . & + & . & . & + & + & + & . & . & . \\
\hline & Phyteuma ovatum & E1 & . & + & + & + & . & . & . & + & . & . \\
\hline & Hypericum maculatum & E1 & . & . & . & . & . & . & . & . & . & . \\
\hline & Senecio cacaliaster & E1 & . & . & + & . & + & . & . & . & . & . \\
\hline & Senecio ovatus & E1 & . & . & + & . & . & . & . & . & . & . \\
\hline & Chaerophyllum villarsii & E1 & . & . & . & . & + & 1 & . & . & . & . \\
\hline & Heracleum montanum & E1 & + & . & . & + & . & . & . & . & . & . \\
\hline & Thalictrum aquilegiifolium & E1 & . & . & . & . & + & . & . & . & + & . \\
\hline & Chaerophyllum hirsutum & E1 & . & . & . & . & . & . & . & . & . & . \\
\hline & Primula elatior & E1 & . & . & . & . & . & . & . & . & . & . \\
\hline & Adenostyles alliariae & E1 & . & . & . & . & . & . & . & . & . & . \\
\hline & Cirsium $x$ benacense & E1 & . & . & . & . & . & . & . & . & . & . \\
\hline & Ranunculus platanifolius & E1 & . & + & . & . & . & . & . & . & . & . \\
\hline & Athyrium distentifolium & E1 & . & . & . & + & . & . & . & . & . & . \\
\hline & Rumex arifolius & E1 & . & . & . & . & . & . & + & . & . & . \\
\hline & Aconitum angustifolium & E1 & . & . & . & . & . & . & . & . & . & . \\
\hline & Epilobium alpestre & E1 & . & . & . & . & . & . & . & . & . & . \\
\hline & Aconitum degenii subsp. paniculatum & E1 & . & . & . & . & . & . & . & . & . & . \\
\hline \multirow[t]{3}{*}{ TG } & Trifolio-Geranietea & & & & & & & & & & & \\
\hline & Laserpitium siler & E1 & + & . & . & . & . & . & . & . & . & . \\
\hline & Silene nutans & E1 & . & . & . & . & . & . & + & . & . & . \\
\hline \multirow[t]{4}{*}{$\mathrm{CA}$} & Caricion austroalpinae & & & & & & & & & & & \\
\hline & Heracleum austriacum subsp. siifolium & E1 & + & . & . & . & . & + & . & . & . & . \\
\hline & Pulsatilla alpina subsp. austroalpina & E1 & . & . & . & $\mathrm{r}$ & . & + & + & . & . & . \\
\hline & Arabis vochinensis & E1 & . & . & . & . & . & . & . & . & . & . \\
\hline \multirow[t]{6}{*}{ Cfir } & Caricion firmae & & & & & & & & & & & \\
\hline & Carex firma & E1 & . & . & . & . & . & . & . & + & + & + \\
\hline & Dryas octopetala & E1 & . & . & . & . & . & . & . & . & . & . \\
\hline & Ranunculus hybridus & E1 & + & . & . & . & . & . & . & . & . & . \\
\hline & Pedicularis rostratocapitata & E1 & . & . & . & . & . & . & . & . & . & + \\
\hline & Sesleria sphaerocephala & E1 & . & . & . & . & . & . & . & . & . & . \\
\hline \multirow[t]{3}{*}{ CF } & Caricion ferrugineae & & & & & & & & & & & \\
\hline & Knautia longifolia & E1 & . & . & . & . & + & + & + & . & . & . \\
\hline & Crepis bocconi & E1 & . & . & . & $\mathrm{r}$ & . & . & . & . & . & . \\
\hline \multirow[t]{5}{*}{ ES } & Elyno-Seslerietea & & & & & & & & & & & \\
\hline & Sesleria caerulea & E1 & + & + & 2 & + & . & 1 & 2 & 1 & 1 & 2 \\
\hline & Aster bellidiastrum & E1 & + & . & 1 & + & + & + & + & 1 & + & + \\
\hline & Selaginella selaginoides & E1 & + & + & + & + & . & . & . & + & + & . \\
\hline & Betonica alopecuros & E1 & 1 & . & + & . & . & + & 1 & . & + & . \\
\hline
\end{tabular}




\section{$\begin{array}{llllllllllllll}11 & 12 & 13 & 14 & 15 & 16 & 17 & 18 & 19 & 20 & 21 & 22 & \text { Pr. } & \text { Fr. }\end{array}$

$\begin{array}{ll}2 & 9 \\ 1 & 5 \\ 1 & 5\end{array}$

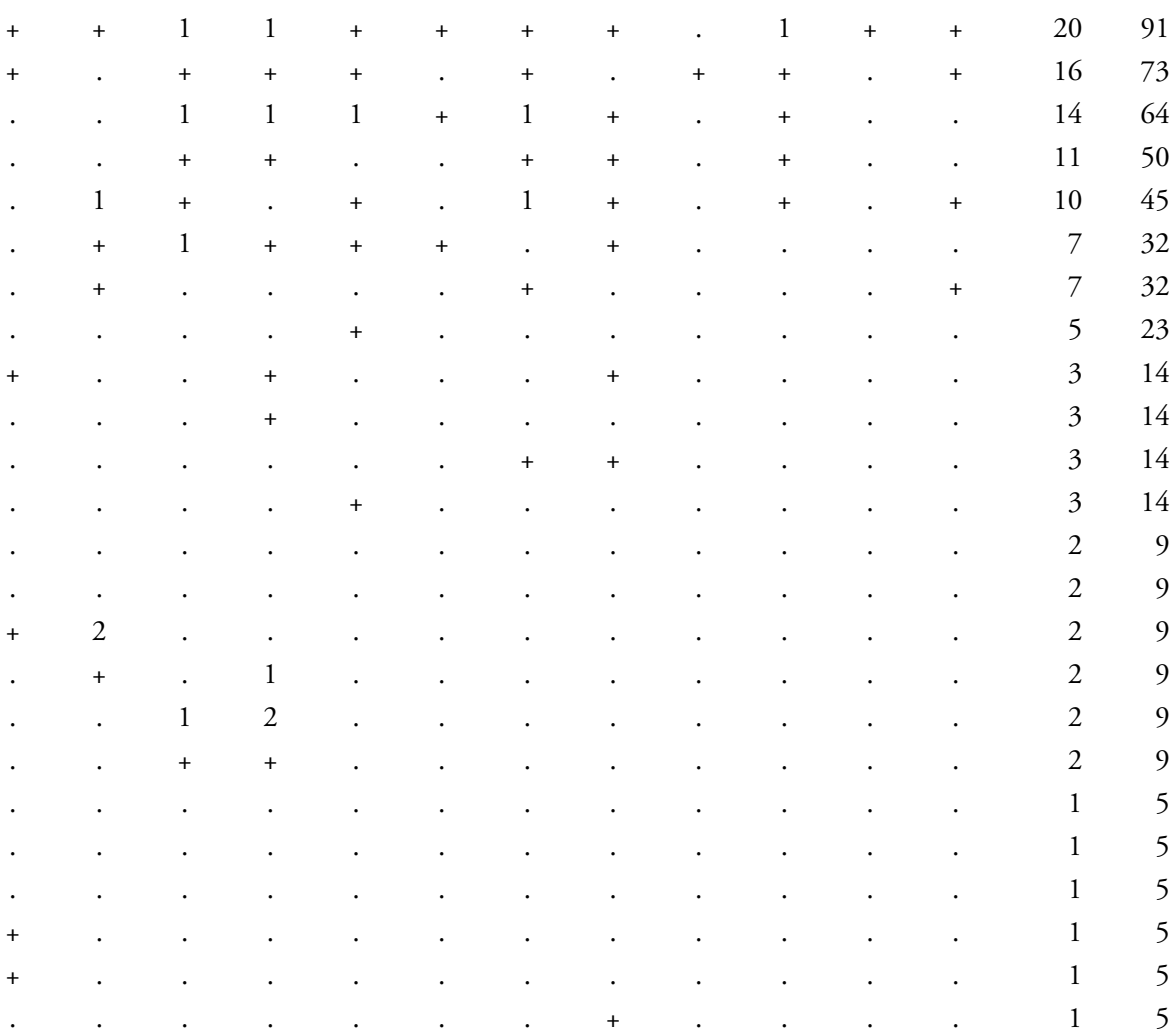

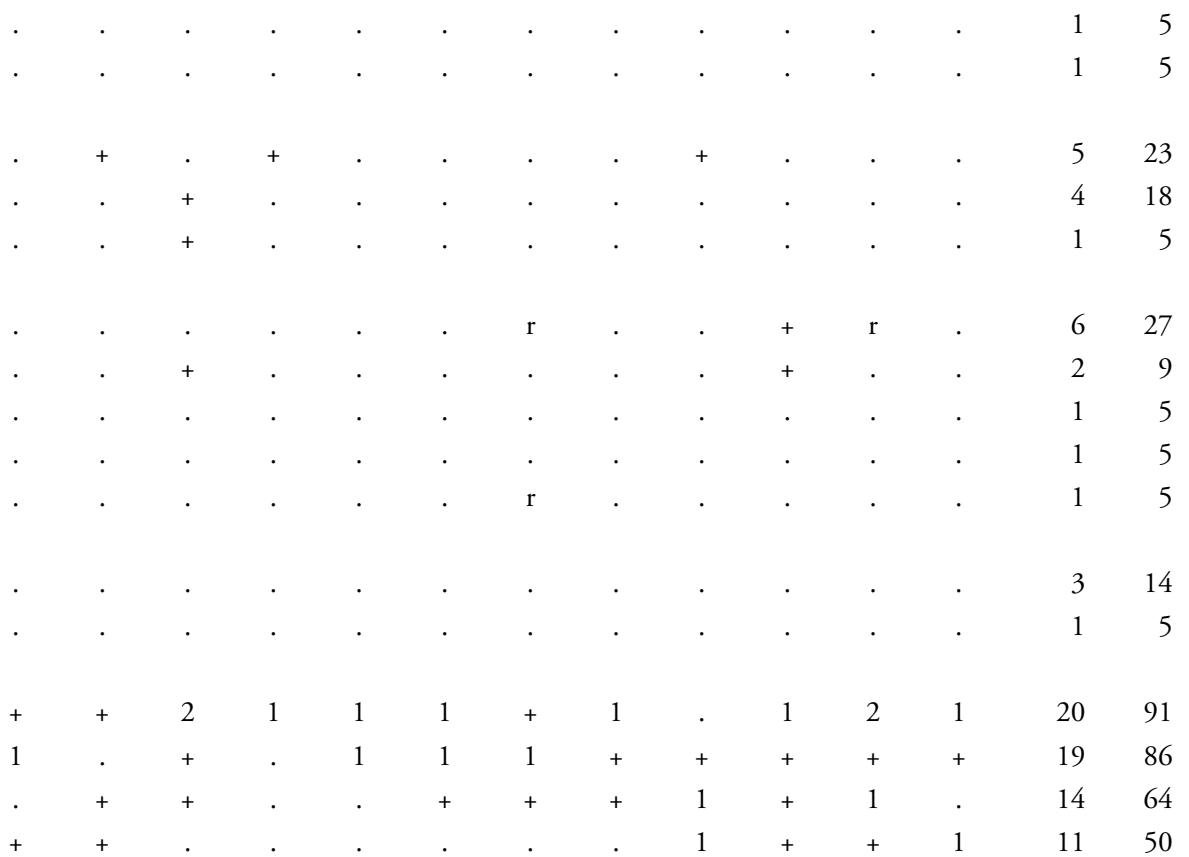




\section{Number of relevé (Zaporedna številka popisa)}

Phyteuma orbiculare

Juncus monanthos

Festuca calva

Bartsia alpina

Galium anisophyllum

Carduus crassifolius

Campanula witasekiana

Polygonum viviparum

Senecio abrotanifolius

Homogyne discolor

Hieracium villosum

Lotus alpinus

Helianthemum nummularium subsp. grandiflorum E1

Euphrasia picta

Gentiana clusii

E1

Alchemilla alpigena

E1

Carex sempervirens

Globularia nudicaulis

Anemone narcissiflora

Leucanthemum heterophyllum (L.maximum agg.) E1

Scabiosa lucida subsp. lucida

Hieracium pilosum

Ranunculus montanus

Alchemilla sp.

Gentiana lutea subsp. symphyandra

Ranunculus carinthiacus

Polygala alpestris

Thymus praecox subsp. polytrichus

Juncetea trifidi

Campanula scheuchzeri

\section{Calluno-Ulicetea}

Pseudorchis albida

Gentiana pannonica

Coeloglossum viride

Meum athamanticum

Potentilla erecta

\section{E1}

Festuca nigrescens

\section{Festuco-Brometea}

Cirsium erisithales

Buphthalmum salicifolium

Prunella grandiflora

Carlina acaulis

Koeleria pyramidata

Linum catharticum

$\mathrm{PaT}$ Poo alpinae-Trisetetalia

Trollius europaeus

Poa alpina

MA Molinio-Arrbenatheretea

Crepis paludosa

Leontodon hispidus 


\begin{tabular}{|c|c|c|c|c|c|c|c|c|c|c|c|c|c|}
\hline . & . & + & + & 1 & 1 & 1 & + & 1 & & . & . & 11 & 50 \\
\hline+ & + & + & . & . & + & + & . & + & . & . & . & 7 & 32 \\
\hline . &. & . & . & . & . & . & . & 1 & . & + & + & 5 & 23 \\
\hline . & . & . & . & . & + & . & . & . & + & . & . & 5 & 23 \\
\hline . & . & + & 1 & . & . & . & + & + & . & . & . & 5 & 23 \\
\hline . & . & . & . & r & . & . & . & . & r & $\mathrm{r}$ & . & 4 & 18 \\
\hline . & . & + & . & . & . & . & . & . & . & . & . & 3 & 14 \\
\hline . & . & + & . & . & . & . & . & . & . & . & . & 3 & 14 \\
\hline+ & . & . & . & . & . & . & . & 1 & . & . & . & 3 & 14 \\
\hline+ & . & . & + & . & . & . & . & . & . & . & . & 3 & 14 \\
\hline . & . & . & . & . & . & . & . & . & . & . & . & 2 & 9 \\
\hline . & . & . & . & . & . & . & . & . & . & . & . & 2 & 9 \\
\hline . & . & . & . & . & . & . & . & . & . & . & . & 2 & 9 \\
\hline . & . & . & . & . & + & . & . & . & . & . & . & 2 & 9 \\
\hline . & . & . & . & . & . & . & . & . & . & . & . & 2 & 9 \\
\hline 1 & + & . & . & . & . & . & . & . & . & . & . & 2 & 9 \\
\hline . & + & . & . & . & . & . & . & . & 1 & . & . & 2 & 9 \\
\hline . & . & . & . & . & . & . & . & $\mathrm{r}$ & + & . & . & 2 & 9 \\
\hline . & . & . & . & . & . & . & . & . & . & . & . & 1 & 5 \\
\hline . & . & . & . & . & . & . & . & . & . & . & . & 1 & 5 \\
\hline . & . & . & . & . & . & . & . & . & . & . & . & 1 & 5 \\
\hline . & . & . & . & . & . & . & . & . & . & . & . & 1 & 5 \\
\hline . & + & . & . & . & . & . & . & . & . & . & . & 1 & 5 \\
\hline . & . & . & + & . & . & . & . & . & . & . & . & 1 & 5 \\
\hline . & . & . & + & . & . & . & . & . & . & . & . & 1 & 5 \\
\hline . & . & . & + & . & . & . & . & . & . & . & . & 1 & 5 \\
\hline . & . & . & . & . & . & . & + & . & . & . & . & 1 & 5 \\
\hline . & . & $\cdot$ & . & . & . & . & . & + & . & . & . & 1 & 5 \\
\hline+ & . & 1 & + & . & . & . & . & + & + & + & + & 13 & 59 \\
\hline . & . & . & . & + & . & + & . & . & . & . & . & 3 & 14 \\
\hline . & . & + & . & . & . & . & . & . & . & . & . & 3 & 14 \\
\hline . & . & . & . & . & . & . & + & . & . & . & . & 2 & 9 \\
\hline . & . & . & 1 & . & . & . & . & . & . & . & . & 2 & 9 \\
\hline . & . & . & . & . & . & . & . & . & . & . & . & 1 & 5 \\
\hline . & . & . & + & . & . & . & . & . & . & . & . & 1 & 5 \\
\hline . & . & . & . & . & + & . & + & + & . & . & . & 10 & 45 \\
\hline . & . & . & . & . & . & . & . & . & . & . & . & 4 & 18 \\
\hline . & . & + & + & . & . & . & . & . & . & . & . & 4 & 18 \\
\hline . & . & $\cdot$ & . & . & . & . & . & . & . & . & . & 2 & 9 \\
\hline . & . & . & . & . & . & . & . & . & . & . & . & 1 & 5 \\
\hline . & . & . & . & . & . & $r$ & . & . & . & . & . & 1 & 5 \\
\hline . & . & + & + & . & + & + & . & $\mathrm{r}$ & . & . & . & 7 & 32 \\
\hline . & . & + & 1 & . & . & . & . & . & . & . & . & 2 & 9 \\
\hline . & . & & . & r & & + & . & . & . & . & . & 3 & 14 \\
\hline · & + & . & + & . & . & . & . & . & + & . & . & 3 & 14 \\
\hline
\end{tabular}




\section{Number of relevé (Zaporedna števillka popisa)}

Anthoxanthum odoratum

Festuca rubra

Angelica sylvestris

Ajuga reptans

Crepis sp.

Dactylis glomerata s.str.

Cerastium fontanum

Trifolium pratense

Deschampsia cespitosa

MC Montio-Cardaminetea

Saxifraga aizoides

CD Caricetalia davallianae

Parnassia palustris

Tofieldia calyculata

Pinguicula alpina

EA Epilobietea angustifolii

Fragaria vesca

Rubus idaeus

Galeopsis speciosa

AC Arabidetalia caeruleae

Soldanella alpina

TR Thlaspietea rotundifolii

Gymnocarpium robertianum

Dryopteris villarii

Cystopteris montana

Biscutella laevigata

Valeriana montana

Saxifraga caesia

Cerastium carinthiacum

Trisetum argenteum

Rumex scutatus

Heracleum pollinianum

Euphrasia cuspidata

Hieracium bifidum

Rhodiola rosea

Minuartia austriaca

\section{Cystopteridion fragilis}

Cystopteris fragilis

Carex brachystachys

E1

Heliosperma pusillum

Physoplexido comosae-Saxifragion petraeae

Saxifraga crustata

Saxifraga squarrosa

Potentilletalia caulescentis

Campanula cochleariifolia

Primula auricula

Potentilla clusiana

Festuca stenantha 


\begin{tabular}{|c|c|c|c|c|c|c|c|c|c|c|c|c|}
\hline & \multicolumn{2}{|c|}{ Number of relevé (Zaporedna številka popisa) } & 1 & 2 & 3 & 4 & 5 & 6 & 7 & 8 & 9 & 10 \\
\hline & Moehringia muscosa & E1 & . & . & + & . & . & . & . & . & . & . \\
\hline & Asplenium ruta-muraria & E1 & . & . & + & . & . & . & + & . & . & . \\
\hline & Polypodium vulgare & E1 & . & . & . & . & . & . & . & + & . & . \\
\hline & Asplenium trichomanes & E1 & . & . & . & . & . & . & . & . & . & . \\
\hline & Festuca sp. & E1 & . & . & . & . & . & . & . & . & . & . \\
\hline & Hieracium dollineri & E1 & . & . & . & . & . & . & + & . & . & . \\
\hline & Gypsophila repens & E1 & . & . & . & . & . & . & . & . & + & . \\
\hline \multirow[t]{32}{*}{ ML } & \multicolumn{12}{|c|}{ Mosses and lichens (Mahovi in lišaji) } \\
\hline & Tortella tortuosa & E0 & 1 & + & 1 & 1 & + & + & + & + & + & + \\
\hline & Rhytidiadelphus triquetrus & E0 & + & 1 & . & + & . & + & . & . & + & + \\
\hline & Ctenidium molluscum & E0 & 1 & 1 & + & + & . & . & + & . & + & . \\
\hline & Hylocomium splendens & E0 & . & + & . & + & . & + & . & . & . & + \\
\hline & Dicranum scoparium & E0 & . & + & + & + & + & . & . & . & + & . \\
\hline & Fissidens dubius & E0 & 1 & . & + & 1 & . & . & + & . & . & . \\
\hline & Peltigera leucophlebia & E0 & . & + & . & . & . & . & . & . & + & . \\
\hline & Polytrichum formosum & E0 & + & + & + & . & · & . & . & . & . & . \\
\hline & Plagiochila porelloides & E0 & 1 & . & + & . & . & . & . & . & . & . \\
\hline & Sphagnum sp. & E0 & . & 1 & . & + & . & . & . & . & . & + \\
\hline & Orthothecium rufescens & E0 & . & . & + & + & . & . & . & . & . & . \\
\hline & Schistidium sp. & E0 & . & . & . & . & . & . & + & . & . & . \\
\hline & Paraleucobryum sauteri & E0 & . & . & + & 1 & . & . & . & . & . & . \\
\hline & Conocephalum conicum & E0 & + & . & . & + & . & . & . & . & . & . \\
\hline & Dicranum majus & E0 & + & . & . & + & . & . & . & . & . & . \\
\hline & Plagiothecium sylvaticum & E0 & . & + & . & . & . & . & . & . & . & . \\
\hline & Isothecium alopecuroides & E0 & . & . & + & . & . & . & . & . & . & . \\
\hline & Marchantia polymorpha & E0 & . & . & . & . & . & . & . & . & . & . \\
\hline & Metzgeria furcata & E0 & . & . & + & . & . & . & . & . & $\cdot$ & . \\
\hline & Plagiopus oederi & E0 & . & . & + & . & . & . & . & . & . & . \\
\hline & Plagiothecium undulatum & E0 & . & $\cdot$ & . & . & · & . & · & . & . & + \\
\hline & Eurhynchium angustirete & E0 & . & . & . & . & · & $\cdot$ & . & . & . & . \\
\hline & Plagiomnium undulatum & E0 & . & . & . & . & . & . & . & . & . & . \\
\hline & Hypogymnia physodes & E4 & . & . & . & . & . & . & . & . & . & $\cdot$ \\
\hline & Bryoria fuscescens & E4 & . & $\cdot$ & . & . & . & . & . & . & . & . \\
\hline & Parmelia sulcata & E3c & . & . & . & . & . & . & . & $\cdot$ & . & . \\
\hline & Pseudoleskea incurvata & E0 & . & · & · & . & · & . & · & . & . & $\cdot$ \\
\hline & Rhytidiadelphus loreus & E0 & . & . & . & . & . & . & · & . & . & · \\
\hline & Bazzania trilobata & E0 & . & . & . & . & · & . & $\cdot$ & . & . & · \\
\hline & Dicranum sp. & E0 & . & . & . & . & . & . & · & . & . & . \\
\hline & Rhizomnium punctatum & E0 & . & . & . & . & . & . & . & . & . & . \\
\hline
\end{tabular}

\section{Legend - Legenda}

ID Igor Dakskobler

AR Andrej Rozman

AS Andrej Seliškar

A Limestone - apnenec

D Dolomite - dolomit

Gr Gravel - grušč

Mo Moraine (Til) - morena (til)

Re Rendzina - rendzina

JA Julian Alps - Julijske Alpe

K Karavanke - Karavanke

Pr. Presence (number of relevés in which the species is presented) - število popisov, v katerih se pojavlja vrsta

Fr. Frequency in $\%-$ frekvenca $\mathrm{v} \%$ 


\begin{tabular}{|c|c|c|c|c|c|c|c|c|c|c|c|c|}
\hline+ & $+\quad+$ & + & + & + & + & 1 & + & 1 & 1 & 1 & 22 & 100 \\
\hline 2 & + & + & + & . & . & 1 & + & 1 & + & 1 & 16 & 73 \\
\hline 1 & + & . & + & + & + & 1 & . & . & + & + & 15 & 68 \\
\hline 1 & . & . & . & . & + & 1 & . & + & + & + & 11 & 50 \\
\hline . & + & . & + & . & . & 1 & . & + & . & + & 10 & 45 \\
\hline . & . & . & + & . & . & . & + & + & + & + & 10 & 45 \\
\hline+ & + & . & . & + & + & + & . & + & . & + & $S$ & 41 \\
\hline . & . & . & + & . & . & . & . & + & + & + & $\varepsilon$ & 36 \\
\hline 1 & . & . & . & . & . & . & + & . & . & + & 5 & 23 \\
\hline . & . & . & + & . & . & + & . & . & . & . & 5 & 23 \\
\hline . & . & . & . & . & + & . & . & . & + & . & 5 & 23 \\
\hline . & + & + & . & . & . & . & . & . & . & + & 4 & 18 \\
\hline . & . & . & . & . & . & . & . & + & . & . & 3 & 14 \\
\hline . & . & . & . & . & . & . & . & . & . & . & 2 & 9 \\
\hline . & . & . & . & . & . & . & . & . & . & . & 2 & 9 \\
\hline . & . & . & . & . & . & + & . & . & . & . & 2 & 9 \\
\hline . & . & + & . & . & . & . & . & . & . & . & 2 & 9 \\
\hline . & . & . & . & . & + & . & . & . & . & . & 2 & 9 \\
\hline . & . & . & . & . & . & . & . & . & . & . & & 5 \\
\hline . & . & . & . & . & . & . & . & . & . & . & & 5 \\
\hline . & . & . & . & . & . & . & . & . & . & . & & 5 \\
\hline+ & . & . & . & . & . & . & . & . & . & . & & 5 \\
\hline+ & . & . & . & . & . & . & . & . & . & . & & 5 \\
\hline . & . & . & . & . & . & . & . & . & . & . & & 5 \\
\hline . & + & . & . & . & . & . & . & . & . & . & & 5 \\
\hline . & . & . & . & . & . & . & . & . & . & . & & 5 \\
\hline . & . & + & . & . & . & . & . & . & . & . & & 5 \\
\hline . & . & . & . & . & . & + & . & . & . & . & & 5 \\
\hline . & . & . & . & & . & + & . & . & . & . & & 5 \\
\hline . & . & . & . & . & . & . & . & + & . & . & & 5 \\
\hline & . & & & & & & & & & & & \\
\hline
\end{tabular}


Table 6: Rhodothamno-Laricetum sorbetosum chamaemespili var. Saxifraga cuneifolia

\begin{tabular}{|c|c|c|c|c|c|c|c|c|c|c|c|c|c|c|c|c|c|c|c|}
\hline & \multicolumn{2}{|l|}{ Number of relevé (Zaporedna številka popisa) } & 1 & 2 & 3 & 4 & 5 & 6 & 7 & 8 & 9 & 10 & 11 & 12 & 13 & 14 & 15 & 16 & Pr. \\
\hline & \multicolumn{2}{|l|}{$\begin{array}{l}\text { Database number of relevé } \\
\text { (Delovna številka popisa) }\end{array}$} & 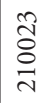 & 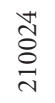 & 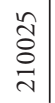 & 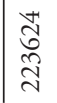 & 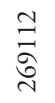 & $\underset{\sim}{\stackrel{\infty}{\sigma}}$ & 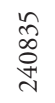 & $\begin{array}{l}\stackrel{\aleph}{\mathrm{D}} \\
\text { }\end{array}$ & 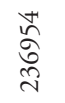 & $\begin{array}{l}\hat{\sigma} \\
\hat{\sigma}\end{array}$ & 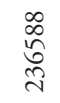 & 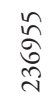 & $\vec{\Xi}$ & $\begin{array}{l}\infty \\
\stackrel{\infty}{\sigma} \\
\stackrel{\sim}{2}\end{array}$ & 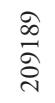 & $\stackrel{\curvearrowright}{\stackrel{े}{े}}$ & \\
\hline & \multicolumn{2}{|l|}{ Author of the relevé (Avtor popisa) } & ID & ID & ID & ID & ID & ID & ID & ID & ID & ID & IDAS & ID & ID & ID & ID & ID & \\
\hline & \multicolumn{2}{|l|}{ Elevation in m (Nadmorska višina $\mathrm{v} \mathrm{m}$ ) } & 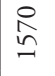 & $\stackrel{\text { }}{\sim}$ & 원 & $\stackrel{ }{\stackrel{ }{\beth}}$ & $\stackrel{n}{\underline{\Xi}}$ & $\stackrel{\circ}{0}$ & వి & 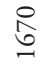 & 웜 & $\stackrel{n}{\sigma}$ & $\stackrel{R}{\stackrel{R}{n}}$ & $\stackrel{\circ}{6}$ & $\stackrel{2}{\cong}$ & $\stackrel{\nwarrow}{\bumpeq}$ & $\stackrel{\circ}{\stackrel{\sigma}{-}}$ & $\begin{array}{l}\infty \\
\stackrel{\sim}{\simeq}\end{array}$ & \\
\hline & \multicolumn{2}{|l|}{ Aspect (Lega) } & 急 & 之 & 急 & 圣 & 罠 & 㟐 & z & 㞱 & 之 & 学 & 急 & 더 & 㞱 & 之 & 吕 & 㐏 & \\
\hline & \multicolumn{2}{|l|}{ Slope in degrees (Nagib v stopinjah) } & 45 & 40 & 30 & 45 & 45 & 35 & 30 & 35 & 15 & 30 & 10 & 10 & 30 & 20 & 20 & 20 & \\
\hline & \multicolumn{2}{|l|}{ Parent material (Matična podlaga) } & DA & A & A & DA & A & A & $\mathrm{D}$ & A & A & A & DA & A & A & A & A & A & \\
\hline & \multicolumn{2}{|l|}{ Soil (Tla) } & $\operatorname{Re}$ & $\operatorname{Re}$ & $\operatorname{Re}$ & $\operatorname{Re}$ & $\operatorname{Re}$ & $\operatorname{Re}$ & $\operatorname{Re}$ & $\operatorname{Re}$ & $\operatorname{Re}$ & $\operatorname{Re}$ & $\operatorname{Re}$ & $\operatorname{Re}$ & $\operatorname{Re}$ & $\operatorname{Re}$ & $\operatorname{Re}$ & $\operatorname{Re}$ & \\
\hline & \multicolumn{2}{|l|}{ Stoniness in \% (Kamnitost v \%) } & 10 & 20 & 10 & 10 & 10 & 5 & 0 & 5 & 10 & 10 & 10 & 20 & 10 & 30 & 30 & 30 & \\
\hline & \multicolumn{19}{|l|}{ Cover in \% (Zastiranje v \%): } \\
\hline & Upper tree layer (Zgornja drevesna plast) & E3b & 60 & 60 & 60 & 60 & 70 & 70 & 60 & 60 & 60 & 70 & 60 & 40 & 70 & 60 & 60 & 60 & \\
\hline & Lower tree layer (Spodnja drevesna plasti) & E3a & 10 & 10 & 5 & 5 & 10 & 5 & 10 & 10 & 10 & 5 & 10 & 10 & 10 & 0 & 20 & 30 & \\
\hline & Shrub layer (Grmovna plast) & E2 & 30 & 30 & 40 & 50 & 45 & 20 & 30 & 30 & 20 & 20 & 20 & 30 & 40 & 40 & 30 & 40 & \\
\hline & Herb layer (Zeliščna plast) & E1 & 80 & 80 & 80 & 80 & 90 & 90 & 90 & 90 & 80 & 80 & 90 & 70 & 90 & 70 & 80 & 80 & \\
\hline & Moss layer (Mahovna plast) & E0 & 10 & 20 & 10 & 10 & 10 & 10 & 10 & 5 & 5 & 5 & 10 & 5 & 10 & 20 & 20 & 20 & \\
\hline & Number of species (Število vrst) & & 67 & 76 & 69 & 60 & 50 & 50 & 61 & 75 & 81 & 63 & 79 & 91 & 54 & 80 & 76 & 62 & \\
\hline & Relevé area (Velikost popisne ploskve) & $\mathrm{m}^{2}$ & 200 & 400 & 400 & 200 & 400 & 400 & 400 & 400 & 400 & 400 & 400 & 400 & 400 & 200 & 200 & 200 & \\
\hline & \multicolumn{2}{|l|}{ Date of taking relevé (Datum popisa) } & 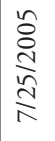 & $\begin{array}{l}\stackrel{n}{o} \\
\stackrel{d}{N} \\
\stackrel{n}{N} \\
\end{array}$ & 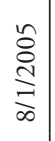 & 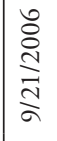 & 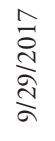 & 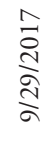 & 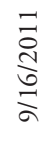 & 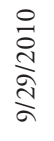 & 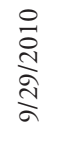 & 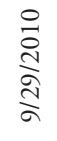 & 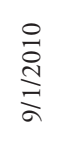 & 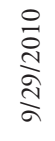 & 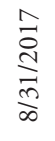 & $\frac{\widehat{a}}{\frac{\widehat{a}}{a}}$ & 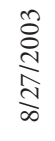 & 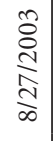 & \\
\hline & \multicolumn{2}{|l|}{ Locality (Nahajališče) } & $\begin{array}{l}\frac{y}{\vec{z}} \\
\frac{\vec{u}}{\vec{u}} \\
\frac{\vec{u}}{\vec{a}}\end{array}$ & $\begin{array}{l}\frac{y}{\vec{z}} \\
\frac{\vec{u}}{\vec{y}} \\
\frac{\vec{y}}{\vec{a}}\end{array}$ & $\begin{array}{l}\frac{y}{\vec{z}} \\
\frac{\vec{y}}{\vec{y}} \\
\frac{\vec{y}}{\vec{a}}\end{array}$ & 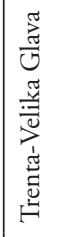 & 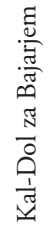 & 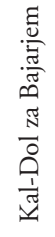 & $\frac{\stackrel{\widetilde{J}}{\mathbb{N}}}{\frac{\mathbb{N}}{N}}$ & 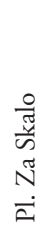 & 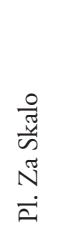 & $\begin{array}{l}\frac{O}{\pi} \\
\frac{\pi}{\omega} \\
\tilde{N} \\
N \\
\dot{a}\end{array}$ & 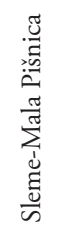 & $\begin{array}{l}\frac{O}{\pi} \\
\frac{\pi}{N} \\
\tilde{N} \\
N \\
\dot{I}\end{array}$ & 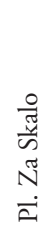 & 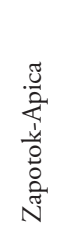 & 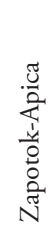 & 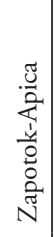 & \\
\hline & \multicolumn{2}{|l|}{ Mountain range (Pogorje) } & JA & JA & JA & JA & JA & JA & K & JA & JA & JA & JA & JA & JA & JA & JA & JA & \\
\hline & \multicolumn{2}{|l|}{ Quadrant (Kvadrant) } & $\begin{array}{l}\frac{m}{\infty} \\
\substack{\infty \\
\curvearrowleft}\end{array}$ & $\begin{array}{l}\frac{n}{\infty} \\
\stackrel{+}{0} \\
\swarrow\end{array}$ & $\begin{array}{l}\frac{m}{\infty} \\
\stackrel{\infty}{0} \\
\stackrel{2}{2}\end{array}$ & 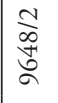 & 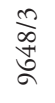 & 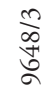 & 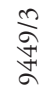 & $\begin{array}{l}\frac{n}{\infty} \\
\underset{\infty}{\infty} \\
\sigma\end{array}$ & $\begin{array}{l}\stackrel{m}{\infty} \\
\stackrel{\infty}{d} \\
\stackrel{0}{\alpha}\end{array}$ & \begin{tabular}{l}
$m$ \\
$\infty$ \\
\multirow{\infty}{\infty}{} \\
$\sigma$
\end{tabular} & \begin{tabular}{l}
$\stackrel{n}{\infty}$ \\
$\stackrel{+}{*}$ \\
\multirow{\alpha}{\alpha}{}
\end{tabular} & 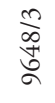 & $\begin{array}{l}\stackrel{n}{\infty} \\
\stackrel{\infty}{+1} \\
\swarrow\end{array}$ & $\begin{array}{l}\underset{\infty}{\infty} \\
\underset{+}{0} \\
\sigma\end{array}$ & $\begin{array}{l}\underset{\infty}{\infty} \\
\underset{+}{0} \\
\sigma\end{array}$ & $\begin{array}{l}\underset{⿱}{\infty} \\
\stackrel{+}{0} \\
\swarrow\end{array}$ & \\
\hline & \multirow[t]{2}{*}{ Coordinate GK-Y (D-48) } & $\mathrm{m}$ & 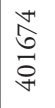 & $\begin{array}{l}\stackrel{ }{N} \\
\stackrel{ }{\circ}\end{array}$ & 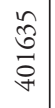 & 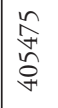 & $\begin{array}{l}\stackrel{+}{0} \\
\stackrel{0}{0} \\
\stackrel{0}{+}\end{array}$ & 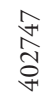 & 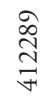 & $\begin{array}{l}\stackrel{ }{a} \\
\stackrel{ }{\circ}\end{array}$ & 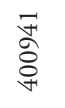 & $\begin{array}{l}\vec{\vdots} \\
\text { I } \\
\stackrel{+}{+}\end{array}$ & 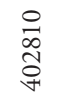 & હે & $\begin{array}{l}\infty \\
\infty \\
\vec{\sim} \\
\stackrel{+}{+}\end{array}$ & $\begin{array}{l}\stackrel{n}{\&} \\
\stackrel{\sigma}{\sigma}\end{array}$ & 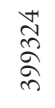 & 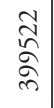 & \\
\hline & & $\mathrm{m}$ & $\begin{array}{l}\stackrel{\curvearrowright}{\curvearrowright} \\
\stackrel{n}{n}\end{array}$ & 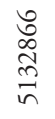 & $\begin{array}{l}\overrightarrow{0} \\
\mathbb{0} \\
\tilde{n} \\
\vec{n}\end{array}$ & 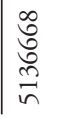 & $\begin{array}{l}\infty \\
\stackrel{0}{0} \\
\stackrel{\infty}{n}\end{array}$ & $\begin{array}{l}\stackrel{8}{\infty} \\
\stackrel{\infty}{ } \\
\stackrel{0}{n}\end{array}$ & 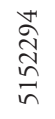 & $\begin{array}{l}\hat{\hat{\sigma}} \\
\stackrel{0}{n} \\
\stackrel{n}{n}\end{array}$ & 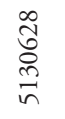 & $\begin{array}{l}\infty \\
\infty \\
\hat{\sigma} \\
\stackrel{n}{n}\end{array}$ & 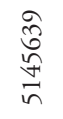 & 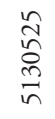 & $\begin{array}{l}\infty \\
\infty \\
\infty \\
\infty \\
\infty \\
i n\end{array}$ & $\begin{array}{l}\stackrel{0}{\Omega} \\
\stackrel{n}{n} \\
\text { n }\end{array}$ & $\begin{array}{l}\stackrel{+}{ } \\
\infty \\
\infty \\
n \\
n\end{array}$ & $\begin{array}{l}\stackrel{*}{n} \\
\hat{\curvearrowright} \\
\stackrel{n}{n}\end{array}$ & \\
\hline & \multicolumn{18}{|c|}{ Character and differential species of the association (Značilnice in razlikovalnice asociacije) } & Pr. Fr. \\
\hline VP & Larix decidua & E3 & 4 & 4 & 4 & 4 & 4 & 4 & 4 & 4 & 4 & 4 & 3 & 3 & 4 & 4 & 4 & 4 & 16100 \\
\hline VP & Larix decidua & E2 & 1 & . & 1 & 1 & 1 & 1 & 1 & 1 & 1 & 1 & + & 1 & 2 & 1 & 1 & 1 & 1594 \\
\hline VP & Larix decidua & E1 & + & . & + & . & . & . & . & . & . & . & . & . & . & . & + & + & 425 \\
\hline $\mathrm{EP}$ & Rhododendron hirsutum & E2 & 3 & 3 & 3 & 4 & 4 & 3 & 3 & 2 & 3 & 3 & 2 & 3 & 3 & 3 & 4 & 4 & 16100 \\
\hline EP & Rhodothamnus chamaecistus & E1 & 2 & + & + & 2 & 1 & + & + & + & + & + & 1 & 1 & 2 & 1 & 3 & 3 & 16100 \\
\hline CA & Laserpitium peucedanoides & E1 & + & + & + & + & . & . & + & + & + & + & + & 1 & + & + & + & + & 1488 \\
\hline PS & Paederota lutea & E1 & 1 & + & + & + & + & . & . & + & + & . & + & 1 & 1 & + & + & + & 13 \\
\hline ES & Astrantia bavarica & E1 & + & . & + & + & + & . & . & + & + & + & + & + & 1 & 1 & 1 & 1 & 13 \\
\hline $\mathrm{AF}$ & Anemone trifolia & E1 & 1 & 1 & 1 & 1 & . & + & + & . & + & . & 1 & . & + & + & . & 1 & 11 \\
\hline TR & Festuca nitida & E1 & . & . & . & + & . & + &. & + & + & . & + & + & 1 & + & + & + & $10 \quad 63$ \\
\hline TR & Heliosperma alpestre & E1 & & . & + & + & & & $\mathrm{r}$ & . & . & . & + & . & . & $\mathrm{r}$ & + & + & 744 \\
\hline
\end{tabular}




\begin{tabular}{|c|c|c|c|c|c|c|c|c|c|c|c|c|c|c|c|c|c|c|c|c|}
\hline \multicolumn{3}{|c|}{ Number of relevé (Zaporedna številka popisa) } & \multirow{2}{*}{$\frac{1}{1}$} & \multirow{2}{*}{\multicolumn{2}{|c|}{23}} & \multirow{2}{*}{$\begin{array}{l}4 \\
+\end{array}$} & \multirow{2}{*}{$\frac{5}{.}$} & \multirow[t]{2}{*}{6} & \multirow{2}{*}{$\begin{array}{l}7 \\
.\end{array}$} & \multirow{2}{*}{$\begin{array}{l}8 \\
.\end{array}$} & \multirow{2}{*}{$\frac{9}{.}$} & \multirow{2}{*}{10} & \multirow{2}{*}{$\frac{11}{+}$} & \multirow{2}{*}{$\frac{12}{+}$} & \multirow{2}{*}{$\frac{13}{.}$} & 14 & 15 & 16 & Pr. & Fr. \\
\hline $\mathrm{PC}$ & Valeriana saxatilis & E1 & & + & & & & & & & & & & & & 1 & . &. & 6 & 38 \\
\hline VP & Homogyne sylvestris & E1 & + & . & + & + & . & . & 1 & . & . & . & . & . & . & . & + & . & 5 & 31 \\
\hline $\mathrm{CF}$ & Carex ferruginea & E1 & . & 1 & . & . & . & . & $\mathrm{r}$ & . & . & . & + & . & . & . & . & . & 3 & 19 \\
\hline TR & Astrantia carniolica & E1 & . & 1 & . & . & . & . & . & . & . & . & . & . & . & . & . & . & 1 & 6 \\
\hline & Differential species of the variant & ant $(1$ & Razli & rova & Ine $v$ & sta & rari & nte : & n su & vari & ante & & & & & & & & & \\
\hline VP & Saxifraga cuneifolia & E1 & 1 & + & + & + & 1 &. & + & + & + & + & . & + & . & + & . & 1 & 12 & 75 \\
\hline $\mathrm{MuA}$ & Athyrium filix-femina & E1 & . & . & . & . & . & + & + & 1 & 1 & + & 1 & 1 & 1 & + & + & + & 11 & 69 \\
\hline $\mathrm{AC}$ & Soldanella alpina & E1 & . & . & . & + & . & . & + & + & + & + & + & . & . & + & + & + & 9 & 56 \\
\hline VP & Vaccinio-Piceetea & & & & & & & & & & & & & & & & & & & \\
\hline & Vaccinium myrtillus & E1 & 3 & 3 & 3 & 2 & 3 & 2 & 3 & 3 & 3 & 3 & 4 & 3 & 3 & 2 & 2 & 3 & 16 & 100 \\
\hline & Vaccinium vitis-idaea & E1 & 1 & 1 & 1 & 2 & 3 & 1 & 2 & + & 1 & 1 & 2 & 1 & 3 & 1 & 1 & 2 & 16 & 100 \\
\hline & Polystichum lonchitis & E1 & 1 & 1 & 1 & + & 1 & 2 & + & + & + & 1 & 1 & 1 & 1 & 1 & 1 & 1 & 16 & 100 \\
\hline & Clematis alpina & E2 & 1 & 1 & 1 & 1 & + & 1 & + & + & + & 1 & + & + & 1 & + & + & + & 16 & 100 \\
\hline & Calamagrostis villosa & E1 & 2 & 2 & 3 & 3 & 4 & 4 & 3 & 3 & 3 & 3 & 3 & 3 & 4 & 2 & 3 & 3 & 16 & 100 \\
\hline & Hieracium murorum & E1 & + & + & + & 1 & + & + & + & 1 & + & 1 & . & 1 & 1 & + & + & + & 15 & 94 \\
\hline & Lycopodium annotinum & E1 & 1 & 1 & 1 & 2 & 2 & 2 & 2 & 1 & 1 & 1 & 1 & + & . & + & + & 1 & 15 & 94 \\
\hline & Luzula sylvatica & E1 & 1 & . & 1 & 1 & 1 & 2 & . & 1 & 2 & 1 & 1 & 1 & 2 & 1 & 1 & 1 & 14 & 88 \\
\hline & Valeriana tripteris & E1 & 1 & 1 & 1 & 1 & 1 & 2 & 1 & 1 & 1 & 1 & + & . & 1 & + & . & + & 14 & 88 \\
\hline & Homogyne alpina & E1 & + & . & 1 & + & + & + & 1 & 1 & 1 & 1 & 1 & 1 & + & 1 & . & 2 & 14 & 88 \\
\hline & Oxalis acetosella & E1 & 1 & + & 1 & + & 1 & 1 & 1 & 1 & 1 & 1 & + & 1 & . & . & 1 & 1 & 14 & 88 \\
\hline & Picea abies & E3 & . & + & $\mathrm{r}$ & . & + & . & . & + & + & + & 1 & 1 & + & . & $\mathrm{r}$ & . & 10 & 63 \\
\hline & Picea abies & E2 & + & + & . & + & + & + & $\mathrm{r}$ & + & . & + & 1 & 1 & . & + & $\mathrm{r}$ & + & 13 & 81 \\
\hline & Picea abies & E1 & . & . & . & . & . & . & . & + & . & . & . & + & . & . & . & . & 2 & 13 \\
\hline & Aposeris foetida & E1 & + & . & + & + & + & . & 1 & 1 & 1 & 1 & . & 1 & + & 1 & 1 & 1 & 13 & 81 \\
\hline & Dryopteris dilatata & E1 & . & + & + & + & 1 & 1 & + & 1 & 1 & 1 & 1 & 1 & 1 & . & . & + & 13 & 81 \\
\hline & Rosa pendulina & E2 & + & + & + & + & . & + & . & 1 & + & 1 & . & + & + & + & . & + & 12 & 75 \\
\hline & Solidago virgaurea subsp. virgaurea & E1 & 1 & 1 & + & . & . & . & . & + & + & + & + & 1 & 1 & + & + & + & 12 & 75 \\
\hline & Gymnocarpium dryopteris & E1 & + & 1 & 1 & + & + & + & + & + & + & + & . & . & 1 & + & . & . & 12 & 75 \\
\hline & Maianthemum bifolium & E1 & 1 & 1 & 1 & . & . & + & . & . & + & + & 1 & . & 1 & + & + & + & 11 & 69 \\
\hline & Phegopteris connectilis & E1 & 2 & 2 & 1 & + & . & . & . & + & + & . & . & . & 1 & + & + & 1 & 10 & 63 \\
\hline & Dryopteris expansa & E1 & . & . & . & . & + & . & . & + & + & + & + & + & + & + & . & . & 8 & 50 \\
\hline & Abies alba & E2 & + & 1 & . & $\mathrm{r}$ & . & . & . & + & + & + & . & + & . & $\mathrm{r}$ & . & . & 8 & 50 \\
\hline & Abies alba & E1 & . & . & . & . & . & . & . & . & . & . & + & . & . & . & . & . & 1 & 6 \\
\hline & Abies alba & E3 & . & + & . & . & . & . & . & + & . & . & + & . & . & . & . & . & 3 & 19 \\
\hline & Melampyrum sylvaticum & E1 & . & + & . & + & . & . & + & . & + & . & 1 & . & + & . & + & . & 7 & 44 \\
\hline & Huperzia selago & E1 & + & . & . & . & . & . & . & . & . & + & . & + & . & . & + & + & 5 & 31 \\
\hline & Gentiana asclepiadea & E1 & . & + & . & . & . & . & . & . & . & + & + & . & . & + & . & . & 4 & 25 \\
\hline & Veronica urticifolia & E1 & 1 & + & . & . & . & . & . & . & . & + & . & . & . & . & . & . & 3 & 19 \\
\hline & Lonicera caerulea & E2 & + & . & . & . & + & . & . & . & . & . & + & . & . & . & . & . & 3 & 19 \\
\hline & Lonicera nigra & E2 & . & + & + & . & . & . & . & . & . & . & . & . & . & . & . & . & 2 & 13 \\
\hline & Calamagrostis arundinacea & E1 & . & . & 1 & + & . & . & . & . & . & . & . & . & . & . & . & . & 2 & 13 \\
\hline & Luzula pilosa & E1 & . & . & . & . & . & + & + & . & . & . & . & . & . & . & . & . & 2 & 13 \\
\hline & Ajuga pyramidalis & E1 & . & . & . & . & . & . & . & + & . & . & . & + & . & . & . & . & 2 & 13 \\
\hline & Pyrola minor & E1 & . & . & . & . & + & . & . & . & . & . & . & . & . & . & . & . & 1 & 6 \\
\hline EP & Erico-Pinetea & & & & & & & & & & & & & & & & & & & \\
\hline & Pinus mugo & E2 & + & 1 & 3 & 1 & 2 & 1 & 1 & 1 & + & $\mathrm{r}$ & + & 1 & 2 & + & $\mathrm{r}$ & 1 & 16 & 100 \\
\hline & Rubus saxatilis & E1 & + & 1 & 1 & 1 & . & 1 & + & 1 & 1 & 1 & + & 1 & 1 & 1 & 1 & 1 & 15 & 94 \\
\hline & Calamagrostis varia & E1 & . & 1 & + & 1 & . & + & + & + & + & + & + & + & . & 1 & + & + & 13 & 81 \\
\hline & Erica carnea & E1 & . & 1 & + & . & . & 2 & . & + & + & + & 1 & 1 & . & . & . & . & 8 & 50 \\
\hline & Aquilegia nigricans & E1 & + & + & . & . & + & . & + & . & . & . & . & . & . & . & . & . & 4 & 25 \\
\hline & Carex ornithopoda & E1 & + & + & . & . & . & . & . & . & . & . & . & . & . & . & + & + & 4 & 25 \\
\hline
\end{tabular}


\begin{tabular}{|l|lll|llllllllllllll|ll} 
Number of relevé (Zaporedna številka popisa) & 1 & 2 & 3 & 4 & 5 & 6 & 7 & 8 & 9 & 10 & 11 & 12 & 13 & 14 & 15 & 16 & Pr. & Fr.
\end{tabular}

AF Aremonio-Fagion

Cardamine enneaphyllos

Knautia drymeia

Helleborus niger

TA Tilio-Acerion

Acer pseudoplatanus

Acer pseudoplatanus

FS Fagetalia sylvaticae

Melica nutans

Daphne mezereum

Dryopteris filix-mas

Galeobdolon flavidum

Fagus sylvatica

Fagus sylvatica

Fagus sylvatica

Luzula nivea

Prenanthes purpurea

Lonicera alpigena

Paris quadrifolia

Lilium martagon

Symphytum tuberosum

Euphorbia amygdaloides

Polystichum aculeatum

QP Quercetalia pubescenti-petraeae

Sorbus aria (Aria edulis)

QF Querco-Fagetea

Festuca heterophylla

Hepatica nobilis

Carex digitata

Anemone nemorosa

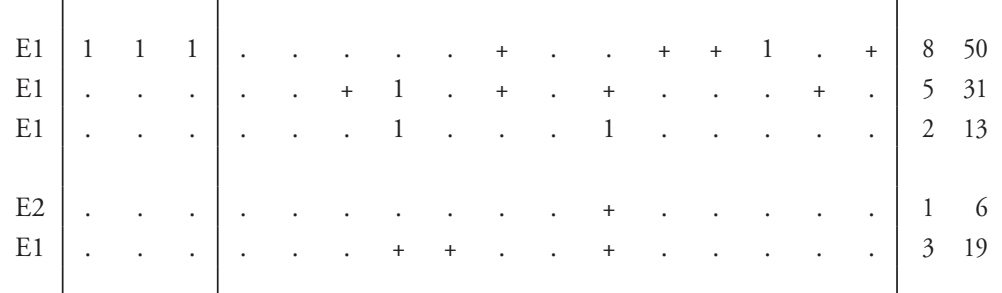

Sambuco-Salicion capreae, Rhamno-Prunetea

Sorbus aucuparia subsp. aucuparia

Sorbus aucuparia subsp. aucuparia

Sorbus aucuparia subsp. aucuparia

BA Betulo-Alnetea

Sorbus chamaemespilus

Salix appendiculata

Salix appendiculata

Salix glabra

Juniperus sibirica

Alnus viridis

Sorbus aucuparia subsp. glabrata

MuA Mulgedio-Aconitetea

Geranium sylvaticum

Viola biflora

Aconitum lycoctonum subsp. ranunculifolium

Veratrum album subsp. lobelianum

Polygonatum verticillatum

Saxifraga rotundifolia

Hypericum maculatum

Chaerophyllum villarsii

Adenostyles alliariae

Aconitum angustifolium

E1

E2

E1

E1

E3

E2

E1

E1

E1

E1

E1

E2

E1

E1

E1

E3

E2

E1

E2

E3

E2

E2

E2

E2

E2

E1

E1

E1

E1

E1

E1

E1

E1

E1

E1 
Number of relevé (Zaporedna številka popisa)

Chaerophyllum hirsutum

Peucedanum ostruthium

Poa hybrida

Pedicularis recutita

Primula elatior

Cicerbita alpina

Alchemilla xanthochlora

CA Caricion austroalpinae

Festuca calva

Arabis vochinensis

Pulsatilla alpina subsp. austroalpina

Koeleria eriostachya

Cfir Caricion firmae

Dryas octopetala

CF Caricion ferrugineae

Knautia longifolia

ES Elyno-Seslerietea

Sesleria caerulea

Aster bellidiastrum

Selaginella selaginoides

Campanula witasekiana

Betonica alopecuros

Thymus praecox subsp. polytrichus

Juncus monanthos

Senecio abrotanifolius

Alchemilla alpigena

Bartsia alpina

Carex sempervirens

Helianthemum nummularium subsp. grandiflorum E1

Galium anisophyllum

Polygonum viviparum

Ranunculus montanus

Ranunculus carinthiacus

Globularia nudicaulis

Gentianella anisodonta

Lotus alpinus

Helictotrichon parlatorei

Leucanthemum heterophyllum (L. maximum agg.) E1

Achillea clavenae

Potentilla crantzii

Globularia cordifolia

Scabiosa lucida subsp. lucida

Homogyne discolor

Hieracium villosum

JT Juncetea trifidi

Campanula scheuchzeri

LV Loiseleurio-Vaccinietea

Vaccinium gaultherioides

Arctostaphylos alpinus

CU Calluno-Ulicetea

Potentilla erecta

Festuca nigrescens

Gentiana pannonica

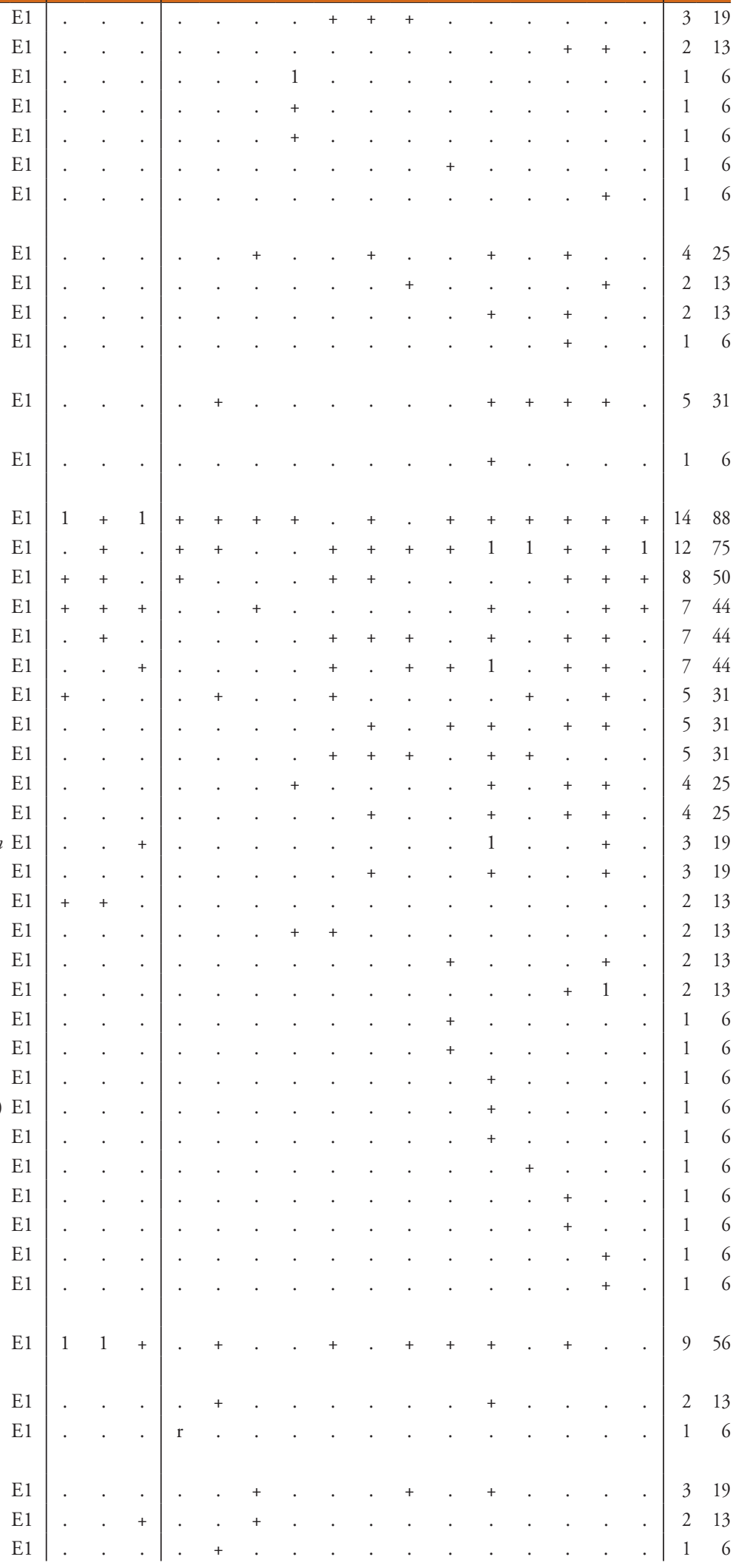


\begin{tabular}{l|lll|llllllllllllll|lll} 
Number of relevé (Zaporedna številka popisa) & 1 & 2 & 3 & 4 & 5 & 6 & 7 & 8 & 9 & 10 & 11 & 12 & 13 & 14 & 15 & 16 & Pr. & Fr
\end{tabular}

FB Festuco-Brometea

Cirsium erisithales

Carlina acaulis

PaT Poo alpinae-Trisetetalia

Trollius europaeus

Poa alpina

MA Molinio-Arrbenatheretea

Leontodon hispidus

Galium mollugo

Dactylis glomerata s. str.

Veronica chamaedrys

Deschampsia cespitosa

Anthoxanthum odoratum

MC Montio-Cradaminetea

Saxifraga aizoides

CD Caricetalia davallianae

Parnassia palustris

Pinguicula alpina

Carex capillaris

EA Epilobietea angustifolii

Fragaria vesca

Urtica dioica

Rubus idaeus

AC Arabidetalia caeruleae

Salix serpyllifolia

Ranunculus traunfellneri

TR Thlaspietea rotundifolii

Adenostyles glabra

Dryopteris villarii

Trisetum argenteum

Cystopteris montana

Gymnocarpium robertianum

Saxifraga sedoides

Valeriana montana

Cy Cystopteridion fragilis

Cystopteris fragilis

Carex brachystachys

PC Potentilletalia caulescentis

Campanula cochleariifolia

AT Asplenietea trichomanis

Asplenium viride

Asplenium ruta-muraria

Polypodium vulgare

$\mathrm{Fu} \quad$ Fungi (Glive)

Laetiporus sulphureus agg. (L. horoniensis)

ML Mosses and lichens (Mahovi in lišaji)

Rhytidiadelphus triquetrus

Dicranum scoparium

Tortella tortuosa

Polytrichum formosum

Hylocomium splendens

Fissidens dubius

Peltigera leucophlebia
E1

E

E1

E1

E1

E1

E1

E1

E1

E1

E1

E1

E1

E1

E1

E1

E2

E1

E1

E1

E1

E1

E1

E1

E1

E1

E1

E1

E1

E1

E1

E3c

E0

E0

E0

E0

E0

E0

E0
213

16

319

319

638

16

16

16

16

16

16

425

213

16

319

213

16

16

16

850

531

425

213

213

16

16

$8 \quad 50$

213

213

$16 \quad 100$

319

213

213

16100

1594

1488

169

956

9 56

956 
Number of relevé (Zaporedna številka popisa)

Ctenidium molluscum

Orthothecium rufescens

Rhytidiadelphus loreus

Letharia vulpina

Schistidium sp.

Plagiothecium sylvaticum

Cladonia pyxidata

Distichium capillaceum

Plagiothecium undulatum

Marchantia polymorpha

Sanionia uncinata

Sphagnum sp.

Vulpicida pinastri

Eurhynchium angustirete

Hypogymnia physodes

Cladonia furcata

Rhizomnium punctatum

\begin{tabular}{|c|c|c|c|c|c|c|c|c|c|c|c|c|c|c|c|c|c|}
\hline & 1 & 2 & 3 & 4 & 5 & 6 & 7 & 8 & 9 & 10 & 11 & 12 & 13 & 14 & 15 & 16 & Pr. \\
\hline E0 & . & . & + & 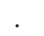 & . & 1 & & . & + & . & + & + & . & 1 & 1 & 1 & 8 \\
\hline E0 & . & + & . & + & . & . & . & + & . & . & . & . & . & + & + & . & 5 \\
\hline E0 & . & . & . & + & . & . & . & . & . & . & . & . & . & . & . & + & 2 \\
\hline $\mathrm{E} 3 \mathrm{c}$ & . & . & . & . & . & . & + & . & . & . & + & . & . & . & . & . & 2 \\
\hline E0 & . & . & . & . & . & . & . & . & + & . & + & . & . & . & . & . & 2 \\
\hline E0 & . & . & . & . & . & . & . & + & . & + & . & . & . & . & . & . & 2 \\
\hline E0 & . & . & . & . & . & . & . & . & . & . & + & + & . & . & . & . & 2 \\
\hline E0 & + & . & . & . & . & . & . & . & . & . & . & . & . & . & . & . & 1 \\
\hline E0 & . & + & . & . & . & . & . & . & . & . & . & . & . & . & . & . & 1 \\
\hline E0 & . & + & . & . & . & . & . & . & . & . & . & . & . & . & . & . & 1 \\
\hline E0 & . & . & + & . & . & . & . & . & . & . & . & . & . & . & . & . & 1 \\
\hline E0 & . & . & . & + & . & . & . & . & . & . & . & . & . & . & . & . & 1 \\
\hline E3c & . & . & . & . & . & . & . & . & + & . & . & . & . & . & . & . & 1 \\
\hline E0 & . & . & . & . & . & . & . & . & + & . & . & . & . & . & . & . & 1 \\
\hline E3c & $\cdot$ & . & . & . & $\cdot$ & . & . & . & . & . & + & . & . & . & . & . & 1 \\
\hline E0 & . & . & . & . & . & . & . & . & . & . & . & + & . & . & . & . & 1 \\
\hline E0 & . & . & . & . & . & . & . & . & . & . & . & . & . & . & . & + & 1 \\
\hline
\end{tabular}

\section{Legend - Legenda}

ID Igor Dakskobler

AS Andrej Seliškar

A Limestone - apnenec

D Dolomite-dolomit

Re Rendzina - rendzina

JA Julian Alps - Julijske Alpe

K Karavanke - Karavanke

Pr. Presence (number of relevés in which the species is presented) - število popisov, v katerih se pojavlja vrsta

Fr. Frequency in $\%$ - frekvenca v \% 
Table 7 (Tabela 7): Rhodothamno-Laricetum sorbetosum chamaemespili var. Anemone trifolia

Number of relevé (Zaporedna številka popisa)

Database number of relevé

(Delovna številka popisa)

Author of the relevé (Avtor popisa)

Elevation in $\mathrm{m}$ (Nadmorska višina $\mathrm{v} \mathrm{m}$ )

Aspect (Lega)

Slope in degrees (Nagib v stopinjah)

Parent material (Matična podlaga)

Soil (Tla)

Stoniness in \% (Kamnitost v \%)

Cover in \% (Zastiranje v \%):

Upper tree layer (Zgornja drevesna plast)

Lower tree layer (Spodnja drevesna plasti)

Shrub layer (Grmovna plast)

Herb layer (Zeliščna plast)

Moss layer (Mahovna plast)

Number of species (Število vrst)

Relevé area (Velikost popisne ploskve)

Date of taking relevé

(Datum popisa)

Locality (Nahajališče)

Mountain range (Pogorje)

Quadrant (Kvadrant)

Coordinate GK-Y (D-48)

Coordinate GK-X (D-48)

Character and differential species of the association

VP

$\mathrm{VP}$

$\mathrm{EP}$

$\mathrm{EP}$

$\mathrm{AF}$

TR Heliosperma alpestre

CA Laserpitium peucedanoides

PS Paederotalutea

CF Carex ferruginea

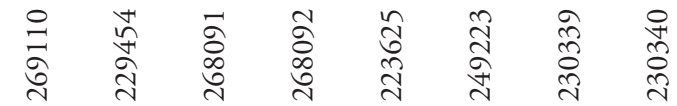

ID IDAR ID ID ID IDAP ID ID

$\begin{array}{llllllll}1590 & 1650 & 1754 & 1735 & 1650 & 1650 & 1650 & 1760\end{array}$

NE NE NW NW SW $\quad \mathrm{N}$ NE SEE

$\begin{array}{llllllll}40 & 40 & 25 & 30 & 40 & 15 & 5 & 15\end{array}$

A DA D D DA DA DA D

$\operatorname{Re} \operatorname{Re} \operatorname{Re} \operatorname{Re} \operatorname{Re} \operatorname{Re} \operatorname{Re} \operatorname{Re}$

$\begin{array}{llllllll}10 & 20 & 5 & 5 & 20 & 5 & 20 & 0\end{array}$

$\begin{array}{lcccccccc}\text { E3b } & 60 & 60 & 55 & 70 & 40 & 60 & 60 & 50 \\ \text { E3a } & 5 & 10 & 5 & 10 & 10 & 10 & 20 & 5 \\ \text { E2 } & 45 & 60 & 50 & 30 & 20 & 80 & 30 & 30 \\ \text { E1 } & 90 & 80 & 90 & 90 & 80 & 90 & 70 & 90 \\ \text { E0 } & 10 & 10 & 5 & 10 & 10 & 10 & 20 & 10 \\ & 82 & 72 & 59 & 63 & 63 & 47 & 58 & 49 \\ \mathrm{~m}^{2} & 400 & 400 & 400 & 400 & 200 & 400 & 400 & 400 \\ & 8 / 31 & 7 / 27 & 8 / 8 & 8 / 8 & 9 / 21 & 7 / 24 & 9 / 30 & 9 / 30 \\ & 2017 & 2009 & 2017 & 2017 & 2006 & 2013 & 2009 & 2009\end{array}$

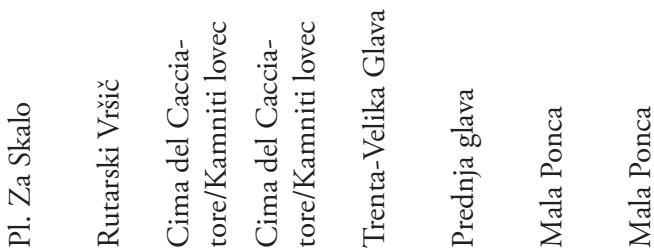

JA JA JA JA JA JA JA JA

$\begin{array}{llllllll}9648 & 9549 & 9547 & 9547 & 9648 & 9548 & 9548 & 9548\end{array}$

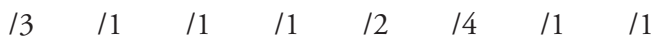

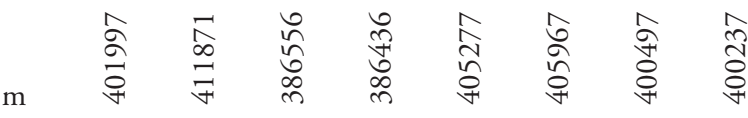

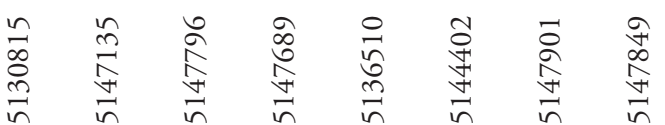

Pr. Fr.

\begin{tabular}{lllllllllll} 
E3 & 4 & 4 & 4 & 4 & 3 & 4 & 4 & 3 & 8 & 100 \\
E2 & 1 & + & 1 & 1 & 1 & 2 & 1 & 1 & 8 & 100 \\
E2 & 3 & 4 & 2 & 3 & 2 & 3 & 3 & 4 & 8 & 100 \\
E1 & 1 & 1 & + & 1 & 1 & + & 1 & + & 8 & 100 \\
\cline { 2 - 7 } & 1 & 1 & 1 & 1 & + & + & + & + & 8 & 100 \\
E1 & + & + &. & 1 & 1 & 1 & + &. & 6 & 75 \\
E1 & + & + &. &. & + & + & + &. & 5 & 63 \\
E1 & + & + &. & + & + &. &. & + & 5 & 63 \\
E1 & + &. & + & + &. & + & + &. & 5 & 63
\end{tabular}




\begin{tabular}{|c|c|c|c|c|c|c|c|c|c|c|c|c|}
\hline & Number of relevé (Zaporedna številka popisa) & & 1 & 2 & 3 & 4 & 5 & 6 & 7 & 8 & Pr. & Fr. \\
\hline TR & Festuca nitida & E1 & + & . & . & . & . & + & + & + & 4 & 50 \\
\hline $\mathrm{VP}$ & Homogyne sylvestris & E1 & 1 & + & + & . & . & . & . & . & 3 & 38 \\
\hline PC & Valeriana saxatilis & E1 & + & + & . & . & + & . & . & . & 3 & 38 \\
\hline ES & Astrantia bavarica & E1 & + & . & . & & + & . & . & . & 2 & 25 \\
\hline TR & Astrantia carniolica & E1 & + & . & . & . & . & . & . & . & 1 & 13 \\
\hline \multirow[t]{37}{*}{ VP } & Vaccinio-Piceetea & & & & & & & & & & & \\
\hline & Vaccinium myrtillus & E1 & 3 & 2 & 3 & 2 & 3 & 3 & 4 & 3 & 8 & 100 \\
\hline & Vaccinium vitis-idaea & E1 & 3 & + & 2 & 2 & 2 & 3 & 2 & 2 & 8 & 100 \\
\hline & Luzula sylvatica & E1 & 1 & 1 & 1 & 1 & 2 & 1 & 1 & + & 8 & 100 \\
\hline & Picea abies & E3 & . & . & + & $\mathrm{r}$ & + & + & . & . & 4 & 50 \\
\hline & Picea abies & $\mathrm{E} 2$ & + & + & 1 & + & 1 & + & + & + & 8 & 100 \\
\hline & Picea abies & E1 & . & . & . & + & + & . & . & . & 2 & 25 \\
\hline & Polystichum lonchitis & E1 & 1 & + & + & 1 & 1 & 1 & 1 & + & 8 & 100 \\
\hline & Calamagrostis villosa & E1 & 2 & 2 & 4 & 3 & 3 & 3 & 3 & 2 & 8 & 100 \\
\hline & Homogyne alpina & E1 & 1 & + & + & 1 & + & 1 & 1 & 1 & 8 & 100 \\
\hline & Lycopodium annotinum & E1 & 1 & 1 & 1 & 1 & 1 & 1 & 1 & 2 & 8 & 100 \\
\hline & Aposeris foetida & E1 & . & + & 1 & 1 & + & 1 & + & + & 7 & 88 \\
\hline & Hieracium murorum & E1 & 1 & + & + & + & + & . & + & + & 7 & 88 \\
\hline & Clematis alpina & $\mathrm{E} 2$ & 1 & 1 & . & . & 1 & $\mathrm{r}$ & + & + & 6 & 75 \\
\hline & Melampyrum sylvaticum & E1 & + & 1 & + & 1 & . & + & . & . & 5 & 63 \\
\hline & Huperzia selago & E1 & + & . & . & + & + & + & . & + & 5 & 63 \\
\hline & Rosa pendulina & $\mathrm{E} 2$ & 1 & + & + & . & 1 & . & . & . & 4 & 50 \\
\hline & Gymnocarpium dryopteris & E1 & . & 1 & + & . & . & + & + & . & 4 & 50 \\
\hline & Oxalis acetosella & E1 & . & + & . & . & . & + & + & + & 4 & 50 \\
\hline & Phegopteris connectilis & E1 & 2 & 1 & . & + & . & . & . & . & 3 & 38 \\
\hline & Valeriana tripteris & E1 & 2 & . & + & 1 & . & . & . & . & 3 & 38 \\
\hline & Solidago virgaurea subsp. virgaurea & E1 & 1 & + & + & . & . & . & . & . & 3 & 38 \\
\hline & Lonicera caerulea & $\mathrm{E} 2$ & + & . & . & . & . & . & + & + & 3 & 38 \\
\hline & Abies alba & E3 & + & . & . & . & . & . & + & . & 2 & 25 \\
\hline & Abies alba & $\mathrm{E} 2$ & + & . & . & . & . & + & + & . & 3 & 38 \\
\hline & Abies alba & E1 & . & . & . & + & . & & . & . & 1 & 13 \\
\hline & Dryopteris dilatata & E1 & 1 & . & . & + & . & . & . & . & 2 & 25 \\
\hline & Maianthemum bifolium & E1 & + & 1 & . & . & . & . & . & . & 2 & 25 \\
\hline & Dryopteris expansa & E1 & + & . & + & . & . & . & . & . & 2 & 25 \\
\hline & Veronica urticifolia & E1 & . & + & . & . & + & . & . & . & 2 & 25 \\
\hline & Gentiana asclepiadea & E1 & . & . & 1 & + & . & . & . & . & 2 & 25 \\
\hline & Lonicera nigra & $\mathrm{E} 2$ & + & . & . & . & . & . & . & . & 1 & 13 \\
\hline & Luzula luzulina & E1 & + & . & . & . & . & . & . & . & 1 & 13 \\
\hline & Luzula pilosa & E1 & . & + & . & . & . & . & . & . & 1 & 13 \\
\hline & Thelypteris limbosperma & E1 & . & . & + & . & . & . & . & . & 1 & 13 \\
\hline & Saxifraga cuneifolia & E1 & . & . & . & . & + & . & . & . & 1 & 13 \\
\hline & Ajuga pyramidalis & E1 & . & . & . & . & . & . & + & . & 1 & 13 \\
\hline \multirow[t]{2}{*}{ EP } & Erico-Pinetea & & & & & & & & & & & \\
\hline & Erica carnea & E1 & 2 & 3 & 3 & 2 & 3 & 3 & 3 & 3 & 8 & 100 \\
\hline
\end{tabular}


Pinus mugo

Calamagrostis varia

Rubus saxatilis

Aquilegia nigricans

AF Aremonio-Fagion

Knautia drymeia

Cardamine enneaphyllos

Cyclamen purpurascens

Helleborus niger

TA Tilio-Acerion

Acer pseudoplatanus

E2

E1

E1

E1

E1

E1

E1

E1

E1

FS Fagetalia sylvaticae

Daphne mezereum

Melica nutans

Prenanthes purpurea

Fagus sylvatica

Fagus sylvatica

Lilium martagon

Paris quadrifolia

Mercurialis perennis

Galeobdolon flavidum

Dryopteris filix-mas

Luzula nivea

Lonicera alpigena

QR Quercetalia roboris

Melampyrum pratense subsp. vulgatum

QF Querco-Fagetea

Carex digitata

Anemone nemorosa

Hepatica nobilis

Viola riviniana

SSC Sambuco-Salicion capreae, Rhamno-Prunetea

Sorbus aucuparia subsp. aucuparia

Sorbus aucuparia subsp. aucuparia

Sorbus aucuparia subsp. aucuparia

BA Betulo-Alnetea

Sorbus chamaemespilus

Salix appendiculata

Alnus viridis

Juniperus sibirica

Salix glabra

Sorbus aucuparia subsp. glabrata

Salix waldsteiniana

MuA Mulgedio-Aconitetea

E2

E1

E3

E2

E1

E1

E1

E1

E1

E1

E2

E1

E1

E1

E1

E1

E3

E2

E1

2

E1

1

1

1

E1

$\begin{array}{llllllll}\text { E1 } & 2 & + & 1 & 6\end{array}$

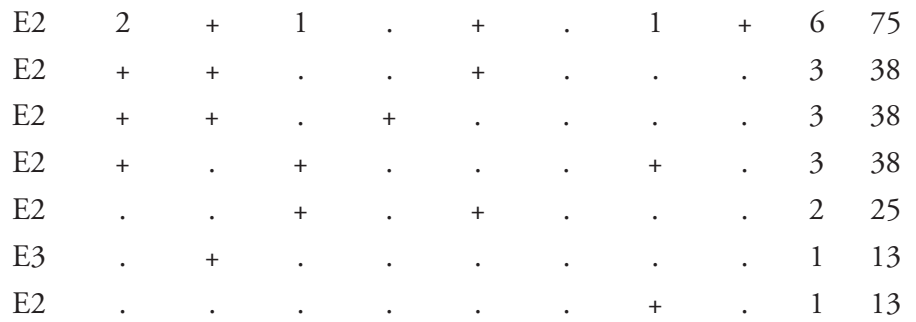

Viola biflora 
Number of relevé (Zaporedna številka popisa)

$\begin{array}{llllllllll}1 & 2 & 3 & 4 & 5 & 6 & 7 & 8 & \text { Pr. } & \text { Fr. }\end{array}$

Geranium sylvaticum

E1

Athyrium filix-femina

E1

Polygonatum verticillatum

Veratrum album subsp. lobelianum

Aconitum lycoctonum subsp. ranunculifolium

E1

Geum rivale

Chaerophyllum villarsii

E1

E1

E1

E1

E1

Chaerophyllum hirsutum

E1

Adenostyles alliariae

E1

E1

Festuca calva

Cfir Caricion firmae

Dryas octopetala

E1

Carex firma

Helianthemum alpestre

CF Caricion ferrugineae

Knautia longifolia

E1

ES Elyno-Seslerietea

Sesleria caerulea

Aster bellidiastrum

E1

Selaginella selaginoides

E1

Helianthemum nummularium subsp. grandiflorum

Carex sempervirens

Ranunculus montanus

E1

Betonica alopecuros

E1

Bartsia alpina

E1

Daphne striata

E1

Senecio abrotanifolius

E1

Alchemilla alpigena

Phyteuma orbiculare

Polygonum viviparum

Thymus praecox subsp. polytrichus

E1

Gentianella anisodonta

E1

Rhinanthus glacialis

Lotus alpinus

E1

Scabiosa lucida subsp. lucida

Gentiana clusii

Galium anisophyllum

Campanula scheuchzeri

Rhododendron ferrugineum

Coeloglossum viride

Veronica officinalis

Festuca nigrescens

\section{8}

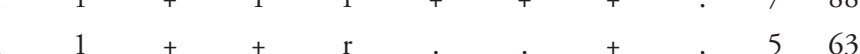

+ $1+$ + . . . 450

$+\quad+\quad 1 \quad . \quad$. $\quad . \quad 38$

+ . . . . $\quad+\quad 25$

. + . . . . . . . . 113

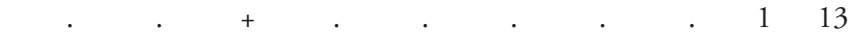

(20)

. + . . . . .25

$+\quad \cdot+2+25$

.+5.

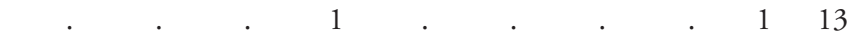

$1+$ + + + $1+788$

- . . . 1.4 .538

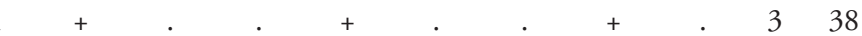

. $+\quad+\quad . \quad$. $\quad$. 38

..$+2 . \quad . \quad 25$

..$+. \quad . \quad 2525$

..$+. \quad . \quad 25$

..$+2 . \quad . \quad 25$

. . . . . . . $1 .+25$

. . . . . $1 .+25$

+ . . . . . . . . . . 1313

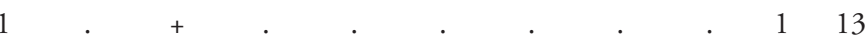

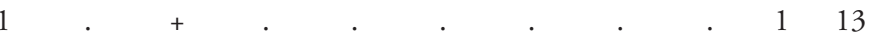

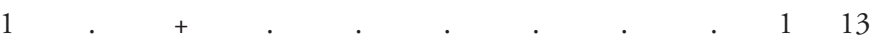

1..$\quad$. . . . . . . 1313

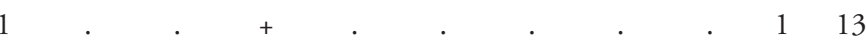

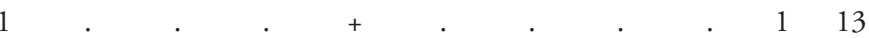

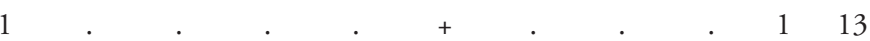

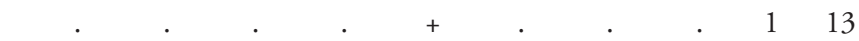

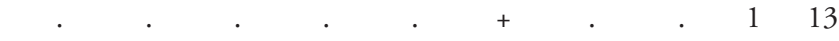

$2 . \quad 3+. \quad . \quad . \quad 25$

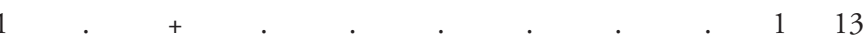


$\begin{array}{lllllllllllll}\text { Number of relevé (Zaporedna številka popisa) } & 1 & 2 & 3 & 4 & 5 & 6 & 7 & 8 & \text { Pr. } & \text { Fr. }\end{array}$

Gentiana pannonica

E1

Potentilla erecta

E1

Agrostis capillaris

E1

E1

E1

Galium lucidum

E1

Trollius europaeus

E1

Ranunculus nemorosus

E1

Deschampsia cespitosa

Leontodon hispidus

E1

CD Caricetalia davallianae

Parnassia palustris

Carex capillaris

E1

E1

EA Epilobietea angustifolii

Fragaria vesca

E1

AC Arabidetalia caeruleae

Soldanella alpina

TR Thlaspietea rotundifolii

Gymnocarpium robertianum

Trisetum argenteum

Biscutella laevigata

Adenostyles glabra

Festuca laxa

Saxifraga caesia

Cy Cystopteridion fragilis

Cystopteris fragilis

E1

E1

E1

E1

E1

E1

E1

E1

PS Physoplexido comosae-Saxifragion petraeae

Potentilla nitida

E1

PC Potentilletalia caulescentis

Campanula cochleariifolia

E1

Primula auricula

AT Asplenietea trichomanis

Asplenium viride

Moehringia muscosa

E1

E1

ML Mosses and lichens (Mahovi in lišaji)

Tortella tortuosa

Rhytidiadelphus triquetrus

Ctenidium molluscum

Polytrichum formosum

Hylocomium splendens

Schistidium sp.

Dicranum scoparium

Orthothecium rufescens

$\begin{array}{lllllllllll}\text { E0 } & + & 1 & . & + & + & + & 1 & + & 7 & 88 \\ \text { E0 } & 1 & 1 & 1 & + & + & 1 & \cdot & . & 6 & 75 \\ \text { E0 } & + & . & 1 & . & . & + & 1 & + & 5 & 63 \\ \text { E0 } & 1 & + & . & + & . & . & \cdot & + & 4 & 50 \\ \text { E0 } & 1 & 1 & . & . & + & . & . & . & 3 & 38 \\ \text { E0 } & . & . & . & . & + & . & + & + & 3 & 38 \\ \text { E0 } & + & . & . & . & + & . & . & . & 2 & 25 \\ \text { E0 } & + & . & . & . & . & . & . & + & 2 & 25\end{array}$




\begin{tabular}{|c|c|c|c|c|c|c|c|c|c|c|c|}
\hline Number of relevé (Zaporedna številka popisa) & & 1 & 2 & 3 & 4 & 5 & 6 & 7 & 8 & Pr. & Fr. \\
\hline Peltigera leucophlebia & E0 & . & + & . & . & + & . & . & . & 2 & 25 \\
\hline Fissidens dubius & E0 & . & + & . & . & . & + & . & . & 2 & 25 \\
\hline Sphagnum sp. & E0 & 1 & . & . & . & . & . & . & . & 1 & 13 \\
\hline Plagiothecium sylvaticum & E0 & 1 & . & . & . & . & . & . & . & 1 & 13 \\
\hline Rhizomnium punctatum & E0 & + & . & . & . & . & . & . & . & 1 & 13 \\
\hline Marchantia polymorpha & E0 & . & + & . & . & . & . & . & . & 1 & 13 \\
\hline Letharia vulpina & E3c & . & . & . & + & . & . & . & . & 1 & 13 \\
\hline Mnium thomsonii & E0 & . & . & . & . & + & . & . & . & 1 & 13 \\
\hline Cladonia sp. & E0 & . & . & . & . & . & . & + & . & 1 & 13 \\
\hline Cladonia pyxidata & E0 & . & . & . & . & . & . & . & + & 1 & 13 \\
\hline
\end{tabular}

\section{Legend - Legenda}

ID Igor Dakskobler

AP Aleš Poljanec

A Limestone - apnenec

D Dolomite - dolomit

Re Rendzina - rendzina

JA Julian Alps - Julijske Alpe

Pr. Presence (number of relevés in which the species is presented) - število popisov, v katerih se pojavlja vrsta

Fr. Frequency in $\%$ - frekvenca $\mathrm{\%} \%$ 
Table 8 (Tabela 8): Rhodothamno-Laricetum sorbetosum chamaemespili var. Homogyne alpina

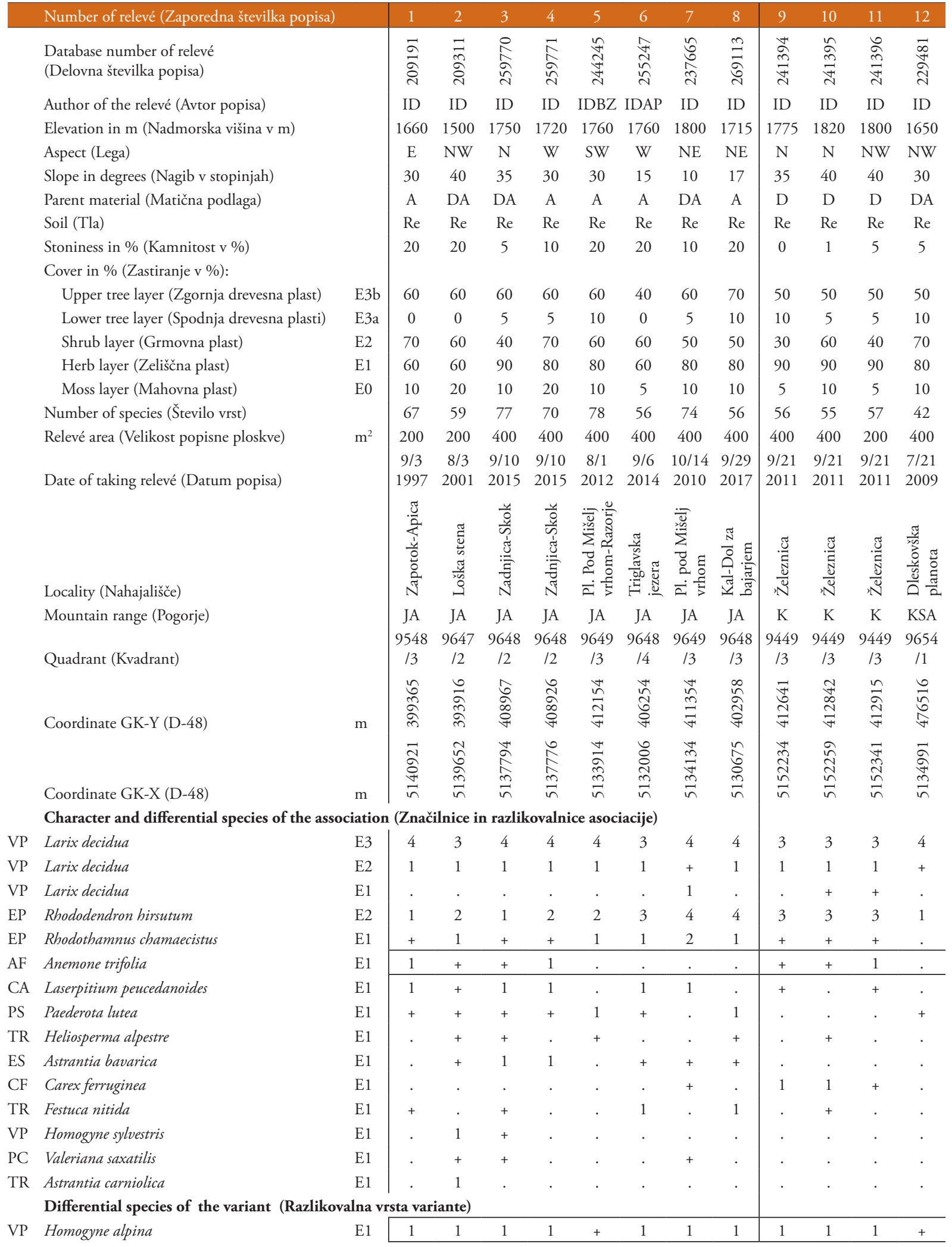


\begin{tabular}{|l|llllllll|llll} 
Number of relevé (Zaporedna številka popisa) & 1 & 2 & 3 & 4 & 5 & 6 & 7 & 8 & 9 & 10 & 11 & 12
\end{tabular}

VP Vaccinio-Piceetea

Luzula sylvatica

Vaccinium myrtillus

Vaccinium vitis-idaea

Valeriana tripteris

Calamagrostis villosa

Lycopodium annotinum

Polystichum lonchitis

Dryopteris dilatata

Aposeris foetida

Rosa pendulina

Oxalis acetosella

Clematis alpina

Lonicera caerulea

Gymnocarpium dryopteris

Hieracium murorum

Picea abies

Picea abies

Picea abies

Solidago virgaurea subsp. virgaurea

Huperzia selago

Saxifraga cuneifolia

Melampyrum sylvaticum

Dryopteris expansa

Lonicera nigra

Phegopteris connectilis

Maianthemum bifolium

Veronica urticifolia

Abies alba

Abies alba

Avenella flexuosa

Luzula pilosa

Pyrola rotundifolia

Luzula luzulina

Luzula luzuloides

Orthilia secunda

Ajuga pyramidalis

Listera cordata

Pyrola minor

Calamagrostis arundinacea

Gentiana asclepiadea

Pyrola chlorantha

EP

Erico-Pinetea

Pinus mugo

Erica carnea

Rubus saxatilis

Calamagrostis varia

Carex ornithopoda

Aquilegia nigricans

Polygala chamaebuxus

Chamaecytisus hirsutus

Arctostaphylos uva-ursi

\section{E1}

E1

E1

E1

E1

E1

E1

E1

E1

E2

E1

E2

E2

E1

E1

E3

E2

E1

E1

E1

E1

E1

E1

E2

E1

E1

E1

E3

E2

E1

E1

E1

E1

E1

E1

E1

E1

E1

E1

E1

E1

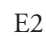

E1

E1

E1

E1

E1

E1

E1

E1

\begin{tabular}{|c|c|c|c|c|c|c|c|c|c|c|c|}
\hline 1 & 1 & 1 & 1 & 1 & + & 1 & 1 & 2 & 3 & 2 & + \\
\hline 2 & 2 & 2 & + & 3 & 3 & 3 & 3 & 1 & & 2 & 4 \\
\hline . & + & 1 & 1 & 1 & 2 & 2 & 2 & 2 & & 2 & 1 \\
\hline 1 & + & 1 & 1 & 1 & 1 & 1 & 1 & 1 & & 2 & + \\
\hline 1 & 2 & 2 & 2 & 1 & 1 & 2 & 3 & 4 & & 3 & 2 \\
\hline 1 & + & 1 & 1 & 1 & . & + & 1 & 2 & & 1 & 2 \\
\hline+ & + & + & + & 1 & 1 & 1 & 1 & + & & + & + \\
\hline+ & . & + & . & + & . & + & + & + & & + & + \\
\hline 1 & 1 & 1 & . & . & + & . & + & 1 & & 1 & + \\
\hline+ & + & 1 & 1 & 2 & 1 & 1 & + & . & & . & + \\
\hline 1 & . & 1 & . & . & + & . & . & + & & + & 1 \\
\hline+ & 1 & 1 & 1 & + & + & + & . & . & & . & . \\
\hline+ & . & 1 & 1 & 2 & 1 & + & . & + & & + & . \\
\hline . & + & + & + & + & . & . & . & + & & . & + \\
\hline+ & . & + & + & + & . & + & + & 1 & & . & + \\
\hline . & . & . & . & $\mathrm{r}$ & . & . & + & + & & $\mathrm{r}$ & . \\
\hline+ & . & + & . & . & . & . & . & + & & + & + \\
\hline . & . & . & . & . & . & . & . & . & & . & . \\
\hline+ & + & + & 1 & 1 & 1 & 1 & . & . & & . & . \\
\hline+ & + & + & . & + & + & + & . & + & & . & + \\
\hline+ & + & 1 & + & . & . & + & + & + & & . & . \\
\hline . & . & . & . & + & . & . & . & + & & + & . \\
\hline+ & . & + & + & . & . & . & + & . & & . & + \\
\hline+ & + & + & + & $\cdot$ & . & . & . & . & & . & . \\
\hline . & . & . & + & . & . & . & . & . & & . & . \\
\hline+ & . & . & + & 1 & . & . & . & . & & . & . \\
\hline+ & . & . & . & + & . & . & . & . & & . & . \\
\hline$\cdot$ & . & . & . & $\cdot$ & . & . & . & . & & $\mathrm{r}$ & . \\
\hline$\cdot$ & . & . & . & . & . & . & . & . & & . & . \\
\hline . & . & . & . & . & . & . & . & 1 & & 1 & . \\
\hline . & . & . & . & . & . & . & . & . & & . & . \\
\hline . & . & . & . & . & . & . & . & . & & . & . \\
\hline . & . & . & + & . & + & . & . & . & & . & . \\
\hline . & . & . & . & + & . & . & . & . & & . & + \\
\hline . & . & . & . & . & . & . & . & . & & . & . \\
\hline$\cdot$ & . & . & . & . & . & . & + & . & & . & . \\
\hline . & . & . & . & . & . & . & . & . & & . & + \\
\hline . & . & . & . & . & . & . & . & . & & . & . \\
\hline . & . & . & . & . & . & . & . & . & & . & . \\
\hline . & . & . & . & . & . & . & . & . & & . & . \\
\hline$\cdot$ & . & . & . & . & . & . & . & . & & . & . \\
\hline 3 & 3 & 1 & 4 & 3 & 5 & 3 & 4 & 2 & & 3 & 4 \\
\hline+ & 1 & . & 1 & 1 & 1 & . & . & 1 & & 2 & 2 \\
\hline 1 & 1 & + & 1 & 2 & . & 1 & 1 & . & & . & + \\
\hline . & + & + & 1 & + & . & 1 & . & + & & + & . \\
\hline . & . & . & . & + & . & + & . & . & & . & . \\
\hline . & . & $\cdot$ & + & . & . & . & . & . & & . & . \\
\hline . & . & . & . & . & . & . & . & . & & . & . \\
\hline . & . & . & . & $\cdot$ & . & . & . & • & & . & . \\
\hline . & . & . & . & . & . & . & . & & & . & \\
\hline
\end{tabular}


\begin{tabular}{|l|l|l|l|l|lllll|lll|l} 
Number of relevé (Zaporedna številka popisa) & 1 & 2 & 3 & 4 & 5 & 6 & 7 & 8 & 9 & 10 & 11 & 12
\end{tabular}

AF Aremonio-Fagion

Knautia drymeia

Cardamine enneaphyllos

Helleborus niger

Cyclamen purpurascens

Cardamine trifolia

TA Tilio-Acerion

Acer pseudoplatanus

Acer pseudoplatanus

Chrysosplenium alternifolium

FS Fagetalia sylvaticae

Daphne mezereum

Melica nutans

Prenanthes purpurea

Galeobdolon flavidum

Lilium martagon

Dryopteris filix-mas

Lonicera alpigena

Fagus sylvatica

Fagus sylvatica

E1

E1

E1

E1

Fagus sylvatica

Galium laevigatum

Paris quadrifolia

Polystichum aculeatum

Mercurialis perennis

Luzula nivea

Mycelis muralis

Symphytum tuberosum

QP Quercetalia pubescenti-petraeae

Sorbus aria (Aria edulis)

Convallaria majalis

QF Querco-Fagetea

Hepatica nobilis

Poa nemoralis

Hieracium lachenalii

Anemone nemorosa

Carex digitata

Platanthera bifolia

SSC Sambuco-Salicion capreae, Rhamno-Prunetea

Sorbus aucuparia subsp. aucuparia

Sorbus aucuparia subsp. aucuparia

Sorbus aucuparia subsp. aucuparia

BA Betulo-Alnetea

Sorbus chamaemespilus

Salix appendiculata

Alnus viridis

Salix glabra

Juniperus sibirica

Sorbus aucuparia subsp. glabrata

Sorbus aucuparia subsp. glabrata

Sorbus aucuparia subsp. glabrata

Salix waldsteiniana

\begin{tabular}{|c|c|c|c|c|c|c|c|c|c|c|c|}
\hline 1 & 1 & 1 & + & 2 & 1 & 1 & 1 & + & + & + & 3 \\
\hline+ & + & 1 & 1 & 1 & . & + & + & . & . & . & + \\
\hline . & . & + & . & 1 & . & + & . & . & . & . & . \\
\hline . & + & + & + & + & . & . & . & . & . & . & + \\
\hline+ & . & . & . & 1 & 2 & 1 & + & . & . & . & + \\
\hline . & . & . & . & . & . & . & . & . & . & . & + \\
\hline . & . & . & . & . & . & . & . & . & + & . & . \\
\hline . & . & . & . & . & . & . & . & . & . & . & 1 \\
\hline . & . & + & . & . & 1 & . & . & . & . & . & \\
\hline
\end{tabular}




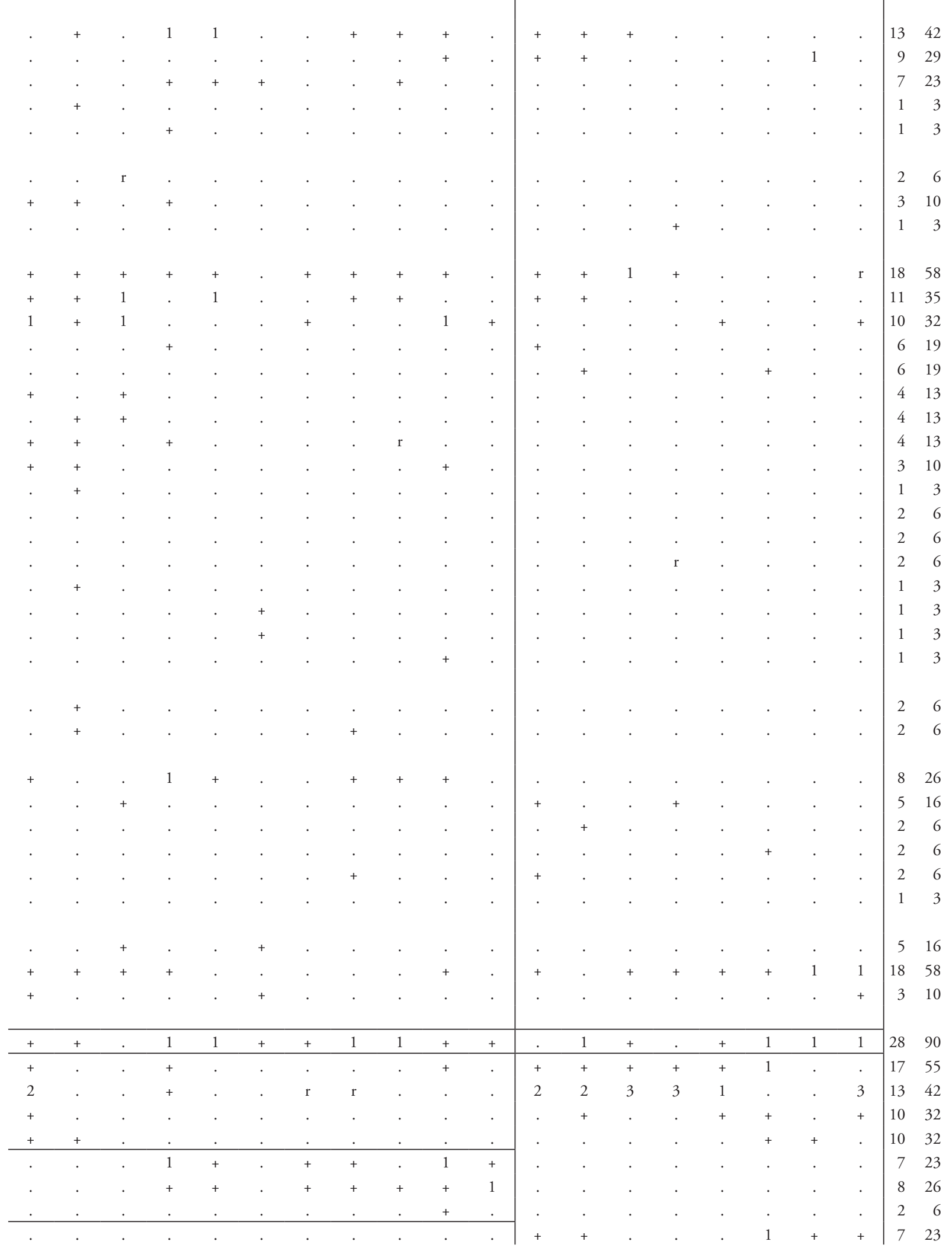


\begin{tabular}{|l|llllllll|llll} 
Number of relevé (Zaporedna številka popisa) & 1 & 2 & 3 & 4 & 5 & 6 & 7 & 8 & 9 & 10 & 11 & 12
\end{tabular} MuA Mulgedio-Aconitetea

Viola biflora

Geranium sylvaticum

Saxifraga rotundifolia

Veratrum album subsp. lobelianum

Athyrium filix-femina

Polygonatum verticillatum

Aconitum lycoctonum subsp. ranunculifolium

Phyteuma ovatum

Chaerophyllum villarsii

Adenostyles alliariae

Poa hybrida

Geum rivale

Chaerophyllum hirsutum

Peucedanum ostruthium

Primula elatior

Hypericum maculatum

Senecio cacaliaster

Ranunculus platanifolius

Alchemilla monticola

Veratrum album subsp. album

Rumex arifolius

Stellaria nemorum

Pedicularis recutita

Allium victorialis

TG Trifolio-Geranietea

Polygonatum odoratum

CA Caricion austroalpinae

Pulsatilla alpina subsp. austroalpina

Festuca calva

Heracleum austriacum subsp. siifolium

Koeleria eriostachya

Cfir Caricion firmae

Carex firma

Dryas octopetala

Helianthemum alpestre

Veronica aphylla

Pedicularis rostratocapitata

E1

E1

E1

E1

E1

E1

E1

E1

E1

E1

E1

E1

E1

E1

E1

E1

E1

E1

E1

E1

E1

E1

E1

E1

E1

E1

E1

E1

E1

E1

E1

E1

E1

E1

E1

Sesleria sphaerocephala

CF Caricion ferrugineae

Knautia longifolia

ES Elyno-Seslerietea

Sesleria caerulea

Aster bellidiastrum

Selaginella selaginoides

Phyteuma orbiculare

Senecio abrotanifolius

Helianthemum nummularium subsp. grandiflorum El Galium anisophyllum

Thymus praecox subsp. polytrichus

Juncus monanthos

Bartsia alpina

\begin{tabular}{cccccccc|cccc}
1 & 1 & 1 & 1 & 1 & 1 &. & 1 & 1 &. & 1 &. \\
+ & $\mathrm{r}$ & 1 & 1 & 1 & 1 &. & + & 1 & + & 1 &. \\
+ & $\cdot$ & + & + & + &. & + & + & 1 & + & + & + \\
$\cdot$ & + & 1 & + & 1 & 1 & + & $\cdot$ & $\cdot$ & + & $\cdot$ &. \\
$\cdot$ & + & + & + & + &. & $\cdot$ & + &. &. &. &.
\end{tabular}




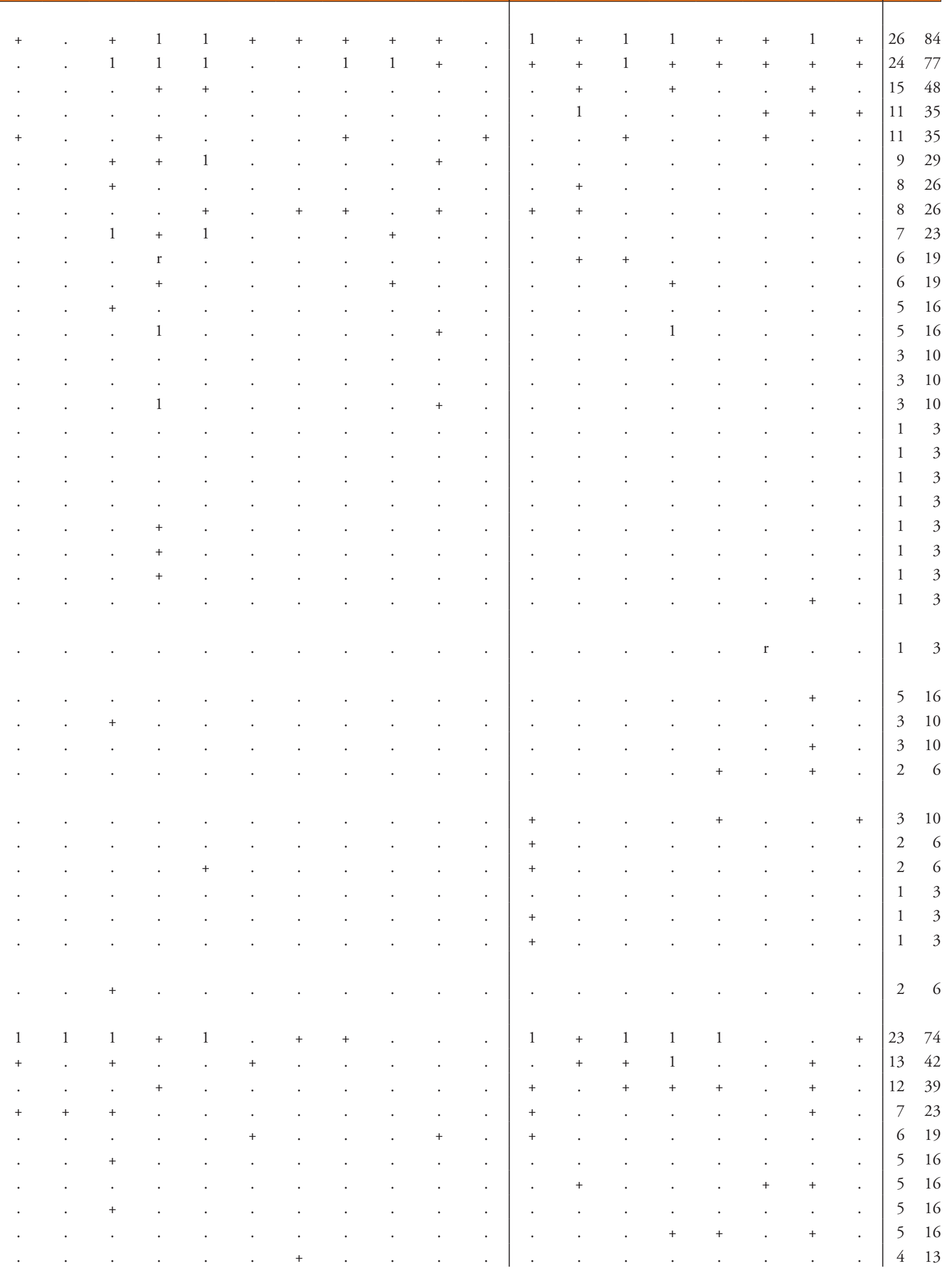


Polygonum viviparum

Betonica alopecuros

Carex sempervirens

Campanula witasekiana

Scabiosa lucida subsp. lucida

Ranunculus montanus

Saussurea discolor

Myosotis alpestris

Homogyne discolor

Potentilla crantzii

Gentianella anisodonta

Daphne striata

Carex mucronata

Cerastium strictum

Alchemilla sp.

Polygala alpestris

JT Juncetea trifidi

Campanula scheuchzeri

Anthoxanthum nipponicum

Solidago virgaurea subsp. minuta

LV Loiseleurio-Vaccinietea

Arctostaphylos alpinus

Vaccinium gaultherioides

Empetrum hermaphroditum

CU Calluno-Ulicetea

Gentiana pannonica

Festuca nigrescens

Coeloglossum viride

Meum athamanticum

Potentilla aurea

Veronica officinalis

FB Festuco-Brometea

Cirsium erisithales

Gymnadenia conopsea

Gentianella ciliata

Linum catharticum

Buphthalmum salicifolium

Carlina acaulis

Briza media

PaT Poo alpinae-Trisetetalia

Poa alpina

Trollius europaeus

MA Molinio-Arrhenetheretea

Leontodon hispidus

Anthoxanthum odoratum

Veronica chamaedrys

Cerastium fontanum

Galium album

MC Montio-Cardaminetea

Saxifraga aizoides

CD Caricetalia davallianae

Parnassia palustris

\section{E1}

E1

E1

E1

E1

E1

E1

E1

E1

E1

E1

E1

E1

E1

E1

E1

E1

E1

E1

E1

E1

E1

E1

E1

E1

E1

E1

E1

E1

E1

E1

E1

E1

E1

E1

E1

E1

E1

E1

E1

E1

E1

E1

E1

.

$\cdot$
+
$\cdot$




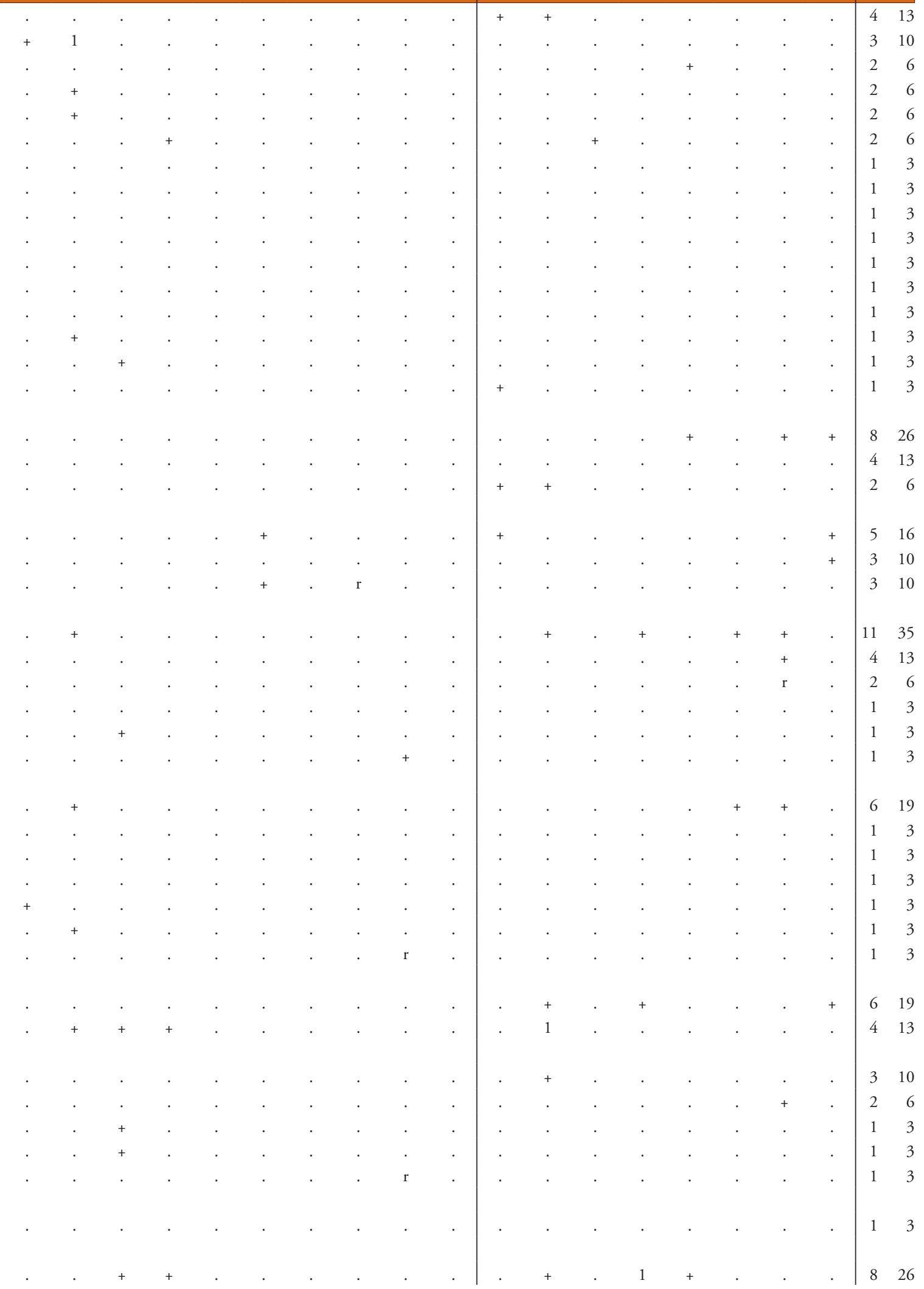


Number of relevé (Zaporedna števillka popisa)

Tofieldia calyculata

Pinguicula alpina

EA Epilobietea angustifolii

Rubus idaeus

Fragaria vesca

Chamaenerion angustifolium

AC Arabidetea caeruleae

Soldanella alpina

Ranunculus traunfellneri

Salix retusa

Alchemilla fissa

TR Thlaspietea rotundifolii

Gymnocarpium robertianum

Adenostyles glabra

Dryopteris villarii

Biscutella laevigata

Cystopteris montana

Valeriana montana

Rumex scutatus

Heracleum sphondxlium subsp. pollinianum

Rhodiola rosea

Arabis alpina

Festuca laxa

Minuartia austriaca

Saxifraga caesia

Cerastium carinthiacum

Poa minor

Cy Cystopteridion fragilis

Cystopteris fragilis

Heliosperma pusillum

Carex brachystachys

Cystopteris regia

PS Physoplexido comosae-Saxifragion petraeae

Saxifraga squarrosa

Campanula zoysii

Saxifraga crustata

PC Potentilletalia caulescentis

Campanula cochleariifolia

Primula auricula

Potentilla clusiana

AT Asplenietea trichomanis

Asplenium viride

Moehringia muscosa

$\mathrm{Fu}$ Fungi (Glive)

Laricifomes officinalis

Laetiporus sulphureus agg. (L. horoniensis)

ML Mosses and lichens (Mahovi in lišaji)

Rhytidiadelphus triquetrus

Hylocomium splendens

Tortella tortuosa

Dicranum scoparium

Ctenidium molluscum
E1

E2

E1

E1

E1

E1

E1

E1

E1

E1

E1

E1

E1

E1

E1

E1

E1

E1

E1

E1

E1

E1

E1

E1

E1

E1

E1

E1

E1

E1

E1

E1

E1

E1

E1

E3c

$\mathrm{E} 3 \mathrm{c}$

E0

E0

E0

E0

E0

1

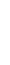

(1)

\section{$\begin{array}{lllll}1 & 2 & 3 & 4 & 5\end{array}$}

\begin{tabular}{lll|llll}
7 & 8 & 9 & 10 & 11 & 12
\end{tabular} 


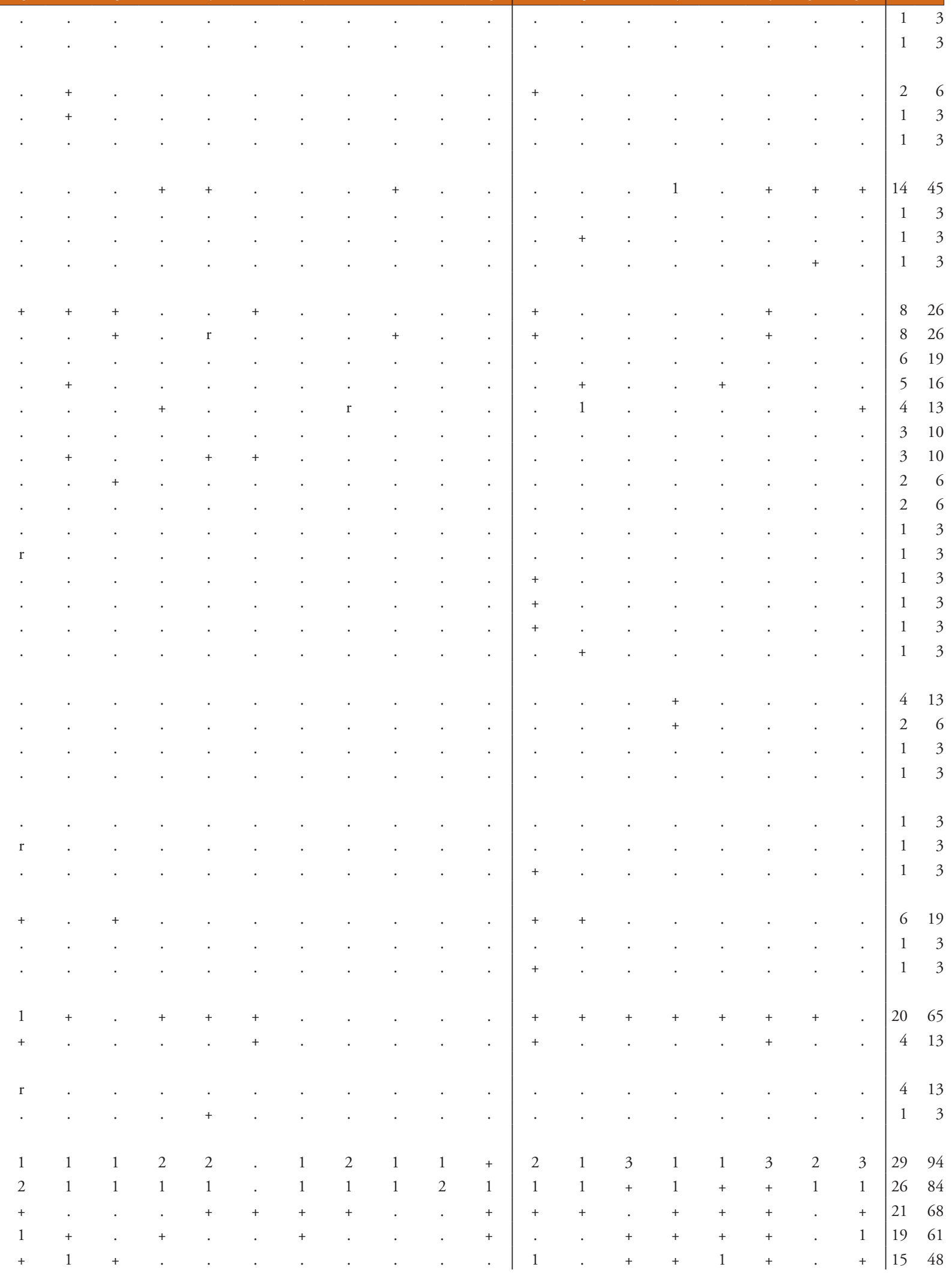


Number of relevé (Zaporedna števillka popisa)

Polytrichum formosum

Plagiothecium undulatum

Peltigera leucophlebia

Letharia vulpina

Sphagnum sp.

Fissidens dubius

Rhytidiadelphus loreus

Schistidium sp.

Orthothecium rufescens

Cetraria islandica

Eurhynchium angustirete

Cladonia sp.

Mnium thomsonii

Dicranum majus

Rhizomnium punctatum

Peltigera canina

Cladonia furcata

Hypogymnia physodes

Plagiothecium sylvaticum

Cladonia pyxidata

Plagiochila asplenioides

Usnea sp.

Plagiomnium undulatum

Polytrichum commune

Plagiochila porelloides

Mnium sp.

\begin{tabular}{|c|c|c|c|c|c|c|c|c|c|c|c|c|}
\hline & 1 & 2 & 3 & 4 & 5 & 6 & 7 & 8 & 9 & 10 & 11 & 12 \\
\hline E0 & 1 & . & + & . & . & . & . & + & . & + & . & . \\
\hline E0 & . & . & . & . & . & . & . & . & . & . & . & . \\
\hline E0 & + & + & . & + & . & . & + & . & . & + & . & . \\
\hline $\mathrm{E} 3 \mathrm{c}$ & . & . & . & . & + & . & + & . & + & + & 1 & . \\
\hline E0 & . & . & . & . & . & . & . & . & . & . & . & . \\
\hline E0 & . & + & . & . & . & . & . & + & . & + & . & . \\
\hline E0 & . & + & . & . & . & . & + & . & . & . & . & + \\
\hline E0 & . & . & . & . & + & . & . & . & . & . & . & . \\
\hline E0 & . & + & . & . & . & . & . & . & . & . & . & . \\
\hline E0 & . & . & . & . & . & . & + & . & . & . & . & . \\
\hline E0 & . & . & . & . & . & . & . & . & . & . & . & . \\
\hline E0 & . & . & . & . & . & . & . & . & . & . & . & . \\
\hline E0 & + & . & . & . & . & . & . & . & . & . & . & . \\
\hline E0 & + & . & . & . & . & . & . & . & . & . & . & . \\
\hline E0 & . & . & + & . & . & . & . & . & . & . & . & . \\
\hline E0 & . & . & . & . & + & . & . & . & . & . & . & . \\
\hline E0 & . & . & . & . & . & . & + & . & . & . & . & . \\
\hline E3c & . & . & . & . & . & . & $r$ & . & . & . & . & . \\
\hline E0 & . & . & . & . & . & . & . & . & . & . & . & + \\
\hline E0 & . & . & . & . & . & . & . & . & . & . & . & . \\
\hline E0 & . & . & . & . & . & . & . & . & . & . & . & . \\
\hline E3c & . & . & . & . & . & . & . & . & . & . & . & . \\
\hline E0 & . & . & . & . & . & . & . & . & . & . & . & $\cdot$ \\
\hline E0 & . & . & . & . & . & . & . & . & . & . & . & . \\
\hline E0 & . & . & . & . & . & . & . & . & . & . & . & . \\
\hline E0 & . & . & . & . & . & . & . & . & . & . & . & . \\
\hline
\end{tabular}

\section{Legend - Legenda}

ID Igor Dakskobler

BZ Branko Zupan

AP Aleš Poljanec

A Limestone - apnenec

D Dolomite - dolomit

Gr Gravel-grušč

Re Rendzina - rendzina

JA Julian Alps - Julijske Alpe

KSA Kamnik-Savinja Alps - Kamniško-Savinjske Alpe

K Karavanke - Karavanke

Pr. Presence (number of relevés in which the species is presented) - število popisov, v katerih se pojavlja vrsta

Fr. Frequency in $\%$ - frekvenca $v \%$ 


\begin{tabular}{|c|c|c|c|c|c|c|c|c|c|c|c|c|c|c|c|c|c|c|c|c|}
\hline 13 & 14 & 15 & 16 & 17 & 18 & 19 & 20 & 21 & 22 & 23 & 24 & 25 & 26 & 27 & 28 & 29 & 30 & 31 & Pr. & $\mathrm{Fr}$. \\
\hline+ & + & . & . & & + & + & + & . & . & . & . & . & . & + & + & . & 2 & 1 & 13 & 42 \\
\hline 1 & + & . & . & + & + & . & + & + & 1 & 1 & . & . & . & + & + & . & . & . & 10 & 32 \\
\hline 1 & + & . & . & . & . & + & . & . & . & . & . & . & . & . & . & . & . & . & 8 & 26 \\
\hline . & . & . & + & . & . & & . & . & & . & . & & . & . & . & . & + & . & 7 & 23 \\
\hline+ & + & . & . & + & . & + & & . & . & + & . & . & + & . & 1 & . & . & & 7 & 23 \\
\hline+ & . & . & . & . & . & . & . & . & . & . & . & . & . & . & + & + & & . & 6 & 19 \\
\hline+ & . & + & . & . & . & . & . & . & & . & . & . & . & . & . & . & . & . & 5 & 16 \\
\hline+ & . & . & . & . & . & . & + & . & & . & . & + & . & . & . & . & . & . & 4 & 13 \\
\hline+ & & . & . & & . & . & . & . & . & . & . & . & . & + & . & . & . & & 3 & 10 \\
\hline . & . & . & . & . & . & + & . & . & . & + & . & . & . & . & . & . & . & . & 3 & 10 \\
\hline+ & . & . & 1 & . & . & . & . & . & . & . & . & . & . & + & . & . & . & . & 3 & 10 \\
\hline . & . & . & . & . & . & . & . & . & & . & . & . & . & + & + & . & . & . & 2 & 6 \\
\hline . & . & . & . & . & . & . & . & . & . & . & . & . & . & . & . & . & . & . & 1 & 3 \\
\hline . & . & . & . & . & . &. & . & . & . & . & . & . & . & . & . & . & . & . & 1 & 3 \\
\hline . & & . & . & & . & . & & . & . & . & . & . & . & . & . & . & . & & 1 & 3 \\
\hline . & . & . & & . & . & & . & . & & . & . & & . & . & . & . & . & . & 1 & 3 \\
\hline . & . & . & . & . & . & . & . & . & . & . & . & . & . & . & . & . & & . & 1 & 3 \\
\hline . & . & . & . & . & . & . & . & . & . & . & . & . & . & . & . & . & & . & 1 & 3 \\
\hline . & & . & . & & . & . & . & . & . & . & . & . & . & . & . & . & . & & 1 & 3 \\
\hline+ & . & . & & . & . & & . & . & & & & & . & . & & & . & . & 1 & 3 \\
\hline . & . & . & + & . & . & . & . & . & . & . & . & . & . & . & . & . & & . & 1 & 3 \\
\hline . & . & . & + & . & . & . & . & . & . & . & . & . & . & . & . & . & & . & 1 & 3 \\
\hline . & . & . & . & . & . & + & . & . & . & . & . & . & . & . & . & . & & . & 1 & 3 \\
\hline & . & & & . & & & . & & & & & + & & & & & & . & 1 & 3 \\
\hline . & & . & . & . & . & . & . & . & . & . & . & . & . & . & . & + & & . & 1 & 3 \\
\hline & . & & & & & & & & & & & & & & & + & & & 1 & \\
\hline
\end{tabular}


Table 9 (Tabela 9): Rhodothamno-Laricetum sorbetosum chamaemespili var. Poa alpina

\begin{tabular}{|c|c|c|c|c|c|c|c|c|c|c|c|}
\hline Number of relevé (Zaporedna številka popisa) & & 1 & 2 & 3 & 4 & 5 & 6 & 7 & 8 & 9 & 10 \\
\hline $\begin{array}{l}\text { Database number of relevé } \\
\text { (Delovna številka popisa) }\end{array}$ & & 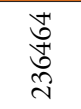 & $\begin{array}{l}\text { ज্ } \\
\stackrel{0}{0} \\
\text { त }\end{array}$ & $\begin{array}{l}\hat{\approx} \\
\stackrel{\text { ेे }}{2}\end{array}$ & 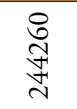 & 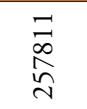 & $\begin{array}{l}\stackrel{n}{n} \\
\stackrel{n}{v}\end{array}$ & $\begin{array}{l}\vec{n} \\
\stackrel{n}{n} \\
\approx\end{array}$ & 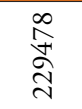 & 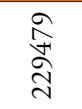 & 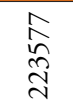 \\
\hline Author of the relevé (Avtor popisa) & & IDAS & ID & ID & ID & IDAR & ID & ID & ID & ID & ID \\
\hline Elevation in $\mathrm{m}$ (Nadmorska višina $\mathrm{v} \mathrm{m})$ & & 1570 & 1400 & 1510 & 1550 & 1610 & 1605 & 1580 & 1590 & 1600 & 1700 \\
\hline Aspect (Lega) & & $\mathrm{NE}$ & SE & $\mathrm{E}$ & $\mathrm{NE}$ & $\mathrm{NE}$ & $\mathrm{N}$ & $\mathrm{NE}$ & $\mathrm{E}$ & W & SEE \\
\hline Slope in degrees (Nagib v stopinjah) & & 30 & 25 & 45 & 45 & 45 & 25 & 10 & 30 & 25 & 25 \\
\hline Parent material (Matična podlaga) & & $\mathrm{D}$ & A & A & A & A & A & A & DA & DA & A \\
\hline Soil (Tla) & & $\operatorname{Re}$ & $\operatorname{Re}$ & $\operatorname{Re}$ & $\operatorname{Re}$ & $\operatorname{Re}$ & $\operatorname{Re}$ & $\operatorname{Re}$ & $\operatorname{Re}$ & $\operatorname{Re}$ & $\operatorname{Re}$ \\
\hline Stoniness in \% (Kamnitost v \%) & & 20 & 30 & 20 & 5 & 20 & 60 & 40 & 10 & 10 & 30 \\
\hline \multicolumn{12}{|l|}{ Cover in \% (Zastiranje v \%): } \\
\hline Upper tree layer (Zgornja drevesna plast) & $\mathrm{E} 3 \mathrm{~b}$ & 60 & 70 & 60 & 60 & 40 & 50 & 60 & 50 & 70 & 60 \\
\hline Lower tree layer (Spodnja drevesna plasti) & E3a & 10 & 10 & 10 & 10 & 30 & 10 & 5 & 10 & 10 & 10 \\
\hline Shrub layer (Grmovna plast) & E2 & 15 & 20 & 20 & 20 & 35 & 40 & 40 & 30 & 20 & 30 \\
\hline Herb layer (Zeliščna plast) & E1 & 80 & 70 & 80 & 90 & 90 & 70 & 70 & 90 & 90 & 70 \\
\hline Moss layer (Mahovna plast) & E0 & 5 & 10 & 10 & 20 & 10 & 30 & 10 & 10 & 5 & 10 \\
\hline Number of species (Število vrst) & & 77 & 57 & 62 & 75 & 93 & 79 & 84 & 59 & 59 & 72 \\
\hline Relevé area (Velikost popisne ploskve) & $\mathrm{m}^{2}$ & 400 & 400 & 200 & 400 & 400 & 400 & 400 & 200 & 400 & 400 \\
\hline Date of taking relevé (Datum popisa) & & $\begin{array}{l}8 / 18 \\
2010\end{array}$ & $\begin{array}{c}7 / 6 \\
2010\end{array}$ & $\begin{array}{l}9 / 17 \\
2002\end{array}$ & $\begin{array}{c}8 / 8 \\
2012\end{array}$ & $\begin{array}{l}8 / 27 \\
2015\end{array}$ & $\begin{array}{c}8 / 6 \\
2015\end{array}$ & $\begin{array}{c}8 / 6 \\
2015\end{array}$ & $\begin{array}{l}7 / 21 \\
2009\end{array}$ & $\begin{array}{l}7 / 21 \\
2009\end{array}$ & $\begin{array}{l}9 / 14 \\
2006\end{array}$ \\
\hline Locality (Nahajališče) & & 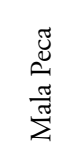 & 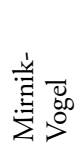 & 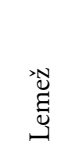 & 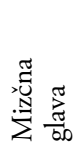 & 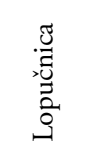 & 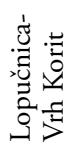 & 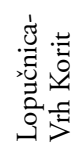 & 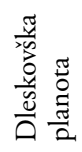 & 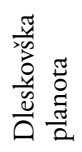 & $\begin{array}{l}\frac{y}{a} \\
\vec{a} \\
\dot{a}\end{array}$ \\
\hline Mountain range (Pogorje) & & K & JA & JA & JA & JA & JA & JA & KSA & KSA & JA \\
\hline & & 9554 & 9749 & 9748 & 9649 & 9648 & 9748 & 9648 & 9654 & 9654 & 9649 \\
\hline Quadrant (Kvadrant) & & 12 & $/ 1$ & $/ 1$ & 13 & 14 & 12 & 14 & $/ 1$ & $/ 1$ & 12 \\
\hline Coordinate GK-Y (D-48) & $\mathrm{m}$ & 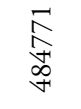 & 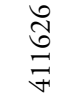 & $\begin{array}{l}\infty \\
\infty \\
\infty \\
\check{m}\end{array}$ & 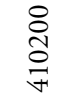 & \begin{tabular}{l}
$\vec{n}$ \\
\multirow{2}{\hat{q}}{} \\
+⿱
\end{tabular} & 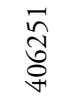 & 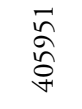 & $\begin{array}{l}\infty \\
\stackrel{\infty}{\sigma} \\
\stackrel{+}{ }\end{array}$ & $\begin{array}{l}\stackrel{+}{\infty} \\
\hat{\sigma} \\
\stackrel{+}{+}\end{array}$ & $\begin{array}{l}\text { ڤે } \\
\stackrel{2}{F}\end{array}$ \\
\hline Coordinate GK-X (D-48) & $\mathrm{m}$ & 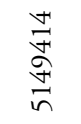 & 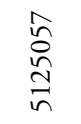 & 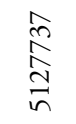 & $\begin{array}{l}\stackrel{0}{1} \\
\infty \\
0 \\
\infty \\
i n\end{array}$ & 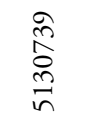 & \begin{tabular}{l}
$\infty$ \\
$\infty$ \\
$\infty$ \\
$\stackrel{\infty}{n}$ \\
\multirow{n}{n}{}
\end{tabular} & 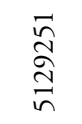 & 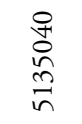 & 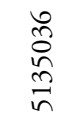 & $\begin{array}{l}\hat{\Xi} \\
\infty \\
\grave{n} \\
\vec{n}\end{array}$ \\
\hline
\end{tabular}

Character and differential species of the association (Značilnice in razlikovalnice asociacije)

VP Larix decidua

VP Larix decidua

VP Larix decidua

EP Rhododendron hirsutum

CA Laserpitium peucedanoides

PS Paederota lutea

EP Rhodothamnus chamaecistus

ES Astrantia bavarica

CF Carex ferruginea

TR Festuca nitida

TR Heliosperma alpestre

VP Homogyne sylvestris

AF Anemone trifolia

PC Valeriana saxatilis

PS Campanula carnica

Differential species of the variant (Razlikovalna vrsta variante)

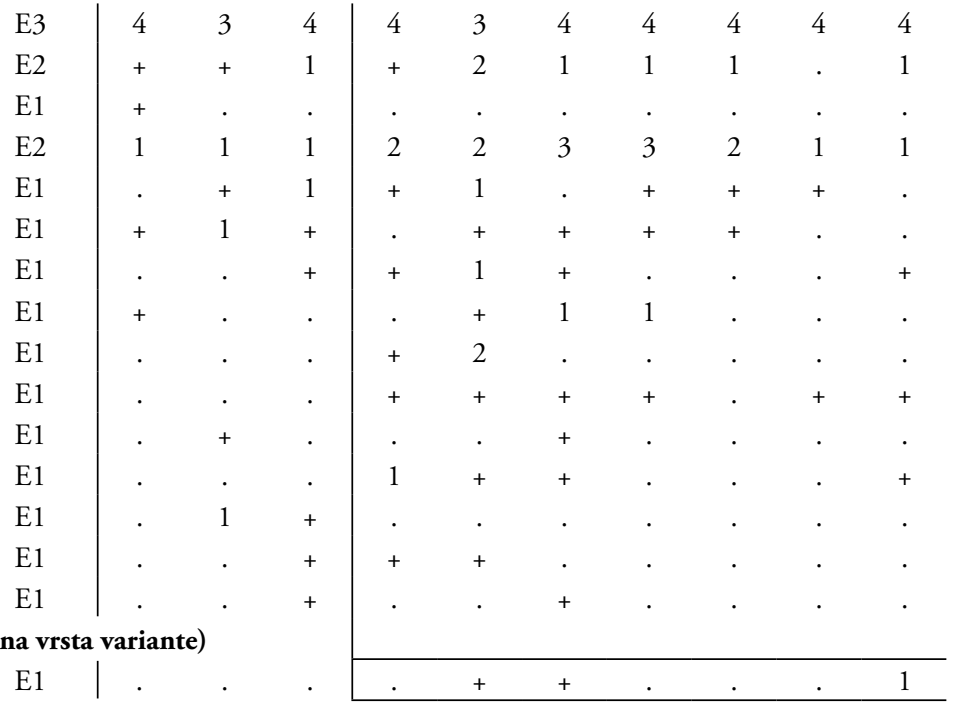




\begin{tabular}{|c|c|c|c|c|c|c|c|c|c|c|c|c|c|c|c|c|c|}
\hline 11 & 12 & 13 & 14 & 15 & 16 & 17 & 18 & 19 & 20 & 21 & 22 & 23 & 24 & 25 & 26 & Pr. & $F_{1}$ \\
\hline 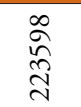 & 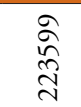 & 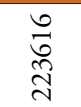 & $\begin{array}{l}\stackrel{2}{2} \\
\stackrel{\text { †े }}{\text { ते }}\end{array}$ & $\begin{array}{l}\text { त్రి } \\
\text { तె } \\
\text { ते }\end{array}$ & 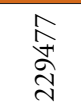 & $\begin{array}{l}\text { तె } \\
\text { तె } \\
\text { הి }\end{array}$ & $\begin{array}{l}\text { तె } \\
\text { तె } \\
\text { הి }\end{array}$ & 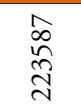 & $\begin{array}{l}\stackrel{+}{\infty} \\
\underset{\sim}{\sim}\end{array}$ & $\begin{array}{l}\hat{\Xi} \\
\stackrel{N}{N} \\
\text { Nan }\end{array}$ & 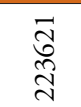 & $\begin{array}{c}\stackrel{8}{尺} \\
\stackrel{\sim}{\sim}\end{array}$ & $\begin{array}{l}\text { त్ర } \\
\text { तె } \\
\text { ה }\end{array}$ & $\begin{array}{l}\vec{\infty} \\
\hat{\tilde{N}} \\
\tilde{N}\end{array}$ & $\begin{array}{l}\stackrel{+}{0} \\
\stackrel{2}{\hat{~}}\end{array}$ & & \\
\hline ID & ID & ID & ID & ID & ID & ID & ID & ID & ID & ID & ID & ID & ID & ID & ID & & \\
\hline 1660 & 1590 & 1590 & 1610 & 1625 & 1600 & 1700 & 1750 & 1650 & 1652 & 1600 & 1620 & 1630 & 1620 & 1660 & 1600 & & \\
\hline SEE & NEE & E & NW & $\mathrm{N}$ & E & SE & SE & SW & SE & $\mathrm{N}$ & NW & NW & W & SW & $\mathrm{NE}$ & & \\
\hline 10 & 25 & 15 & 10 & 10 & 25 & 25 & 20 & 10 & 20 & 20 & 20 & 5 & 30 & 20 & 10 & & \\
\hline A & A & DA & DA & DA & DA & DA & A & A & A & DA & DA & A & DA & A & A & & \\
\hline $\operatorname{Re}$ & $\operatorname{Re}$ & $\operatorname{Re}$ & $\operatorname{Re}$ & $\operatorname{Re}$ & $\mathrm{Re}$ & $\operatorname{Re}$ & $\mathrm{Re}$ & $\operatorname{Re}$ & $\operatorname{Re}$ & $\operatorname{Re}$ & $\operatorname{Re}$ & $\operatorname{Re}$ & $\operatorname{Re}$ & $\operatorname{Re}$ & $\operatorname{Re}$ & & \\
\hline 20 & 0 & 0 & 0 & 10 & 10 & 20 & 30 & 10 & 30 & 10 & 20 & 20 & 10 & 30 & 30 & & \\
\hline 60 & 40 & 50 & 60 & 50 & 60 & 60 & 50 & 60 & 60 & 50 & 60 & 30 & 50 & 50 & 60 & & \\
\hline 10 & 5 & 5 & 0 & 0 & 0 & 0 & 0 & 0 & 10 & 5 & 0 & 5 & 10 & 0 & 10 & & \\
\hline 50 & 70 & 80 & 40 & 60 & 50 & 40 & 50 & 50 & 40 & 70 & 60 & 80 & 20 & 20 & 30 & & \\
\hline 70 & 60 & 60 & 90 & 60 & 90 & 60 & 60 & 70 & 70 & 50 & 60 & 10 & 80 & 70 & 80 & & \\
\hline 10 & 5 & 10 & 0 & 10 & 10 & 10 & 10 & 10 & 10 & 5 & 10 & 0 & 5 & 10 & 10 & & \\
\hline 55 & 70 & 47 & 55 & 56 & 78 & 64 & 71 & 57 & 88 & 58 & 58 & 62 & 80 & 65 & 78 & & \\
\hline 400 & 400 & 400 & 400 & 400 & 400 & 400 & 400 & 400 & 400 & 400 & 400 & 400 & 400 & 400 & 400 & & \\
\hline $9 / 14$ & $9 / 14$ & $9 / 12$ & $9 / 7$ & $9 / 12$ & $7 / 21$ & $9 / 12$ & $9 / 12$ & $9 / 14$ & $9 / 14$ & $9 / 12$ & $9 / 12$ & $8 / 7$ & $9 / 21$ & $9 / 14$ & $8 / 7$ & & \\
\hline 2006 & 2006 & 2006 & 2009 & 2006 & 2009 & 2006 & 2006 & 2006 & 2006 & 2006 & 2006 & 2015 & 2006 & 2006 & 2015 & & \\
\hline $\begin{array}{l}\frac{y}{a} \\
\vec{a} \\
\dot{a}\end{array}$ & $\begin{array}{l}\frac{y}{a} \\
\underline{a} \\
\dot{a}\end{array}$ & $\begin{array}{l}\frac{\ddot{v}}{\underline{\underline{u}}} \\
\overrightarrow{\underline{a}}\end{array}$ & $\begin{array}{l}\frac{y}{a} \\
\vec{a} \\
\dot{a}\end{array}$ & $\begin{array}{l}\frac{y}{a} \\
\vec{a} \\
\dot{a}\end{array}$ & 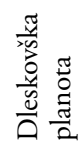 & $\begin{array}{l}\frac{y}{\Delta} \\
\vec{a} \\
\dot{a}\end{array}$ & $\begin{array}{l}\frac{y}{\Delta} \\
\vec{a} \\
\dot{a}\end{array}$ & $\begin{array}{l}\frac{u}{a} \\
\vec{a} \\
\dot{a}\end{array}$ & $\begin{array}{l}\frac{u}{\Delta} \\
\vec{a} \\
\dot{a}\end{array}$ & $\begin{array}{l}\frac{u}{\vec{a}} \\
\dot{\vec{a}}\end{array}$ & $\begin{array}{l}\frac{u}{\vec{a}} \\
\dot{a} \\
\dot{a}\end{array}$ & 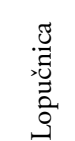 & 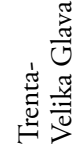 & $\begin{array}{l}\frac{y}{\Delta} \\
\vec{a} \\
\vec{a}\end{array}$ & 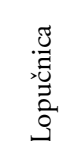 & & \\
\hline JA & JA & JA & JA & JA & KSA & JA & JA & JA & JA & JA & JA & JA & $\mathrm{JA}$ & JA & JA & & \\
\hline $\begin{array}{c}9549 \\
14\end{array}$ & $\begin{array}{c}9649 \\
12\end{array}$ & $\begin{array}{c}9649 \\
12\end{array}$ & $\begin{array}{c}9649 \\
12\end{array}$ & $\begin{array}{c}9649 \\
12\end{array}$ & $\begin{array}{c}9654 \\
/ 1\end{array}$ & $\begin{array}{c}9649 \\
12\end{array}$ & $\begin{array}{c}9649 \\
12\end{array}$ & $\begin{array}{c}9649 \\
12\end{array}$ & $\begin{array}{c}9649 \\
12\end{array}$ & $\begin{array}{c}9649 \\
12\end{array}$ & $\begin{array}{c}9649 \\
12\end{array}$ & $\begin{array}{c}9648 \\
14\end{array}$ & 9648 & 9649 & $\begin{array}{c}9648 \\
14\end{array}$ & & \\
\hline$\underset{\precsim}{\vec{\sigma}}$ & 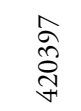 & 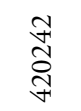 & 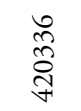 & $\begin{array}{l}\widetilde{\sigma} \\
\stackrel{\vec{\gamma}}{\sigma}\end{array}$ & 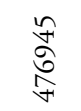 & \begin{tabular}{l} 
ثै \\
\multirow{\sigma}{*}{} \\
$\vec{\nabla}$
\end{tabular} & 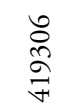 & 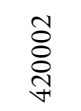 & 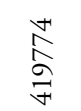 & 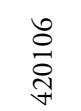 & $\begin{array}{l}\approx \\
\infty \\
\stackrel{\sigma}{F}\end{array}$ & 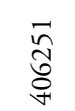 & $\begin{array}{l}\hat{n} \\
\hat{n} \\
\hat{\sigma}\end{array}$ & 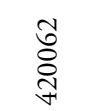 & $\begin{array}{l}\overrightarrow{5} \\
\hat{n} \\
\hat{a}\end{array}$ & & \\
\hline 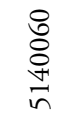 & 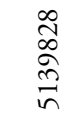 & $\begin{array}{l}\stackrel{+}{\infty} \\
\stackrel{\infty}{n} \\
\stackrel{n}{n}\end{array}$ & $\begin{array}{l}\stackrel{n}{\hat{a}} \\
\hat{\sigma} \\
\hat{n}\end{array}$ & $\begin{array}{l}\vec{a} \\
\vec{a} \\
\vec{n}\end{array}$ & $\begin{array}{l}\tilde{n} \\
\infty \\
\infty \\
\tilde{n} \\
\tilde{n}\end{array}$ & $\begin{array}{l}\infty \\
\stackrel{\infty}{n} \\
\stackrel{n}{n}\end{array}$ & \begin{tabular}{l}
$\stackrel{0}{n}$ \\
$\hat{n}$ \\
\multirow{n}{n}{}
\end{tabular} & 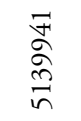 & $\begin{array}{l}\overline{\hat{\sigma}} \\
\hat{n} \\
\tilde{n}\end{array}$ & $\begin{array}{l}\tilde{N} \\
\underset{n}{n} \\
\tilde{n}\end{array}$ & $\begin{array}{l}\widetilde{\partial} \\
\stackrel{\vec{n}}{n}\end{array}$ & $\begin{array}{l}\text { के } \\
\stackrel{+}{0} \\
\infty \\
\stackrel{n}{n}\end{array}$ & 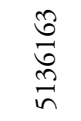 & $\begin{array}{l}\hat{\tilde{\alpha}} \\
\hat{\infty} \\
\hat{n} \\
\hat{n}\end{array}$ & \begin{tabular}{l}
$\infty$ \\
$\infty$ \\
$\infty$ \\
\multirow{0}{0}{} \\
$\stackrel{n}{n}$ \\
\multirow{n}{n}{}
\end{tabular} & & \\
\hline 4 & 3 & 4 & 4 & 4 & 4 & 4 & 4 & 4 & 4 & 4 & 4 & 2 & 4 & 3 & 4 & Pr. & 100 \\
\hline 1 & 1 & + & + & 1 & 1 & 1 & 1 & 1 & 1 & 1 & 1 & + & 1 & 1 & 1 & 25 & 96 \\
\hline . & & . & . & . & & . & + & + & 1 & . & . & . & & + & . & 5 & 19 \\
\hline 2 & 4 & 4 & 3 & 3 & 3 & 3 & 3 & 2 & 3 & 3 & 3 & + & 1 & + & + & 26 & 100 \\
\hline . & + & + & + & 1 & 1 & 1 & + & + & 1 & 1 & + & + & 1 & + & + & 22 & 85 \\
\hline+ & . & . & . & . & + & + & + & + & + & . & . & + & + & + & + & 17 & 65 \\
\hline . & & . & . & + & . & + & + & . & + & . & + & 1 & + & + & 1 & 14 & 54 \\
\hline+ & 1 & . & + & + & + & . & . & . & . & . & . & 1 & . & . & 1 & 11 & 42 \\
\hline . & 1 & + & + & + & + & . & . & . & + & + & + & . & & + & . & 11 & 42 \\
\hline . & . & . & . & . & + & . & + & . & + & . & . & + & . & . & + & 11 & 42 \\
\hline+ & + & . & . & . & + & . & + & . & + & + & + & . & . & + & . & 10 & 38 \\
\hline . & + & . & . & . & & + & . & . & + & . & . & . & . & . & + & 8 & 31 \\
\hline . & . & . & . & . & . & . & . & . & . & . & . & . & + & + & . & 4 & 15 \\
\hline . & 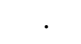 & . & . & . & . & . & . & . & . & . & . & . & . & . & . & 3 & 12 \\
\hline . & & . & . & . & . & . & . & . & . & . & . & . & . & . & . & 2 & 8 \\
\hline 1 & + & + & + & + & & . & 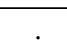 & + & + & + & + & . & & 1 & + & 14 & 54 \\
\hline
\end{tabular}


\begin{tabular}{l|l|ll|lllllll} 
Number of relevé (Zaporedna številka popisa) & 1 & 2 & 3 & 4 & 5 & 6 & 7 & 8 & 9 & 10
\end{tabular}

\section{VP Vaccinio-Piceetea}

Vaccinium myrtillus

Vaccinium vitis-idaea

Polystichum lonchitis

Luzula sylvatica

Valeriana tripteris

Picea abies

Picea abies

Picea abies

Calamagrostis villosa

Aposeris foetida

Clematis alpina

Homogyne alpina

Hieracium murorum

Dryopteris dilatata

Rosa pendulina

Melampyrum sylvaticum

Lycopodium annotinum

Oxalis acetosella

Solidago virgaurea subsp. virgaurea

Calamagrostis arundinacea

Maianthemum bifolium

Abies alba

Abies alba

Saxifraga cuneifolia

Gymnocarpium dryopteris

Lonicera caerulea

Gentiana asclepiadea

Veronica urticifolia

Huperzia selago

Dryopteris expansa

Luzula luzuloides

Lonicera nigra

Ajuga pyramidalis

Phegopteris connectilis

Luzula pilosa

Pyrola rotundifolia

Luzula luzulina

Pyrola minor

EP Erico-Pinetea

Erica carnea

Rubus saxatilis

Calamagrostis varia

Pinus mugo

Carex ornithopoda

Polygala chamaebuxus

Amelanchier ovalis

Epipactis atrorubens

Aquilegia nigricans

Galium austriacum

Gymnadenia odoratissima

Molinia arundinacea
E1

E1

E1

E1

E1

E3

E2

E1

E1

E1

E2

E1

E1

E1

E2

E1

E1

E1

E1

E1

E1

E3

E2

E1

E1

E2

E1

E1

E1

E1

E1

E2

E1

E1

E1

E1

E1

E1

E1

E1

E1

E2

E1

E1

E2

E1

E1

E1

E1

E1

\begin{tabular}{lll|lllllll}
2 & 2 & 2 & 2 & 2 & 2 & 3 & 3 & 3 & + \\
1 & 1 & 1 & 2 & 1 & 1 & 1 & 2 & 1 & 2
\end{tabular}

$\begin{array}{lllllllll}1 & + & + & 1 & 1 & 2 & 1 & 1 & +\end{array}$

\begin{tabular}{lll|lllll}
+ & 1 & 1 & $\mathrm{r}$ & + & + & +
\end{tabular}

(1)

.




\begin{tabular}{l|lll|lllllll} 
& 1 & 2 & 3 & 4 & 5 & 6 & 7 & 8 & 9 & 10
\end{tabular}

AF Aremonio-Fagion

Knautia drymeia

Helleborus niger

Cardamine enneaphyllos

Cyclamen purpurascens

Cardamine trifolia

Aremonia agrimonoides

TA Tilio-Acerion

Acer pseudoplatanus

Acer pseudoplatanus

Acer pseudoplatanus

Dryopteris affinis

FS Fagetalia sylvaticae

Melica nutans

Daphne mezereum

Euphorbia amygdaloides

E1

E1

E1

E1

E1

E1

Dryopteris flix-mas

Galium laevigatum

Galeobdolon flavidum

Paris quadrifolia

Mercurialis perennis

Fagus sylvatica

Fagus sylvatica

Fagus sylvatica

Lilium martagon

Lonicera alpigena

Polystichum aculeatum

Epilobium montanum

Symphytum tuberosum

Prenanthes purpurea

Luzula nivea

Myosotis sylvatica

Mycelis muralis

Ranunculus lanuginosus

QP Quercetalia pubescenti-petraeae

Convallaria majalis

Sorbus aria (Aria edulis)

Melampyrum pratense subsp. vulgatum

QF Querco-Fagetea

Hepatica nobilis

Anemone nemorosa

Poa nemoralis

Carex digitata

Festuca heterophylla

Hieracium lachenalii

E2

E1

E1

E1

E2

E1

E1

E1

E1

E1

E1

E3

E2

E1

E1

E2

E1

E1

E1

E1

E1

E1

E1

E1

E1

E2

E1

Galium sylvaticum

Listera ovata

Platanthera bifolia

SSC Sambuco-Salicion capreae, Rhamno-Prunetea

Sorbus aucuparia subsp. aucuparia

Sorbus aucuparia subsp. aucuparia

Populus tremula

E1

E1

E1

E1

E1

E1

E1

E1 
\begin{tabular}{l|lll|lllllll} 
Number of relevé (Zaporedna številka popisa) & 1 & 2 & 3 & 4 & 5 & 6 & 7 & 8 & 9 & 10
\end{tabular}

BA Betulo-Alnetea

Sorbus chamaemespilus

Salix appendiculata

Salix appendiculata

Alnus viridis

Salix glabra

Juniperus sibirica

Salix waldsteiniana

Ribes alpinum

MuA Mulgedio-Aconitetea

Geranium sylvaticum

Hypericum maculatum

Athyrium flix-femina

Saxifraga rotundifolia

Viola biflora

Polygonatum verticillatum

Veratrum album subsp. lobelianum

Geum rivale

Chaerophyllum villarsii

Primula elatior

Aconitum lycoctonum subsp. ranunculifolium

Senecio cacaliaster

Poa hybrida

Phyteuma ovatum

Adenostyles alliariae

Ranunculus platanifolius

Chaerophyllum hirsutum

Senecio ovatus

Rumex arifolius

Thalictrum aquilegiifolium

Tanacetum corymbosum subsp. clusii

Orobanche lycoctoni

Peucedanum ostruthium

Milium effusum

Scrophularia scopolii

Silene vulgaris subsp. antelopum

\section{CA Caricion austroalpinae}

Pulsatilla alpina subsp. austroalpina

Heracleum austriacum subsp. siifolium

Festuca calva

Koeleria eriostachya

Linum julicum

Gentiana lutea subsp. vardjanii

Cfir Caricion firmae

Dryas octopetala

Helianthemum alpestre

Carex firma

Pedicularis rostratocapitata

CF Caricion ferrugineae

Knautia longifolia
E2

E3

E2

E2

E2

E2

E2

E2

E1

E1

E1

E1

E1

E1

E1

E1

E1

E1

E1

E1

E1

E1

E1

E1

E1

E1

E1

E1

E1

E1

E1

E1

E1

E1

E1

E1

E1

E1

E1

E1

E1

E1

E1

E1

E1

\begin{tabular}{|ccc|ccccccc}
\hline+ & + & + & 1 & + & 2 & 1 & 1 & + & 2 \\
\hline. &. &. &. &. &. &. &. & &
\end{tabular}

.

1

1

1

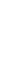

1

1 1 1 
Number of relevé (Zaporedna številka popisa)

\begin{tabular}{|lll|lllllll}
1 & 2 & 3 & 4 & 5 & 6 & 7 & 8 & 9 & 10
\end{tabular}

ES Elyno-Seslerietea

Aster bellidiastrum

Senecio abrotanifolius

Sesleria caerulea

Selaginella selaginoides

Thymus praecox subsp. polytrichus

Phyteuma orbiculare

Betonica alopecuros

Campanula witasekiana

Potentilla crantzii

Helianthemum nummularium subsp. grandiflorum E1

Lotus alpinus

Ranunculus montanus

Polygonum viviparum

Galium anisophyllum

Gentianella anisodonta

Scabiosa lucida subsp. lucida

Thesium alpinum

Euphrasia salisburgensis

Alchemilla vulgaris agg.

Carduus crassifolius

Leucanthemum heterophyllum (L.maximum agg.)

Alchemilla alpigena

Carex sempervirens

Hieracium villosum

Rhinanthus glacialis

Alchemilla venosula

Hieracium pilosum

Arabis ciliata

Juncus monanthos

Polygala alpestris

Ranunculus carinthiacus

JT Juncetea trifidi

Campanula scheuchzeri

Solidago virgaurea subsp. minuta

\section{CU Calluno-Ulicetea}

Festuca nigrescens

Gentiana pannonica

Potentilla erecta

Meum athamanticum

Veronica officinalis

Pseudorchis albida

Coeloglossum viride

Agrostis capillaris

Festuco-Brometea

Cirsium erisithales

Thlaspi praecox

Buphthalmum salicifolium

Carlina acaulis

Bromus erecta agg. (Bromopsis transsilvanica)

Hippocrepis comosa
E1

E1

E1

E1

E1

E1

E1

E1

E1

E1

E1

E1

E1

E1

E1

E1

E1

E1

E1

E1

E1

E1

E1

E1

E1

E1

E1

E1

E1

E1

E1

E1

E1

E1

E1

E1

E1

E1

E1

E1

E1

E1

E1

E1

\section{$\begin{array}{lllllll}4 & 5 & 6 & 7 & 8 & 9 & 10\end{array}$}

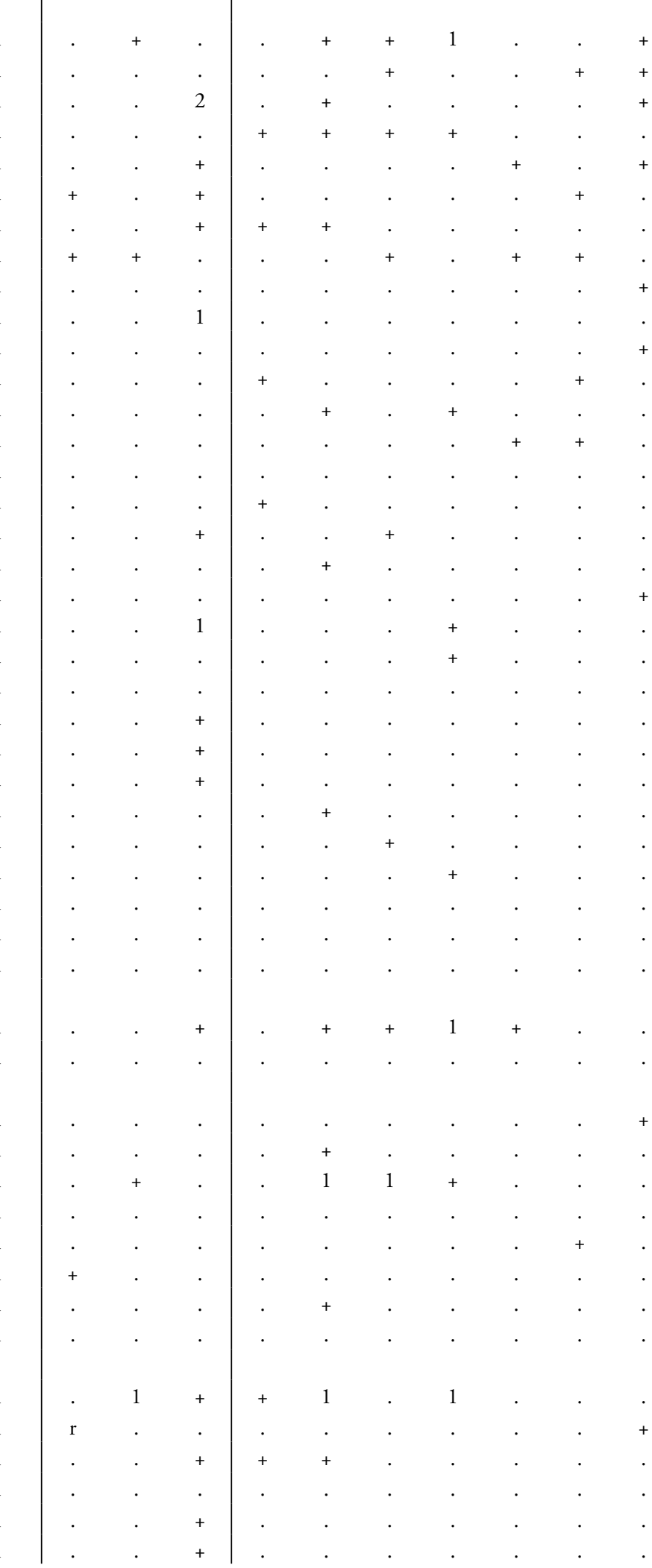


Number of relevé (Zaporedna številka popisa)

Stachys recta

Koeleria pyramidata

Prunella grandiflora

PaT Poo alpinae-Trisetetalia

Trollius europaeus

Ranunculus nemorosus

MA Molinio-Arrbenatheretea

Anthoxanthum odoratum

Leontodon hispidus

Achillea millefolium

Cerastium fontanum

Crepis paludosa

Veronica chamaedrys

Prunella vulgaris

Deschampsia cespitosa

Dactylis glomerata s. str.

MC Montio-Cardaminetea

Saxifraga aizoides

CD Caricetallia davallianae

Parnassia palustris

Pinguicula alpina

Carex capillaris

EA Epilobietea angustifolii

Fragaria vesca

Chamaenerion angustifolium

Myosotis arvensis

Rubus idaeus

AC Arabidetea caeruleae

Soldanella alpina

TR Thlaspietea rotundifolii

Gymnocarpium robertianum

Adenostyles glabra

Hieracium bifidum

Dryopteris villarii

Astrantia carniolica

Sedum atratum

Trisetum argenteum

Cystopteris montana

Arabis alpina

Rhodiola rosea

Cy Cystopteridion fragilis

Cystopteris fragilis

Cystopteris regia

PS Physoplexido comosae-Saxifragion petraeae

Saxifraga hostii

Saxifraga crustata

Athamanta turbith

E1

E1

E1

E1

E1

E1

E1

E1

E1

E1

E1

E1

E1

E1

E1

E1

E1

E1

E1

E1

E1

E2

E1

E1

E1

E1

E1

E1

E1

E1

E1

E1

E1

E1

E1

E1

E1

E1

E1

\section{\begin{tabular}{ll|lllllll}
2 & 3 & 4 & 5 & 6 & 7 & 8 & 9 & 10
\end{tabular} \\ 10}

\begin{tabular}{l|lll|l} 
& 1 & 2 & 3 & 4 \\
1 & $\cdot$ & $\cdot$ & + & $\cdot$ \\
1 & $\cdot$ & $\cdot$ & $\cdot$ & $\cdot$ \\
& $\cdot$ & $\cdot$ & $\cdot$ &.
\end{tabular}

(1)

(1)

(1)

1

1

1

1

1

1

2

E1

\section{Potentilletalia caulescentis}

Campanula cochleariifolia

Primula auricula

Festuca stenantha 
Number of relevé (Zaporedna številka popisa)

Campanula cespitosa

Arabis bellidiofolia subsp. stellulata

AT Asplenietea trichomanis

Asplenium viride

Moehringia muscosa

Asplenium trichomanes

Asplenium ruta-muraria

Polypodium vulgare

Gypsophila repens

Festuca sp.

$\mathrm{Fu} \quad$ Fungi (Glive)

Laricifomes officinalis

Laetiporus sulphureus agg. (L. horoniensis)

ML Mosses and lichens (Mahovi in lišaji)

Tortella tortuosa

Rhytidiadelphus triquetrus

Ctenidium molluscum

Dicranum scoparium

Schistidium sp.

Hylocomium splendens

Peltigera leucophlebia

Fissidens dubius

Polytrichum formosum

Cladonia pyxidata

Peltigera canina

Polytrichum sp.

Isothecium alopecuroides

Pseudoleskea incurvata

Plagiochila asplenioides

Cetraria islandica

\begin{tabular}{|c|c|c|c|c|c|c|c|c|c|c|}
\hline & 1 & 2 & 3 & 4 & 5 & 6 & 7 & 8 & 9 & 10 \\
\hline E1 & . & + & . & . & . & . & . & . & . & . \\
\hline E1 & . & . & . & . & . & . & . & . & . & . \\
\hline E1 & + & + & + & + & + & + & . & + & + & + \\
\hline E1 & . & + & . & . & + & + & . & . & . & . \\
\hline E1 & . & + & . & . & . & + & + & + & . & . \\
\hline E1 & . & + & . & . & . & + & . & . & . & . \\
\hline E1 & + & . & . & . & . & . & . & . & . & . \\
\hline E1 & . & . & + & . & . & . & . & . & . & . \\
\hline E1 & . & . & . & . & . & . & . & . & . & . \\
\hline E3c & . & . & . & . & r & . & . & . & . & . \\
\hline $\mathrm{E} 3 \mathrm{c}$ & . & . & . & . & . & . & . & . & . & . \\
\hline E0 & + & . & + & . & + & + & + & + & + & 1 \\
\hline E0 & + & . & + & 1 & + & 2 & + & . & . & + \\
\hline E0 & 1 & 1 & + & . & 1 & 1 & 1 & + & . & + \\
\hline E0 & . & . & . & + & + & 1 & + & . & . & . \\
\hline E0 & . & . & . & + & . & . & + & + & + & + \\
\hline E0 & + & . & + & 2 & + & . & . & . & . & . \\
\hline E0 & + & . & . & . & . & . & . & . & . & . \\
\hline E0 & . & . & . & . & . & + & + & . & . & . \\
\hline E0 & + & . & . & . & + & . & + & . & . & . \\
\hline E0 & + & . & . & . & . & . & . & . & . & . \\
\hline E0 & . & + & . & . & . & . & . & . & . & . \\
\hline E0 & + & . & . & . & . & . & . & . & . & . \\
\hline E0 & . & . & . & . & . & . & . & . & . & + \\
\hline E0 & . & . & . & . & . & . & . & . & . & . \\
\hline E0 & . & . & . & . & . & . & . & . & . & . \\
\hline E0 & . & . & . & . & . & . & . & . & . & . \\
\hline
\end{tabular}

\section{Legend - Legenda}

ID Igor Dakskobler

AS Andrej Seliškar

AR Andrej Rozman

A Limestone - apnenec

D Dolomite - dolomit

Gr Gravel - grušč

Re Rendzina - rendzina

JA Julian Alps - Julijske Alpe

KSA Kamnik-Savinja Alps - Kamniško-Savinjske Alpe

K Karavanke - Karavanke

Pr. Presence (number of relevés in which the species is presented) - število popisov, $\mathrm{v}$ katerih se pojavlja vrsta

Fr. Frequency in $\%$ - frekvenca $v \%$ 


\begin{tabular}{|c|c|c|c|c|c|c|c|c|c|c|c|c|c|c|c|c|c|}
\hline 11 & 12 & 13 & 14 & 15 & 16 & 17 & 18 & 19 & 20 & 21 & 22 & 23 & 24 & 25 & 26 & Pr. & Fr. \\
\hline . & . & . & . & . & . & . & $\cdot$ & . & $\cdot$ & . & . & . & . & . & . & 1 & 4 \\
\hline . & . & . & . & . & . & . & . & . & . & . & . & . & + & . & . & 1 & 4 \\
\hline+ & . & . & . & . & + & + & + & + & + & . & 1 & . & + & + & + & 19 & 73 \\
\hline . & . & . & . & . & + & + & + & . & + & . & . & . & . & . & . & 7 & 27 \\
\hline+ & . & . & . & . & . & . & + & . & . & . & . & . & + & . & . & 7 & 27 \\
\hline . & $\cdot$ & . & . & . & . & . & . & . & . & . & . & . & + & . & + & 4 & 15 \\
\hline . & . & . & . & . & . & . & . & . & . & . & . & . & . & . & . & 1 & 4 \\
\hline . & . & . & . & . & . & . & . & . & . & . & . & . & . & . & . & 1 & 4 \\
\hline . & . & . & . & . & . & . & + & . & . & . & . & . & . & . & . & 1 & 4 \\
\hline . & $\cdot$ & . & . & . & . & . & . & . & . & . & . & . & . & . & . & 1 & 4 \\
\hline . & . & . & . & . & . & . & . & . & . & . & . & + & . & . & . & 1 & 4 \\
\hline+ & + & . & . & + & + & 1 & 1 & . & 1 & 1 & 1 & + & 1 & 1 & 1 & 21 & 81 \\
\hline . & . & . & . & + & 1 & + & + & + & + & + & + & + & + & + & 1 & 19 & 73 \\
\hline 1 & . & . & . & . & + & + & + & + & 1 & 1 & . & + & + & + & . & 18 & 69 \\
\hline . & . & . & . & . & . & + & . & . & + & . & + & + & + & . & + & 10 & 38 \\
\hline+ & . & . & . & . & . & . & . & + & . & . & . & . & 1 & . & + & 9 & 35 \\
\hline . & . & + & . & + & . & . & . & . & . & . & + & . & + & . & . & 8 & 31 \\
\hline . & . & . & . & . & . & . & . & . & + & . & . & . & + & + & . & 4 & 15 \\
\hline . & . & . & . & + & . & . & . & . & . & . & . & . & + & . & . & 4 & 15 \\
\hline . & . & . & . & . & . & . & . & . & . & . & . & . & . & . & . & 3 & 12 \\
\hline+ & . & . & . & . & . & . & . & . & + & . & . & . & . & . & . & 3 & 12 \\
\hline . & . & . & . & . & . & . & + & . & . & . & . & . & . & + & . & 3 & 12 \\
\hline . & . & . & . & . & . & . & . & . & . & . & . & . & . & . & . & 1 & 4 \\
\hline . & . & . & . & . & . & . & . & . & . & . &. & . & . & r &. & 1 & 4 \\
\hline . & . & . & . & . & . & + & . & . & . & . & . & . & . & . & . & 1 & 4 \\
\hline . & . & . & . & . & . & . & . & . & . & . & . & . & . & + & . & 1 & 4 \\
\hline & . & . & & . & . & . & . & . & . & . & . & . & . & + & . & 1 & 4 \\
\hline
\end{tabular}


Table 10 (Tabela 10): Rhodothamno-Laricetum sorbetosum chamaemespili var. Pinus mugo

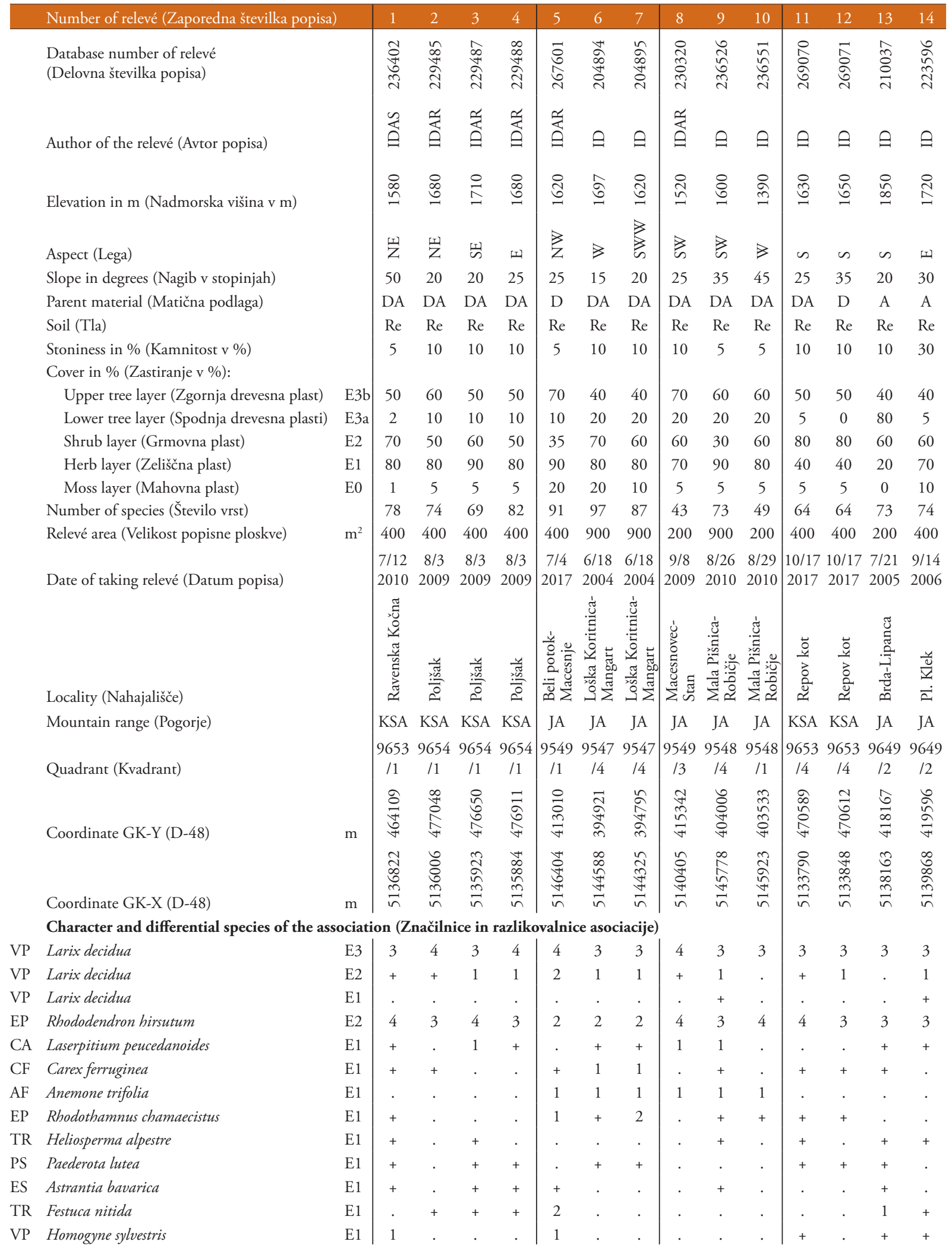


\begin{tabular}{|l|llll|lll|llll|llll} 
Number of relevé (Zaporedna številka popisa) & 1 & 2 & 3 & 4 & 5 & 6 & 7 & 8 & 9 & 10 & 11 & 12 & 13 & 14
\end{tabular}

PC Valeriana saxatilis

TR Astrantia carniolica

\begin{tabular}{l|l} 
E1 & E \\
E & +
\end{tabular}

Differential species of the variant (Razlikovalna vrsta variante)

EP Pinus mugo

VP Vaccinio-Piceetea

Vaccinium vitis-idaea

Luzula sylvatica

Polystichum lonchitis

Aposeris foetida

Vaccinium myrtillus

Rosa pendulina

Picea abies

Picea abies

Picea abies

Calamagrostis villosa

Valeriana tripteris

Homogyne alpina

Clematis alpina

Oxalis acetosella

Hieracium murorum

Lycopodium annotinum

Dryopteris dilatata

Gymnocarpium dryopteris

Melampyrum sylvaticum

Lonicera caerulea

Ajuga pyramidalis

Phegopteris connectilis

Dryopteris expansa

Huperzia selago

Abies alba

Abies alba

Solidago virgaurea subsp. virgaurea

Gentiana asclepiadea

Lonicera nigra

Veronica urticifolia

Luzula luzuloides

Luzula luzulina

Calamagrostis arundinacea

Luzula pilosa

Maianthemum bifolium

Saxifraga cuneifolia

Circaea alpina

Moneses uniflora

Blechnum spicant

Pyrola rotundifolia

Avenella flexuosa

Listera cordata

\section{Erico-Pinetea}

Erica carnea

Calamagrostis varia

Rubus saxatilis

Polygala chamaebuxus

Carex ornithopoda

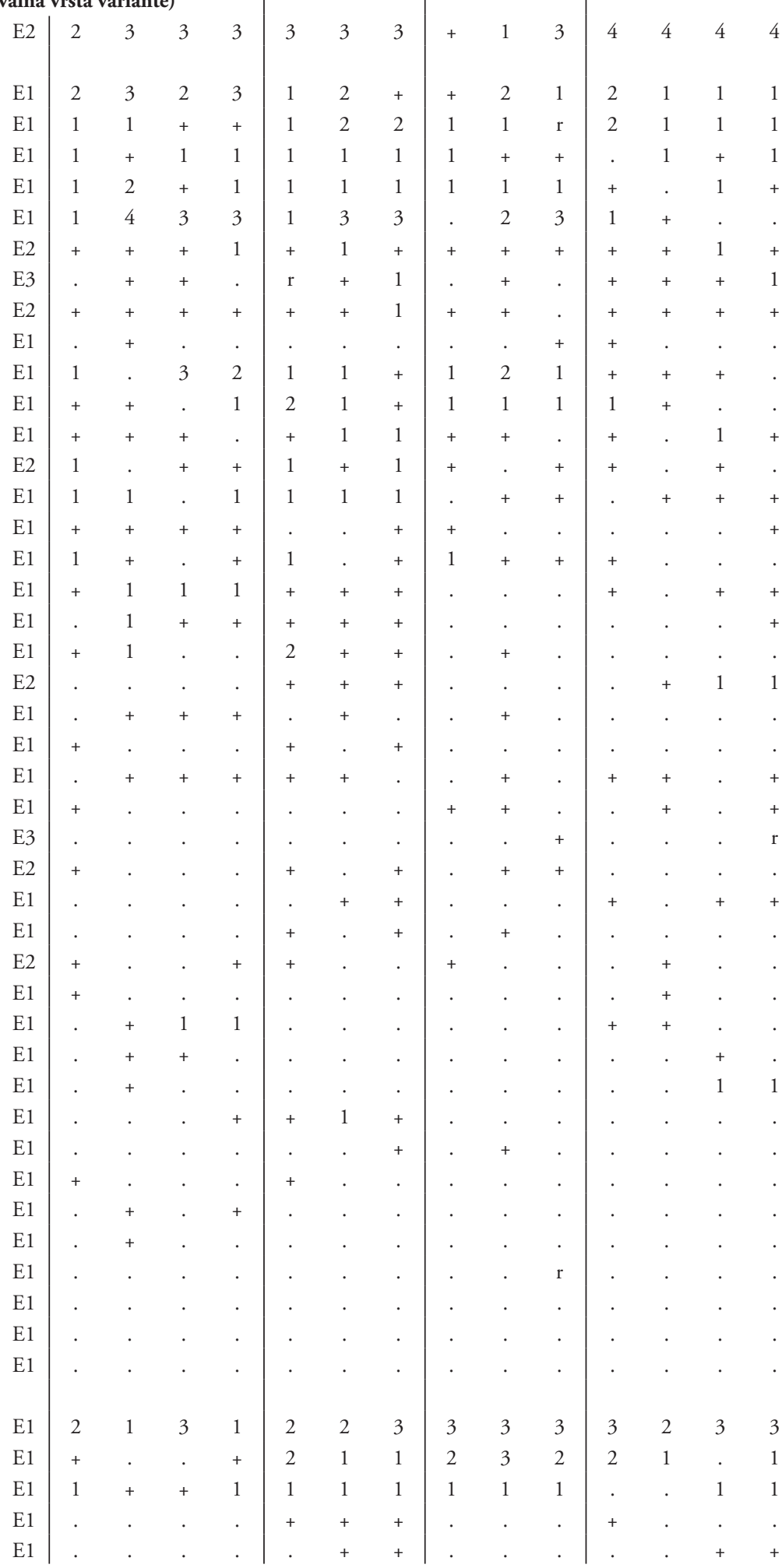




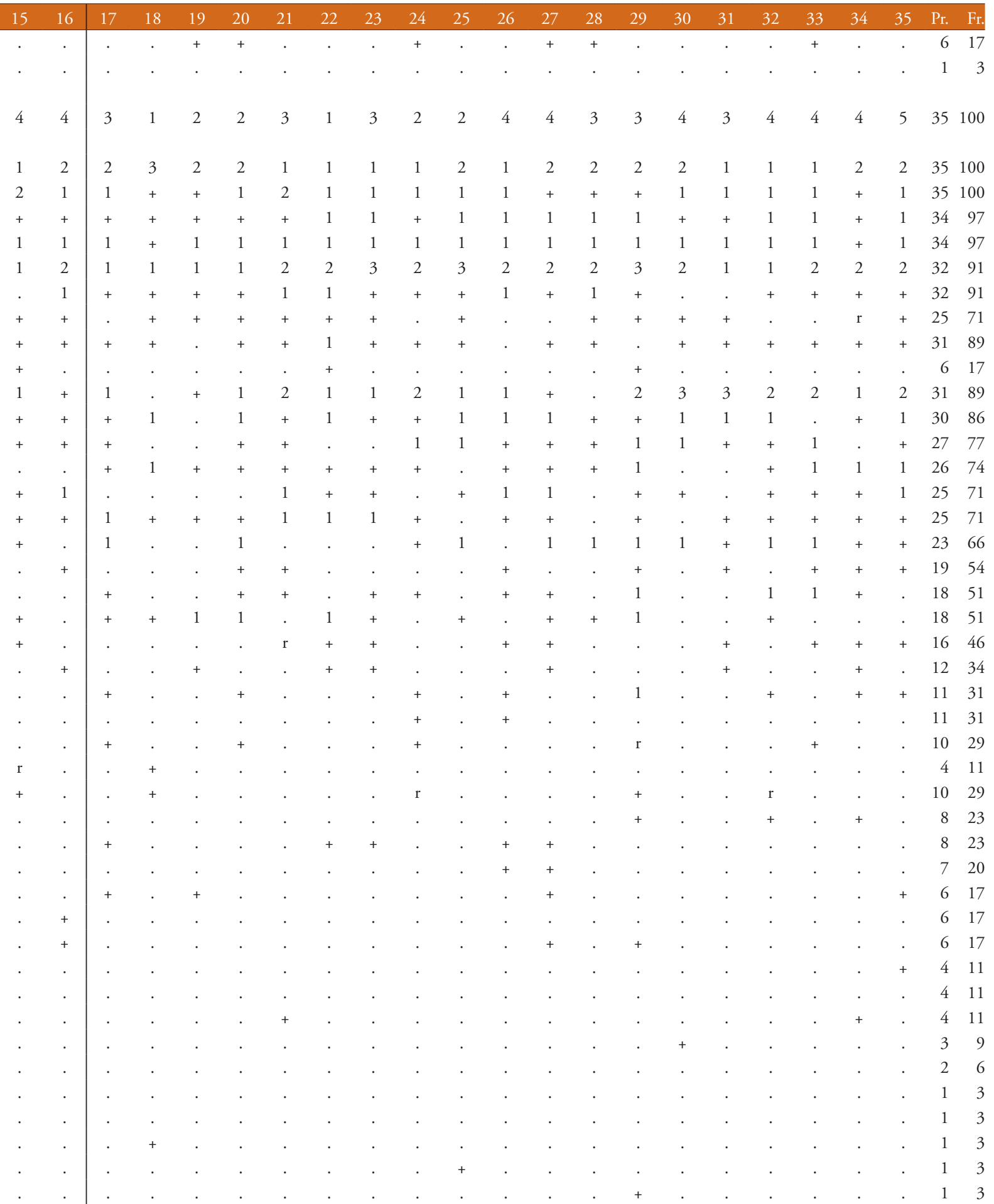

\begin{tabular}{rl|lllllllllllllllllllll}
4 & 3 & 2 & 4 & 3 & 3 & 3 & 4 & 4 & 2 & 3 & 3 & 3 & 3 & 3 & 3 & 2 & 3 & 3 & 3 & 3 & 35 & 100 \\
+ & + & 2 & 1 & 3 & 2 & 2 & 3 & 3 & 1 & 1 & 2 & 1 & 3 & 1 & + & 1 & 2 & + & 2 & + & 32 & 91 \\
1 & 1 & 1 &. & + &. & + & + & + &. & 1 & + & 1 & + & + &. & + & + & + & + & + & 29 & 83 \\
1 &. &. &. &. &. & + & + &. &. &. &. &. &. &. &. &. &. &. &. &. & 7 & 20 \\
. &. &. &. &. &. & + &. &. & + &. &. &. &. & + &. &. &. &. &. &. & 7 & 20
\end{tabular}


Aquilegia nigricans

Carex alba

Genista radiata

Cotoneaster tomentosus

Molinia arundinacea

Asperula aristata

AF Aremonio-Fagion

Knautia drymeia

Cyclamen purpurascens

Cardamine enneaphyllos

Helleborus niger

Rhamnus fallax

TA Tilio-Acerion

Acer pseudoplatanus

Acer pseudoplatanus

Acer pseudoplatanus

Adoxa moschatellina

Geranium robertianum

FS Fagetalia sylvaticae

Daphne mezereum

Melica nutans

Galeobdolon flavidum

Lonicera alpigena

Lilium martagon

Fagus sylvatica

Fagus sylvatica

Fagus sylvatica

Prenanthes purpurea

Mercurialis perennis

Paris quadrifolia

Euphorbia amygdaloides

Dryopteris filix-mas

Polystichum aculeatum

Phyteuma spicatum

Epilobium montanum

Myosotis sylvatica

Galium laevigatum

Laburnum alpinum

Laburnum alpinum

Symphytum tuberosum

Petasites albus

Mycelis muralis

Luzula nivea

QP Quercetalia pubescenti-petraeae

Sorbus aria (Aria edulis)

Sorbus aria (Aria edulis)

$\mathrm{QR}$ Quercetalia roboris

Melampyrum pratense subsp. vulgatum

QF

Querco-Fagetea

Carex digitata

Hepatica nobilis

Poa nemoralis

Anemone nemorosa

E1

E2

E2

E1

E1

E1

E1

E

E

E2

E3

E2

E1

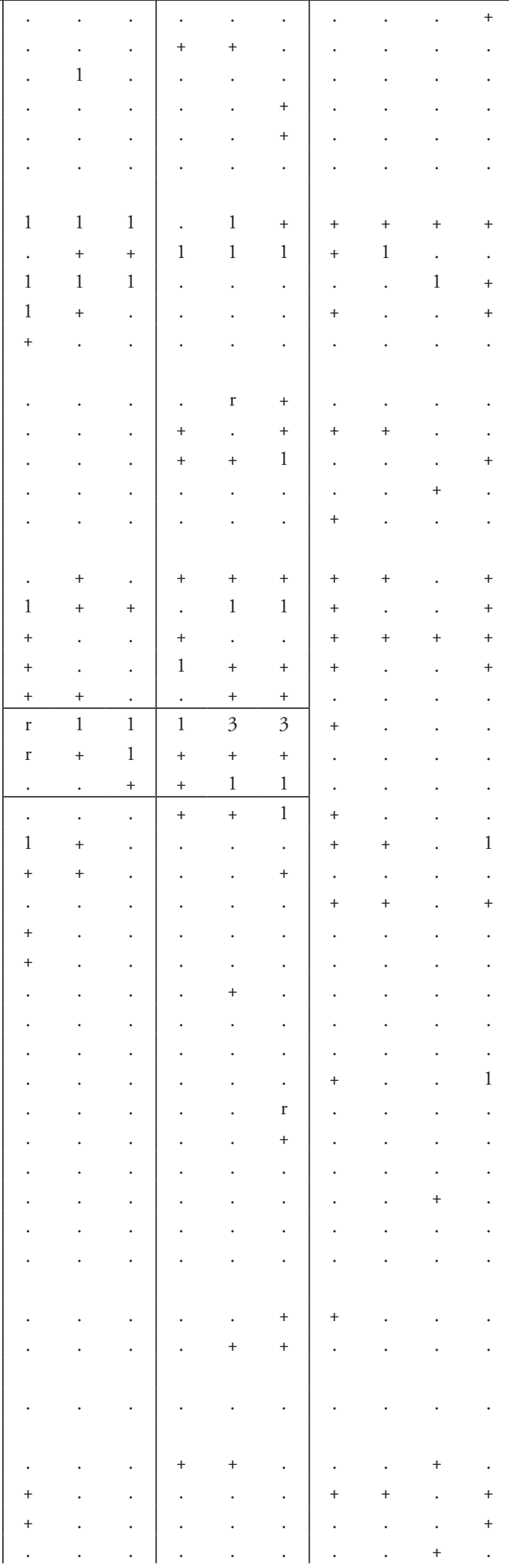


Viola riviniana

Hieracium lachenalii

E1

E1

SSC Sambuco-Salicion capreae, Rhamno-Prunetea

Sorbus aucuparia subsp. aucuparia

Sorbus aucuparia subsp. aucuparia

Sorbus aucuparia subsp. aucuparia

Populus tremula

BA Betulo-Alnetea

Sorbus chamaemespilus

Salix appendiculata

Salix appendiculata

Juniperus sibirica

Alnus viridis

Salix glabra

Ribes alpinum

Salix waldsteiniana

Ribes petraeum

MuA Mulgedio-Aconitetea

Viola biflora

Geranium sylvaticum

Veratrum album subsp. lobelianum

Athyrium filix-femina

Polygonatum verticillatum

Saxifraga rotundifolia

Aconitum lycoctonum subsp. ranunculifolium

Hypericum maculatum

Chaerophyllum hirsutum

Geum rivale

Senecio cacaliaster

Adenostyles alliariae

Primula elatior

Rumex arifolius

Chaerophyllum villarsii

Poa hybrida

Ranunculus platanifolius

Senecio ovatus

Phyteuma ovatum

Aconitum tauricum

Peucedanum ostruthium

Aconitum angustifolium

Alchemilla monticola

Stellaria nemorum

Alchemilla xanthochlora

Epilobium alpestre

Doronicum austriacum

Veratrum album subsp. album

Thalictrum aquilegiifolium

CA Caricion austroalpinae

Heracleum austriacum subsp. siifolium

Pulsatilla alpina subsp. austroalpina

Festuca calva

Gentiana lutea subsp. vardjanii

Arabis vochinensis
E2

E1

E1

E1

E1

E1

E1

E1

E1

E1

E1

E1

E1

E1

E1

E1

E1

E1

E1

E1

E1

E1

E1

E1

E1

E1

E1

E1

E1

E1

E1

\begin{tabular}{l|lll|}
$\cdot$ & $\cdot$ & $\cdot$ & $\cdot$ \\
. & $\cdot$ &. &.
\end{tabular}

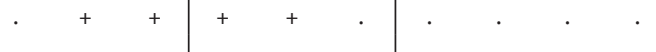




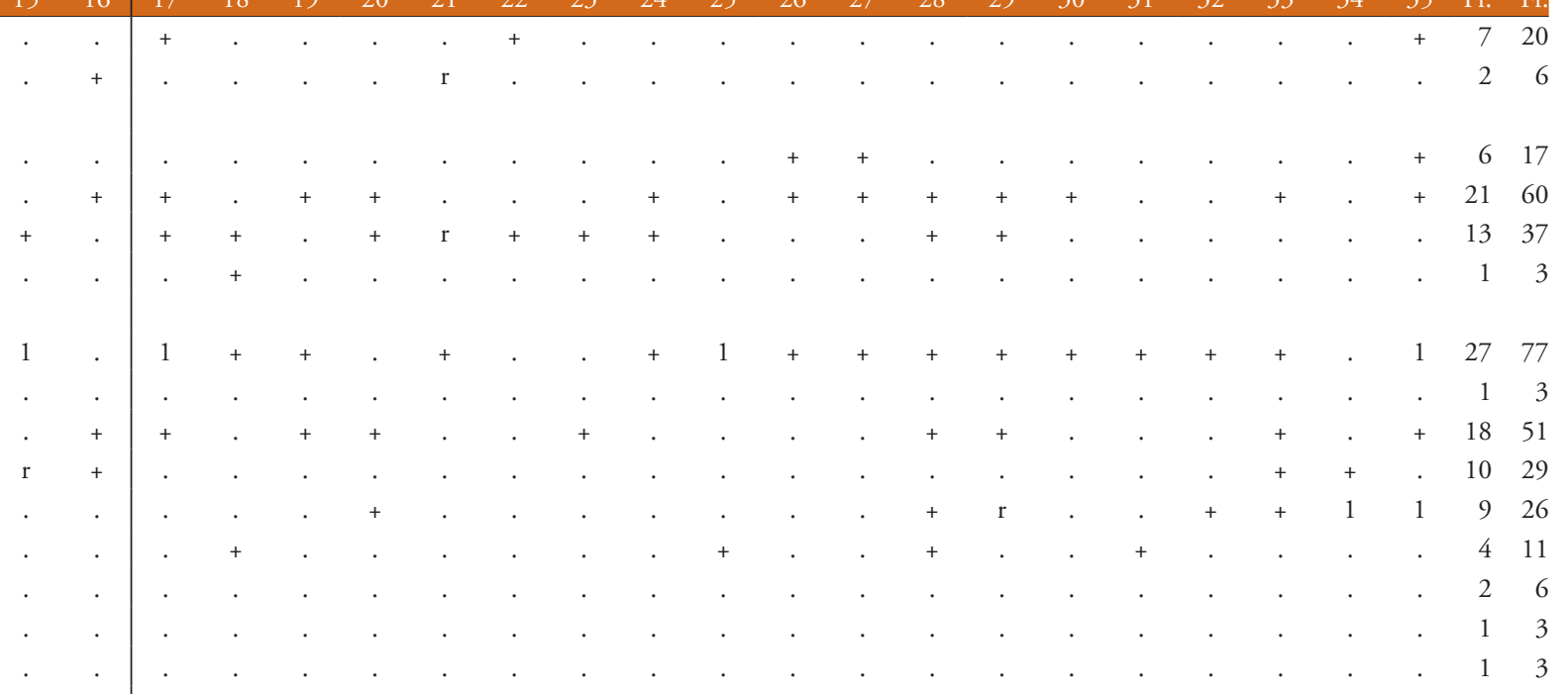


\begin{tabular}{|l|llll|lll|lll|llll} 
Number of relevé (Zaporedna številka popisa) & 1 & 2 & 3 & 4 & 5 & 6 & 7 & 8 & 9 & 10 & 11 & 12 & 13 & 14
\end{tabular} Cfir Caricion firmae

Dryas octopetala

Carex firma

Leontopodium alpinum

Helianthemum alpestre

ES Elyno-Seslerietea

Sesleria caerulea

Phyteuma orbiculare

Senecio abrotanifolius

Aster bellidiastrum

Betonica alopecuros

Galium anisophyllum

Lotus alpinus

Campanula witasekiana

Thymus praecox subsp. polytrichus

Polygonum viviparum

Helianthemum nummularium subsp. grandiflorum E1

Polygala alpestris

Carex sempervirens

Selaginella selaginoides

Scabiosa lucida subsp. stricta

Ranunculus carinthiacus

Euphrasia picta

Scabiosa lucida subsp. lucida

Daphne striata

Gentianella anisodonta

Euphrasia salisburgensis

Homogyne discolor

Juncus monanthos

Bartsia alpina

Ranunculus montanus

Carex mucronata

Arabis ciliata

Alchemilla sp.

Globularia nudicaulis

Myosotis alpestris

Potentilla crantzi

Gentiana lutea subsp. symphyandra

Acinos alpinus

Alchemilla exigua

Carduus crassifolius

JT Juncetea trifidi

Campanula scheuchzeri

Anthoxanthum nipponicum

CU Calluno-Ulicetea

Gentiana pannonica

Festuca nigrescens

Potentilla erecta

Veronica officinalis

Alchemilla flabellata

Coeloglossum viride

Pseudorchis albida

Agrostis capillaris

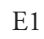

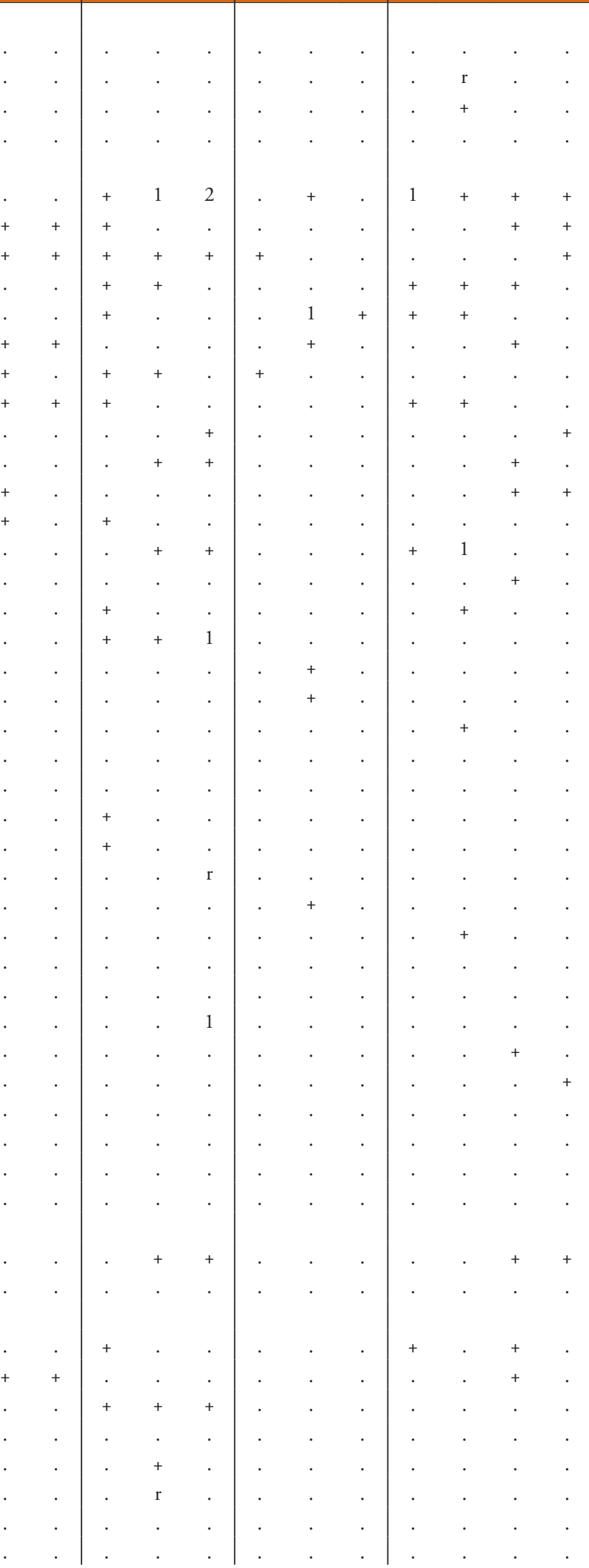




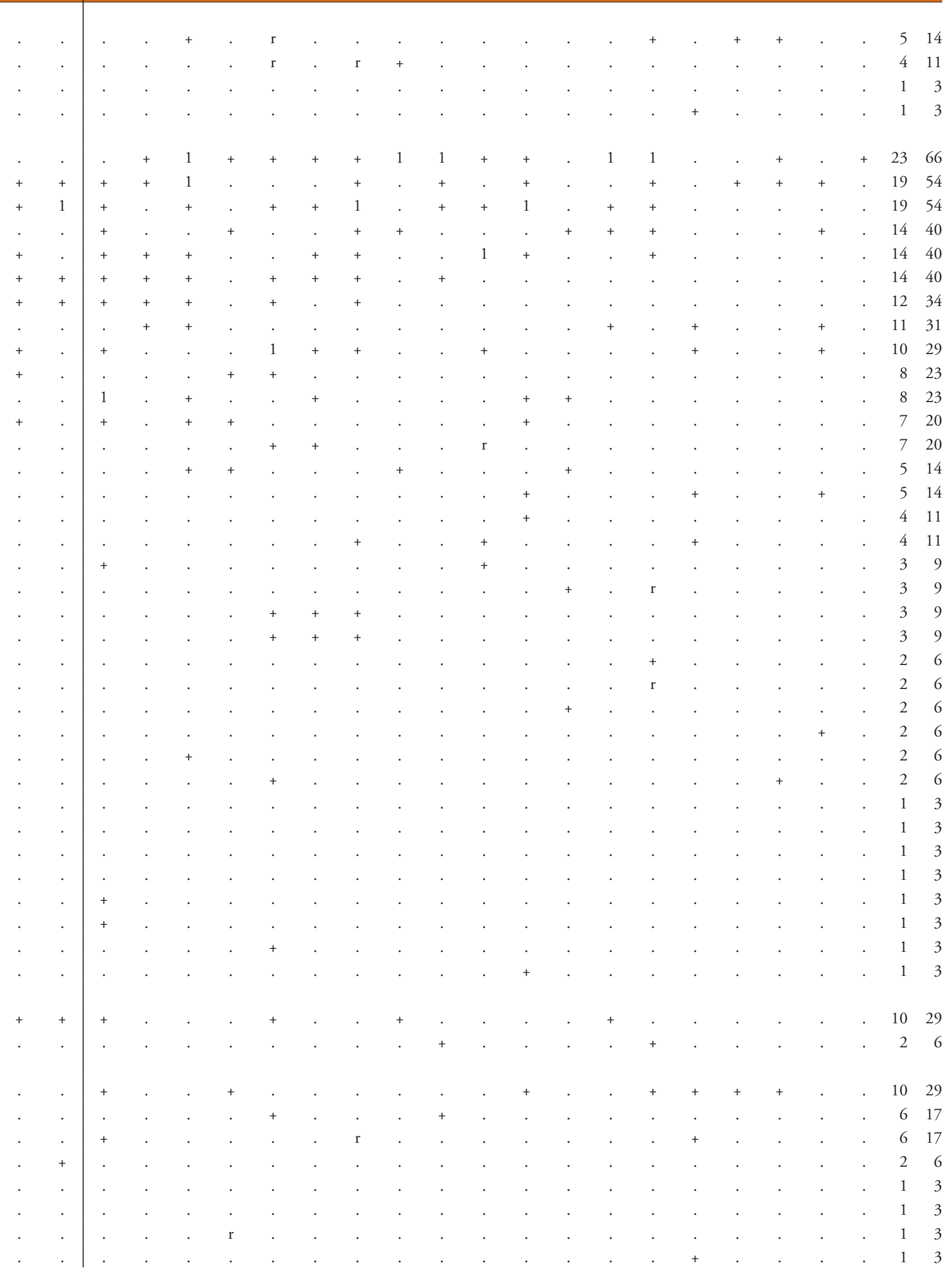


\begin{tabular}{|l|l|l|l|l|l|ll|lll|llll} 
Number of relevé (Zaporedna številka popisa) & 1 & 2 & 3 & 4 & 5 & 6 & 7 & 8 & 9 & 10 & 11 & 12 & 13 & 14
\end{tabular}

FB Festuco-Brometea

Carlina acaulis

Cirsium erisithales

Buphthalmum salicifolium

Linum catharticum

PaT Poo alpinae-Trisetetalia

Poa alpina

Trollius europaeus

Ranunculus nemorosus

Crocus albiflorus

MA Molinio-Arrbenatheretea

Leontodon hispidus

Veronica chamaedrys

Galium album

Anthoxanthum odoratum

Crepis paludosa

Cerastium fontanum

Deschampsia cespitosa

MC Montio-Cardaminetea

Saxifraga aizoides

Cardamine flexuosa

CD Caricetalia davallianae

Parnassia palustris

Carex capillaris

EA Epilobietea angustifolii

Fragaria vesca

Chamaenerion angustifolium

Rubus idaeus

Urtica dioica

Myosotis arvensis

Tussilago farfara

AC Arabidetalia caeruleae

Soldanella alpina

Soldanella minima

TR Thlaspietea rotundifolii

Gymnocarpium robertianum

Adenostyles glabra

Biscutella laevigata

Valeriana montana

Trisetum argenteum

Hieracium bifidum

Cirsium spinosissimum

Rhodiola rosea

Festuca laxa

Dryopteris villarii

Cystopteris montana

Rumex scutatus

Silene vulgaris subsp. glareosa

Cy Cystopteridion fragilis

Cystopteris fragilis

Cystopteris regia

E1

E1

E1

E1

E1

E1

E1

E1

E1

E1

E1

E1

E1

E1

E1

E1

E1

E1

E1

E1

E1

E2

E1

E1

E1

E1

E1

E1

E1

E1

E1

E1

E1

E1

E1

E1

E1

E1

E1

E1

E1

E1

E1

Carex brachystachys

PS Physoplexido comosae-Saxifragion petraeae

Campanula zoysii

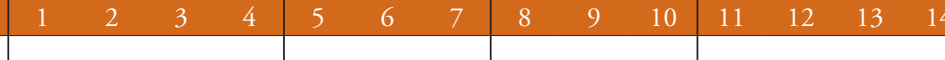
14 . 


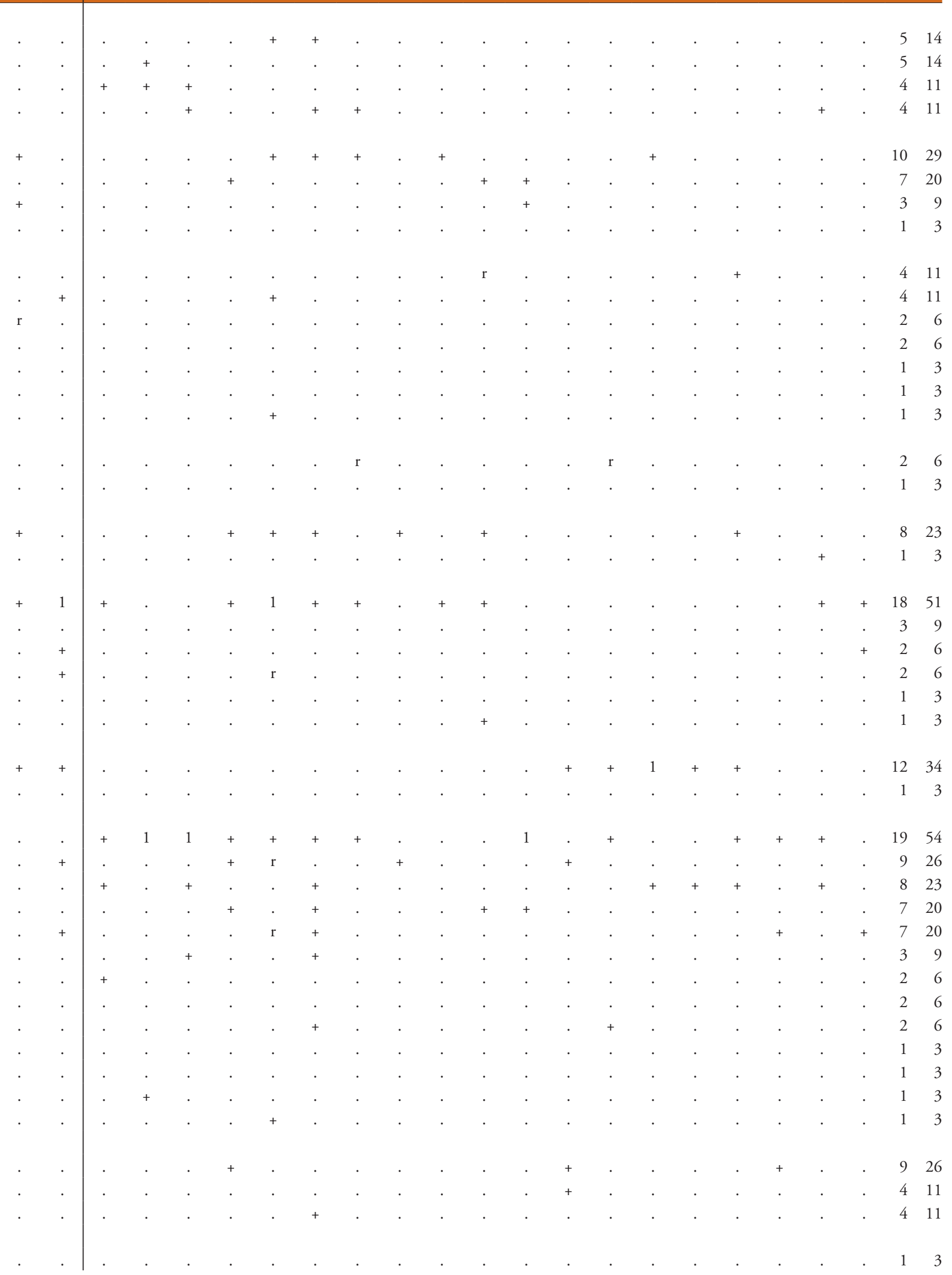


\begin{tabular}{|l|llll|lll|llll|llll} 
Number of relevé (Zaporedna številka popisa) & 1 & 2 & 3 & 4 & 5 & 6 & 7 & 8 & 9 & 10 & 11 & 12 & 13 & 14
\end{tabular}

PC Potentilletalia caulescentis

Campanula cochleariifolia

Festuca stenantha

Potentilla caulescens

Festuca alpina

Campanula cespitosa

AT Asplenietea trichomanis

Asplenium viride

Moehringia muscosa

Asplenium trichomanes

Kernera saxatilis

Asplenium ruta-muraria

Festuca sp.

Polypodium vulgare

Fu Fungi (Glive)

Laetiporus sulphureus agg. (L. horoniensis)

Laricifomes officinalis

ML Mosses and lichens (Mahovi in lišaji)

Tortella tortuosa

Ctenidium molluscum

Rhytidiadelphus triquetrus

Hylocomium splendens

Schistidium sp.

Letharia vulpina

Polytrichum formosum

Dicranum scoparium

Fissidens dubius

Peltigera leucophlebia

Cetraria islandica

Paraleucobryum sauteri

Encalypta streptocarpa

Rhytidiadelphus loreus

Plagiothecium undulatum

Hypogymnia physodes

Cladonia pyxidata

Plagiochila porelloides

Peltigera canina

Plagiochila asplenioides

Mnium sp.

Distichium capillaceum

Orthothecium rufescens

Bartramia pomiformis

Eurhynchium angustirete

Conocephalum conicum

Plagiothecium sylvaticum

Sanionia uncinata

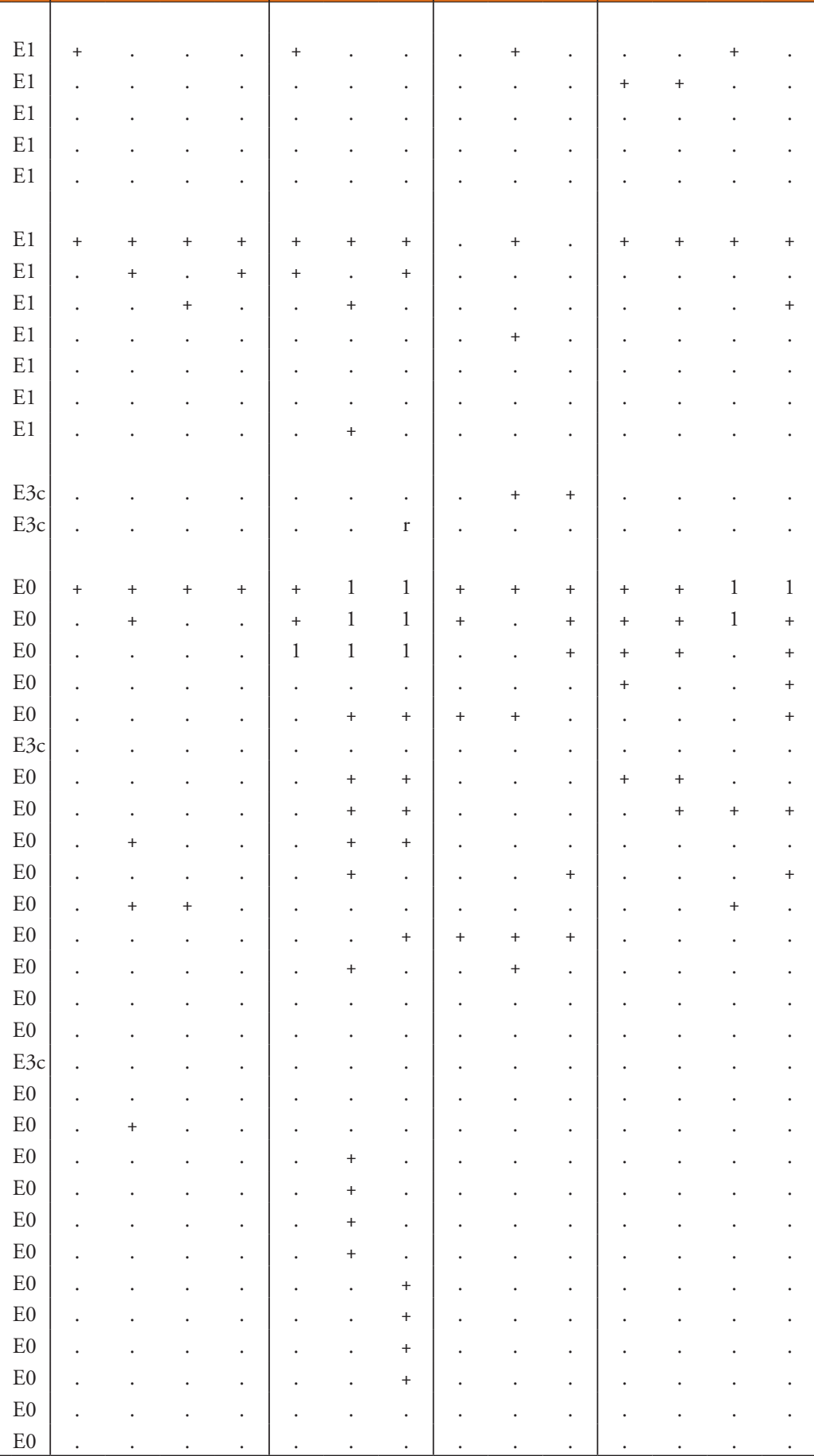

Legend - Legenda

ID Igor Dakskobler

AS Andrej Seliškar

AR Andrej Rozman

AP Aleš Poljanec

BZ Branko Zupan

A Limestone - apnenec
D Dolomite - dolomit

Re Rendzina - rendzina

JA Julian Alps - Julijske Alpe

KSA Kamnik-Savinja Alps - Kamniško-Savinjske Alpe

K Karavanke - Karavanke

Pr. Presence (number of relevés in which the species is presented) - število popisov, v katerih se pojavlja vrsta

Fr. Frequency in $\%$ - frekvenca $v \%$ 


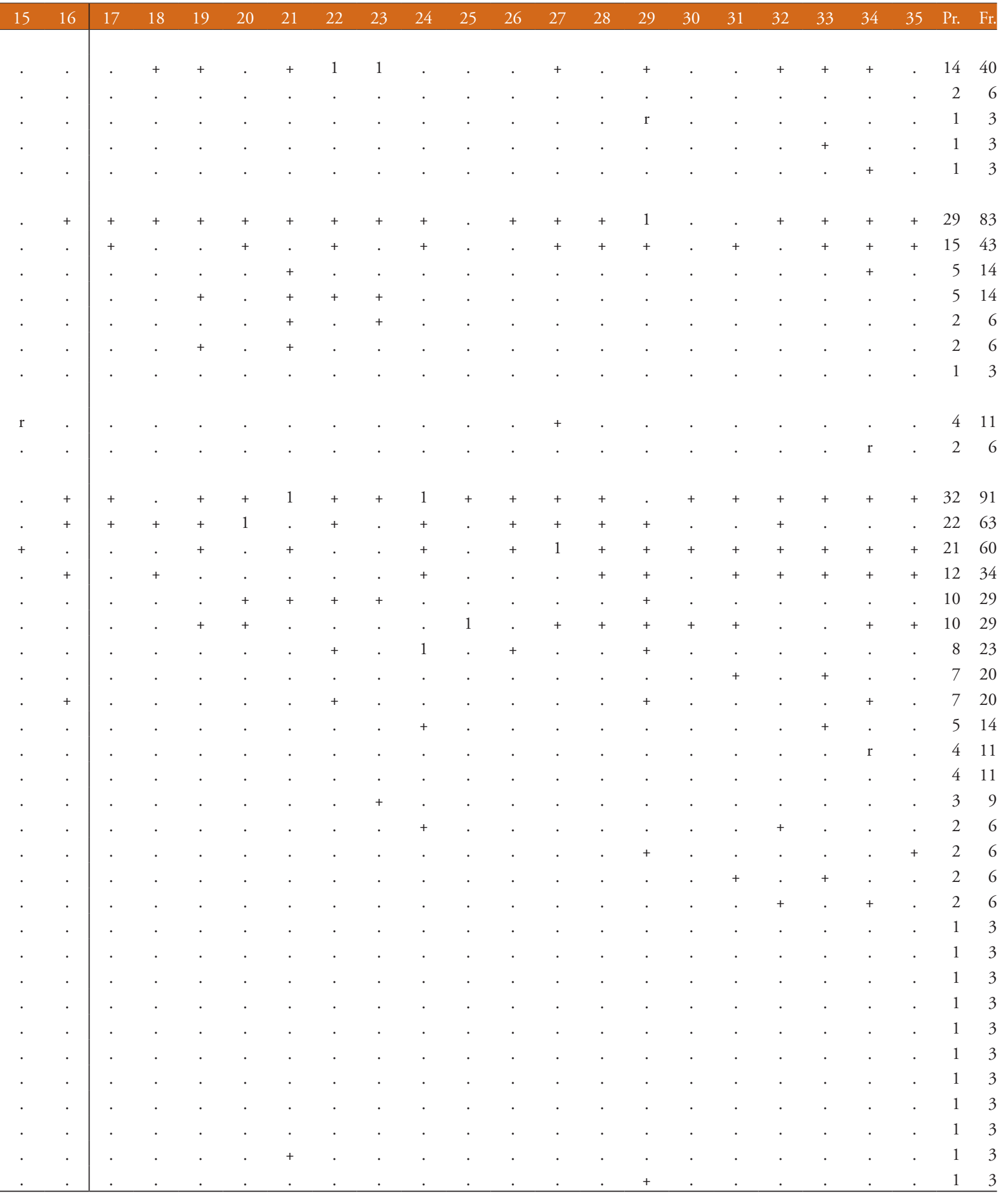


Table 11 (Tabela 11): Rhodothamno-Laricetum sorbetosum chamaemespili var. Calamagrostis varia

\begin{tabular}{|c|c|c|c|c|c|c|c|c|c|c|c|c|c|c|}
\hline Number of relevé (Zaporedna številka popisa) & & 1 & 2 & 3 & 4 & 5 & 6 & 7 & 8 & 9 & 10 & 11 & 12 & 13 \\
\hline $\begin{array}{l}\text { Database number of relevé } \\
\text { (Delovna številka popisa) }\end{array}$ & & 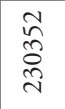 & 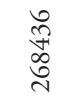 & 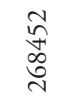 & 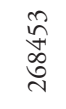 & $\begin{array}{l}n \\
\infty \\
\infty \\
0 \\
\end{array}$ & 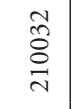 & 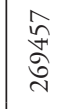 & \begin{tabular}{l}
$\stackrel{2}{n}$ \\
\multirow{\sigma}{*}{}
\end{tabular} & 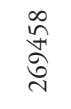 & 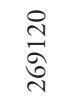 & 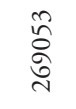 & 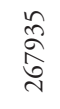 & 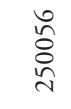 \\
\hline Author of the relevé (Avtor popisa) & & $\stackrel{\text { 究 }}{\varrho}$ & $\begin{array}{l}N \\
\stackrel{\cong}{\ominus}\end{array}$ & 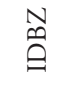 & $\begin{array}{l}N \\
\text { ڤิ }\end{array}$ & $\ominus$ & $\theta$ & 田 & $\theta$ & $\Leftrightarrow$ & $\Theta$ & $\Theta$ & $\theta$ & $\theta$ \\
\hline Elevation in $\mathrm{m}$ (Nadmorska višina $\mathrm{v} \mathrm{m})$ & & $\stackrel{2}{\sim}$ & 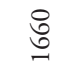 & 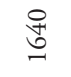 & $\underset{ٍ}{\stackrel{n}{0}}$ & $\stackrel{\Re}{\cong}$ & $\underset{-}{8}$ & $\stackrel{0}{\stackrel{2}{n}}$ & 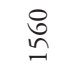 & $\stackrel{\text { }}{\sim}$ & 余 & 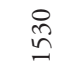 & 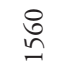 & \&્ఠ \\
\hline Aspect (Lega) & & $\mathrm{NE}$ & SE & SE & $\mathrm{E}$ & SE & SW & $\mathrm{NE}$ & NNE & NNE & SEE & NNW & SE & $\mathrm{E}$ \\
\hline Slope in degrees (Nagib v stopinjah) & & 40 & 10 & 30 & 35 & 40 & 25 & 5 & 20 & 35 & 30 & 30 & 30 & 25 \\
\hline Parent material (Matična podlaga) & & A & A & A & $\mathrm{DA}$ & DA & DA & Gr & DA & DA & DA & DA & $\mathrm{DA}$ & DA \\
\hline Soil (Tla) & & $\operatorname{Re}$ & $\operatorname{Re}$ & $\operatorname{Re}$ & $\operatorname{Re}$ & $\mathrm{Re}$ & $\operatorname{Re}$ & $\operatorname{Re}$ & $\operatorname{Re}$ & $\operatorname{Re}$ & $\operatorname{Re}$ & $\operatorname{Re}$ & $\operatorname{Re}$ & $\operatorname{Re}$ \\
\hline Stoniness in \% (Kamnitost v \%) & & 30 & 10 & 30 & 30 & 15 & 10 & 5 & 20 & 5 & 10 & 10 & 5 & 5 \\
\hline Cover in \% (Zastiranje v \%): & & & & & & & & & & & & & & \\
\hline Upper tree layer (Zgornja drevesna plast) & $\mathrm{E} 3 \mathrm{~b}$ & 60 & 60 & 70 & 70 & 50 & 50 & 70 & 70 & 40 & 70 & 70 & 70 & 60 \\
\hline Lower tree layer (Spodnja drevesna plasti) & E3a & 10 & 5 & 5 & 5 & 5 & 20 & 5 & 5 & 20 & 10 & 10 & 10 & 5 \\
\hline Shrub layer (Grmovna plast) & $\mathrm{E} 2$ & 40 & 45 & 25 & 40 & 60 & 70 & 30 & 40 & 50 & 40 & 40 & 45 & 40 \\
\hline Herb layer (Zeliščna plast) & E1 & 70 & 90 & 70 & 80 & 80 & 80 & 90 & 80 & 80 & 80 & 90 & 70 & 90 \\
\hline Moss layer (Mahovna plast) & E0 & 10 & 10 & 10 & 10 & 5 & 10 & 5 & 5 & 10 & 10 & 10 & 10 & 3 \\
\hline Number of species (Število vrst) & & 92 & 68 & 71 & 75 & 97 & 80 & 44 & 60 & 60 & 56 & 43 & 45 & 49 \\
\hline Relevé area (Velikost popisne ploskve) & $\mathrm{m}^{2}$ & 400 & 400 & 400 & 400 & 400 & 400 & 400 & 400 & 400 & 400 & 400 & 400 & 200 \\
\hline Date of taking relevé (Datum popisa) & & $\begin{array}{c}8 / 6 \\
2009\end{array}$ & $\begin{array}{l}8 / 17 \\
2017\end{array}$ & $\begin{array}{l}8 / 17 \\
2017\end{array}$ & $\begin{array}{l}8 / 17 \\
2017\end{array}$ & $\begin{array}{l}9 / 22 \\
2010\end{array}$ & $\begin{array}{l}6 / 21 \\
2005\end{array}$ & $\begin{array}{l}8 / 23 \\
2017\end{array}$ & $\begin{array}{l}8 / 23 \\
2017\end{array}$ & $\begin{array}{l}8 / 23 \\
2017\end{array}$ & $\begin{array}{l}8 / 21 \\
2017\end{array}$ & $\begin{array}{l}10 / 17 \\
2017\end{array}$ & $\begin{array}{l}7 / 17 \\
2017\end{array}$ & $\begin{array}{r}9 / 24 \\
2013\end{array}$ \\
\hline 更 & & 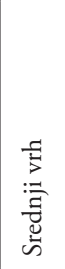 & $\frac{\mathscr{U}}{\tilde{n}}$ & $\frac{\mathscr{U}}{\tilde{U}}$ & $\frac{\tilde{u}}{\tilde{v}}$ & 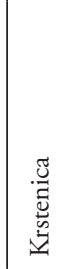 & 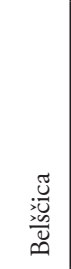 & 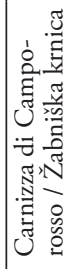 & 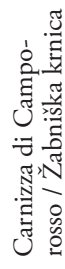 & 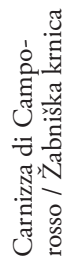 & 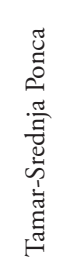 & $\begin{array}{l}\frac{\overrightarrow{0}}{4} \\
\overrightarrow{0} \\
\frac{\partial}{2}\end{array}$ & 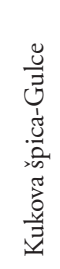 & 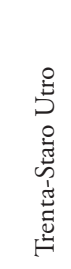 \\
\hline Mountain range (Pogorje) & & K & KSA & KSA & KSA & JA & $\mathrm{K}$ & JA & JA & JA & JA & KSA & JA & JA \\
\hline Quadrant (Kvadrant) & & $\begin{array}{c}9551 \\
13\end{array}$ & $\begin{array}{c}9653 \\
13\end{array}$ & $\begin{array}{l}9653 \\
13\end{array}$ & $\begin{array}{c}9653 \\
13\end{array}$ & $\begin{array}{c}9649 \\
13\end{array}$ & $\begin{array}{c}9550 \\
12\end{array}$ & $\begin{array}{l}9547 \\
13\end{array}$ & $\begin{array}{c}9547 \\
13\end{array}$ & $\begin{array}{c}9547 \\
13\end{array}$ & $\begin{array}{l}9548 \\
/ 1\end{array}$ & $\begin{array}{l}9653 \\
14\end{array}$ & $\begin{array}{c}9549 \\
13\end{array}$ & $\begin{array}{l}9648 \\
/ 1\end{array}$ \\
\hline Coordinate GK-Y (D-48) & $\mathrm{m}$ & $\begin{array}{l}\stackrel{n}{+} \\
\stackrel{\infty}{+} \\
\stackrel{f}{f}\end{array}$ & 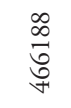 & $\begin{array}{l}\infty \\
\text { స్ర } \\
\text { ర্+ } \\
+\end{array}$ & 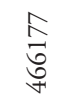 & $\begin{array}{l}\infty \\
\infty \\
\infty \\
\exists \\
\exists\end{array}$ & \begin{tabular}{l}
$\infty$ \\
$\infty$ \\
$\infty$ \\
$\approx$ \\
\multirow{+}{+}{}
\end{tabular} & $\begin{array}{l}\vec{\infty} \\
n \\
\infty \\
\infty\end{array}$ & $\begin{array}{l}\stackrel{+}{1} \\
\stackrel{0}{+} \\
\stackrel{+}{\infty} \\
\infty\end{array}$ & \begin{tabular}{l}
$\overrightarrow{7}$ \\
\multirow{n}{n}{} \\
$\infty$
\end{tabular} & $\begin{array}{l}\stackrel{n}{\hat{s}} \\
\stackrel{8}{+}\end{array}$ & 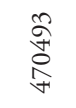 & 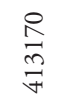 & 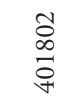 \\
\hline Coordinate GK-X (D-48) & $\mathrm{m}$ & 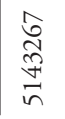 & $\begin{array}{l}\stackrel{n}{*} \\
\stackrel{n}{n} \\
\tilde{n}\end{array}$ & 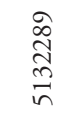 & 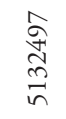 & 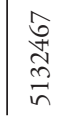 & $\begin{array}{l}\stackrel{a}{n} \\
\stackrel{\hbar}{n}\end{array}$ & $\begin{array}{l}\stackrel{a}{0} \\
\stackrel{\circ}{+} \\
i\end{array}$ & $\begin{array}{l}\hat{a} \\
\stackrel{\circ}{\infty} \\
\stackrel{\infty}{n}\end{array}$ & $\begin{array}{l}\circ \\
\stackrel{0}{0} \\
\stackrel{\circ}{f} \\
i n\end{array}$ & $\begin{array}{l}\stackrel{n}{a} \\
\stackrel{a}{f} \\
i n\end{array}$ & 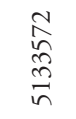 & $\begin{array}{l}\stackrel{\infty}{+} \\
\stackrel{\infty}{*} \\
\stackrel{\sim}{n}\end{array}$ & $\begin{array}{l}\infty \\
\stackrel{\sim}{\alpha} \\
\stackrel{\infty}{n}\end{array}$ \\
\hline
\end{tabular}

Character and differential species of the association (Značilnice in razlikovalnice asociacije)

Laserpitium peucedanoides

Rhodothamnus chamaecistus

Anemone trifolia

Paederota lutea

Festuca nitida

Homogyne sylvestris

Heliosperma alpestre

Astrantia bavarica

Valeriana saxatilis

Carex ferruginea

\begin{tabular}{|c|c|c|c|c|c|c|c|c|c|c|c|c|c|c|c|}
\hline E3 & 4 & 4 & 4 & 4 & 3 & 4 & 4 & 4 & 3 & 4 & 4 & 4 & 4 & 13 & 100 \\
\hline E2 & + & + & . & + & 1 & 1 & 1 & 1 & 2 & . & . & + & + & 10 & 77 \\
\hline E1 & + & + & + & + & 1 & + & . & 1 & + & + & . & 1 & 1 & 11 & 85 \\
\hline E2 & 2 & 2 & 2 & 3 & 1 & + & 2 & . & . & + & . & 1 & + & 10 & 77 \\
\hline E1 & + & . & + & + & . & & + & + & 1 & . & 1 & . & . & 7 & 54 \\
\hline E1 & . & . & . & . & . & 1 & 1 & 1 & 1 & 1 & . & 1 & 1 & 7 & 54 \\
\hline E1 & 1 & + & 1 & + & . & . & . & + & . & . & + & . & . & 6 & 46 \\
\hline E1 & + & + & + & 1 & + & & . & . & . & . & . & . & . & 5 & 38 \\
\hline E1 & + & . & . & + & 1 & & . & . & 1 & . & + & . & . & 5 & 38 \\
\hline E1 & + & . & . & + & + & & . & . & . & . & . & + & . & 4 & 31 \\
\hline E1 & + & + & . & + & + & & . & . & . & . & . & . & . & 4 & 31 \\
\hline E1 & . & . & . & . & . & 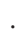 & . & + & + & . & . & . & . & 2 & 15 \\
\hline E1 & . & . & . & . & . & & . & + & + & . & . & . & . & 2 & 15 \\
\hline
\end{tabular}




\begin{tabular}{|c|c|c|c|c|c|c|c|c|c|c|c|c|c|c|c|c|c|}
\hline & \multicolumn{2}{|c|}{ Number of relevé (Zaporedna številka popisa) } & 1 & 2 & 3 & 4 & 5 & 6 & 7 & 8 & 9 & 10 & 11 & 12 & 13 & Pr. & Fr. \\
\hline \multirow[t]{2}{*}{ TR } & Astrantia carniolica & E1 & . & . & . & . & . & . & . & . & . & . & + & . & . & 1 & 8 \\
\hline & \multicolumn{17}{|c|}{ Differential species of the variant (Razlikovalna vrsta variante) } \\
\hline EP & Calamagrostis varia & E1 & 2 & 4 & 3 & 3 & 2 & 2 & 2 & 3 & 3 & 3 & 4 & 4 & 4 & 13 & 100 \\
\hline \multirow[t]{39}{*}{ VP } & Vaccinio-Piceetea & & & & & & & & & & & & & & & & \\
\hline & Vaccinium myrtillus & E1 & + & 3 & 2 & 2 & 1 & 2 & 2 & 3 & 3 & 1 & 1 & + & 2 & 13 & 100 \\
\hline & Vaccinium vitis-idaea & E1 & 1 & 1 & . & + & 2 & 1 & 1 & 1 & 2 & 1 & 2 & 3 & 2 & 12 & 92 \\
\hline & Picea abies & E3 & + & . & 1 & + & + & 1 & + & + & + & 1 & + & + & + & 12 & 92 \\
\hline & Picea abies & E2 & 1 & + & + & . & + & 1 & . & + & . & + & 1 & 1 & + & 10 & 77 \\
\hline & Picea abies & E1 & + & . & . & . & . & . & . & . & . & . & + & . & . & 2 & 15 \\
\hline & Polystichum lonchitis & E1 & 1 & + & 1 & + & 1 & + & . & + & . & 1 & 1 & + & 1 & 11 & 85 \\
\hline & Calamagrostis villosa & E1 & + & 1 & 1 & 1 & . & . & 1 & 2 & 1 & + & + & 1 & 1 & 11 & 85 \\
\hline & Lycopodium annotinum & E1 & + & 1 & . & + & + & . & 1 & 1 & 1 & + & 2 & + & + & 11 & 85 \\
\hline & Luzula sylvatica & E1 & . & . & + & + & . & + & 2 & 1 & 1 & 1 & 1 & . & 2 & 9 & 69 \\
\hline & Valeriana tripteris & E1 & + & + & + & . & 1 & 1 & . & 2 & 1 & + & 1 & . & . & 9 & 69 \\
\hline & Clematis alpina & E2 & 1 & 1 & 1 & 1 & 1 & 2 & . & + & . & + & . & . & . & 8 & 62 \\
\hline & Aposeris foetida & E1 & . & + & 1 & . & + & . & + & . & + & 1 & . & + & 1 & 8 & 62 \\
\hline & Rosa pendulina & E2 & + & 1 & + & . & 1 & + & . & $\mathrm{r}$ & . & + & + & . & . & 8 & 62 \\
\hline & Maianthemum bifolium & E1 & + & 1 & + & + & + & . & 1 & + & . & + & . & . & . & 8 & 62 \\
\hline & Lonicera caerulea & E2 & . & + & . & + & 1 & + & + & + & + & . & . & + & . & 8 & 62 \\
\hline & Homogyne alpina & E1 & + & + & . & . & . & . & + & 1 & + & + & + & . & . & 7 & 54 \\
\hline & Oxalis acetosella & E1 & + & . & + & 1 & + & 1 & . & . & + & + & . & . & . & 7 & 54 \\
\hline & Gymnocarpium dryopteris & E1 & + & . & + & + & . & + & . & + & + & + & . & . & . & 7 & 54 \\
\hline & Ajuga pyramidalis & E1 & + & + & + & + & . & + & . & . & + & . & . & . & + & 7 & 54 \\
\hline & Solidago virgaurea subsp. virgaurea & E1 & + & + & + & + & 1 & 1 & . & . & . & . & . & . & . & 6 & 46 \\
\hline & Melampyrum sylvaticum & E1 & + & . & + & + & . & . & . & + & . & + & . & 1 & . & 6 & 46 \\
\hline & Dryopteris expansa & E1 & . & 1 & + & 1 & 1 & + & . & . & . & + & . & . & . & 6 & 46 \\
\hline & Dryopteris dilatata & E1 & + & + & . & . & + & + & . & . & . & . & . & . & + & 5 & 38 \\
\hline & Luzula luzuloides & E1 & + & . & 1 & + & + & + & . & . & . & . & . & . & . & 5 & 38 \\
\hline & Lonicera nigra & E2 & + & . & . & . & + & + & . & + & . & . & . & + & . & 5 & 38 \\
\hline & Hieracium murorum & E1 & + & . & . & . & + & 1 & . & . & . & . & + & . & . & 4 & 31 \\
\hline & Abies alba & E3 & . & . & . & . & . & . & . & . & . & . & + & . & . & 1 & 8 \\
\hline & Abies alba & E2 & . & + & . & . & . & . & . & $\mathrm{r}$ & $\mathrm{r}$ & . & + & . & . & 4 & 31 \\
\hline & Abies alba & E1 & . & . & . & . & . & . & + & . & . & . & . & . & . & 1 & 8 \\
\hline & Phegopteris connectilis & E1 & + & . & . & . & . & . & . & + & + & . & . & . & . & 3 & 23 \\
\hline & Gentiana asclepiadea & E1 & . & . & . & . & + & . & . & + & + & . & . & . & . & 3 & 23 \\
\hline & Saxifraga cuneifolia & E1 & 1 & . & . & . & + & . & . & . & . & . & . & . & . & 2 & 15 \\
\hline & Veronica urticifolia & E1 & 1 & . & . & . & . & . & . & . & + & . & . & . & . & 2 & 15 \\
\hline & Huperzia selago & E1 & . & . & . & . & + & . & . & . & + & . & . & . & . & 2 & 15 \\
\hline & Luzula luzulina & E1 & . & . & . & . & + & 1 & . & . & . & . & . & . & . & 2 & 15 \\
\hline & Luzula pilosa & E1 & . & . & . & . & . & + & . & . & . & + & . & . & . & 2 & 15 \\
\hline & Calamagrostis arundinacea & E1 & . & . & . & . & . & + & . & . & . & . & . & . & . & 1 & 8 \\
\hline & Pyrola minor & E1 & . & . & . & . & . & . & + & . & . & . & . & . & . & 1 & 8 \\
\hline \multirow[t]{9}{*}{ EP } & Erico-Pinetea & & & & & & & & & & & & & & & & \\
\hline & Pinus mugo & E2 & 2 & 3 & 2 & 3 & 4 & 4 & 1 & 3 & 3 & 3 & 4 & 3 & 2 & 13 & 100 \\
\hline & Erica carnea & E1 & 1 & . & 2 & 2 & 2 & 1 & 1 & 2 & 2 & . & 3 & 3 & 1 & 11 & 85 \\
\hline & Rubus saxatilis & E1 & + & 1 & . & 1 & 1 & 2 & + & 2 & 2 & + & . & 1 & 1 & 11 & 85 \\
\hline & Carex ornithopoda & E1 & . & . & + & . & . & + & . & . & . & . & . & . & . & 2 & 15 \\
\hline & Aquilegia nigricans & E1 & + & . & . & . & + & . & . & . & . & . & . & . & . & 2 & 15 \\
\hline & Carex alba & E1 & . & . & . & . & + & . & . & . & . & . & . & . & . & 1 & 8 \\
\hline & Polygala chamaebuxus & E1 & . & . & . & . & . & . & + & . & . & . & . & . & . & 1 & 8 \\
\hline & Genista radiata & E2 & . & . & . & . & . & . & . & . & . & . & . & . & + & 1 & 8 \\
\hline
\end{tabular}


\begin{tabular}{l|lllll|ll|llllllllll} 
Number of relevé (Zaporedna številka popisa) & 1 & 2 & 3 & 4 & 5 & 6 & 7 & 8 & 9 & 10 & 11 & 12 & 13 & Pr. & Fr.
\end{tabular}

AF Aremonio-Fagion

Knautia drymeia

Cyclamen purpurascens

Cardamine enneaphyllos

Rhamnus fallax

Helleborus niger

TA Tilio-Acerion

Acer pseudoplatanus

Acer pseudoplatanus

Acer pseudoplatanus

FS Fagetalia sylvaticae

Daphne mezereum

Melica nutans

Dryopteris filix-mas

Lilium martagon

Mercurialis perennis

Galeobdolon flavidum

Lonicera alpigena

Paris quadrifolia

Fagus sylvatica

Fagus sylvatica

Fagus sylvatica

Galium laevigatum

Polystichum aculeatum

Euphorbia amygdaloides

Prenanthes purpurea

Epilobium montanum

Luzula nivea

Laburnum alpinum

Mycelis muralis

Epipactis helleborine

Myosotis sylvatica

Symphytum tuberosum

Phyteuma spicatum

QP Quercetalia pubescenti-petraeae

Sorbus aria (Aria edulis)

Convallaria majalis

QR Quercetalia roboris

Melampyrum pratense subsp. vulgatum

QF Querco-Fagetea

Anemone nemorosa

Hepatica nobilis

Poa nemoralis

Viola riviniana

Carex digitata

SSC Sambuco-Salicion capreae, Rhamno prunetea Sorbus aucuparia subsp. aucuparia Sorbus aucuparia subsp. aucuparia Sorbus aucuparia subsp. aucuparia

BA

Betulo-Alnetea

Sorbus chamaemespilus

Salix appendiculata

Juniperus sibirica

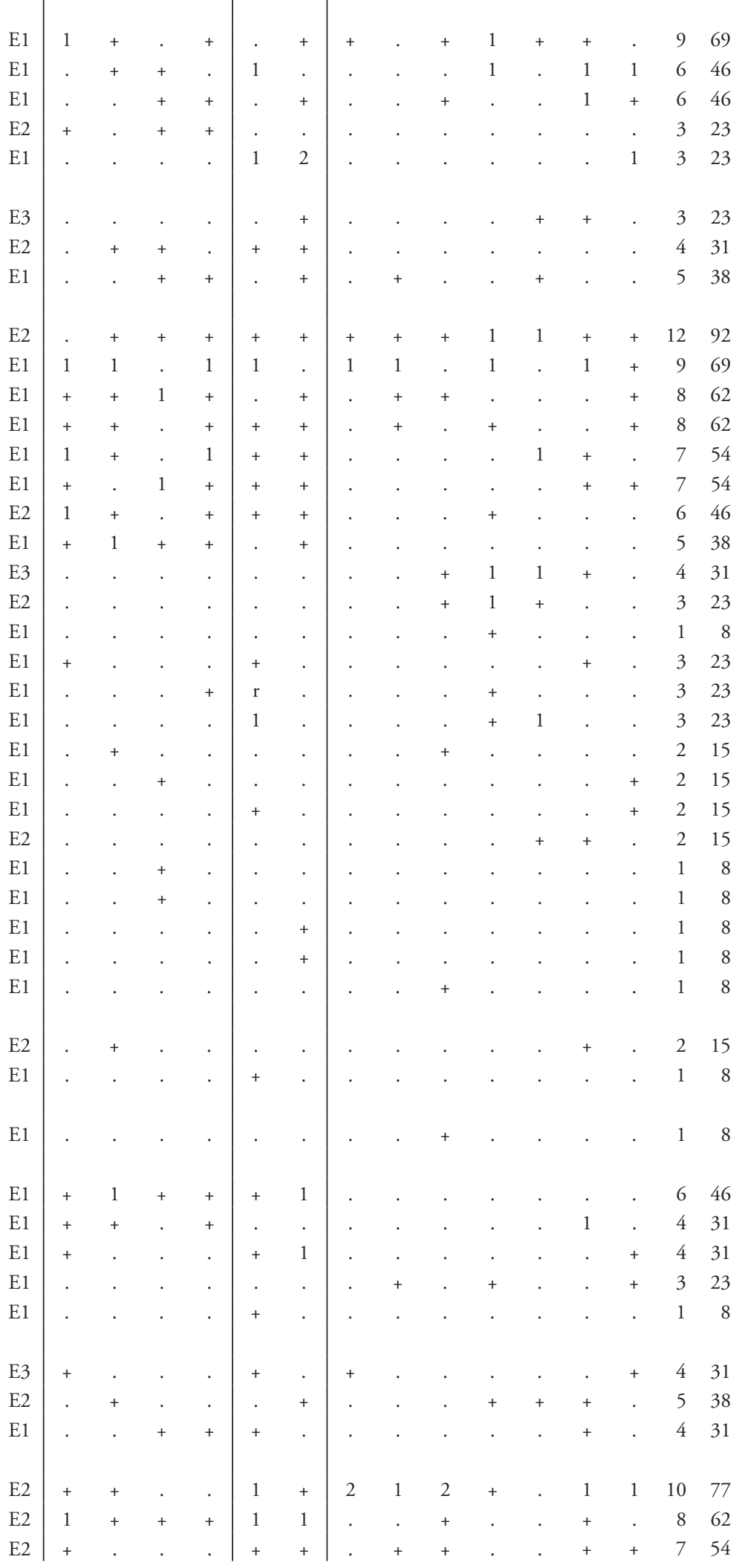


\begin{tabular}{l|l|llll|ll|llllllllll} 
Number of relevé (Zaporedna številka popisa) & 1 & 2 & 3 & 4 & 5 & 6 & 7 & 8 & 9 & 10 & 11 & 12 & 13 & Pr. & Fr.
\end{tabular}

Alnus viridis

Salix glabra

Ribes alpinum

MuA Mulgedio-Aconitetea

Athyrium filix-femina

Geranium sylvaticum

Polygonatum verticillatum

Hypericum maculatum

Viola biflora

Aconitum lycoctonum subsp. ranunculifolium

Saxifraga rotundifolia

Veratrum album subsp. lobelianum

Aconitum tauricum

Chaerophyllum villarsii

Senecio ovatus

Chaerophyllum hirsutum

Senecio cacaliaster

Ranunculus platanifolius

Phyteuma ovatum

Veratrum album subsp. album

Primula elatior

Geum rivale

Aconitum degenii subsp. paniculatum

Lamium maculatum

Adenostyles alliariae

TG Trifolio-Geranietea

Digitalis grandiflora

CA Caricion austroalpinae

Pulsatilla alpina subsp. austroalpina

Koeleria eriostachya

Heracleum austriacum subsp. siifolium

Festuca calva

ES Elyno-Seslerietea

Betonica alopecuros

Sesleria caerulea

Lotus alpinus

Helianthemum nummularium subsp. grandiflorum E

Aster bellidiastrum

Selaginella selaginoides

Galium anisophyllum

Campanula witasekiana

Ranunculus carinthiacus

Senecio abrotanifolius

Carex sempervirens

Phyteuma orbiculare

Scabiosa lucida subsp. stricta

Scabiosa lucida subsp. lucida

Thymus praecox subsp. polytrichus

Leucanthemum heterophyllum (L.maximum agg.)

Euphrasia picta

Gentiana lutea subsp. symphyandra

Saxifraga adscendens

Polygala alpestris

\begin{tabular}{|c|c|c|c|c|c|c|c|c|c|c|c|c|c|c|}
\hline E2 & + & 1 & + & 1 & . & + & . & . & + & . & & & & 6 \\
\hline E2 & . & 1 & . & + & . & . & + & . & 2 & + & & & & 5 \\
\hline $\mathrm{E} 2$ & + & . & . & . & . & 1 & . & . & . & . & & & . & 2 \\
\hline E1 & + & + & + & . & + & + & + & + & + & + & & + & . & 10 \\
\hline E1 & + & + & + & 1 & + & + & + & + & . & + & & & . & 9 \\
\hline E1 & + & 1 & . & . & + & + & . & + & + & 1 & & . & . & 7 \\
\hline E1 & + & + & + & 1 & + & 1 & . & . & . & . & & . & . & 6 \\
\hline E1 & . & . & . & + & + & + & 1 & 1 & 2 & . & & . & . & 6 \\
\hline E1 & . & . & . & . & 1 & . & 2 & 1 & + & + & & & + & 6 \\
\hline E1 & + & . & + & 1 & . & . & . & . & . & . & & & . & 3 \\
\hline E1 & . & + & . & + & . & . & . & + & . & . & & . & . & 3 \\
\hline E1 & . & + & + & 1 & . & . & . & . & . & . & & . & . & 3 \\
\hline E1 & . & . & . & . & + & . & + & . & + & . & & . & . & 3 \\
\hline E1 & . & + & + & . & . & . & . & . & . & . & & & . & 2 \\
\hline E1 & . & . & . & + & . & . & . & + & & . & & & . & 2 \\
\hline E1 & . & . & . & . & + & . & . & . & . & . & & + & . & 2 \\
\hline E1 & . & . & . & . & + & + & . & . & . & . & & . & . & 2 \\
\hline E1 & + & . & . & . & . & . & . & . & . & . & & . & . & 1 \\
\hline E1 & + & . & . & . & . & . & . & . & . & . & & . & . & 1 \\
\hline E1 & . & . & . & + & . & . & $\cdot$ & . & . & . & & & . & 1 \\
\hline E1 & . & . & . & . & + & . & . & . & & . & & & . & 1 \\
\hline E1 & . & . & . & . & + & . & . & . & . & . & & & . & 1 \\
\hline E1 & . & . & . & . & . & 1 & . & . & . & . & & . & . & 1 \\
\hline E1 & . & . & . & . & . & . & . & + & . & . & & . & . & 1 \\
\hline E1 & + & . & . & . & . & . & . & . & . & . & & & . & 1 \\
\hline E1 & + & . & . & . & . & + & + & + & . & . & & & . & 4 \\
\hline E1 & . & . & + & + & + & . & . & . & . & . & & . & . & 3 \\
\hline E1 & . & . & . & . & + & + & . & . & . & . & & . & . & 2 \\
\hline E1 & . & . & . & . & . & . & . & . & . & . & & + & + & 2 \\
\hline E1 & . & + & + & . & 1 & + & . & . & + & 1 & & 1 & 1 & 8 \\
\hline E1 & + & + & . & . & 1 & . & + & + & . & . & & & . & 6 \\
\hline E1 & + & . & . & + & + & + & . & . & . & + & & . & . & 5 \\
\hline E1 & . & + & + & + & + & . & . & . & . & + & & . & . & 5 \\
\hline E1 & + & . & + & . & . & . & . & + & + & . & & . & . & 4 \\
\hline E1 & + & . & . & . & . & . & + & + & + & . & & & . & 4 \\
\hline E1 & . & + & + & . & . & + & . & . & . & . & & & . & 4 \\
\hline E1 & . & + & + & 1 & . & . & . & . & . & . & & & . & 4 \\
\hline E1 & . & . & + & + & . & + & . & . & . & . & & & . & 3 \\
\hline E1 & . & . & . & . & + & . & . & . & . & 1 & & & + & 3 \\
\hline E1 & . & . & . & . & + & . & + & . & . & 1 & & & . & 3 \\
\hline E1 & + & + & . & . & . & . & . & . & . & . & & & . & 2 \\
\hline E1 & . & + & . & . & + & . & . & . & & . & & & . & 2 \\
\hline E1 & . & . & . & + & . & . & . & . & . & . & & & . & 1 \\
\hline E1 & . & . & . & . & + & . & . & . & . & . & & & . & 1 \\
\hline E1 & . & . & . & . & + & . & . & . & . & . & & & & 1 \\
\hline E1 & . & . & . & . & + & . & . & . & . & . & & & & 1 \\
\hline E1 & . & . & . & . & + & . & . & . & . & . & & & & 1 \\
\hline E1 & . & . & . & . & + & . & . & . & . & . & & & & 1 \\
\hline E1 & . & . & . & . & . & + & . & . & . & . & & & & 1 \\
\hline
\end{tabular}


Number of relevé (Zaporedna številka popisa)

Myosotis alpestris

Homogyne discolor

Juncus monanthos

Globularia nudicaulis

JT Juncetea trifidi

Campanula scheuchzeri

CU Calluno-Ulicetea

Gentiana pannonica

Coeloglossum viride

Festuca nigrescens

Potentilla erecta

FB Festuco-Brometea

Cirsium erisithales

Buphthalmum salicifolium

Carlina acaulis

Gentianella ciliata

PaT Poo alpinae-Trisetetalia

Trollius europaeus

Ranunculus nemorosus

Poa alpina

MA Molinio-Arrbenatheretea

Crepis paludosa

Veronica chamaedrys

Galium mollugo

Lathyrus pratensis

Dactylis glomerata s.str.

CD Caricetalia davallianae

Parnassia palustris

Tofieldia calyculata

EA Epilobietea angustifoliae

Fragaria vesca

Chamaenerion angustifolium

Urtica dioica

Rubus idaeus

AC Arabidetalia caeruleae

Soldanella alpina

Soldanella minima

TR Thlaspietea rotundifolii

Gymnocarpium robertianum

Hieracium bifidum

Adenostyles glabra

Trisetum argenteum

Valeriana montana

Dryopteris villarii

Cystopteris montana

Biscutella laevigata

Cy Cystopteridion fragilis

Cystopteris fragilis

Heliosperma pusillum

PC Potentilletalia caulescentis

Campanula cochleariifolia

AT Asplenietea trichomanis

Asplenium viride

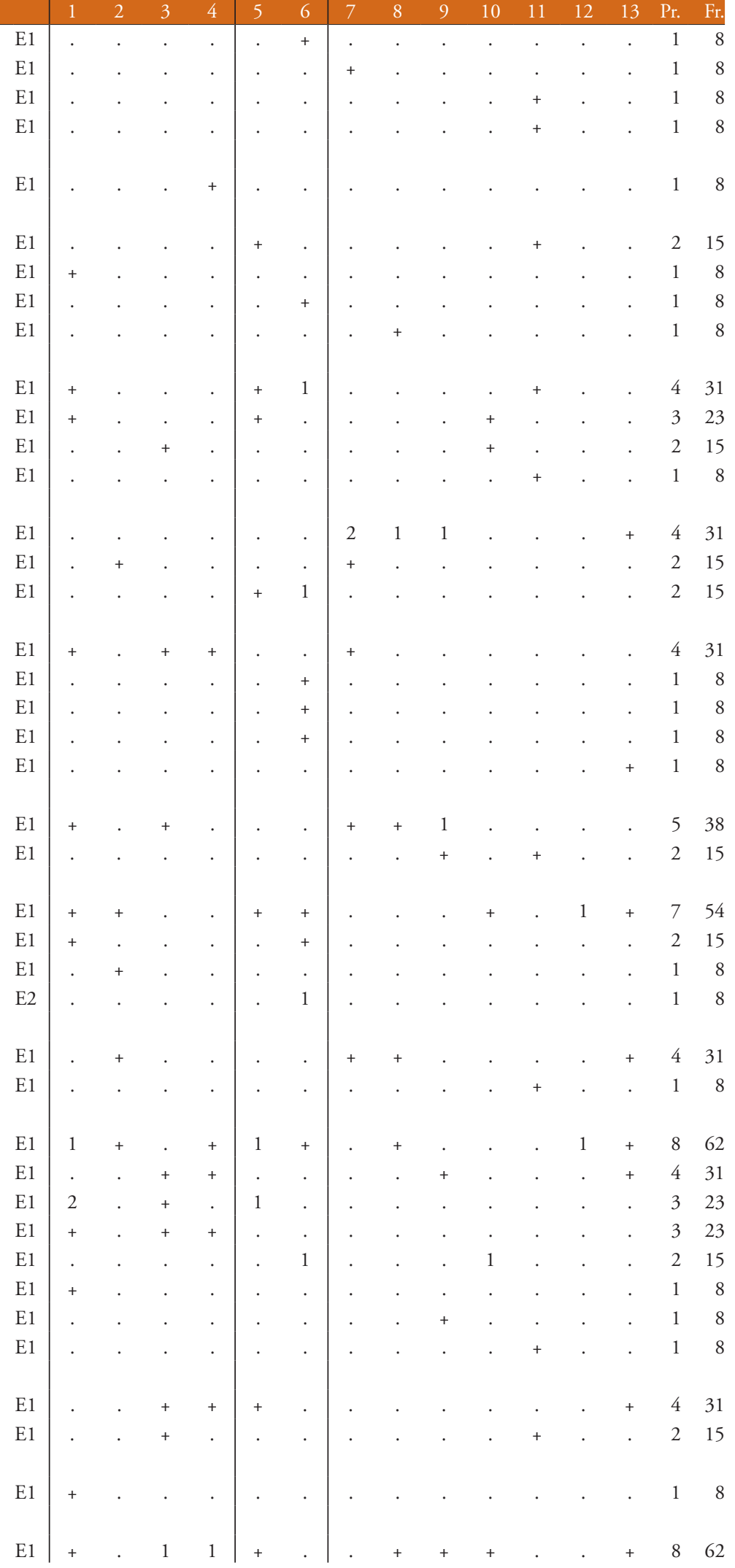


Number of relevé (Zaporedna številka popisa)

Asplenium ruta-muraria

Asplenium trichomanes

Moehringia muscosa

ML Mosses and lichens (Mahovi in lišaji)

Tortella tortuosa

Ctenidium molluscum

Dicranum scoparium

Rhytidiadelphus triquetrus

Fissidens dubius

Schistidium sp.

Hylocomium splendens

Paraleucobryum sauteri

Polytrichum formosum

Peltigera leucophlebia

Homalothecium philippeanum

Isothecium alopecuroides

Peltigera canina

Cetraria islandica

Orthothecium rufescens

Marchantia polymorpha

Homalothecium lutescens

\begin{tabular}{|c|c|c|c|c|c|c|c|c|c|c|c|c|c|c|c|}
\hline & 1 & 2 & 3 & 4 & 5 & 6 & 7 & 8 & 9 & 10 & 11 & 12 & 13 & Pr. & Fr. \\
\hline E1 & + & . & + & . & . & . & . & . & . & . & . & . & . & 2 & 15 \\
\hline E1 & + & . & + & . & . & . & . & . & . & . & . & . & . & 2 & 15 \\
\hline E1 & + & . & . & . & . & . & . & . & . & . & . & . & . & 1 & 8 \\
\hline E0 & + & . & + & + & + & + & + & + & + & + & + & . & + & 11 & 85 \\
\hline E0 & + & 1 & 1 & 1 & + & . & . & + & . & + & + & 1 & + & 10 & 77 \\
\hline E0 & . & + & . & + & + & + & . & + & . & . & + & . & . & 6 & 46 \\
\hline E0 & . & 1 & . & . & + & . & + & . & + & . & + & . & . & 5 & 38 \\
\hline E0 & + & . & + & . & . & . & . & . & + & + & . & . & . & 4 & 31 \\
\hline E0 & + & . & . & + & . & + & . & . & . & . & . & . & . & 3 & 23 \\
\hline E0 & . & . & . & + & + & . & . & . & . & . & . & . & . & 2 & 15 \\
\hline E0 & . & . & . & . & . & . & . & . & . & + & . & . & + & 2 & 15 \\
\hline E0 & + & . & . & . & . & . & . & . & . & . & . & . & . & 1 & 8 \\
\hline E0 & + & . & . & . & . & . & . & . & . & . & . & . & . & 1 & 8 \\
\hline E0 & . & . & + & . & . & . & . & . & . & . & . & . & . & 1 & 8 \\
\hline E0 & . & . & + & . & . & . & . & . & . & . & . & . & . & 1 & 8 \\
\hline E0 & . & . & . & . & + & . & . & . & . & . & . & . & . & 1 & 8 \\
\hline E0 & . & . & . & . & + & . & . & . & . & . & . & . & . & 1 & 8 \\
\hline E0 & . & . & . & . & . & . & . & + & . & . & . & . & . & 1 & 8 \\
\hline E0 & . & . & . & . & . & . & . & . & + & . & . & . & . & 1 & 8 \\
\hline E0 & . & . & . & . & . & . & . & . & . & . & + & . & . & 1 & 8 \\
\hline
\end{tabular}

Legend - Legenda

ID Igor Dakskobler

BZ Branko Zupan

AR Andrej Rozman

A Limestone - apnenec

D Dolomite - dolomit

Gr Gravel - grušč

Re Rendzina - rendzina

JA Julian Alps - Julijske Alpe

KSA Kamnik-Savinja Alps - Kamniško-Savinjske Alpe

K Karavanke - Karavanke

Pr. Presence (number of relevés in which the species is presented) - število popisov, $\mathrm{v}$ katerih se pojavlja vrsta

Fr. Frequency in $\%$ - frekvenca $v \%$ 
Table 12 (Tabela 12): Rhodothamno-Laricetum sorbetosum chamaemespili var. Rubus saxatilis

Number of relevé (Zaporedna številka popisa)

Database number of relevé

(Delovna številka popisa)

Author of the relevé (Avtor popisa)

Elevation in $\mathrm{m}$ (Nadmorska višina $\mathrm{v} \mathrm{m}$ )

Aspect (Lega)

Slope in degrees (Nagib v stopinjah)

Parent material (Matična podlaga)

Soil (Tla)

Stoniness in \% (Kamnitost v \%)

Cover in \% (Zastiranje v \%):

Upper tree layer (Zgornja drevesna plast)

Lower tree layer (Spodnja drevesna plasti)

Shrub layer (Grmovna plast)

Herb layer (Zeliščna plast)

Moss layer (Mahovna plast)

Number of species (Število vrst)

Relevé area (Velikost popisne ploskve)

Date of taking relevé (Datum popisa)

Locality (Nahajališče)

Mountain range (Pogorje)

Quadrant (Kvadrant)

Coordinate GK-Y (D-48)

Coordinate GK-X (D-48)

Character and differential species of the association

VP

Larix decidua

VP Larix decidua

RP Rhododendron hirsutum

PS Paederota lutea

AF Anemone trifolia

TR Heliosperma alpestre

TR Festuca nitida

ES Astrantia bavarica

EP Rhodothamnus chamaecistus

CF Carex ferruginea

VP Homogyne sylvestris

CA Laserpitium peucedanoides

PC Valeriana saxatilis

TR Astrantia carniolica

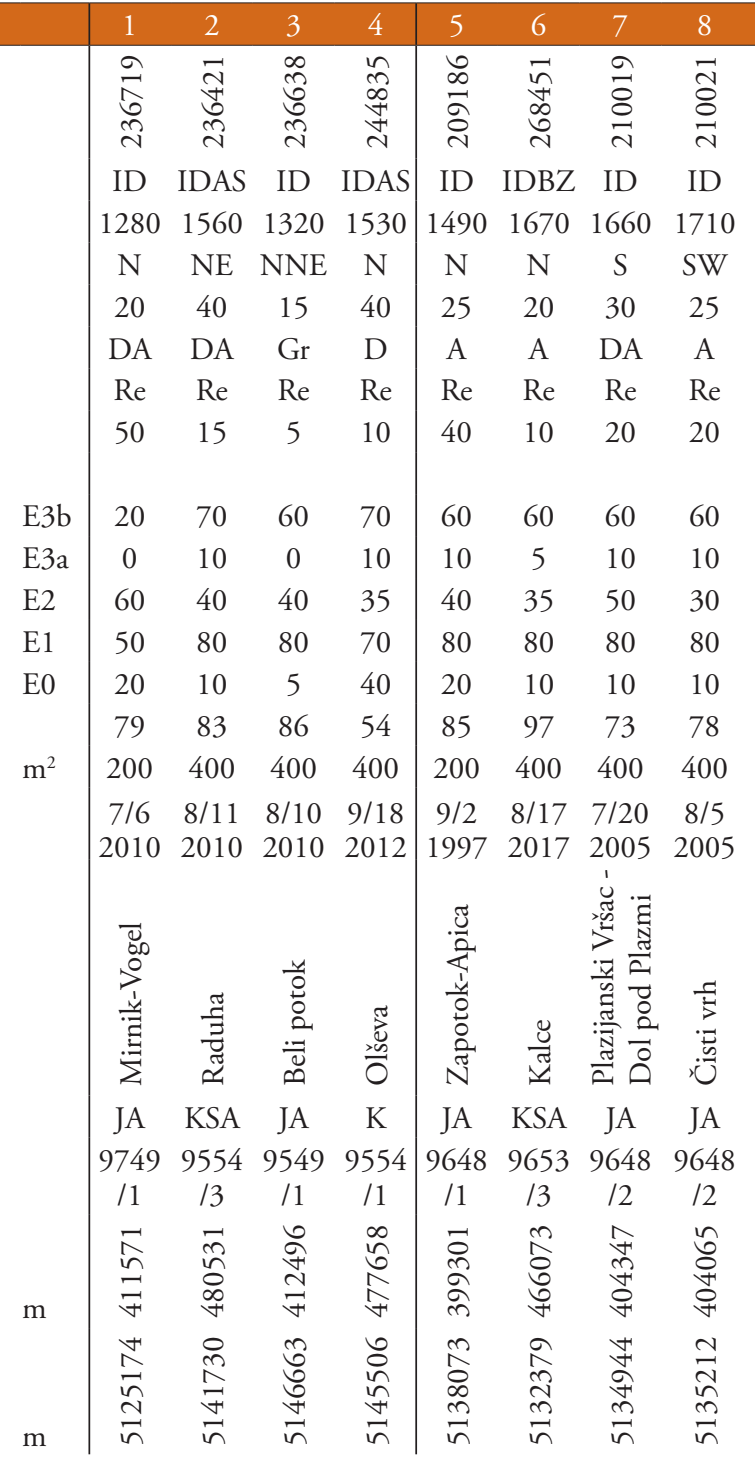

Pr. Fr.

\begin{tabular}{|c|c|c|c|c|c|c|c|c|c|c|}
\hline E3 & 2 & 4 & 4 & 4 & 4 & 4 & 4 & 4 & 8 & 100 \\
\hline E2 & 1 & 1 & 1 & + & + & . & 1 & 1 & 7 & 88 \\
\hline $\mathrm{E} 2$ & 4 & 3 & 3 & 4 & 1 & 2 & 1 & 2 & 8 & 100 \\
\hline $\mathrm{E} 1$ & + & . & + & 1 & + & 1 & + & + & 7 & 88 \\
\hline E1 & 1 & . & 1 & . & 1 & . & 1 & + & 5 & 63 \\
\hline E1 & . & + & + & . & + & . & + & 1 & 5 & 63 \\
\hline E1 & . & + & $\mathrm{r}$ & . & + & + & . & + & 5 & 63 \\
\hline E1 & . & . & + & . & + & + & + & 1 & 5 & 63 \\
\hline E1 & 1 & . & . & + & + & + & . & . & 4 & 50 \\
\hline E1 & . & 1 & 1 & . & + & + & . & . & 4 & 50 \\
\hline E1 & . & + & + & 1 & . & + & . & . & 4 & 50 \\
\hline E1 & . & + & . & . & . & + & 1 & + & 4 & 50 \\
\hline $\mathrm{E} 1$ & 1 & . & . & . & + & . & . & . & 2 & 25 \\
\hline E1 & . & . & + & . & . & . & . & . & 1 & 13 \\
\hline
\end{tabular}




\begin{tabular}{|c|c|c|c|c|c|c|c|c|c|c|c|c|}
\hline & Number of relevé (Zaporedna številka popisa) & & 1 & 2 & 3 & 4 & 5 & 6 & 7 & 8 & Pr. & Fr. \\
\hline & \multicolumn{12}{|c|}{ Differential species of the variant (Razlikovalna vrsta variante) } \\
\hline $\mathrm{EP}$ & Rubus saxatilis & E1 & 1 & + & + & 1 & 1 & 1 & 1 & 2 & 8 & 100 \\
\hline \multirow[t]{39}{*}{ VP } & Vaccinio-Piceetea & & & & & & & & & & & \\
\hline & Vaccinium myrtillus & E1 & 3 & 4 & 3 & 3 & 3 & 4 & 2 & 2 & 8 & 100 \\
\hline & Vaccinium vitis-idaea & E1 & 2 & 2 & 2 & 3 & + & 2 & 1 & 1 & 8 & 100 \\
\hline & Polystichum lonchitis & E1 & + & 1 & + & + & 1 & 1 & + & 1 & 8 & 100 \\
\hline & Calamagrostis villosa & E1 & 1 & + & 1 & 1 & 3 & 3 & 2 & 3 & 8 & 100 \\
\hline & Homogyne alpina & E1 & 1 & + & 1 & 1 & 1 & + & + & 1 & 8 & 100 \\
\hline & Oxalis acetosella & E1 & 1 & 1 & 1 & 1 & 1 & 1 & + & 1 & 8 & 100 \\
\hline & Luzula sylvatica & E1 & . & 1 & 1 & + & 1 & 1 & 1 & 2 & 7 & 88 \\
\hline & Picea abies & E3 & . & + & $\mathrm{r}$ & 1 & + & + & + & + & 7 & 88 \\
\hline & Picea abies & E2 & 1 & 1 & + & 1 & 1 & + & . & . & 6 & 75 \\
\hline & Picea abies & E1 & + & . & . & . & + & . & . & . & 2 & 25 \\
\hline & Clematis alpina & E2 & 1 & 1 & 1 & 1 & . & 1 & 1 & 1 & 7 & 88 \\
\hline & Aposeris foetida & E1 & . & + & + & + & 1 & 1 & 1 & 1 & 7 & 88 \\
\hline & Dryopteris dilatata & E1 & + & + & + & . & + & + & + & + & 7 & 88 \\
\hline & Gymnocarpium dryopteris & E1 & + & 2 & 1 & 1 & 1 & 1 & 1 & . & 7 & 88 \\
\hline & Maianthemum bifolium & E1 & + & + & + & . & + & 1 & 1 & 1 & 7 & 88 \\
\hline & Hieracium murorum & E1 & + & + & . & . & + & + & 1 & 1 & 6 & 75 \\
\hline & Melampyrum sylvaticum & E1 & + & . & . & + & + & + & 1 & + & 6 & 75 \\
\hline & Phegopteris connectilis & E1 & 1 & . & + & + & 1 & + & . & + & 6 & 75 \\
\hline & Dryopteris expansa & E1 & + & + & . & . & + & 1 & + & + & 6 & 75 \\
\hline & Valeriana tripteris & E1 & + & 1 & . & + & + & 1 & . & . & 5 & 63 \\
\hline & Lycopodium annotinum & E1 & 2 & . & . & 1 & . & 1 & + & + & 5 & 63 \\
\hline & Solidago virgaurea subsp. virgaurea & E1 & + & . & . & . & . & + & + & + & 4 & 50 \\
\hline & Saxifraga cuneifolia & E1 & 1 & . & . & + & + & . & + & . & 4 & 50 \\
\hline & Huperzia selago & E1 & + & . & . & . & + & . & + & . & 3 & 38 \\
\hline & Lonicera caerulea & E2 & 1 & . & + & . & . & + & . & . & 3 & 38 \\
\hline & Abies alba & E3 & . & . & . & . & . & . & . & + & 1 & 13 \\
\hline & Abies alba & E2 & 1 & + & . & + & . & . & . & . & 3 & 38 \\
\hline & Abies alba & E1 & + & . & . & . & . & . & . & . & 1 & 13 \\
\hline & Luzula pilosa & E1 & + & . & + & . & . & + & . & . & 3 & 38 \\
\hline & Gentiana asclepiadea & E1 & + & . & . & . & . & . & + & . & 2 & 25 \\
\hline & Calamagrostis arundinacea & E1 & + & . & . & . & + & . & . & . & 2 & 25 \\
\hline & Pyrola rotundifolia & E1 & + & . & + & . & . & . & . & . & 2 & 25 \\
\hline & Lonicera nigra & E2 & . & + & . & . & . & . & + & . & 2 & 25 \\
\hline & Luzula luzuloides & E1 & . & . & . & + & . & 2 & . & . & 2 & 25 \\
\hline & Veronica urticifolia & E1 & . & . & . & . & + & . & . & + & 2 & 25 \\
\hline & Rosa pendulina & E2 & . & . & . & . & . & + & + & . & 2 & 25 \\
\hline & Moneses uniflora & E1 & + & . & . & . & . & . & . & . & 1 & 13 \\
\hline & Blechnum spicant & E1 & . & . & . & . & . & $\mathrm{r}$ & . & . & 1 & 13 \\
\hline \multirow[t]{7}{*}{ EP } & Erico-Pinetea & & & & & & & & & & & \\
\hline & Pinus mugo & E2 & + & . & 2 & . & + & 3 & 3 & 2 & 6 & 75 \\
\hline & Erica carnea & E1 & . & 1 & 2 & 4 & + & 1 & 1 & . & 6 & 75 \\
\hline & Calamagrostis varia & E1 & . & . & 1 & + & . & + & + & . & 4 & 50 \\
\hline & Carex ornithopoda & E1 & + & . & . & + & . & . & + & . & 3 & 38 \\
\hline & Epipactis atrorubens & E1 & . & . & + & . & . & . & . & . & 1 & 13 \\
\hline & Genista radiata & E2 & . & . & . & . & . & . & + & . & 1 & 13 \\
\hline
\end{tabular}




\begin{tabular}{|c|c|c|c|c|c|c|c|c|c|c|c|c|}
\hline & Number of relevé (Zaporedna številka popisa) & & 1 & 2 & 3 & 4 & 5 & 6 & 7 & 8 & Pr. & Fr. \\
\hline \multirow[t]{7}{*}{$\mathrm{AF}$} & Aremonio-Fagion & & & & & & & & & & & \\
\hline & Cardamine enneaphyllos & E1 & + & 1 & + & . & 1 & + & 1 & + & 7 & 88 \\
\hline & Knautia drymeia & E1 & . & + & + & . & + & + & + & + & 6 & 75 \\
\hline & Cyclamen purpurascens & E1 & . & + & + & . & . & + & 1 & . & 4 & 50 \\
\hline & Helleborus niger & E1 & . & 1 & 1 & . & + & . & . & . & 3 & 38 \\
\hline & Cardamine trifolia & E1 & 1 & . & . & . & . & . & . & . & 1 & 13 \\
\hline & Rhamnus fallax & E2 & . & . & + & . & . & . & . & . & 1 & 13 \\
\hline \multirow[t]{4}{*}{ TA } & Tilio-Acerion & & & & & & & & & & & \\
\hline & Acer pseudoplatanus & E2 & . & + & . & . & . & 1 & . & . & 2 & 25 \\
\hline & Acer pseudoplatanus & E1 & . & . & + & . & . & . & . & . & 1 & 13 \\
\hline & Chrysosplenium alternifolium & E1 & . & + & . & . & . & . & . & . & 1 & 13 \\
\hline \multirow[t]{16}{*}{ FS } & Fagetalia sylvaticae & & & & & & & & & & & \\
\hline & Melica nutans & E1 & + & + & 1 & + & + & 1 & 2 & 1 & 8 & 100 \\
\hline & Paris quadrifolia & E1 & + & + & . & . & + & + & + & + & 6 & 75 \\
\hline & Daphne mezereum & E2 & . & + & + & . & . & 1 & . & + & 4 & 50 \\
\hline & Dryopteris filix-mas & E1 & . & + & . & . & + & + & . & + & 4 & 50 \\
\hline & Lilium martagon & E1 & . & . & + & . & + & + & . & + & 4 & 50 \\
\hline & Mercurialis perennis & E1 & . & . & + & . & . & + & 1 & . & 3 & 38 \\
\hline & Polystichum aculeatum & E1 & . & . & + & . & + & + & . & . & 3 & 38 \\
\hline & Lonicera alpigena & E2 & + & . & . & . & . & . & + & . & 2 & 25 \\
\hline & Galeobdolon flavidum & E1 & . & + & . & . & . & . & . & + & 2 & 25 \\
\hline & Fagus sylvatica & E3 & . & . & . & . & 1 & . & + & . & 2 & 25 \\
\hline & Fagus sylvatica & E2 & . & . & . & $\mathrm{r}$ & 2 & . & . & . & 2 & 25 \\
\hline & Prenanthes purpurea & E1 & . & . & . & + & . & + & . & . & 2 & 25 \\
\hline & Actaea spicata & E1 & + & . & . & . & . & . & . & . & 1 & 13 \\
\hline & Myosotis sylvatica & E1 & . & + & . & . & . & . & . & . & 1 & 13 \\
\hline & Symphytum tuberosum & E1 & . & 1 & . & . & . & . & . & . & 1 & 13 \\
\hline \multirow[t]{2}{*}{ QP } & Quercetalia pubescenti-petraeae & & & & & & & & & & & \\
\hline & Sorbus aria (Aria edulis) & E2 & . & . & . & . & . & + & . & . & 1 & 13 \\
\hline \multirow[t]{2}{*}{ QR } & Quercetalia roboris & & & & & & & & & & & \\
\hline & Melampyrum pratense subsp. vulgatum & E1 & . & . & . & . & . & + & . & . & 1 & 13 \\
\hline \multirow[t]{6}{*}{ QF } & Querco-Fagetea & & & & & & & & & & & \\
\hline & Hepatica nobilis & E1 & + & + & . & 1 & . & . & . & . & 3 & 38 \\
\hline & Carex digitata & E1 & . & . & . & . & + & . & . & + & 2 & 25 \\
\hline & Anemone nemorosa & E1 & + & . & . & . & . & . & . & . & 1 & 13 \\
\hline & Poa nemoralis & E1 & . & + & . & . & . & . & . & . & 1 & 13 \\
\hline & Listera ovata & E1 & . & . & + & . & . & . & . & . & 1 & 13 \\
\hline \multirow[t]{5}{*}{ SSC } & Sambuco-Salicion capreae, Rhamno-Prunetea & & & & & & & & & & & \\
\hline & Sorbus aucuparia subsp. aucuparia & E3 & + & + & . & + & . & + & . & . & 4 & 50 \\
\hline & Sorbus aucuparia subsp. aucuparia & E2 & 1 & 1 & + & 1 & + & 1 & + & + & 8 & 100 \\
\hline & Sorbus aucuparia subsp. aucuparia & E1 & + & + & 1 & + & . & . & . & . & 4 & 50 \\
\hline & Betula pendula & E3 & . & . & . & $\mathrm{r}$ & . & . & . & . & 1 & 13 \\
\hline \multirow[t]{8}{*}{ BA } & Betulo-Alnetea & & & & & & & & & & & \\
\hline & Sorbus chamaemespilus & E2 & . & 1 & + & + & 1 & 1 & 2 & 2 & 7 & 88 \\
\hline & Salix appendiculata & E2 & 1 & + & 1 & 1 & + & 1 & . & . & 6 & 75 \\
\hline & Alnus viridis & E2 & 1 & + & . & 1 & . & + & . & . & 4 & 50 \\
\hline & Salix glabra & E2 & 1 & + & 1 & + & . & & . & . & 4 & 50 \\
\hline & Juniperus sibirica & E2 & . & . & . & . & . & + & + & 1 & 3 & 38 \\
\hline & Salix waldsteiniana & E2 & + & . & . & . & . & . & . & . & 1 & 13 \\
\hline & Betula pubescens subsp. carpatica & E1 & . & & + & . & . & . & . & . & 1 & 13 \\
\hline
\end{tabular}


Number of relevé (Zaporedna številka popisa)

\begin{tabular}{l|llll|llllll} 
& 1 & 2 & 3 & 4 & 5 & 6 & 7 & 8 & Pr. & Fr.
\end{tabular}

MuA Mulgedio-Aconitetea

Veratrum album subsp. lobelianum

Athyrium filix-femina

Viola biflora

Geranium sylvaticum

Polygonatum verticillatum

Saxifraga rotundifolia

Hypericum maculatum

Chaerophyllum hirsutum

Senecio ovatus

Aconitum lycoctonum subsp. ranunculifolium

Geum rivale

Thalictrum aquilegiifolium

Adenostyles alliariae

Senecio cacaliaster

Centaurea montana

Scrophularia scopolii

Primula elatior

Rumex arifolius

Epilobium alpestre

Pedicularis recutita

Agropyron caninum

Alchemilla xanthochlora

Aconitum tauricum

CA Caricion austroalpinae

Heracleum austriacum subsp. siifolium

Koeleria eriostachya

Arabis vochinensis

Pulsatilla alpina subsp. austroalpina

Festuca calva

Cfir Caricion firmae

Helianthemum alpestre

Dryas octopetala

CF Caricion ferrugineae

Cerastium subtriflorum

ES

\section{Elyno-Seslerietea}

Sesleria caerulea

Campanula witasekiana

Aster bellidiastrum

Polygonum viviparum

Betonica alopecuros

Senecio abrotanifolius

Phyteuma orbiculare

Selaginella selaginoides

Ranunculus montanus

Juncus monanthos

Thymus praecox subsp. polytrichus

Ranunculus carinthiacus

Helianthemum nummularium subsp. grandiflorum

Leucanthemum heterophyllum (L.maximum agg.)

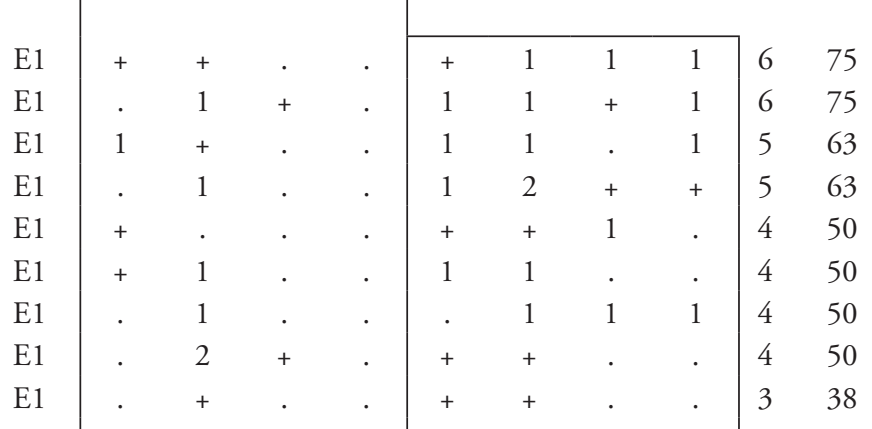

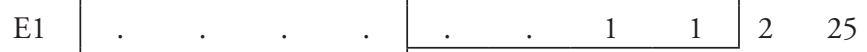

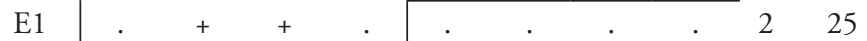

$\mathrm{E} 1$. . . . . . . . 252

E1 . . . . . + . . 25

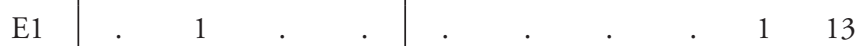

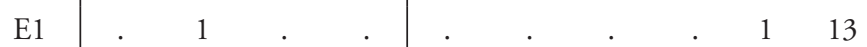

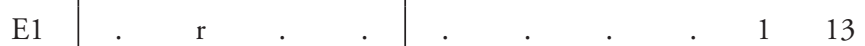

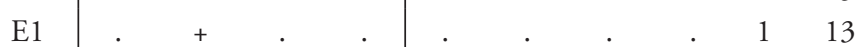

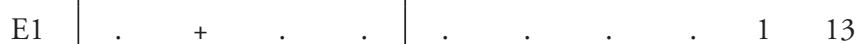

E1 . + . . . . . . . . .

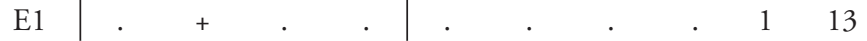

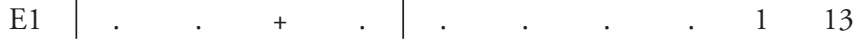

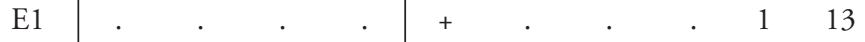

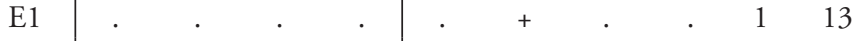

E1 . . . . . $\quad+\quad+\quad 25$

E1 . . . . . $\quad+\quad+\quad+\quad 25$

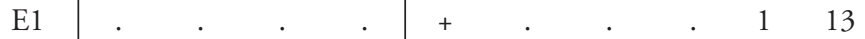

E1 . . . . . . . . .

E1 . . . . . . . . . . .

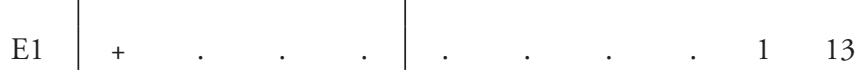

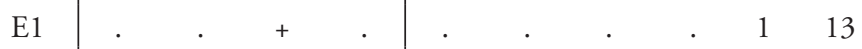

E1 
Number of relevé (Zaporedna številka popisa)

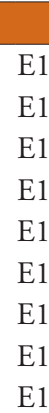

alpigena

Hieracium pilosum

Galium anisophyllum

Lotus alpinus

Homogyne discolor

Carduus crassifolius

Myosotis alpestris

Potentilla crantzii

Phleum hirsutum

JT Juncetea trifidi

Campanula scheuchzeri

Solidago virgaurea subsp. minuta

CU Calluno-Ulicetea

Gentiana pannonica

Festuca nigrescens

Potentilla erecta

Agrostis capillaris

Potentilla aurea

FB Festuco-Brometea

Gymnadenia conopsea

PaT Poo alpinae-Trisetetalia

Poa alpina

Ranunculus nemorosus

MA Molinio-Arrhenatheretea

Crepis paludosa

Deschampsia cespitosa

Cerastium fontanum

Caltha palustris

Prunella vulgaris

Festuca rubra

Anthoxanthum odoratum

CD Caricetalia davallianae

Carex capillaris

Parnassia palustris

Tofieldia calyculata

EA Epilobietea angustifoliae

Fragaria vesca

Rubus idaeus

Urtica dioica

AC Arabidetea coeruleae

Soldanella alpina

Salix retusa

TR Thlaspietea rotundifolii

Gymnocarpium robertianum

Adenostyles glabra

Cystopteris montana

Hieracium bifidum

Saxifraga sedoides

Rumex scutatus

Trisetum argenteum

Valeriana montana

\begin{tabular}{|c|c|c|c|c|c|c|c|c|c|c|}
\hline & 1 & 2 & 3 & 4 & 5 & 6 & 7 & 8 & Pr. & Fr. \\
\hline E1 & . & . & . & . & . & . & + & 1 & 2 & 25 \\
\hline E1 & + & . & . & . & . & . & . & . & 1 & 13 \\
\hline E1 & . & . & + & . & . & . & . & . & 1 & 13 \\
\hline E1 & . & . & + & . & . & . & . & . & 1 & 13 \\
\hline E1 & . & . & + & . & . & . & . & . & 1 & 13 \\
\hline E1 & . & . & . & . & . & + & . & . & 1 & 13 \\
\hline E1 & . & . & . & . & . & . & . & + & 1 & 13 \\
\hline E1 & . & . & . & . & . & . & . & + & 1 & 13 \\
\hline E1 & . & . & . & . & . & . & . & + & 1 & 13 \\
\hline E1 & . & . & . & . & + & + & . & + & 3 & 38 \\
\hline E1 & + & . & . & . & . & . & . & . & 1 & 13 \\
\hline E1 & . & . & . & . & . & + & . & + & 2 & 25 \\
\hline E1 & . & . & . & . & . & . & + & + & 2 & 25 \\
\hline E1 & . & . & 1 & . & . & . & . & . & 1 & 13 \\
\hline E1 & . & . & + & . & . & . & . & . & 1 & 13 \\
\hline E1 & . & . & . & . & . & . & . & + & 1 & 13 \\
\hline E1 & . & . & + & . & . & . & . & . & 1 & 13 \\
\hline E1 & . & . & . & . & + & . & . & + & 2 & 25 \\
\hline E1 & . & . & . & . & + & + & . & . & 2 & 25 \\
\hline E1 & . & + & + & . & . & + & . & . & 3 & 38 \\
\hline E1 & + & + & . & . & . & . & . & . & 2 & 25 \\
\hline E1 & . & + & . & . & . & . & . & . & 1 & 13 \\
\hline E1 & . & + & . & . & . & . & . & . & 1 & 13 \\
\hline E1 & . & . & + & . & . & . & . & . & 1 & 13 \\
\hline E1 & . & . & . & . & . & + & . & . & 1 & 13 \\
\hline E1 & . & . & . & . & . & . & . & + & 1 & 13 \\
\hline E1 & + & . & . & . & . & . & . & . & 1 & 13 \\
\hline E1 & . & . & + & . & . & . & . & . & 1 & 13 \\
\hline E1 & . & . & + & . & . & . & . & . & 1 & 13 \\
\hline E1 & . & + & + & . & + & . & + & . & 4 & 50 \\
\hline E2 & . & + & . & . & . & . & . & . & 1 & 13 \\
\hline E1 & . & + & . & . & . & . & . & . & 1 & 13 \\
\hline E1 & . & . & . & . & + & + & . & . & 2 & 25 \\
\hline E1 & + & . & . & . & . & . & . & . & 1 & 13 \\
\hline E1 & + & . & 1 & 1 & . & . & + & . & 4 & 50 \\
\hline E1 & . & . & + & + & + & + & . & . & 4 & 50 \\
\hline E1 & 1 & 1 & . & . & + & . & . & . & 3 & 38 \\
\hline E1 & + & . & + & . & . & . & . & . & 2 & 25 \\
\hline E1 & + & . & . & . & . & . & . & . & 1 & 13 \\
\hline E1 & . & . & + & . & . & . & . & . & 1 & 13 \\
\hline E1 & . & . & . & + & . & . & . & . & 1 & 13 \\
\hline E1 & . & . & . & . & + & . & . & . & 1 & 13 \\
\hline
\end{tabular}




\begin{tabular}{|c|c|c|c|c|c|c|c|c|c|c|c|c|}
\hline & Number of relevé (Zaporedna številka popisa) & & 1 & 2 & 3 & 4 & 5 & 6 & 7 & 8 & Pr. & Fr. \\
\hline & Dryopteris villarii & E1 & . & . & . & . & . & + & . & . & 1 & 13 \\
\hline & Rhodiola rosea & E1 & . & . & . & . & . & + & . & . & 1 & 13 \\
\hline \multirow[t]{4}{*}{ Сy } & Cystopteridion fragilis & & & & & & & & & & & \\
\hline & Cystopteris fragilis & E1 & + & + & . & . & + & + & + & + & 6 & 75 \\
\hline & Carex brachystachys & E1 & + & . & . & . & . & . & . & . & 1 & 13 \\
\hline & Cystopteris regia & E1 & . & . & . & . & . & . & . & + & 1 & 13 \\
\hline \multirow[t]{4}{*}{ PC } & Potentilletalia caulescentis & & & & & & & & & & & \\
\hline & Campanula cochleariifolia & E1 & + & + & + & + & . & + & . & . & 5 & 63 \\
\hline & Arabis bellidiofolia subsp. stellulata & E1 & . & . & r & . & . & . & . & . & 1 & 13 \\
\hline & Festuca stenantha & E1 & . & . & . & . & . & . & + & . & 1 & 13 \\
\hline \multirow[t]{7}{*}{ AT } & Asplenietea trichomanis & & & & & & & & & & & \\
\hline & Asplenium viride & E1 & + & + & . & + & 1 & 1 & + & 1 & 7 & 88 \\
\hline & Asplenium ruta-muraria & E1 & . & . & . & . & + & . & + & + & 3 & 38 \\
\hline & Asplenium trichomanes & E1 & . & . & . & . & + & . & . & . & 1 & 13 \\
\hline & Potentilla sp. & E1 & . & . & . & . & + & . & . & . & 1 & 13 \\
\hline & Hieracium dollineri & E1 & . & . & . & . & . & + & . & . & 1 & 13 \\
\hline & Moehringia muscosa & E1 & . & . & . & . & . & . & . & + & 1 & 13 \\
\hline \multirow[t]{26}{*}{ ML } & Mosses and lichens (Mahovi in lišaji) & & & & & & & & & & & \\
\hline & Tortella tortuosa & E0 & . & + & + & + & 1 & 1 & 1 & 1 & 7 & 88 \\
\hline & Rhytidiadelphus triquetrus & E0 & 2 & 2 & 2 & 2 & + & 1 & . & . & 6 & 75 \\
\hline & Ctenidium molluscum & E0 & . & . & . & + & 1 & + & 1 & 1 & 5 & 63 \\
\hline & Dicranum scoparium & E0 & . & . & + & + & . & + & + & + & 5 & 63 \\
\hline & Peltigera leucophlebia & E0 & + & + & . & . & + & + & . & . & 4 & 50 \\
\hline & Hylocomium splendens & E0 & 1 & + & . & 2 & . & . & . & . & 3 & 38 \\
\hline & Polytrichum formosum & E0 & 1 & . & . & . & 1 & 1 & . & . & 3 & 38 \\
\hline & Fissidens dubius & E0 & + & . & . & . & 1 & . & . & . & 2 & 25 \\
\hline & Plagiothecium sylvaticum & E0 & . & + & . & . & . & + & . & . & 2 & 25 \\
\hline & Schistidium sp. & E0 & . & . & + & . & . & . & . & + & 2 & 25 \\
\hline & Rhytidiadelphus loreus & E0 & . & . & . & . & + & + & . & . & 2 & 25 \\
\hline & Orthothecium rufescens & E0 & + & . & . & . & . & . & . & . & 1 & 13 \\
\hline & Sphagnum sp. & E0 & + & . & . & . & . & . & . & . & 1 & 13 \\
\hline & Cetraria islandica & E0 & + & . & . & . & . & . & . & . & 1 & 13 \\
\hline & Sanionia uncinata & E0 & + & . & . & . & . & . & . & . & 1 & 13 \\
\hline & Polytrichum commune & E0 & + & . & . & . & . & . & . & . & 1 & 13 \\
\hline & Marchantia polymorpha & E0 & . & . & + & . & . & . & . & . & 1 & 13 \\
\hline & Sphagnum quinquefarium & E0 & . & . & . & 1 & . & . & . & . & 1 & 13 \\
\hline & Plagiothecium undulatum & E0 & . & . & . & 2 & . & . & . & . & 1 & 13 \\
\hline & Eurhynchium angustirete & E0 & . & . & . & + & . & . & . & . & 1 & 13 \\
\hline & Scapania aequiloba & E0 & . & . & . & + & . & . & . & . & 1 & 13 \\
\hline & Plagiochila porelloides & E0 & . & . & . & . & 1 & . & . & . & 1 & 13 \\
\hline & Rhizomnium punctatum & E0 & . & . & . & . & + & . & . & . & 1 & 13 \\
\hline & Peltigera canina & E0 & . & . & . & . & . & . & . & + & 1 & 13 \\
\hline & Vulpicida pinastri & E3c & . & & . & . & . & . & . & + & 1 & 13 \\
\hline
\end{tabular}

\section{Legend - Legenda}

ID Igor Dakskobler

AS Andrej Seliškar

BZ Branko Zupan

A Limestone - apnenec

D Dolomite - dolomit

Gr Gravel - grušč
Re Rendzina - rendzina

JA Julian Alps - Julijske Alpe

KSA Kamnik-Savinja Alps - Kamniško-Savinjske Alpe

K Karavanke - Karavanke

Pr. Presence (number of relevés in which the species is presented) - število popisov, $\mathrm{v}$ katerih se pojavlja vrsta

Fr. Frequency in $\%-$ frekvenca $v \%$ 
Table 13 (Tabela 13): Rhodothamno-Laricetum anemonetosum trifoliae var. typica

\begin{tabular}{|c|c|c|c|c|c|c|c|c|c|c|c|c|c|c|c|c|c|}
\hline Number of relevé (Zaporedna številka popisa) & & 1 & 2 & 3 & 4 & 5 & 6 & 7 & 8 & 9 & 10 & 11 & 12 & 13 & 14 & 15 & 16 \\
\hline $\begin{array}{l}\text { Database number of relevé } \\
\text { (Delovna številka popisa) }\end{array}$ & & $\begin{array}{l}\vec{\Xi} \\
\stackrel{+}{~} \\
\underset{d}{J}\end{array}$ & 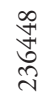 & $\frac{\stackrel{n}{F}}{\underset{\overbrace{}}{\tilde{D}}}$ & $\begin{array}{l}\text { त్ర } \\
\text { तై } \\
\end{array}$ & 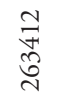 & 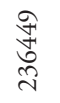 & 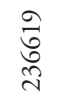 & \begin{tabular}{l} 
के \\
\multirow{ָ}{0}{} \\
$\underset{\sim}{ }$
\end{tabular} & $\begin{array}{l}\stackrel{+}{\infty} \\
\stackrel{\sim}{\stackrel{\sim}{*}}\end{array}$ & $\begin{array}{l}\text { ָิ } \\
\stackrel{\Xi}{\sim}\end{array}$ & 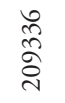 & 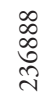 & $\begin{array}{l}\overline{\widehat{ล}} \\
\text { } \\
\text { ते }\end{array}$ & $\begin{array}{l}\infty \\
\stackrel{\infty}{\hat{~}} \\
\hat{\tilde{\nu}}\end{array}$ & $\begin{array}{l}\hat{\tilde{n}} \\
\stackrel{\hat{े}}{ }\end{array}$ & 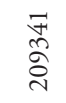 \\
\hline Author of the relevé (Avtor popisa) & & $\theta$ & $\stackrel{\infty}{\varrho}$ & 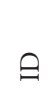 & $\sum^{\prime}$ & $\ominus$ & 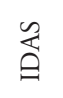 & 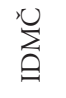 & $\stackrel{\infty}{\varrho}$ & $\stackrel{\infty}{\stackrel{c}{\epsilon}}$ & $\ominus$ & $\ominus$ & 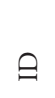 & $\ominus$ & $\Theta$ & $\theta$ & \\
\hline Elevation in $\mathrm{m}$ (Nadmorska višina $\mathrm{v} \mathrm{m})$ & & 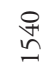 & $\tilde{\check{\check{\sigma}}}$ & $\stackrel{8}{\&}$ & $\stackrel{8}{\circledR}$ & $\stackrel{R}{\stackrel{R}{n}}$ & 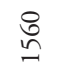 & 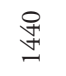 & $\stackrel{n}{n}$ & $\stackrel{\overbrace{}}{\underset{7}{-}}$ & 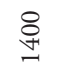 & $\underset{-}{8}$ & 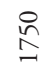 & 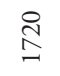 & $\stackrel{8}{\circledR}$ & $\underset{+}{\stackrel{+}{Z}}$ & $\stackrel{尺}{\underset{t}{f}}$ \\
\hline Aspect (Lega) & & 点 & Z & Z & Z & Z & 妾 & Z & z & Z & 崩 & $\mathrm{z}$ & Z & 㐎 & 㟧 & Z & Z \\
\hline Slope in degrees (Nagib v stopinjah) & & 50 & 60 & 45 & 45 & 40 & 35 & 40 & 70 & 45 & 45 & 45 & 50 & 45 & 45 & 25 & 40 \\
\hline Parent material (Matična podlaga) & & DA & A & $\mathrm{D}$ & DA & $\mathrm{D}$ & DA & DA & A & $\mathrm{D}$ & DA & A & DA & DA & A & A & A \\
\hline Soil (Tla) & & $\operatorname{Re}$ & $\operatorname{Re}$ & $\operatorname{Re}$ & $\operatorname{Re}$ & $\operatorname{Re}$ & $\operatorname{Re}$ & $\mathrm{Re}$ & $\operatorname{Re}$ & $\operatorname{Re}$ & $\operatorname{Re}$ & $\operatorname{Re}$ & $\operatorname{Re}$ & $\mathrm{Re}$ & $\operatorname{Re}$ & $\operatorname{Re}$ & $\operatorname{Re}$ \\
\hline Stoniness in \% (Kamnitost v \%) & & 40 & 50 & 50 & 20 & 30 & 30 & 30 & 40 & 40 & 20 & 30 & 20 & 40 & 40 & 50 & 40 \\
\hline \multicolumn{18}{|l|}{ Cover in \% (Zastiranje v \%): } \\
\hline Upper tree layer (Zgornja drevesn & E3b & 50 & 60 & 60 & 60 & 60 & 70 & 60 & 40 & 50 & 60 & 50 & 50 & 60 & 60 & 60 & 50 \\
\hline Lower tree layer (Spodnja drevesna plasti) & E3a & 10 & 10 & 5 & 5 & 10 & 5 & 5 & 5 & 10 & 10 & 10 & 5 & 10 & 0 & 10 & 10 \\
\hline Shrub layer (Grmovna plast) & E2 & 20 & 15 & 10 & 20 & 10 & 10 & 10 & 15 & 30 & 20 & 20 & 20 & 30 & 20 & 40 & 0 \\
\hline Herb layer (Zeliščna plast) & E1 & 70 & 60 & 70 & 70 & 80 & 70 & 70 & 60 & 60 & 70 & 80 & 70 & 70 & 70 & 70 & 60 \\
\hline Moss layer (Mahovna plast) & E0 & 10 & 5 & 10 & 5 & 10 & 2 & 10 & 10 & 10 & 20 & 20 & 10 & 10 & 10 & 30 & 20 \\
\hline Number of species (Število vrst) & & 75 & 83 & 62 & 73 & 57 & 58 & 69 & 52 & 41 & 51 & 75 & 71 & 63 & 67 & 84 & 68 \\
\hline Relevé area (Velikost popisne ploskve) & $\mathrm{m}^{2}$ & 400 & 400 & 400 & 400 & 400 & 400 & 400 & 200 & 200 & 200 & 200 & 400 & 200 & 200 & 200 & 200 \\
\hline Date of taking relevé (Datum popisa) & & $\begin{array}{c}8 / 8 \\
2012\end{array}$ & $\begin{array}{l}7 / 7 \\
2010\end{array}$ & $\begin{array}{l}7 / 4 \\
2016\end{array}$ & $\begin{array}{l}8 / 23 \\
2010\end{array}$ & $\begin{array}{l}7 / 4 \\
2016\end{array}$ & $\begin{array}{l}7 / 7 \\
2010\end{array}$ & $\begin{array}{l}8 / 23 \\
2010\end{array}$ & $\begin{array}{l}8 / 16 \\
2010\end{array}$ & $\begin{array}{l}9 / 12 \\
2012\end{array}$ & $\begin{array}{l}7 / 25 \\
2005\end{array}$ & $\begin{array}{l}9 / 16 \\
2002\end{array}$ & $\begin{array}{l}9 / 22 \\
2010\end{array}$ & & & $\begin{array}{l}9 / 17 \\
2002\end{array}$ & \\
\hline
\end{tabular}

Locality (Nahajališče)

Mountain range (Pogorje)

Quadrant (Kvadrant)

Coordinate GK-Y (D-48)

Coordinate GK-X (D-48)

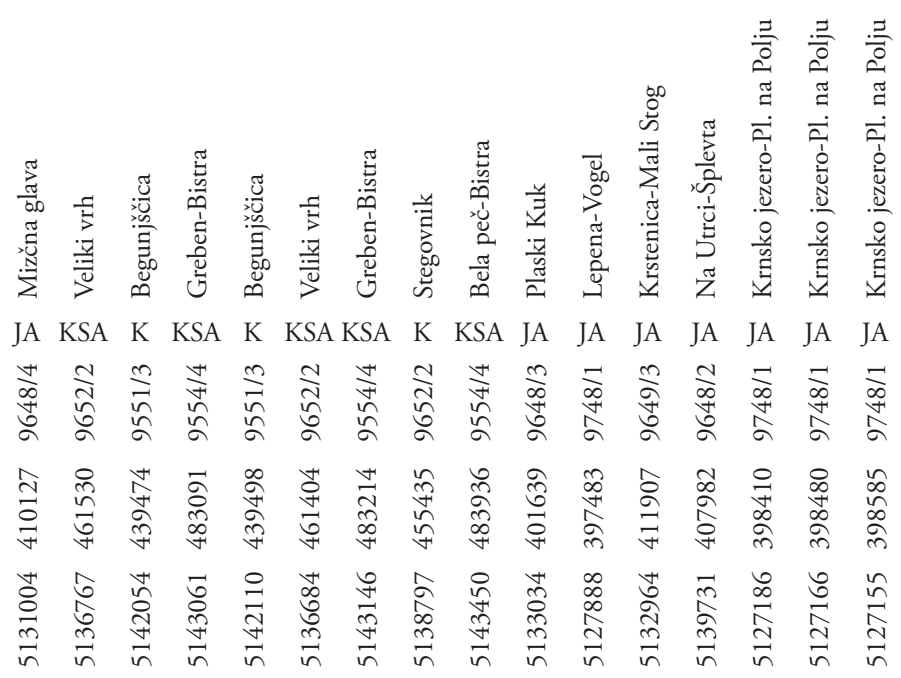

Character and differential species of the association (Značilnice in razlikovalnice asociacije)

VP Larix decidua

EP Rhododendron hirsutum

EP Rhodothamnus chamaecistus

PS Paederota lutea

PC Valeriana saxatilis

VP Homogyne sylvestris

CA Laserpitium peucedanoides

TR Heliosperma alpestre

$\begin{array}{lllllllllllllllll}\text { E3 } & 3 & 3 & 4 & 4 & 4 & 4 & 4 & 3 & 3 & 4 & 3 & 3 & 4 & 4 & 4 & 3 \\ \text { E2 } & 1 & + & + & + & + & + & + & 1 & 1 & 1 & 1 & 1 & 1 & 1 & 1 & 1 \\ \text { E1 } & . & . & . & + & + & . & . & . & . & . & . & + & . & + & . & . \\ \text { E2 } & 1 & + & \mathrm{r} & 1 & + & . & + & 1 & + & 3 & 3 & 3 & 3 & 3 & 3 & 3 \\ \text { E1 } & 2 & 1 & + & 1 & 1 & + & + & 2 & 1 & 3 & 2 & 2 & 2 & 3 & 1 & 2 \\ \text { E1 } & 1 & 2 & 1 & + & 1 & 1 & + & 1 & + & 1 & 1 & 1 & 1 & 1 & 1 & 1 \\ \text { E1 } & 1 & + & 1 & . & 1 & 1 & 1 & 1 & 1 & + & 1 & 1 & + & 1 & 1 & 1 \\ \text { E1 } & + & 1 & + & 1 & 1 & . & 1 & + & + & + & + & 1 & 1 & . & + & + \\ \text { E1 } & 1 & . & . & 1 & 1 & + & + & 1 & + & 1 & 1 & + & 1 & 1 & 1 & 1 \\ \text { E1 } & . & + & . & + & + & + & + & + & + & . & + & 1 & 1 & + & + & .\end{array}$


$\begin{array}{llllllllllllllllllllllllllllll}17 & 18 & 19 & 20 & 21 & 22 & 23 & 24 & 25 & 26 & 27 & 28 & 29 & 30 & 31 & 32 & 33 & 34 & 35 & 36 & 37 & 38 & 39 & 40 & 41 & 42 & 43 & 44 & \text { Pr. } & \text { Fr. }\end{array}$

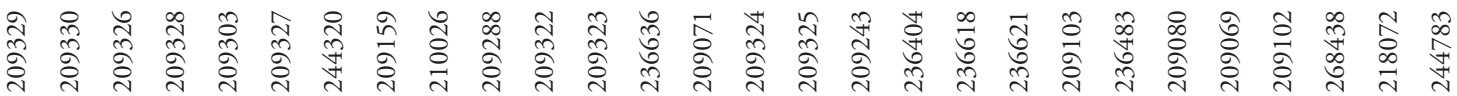

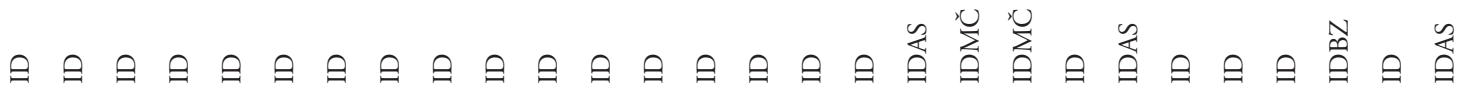

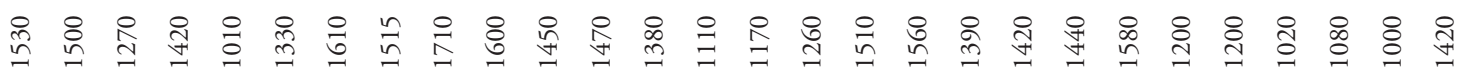

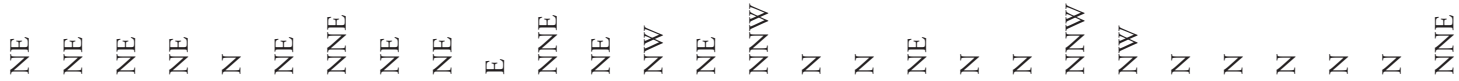
$\begin{array}{llllllllllllllllllllllllllll}45 & 45 & 50 & 45 & 45 & 50 & 47 & 45 & 45 & 45 & 45 & 40 & 60 & 45 & 45 & 45 & 45 & 60 & 60 & 50 & 45 & 45 & 40 & 50 & 45 & 45 & 45 & 45\end{array}$ A A A A A A DA A A A A A DA DR A A A DA DA DA DA A AR ALR AR D DA D $\operatorname{Re} \operatorname{Re} \operatorname{Re} \operatorname{Re} \operatorname{Re} \operatorname{Re} \operatorname{Re} \operatorname{Re} \operatorname{Re} \operatorname{Re} \operatorname{Re} \operatorname{Re} \operatorname{Re} \operatorname{Re} \operatorname{Re} \operatorname{Re} \operatorname{Re} \operatorname{Re} \operatorname{Re} \operatorname{Re} \operatorname{Re} \operatorname{Re} \operatorname{Re} \operatorname{Re} \operatorname{Re} L i{ }^{2} \operatorname{Re} \operatorname{Re}$

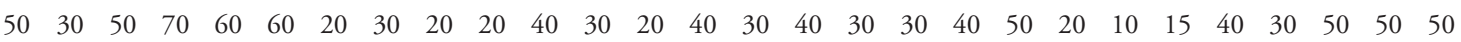

$\begin{array}{llllllllllllllllllllllllllll}60 & 70 & 60 & 60 & 70 & 50 & 50 & 80 & 70 & 70 & 60 & 80 & 50 & 40 & 60 & 60 & 60 & 60 & 60 & 50 & 50 & 50 & 70 & 60 & 50 & 50 & 60 & 50\end{array}$ $\begin{array}{llllllllllllllllllllllllllll}10 & 10 & 20 & 20 & 20 & 10 & 10 & 0 & 0 & 0 & 0 & 0 & 10 & 20 & 10 & 0 & 10 & 0 & 5 & 5 & 0 & 5 & 10 & 20 & 20 & 10 & 0 & 10\end{array}$ $\begin{array}{lllllllllllllllllllllllllllll}30 & 20 & 20 & 10 & 20 & 10 & 20 & 5 & 30 & 20 & 20 & 20 & 20 & 40 & 30 & 30 & 20 & 20 & 20 & 10 & 60 & 10 & 20 & 15 & 40 & 20 & 20 & 20\end{array}$ $\begin{array}{lllllllllllllllllllllllllllll}60 & 60 & 50 & 60 & 60 & 50 & 80 & 60 & 70 & 70 & 70 & 70 & 70 & 70 & 70 & 70 & 70 & 70 & 60 & 50 & 50 & 80 & 80 & 60 & 80 & 60 & 60 & 50\end{array}$ $\begin{array}{llllllllllllllllllllllllllll}20 & 10 & 10 & 20 & 10 & 10 & 0 & 10 & 10 & 10 & 10 & 10 & 10 & 10 & 20 & 20 & 10 & 2 & 5 & 5 & 10 & 10 & 10 & 10 & 10 & 10 & 10 & 20\end{array}$ $\begin{array}{llllllllllllllllllllllllllll}122 & 96 & 63 & 89 & 57 & 76 & 61 & 72 & 58 & 68 & 70 & 101 & 70 & 60 & 69 & 60 & 70 & 61 & 65 & 58 & 42 & 49 & 66 & 58 & 73 & 55 & 39 & 41\end{array}$ 400400200400200200400200200200400400200200200400200400400400200200200200200200100200

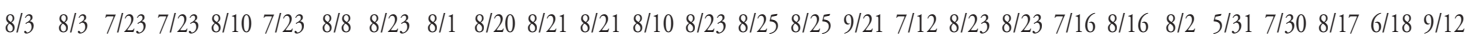
1999199919991999200019992012200420052002199819982010199119981998200120102010201019972010199020021992201720052012

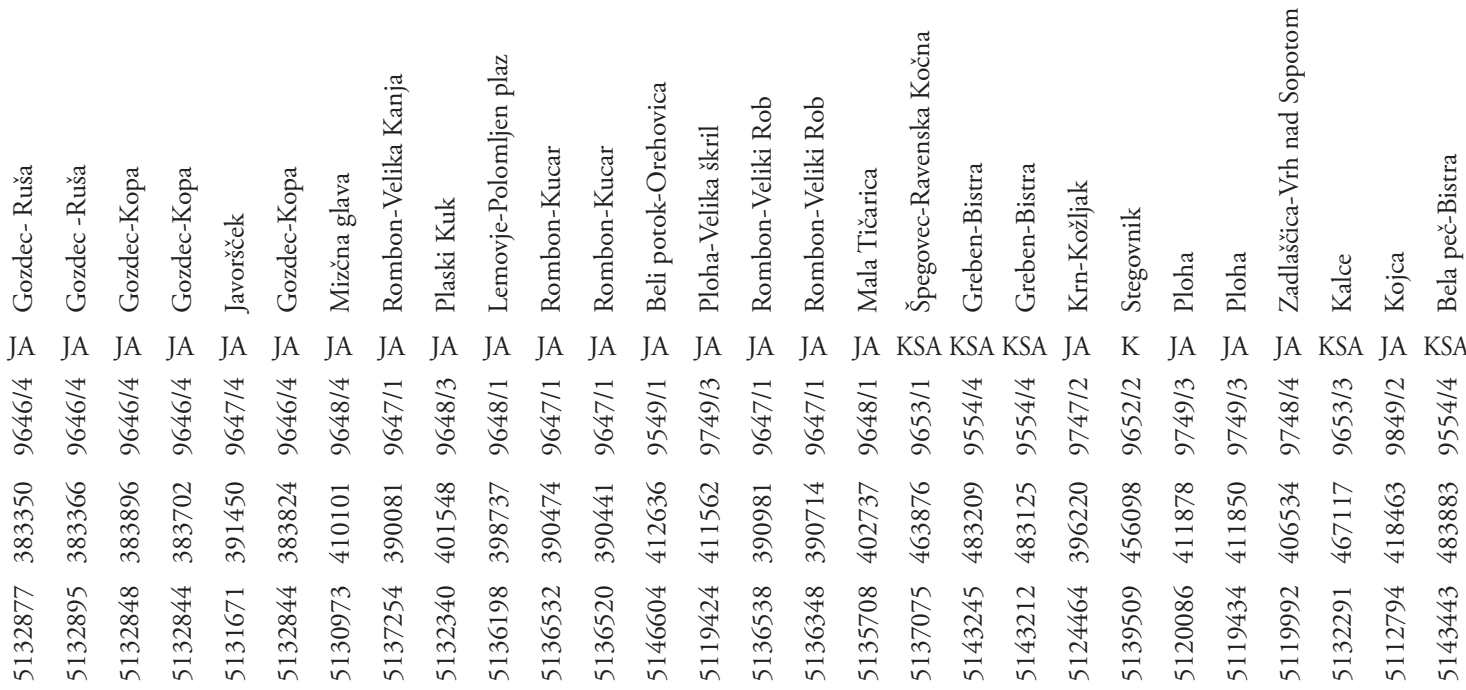

Pr. Fr.

$\begin{array}{rlllllllllllllllllllllllllllllllllllll}4 & 4 & 4 & 4 & 4 & 3 & 4 & 4 & 4 & 4 & 4 & 4 & 3 & 3 & 4 & 3 & 4 & 4 & 3 & 3 & 3 & 3 & 3 & 4 & 3 & 3 & 4 & 3 & 44 & 100 \\ + & + & + & 1 & + & + & 1 & 2 & 1 & 1 & 1 & 1 & 1 & + & 1 & 1 & + & + & 1 & 1 & 1 & 1 & + & . & + & 1 & + & 1 & 43 & 98 \\ + & + & . & + & . & . & . & + & . & . & + & . & . & . & . & . & + & . & + & . & . & + & . & . & . & . & . & . & 12 & 27 \\ 1 & + & + & + & + & + & 2 & 2 & 2 & + & 1 & 1 & 1 & 2 & 2 & 2 & 1 & 1 & 1 & 1 & 3 & 2 & 1 & 1 & 1 & + & . & + & 42 & 95 \\ + & + & 1 & 1 & + & 1 & 1 & 1 & 2 & 1 & 1 & 1 & 2 & 1 & 1 & 1 & 2 & 2 & 2 & 1 & + & + & + & . & . & + & . & + & 42 & 95 \\ 1 & 1 & 1 & 1 & + & + & 1 & + & 1 & + & + & + & 1 & + & + & + & 1 & 1 & + & 1 & + & 1 & . & + & + & 1 & . & + & 42 & 95 \\ + & + & + & 1 & + & + & + & . & . & + & 1 & + & 1 & 1 & + & 1 & 1 & . & 1 & + & + & 1 & 1 & + & . & + & 1 & 1 & 40 & 91 \\ \mathrm{r} & + & 1 & 1 & 1 & + & + & 1 & 1 & . & + & + & + & + & 1 & + & 1 & 1 & 1 & 1 & + & + & 1 & . & + & + & . & . & 38 & 86 \\ + & + & + & 1 & . & + & 1 & 1 & 1 & 1 & 1 & 1 & + & + & + & + & 1 & + & 1 & . & 1 & 1 & + & + & . & + & . & . & 37 & 84 \\ . & . & . & . & . & . & + & + & . & . & + & . & + & . & + & . & + & + & + & . & . & + & . & . & . & . & . & + & 22 & 50\end{array}$




\section{Number of relevé (Zaporedna številka popisa)}

\begin{tabular}{ll}
\hline AF Anemone trifolia & E1 \\
ES Astrantia bavarica & E1 \\
TR Festuca nitida & E1 \\
TR Astrantia carniolica & E1 \\
PS Campanula carnica & E1 \\
CF Carex ferruginea & E1
\end{tabular}

Differential species of the subassociation (Razlikovalne vrste subasociacije)

$\begin{array}{ll}\text { ES } & \text { Sesleria caerulea } \\ \text { PC } & \text { Primula auricula } \\ \text { PS } & \text { Saxifraga crustata } \\ \text { Cfr } & \text { Carex brachystachys } \\ \text { VP } & \text { Vaccinio-Piceetea }\end{array}$

Clematis alpina

Picea abies

Picea abies

Picea abies

Valeriana tripteris

Vaccinium vitis-idaed

Solidago virgaurea subsp. virgaurea

Vaccinium myrtillus

Rosa pendulina

Oxalis acetosella

Luzula sylvatica

Polystichum lonchitis

Huperzia selago

Calamagrostis villosa

Homogyne alpina

Hieracium murorum

Saxifraga cuneifolia

Melampyrum sylvaticum

Maianthemum bifolium

Phegopteris connectilis

Gymnocarpium dryopteris

Lycopodium annotinum

Veronica urticifolia

Abies alba

Abies alba

Abies alba

Aposeris foetida

Luzula luzuloides

Calamagrostis arundinacea

Dryopteris dilatata

Gentiana asclepiadea

Lonicera caerulea

Dryopteris expansa

Lonicera nigra

EP Erico-Pinetea

Calamagrostis varia

Erica carnea

Pinus mugo

Rubus saxatilis

Carex ornithopoda

Aquilegia nigricans

Polygala chamaebuxus

Amelanchier ovalis

Asperula aristata

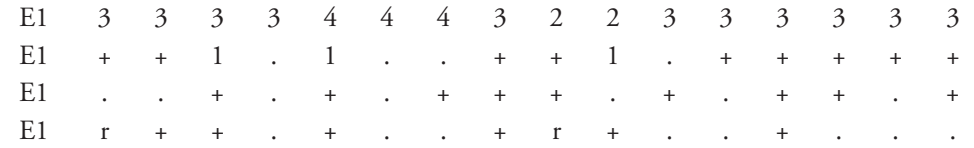

$\mathrm{E} 2+1 .+1++\ldots+1 \quad 1 \quad 1 \quad 1 \quad 1 \quad 1 \quad 1 \quad 1$

$\mathrm{E} 3+++\mathrm{r}++.+++\ldots+++$

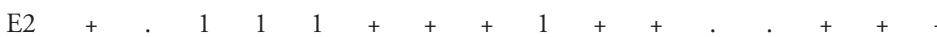

E1

E1

E1

E1

E1

E1

E1

E1

E1

E1

E1

E1

E1

E1

E1

E1

E2

E2

E

E1

E1 
Pinus sylvestris

Pinus sylvestris

Gymnadenia odoratissima

Cotoneaster tomentosus

Genista radiata

Chamaecytisus hirsutus

Carex alba

AF Aremonio-Fagion

Cyclamen purpurascens

Helleborus niger

Cardamine trifolia

Knautia drymeia

Cardamine enneaphyllos

Rhamnus fallax

Laserpitium krapfii

TA Tilio-Acerion

Acer pseudoplatanus

Acer pseudoplatanus

Acer pseudoplatanus

Aruncus dioicus

FS Fagetalia sylvaticae

Daphne mezereum

Lonicera alpigena

Melica nutans

Fagus sylvatica

Fagus sylvatica

Fagus sylvatica

Luzula nivea

Mercurialis perennis

Lilium martagon

Laburnum alpinum

Laburnum alpinum

Galium laevigatum

Dryopteris filix-mas

Paris quadrifolia

Galeobdolon flavidum

Prenanthes purpurea

Lathyrus vernus subsp. flaccidus

Polystichum aculeatum

Viola reichenbachiana

Lathyrus vernus subsp. vernus

Tilia cordata

Salvia glutinosa

Myosotis sylvatica

Epilobium montanum

Symphytum tuberosum

Euphorbia amygdaloides

Actaea spicata

E3

E1

E2

E2

E1

E1

E1

E1

E1

E1

E1

E1

E3

E

E2

E2

E1

E3

E2

E1

E1

E1

E3

E2

E1

E1

E1

E1

E1

E1

E1

E1

E3

E1

E1

E1

E1

E1

E3

E2

E1

E3

E2

E1

E1 

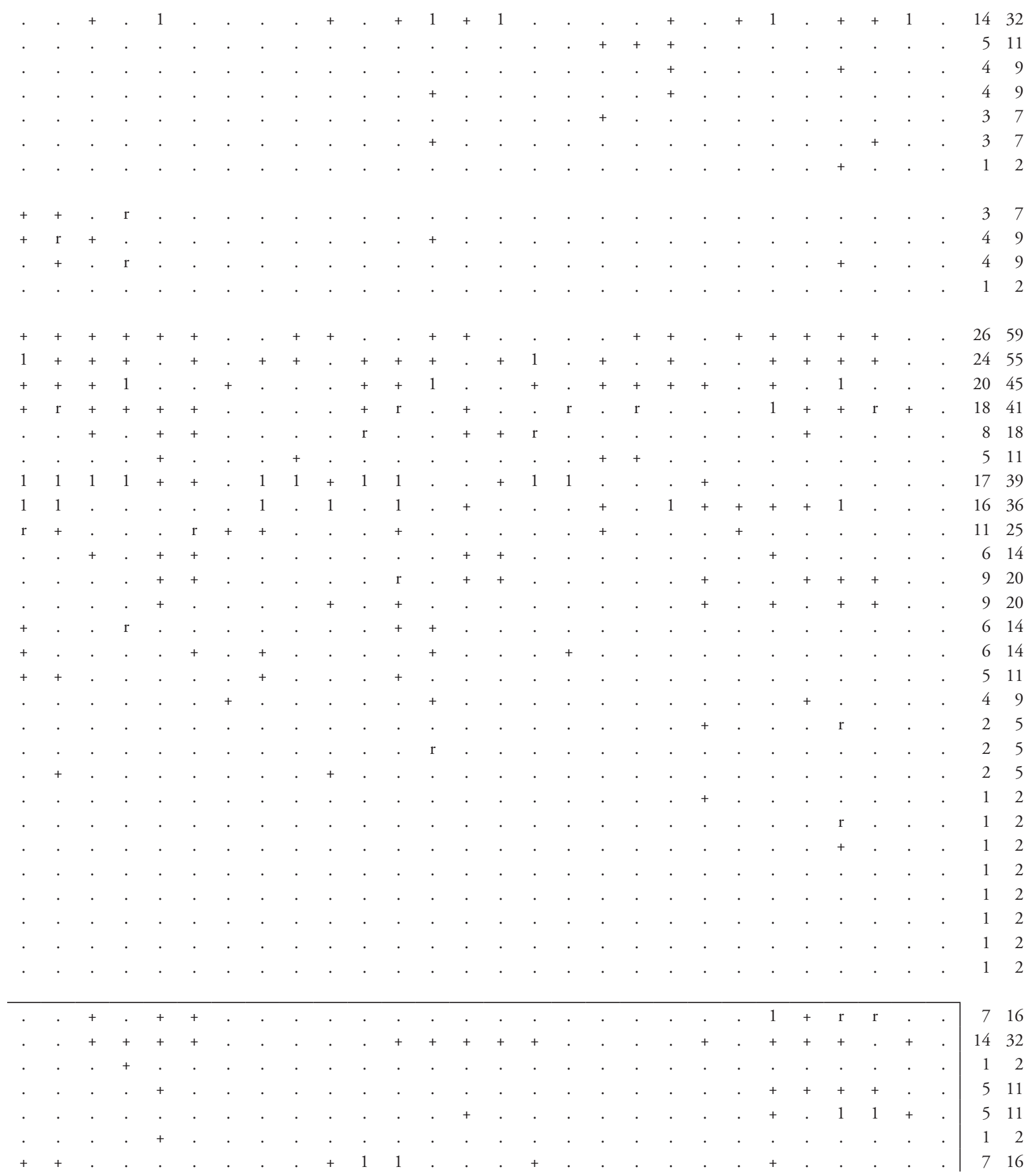
Number of relevé (Zaporedna številka popisa)

Primula veris subsp. columnae

E1

Fraxinus ornus

Arabis turrita

QR Quecetalia roboris

Melampyrum pratense subsp. vulgatum

E1

F Querco-Fagetea

Hepatica nobilis

Carex digitata

Anemone nemorosa

Poa nemoralis

Corylus avellana

Dactylorhiza fuchsii

SSC Sambuco-Salicion capreae, Rhamno-Prunetea

Sorbus aucuparia subsp. aucuparia

Sorbus aucuparia subsp. aucuparia

Sorbus aucuparia subsp. aucuparia

Betula pendula

Betula pendula

RP Juniperus communis

Sambucus racemosa

E1

E1

E1

E1

E1

BA Betulo-Alnetea

Salix appendiculata

Salix appendiculata

Salix glabra

Alnus viridis

Sorbus chamaemespilus

Juniperus sibirica

Ribes alpinum

Sorbus mougeotii

Sorbus mougeotii

Salix waldsteiniana

MuA Mulgedio-Aconitetea

Viola biflora

Veratrum album subsp. lobelianum

Polygonatum verticillatum

Aconitum lycoctonum subsp. ranunculifolium

Athyrium filix-femina

Aconitum angustifolium

Geranium sylvaticum

Ranunculus platanifolius

Saxifraga rotundifolia

Senecio ovatus

Hypericum maculatum

Phyteuma ovatum

Thalictrum aquilegiifolium

Geum rivale

Chaerophyllum hirsutum

Aconitum tauricum

Primula elatior

Doronicum austriacum

Alchemilla xanthochlora

Aconitum degenii subsp. paniculatum

Chaerophyllum villarsii

Heracleum montanum

Senecio cacaliaster

E3

E2

E2

E2

E2

E2

E2

E3

E2

E1

E1

E1

E1

E1

E1

E1

E1

E1

E1

E1

E1

E1

E1

E1

E1

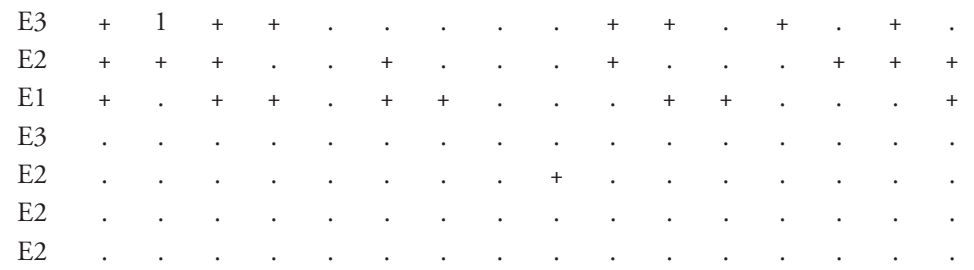


Number of relevé (Zaporedna številka popisa)

TG Trifolio-Geranietea

Achillea distans

Campanula rapunculoides

Thalictrum minus

E1

CA Caricion austroalpinae

Pulsatilla alpina subsp. austroalpina

Koeleria eriostachya

Linum julicum

Festuca calva

Arabis vochinensis

Cfir Caricion firmae

Carex firma

Pedicularis rostratocapitata

Phyteuma sieberi

Dryas octopetala

Sesleria sphaerocephala

Primula wulfeniana

Pedicularis rosea

Leontopodium alpinum

Oxytropis neglecta

Ranunculus hybridus

Helianthemum alpestre

CF Caricion ferrugineae

Hedysarum hedysaroides subsp. exaltatum E1

Cerastium subtriflorum

Allium kermesinum

ES Elyno-Seslerietea

Aster bellidiastrum

Selaginella selaginoides

Phyteuma orbiculare

Hieracium villosum

Carex sempervirens

Betonica alopecuros

Galium anisophyllum

Polygonum viviparum

Campanula witasekiana

Alchemilla alpigena

Juncus monanthos

Achillea clavenae

Ranunculus carinthiacus

Euphrasia salisburgensis

Lotus alpinus

Polygala alpestris

Thymus praecox subsp. polytrichus

Pedicularis verticillata

Erigeron glabratus

Ranunculus montanus

Helianthemum nummularium subsp. grandiflorum

Bartsia alpina

Senecio abrotanifolius

Thesium alpinum

Scabiosa lucida subsp. stricta

Hieracium pilosum

Carduus crassifolius

Gentianella anisodonta

Carex mucronata
E1

E1

E1

E1

E1

E1

E1

E1

E1

E1

E1

E1

E1

E1

E1

E1

E1

E1

E1

E1

E1

E1

E1

E1

E1

E1

E1

E1

E1 


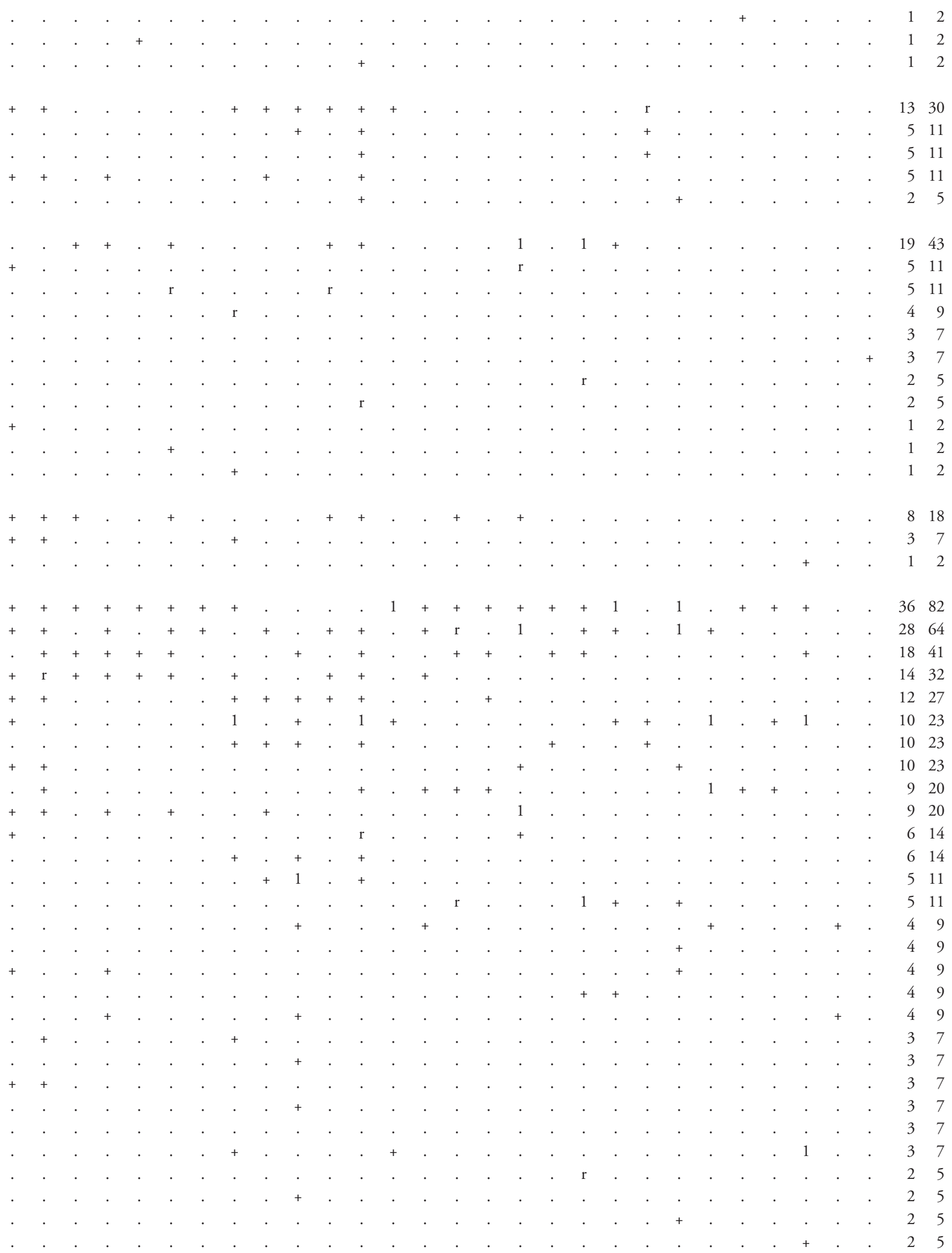


Gentiana clusii

Homogyne discolor

Gentiana sp.

Myosotis alpestris

Gentiana lutea subsp. symphyandra

Rhinanthus glacialis

Leucanthemum heterophyllum (L.maximum agg.)

Scabiosa lucida subsp. lucida

Phleum hirsutum

Carduus defloratus

$\mathrm{JT}$ Juncetea trifidi

Campanula scheuchzeri

LV Loiseleurio-Vaccinietea

Arctostaphylos alpinus

CU Calluno-Ulicetea

Coeloglossum viride

Festuca nigrescens

Gentiana pannonica

FB Festuco-Brometea

Cirsium erisithales

Carex humilis

Buphthalmum salicifolium

Bromus erectus agg. (Bromopsis transsilvanica)

Prunella grandiflora

Helictotrichon praeustum

Dianthus monspessulanus

Gymnadenia conopsea

Hieracium pilosella

Euphorbia cyparissias

Ranunculu.

MA Molinio-Arrhenatheretea

Festuca rubra

Dactylis glomerata s. str.

Centaurea jacea agg.

Taraxacum officinale agg.

Crepis paludosa

Anthoxanthum odoratum

Lathyrus pratensis

Leontodon hispidus

Angelica sylvestris

Saxifraga aizoides

CD Caricetalia davallianae

Parnassia palustris

Tofieldia calyculata

Pinguicula alpina

EA Epilobietea angustifolii

Fragaria vesca

Rubus idaeus

Urtica dioica
E1

E1

E1

E1

E1

E1

E1

E1

E1

E1

E1

E1

E1

E1

E1

E1

E1

E1

E1

E1

E1

E1

E1

E1

E1

E1

E1

E1

E1

E1

E1

E1

E1

E1

E1

E1

E1

E1

$\begin{array}{llll}\cdot & \cdot & \cdot & \cdot \\ \cdot & \cdot & \cdot & \cdot \\ \cdot & \cdot & \cdot & \cdot \\ \cdot & \cdot & \cdot & \cdot \\ \cdot & \cdot & \cdot & \cdot \\ \cdot & \cdot & \cdot & \cdot \\ \cdot & \cdot & \cdot & \cdot \\ \cdot & \cdot & \cdot & \cdot \\ \cdot & \cdot & \cdot & \cdot \\ \cdot & \cdot & \cdot & \cdot\end{array}$

.

.

E1

.

.

1

1

,

1

1

1

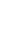

1

.

1

1

E1

E1

E1

E1

E1

E2

E1 
Number of relevé (Zaporedna številka popisa)

AC Arabidetalia caeruleae

Soldanella alpina

Ranunculus traunfellneri

Soldanella minima

Carex atrata

TR Thlaspietea rotundifolii

Gymnocarpium robertianum

Adenostyles glabra

Hieracium bifidum

Dryopteris villarii

Trisetum argenteum

Molopospermum peloponnesiacum

Biscutella laevigata

Athamanta cretensis

Pimpinella alpina

Minuartia austriaca

Saxifraga caesia

Arabis alpina

Geranium macrorrhizum

Valeriana montana

Cystopteris montana

Hieracium glaucum

Hieracium sp.

Petasites paradoxus

Hieracium porrifolium

Euphrasia cuspidata

Heliosperma pusillum

Cystopteris regia

PS Physoplexido comosae-Saxifragion petraeae

Campanula zoysii

Saxifraga squarrosa

Athamanta turbith

Bupleurum petraeum

Phyteuma scheuchzeri subsp. columnae Silene hayekiana

PC Potentilletalia caulescentis

Campanula cochleariifolia

Festuca stenantha

Campanula cespitosa

Potentilla clusiana

Androsace lactea

Potentilla caulescens

Rhamnus pumilus

Saxifraga burseriana

Arabis bellidiofolia subsp. stellulata

AT Asplenietea trichomanis

Asplenium viride

Asplenium ruta-muraria

Polypodium vulgare

Kernera saxatilis

Asplenium trichomanes

Moehringia muscosa

Festuca sp.

Draba sp.
E1

E1

E1

E1

E1

E1

E1

E1

E1

E1

E1

E1

E1

E1

E1

E1

E1

E1

E1

E1

E1

E1

E1

E1

(1)

El

(2)

2

(1)

1

.

.

E1

1

1

1

(1)

E1

E1

E1

E1

E1

E1

E1

E1

E1

E1

E1

E1

E1

E1

E1

E1 

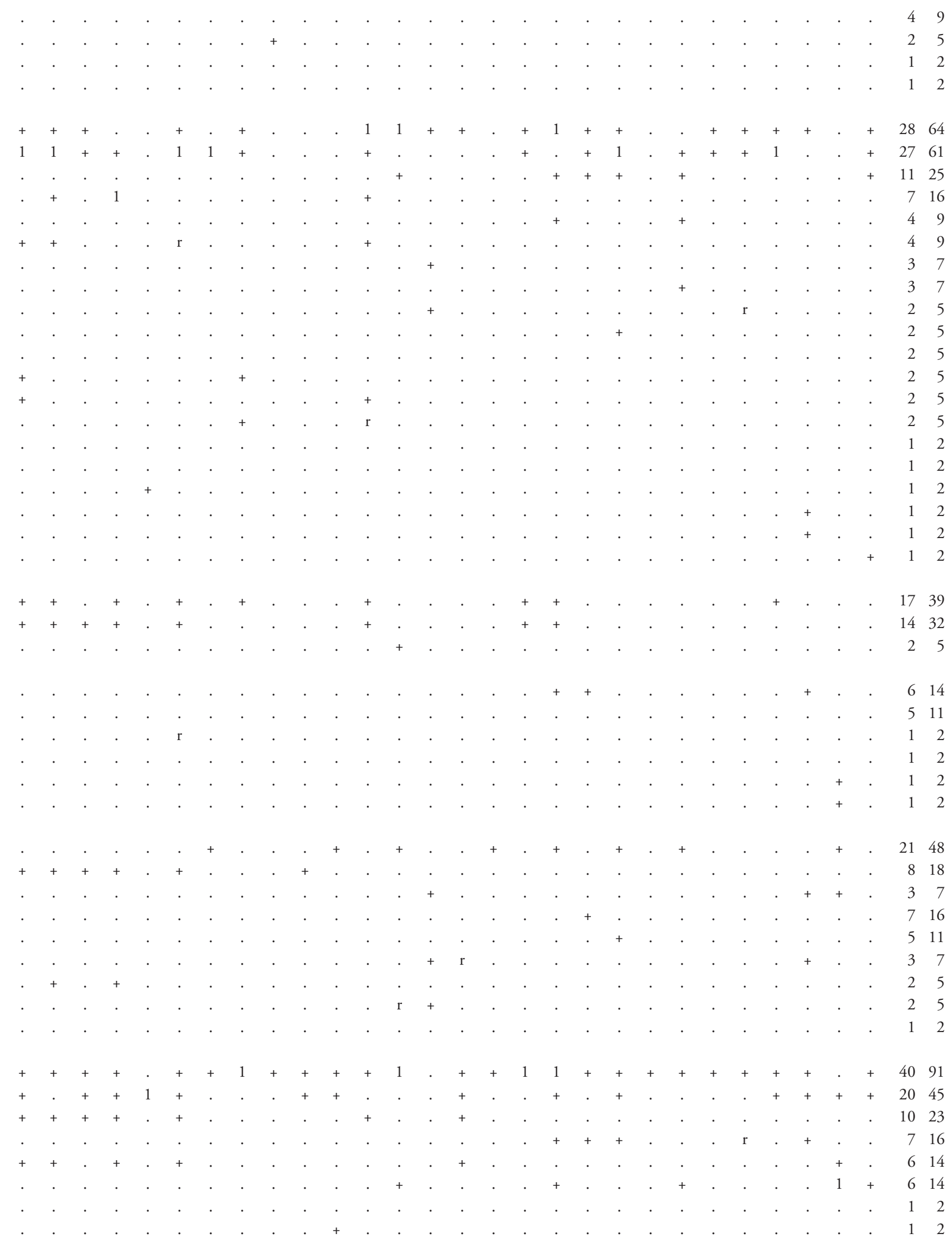
$\begin{array}{lllllllllllllllll}\text { Number of relevé (Zaporedna številka popisa) } & 1 & 2 & 3 & 4 & 5 & 6 & 7 & 8 & 9 & 10 & 11 & 12 & 13 & 14 & 15 & 16\end{array}$

Fu Fungi (Glive)

Laetiporus sulphureus agg. (L. horoniensis)

ML Mosses and lichens (Mahovi in lišaji)

Ctenidium molluscum

Tortella tortuosa

Rhytidiadelphus triquetrus

Hylocomium splendens

Dicranum scoparium

Neckera crispa

Polytrichum formosum

Peltigera leucophlebia

Schistidium sp.

Fissidens dubius

Orthothecium rufescens

Plagiochila porelloides

Peltigera canina

Hypnum cupressiforme

Bartramia pomiformis

Homalothecium philippeanum

Marchantia polymorpha

Cladonia sp.

Solorina saccata

Eurhynchium angustirete

Conocephalum conicum

Plagiomnium undulatum

Dicranum sp.

Metzgeria furcata

Bryum argenteum

Campylium stellatum

Mnium marginatum

Brachythecium velutinum

Musci spp.

Plagiothecium sylvaticum

Scleropodium purum

Cladonia pyxidata

Homalothecium lutescens

Rhizomnium punctatum

Leucobryum glaucum

Sphagnum sp.

Distichium capillaceum

Hypogymnia physodes

Cetraria islandica

Thuidium tamariscinum

Rhytidiadelphus loreus

\begin{tabular}{|c|c|c|c|c|c|c|c|c|c|c|c|c|c|c|}
\hline $\mathrm{E} 3 \mathrm{c}$ & $\mathrm{r}$ & & . & & . & & . & & & & . & & & \\
\hline E0 & + & + & 1 & 1 & + & + & + & 1 & 1 & 1 & 1 & & & \\
\hline E0 & + & + & + & 1 & + & . & + & 1 & + & + & + & + & & \\
\hline E0 & 1 & + & . & + & 1 & + & + & + & + & 1 & 1 & & & \\
\hline E0 & 1 & + & 1 & + & . & + & + & . & + & + & 2 & + & & \\
\hline E0 & + & + & + & 1 & + & . & + & . & 1 & + & 1 & & & \\
\hline E0 & + & + & . & . & . & + & + & 1 & . & + & . & 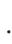 & & \\
\hline E0 & . & + & + & + & . & . & . & . & + & 1 & + & + & & \\
\hline E0 & + & . & + & + & + & . & + & . & - & + & + & + & & \\
\hline E0 & . & . & . & . & . & + & + & . & . & $\cdot$ & + & . & & \\
\hline E0 & . & . & . & . & . & . & . & . & . & . & . & & & \\
\hline E0 & + & . & 1 & + & + & . & . & . & . & . & + & 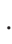 & & \\
\hline E0 & . & . & . & + & . & . & + & . & $\cdot$ & . & r. & ${ }^{\circ}$ & & \\
\hline E0 & . & . & . & . & . & . & . & . & . & . & + & & & \\
\hline E0 & . & . & . & . & . & . & . & . & $\cdot$ & . & . & $\cdot$ & & \\
\hline E0 & . & + & . & . & . & . & . & . & . & + & r. & & & \\
\hline E0 & . & . & . & . & . & + & . & . & . & . & · & & & \\
\hline E0 & . & . & . & . & . & . & . & . & . & . & r & & & \\
\hline E0 & . & . & . & . & . & . & . & . & . & . & . & & & \\
\hline E0 & + & . & . & . & . & . & . & . & . & + & . & & & \\
\hline E0 & . & . & . & + & . & . & . & . & . & $\cdot$ & 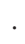 & & & \\
\hline E0 & . & . & . & . & . & . & . & . & . & . & . & & & \\
\hline E0 & . & . & . & . & . & . & . & . & . & · & • & & & \\
\hline E0 & . & . & . & . & . & . & . & . & . & . & . & & & \\
\hline E0 & . & . & . & . & . & . & . & . & . & . & . & & & \\
\hline E0 & . & . & . & . & . & . & . & . & . & . & . & & & \\
\hline E0 & . & . & . & . & . & . & . & . & $\cdot$ & r & . & & & \\
\hline E0 & . & . & . & . & . & . & . & . & . & & . & & & \\
\hline E0 & . & . & . & . & . & . & . & . & . & . & . & . & & \\
\hline E0 & + & . & . & . & . & . & . & . & . & . & . & & & \\
\hline E0 & . & . & . & + & . & . & . & . & . & . & . & . & & \\
\hline E0 & . & . & . & . & . & . & . & . & . & . & + & & & \\
\hline E0 & . & . & . & . & . & . & . & . & . & . & . & & & \\
\hline E0 & . & . & . & . & . & . & . & . & . & & . & & & \\
\hline E0 & . & . & . & . & . & . & . & . & . & 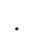 & . & & & \\
\hline E0 & . & . & . & . & . & . & . & . & . & & . & & & \\
\hline E0 & . & . & . & . & . & . & . & . & 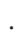 & & , & & & \\
\hline E0 & . & . & . & . & . & . & . & . & . & & . & & & \\
\hline E3c & . & . & . & . & . & . & . & . & & & & & & \\
\hline E0 & . & . & . & . & . & . & . & . & & & 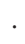 & & & \\
\hline E0 & . & . & . & . & . & . & . & 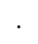 & & & & & & \\
\hline E0 & & & & & . & & & & & & & & & \\
\hline
\end{tabular}

\section{Legend - Legenda}

ID Igor Dakskobler

AS Andrej Seliškar

MČ Miran Čas

A Limestone - apnenec

D Dolomite - dolomit

Li Litosol - litosil
Re Rendzina - rendzina

JA Julian Alps - Julijske Alpe

KSA Kamnik-Savinja Alps - Kamniško-Savinjske Alpe

K Karavanke - Karavanke

Pr. Presence (number of relevés in which the species is presented) - število popisov, v katerih se pojavlja vrsta

Fr. Frequency in $\%-$ frekvenca v $\%$ 


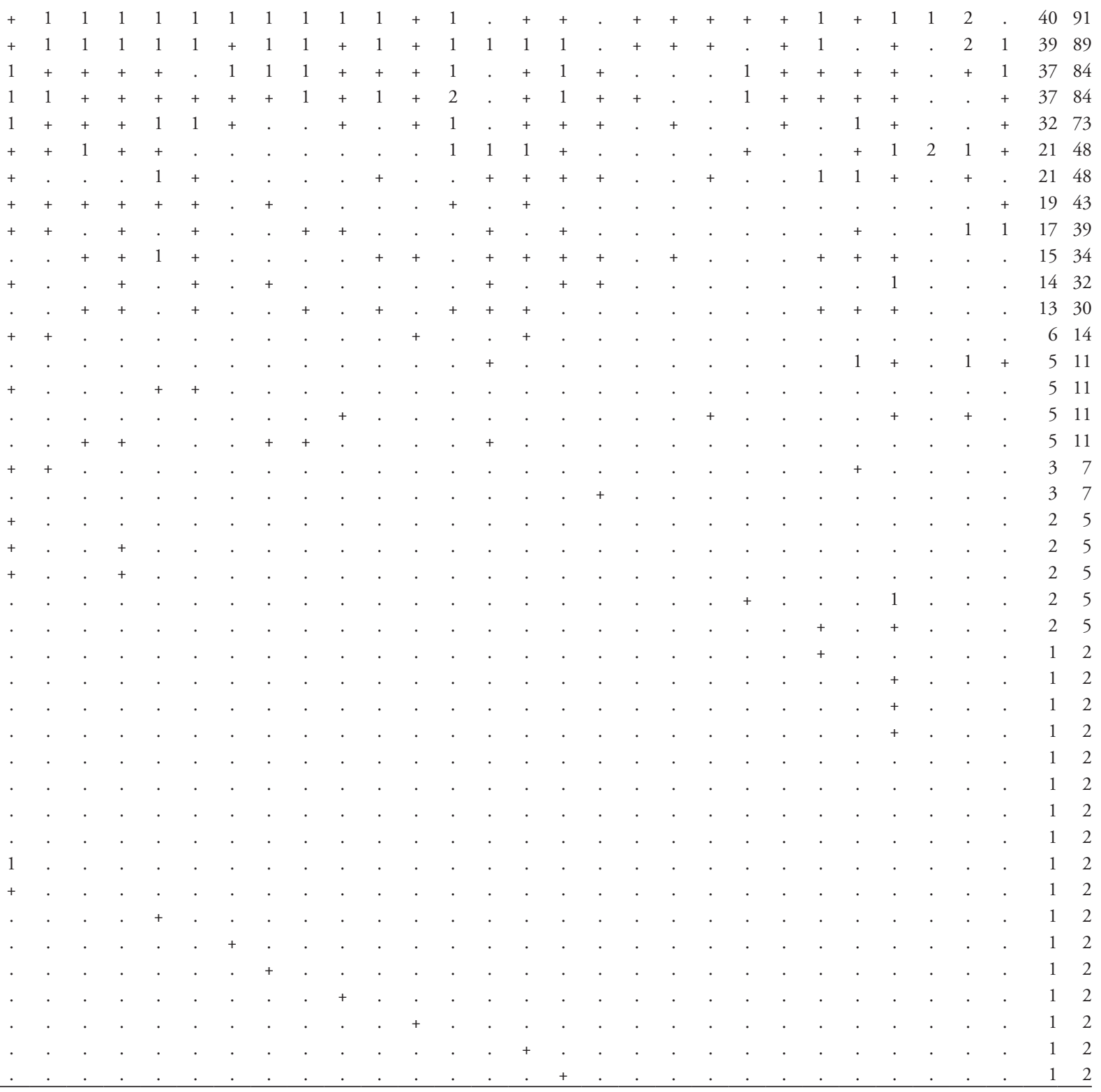


Table 14 (Tabela 14): Rhodothamno-Laricetum anemonetosum trifoliae var. Festuca nitida

\section{Number of relevé (Zaporedna številka popisa)}

Database number of relevé

(Delovna številka popisa)

Author of the relevé (Avtor popisa)

Elevation in $\mathrm{m}$ (Nadmorska višina $\mathrm{v} \mathrm{m}$ )

Aspect (Lega)

Slope in degrees (Nagib v stopinjah)

Parent material (Matična podlaga)

Soil (Tla)

Stoniness in \% (Kamnitost v \%)

Cover in \% (Zastiranje v \%):

Upper tree layer (Zgornja drevesna plast)

Lower tree layer (Spodnja drevesna plasti)

Shrub layer (Grmovna plast)

Herb layer (Zeliščna plast)

Number of species (Število vrst)

Relevé area (Velikost popisne ploskve)

Date of taking relevé (Datum popisa)

Locality (Nahajališče)

Mountain range (Pogorje)

Quadrant (Kvadrant)

Coordinate GK-Y (D-48)

Coordinate GK-X (D-48)
Moss layer (Mahovna plast)

\begin{tabular}{|c|c|c|c|c|c|c|c|c|c|c|}
\hline & 1 & 2 & 3 & 4 & 5 & 6 & 7 & 8 & 9 & 10 \\
\hline & $\underset{\text { ণ }}{\stackrel{\infty}{\sim}}$ & $\begin{array}{l}\hat{\infty} \\
\infty \\
0 \\
\approx \\
\end{array}$ & 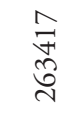 & 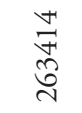 & 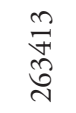 & 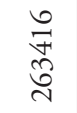 & $\begin{array}{l}0 \\
\infty \\
\infty \\
\stackrel{\nu}{0}\end{array}$ & $\stackrel{m}{\stackrel{n}{\hat{े}}}$ & $\begin{array}{l}\vec{n} \\
\stackrel{n}{\partial} \\
\text { }\end{array}$ & $\begin{array}{l}\text { तै } \\
\text { } \\
\text { } \\
\text { }\end{array}$ \\
\hline & ID & ID & ID & ID & ID & ID & ID & ID & ID & ID \\
\hline & 1390 & 1740 & 1420 & 1340 & 1380 & 1370 & 850 & 1630 & 1590 & 1540 \\
\hline & $\mathrm{N}$ & $\mathrm{N}$ & $\mathrm{N}$ & $\mathrm{N}$ & $\mathrm{N}$ & $\mathrm{N}$ & NW & NNE & $\mathrm{NE}$ & $\mathrm{NE}$ \\
\hline & 30 & 35 & 40 & 40 & 40 & 37 & 35 & 40 & 35 & 35 \\
\hline & DA & $\mathrm{Gr}$ & $\mathrm{Gr}$ & $\mathrm{D}$ & D & Gr & A & A & A & A \\
\hline & $\mathrm{Kd}$ & $\operatorname{Re}$ & $\operatorname{Re}$ & $\operatorname{Re}$ & $\mathrm{Re}$ & $\operatorname{Re}$ & $\operatorname{Re}$ & $\operatorname{Re}$ & $\operatorname{Re}$ & $\operatorname{Re}$ \\
\hline & 30 & 20 & 20 & 29 & 30 & 20 & 20 & 20 & 80 & \\
\hline E3b & 30 & 50 & 60 & 70 & 60 & 70 & 50 & 60 & 60 & 60 \\
\hline E3a & 0 & 20 & 10 & 10 & 10 & 5 & 10 & 10 & 10 & 10 \\
\hline E2 & 50 & 30 & 15 & 20 & 5 & 15 & 30 & 5 & 50 & 40 \\
\hline $\mathrm{E}$ & 70 & 80 & 80 & 80 & 70 & 80 & 50 & 80 & 60 & 7 \\
\hline $\mathrm{F}$ & 10 & 10 & 10 & 5 & 10 & 10 & 5 & 10 & 20 & 10 \\
\hline & 60 & 74 & 76 & 84 & 62 & 76 & 74 & 88 & 91 & 76 \\
\hline$m^{2}$ & 200 & 400 & 400 & 400 & 400 & 400 & 200 & 400 & 200 & 200 \\
\hline & $9 / 23$ & $9 / 22$ & $7 / 4$ & $7 / 4$ & $7 / 4$ & $7 / 4$ & $7 / 6$ & $6 / 17$ & $6 / 15$ & $6 / 1$ \\
\hline & 1997 & 2010 & 2016 & 2016 & 2016 & 2016 & 1991 & 2003 & 2001 & 2001 \\
\hline & 窇离 & 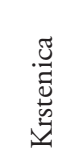 & 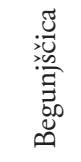 & 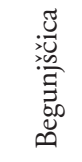 & 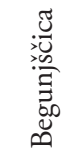 & 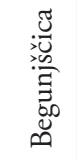 & 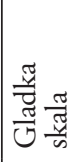 & 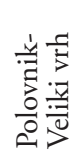 & 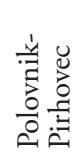 & 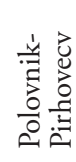 \\
\hline & JA & JA & $\mathrm{K}$ & $\mathrm{K}$ & $\mathrm{K}$ & $\mathrm{K}$ & JA & JA & JA & \\
\hline & 9548 & 9649 & 9551 & 9551 & 9551 & 9551 & 9749 & 9747 & 9747 & 974 \\
\hline & 14 & 13 & 13 & 13 & 13 & 13 & 14 & 12 & $/ 1$ & \\
\hline & 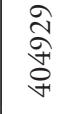 & $\begin{array}{l}n \\
\infty \\
\cdots \\
\exists\end{array}$ & $\begin{array}{l}\hat{n} \\
\hat{\tilde{\sigma}} \\
\stackrel{+}{+}\end{array}$ & $\begin{array}{l}\frac{n}{n} \\
\hat{\sigma}\end{array}$ & $\begin{array}{l}\vec{ర} \\
\stackrel{+}{2} \\
\stackrel{\overbrace{}}{F}\end{array}$ & $\begin{array}{l}\tilde{n} \\
\hat{\tilde{n}} \\
\dot{f}\end{array}$ & 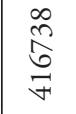 & $\begin{array}{l}\stackrel{n}{2} \\
\stackrel{\sigma}{\sigma}\end{array}$ & 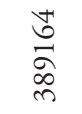 & $\begin{array}{l}\text { ¿' } \\
\curvearrowleft \\
\infty \\
\infty\end{array}$ \\
\hline & $\begin{array}{l}\stackrel{+}{2} \\
\stackrel{+}{+} \\
\stackrel{+}{n}\end{array}$ & $\begin{array}{l}\stackrel{\infty}{\infty} \\
\stackrel{\text { }}{n} \\
\stackrel{n}{n}\end{array}$ & \begin{tabular}{l}
$\infty$ \\
8 \\
\multirow{2}{*}{} \\
$\stackrel{7}{n}$
\end{tabular} & 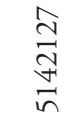 & 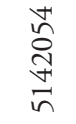 & 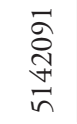 & 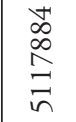 & $\begin{array}{l}\stackrel{+}{N} \\
n \\
\text { N } \\
i n\end{array}$ & 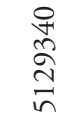 & בิ) \\
\hline
\end{tabular}

Character and differential species of the association (Značilnice in razlikovalnice asociacije)

Pr. Fr.

VP Larix decidua

VP Larix decidua

VP Larix decidua

PS Paederota lutea

VP Homogyne sylvestris

TR Festuca nitida

CA Laserpitium peucedanoides

EP Rhodothamnus chamaecistus

CF Carex ferruginea

EP Rhododendron hirsutum

PC Valeriana saxatilis

ES Astrantia bavarica

\begin{tabular}{|c|c|c|c|c|c|c|c|c|c|c|c|}
\hline E3 & 3 & 4 & 4 & 4 & 4 & 3 & 4 & 4 & 4 & 10 & 100 \\
\hline E2 & 1 & + & + & + & + & + & + & 1 & 1 & 10 & 100 \\
\hline E1 & . & . & . & + & . & . & . & . & . & 2 & 20 \\
\hline E1 & + & + & 2 & 1 & 1 & . & 1 & + & + & 9 & 90 \\
\hline E1 & 1 & 1 & 1 & 1 & 1 & + & 1 & . & . & 8 & 80 \\
\hline E1 & 1 & 1 & 1 & 2 & 2 & . & + & + & 1 & 8 & 80 \\
\hline E1 & + & + & 1 & 1 & + & . & + & . & . & 7 & 70 \\
\hline E1 & + & . & + & + & . & . & 1 & 2 & 1 & 7 & 70 \\
\hline E1 & 2 & 2 & 1 & . & 1 & + & + & . & . & 7 & 70 \\
\hline E2 & 1 & . & . & . & + & 3 & 1 & 2 & 3 & 7 & 70 \\
\hline+ & + & . & + & 1 & . & + & + & + & . & 7 & 70 \\
\hline E1 & + & + & + & + & + & . & + & + & . & 7 & 70 \\
\hline
\end{tabular}




\section{\begin{tabular}{l|llllll|llllll} 
Number of relevé (Zaporedna številka popisa) & 1 & 2 & 3 & 4 & 5 & 6 & 7 & 8 & 9 & 10 & Pr. & Fr
\end{tabular}}

TR Heliosperma alpestre

AF Anemone trifolia

TR Astrantia carniolica

PS Campanula carnica

\begin{tabular}{|c|c|c|c|c|c|c|c|c|}
\hline+ & + & + & + & + & - & . & . & \\
\hline+ & . & . & . & . & . & + & 1 & \\
\hline 1 & + & . & . & . & . & . & . & \\
\hline . & . & + & . & . & . & . & . & \\
\hline
\end{tabular}

Differential species of the subassociation (Razlikovalne vrste subasociacije)

$\begin{array}{ll}\text { ES } & \text { Sesleria caerulea } \\ \text { PC } & \text { Primula auricula } \\ \text { Cfr } & \text { Carex brachystachys } \\ \text { PS } & \text { Saxifraga crustata } \\ \text { VP Vaccinio-Piceetea }\end{array}$

\begin{tabular}{l|llllll|llllll}
$\mathrm{E} 1$ & 2 & 3 & 3 & 4 & 3 & 3 & 3 & 2 & 1 & 1 & 10 & 100
\end{tabular}

Clematis alpina

Luzula sylvatica

Picea abies

Picea abies

Picea abies

Polystichum lonchitis

Oxalis acetosella

Saxifraga cuneifolia

Solidago virgaurea subsp. virgaurea

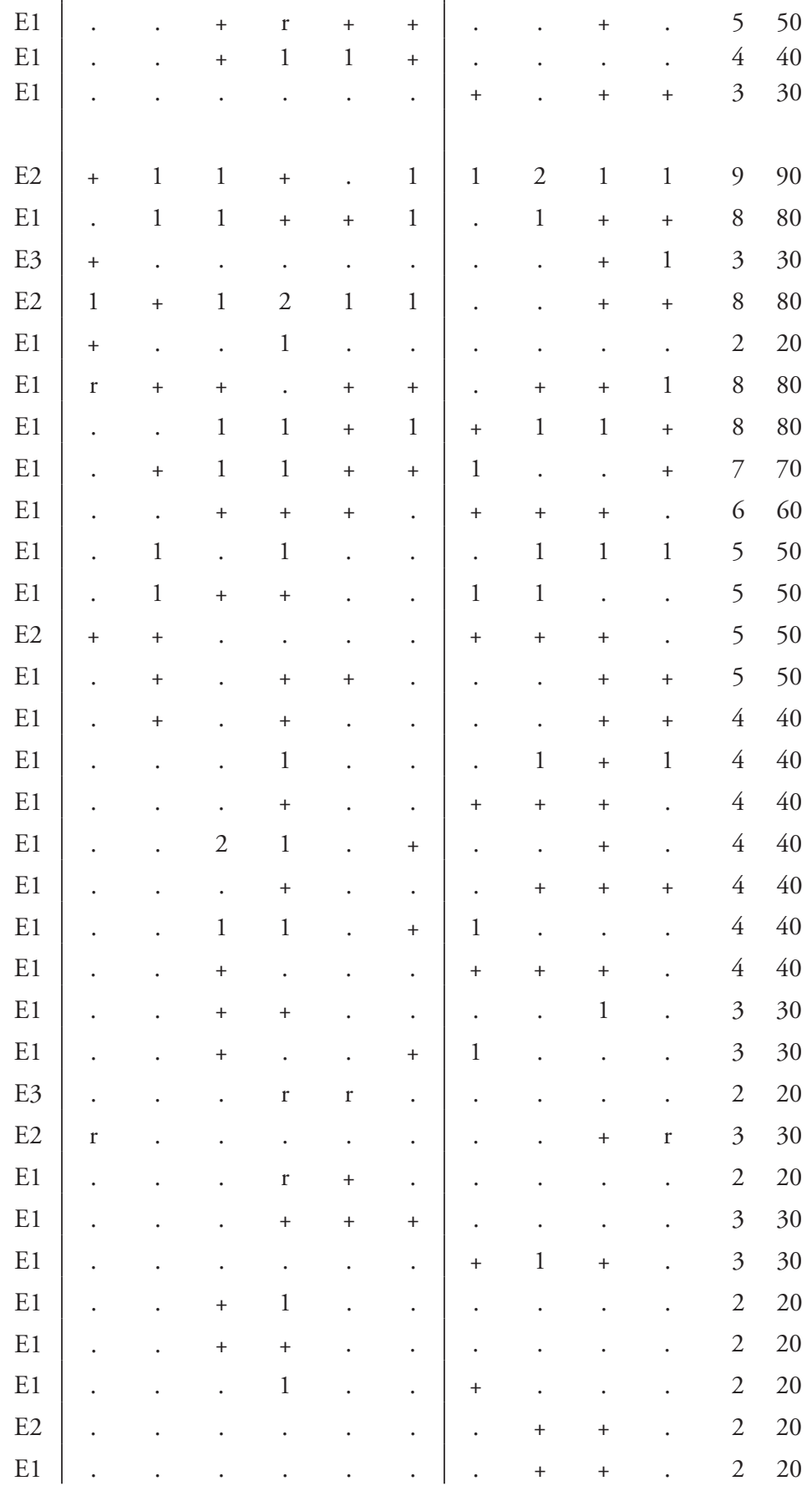

Vaccinium myrtillus

Valeriana tripteris

Rosa pendulina

Huperzia selago

Vaccinium vitis-idaea

Homogyne alpina

Hieracium murorum

Phegopteris connectilis

Maianthemum bifolium

Luzula luzuloides

Gentiana asclepiadea

Lycopodium annotinum

Veronica urticifolia

Abies alba

Abies alba

Abies alba

Moneses uniflora

Calamagrostis arundinacea

Melampyrum sylvaticum

Dryopteris dilatata

Gymnocarpium dryopteris

Lonicera caerulea

Dryopteris expansa 
Number of relevé (Zaporedna številka popisa

Lonicera nigra

Calamagrostis villosa

Aposeris foetida

EP Erico-Pinetea

Rubus saxatilis

Calamagrostis varia

Carex ornithopoda

Pinus mugo

Erica carnea

Aquilegia nigricans

Carex alba

Chamaecytisus hirsutus

AF Aremonio-Fagion

Cardamine enneaphyllos

Cardamine trifolia

Cyclamen purpurascens

Rhamnus fallax

Helleborus odorus

TA Tilio-Acerion

Acer pseudoplatanus

Geranium robertianum

Chrysosplenium alternifolium

Aruncus dioicus

Fagetalia sylvaticae

Dryopteris filix-mas

Galium laevigatum

Lilium martagon

Melica nutans

Daphne mezereum

Mercurialis perennis

Paris quadrifolia

Mycelis muralis

Galeobdolon flavidum

Luzula nivea

Fagus sylvatica

Fagus sylvatica

Fagus sylvatica

Prenanthes purpurea

Lonicera alpigena

Epilobium montanum

Lathyrus vernus subsp. flaccidus

Asarum europaeum

E2

E1

E1

1

23

\section{4}

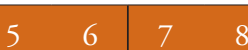

$\begin{array}{lllll}8 & 9 & 10 & \text { Pr. } & \text { Fr }\end{array}$

1

E1

E

E1

E2

E2

E1

E1

E1

E

E1

E1

E1

E2

E1

E1

E1

E1

E1

E1

E1

E1

E1

E1

E2

E1

E1

E1

E1

E1

E3

E2

E1

E1

E2

E1

E1

E1

.

$\begin{array}{llllll}7 & . & . & . & 1 & 10\end{array}$

. $r$

110

110

$11+++\quad+\quad \begin{array}{rrrrr}1 & + & + & 9 & 90\end{array}$

$1 \cdot 11+++.+770$

$\cdot++.+5+4.40$

$3 \quad 30$

330

$3 \quad 30$

110

$\begin{array}{ll}1 & 10\end{array}$

$\begin{array}{lll}6 & 60\end{array}$

330

220

220

110

110

$1 \quad 10$

110

110

$6 \quad 60$

$6 \quad 60$

550

440

$4 \quad 40$

$4 \quad 40$

$4 \quad 40$

330

330

220

220

1 10

110

220

$1 \quad 10$

$1 \quad 10$

110

$1 \quad 10$ 
\begin{tabular}{l|llllll|llllll} 
Number of relevé (Zaporedna številka popisa) & 1 & 2 & 3 & 4 & 5 & 6 & 7 & 8 & 9 & 10 & Pr. & Fr.
\end{tabular}

Festuca altissima

Tilia cordata

Polystichum aculeatum

Lathyrus vernus subsp. vernus

Corydalis cava

QP Quercetalia pubescenti-petraeae

Sorbus aria (Aria edulis)

\section{E1}

Dichoropetalum schottii (Peucedanum schottii)

QF

\section{Querco-Fagetea}

Hepatica nobilis

Anemone nemorosa

Poa nemoralis

Dactylorhiza fuchsii

Festuca heterophylla

Lonicera xylosteum

Carex digitata

Ranunculus auricomus agg.

E1

E1

E1

Sambuco-Salicion capreae, Rhamno-Prunetea

Sorbus aucuparia subsp. aucuparia

Sorbus aucuparia subsp. aucuparia

Sorbus aucuparia subsp. aucuparia

Betula pendula

BA Betulo-Alnetea

Salix appendiculata

Sorbus chamaemespilus

Alnus viridis

Salix glabra

Salix waldsteiniana

Salix appendiculata

MuA Mulgedio-Aconitetea

Viola biflora

Veratrum album subsp. lobelianum

Saxifraga rotundifolia

Aconitum lycoctonum subsp. ranunculifolium

Polygonatum verticillatum

Chaerophyllum hirsutum

Athyrium filix-femina

Geranium sylvaticum

Phyteuma ovatum

Ranunculus platanifolius

Aconitum angustifolium

Hypericum maculatum

E2

E3

E

E1

E1

E1

E1

E1

E1

E1

E1

E1

\begin{tabular}{l|l}
$\mathrm{E} 1$ & $\cdot$ \\
$\mathrm{E} 1$ & $\cdot$ \\
$\mathrm{E} 1$ & $\cdot$ \\
$\mathrm{E} 2$ & $\cdot$ \\
$\mathrm{E} 1$ & $\cdot$ \\
$\mathrm{E} 1$ & $\cdot$ \\
$\mathrm{E} 1$ & $\cdot$ \\
$\mathrm{E} 1$ & $\cdot$ \\
$\mathrm{E} 2$ & $\cdot$ \\
$\mathrm{E} 1$ & $\cdot$ \\
\hline
\end{tabular}

$$
\begin{array}{lll}
\cdot & 1 & 10 \\
\cdot & 1 & 10 \\
\cdot & 1 & 10 \\
+ & 1 & 10 \\
r & 1 & 10 \\
& & \\
+ & 1 & 10 \\
\cdot & 1 & 10 \\
& & \\
+ & 6 & 60 \\
\cdot & 4 & 40 \\
\cdot & 2 & 20 \\
\cdot & 2 & 20 \\
. & 1 & 10 \\
. & 1 & 10 \\
\cdot & 1 & 10 \\
. & 1 & 10
\end{array}
$$

\begin{tabular}{l|cccccc|cccccc} 
E3 & $\cdot$ & $\cdot$ & $\cdot$ & $\mathrm{r}$ & $\mathrm{r}$ & $\cdot$ & + & $\mathrm{r}$ & $\cdot$ & $\cdot$ & 4 & 40 \\
$\mathrm{E} 2$ & $\cdot$ & $\cdot$ & $\cdot$ & $\cdot$ & $\cdot$ & $\cdot$ & + & $\cdot$ & + & $\cdot$ & 2 & 20 \\
$\mathrm{E} 1$ & + & + & $\cdot$ & + & + & + & $\cdot$ & + & $\cdot$ & $\cdot$ & 6 & 60 \\
$\mathrm{E} 3$ & $\cdot$ & $\cdot$ & $\cdot$ & $\cdot$ & $\cdot$ & $\cdot$ & + & $\cdot$ & $\cdot$ & $\cdot$ & 1 & 10
\end{tabular}

E2
E2
E2
E2
E3

$\mathrm{E} 1=+\begin{array}{lllll}+ & 1 & 1 & 1 & 1 \\ \mathrm{E} 1\end{array}$ 
Number of relevé (Zaporedna številka popisa)

Primula elatior

Chaerophyllum villarsii

Alchemilla monticola

Geum rivale

Rumex arifolius

Senecio ovatus

Adenostyles alliariae

Thalictrum aquilegiifolium

Senecio cacaliaster

Alchemilla xanthochlora

TG Trifolio-Geranietea

Polygonatum odoratum

Achillea distans

CA Caricion austroalpinae

Arabis vochinensis

Pulsatilla alpina subsp. austroalpina

Festuca calva

Heracleum austriacum subsp. siifolium

Cfir Caricion firmae

Carex firma

Dryas octopetala

CF Caricion ferrugineae

Knautia longifolia

ES Elyno-Seslerietea

Aster bellidiastrum

Selaginella selaginoides

Polygonum viviparum

Juncus monanthos

Bartsia alpina

Betonica alopecuros

Phyteuma orbiculare

Galium anisophyllum

Alchemilla alpigena

Pedicularis verticillata

Polygala alpestris

Ranunculus carinthiacus

Hieracium villosum

Hieracium pilosum

Carex sempervirens

Lotus alpinus

Euphrasia picta

Rhinanthus glacialis

\begin{tabular}{|c|c|c|c|c|c|c|c|c|c|c|c|c|}
\hline E1 & . & . & + & . & . & 1 & . & . & . & . & 2 & 20 \\
\hline E1 & . & + & . & . & . & . & . & . & . & . & 1 & 10 \\
\hline E1 & . & + & . & . & . & . & . & . & . & . & 1 & 10 \\
\hline E1 & . & . & . & . & . & . & . & + & . & . & 1 & 10 \\
\hline E1 & . & . & + & . & . & . & . & . & . & . & 1 & 10 \\
\hline E1 & . & . & . & . & . & . & + & . & . & . & 1 & 10 \\
\hline E1 & . & . & . & . & . & . & . & + & . & . & 1 & 10 \\
\hline E1 & . & . & . & . & . & . & . & + & . & . & 1 & 10 \\
\hline E1 & . & . & . & . & . & . & . & . & + & . & 1 & 10 \\
\hline E1 & . & . & . & . & . & . & . & . & . & + & 1 & 10 \\
\hline E1 & . & . & . & . & . & . & . & . & . & + & 1 & 10 \\
\hline E1 & . & . & . & . & . & . & . & . & . & + & 1 & 10 \\
\hline E1 & . & . & . & . & . & . & . & + & + & + & 3 & 30 \\
\hline E1 & . & + & . & . & . & . & . & + & . & . & 2 & 20 \\
\hline E1 & . & . & . & . & . & . & . & . & + & + & 2 & 20 \\
\hline E1 & . & + & . & . & . & . & . & . & . & . & 1 & 10 \\
\hline E1 & + & . & + & . & + & + & . & . & . & . & 4 & 40 \\
\hline E1 & . & + & . & . & . & . & . & . & . & . & 1 & 10 \\
\hline E1 & . & . & . & . & . & . & . & $\mathrm{r}$ & . & + & 2 & 20 \\
\hline E1 & + & + & 1 & 1 & 1 & 1 & . & 1 & + & + & 9 & 90 \\
\hline E1 & 1 & + & + & $r$ & 1 & + & . & . & + & . & 7 & 70 \\
\hline E1 & . & . & + & + & + & + & . & + & + & + & 7 & 70 \\
\hline E1 & 1 & + & + & . & . & + & . & + & . & . & 5 & 50 \\
\hline E1 & + & + & . & . & . & . & . & + & + & + & 5 & 50 \\
\hline E1 & + & . & + & + & . & . & . & . & + & . & 4 & 40 \\
\hline E1 & + & . & + & + & + & . & . & . & . & . & 4 & 40 \\
\hline E1 & . & + & . & . & + & . & . & + & + & . & 4 & 40 \\
\hline E1 & . & . & . & . & . & . & . & + & + & + & 3 & 30 \\
\hline E1 & . & . & . & + & . & + & . & . & . & . & 2 & 20 \\
\hline E1 & . & . & . & . & + & . & . & . & . & + & 2 & 20 \\
\hline E1 & . & . & . & . & . & . & . & + & . & + & 2 & 20 \\
\hline E1 & . & . & . & . & . & . & . & + & . & + & 2 & 20 \\
\hline E1 & . & . & . & . & . & . & . & + & + & . & 2 & 20 \\
\hline E1 & . & . & . & . & . & . & . & . & + & + & 2 & 20 \\
\hline E1 & . & + & . & . & . & . & . & . & . & . & 1 & 10 \\
\hline E1 & . & + & . & . & . & . & . & . & . & . & 1 & 10 \\
\hline E1 & . & + & . & . & . & . & . & . & . & . & 1 & 10 \\
\hline
\end{tabular}


\begin{tabular}{l|llllll|llllll} 
Number of relevé (Zaporedna številka popisa) & 1 & 2 & 3 & 4 & 5 & 6 & 7 & 8 & 9 & 10 & Pr. & Fr.
\end{tabular}

Campanula witasekiana

Achillea clavenae

Gentiana verna

E1

E1

JT Juncetea trifidi

Campanula scheuchzeri

CU Calluno-Ulicetea

Pseudorchis albida

Coeloglossum viride

Festuca nigrescens

Meum athamanticum

FB Festuco-Brometea

Cirsium erisithales

Buphthalmum salicifolium

Gentianella ciliata

Linum catharticum

Bromus erectus agg. (Bromopsis transsilvanica)

PaT Poo alpinae-Trisetetalia

Poa alpina

Trollius europaeus

MA Molinio-Arrhenatheretea

Anthoxanthum odoratum

Leontodon hispidus

Dactylis glomerata s.str.

Achillea millefolium

Deschampsia cespitosa

Crepis paludosa

MC Montio-Cardaminetea

Saxifraga aizoides

CD Caricetalia davallianae

Parnassia palustris

Tofieldia calyculata

Pinguicula alpina

EA Epilobietea angustifolii

Fragaria vesca

Rubus idaeus

Urtica dioica

AC Arabidetalia caeruleae

Soldanella minima

Soldanella alpina

Carex atrata

TR

Thlaspietea rotundifolii

Gymnocarpium robertianum

E1

E1

E1

E1

E1

E1

E1

E1

E1

E1

E1

E1

E1

E1

E1

E1

E1

E1

E1

E1

E1

E2

E1

E1

E1

E1 \begin{tabular}{l|llllll|llllll} 
E1 & $\cdot$ & $\cdot$ & $\cdot$ & $\cdot$ & $\cdot$ & $\cdot$ & 1 & $\cdot$ & $\cdot$ & $\cdot$ & 1 & 10 \\
E1 & $\cdot$ & $\cdot$ & $\cdot$ & $\cdot$ & $\cdot$ & $\cdot$ & $\cdot$ & $\cdot$ & $\cdot$ & + & 1 & 10 \\
E1 & $\cdot$ & $\cdot$ & $\cdot$ & $\cdot$ & $\cdot$ & $\cdot$ & $\cdot$ & $\cdot$ & $\cdot$ & + & 1 & 10
\end{tabular}

330

660

330

330

220

220

110

110

110

110

E1 
Number of relevé (Zaporedna številka popisa)

Adenostyles glabra

Dryopteris villarii

Biscutella laevigata

Hieracium bifidum

Rumex scutatus

Minuartia austriaca

Rhodiola rosea

Crepis kerneri

Achnatherum calamagrostis

Aquilegia einseleana

Valeriana montana

Cystopteris montana

Trisetum argenteum

Athamanta cretensis

Arabis alpina

Ligusticum seguieri

Cy Cystopteridion fragilis

Cystopteris fragilis

Heliosperma pusillum

Cystopteris regia

PS Physoplexido comosae-Saxifragion petraeae

Campanula zoysii

Saxifraga squarrosa

Saxifraga hostii

PC Potentilletalia caulescentis

Campanula cochleariifolia

Erysimum sylvestre

Festuca stenantha

Campanula cespitosa

AT Asplenietea trichomanis

Asplenium viride

Moehringia muscosa

Gypsophila repens

Kernera saxatilis

Asplenium ruta-muraria

Asplenium trichomanes

$\mathrm{Fu} \quad$ Fungi (Glive)

Laricifomes officinalis

ML Mosses and lichens (Mahovi in lišaji)

Ctenidium molluscum

Dicranum scoparium

Tortella tortuosa

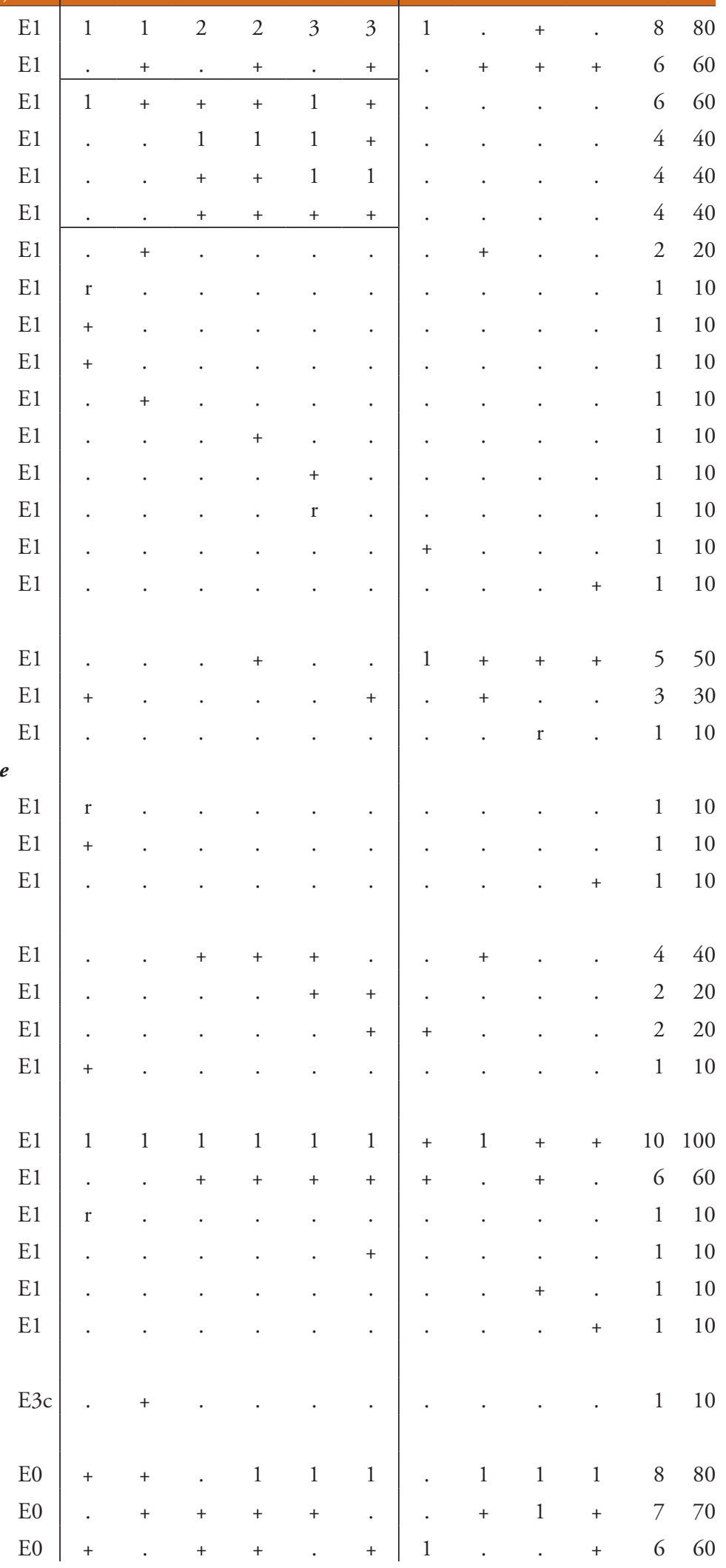


Number of relevé (Zaporedna številka popisa)

Rhytidiadelphus triquetrus

Hylocomium splendens

Peltigera leucophlebia

Polytrichum formosum

Mnium thomsonii

Bryum capillare

Orthothecium rufescens

Neckera crispa

Marchantia polymorpha

Plagiomnium undulatum

Schistidium sp.

Plagiochila porelloides

Conocephalum conicum

Campylium protensum

Fissidens dubius

Letharia vulpina

Peltigera canina

Hypnum cupressiforme

Sanionia uncinata

Isothecium alopecuroides

Hypogymnia physodes

Homalothecium lutescens

Bryum argenteum

Rhytidiadelphus loreus

Plagiochila asplenioides

Rhizomnium punctatum

\begin{tabular}{|c|c|c|c|c|c|c|c|c|c|c|c|c|}
\hline & 1 & 2 & 3 & 4 & 5 & 6 & 7 & 8 & 9 & 10 & Pr. & Fr. \\
\hline E0 & + & + & + & . & + & . & . & . & + & 1 & 6 & 60 \\
\hline E0 & . & . & . & 4 & + & 1 & + & 1 & 1 & . & 6 & 60 \\
\hline E0 & . & . & . & + & . & . & . & + & + & + & 4 & 40 \\
\hline E0 & . & . & . & + & + & . & . & . & + & . & 3 & 30 \\
\hline E0 & . & . & . & . & . & . & + & . & + & + & 3 & 30 \\
\hline E0 & . & . & . & . & . & . & + & . & + & + & 3 & 30 \\
\hline E0 & + & . & . & . & . & . & . & + & . & . & 2 & 20 \\
\hline E0 & + & . & . & . & . & . & + & . & . & . & 2 & 20 \\
\hline E0 & . & . & + & . & . & . & . & . & + & . & 2 & 20 \\
\hline E0 & . & . & + & . & . & . & + & . & . & . & 2 & 20 \\
\hline E0 & . & . & . & . & + & . & + & . & . & . & 2 & 20 \\
\hline E0 & . & . & . & . & . & . & + & + & . & . & 2 & 20 \\
\hline E0 & + & . & . & . & . & . & . & . & . & . & 1 & 10 \\
\hline E0 & + & . & . & . & . & . & . & . & . & . & 1 & 10 \\
\hline E0 & . & + & . & . & . & . & . & . & . & . & 1 & 10 \\
\hline E3c & . & + & . & . & . & . & . & . & . & . & 1 & 10 \\
\hline E0 & . & + & . & . & . & . & . & . & . & . & 1 & 10 \\
\hline E0 & . & . & . & + & . & . & . & . & . & . & 1 & 10 \\
\hline E0 & . & . & . & . & . & + & . & . & . & . & 1 & 10 \\
\hline E0 & . & . & . & . & . & . & + & . & . & . & 1 & 10 \\
\hline E3c & . & . & . & . & . & . & + & . & . & . & 1 & 10 \\
\hline E0 & . & . & . & . & . & . & + & . & . & . & 1 & 10 \\
\hline E0 & . & . & . & . & . & . & 1 & . & . & . & 1 & 10 \\
\hline E0 & . & . & . & . & . & . & . & . & + & . & 1 & 10 \\
\hline E0 & . & . & . & . & . & . & . & . & + & . & 1 & 10 \\
\hline E0 & & & & & . & . & . & . & 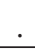 & + & 1 & 10 \\
\hline
\end{tabular}

\section{Legend - Legenda}

ID Igor Dakskobler

A Limestone-apnenec

D Dolomite - dolomit

Gr Gravel - grušč

Re Rendzina - rendzina

Kd Coluvial-deluvial soils - koluvialno-deluvialna tla

JA Julian Alps - Julijske Alpe

K Karavanke - Karavanke

Pr. Presence (number of relevés in which the species is presented) - število popisov, v katerih se pojavlja vrsta

Fr. Frequency in $\%$ - frekvenca v \% 


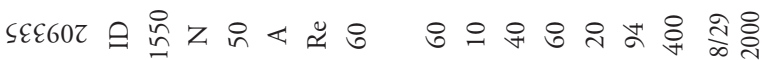

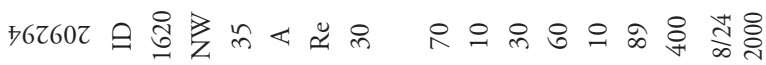

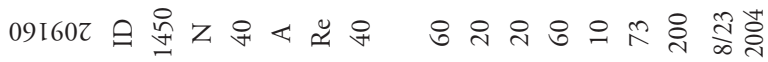

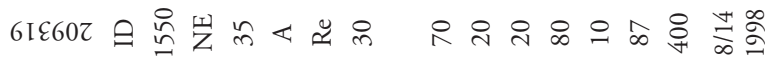

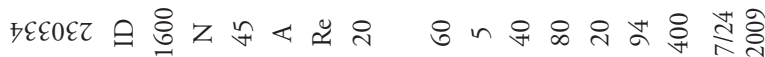

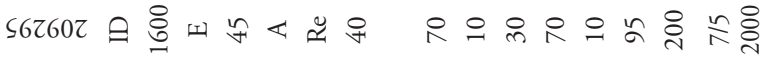

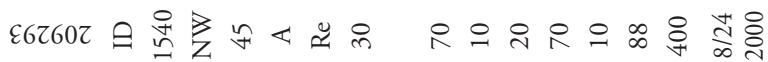

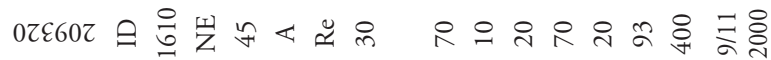

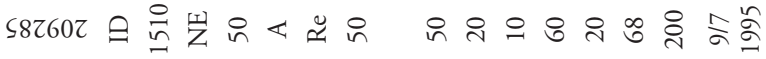
oz001z $\Leftrightarrow$ 근

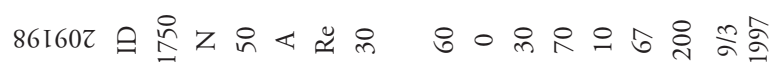

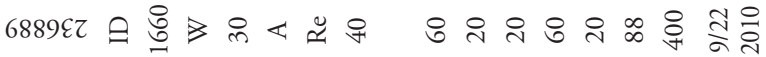

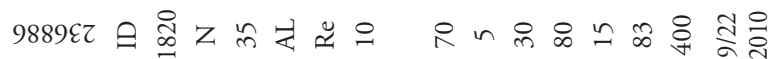

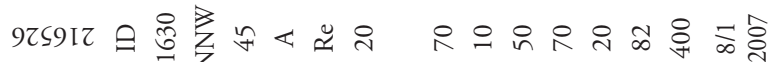

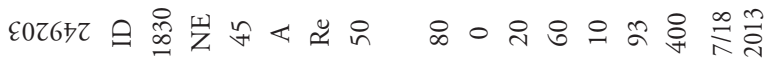

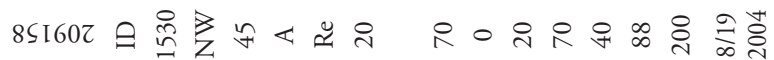

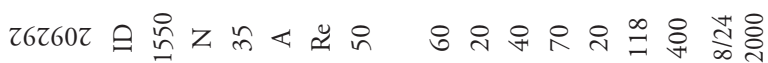

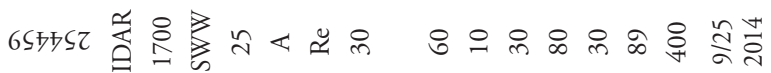

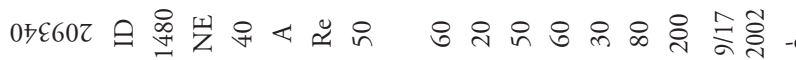

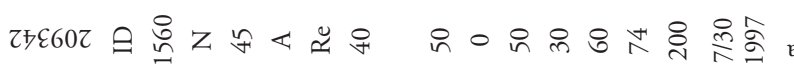

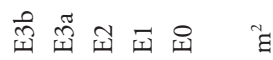

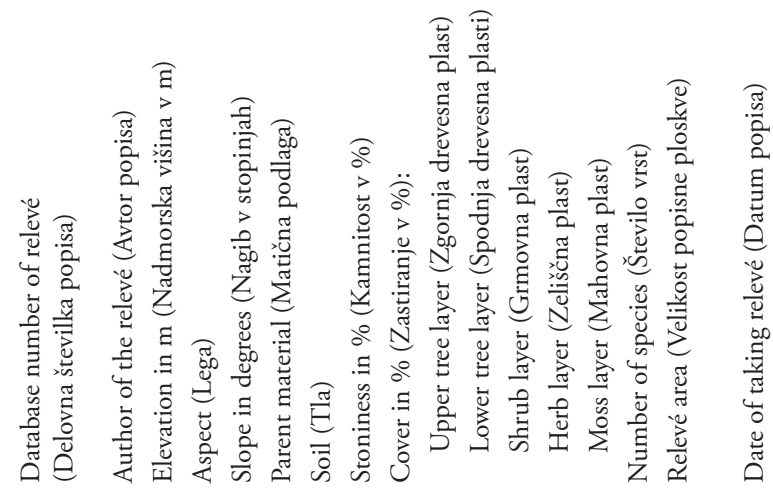

봉ㅇㅇㅇㅡ

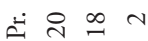

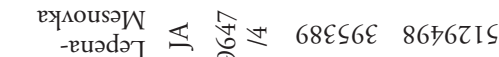

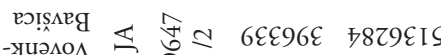

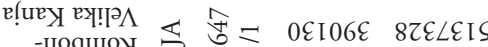

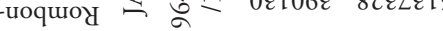

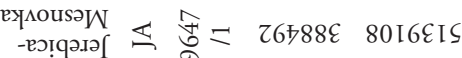

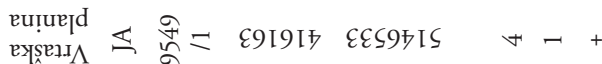

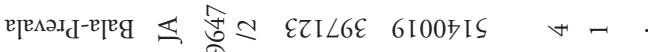

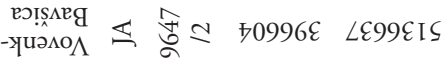

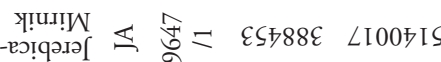

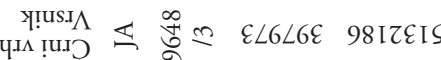

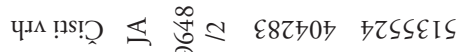
вэнеуіоч

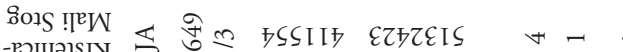

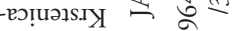

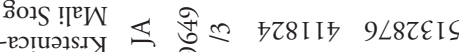

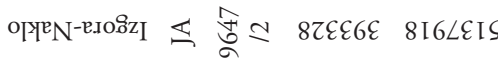

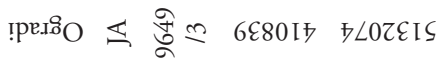

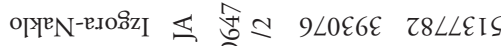

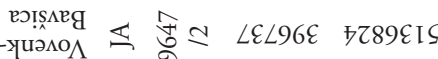

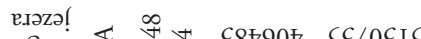

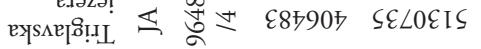

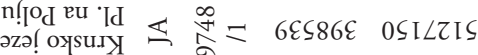

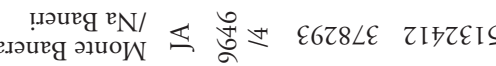
오로되 포 
\&

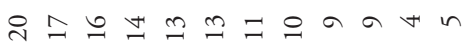

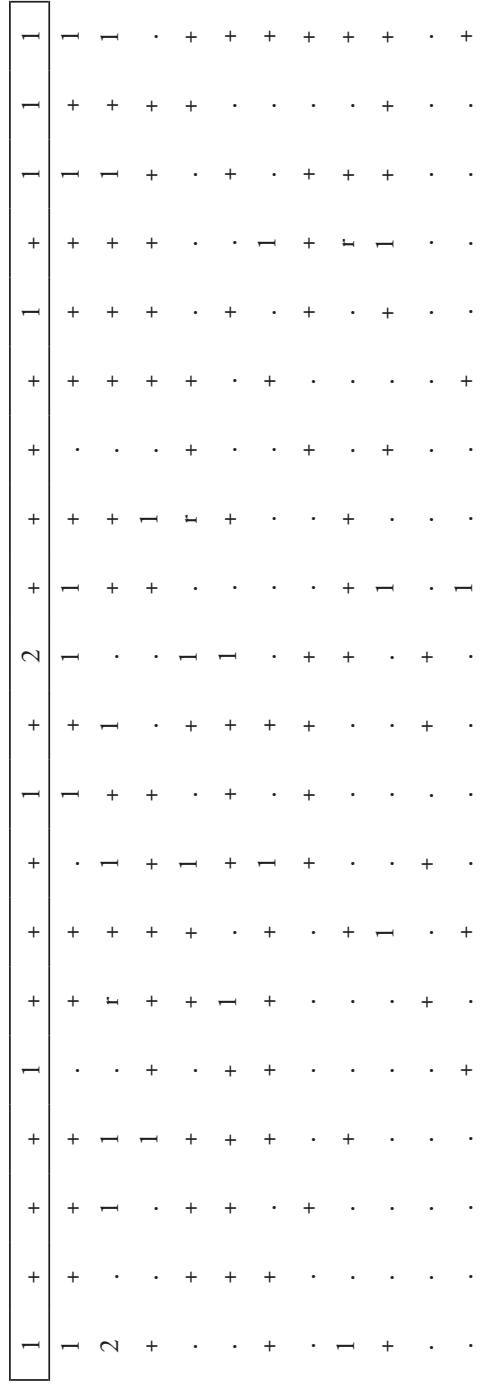

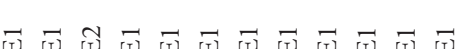

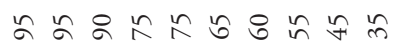
$\curvearrowright \frown \stackrel{\infty}{\sim} \cong \simeq \exists a n$

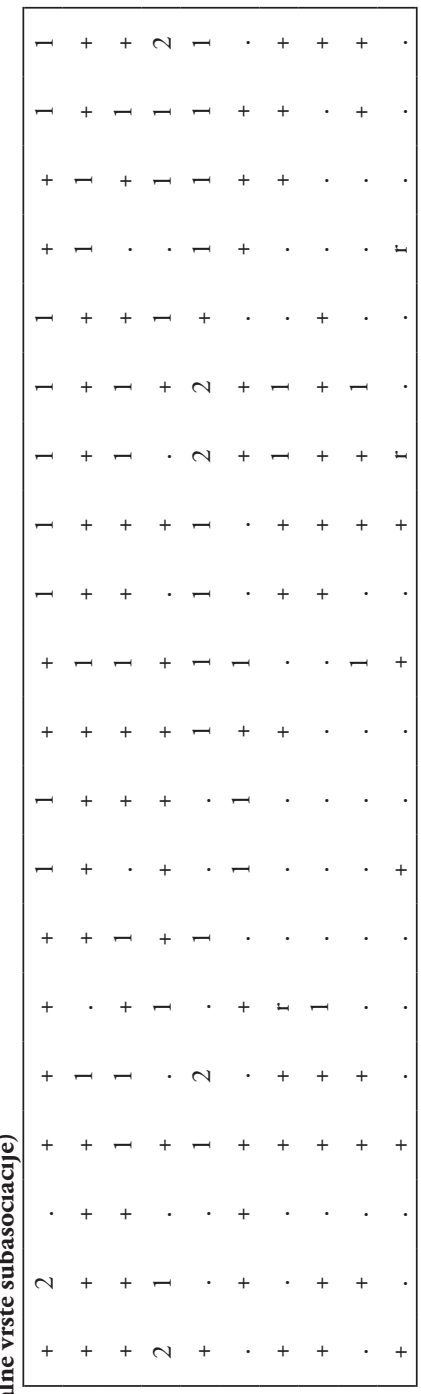

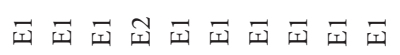

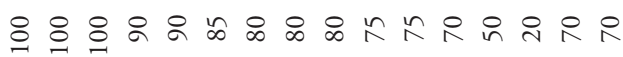

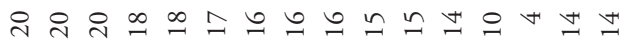
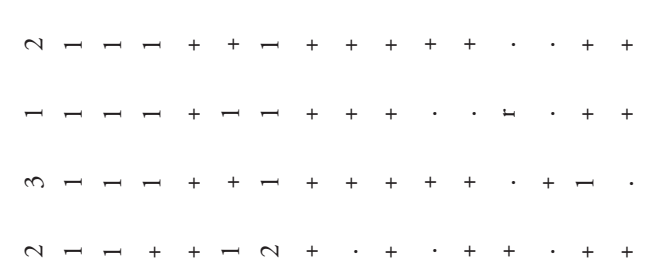

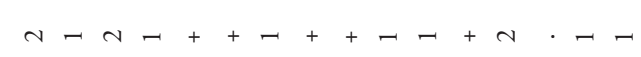
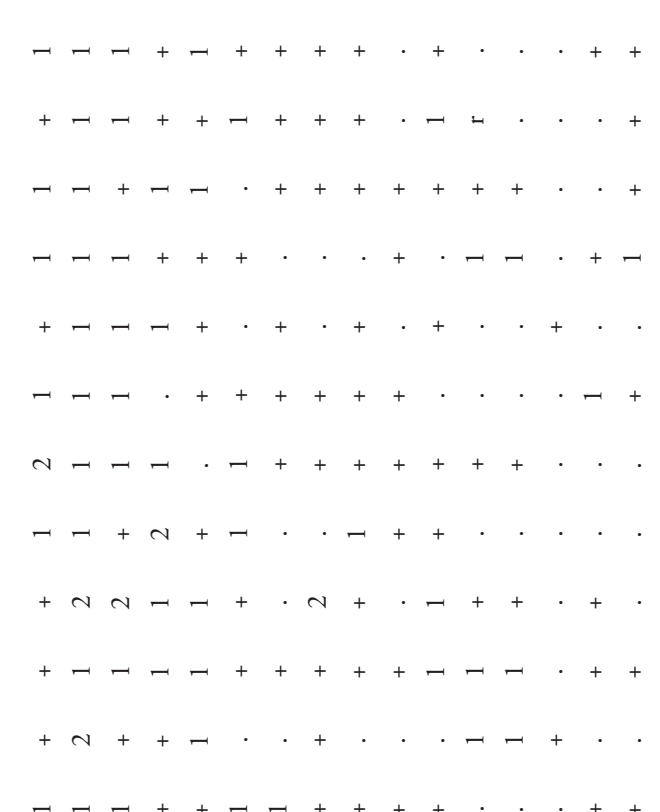

n-

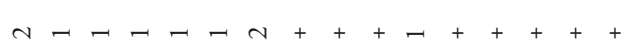

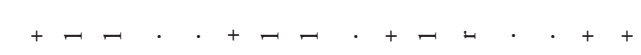

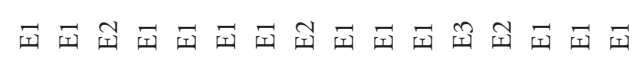

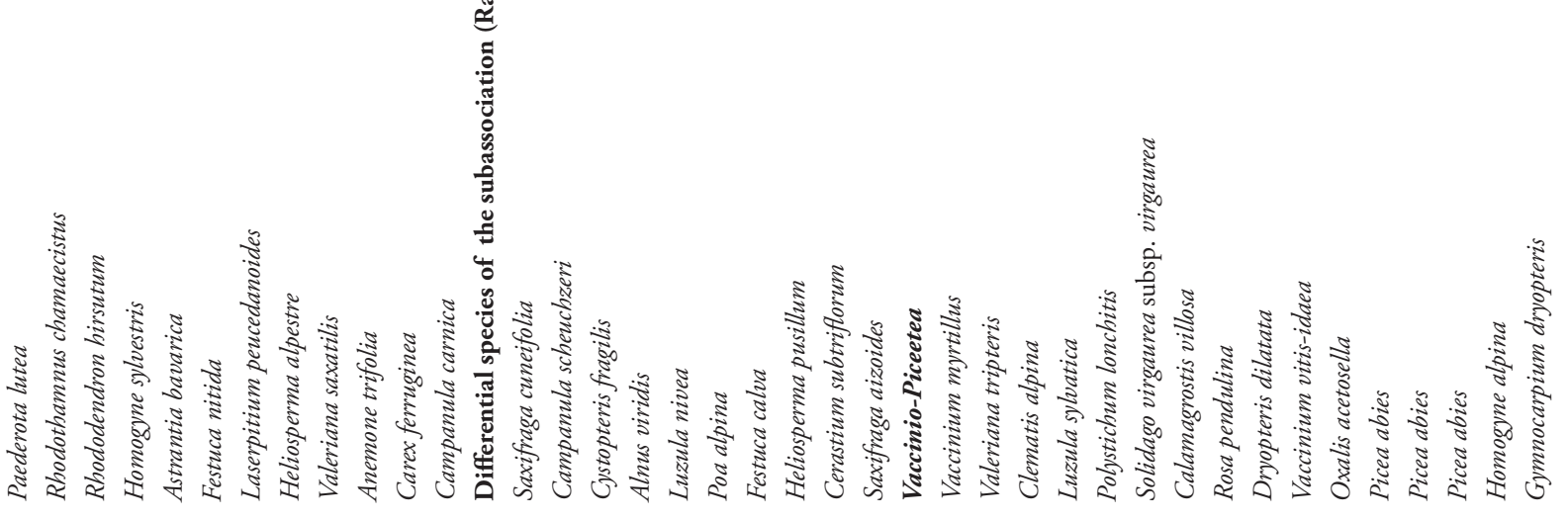

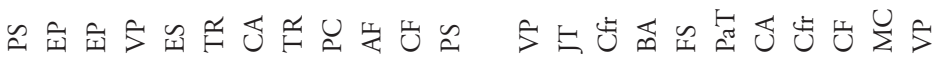




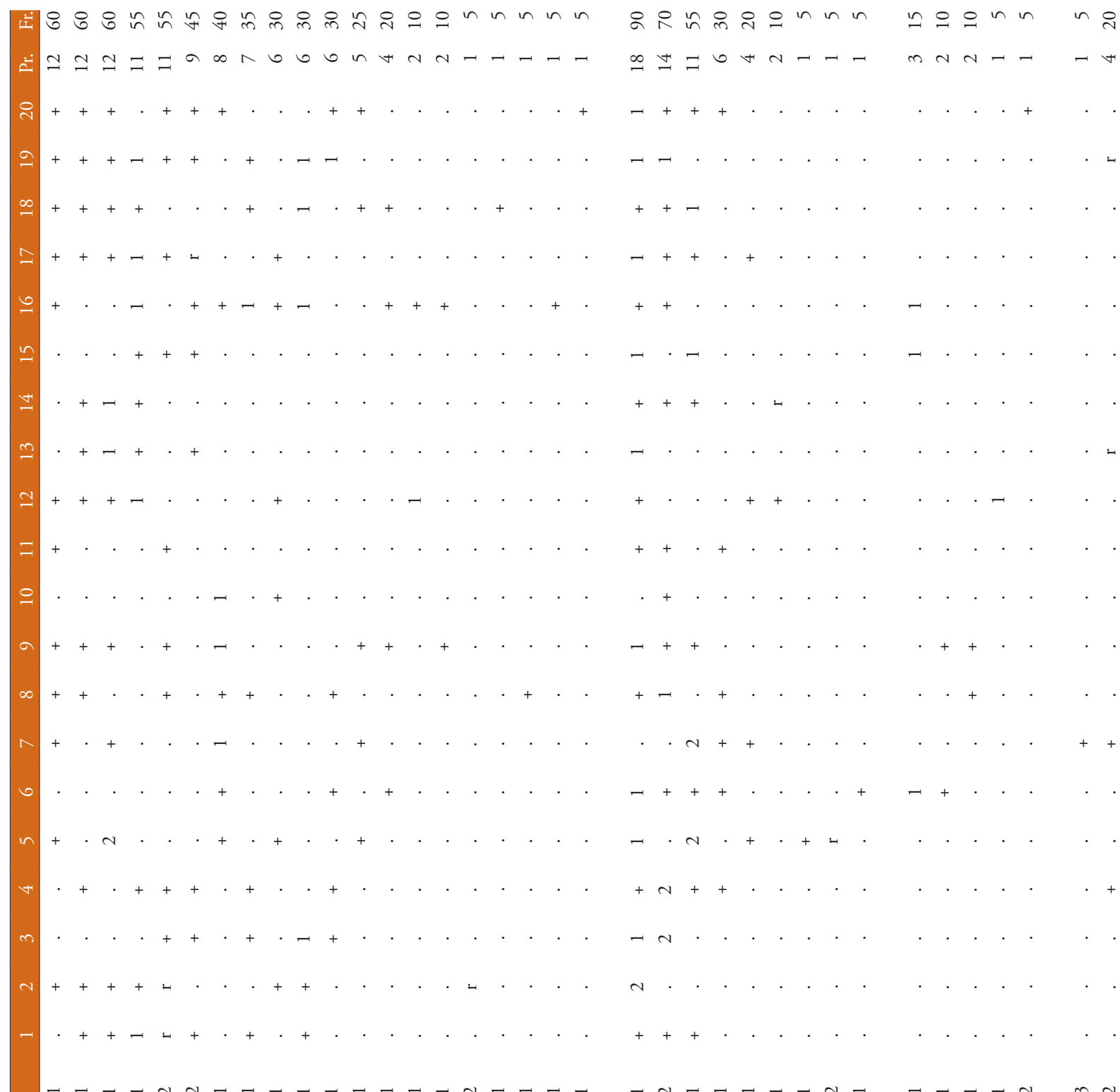

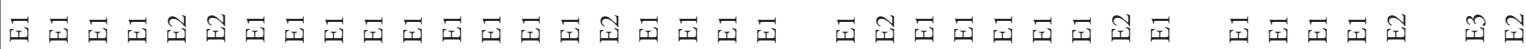




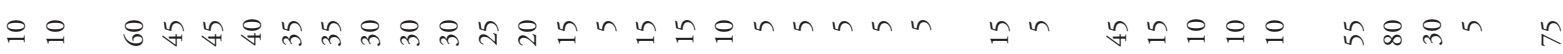

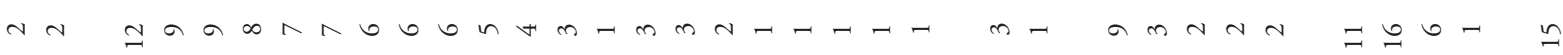

4
7

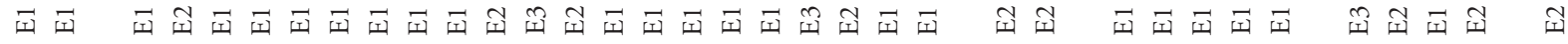

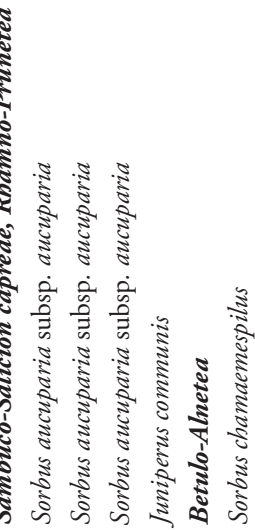

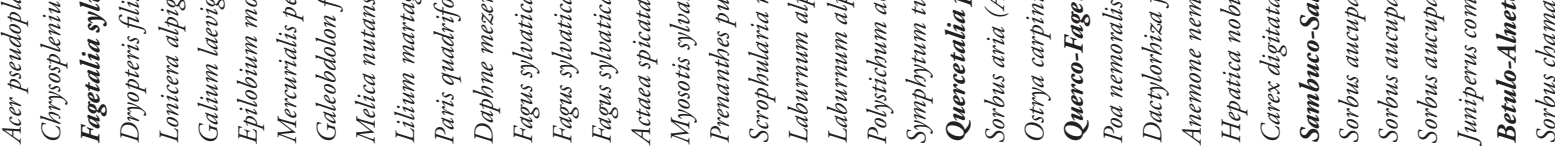
出 $\theta \quad$ ए 


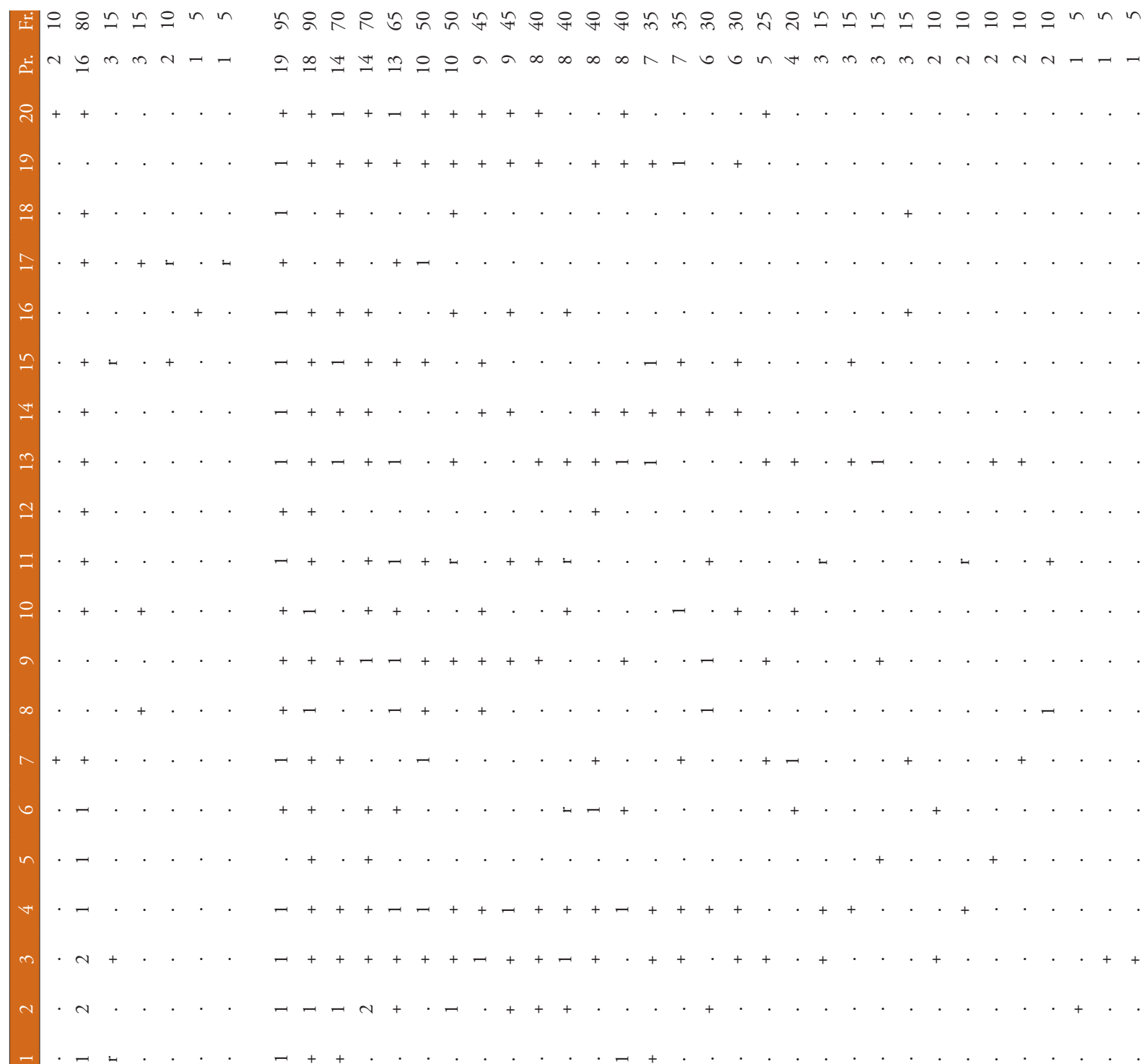

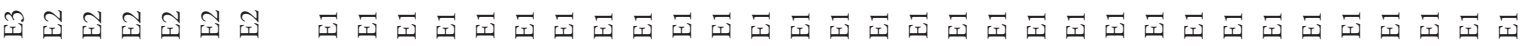
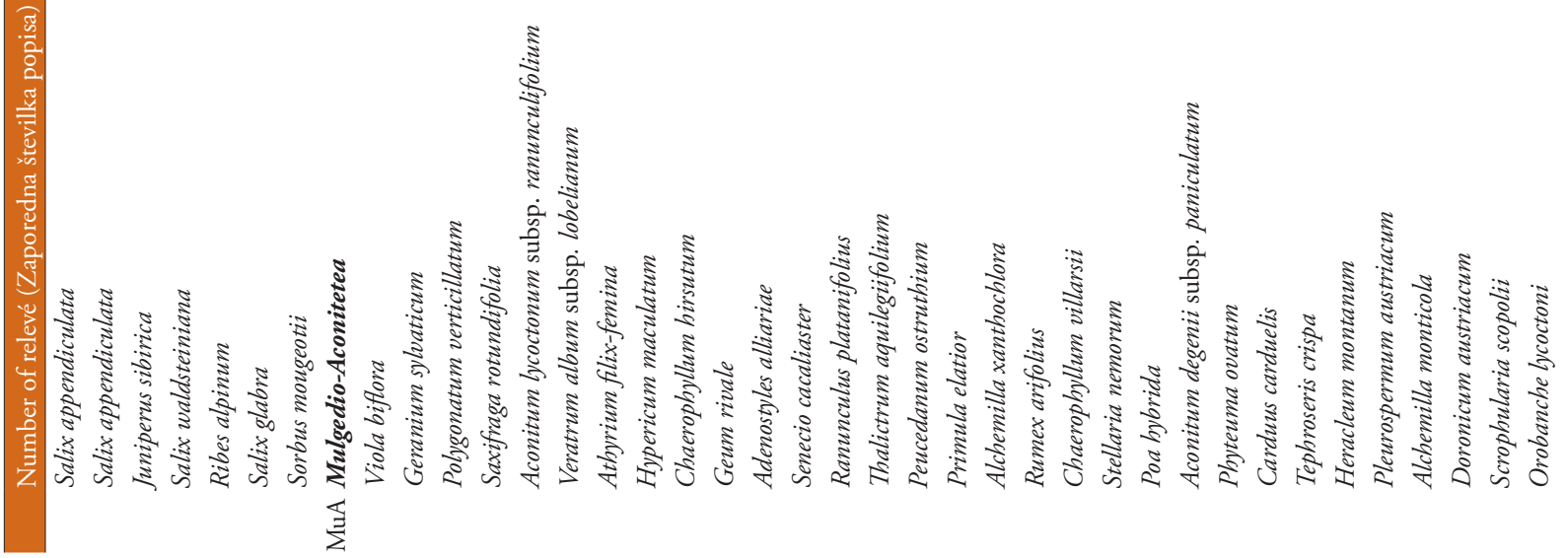


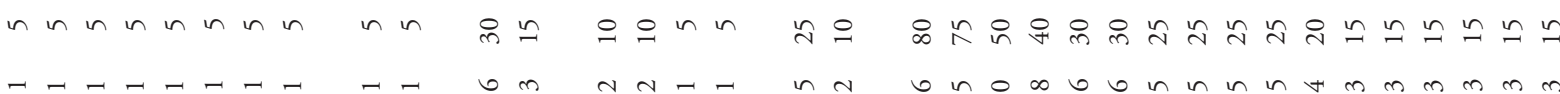

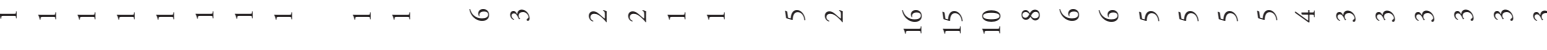

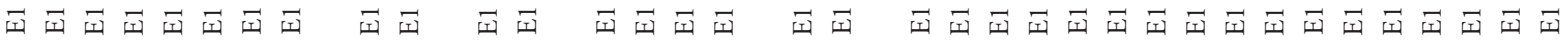

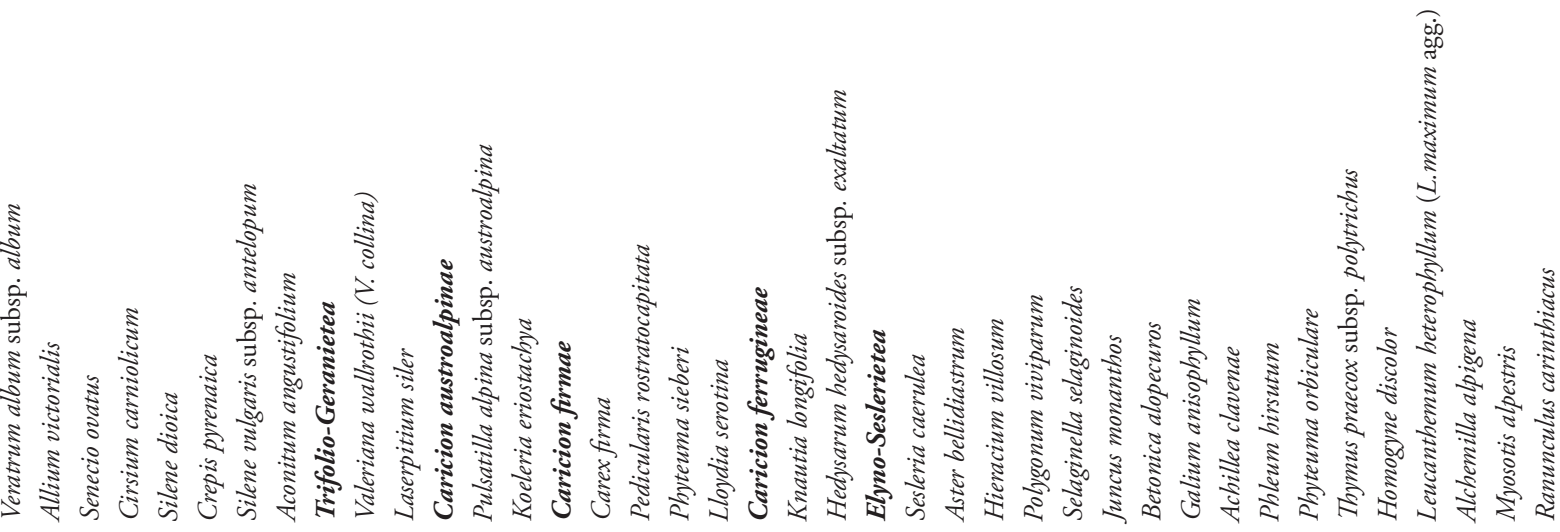

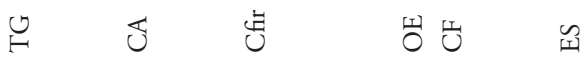




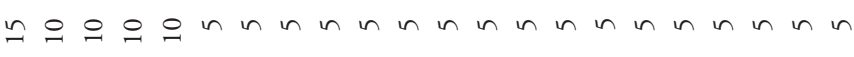

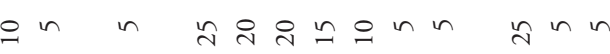

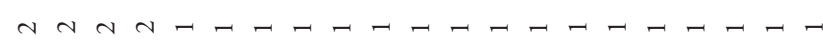

ar -1

in trant

in $\rightarrow-$

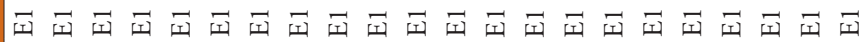

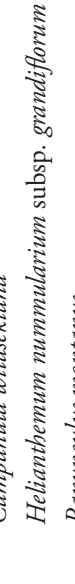

푀 푀

푀

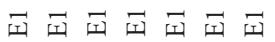

푀포 피

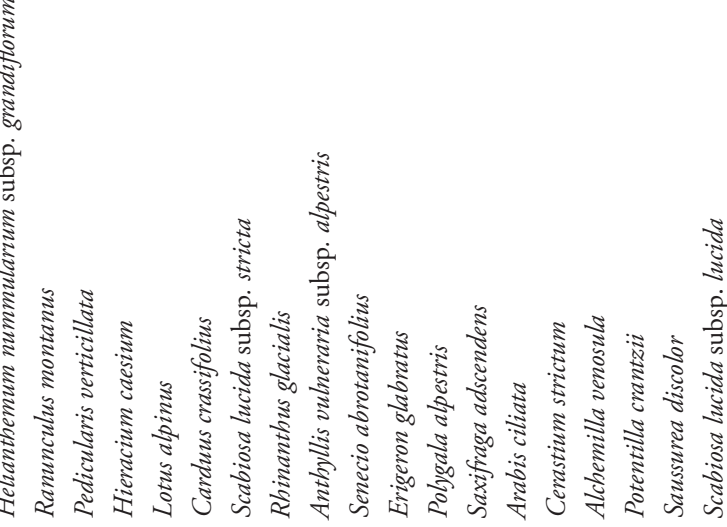




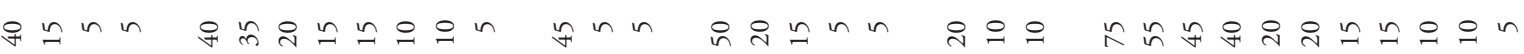

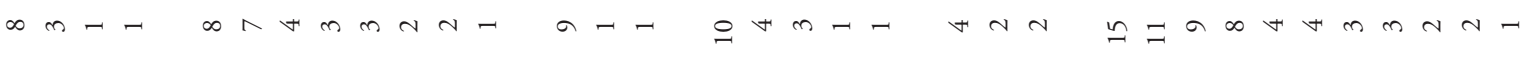

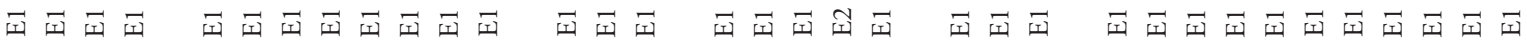

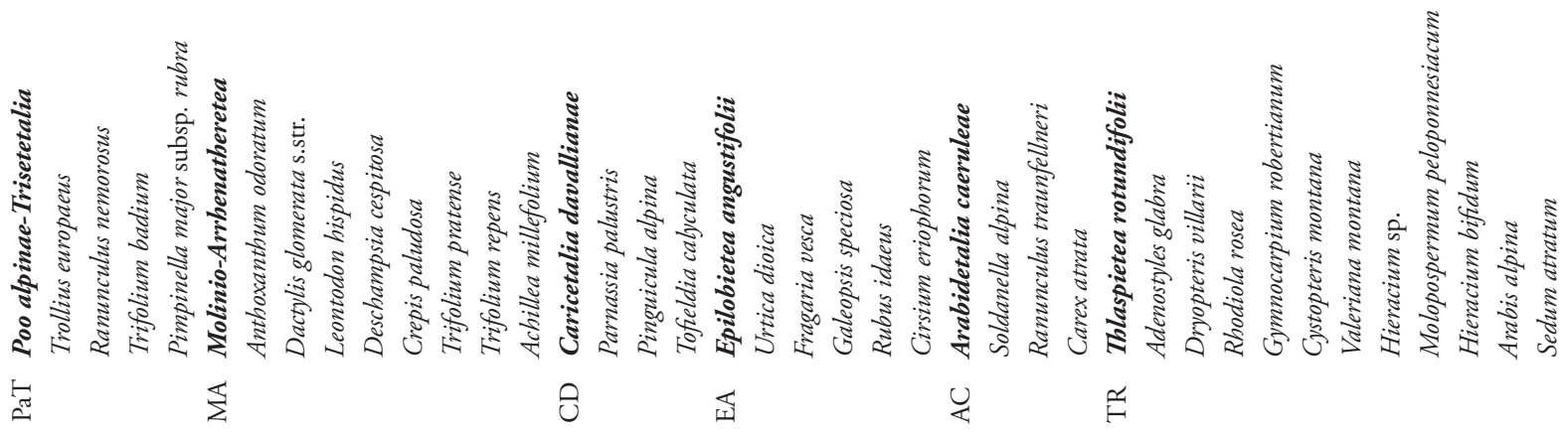




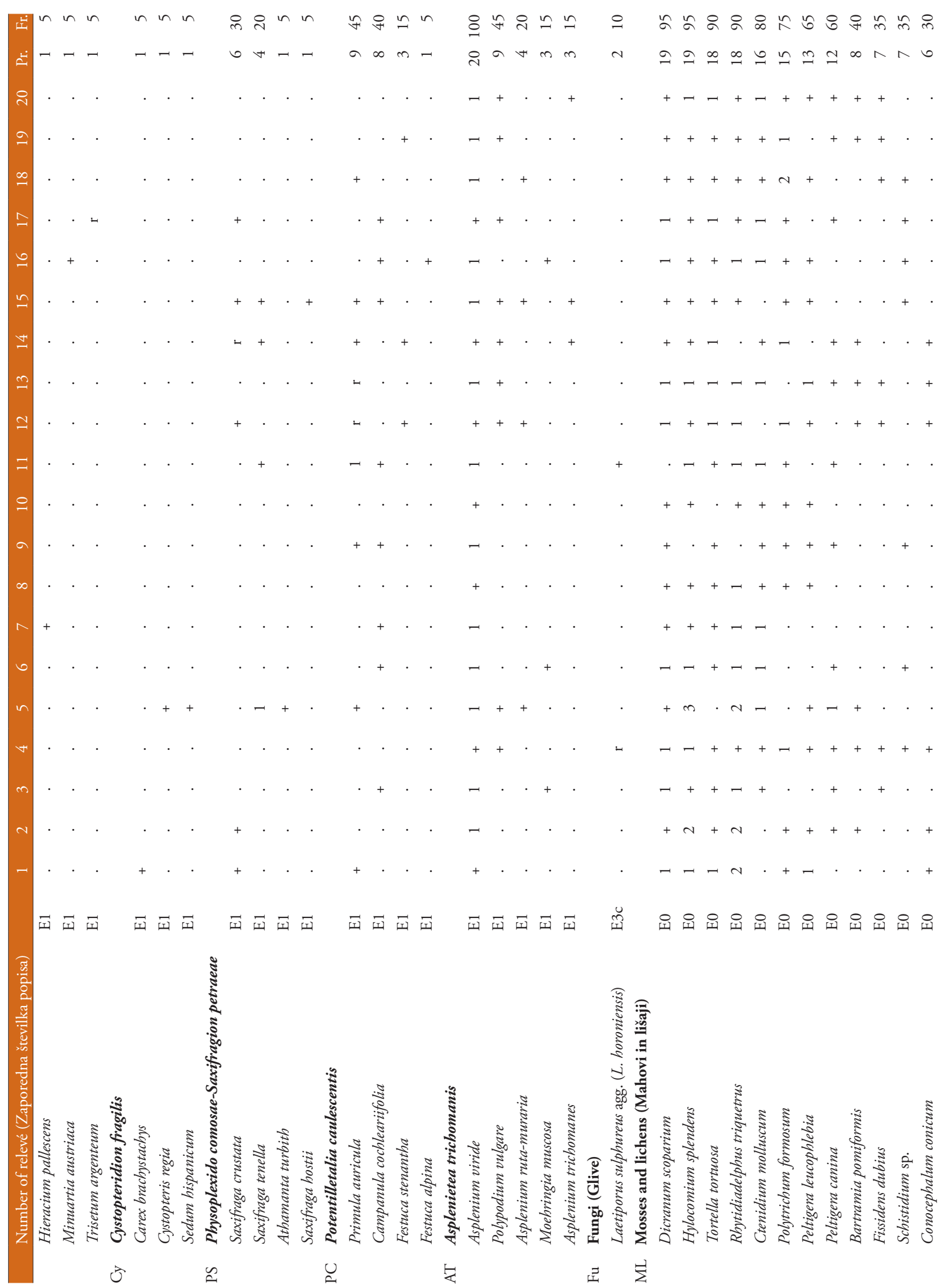




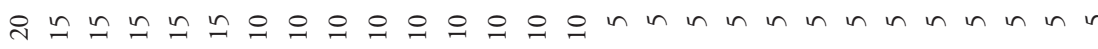

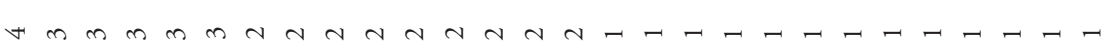


Table 16 (Tabela 16): Rhodothamno-Laricetum piceetosum abietis

\begin{tabular}{|c|c|c|c|c|c|c|c|c|c|c|c|c|}
\hline Number of relevé (Zaporedna številka popisa) & & 1 & 2 & 3 & 4 & 5 & 6 & 7 & 8 & 9 & 10 & 11 \\
\hline $\begin{array}{l}\text { Database number of relevé } \\
\text { (Delovna številka popisa) }\end{array}$ & & $\begin{array}{l}\vec{\approx} \\
\stackrel{0}{1}\end{array}$ & 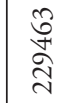 & 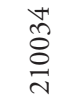 & 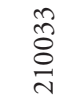 & $\begin{array}{l}0 \\
\stackrel{\overbrace{}}{8} \\
\stackrel{1}{\sim}\end{array}$ & 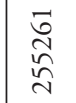 & $\begin{array}{l}\stackrel{n}{\infty} \\
\stackrel{\infty}{n} \\
\end{array}$ & $\begin{array}{l}\hat{\infty} \\
\infty \\
\hat{n}\end{array}$ & 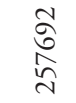 & 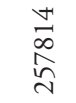 & 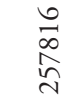 \\
\hline Author of the relevé (Avtor popisa) & & $\Leftrightarrow$ & $\ominus$ & $\ominus$ & $\ominus$ & $\Theta$ & $\stackrel{\text { 究 }}{\varrho}$ & 究 & 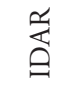 & 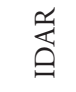 & 究 & $\stackrel{\text { ș }}{\varrho}$ \\
\hline Elevation in $\mathrm{m}$ (Nadmorska višina $\mathrm{v} \mathrm{m})$ & & $\stackrel{\curvearrowright}{\curvearrowleft}$ & 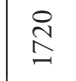 & $\underset{\tilde{\sigma}}{\infty}$ & 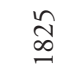 & 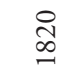 & $\stackrel{8}{2}$ & $\underset{6}{\overparen{G}}$ & $\stackrel{n}{6}$ & 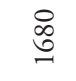 & $\stackrel{\Re}{\cong}$ & $\underset{6}{\stackrel{6}{6}}$ \\
\hline Aspect (Lega) & & 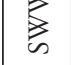 & 贸 & 昞 & 空 & $\infty$ & 紊 & 乩 & 紊 & $\tilde{n}$ & 贸 & 岓 \\
\hline Slope in degrees (Nagib v stopinjah) & & 30 & 25 & 20 & 20 & 15 & 25 & 15 & 25 & 15 & 10 & 10 \\
\hline Parent material (Matična podlaga) & & DA & A & A & A & A & A & A & A & A & A & A \\
\hline Soil (Tla) & & $\operatorname{Re}$ & $\operatorname{Re}$ & $\operatorname{Re}$ & $\operatorname{Re}$ & $\operatorname{Re}$ & $\operatorname{Re}$ & $\operatorname{Re}$ & $\operatorname{Re}$ & $\operatorname{Re}$ & $\operatorname{Re}$ & $\operatorname{Re}$ \\
\hline Stoniness in \% (Kamnitost $\mathrm{v} \%$ ) & & 20 & 30 & 50 & 30 & 40 & 40 & 50 & 30 & 20 & 60 & 30 \\
\hline \multicolumn{13}{|l|}{ Cover in \% (Zastiranje v \%): } \\
\hline Upper tree layer (Zgornja drevesna plast) & E3b & 60 & 50 & 50 & 60 & 50 & 40 & 50 & 55 & 50 & 40 & 50 \\
\hline Lower tree layer (Spodnja drevesna plasti) & E3a & 20 & 10 & 0 & 10 & 0 & 10 & 10 & 10 & 5 & 5 & 5 \\
\hline Shrub layer (Grmovna plast) & E2 & 40 & 30 & 40 & 30 & 30 & 25 & 15 & 15 & 30 & 30 & 30 \\
\hline Herb layer (Zeliščna plast) & E1 & 80 & 80 & 60 & 70 & 60 & 60 & 80 & 90 & 80 & 70 & 80 \\
\hline Moss layer (Mahovna plast) & E0 & 10 & 10 & 10 & 10 & 10 & 10 & 10 & 10 & 10 & 10 & 10 \\
\hline Number of species (Število vrst) & & 89 & 88 & 94 & 83 & 92 & 87 & 72 & 84 & 77 & 81 & 87 \\
\hline Relevé area (Velikost popisne ploskve) & $\mathrm{m}^{2}$ & 400 & 400 & 200 & 400 & 400 & 400 & 400 & 400 & 400 & 400 & 400 \\
\hline & & $6 / 21$ & $7 / 17$ & $7 / 21$ & $7 / 21$ & $7 / 21$ & $9 / 24$ & $8 / 26$ & $8 / 26$ & $8 / 27$ & $8 / 26$ & $8 / 26$ \\
\hline Date of taking relevé (Datum popisa) & & 2005 & 2009 & 2005 & 2005 & 2005 & 2014 & 2015 & 2015 & 2015 & 2015 & 2015 \\
\hline Locality (Nahajališče) & & 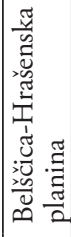 & $\begin{array}{l}\frac{y}{\vec{a}} \\
\dot{a} \\
\dot{a}\end{array}$ & 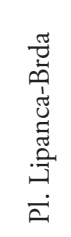 & 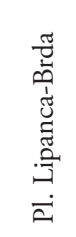 & 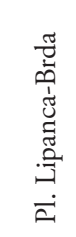 & 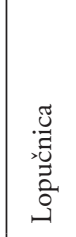 & 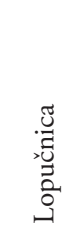 & 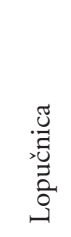 & 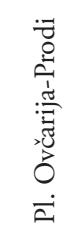 & 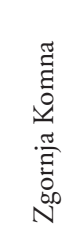 & 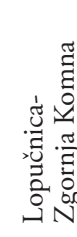 \\
\hline Mountain range (Pogorje) & & $\mathrm{K}$ & JA & JA & JA & JA & JA & JA & JA & JA & JA & JA \\
\hline & & 9550 & 9649 & 9649 & 9649 & 9649 & 9648 & 9648 & 9648 & 9648 & 9648 & 9648 \\
\hline Quadrant (Kvadrant) & & 12 & 12 & 12 & 12 & 12 & 14 & 14 & 14 & 14 & 14 & 14 \\
\hline Coordinate GK-Y (D-48) & $\mathrm{m}$ & $\begin{array}{l}\tilde{m} \\
\tilde{\infty} \\
\sim \\
\widetilde{v}\end{array}$ & $\begin{array}{l}\infty \\
\infty \\
\infty \\
\infty \\
\not \\
\forall\end{array}$ & 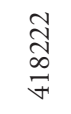 & 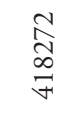 & $\underset{\stackrel{N}{\curvearrowright}}{\stackrel{\infty}{F}}$ & $\begin{array}{l}\text { ㅇ } \\
8 \\
\text { O } \\
\stackrel{+}{1}\end{array}$ & 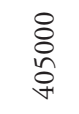 & $\begin{array}{l}8 \\
8 \\
\varnothing \\
o \\
o+\end{array}$ & $\begin{array}{l}\overrightarrow{8} \\
\text { 응 } \\
\text { ᄋ }\end{array}$ & 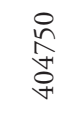 & $\begin{array}{l}\stackrel{0}{n} \\
\stackrel{2}{+} \\
\stackrel{+}{*}\end{array}$ \\
\hline Coordinate GK-X (D-48) & $\mathrm{m}$ & \begin{tabular}{l}
$n$ \\
$\approx$ \\
$n$ \\
\multirow{n}{n}{} \\
\end{tabular} & $\begin{array}{l}m \\
\infty \\
\infty \\
\stackrel{\infty}{n} \\
\stackrel{n}{n}\end{array}$ & 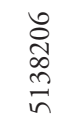 & $\begin{array}{l}\infty \\
\stackrel{\infty}{=} \\
\stackrel{\infty}{n} \\
\stackrel{n}{n}\end{array}$ & $\begin{array}{l}\stackrel{0}{N} \\
\infty \\
\stackrel{\infty}{n} \\
\stackrel{n}{n}\end{array}$ & $\begin{array}{l}\text { o } \\
\stackrel{n}{n} \\
\stackrel{n}{n}\end{array}$ & $\begin{array}{l}\stackrel{n}{0} \\
\stackrel{n}{n} \\
\stackrel{n}{n}\end{array}$ & $\begin{array}{l}\stackrel{0}{n} \\
\stackrel{n}{n} \\
\stackrel{n}{n}\end{array}$ & $\begin{array}{l}\infty \\
\stackrel{m}{\curvearrowright} \\
\stackrel{\overbrace{}}{n}\end{array}$ & $\frac{\stackrel{n}{n}}{\stackrel{n}{n}}$ & $\begin{array}{l}\text { r. } \\
\stackrel{n}{n} \\
\stackrel{n}{n}\end{array}$ \\
\hline
\end{tabular}

$\begin{array}{ll}\text { VP } & \text { Larix decidua } \\ \text { VP } & \text { Larix decidua } \\ \text { VP } & \text { Larix decidua } \\ \text { PS } & \text { Paederota lutea } \\ \text { CA } & \text { Laserpitium peucedanoides } \\ \text { EP } & \text { Rhododendron hirsutum } \\ \text { TR } & \text { Heliosperma alpestre } \\ \text { TR } & \text { Festuca nitida } \\ \text { ES Astrantia bavarica } \\ \text { Cf Carex ferruginea } \\ \text { VP Homogyne sylvestris } \\ \text { EP Rhodothamnus chamaecistus } \\ \text { PC } & \text { Valeriana saxatilis } \\ \text { AF } & \text { Anemone trifolia }\end{array}$

\begin{tabular}{|c|c|c|c|c|c|c|c|c|c|c|c|}
\hline $1 \mathrm{ra}$ & ra & tectiat ia & $1 \mathrm{lKO}$ & Hese & & & & & & & Fr. \\
\hline E3 & 3 & 3 & 4 & 3 & 3 & 3 & 4 & 3 & 3 & 3 & 11100 \\
\hline E2 & + & + & + & 2 & 1 & + & + & + & 1 & 1 & 10 \\
\hline E1 & . & . & + & . & . & . & . & . & . & . & 1 \\
\hline . & + & 1 & + & + & + & + & + & . & + & + & 82 \\
\hline . & 1 & + & . & . & + & 1 & 1 & 1 & 1 & 1 & 73 \\
\hline . & + & 1 & 1 & + & . & . & + & + & + & + & 73 \\
\hline+ & . & 1 & 1 & 1 & . & . & . & + & + & + & 64 \\
\hline . & . & 1 & + & 1 & + & . & . & 1 & 1 & + & 64 \\
\hline . & . & . & + & . & . & + & . & 1 & . & + & 36 \\
\hline . & 1 & . & . & . & + & . & . & + & . & . & 27 \\
\hline . & . & . & + & . & . & + & . & . & . & + & 27 \\
\hline . & . & . & . & . & + & . & + & . & . & + & 27 \\
\hline . & + & . & . & . & . & . & . & . & . & + & 18 \\
\hline 1 & . & . & . & . & . & . & . & . & . & . & 1 \\
\hline
\end{tabular}




\section{\begin{tabular}{l|l|llll|llllllll} 
Number of relevé (Zaporedna številka popisa) & 1 & 2 & 3 & 4 & 5 & 6 & 7 & 8 & 9 & 10 & 11 & Pr. & Fr.
\end{tabular}}

VP

VP Picea abies

VP Picea abies

Fs Daphne mezereum

QF Anemone nemorosa

TR Rhodiola rosea

VP Vaccinio-Piceetea

Vaccinium myrtillus

Vaccinium vitis-idaea

Polystichum lonchitis

Rosa pendulina

Valeriana tripteris

Luzula sylvatica

Homogyne alpina

Hieracium murorum

Melampyrum sylvaticum

Clematis alpina

Aposeris foetida

Solidago virgaurea subsp. virgaurea

Calamagrostis villosa

Oxalis acetosella

Huperzia selago

Dryopteris expansa

Luzula luzulina

Lonicera caerulea

Maianthemum bifolium

Dryopteris dilatata

Lycopodium annotinum

Gentiana asclepiadea

Gymnocarpium dryopteris

Lonicera nigra

Saxifraga cuneifolia

Calamagrostis arundinacea

Veronica urticifolia

Phegopteris connectilis

Luzula luzuloides

Abies alba

Moneses uniflora

Luzula pilosa

EP Erico-Pinetea

Pinus mugo

Rubus saxatilis

Erica carnea

Calamagrostis varia

Carex ornithopoda

Aquilegia nigricans

AF Aremonio-Fagion

Knautia drymeia

Cardamine enneaphyllos

Helleborus niger

Rhamnus fallax

TA Tilio-Acerion

Adoxa moschatellina

Acer pseudoplatanus

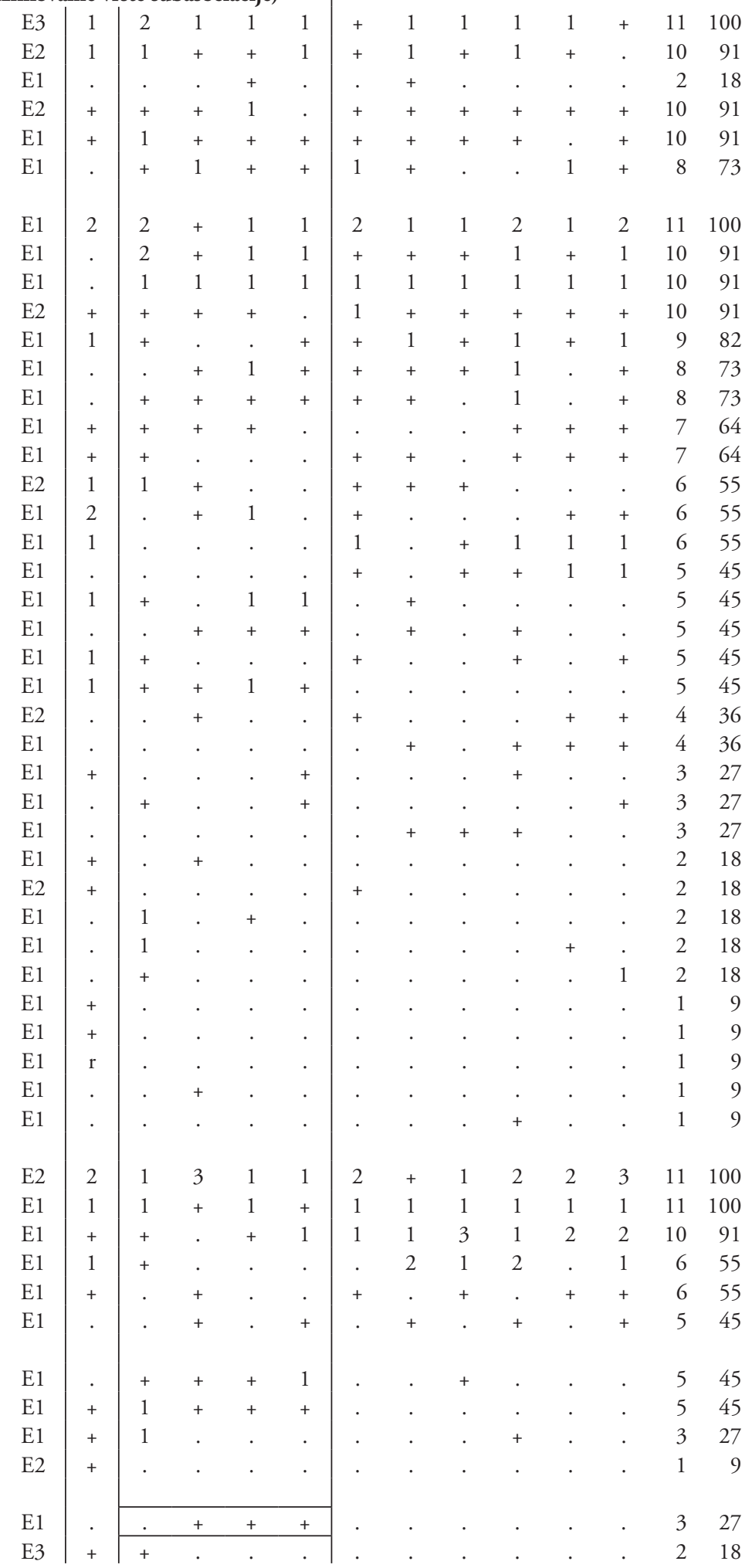




\begin{tabular}{|c|c|c|c|c|c|c|c|c|c|c|c|c|c|c|c|}
\hline & Number of relevé (Zaporedna številka popisa) & & 1 & 2 & 3 & 4 & 5 & 6 & 7 & 8 & 9 & 10 & 11 & Pr. & Fr. \\
\hline & Acer pseudoplatanus & E2 & + & + & . & . & . & . & . & . & . & . & . & 2 & 18 \\
\hline & Acer pseudoplatanus & E1 & + & + & . & . & . & . & . & . & . & . & . & 2 & 18 \\
\hline \multirow[t]{18}{*}{ FS } & Fagetalia sylvaticae & & & & & & & & & & & & & & \\
\hline & Galeobdolon flavidum & E1 & 1 & + & . & . & + & 1 & 1 & . & . & + & + & 7 & 64 \\
\hline & Melica nutans & E1 & 1 & 1 & . & . & . & 1 & + & . & . & + & + & 6 & 55 \\
\hline & Paris quadrifolia & E1 & + & + & + & . & . & + & . & . & . & + & + & 6 & 55 \\
\hline & Dryopteris filix-mas & E1 & 1 & + & . & . & . & . & . & . & . & + & + & 4 & 36 \\
\hline & Galium laevigatum & E1 & + & + & . & . & . & . & . & + & + & . & . & 4 & 36 \\
\hline & Lonicera alpigena & E2 & + & . & . & + & . & + & . & . & . & . & . & 3 & 27 \\
\hline & Lilium martagon & E1 & + & . & . & . & . & + & . & . & . & . & + & 3 & 27 \\
\hline & Mercurialis perennis & E1 & + & . & . & . & + & . & . & . & . & . & . & 2 & 18 \\
\hline & Myosotis sylvatica & E1 & 1 & + & . & . & . & . & . & . & . & . & . & 2 & 18 \\
\hline & Polystichum aculeatum & E1 & 1 & . & . & . & . & . & . & . & + & . & . & 2 & 18 \\
\hline & Fagus sylvatica & E3 & + & . & . & . & . & . & . & . & . & . & . & 1 & 9 \\
\hline & Prenanthes purpurea & E1 & + & . & . & . & . & . & . & . & . & . & . & 1 & 9 \\
\hline & Euphorbia amygdaloides & E1 & + & . & . & . & . & . & . & . & . & . & . & 1 & 9 \\
\hline & Epilobium montanum & E1 & + & . & . & . & . & . & . & . & . & . & . & 1 & 9 \\
\hline & Symphytum tuberosum & E1 & 1 & . & . & . & . & . & . & . & . & . & . & 1 & 9 \\
\hline & Actaea spicata & E1 & + & . & . & . & . & . & . & . & . & . & . & 1 & 9 \\
\hline & Asarum europaeum & E1 & + & . & . & . & . & . & . & . & . & . & . & 1 & 9 \\
\hline \multirow[t]{2}{*}{ QP } & Quercetalia pubescenti-petraeae & & & & & & & & & & & & & & \\
\hline & Convallaria majalis & E1 & + & . & . & . & . & . & + & . & . & . & . & 2 & 18 \\
\hline \multirow[t]{6}{*}{ QF } & Querco-Fagetea & & & & & & & & & & & & & & \\
\hline & Hepatica nobilis & E1 & . & 1 & . & + & . & . & . & . & . & . & . & 2 & 18 \\
\hline & Poa nemoralis & E1 & 1 & + & . & . & . & . & . & . & . & . & . & 2 & 18 \\
\hline & Carex digitata & E1 & . & + & . & . & + & . & . & . & . & . & . & 2 & 18 \\
\hline & Hieracium lachenalii & E1 & . & 1 & . & . & . & . & . & . & . & . & . & 1 & 9 \\
\hline & Moehringia trinervia & E1 & + & . & . & . & . & . & . & . & . & . & . & 1 & 9 \\
\hline \multirow[t]{5}{*}{ SSC } & Sambuco-Salicion capreae, Rhamno-Prunetea & & & & & & & & & & & & & & \\
\hline & Sorbus aucuparia subsp. aucuparia & E3 & 1 & . & . & . & . & + & . & + & . & . & + & 4 & 36 \\
\hline & Sorbus aucuparia subsp. aucuparia & E2 & 1 & . & . & . & . & . & . & + & . & . & 1 & 3 & 27 \\
\hline & Sorbus aucuparia subsp. aucuparia & E1 & . & . & . & . & . & . & . & . & . & . & + & 1 & 9 \\
\hline & Betula pendula & E2 & + & . & . & . & . & . & . & . & . & . & . & 1 & 9 \\
\hline \multirow[t]{6}{*}{$\mathrm{BA}$} & Betulo-Alnetea & & & & & & & & & & & & & & \\
\hline & Sorbus chamaemespilus & E2 & + & 1 & 1 & + & + & 2 & + & + & + & 1 & 1 & 11 & 100 \\
\hline & Juniperus sibirica & E2 & . & + & 1 & + & 1 & + & + & . & + & 1 & 1 & 9 & 82 \\
\hline & Salix appendiculata & E2 & + & + & . & . & . & + & . & + & . & . & 1 & 5 & 45 \\
\hline & Salix waldsteiniana & E2 & . & + & . & . & . & + & + & . & . & + & . & 4 & 36 \\
\hline & Ribes alpinum & E2 & + & + & + & . & . & . & . & . & . & . & . & 3 & 27 \\
\hline \multicolumn{16}{|c|}{ MuA Mulgedio-Aconitetea } \\
\hline & Geranium sylvaticum & E1 & 1 & 1 & + & 1 & + & + & 1 & + & + & 1 & 1 & 11 & 100 \\
\hline & Viola biflora & E1 & + & + & 1 & 1 & 1 & 1 & + & . & + & + & + & 10 & 91 \\
\hline & Veratrum album subsp. lobelianum & E1 & 1 & 1 & 1 & + & 1 & . & 1 & + & + & 1 & + & 10 & 91 \\
\hline & Saxifraga rotundifolia & E1 & + & + & 1 & 1 & 1 & + & + & . & + & + & + & 10 & 91 \\
\hline & Hypericum maculatum & E1 & 1 & + & + & + & 1 & + & 1 & + & 1 & 1 & . & 10 & 91 \\
\hline & Polygonatum verticillatum & E1 & 1 & + & + & . & + & 2 & . & + & 1 & 1 & + & 9 & 82 \\
\hline & Aconitum lycoctonum subsp. ranunculifolium & E1 & + & . & . & . & . & 1 & 2 & + & 1 & 1 & 1 & 7 & 64 \\
\hline & Senecio cacaliaster & E1 & 1 & . & . & . & . & 1 & 2 & + & + & 1 & + & 7 & 64 \\
\hline & Athyrium filix-femina & E1 & 1 & . & . & . & . & + & 1 & + & + & + & . & 6 & 55 \\
\hline & Rumex arifolius & E1 & . & . & + & + & 1 & + & . & + & . & + & . & 6 & 55 \\
\hline & Chaerophyllum hirsutum & E1 & . & + & . & . & + & . & . & + & . & . & . & 3 & 27 \\
\hline & Alchemilla monticola & E1 & . & . & + & + & + & . & . & . & . & . & . & 3 & 27 \\
\hline & Geum rivale & E1 & . & . & . & + & + & . & . & . & + & . & . & 3 & 27 \\
\hline & Thalictrum aquilegiifolium & E1 & . & . & . & . & . & + & . & + & . & + & . & 3 & 27 \\
\hline & Ranunculus platanifolius & E1 & 1 & . & . & . & . & . & . & . & . & + & . & 2 & 18 \\
\hline & Primula elatior & E1 & . & . & . & + & + & . & . & . & . & . & . & 2 & 18 \\
\hline & Peucedanum ostruthium & E1 & . & . & . & . & . & . & . & + & . & + & . & 2 & 18 \\
\hline
\end{tabular}




\begin{tabular}{|c|c|c|c|c|c|c|c|c|c|c|c|c|c|c|c|}
\hline & \multicolumn{2}{|l|}{ Number of relevé (Zaporedna številka popisa) } & 1 & 2 & 3 & 4 & 5 & 6 & 7 & 8 & 9 & 10 & 11 & Pr. & Fr. \\
\hline & Orobanche lycoctoni & E1 & . & . & . & . & . & . & . & $\mathrm{r}$ & . & . & + & 2 & 18 \\
\hline & Lamium maculatum & E1 & 1 & . & . & . & . & . & . & . & . & . & . & 1 & 9 \\
\hline & Scrophularia scopolii & E1 & + & . & . & . & . & . & . & . & . & . & . & 1 & 9 \\
\hline & Cirsium carniolicum & E1 & . & . & . & . & + & . & . & . & . & . & . & 1 & 9 \\
\hline & Carduus carduelis & E1 & . & . & . & . & . & 1 & . & . & . & . & . & 1 & 9 \\
\hline & Silene vulgaris subsp. antelopum & E1 & . & . & . & . & . & + & . & . & . & . & . & 1 & 9 \\
\hline & Aconitum angustifolium & E1 & . & . & . & . & . & . & . & + & . & . & . & 1 & 9 \\
\hline & Orobanche pancicii & E1 & . & . & . & . & . & . & . & + & . & . & . & 1 & 9 \\
\hline & Chaerophyllum villarsii & E1 & . & . & . & . & . & . & . & . & + & . & . & 1 & 9 \\
\hline & Doronicum austriacum & E1 & . & . & . & . & . & . & . & . & + & . & . & 1 & 9 \\
\hline & Epilobium alpestre & E1 & . & . & . & . & . & . & . & . & . & + & . & 1 & 9 \\
\hline \multirow[t]{5}{*}{ TG } & Trifolio-Geranietea & & & & & & & & & & & & & & \\
\hline & Digitalis grandiflora & E1 & + & + & . & . & . & . & . & . & . & . & . & 2 & 18 \\
\hline & Silene nutans & E1 & . & . & . & . & . & . & . & + & . & . & + & 2 & 18 \\
\hline & Dianthus barbatus & E1 & . & . & . & + & . & . & . & . & . & . & . & 1 & 9 \\
\hline & Polygonatum odoratum & E1 & . & . & . & . & . & . & . & . & . & + & . & 1 & 9 \\
\hline \multirow[t]{5}{*}{$\mathrm{CA}$} & Caricion austroalpinae & & & & & & & & & & & & & & \\
\hline & Pulsatilla alpina subsp. austroalpina & E1 & . & + & . & . & . & 1 & 1 & 1 & + & 1 & 1 & 7 & 64 \\
\hline & Heracleum austriacum subsp. siifolium & E1 & . & + & + & + & + & . & . & . & + & . & . & 5 & 45 \\
\hline & Festuca calva & E1 & . & . & + & . & + & . & + & . & . & . & . & 3 & 27 \\
\hline & Arabis vochinensis & E1 & . & . & . & . & + & . & . & . & . & . & + & 2 & 18 \\
\hline \multirow[t]{4}{*}{ Cfir } & Caricion firmae & & & & & & & & & & & & & & \\
\hline & Dryas octopetala & E1 & . & . & . & . & + & . & . & . & + & . & . & 2 & 18 \\
\hline & Helianthemum alpestre & E1 & . & . & . & . & . & . & . & + & . & . & . & 1 & 9 \\
\hline & Leontopodium alpinum & E1 & . & . & . & . & . & + & . & . & . & . & . & 1 & 9 \\
\hline \multirow[t]{2}{*}{$\mathrm{CF}$} & Caricion ferrugineae & & & & & & & & & & & & & & \\
\hline & Knautia longifolia & E1 & . & . & . & . & . & + & + & 1 & + & . & 1 & 5 & 45 \\
\hline \multirow[t]{30}{*}{ ES } & Elyno-Seslerietea & & & & & & & & & & & & & & \\
\hline & Helianthemum nummularium subsp. grandiflorum & E1 & . & + & + & 1 & 1 & + & 2 & 2 & 1 & 1 & 1 & 10 & 91 \\
\hline & Betonica alopecuros & E1 & . & + & . & + & + & + & 1 & 1 & + & 1 & 1 & 9 & 82 \\
\hline & Senecio abrotanifolius & E1 & . & + & 2 & 1 & 2 & . & + & + & + & + & 1 & 9 & 82 \\
\hline & Thymus praecox subsp. polytrichus & E1 & . & . & 1 & + & 1 & 1 & + & + & + & . & + & 8 & 73 \\
\hline & Potentilla crantzii & E1 & . & + & 1 & + & 1 & + & + & + & . & + & . & 8 & 73 \\
\hline & Aster bellidiastrum & E1 & . & + & 1 & + & + & + & . & + & . & . & + & 7 & 64 \\
\hline & Selaginella selaginoides & E1 & . & . & + & + & + & . & + & + & + & . & + & 7 & 64 \\
\hline & Lotus alpinus & E1 & . & + & + & + & . & 1 & + & + & . & . & + & 7 & 64 \\
\hline & Scabiosa lucida subsp. stricta & E1 & . & . & . & . & . & + & + & 2 & + & + & 1 & 6 & 55 \\
\hline & Phyteuma orbiculare & E1 & + & 1 & + & 1 & 1 & . & . & . & . & . & . & 5 & 45 \\
\hline & Galium anisophyllum & E1 & . & + & + & 1 & 1 & . & . & + & . & . & . & 5 & 45 \\
\hline & Leucanthemum heterophyllum (L.maximum agg.) & E1 & . & . & + & . & . & . & + & + & . & + & + & 5 & 45 \\
\hline & Myosotis alpestris & E1 & . & . & + & + & + & + & . & . & . & + & . & 5 & 45 \\
\hline & Sesleria caerulea & E1 & $\mathrm{r}$ & . & + & + & . & . & . & + & . & . & . & 4 & 36 \\
\hline & Ranunculus montanus & E1 & . & + & . & . & . & . & . & . & + & + & + & 4 & 36 \\
\hline & Gentianella anisodonta & E1 & . & . & . & . & . & . & + & + & + & . & + & 4 & 36 \\
\hline & Ranunculus carinthiacus & E1 & . & + & . & . & + & . & . & . & + & . & . & 3 & 27 \\
\hline & Polygala alpestris & E1 & . & + & . & + & + & . & . & . & . & . & . & 3 & 27 \\
\hline & Acinos alpinus & E1 & . & . & 1 & . & + & . & . & . & . & . & . & 2 & 18 \\
\hline & Hieracium villosum & E1 & . & . & + & . & . & + & . & . & . & . & . & 2 & 18 \\
\hline & Polygonum viviparum & E1 & . & . & . & + & + & . & . & . & . & . & . & 2 & 18 \\
\hline & Homogyne discolor & E1 & . & . & . & 1 & + & . & . & . & . & . & . & 2 & 18 \\
\hline & Cerastium strictum & E1 & . & . & . & . & + & . & . & + & . & . & . & 2 & 18 \\
\hline & Hieracium pilosum & E1 & . & . & . & . & . & . & . & + & . & + & . & 2 & 18 \\
\hline & Euphrasia picta & E1 & . & . & . & . & . & . & . & + & + & . & . & 2 & 18 \\
\hline & Gentiana lutea subsp. symphyandra & E1 & . & . & . & . & . & . & . & + & + & . & . & 2 & 18 \\
\hline & Campanula witasekiana & E1 & . & + & . & . & . & . & . & . & . & . & . & 1 & 9 \\
\hline & Carex sempervirens & E1 & . & . & + & . & . & . & . & . & . & . & . & 1 & 9 \\
\hline & Juncus monanthos & E1 & . & . & + & . & . & . & . & . & . & . & . & 1 & 9 \\
\hline
\end{tabular}




\begin{tabular}{|c|c|c|c|c|c|c|c|c|c|c|c|c|c|c|c|}
\hline & Number of relevé (Zaporedna številka popisa) & & 1 & 2 & 3 & 4 & 5 & 6 & 7 & 8 & 9 & 10 & 11 & Pr. & Fr. \\
\hline & Euphrasia salisburgensis & E1 & . & . & . & . & + & . & . & . & . & . & . & 1 & 9 \\
\hline & Carduus crassifolius & E1 & . & . & . & . & . & . & + & . & . & . & . & 1 & 9 \\
\hline & Achillea clavenae & E1 & . & . & . & . & . & . & . & + & . & . & . & 1 & 9 \\
\hline & Rhinanthus glacialis & E1 & . & . & . & . & . & . & . & 1 & . & . & . & 1 & 9 \\
\hline & Alchemilla glaucescens & E1 & . & . & . & . & . & . & . & + & . & . & . & 1 & 9 \\
\hline & Thesium alpinum & E1 & . & . & . & . & . & . & . & . & . & + & . & 1 & 9 \\
\hline \multirow[t]{4}{*}{ JT } & Juncetea trifidi & & & & & & & & & & & & & & \\
\hline & Campanula scheuchzeri & E1 & + & . & + & + & . & + & . & . & . & + & . & 5 & 45 \\
\hline & Centaurea nervosa & E1 & . & . & . & . & . & . & . & . & . & . & + & 1 & 9 \\
\hline & Veronica fruticans & E1 & . & . & . & . & + & . & . & . & . & . & . & 1 & 9 \\
\hline \multirow[t]{7}{*}{$\mathrm{CU}$} & Calluno-Ulicetea & & & & & & & & & & & & & & \\
\hline & Festuca nigrescens & E1 & + & 1 & 1 & 1 & + & 1 & . & . & . & . & . & 6 & 55 \\
\hline & Gentiana pannonica & E1 & . & . & . & . & . & . & + & . & + & + & + & 4 & 36 \\
\hline & Potentilla erecta & E1 & . & . & . & . & . & . & . & + & + & . & + & 3 & 27 \\
\hline & Nardus stricta & E1 & . & . & . & + & . & . & . & . & . & . & . & 1 & 9 \\
\hline & Agrostis capillaris & E1 & . & . & . & . & . & . & . & + & . & . & . & 1 & 9 \\
\hline & Coeloglossum viride & E1 & . & . & . & . & . & . & . & . & + & . & . & 1 & 9 \\
\hline \multirow[t]{9}{*}{$\mathrm{FB}$} & Festuco-Brometea & & & & & & & & & & & & & & \\
\hline & Cirsium erisithales & E1 & 1 & + & . & . & . & 1 & 1 & + & 1 & + & . & 7 & 64 \\
\hline & Buphthalmum salicifolium & E1 & . & + & . & . & . & . & 1 & 2 & 1 & . & 1 & 5 & 45 \\
\hline & Prunella grandiflora & E1 & . & . & . & . & . & . & + & 1 & . & . & + & 3 & 27 \\
\hline & Thlaspi praecox & E1 & . & + & . & + & . & . & . & . & . & . & . & 2 & 18 \\
\hline & Hippocrepis comosa & E1 & . & + & . & . & . & . & . & + & . & . & . & 2 & 18 \\
\hline & Carlina acaulis & E1 & . & . & . & . & . & . & + & . & . & + & . & 2 & 18 \\
\hline & Koeleria pyramidata & E1 & . & . & . & . & . & . & . & 1 & . & . & . & 1 & 9 \\
\hline & Gymnadenia conopsea & E1 & . & . & . & . & . & . & . & . & . & + & . & 1 & 9 \\
\hline \multirow[t]{5}{*}{$\mathrm{PaT}$} & Poo alpinae-Trisetalia & & & & & & & & & & & & & & \\
\hline & Poa alpina & E1 & + & + & 1 & 1 & 1 & . & . & . & + & . & + & 7 & 64 \\
\hline & Crepis aurea & E1 & . & . & + & + & + & . & . & . & . & . & . & 3 & 27 \\
\hline & Ranunculus nemorosus & E1 & . & . & . & + & + & . & . & . & . & . & . & 2 & 18 \\
\hline & Trollius europaeus & E1 & . & . & . & . & . & . & . & + & . & . & . & 1 & 9 \\
\hline \multirow[t]{13}{*}{ MA } & Molinio-Arrbenatheretea & & & & & & & & & & & & & & \\
\hline & Trifolium pratense & E1 & . & . & + & + & + & . & . & + & . & + & . & 5 & 45 \\
\hline & Cerastium fontanum & E1 & . & . & + & + & + & . & . & . & . & . & . & 3 & 27 \\
\hline & Bellis perennis & E1 & . & . & + & + & + & . & . & . & . & . & . & 3 & 27 \\
\hline & Dactylis glomerata s.str. & E1 & . & . & . & . & . & 1 & 2 & . & . & . & + & 3 & 27 \\
\hline & Veronica chamaedrys & E1 & 1 & . & + & . & . & . & . & . & . & . & . & 2 & 18 \\
\hline & Anthoxanthum odoratum & E1 & . & + & . & . & . & . & . & . & + & . & . & 2 & 18 \\
\hline & Taraxacum officinale & E1 & . & + & . & . & + & . & . & . & . & . & . & 2 & 18 \\
\hline & Trifolium repens & E1 & . & . & + & . & + & . & . & . & . & . & . & 2 & 18 \\
\hline & Leontodon hispidus & E1 & . & . & . & . & . & + & . & . & . & + & . & 2 & 18 \\
\hline & Festuca rubra & E1 & . & . & . & . & . & . & . & 2 & . & . & 1 & 2 & 18 \\
\hline & Galium mollugo & E1 & + & . & . & . & . & . & . & . & . & . & . & 1 & 9 \\
\hline & Poa pratensis & E1 & . & . & . & 1 & . & . & . & . & . & . & . & 1 & 9 \\
\hline \multirow[t]{3}{*}{$\mathrm{CD}$} & Caricetalia davallianae & & & & & & & & & & & & & & \\
\hline & Parnassia palustris & E1 & . & . & . & . & . & . & + & . & + & . & . & 2 & 18 \\
\hline & Carex capillaris & E1 & . & . & + & . & . & . & . & . & . & . & . & 1 & 9 \\
\hline \multirow[t]{5}{*}{ EA } & Epilobietea angustifolii & & & & & & & & & & & & & & \\
\hline & Fragaria vesca & E1 & . & + & + & . & + & . & . & . & + & . & . & 4 & 36 \\
\hline & Rubus idaeus & E2 & 1 & . & . & . & . & . & . & . & . & + & . & 2 & 18 \\
\hline & Urtica dioica & E1 & + & . & . & . & . & . & . & + & . & . & . & 2 & 18 \\
\hline & Chamaenerion angustifolium & E1 & . & . & . & . & . & + & . & . & . & . & . & 1 & 9 \\
\hline \multirow[t]{3}{*}{$\mathrm{AC}$} & Arabidetalia caeruleae & & & & & & & & & & & & & & \\
\hline & Soldanella alpina & E1 & . & . & . & 1 & + & . & . & . & . & . & . & 2 & 18 \\
\hline & Soldanella minima & E1 & . & . & + & . & . & . & . & . & . & . & . & 1 & 9 \\
\hline \multirow[t]{2}{*}{ TR } & Thlaspietea rotundifolii & & & & & & & & & & & & & & \\
\hline & Adenostyles glabra & E1 & + & . & . & . & . & 1 & 1 & + & . & + & + & 6 & 55 \\
\hline
\end{tabular}




\begin{tabular}{|c|c|c|c|c|c|c|c|c|c|c|c|c|c|c|c|}
\hline & Number of relevé (Zaporedna številka popisa) & & 1 & 2 & 3 & 4 & 5 & 6 & 7 & 8 & 9 & 10 & 11 & Pr. & Fr. \\
\hline & Dryopteris villarii & E1 & . & . & + & . & + & + & + & . & . & + & + & 6 & 55 \\
\hline & Gymnocarpium robertianum & E1 & . & + & . & . & . & + & + & + & . & . & + & 5 & 45 \\
\hline & Trisetum argenteum & E1 & . & . & . & . & . & + & 1 & + & . & + & + & 5 & 45 \\
\hline & Sedum atratum & E1 & . & . & + & + & + & . & . & . & . & . & + & 4 & 36 \\
\hline & Minuartia austriaca & E1 & . & . & 1 & + & + & . & . & . & . & . & . & 3 & 27 \\
\hline & Arabis alpina & E1 & . & . & . & . & + & . & . & . & . & . & . & 1 & 9 \\
\hline & Hieracium sp. & E1 & . & . & . & . & + & . & . & . & . & . & . & 1 & 9 \\
\hline & Hieracium bifidum & E1 & . & . & . & . & . & + & . & . & . & . & . & 1 & 9 \\
\hline \multirow[t]{3}{*}{ Cy } & Cystopetridion fragilis & & & & & & & & & & & & & & \\
\hline & Cystopteris fragilis & E1 & + & + & + & + & + & + & . & . & . & . & + & 7 & 64 \\
\hline & Cystopteris regia & E1 & . & . & + & . & + & + & . & . & . & . & . & 3 & 27 \\
\hline \multirow[t]{2}{*}{ PS } & Physoplexido comosae-Saxifragion petraeae & & & & & & & & & & & & & & \\
\hline & Saxifraga hostii & E1 & . & + & . & . & + & . & . & . & . & . & . & 2 & 18 \\
\hline \multirow[t]{2}{*}{ PC } & Potentilletalia caulescentis & & & & & & & & & & & & & & \\
\hline & Campanula cochleariifolia & E1 & + & + & 1 & + & . & + & . & . & . & . & . & 5 & 45 \\
\hline \multirow[t]{5}{*}{ AT } & Asplenietea trichomanis & & & & & & & & & & & & & & \\
\hline & Asplenium viride & E1 & + & 1 & 1 & + & + & + & . & + & + & + & + & 10 & 91 \\
\hline & Asplenium ruta-muraria & E1 & . & . & + & . & . & + & + & + & . & + & . & 5 & 45 \\
\hline & Moehringia muscosa & E1 & . & + & + & . & . & + & + & . & . & . & . & 4 & 36 \\
\hline & Asplenium trichomanes & E1 & . & . & . & . & + & + & + & . & . & + & . & 4 & 36 \\
\hline $\mathrm{Fu}$ & Fungi (Glive) & & & & & & & & & & & & & & \\
\hline & Laricifomes officinalis & $\mathrm{E} 3 \mathrm{c}$ & . & . & . & . & . & . & . & . & . & + & . & 1 & 9 \\
\hline \multirow[t]{21}{*}{ ML } & Mosses and lichens (Mahovi in lišaji) & & & & & & & & & & & & & & \\
\hline & Tortella tortuosa & E0 & + & 1 & 1 & 1 & 1 & + & + & + & + & + & + & 11 & 100 \\
\hline & Ctenidium molluscum & E0 & + & 1 & 1 & 1 & 1 & 1 & + & + & 1 & + & + & 11 & 100 \\
\hline & Schistidium sp. & E0 & + & . & + & + & + & + & . & . & + & + & + & 8 & 73 \\
\hline & Dicranum scoparium & E0 & + & . & 1 & + & + & . & . & . & . & + & . & 5 & 45 \\
\hline & Plagiochila porelloides & E0 & + & . & . & . & . & . & + & . & . & + & . & 3 & 27 \\
\hline & Cladonia pyxidata & E0 & . & . & + & + & . & . & . & . & . & . & . & 2 & 18 \\
\hline & Peltigera canina & E0 & . & . & . & + & + & . & . & . & . & . & . & 2 & 18 \\
\hline & Rhytidiadelphus triquetrus & E0 & . & . & + & . & . & . & . & . & . & . & . & 1 & 9 \\
\hline & Hylocomium splendens & E0 & . & . & + & . & . & . & . & . & . & . & . & 1 & 9 \\
\hline & Conocephalum conicum & E0 & . & . & + & . & . & . & . & . & . & . & . & 1 & 9 \\
\hline & Dermatocarpon miniatum & E0 & . & . & + & . & . & . & . & . & . & . & . & 1 & 9 \\
\hline & Anomodon sp. & E0 & . & . & + & . & . & . & . & . & . & . & . & 1 & 9 \\
\hline & Homalothecium sp. & E0 & . & . & + & . & . & . & . & . & . & . & . & 1 & 9 \\
\hline & Homalothecium philippeanum & E0 & . & . & . & + & . & . & . & . & . & . & . & 1 & 9 \\
\hline & Peltigera leucophlebia & E0 & . & . & . & . & + & . & . & . & . & . & . & 1 & 9 \\
\hline & Pseudoleskea incurvata & E0 & . & . & . & . & . & + & . & . & . & . & . & 1 & 9 \\
\hline & Cladonia rangiferina & E0 & . & . & . & . & . & + & . & . & . & . & . & 1 & 9 \\
\hline & Plagiothecium sp. & E0 & . & . & . & . & . & + & . & . & . & . & . & 1 & 9 \\
\hline & Fissidens dubius & E0 & . & . & . & . & . & . & . & . & . & + & . & 1 & 9 \\
\hline & Letharia vulpina & $\mathrm{E} 3 \mathrm{c}$ & . & . & . & . & . & . & . & . & . & + & . & 1 & 9 \\
\hline
\end{tabular}

\section{Legend - Legenda}

ID Igor Dakskobler

AR Andrej Rozman

A Limestone - apnenec

D Dolomite - dolomit

Re Rendzina - rendzina

JA Julian Alps - Julijske Alpe

K Karavanke

Pr. Presence (number of relevés in which the species is presented) - število popisov, v katerih se pojavlja vrsta

Fr. Frequency in $\%$ - frekvenca v \% 
Table 17 (Tabela 17): Rhodothamno-Laricetum cyclaminetosum purpurascentis var. typica

\begin{tabular}{|c|c|c|c|c|c|c|c|c|c|}
\hline Number of relevé (Zaporedna številka popisa) & & 1 & 2 & 3 & 4 & 5 & 6 & 7 & 8 \\
\hline $\begin{array}{l}\text { Database number of relevé } \\
\text { (Delovna številka popisa) }\end{array}$ & & $\begin{array}{l}\curvearrowright \\
\text { ָे } \\
\text { ते }\end{array}$ & 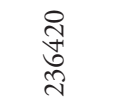 & $\begin{array}{l}\circ \\
\infty \\
\infty \\
\stackrel{N}{N}\end{array}$ & 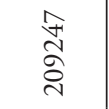 & 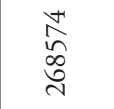 & 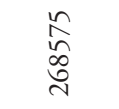 & 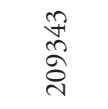 & $\begin{array}{l}\sqrt{\widetilde{\delta}} \\
\delta \\
\text { ते }\end{array}$ \\
\hline Author of the relevé (Avtor popisa) & & IDMČ & IDAS & IDAS & ID & ID & ID & ID & ID \\
\hline Elevation in $\mathrm{m}$ (Nadmorska višina $\mathrm{v} \mathrm{m})$ & & 1710 & 1670 & 1650 & 1680 & 1550 & 1565 & 1610 & 1430 \\
\hline Aspect (Lega) & & E & E & SE & SE & S & SSW & SE & SW \\
\hline Slope in degrees (Nagib v stopinjah) & & 45 & 40 & 40 & 20 & 35 & 35 & 35 & 35 \\
\hline Parent material (Matična podlaga) & & $\mathrm{D}$ & A & DA & A & $\mathrm{D}$ & $\mathrm{D}$ & DA & A \\
\hline Soil (Tla) & & $\operatorname{Re}$ & $\operatorname{Re}$ & $\operatorname{Re}$ & $\operatorname{Re}$ & $\operatorname{Re}$ & $\operatorname{Re}$ & $\operatorname{Re}$ & $\operatorname{Re}$ \\
\hline Stoniness in \% (Kamnitost v \%) & & 10 & 40 & 10 & 30 & 10 & 10 & 10 & 5 \\
\hline \multicolumn{10}{|l|}{ Cover in \% (Zastiranje v \%): } \\
\hline Upper tree layer (Zgor. drevesna plast) & $\mathrm{E} 3 \mathrm{~b}$ & 70 & 60 & 50 & 60 & 60 & 60 & 70 & 70 \\
\hline Lower tree layer (Spod. drevesna plast) & $\mathrm{E} 3 \mathrm{a}$ & 10 & 10 & 20 & 10 & 20 & 10 & 20 & 0 \\
\hline Shrub layer (Grmovna plast) & E2 & 10 & 20 & 80 & 20 & 20 & 30 & 20 & 20 \\
\hline Herb layer (Zeliščna plast) & E1 & 80 & 70 & 5 & 70 & 80 & 90 & 80 & 70 \\
\hline Moss layer (Mahovna plast) & E0 & 5 & 2 & 0 & 10 & 5 & 5 & 5 & 5 \\
\hline Number of species (Število vrst) & & 68 & 78 & 86 & 49 & 55 & 49 & 57 & 47 \\
\hline Relevé area (Velikost popisne ploskve) & $\mathrm{m}^{2}$ & 400 & 400 & 400 & 200 & 400 & 400 & 200 & 200 \\
\hline Date of taking relevé (Datum popisa) & & 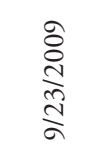 & $\begin{array}{l}\stackrel{0}{0} \\
\stackrel{i}{N} \\
\frac{1}{n}\end{array}$ & $\begin{array}{l}\stackrel{ }{a} \\
\stackrel{d}{\Delta} \\
\stackrel{\Delta}{a}\end{array}$ & $\underset{\sigma}{\stackrel{\curvearrowright}{\sigma}}$ & 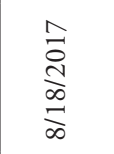 & $\underset{\stackrel{N}{\circ}}{\stackrel{\infty}{\infty}}$ & 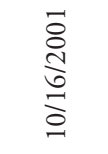 & 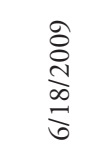 \\
\hline Locality (Nahajališče) & & 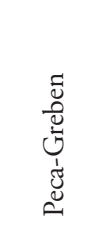 & $\begin{array}{l}\frac{7}{5} \\
\frac{7}{3} \\
\frac{0}{3}\end{array}$ & $\begin{array}{l}\frac{\pi}{0} \\
\frac{\pi}{00} \\
\tilde{\sigma} \\
0 \\
0 \\
0\end{array}$ & 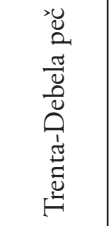 & 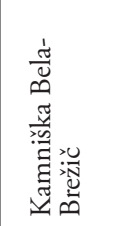 & 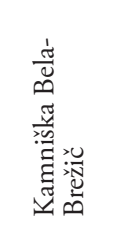 & 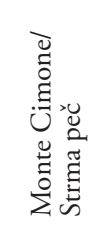 & 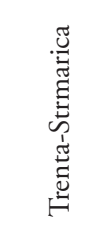 \\
\hline Mountain range (Pogorje) & & $\mathrm{K}$ & KSA & JA & JA & KSA & KSA & JA & JA \\
\hline Quadrant (Kvadrant) & & $9554 / 2$ & $9653 / 1$ & $9549 / 3$ & $9648 / 2$ & $9653 / 4$ & $9653 / 4$ & $9546 / 3$ & $9648 / 1$ \\
\hline Coordinate GK-Y (D-48) & $\mathrm{m}$ & 481591 & 461897 & 412238 & 405219 & 472074 & 472085 & 374901 & 400056 \\
\hline Coordinate GK-X (D-48) & $\mathrm{m}$ & 5150098 & 5136429 & 5143550 & 5139800 & 5132629 & 5132643 & 5143740 & 5137190 \\
\hline
\end{tabular}

VP

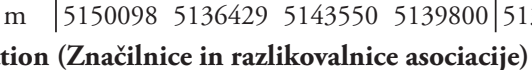

$\mathrm{VP}$

$\mathrm{VP}$

CA Laserpitium peucedanoides

AF Anemone trifolia

EP Rhododendron hirsutum

TR Heliosperma alpestre

PS Paederota lutea

VP Homogyne sylvestris

ES Astrantia bavarica

EP Rhodothamnus chamaecistus

CF Carex ferruginea

TR Festuca nitida

PS Campanula carnica

PC Valeriana saxatilis

Differential species of the subassociation (Razlikovalne vrste subasociacije)

E3
E2
E1
E1
E1
E2
E1
E1
E1
E1
E1
E1
E1
E1
E1

Erica carnea

EP Calamagrostis varia 


\begin{tabular}{|c|c|c|c|c|c|c|c|c|c|c|c|c|c|}
\hline 9 & 10 & 11 & 12 & 13 & 14 & 15 & 16 & 17 & 18 & 19 & 20 & 21 & Pr. \\
\hline $\begin{array}{l}\stackrel{\sigma}{\vec{\sigma}} \\
\text { d }\end{array}$ & 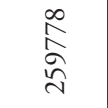 & $\begin{array}{l}\hat{b} \\
\stackrel{2}{0} \\
\stackrel{\sim}{\nu}\end{array}$ & 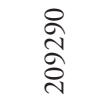 & 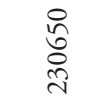 & $\underset{\underset{\sim}{\stackrel{N}{N}}}{\stackrel{N}{N}}$ & $\begin{array}{l}\hat{\alpha} \\
\hat{\tilde{\gamma}} \\
\text { }\end{array}$ & $\begin{array}{l}\stackrel{+}{+} \\
\stackrel{+}{+} \\
\underset{\sim}{+}\end{array}$ & 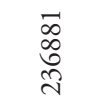 & $\begin{array}{l}\frac{\hat{n}}{\hat{a}} \\
\stackrel{-}{+}\end{array}$ & 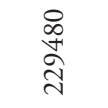 & $\begin{array}{l}\stackrel{n}{0} \\
\stackrel{0}{\sim} \\
\sim\end{array}$ & 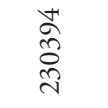 & \\
\hline ID & ID & ID & ID & ID & ID & IDMČ & ID & IDAS & ID & ID & IDAS & IDMČ & \\
\hline 1665 & 1475 & 1290 & 1530 & 1460 & 1700 & 1550 & 1670 & 1670 & 1580 & 1600 & 1430 & 1480 & \\
\hline SE & SW & SW & SSE & SSE & S & E & S & SE & SW & SE & S & S & \\
\hline 35 & 35 & 35 & 30 & 30 & 25 & 35 & 30 & 35 & 25 & 35 & 45 & 30 & \\
\hline DA & DA & A & A & A & D & D & DA & $\mathrm{Gr}$ & A & DA & D & D & \\
\hline $\operatorname{Re}$ & $\operatorname{Re}$ & $\operatorname{Re}$ & $\operatorname{Re}$ & $\operatorname{Re}$ & $\operatorname{Re}$ & $\operatorname{Re}$ & $\operatorname{Re}$ & $\operatorname{Re}$ & $\operatorname{Re}$ & $\operatorname{Re}$ & $\operatorname{Re}$ & $\operatorname{Re}$ & \\
\hline 10 & 10 & 0 & 30 & 5 & 20 & 5 & 20 & 15 & 30 & 20 & 10 & 0 & \\
\hline 70 & 80 & 60 & 60 & 60 & 50 & 60 & 70 & 70 & 70 & 50 & 60 & 70 & \\
\hline 10 & 20 & 0 & 0 & 0 & 10 & 5 & 10 & 10 & 20 & 10 & 5 & 10 & \\
\hline 10 & 5 & 30 & 30 & 60 & 5 & 10 & 30 & 30 & 10 & 30 & 10 & 20 & \\
\hline 90 & 70 & 90 & 80 & 70 & 80 & 80 & 90 & 80 & 80 & 80 & 90 & 80 & \\
\hline 5 & 10 & 0 & 5 & 1 & 5 & 10 & 5 & 5 & 5 & 10 & 2 & 10 & \\
\hline 60 & 46 & 32 & 60 & 47 & 53 & 77 & 64 & 85 & 50 & 61 & 60 & 37 & \\
\hline 400 & 400 & 200 & 200 & 200 & 200 & 400 & 400 & 400 & 200 & 400 & 400 & 400 & \\
\hline 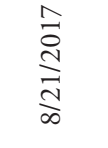 & $\begin{array}{l}\frac{n}{\grave{\lambda}} \\
\frac{\grave{\lambda}}{\grave{\lambda}} \\
\frac{\infty}{0}\end{array}$ & $\frac{\precsim}{\stackrel{Ð}{\sigma}}$ & 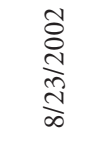 & 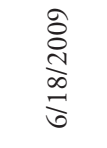 & $\begin{array}{l}\infty \\
\stackrel{0}{0} \\
\frac{N}{n} \\
\frac{n}{n}\end{array}$ & 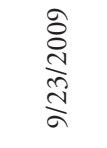 & 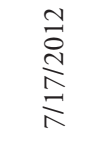 & 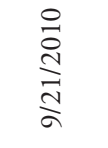 & 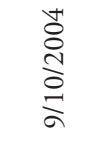 & 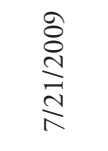 & 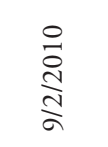 & 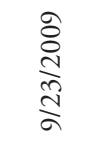 & \\
\hline 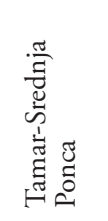 & 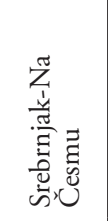 & 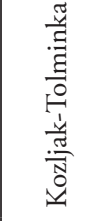 & 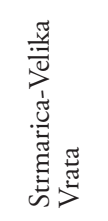 & 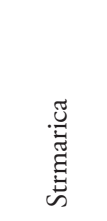 & 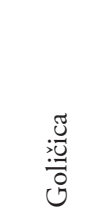 & 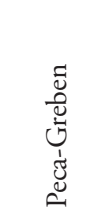 & 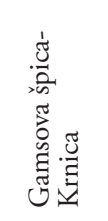 & 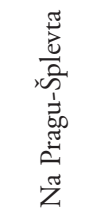 & 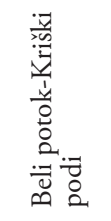 & 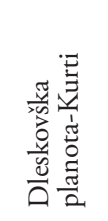 & 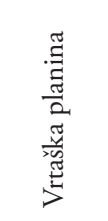 & 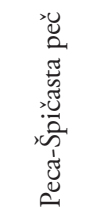 & \\
\hline $\mathrm{JA}$ & $\mathrm{JA}$ & $\mathrm{JA}$ & $\mathrm{JA}$ & $\mathrm{JA}$ & JA & $\mathrm{K}$ & JA & $\mathrm{JA}$ & JA & KSA & $\mathrm{JA}$ & $\mathrm{K}$ & \\
\hline $9548 / 3$ & $9648 / 1$ & $9748 / 2$ & $9648 / 1$ & $9648 / 1$ & $9648 / 2$ & $9554 / 2$ & $9548 / 4$ & $9549 / 3$ & $9648 / 2$ & $9654 / 1$ & 9549/1 & $9554 / 2$ & \\
\hline 400702 & 400742 & 403885 & 399474 & 400060 & 405447 & 481794 & 408005 & 411625 & 407771 & 476676 & 415704 & 481987 & \\
\hline 5145877 & 5137335 & 5124019 & 5137165 & 5137236 & 5139928 & 5150006 & 5144813 & 5143443 & 5139749 & 5135124 & 5145696 & 5150042 & \\
\hline
\end{tabular}

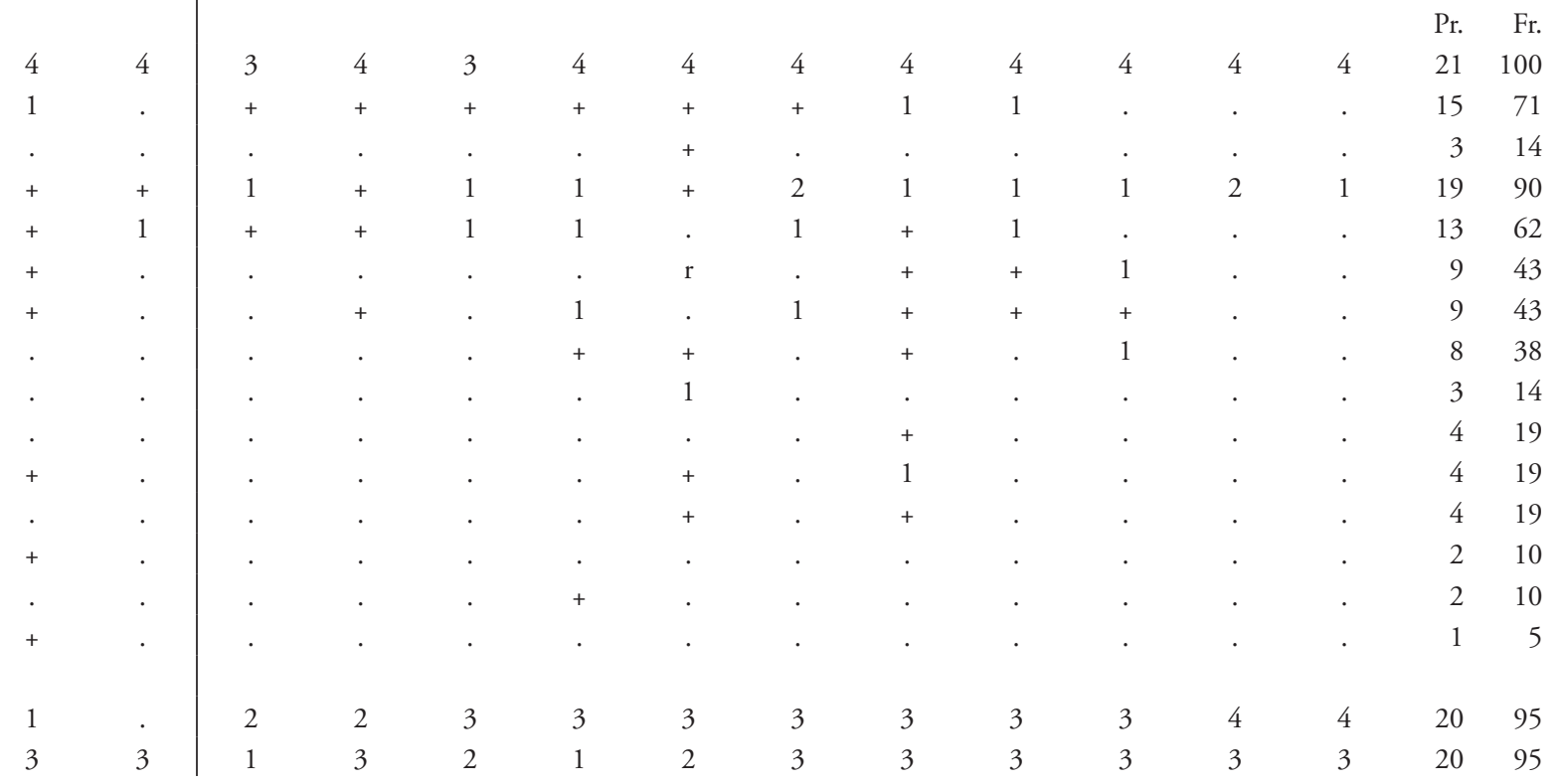




\begin{tabular}{|c|c|c|c|c|c|c|c|c|c|c|}
\hline & \multicolumn{2}{|c|}{ Number of relevé (Zaporedna številka popisa) } & 1 & 2 & 3 & 4 & 5 & 6 & 7 & 8 \\
\hline ES & Betonica alopecuros & E1 & . & . & + & + & 1 & 1 & 1 & + \\
\hline $\mathrm{AF}$ & Cyclamen purpurascens & E1 & 1 & . & . & . & 1 & 1 & + & 1 \\
\hline \multirow[t]{33}{*}{ VP } & \multicolumn{10}{|l|}{ Vaccinio-Piceetea } \\
\hline & Picea abies & E3 & 1 & 1 & 1 & 1 & 1 & 1 & 1 & 1 \\
\hline & Picea abies & E2 & 1 & 1 & 1 & 1 & 1 & 1 & + & 1 \\
\hline & Picea abies & E1 & + & . & . & + & . & + & . & . \\
\hline & Vaccinium vitis-idaea & E1 & + & 1 & + & 2 & + & + & . & 1 \\
\hline & Hieracium murorum & E1 & 1 & + & + & + & . & . & . & 1 \\
\hline & Vaccinium myrtillus & E1 & + & . & 1 & + & + & . & . & 1 \\
\hline & Luzula sylvatica & E1 & 2 & 1 & 1 & 1 & + & + & . & . \\
\hline & Rosa pendulina & E2 & + & . & . & + & . & + & . & . \\
\hline & Clematis alpina & E2 & 1 & + & + & . & . & + & + & + \\
\hline & Polystichum lonchitis & E1 & 1 & + & 1 & 1 & + & . & . & . \\
\hline & Melampyrum sylvaticum & E1 & . & + & + & . & . & . & . & . \\
\hline & Calamagrostis villosa & E1 & + & 1 & . & + & . & . & . & 1 \\
\hline & Aposeris foetida & E1 & . & 1 & 1 & 1 & + & 1 & . & . \\
\hline & Valeriana tripteris & E1 & . & . & 1 & + & . & . & 1 & . \\
\hline & Luzula luzuloides & E1 & 1 & 1 & + & . & + & + & . & . \\
\hline & Oxalis acetosella & E1 & 1 & . & + & 1 & . & . & . & . \\
\hline & Dryopteris dilatata & E1 & + & + & . & . & . & . & . & . \\
\hline & Ajuga pyramidalis & E1 & . & + & . & + & . & + & . & . \\
\hline & Saxifraga cuneifolia & E1 & + & + & . & . & . & . & . & . \\
\hline & Maianthemum bifolium & E1 & . & . & . & . & . & . & . & + \\
\hline & Homogyne alpina & E1 & . & . & . & 1 & . & . & . & . \\
\hline & Lycopodium annotinum & E1 & . & + & . & . & + & . & . & . \\
\hline & Huperzia selago & E1 & . & + & + & . & . & . & . & . \\
\hline & Lonicera caerulea & E2 & . & . & . & . & . & . & . & . \\
\hline & Dryopteris expansa & E1 & . & . & . & . & + & 1 & . & . \\
\hline & Luzula luzulina & E1 & + & . & + & . & . & . & . & . \\
\hline & Veronica urticifolia & E1 & . & + & . & . & . & . & . & . \\
\hline & Abies alba & E3 & . & + & . & . & . & . & . & . \\
\hline & Luzula pilosa & E1 & . & . & . & . & . & . & . & . \\
\hline & Calamagrostis arundinacea & E1 & . & . & . & . & . & . & . & . \\
\hline & Pyrola rotundifolia & E1 & . & . & . & . & . & . & . & . \\
\hline & Monotropa hypopitys & E1 & . & . & . & . & . & . & . & . \\
\hline \multirow[t]{17}{*}{$\mathrm{EP}$} & \multicolumn{10}{|l|}{ Erico-Pinetea } \\
\hline & Rubus saxatilis & E1 & + & + & + & + & 1 & + & 1 & + \\
\hline & Pinus mugo & E2 & . & + & . & + & 1 & 2 & . & + \\
\hline & Polygala chamaebuxus & E1 & . & . & . & $\mathrm{r}$ & . & . & + & + \\
\hline & Carex ornithopoda & E1 & . & + & . & . & . & . & . & . \\
\hline & Carex alba & E1 & . & . & . & + & . & . & . & . \\
\hline & Genista radiata & E2 & . & . & . & . & . & . & + & + \\
\hline & Cotoneaster tomentosus & E2 & . & . & . & . & . & . & . & + \\
\hline & Asperula aristata & E1 & . & . & . & . & . & . & . & . \\
\hline & Amelanchier ovalis & E2 & . & . & . & . & . & . & . & . \\
\hline & Peucedanum austriacum subsp. rablense & E1 & . & . & . & . & . & . & + & . \\
\hline & Epipactis atrorubens & E1 & . & . & . & . & . & . & . & . \\
\hline & Pinus sylvestris & E3 & . & . & . & . & . & . & . & . \\
\hline & Aquilegia nigricans & E1 & . & + & . & . & . & . & . & . \\
\hline & Molinia arundinacea & E1 & . & . & . & . & . & . & . & . \\
\hline & Allium ericetorum & E1 & . & . & . & . & . & . & . & . \\
\hline & Chamaecytisus hirsutus & E1 & . & . & . & . & . & . & . & . \\
\hline
\end{tabular}




\begin{tabular}{|c|c|c|c|c|c|c|c|c|c|c|c|c|c|c|}
\hline 9 & 10 & 11 & 12 & 13 & 14 & 15 & 16 & 17 & 18 & 19 & 20 & 21 & Pr. & $\mathrm{Fr}$ \\
\hline 1 & 1 & 1 & 1 & 1 & + & + & 2 & + & 1 & 1 & 1 & . & 18 & 86 \\
\hline 1 & 1 & 1 & 1 & 1 & . & 1 & 1 & 1 & 1 & . & + & + & 16 & 76 \\
\hline+ & 1 & . & 1 & 1 & . & + & 1 & 1 & 1 & + & + & 1 & 19 & 90 \\
\hline 1 & + & . & 1 & 1 & + & 1 & 1 & 1 & . & 1 & 1 & 1 & 19 & 90 \\
\hline . & . & . & . & . & . & + & + & . & . & . & + & . & 6 & 29 \\
\hline . & . & . & + & + & + & + & 2 & 2 & 1 & + & . & 1 & 16 & 76 \\
\hline . & + & . & + & + & + & + & . & 1 & 1 & 1 & . & + & 14 & 67 \\
\hline . & + & . & + & . & + & + & + & + & + & . & + & . & 13 & 62 \\
\hline+ & + & . & . & . & 1 & 1 & + & + & + & . & . & . & 13 & 62 \\
\hline . & + & . & + & + & + & . & + & + & + & + & + & + & 13 & 62 \\
\hline . & . & . & + & + & . & . & . & + & 1 & 1 & . & . & 11 & 52 \\
\hline 1 & . & . & & . & . & + & + & + & 1 & . & . & . & 10 & 48 \\
\hline+ & . & . & . & . & + & 1 & 1 & + & + & . & + & + & 10 & 48 \\
\hline . & . & . & + & . & . & + & . & + & + & + & . & . & 9 & 43 \\
\hline . & . & . & . & . & . & 1 & 1 & . & 1 & 1 & . & 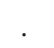 & 9 & 43 \\
\hline . & . & . & + & + & . & . & + & + & . & + & . & 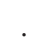 & 8 & 38 \\
\hline . & . & . & . & . & . & + & . & . & . & 1 & . & 1 & 8 & 38 \\
\hline . & . & . & . & . & . & + & . & + & . & . & . & . & 5 & 24 \\
\hline+ & . & . & . & . & . & + & . & . & . & . & . & . & 4 & 19 \\
\hline . & . & . & . & . & . & . & . & . & . & + & . & . & 4 & 19 \\
\hline . & . & . & . & . & . & + & . & . & . & . & . & . & 3 & 14 \\
\hline . & + & . & . & . & + & . & . & . & . & . & . & . & 3 & 14 \\
\hline . & . & . & & & & . & & + & . & . & . & . & 2 & 10 \\
\hline . & . & . & & & & . & . & . & . & . & . & . & 2 & 10 \\
\hline . & . & . & . & . & . & . & . & . & . & . & . & . & 2 & 10 \\
\hline . & . & . & + & . & . & . & . & + & . & . & . & . & 2 & 10 \\
\hline . & . & . & . & . & . & . & . & . & . & . & . & . & 2 & 10 \\
\hline . & . & . & . & . & . & . & . & . & . & . & . & . & 2 & 10 \\
\hline . & . & . & . & . & . & . & . & . & . & . & . & . & 1 & 5 \\
\hline . & . & . & . & . & . & . & . & . & . & . & . & . & 1 & 5 \\
\hline+ & . & . & . & . & . & . & . & . & . & . & . & . & 1 & 5 \\
\hline . & . & . & . & . & . & + & . & . & . & . & . & . & 1 & 5 \\
\hline . & . & . & . & . & . & . & . & + & . & . & . & . & 1 & 5 \\
\hline . & . & . & . & . & . & . & . & . & . & . & . & + & 1 & 5 \\
\hline+ & . & 1 & 1 & + & 1 & . & + & + & 1 & 1 & . & + & 18 & 86 \\
\hline 1 & . & 1 & + & + & . & + & 2 & 1 & 1 & 2 & . & + & 15 & 71 \\
\hline . & . & + & + & + & 1 & + & + & . & . & . & 1 & 1 & 11 & 52 \\
\hline . & . & . & . & . & . & + & + & + & + & . & . & . & 5 & 24 \\
\hline . & + & . & . & . & . & + & + & . & . & . & . & + & 5 & 24 \\
\hline . & . & 2 & 3 & 4 & . & . & . & . & . & . & . & . & 5 & 24 \\
\hline . & . & . & . & + & . & . & $\mathrm{r}$ & . & . & . & + & . & 4 & 19 \\
\hline . & . & + & . & . & . & . & + & . & . & . & 1 & . & 3 & 14 \\
\hline . & . & . & . & + & . & . & + & . & . & . & + & . & 3 & 14 \\
\hline . & . & . & . & . & . & . & . & . & . & . & + & . & 2 & 10 \\
\hline . & . & . & . & . & . & . & . & + & . & . & + & . & 2 & 10 \\
\hline . & . & . & & . & . & . & . & . & . & . & $\mathrm{r}$ & + & 2 & 10 \\
\hline . & . & . & & . & . & . & . & . & . & . & . & . & 1 & 5 \\
\hline . & . & 2 & . & . & . & . & . & . & . & . & . & . & 1 & 5 \\
\hline . & . & + & . & . & . & . & . & . & . & . & . & . & 1 & 5 \\
\hline & . & . & + & . & . & . & . & . & & & & & 1 & 5 \\
\hline
\end{tabular}


Number of relevé (Zaporedna številka popisa)

Galium austriacum

$\mathrm{AF}$

Aremonio-Fagion

Knautia drymeia

Helleborus niger

Cardamine enneaphyllos

Anemone x pittonii

Aremonia agrimonoides

TA Tilio-Acerion

Acer pseudoplatanus

Acer pseudoplatanus

Acer pseudoplatanus

Adoxa moschatellina

FS Fagetalia sylvaticae

Melica nutans

Mercurialis perennis

Daphne mezereum

Dryopteris filix-mas

Lonicera alpigena

Euphorbia amygdaloides

Galeobdolon flavidum

Luzula nivea

Fagus sylvatica

Fagus sylvatica

Fagus sylvatica

Symphytum tuberosum

Epilobium montanum

Mycelis muralis

Lilium martagon

Myosotis sylvatica

Polystichum aculeatum

Paris quadrifolia

Epipactis helleborine

Laburnum alpinum

Laburnum alpinum

Galium laevigatum

Prenanthes purpurea

QP Quercetalia pubescenti-petraeae

Sorbus aria (Aria edulis)

E1

E1

E1

E1

E1

E1

E1

E2

E1

E2

E1

E1

E1

E3

E2

E1

E1

E1

E1

E1

E1

E1

E1

E1

E3

E2

E1

E1

E3

E2

E1

E1

Convallaria majalis

QR Quercetalia roboris

Melampyrum pratense subsp. vulgatum

Hepatica nobilis

Carex digitata

Poa nemoralis

Viola riviniana

Anemone nemorosa

Cruciata glabra

Hieracium lachenalii

Platanthera bifolia

1

(




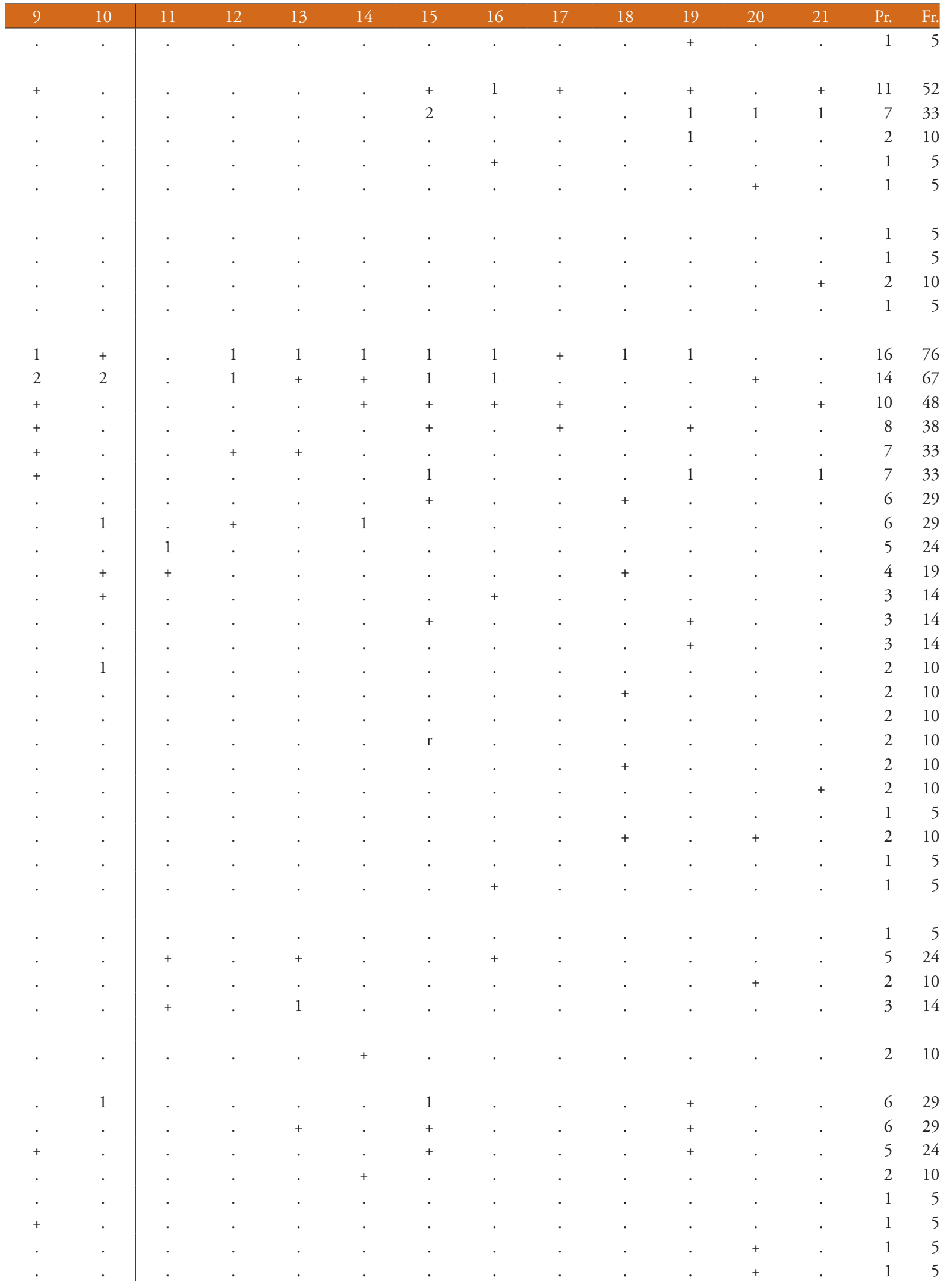




\section{Number of relevé (Zaporedna številka popisa)}

\section{E3}

Sorbus aucuparia subsp. aucuparia

Sorbus aucuparia subsp. aucuparia

Sorbus aucuparia subsp. aucuparia

Betula pendula

BA Betulo-Alnetea

Sorbus chamaemespilus

Salix appendiculata

Salix appendiculata

Juniperus sibirica

Alnus viridis

Sorbus aucuparia subsp. glabrata

Salix glabra

MuA Mulgedio-Aconitetea

Geranium sylvaticum

Senecio ovatus

Viola biflora

Hypericum maculatum

Polygonatum verticillatum

Aconitum lycoctonum subsp. ranunculifolium

Veratrum album subsp. lobelianum

Athyrium filix-femina

Saxifraga rotundifolia

Chaerophyllum villarsii

Geum rivale

Aconitum tauricum

Phyteuma ovatum

Aconitum napellus

Centaurea montana

Chaerophyllum hirsutum

Alchemilla monticola

Heracleum montanum

Silene vulgaris subsp. antelopum

Aconitum angustifolium

Rumex arifolius

Ranunculus platanifolius

TG Trifolio-Geranietea

Viola hirta

Silene nutans

Clinopodium vulgare

Verbascum lanatum

Origanum vulgare

Polygonatum odoratum

Lilium carniolicum

Laserpitium siler

Vicia sylvatica

Laserpitium latifolium

Thalictrum minus

Valeriana wallrothii

Achillea distans

Vincetoxicum hirundinaria

\section{1}

23

\section{5}

\section{6} 8 


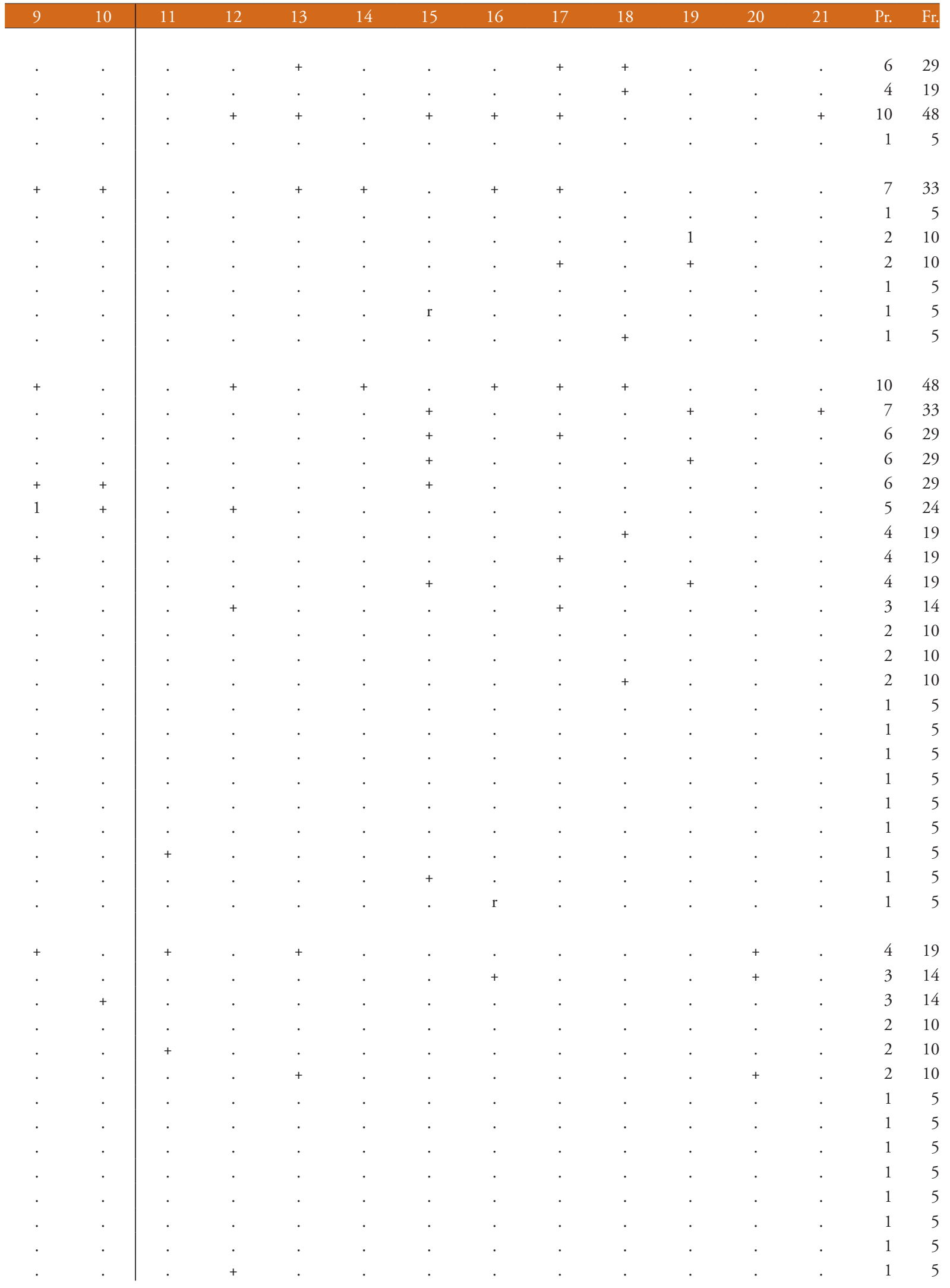


Number of relevé (Zaporedna številka popisa)

\section{E1

Festuca calva

Koeleria eriostachya

Pulsatilla alpina subsp. austroalpina

Heracleum austriacum subsp. siifolium

Centaurea haynaldii subsp. julica

Cfir Caricion firmae

Euphrasia pulchella

Carex firma

Ranunculus hybridus

ES Elyno-Seslerietea

Sesleria caerulea

Lotus alpinus

Helianthemum nummularium subsp. grandiflorum Phyteuma orbiculare

Senecio abrotanifolius

Thymus praecox subsp. polytrichus

Campanula witasekiana

Galium anisophyllum

Leucanthemum heterophyllum (L.maximum agg.)

Ranunculus montanus

Carduus crassifolius

Scabiosa lucida subsp. lucida

Carex sempervirens

Globularia cordifolia

Aster bellidiastrum

Polygala alpestris

Carduus defloratus

Thesium alpinum

Acinos alpinus

Polygonum viviparum

Phleum hirsutum

Achillea clavenae

Rhinanthus glacialis

Euphrasia picta

Selaginella selaginoides

Bartsia alpina

Ranunculus carinthiacus

Gentiana lutea subsp. symphyandra

Daphne striata

Hieracium pilosum

Carex mucronata

Juncus monanthos

Scabiosa lucida subsp. stricta

JT Juncetea trifidi

Campanula scheuchzeri

Veronica fruticans

CU Calluno-Ulicetea

Agrostis capillaris

Gentiana pannonica

Festuca nigrescens

Potentilla erecta

\section{$\begin{array}{lll}1 & 2 & 3\end{array}$}

4

-

$$
1
$$

$+$

.


Number of relevé (Zaporedna številka popisa)

E1

Bromus erectus agg. (Bromopsis transsilvanica)

Centaurea triumfettii

Gentianella ciliata

Prunella grandiflora

Stachys recta

Koeleria pyramidata

Linum catharticum

Gymnadenia conopsea

Hippocrepis comosa

Ajuga genevensis

Coronilla vaginalis

Teucrium montanum

PaT Poo alpinae-Trisetetalia

Poa alpina

Ranunculus nemorosus

MA Molinio-Arrbenatheretea

Veronica chamaedrys

Dactylis glomerata s.str.

Festuca rubra

CD Caricetalia davallianae

Pinguicula alpina

EA Epilobietea angustifoliae

Fragaria vesca

Rubus idaeus

Chamaenerion angustifolium

Myosotis arvensis

E1

E1

E1

E1

E1

E1

E1

E1

E1

E1

E1

E1

E1

E1

E1

E1

E1

E1

E1

E1

E1

E1

E1

E1

E1

E2

E1

E1

AC Arabidetalia caeruleae

Soldanella alpina

TR Thlaspietea rotundifolii

Gymnocarpium robertianum

Adenostyles glabra

Valeriana montana

Hieracium bifidum

Biscutella laevigata

Trisetum argenteum

Scrophularia juratensis

Minuartia austriaca

Senecio rupestris

Achnatherum calamagrostis

Rumex scutatus

Euphrasia cuspidata

Cy Cystopteridion fragilis

Cystopteris fragilis

\section{3}

4

$\begin{array}{llll}5 & 6 & 7 & 8\end{array}$

(1)

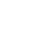

.

.

$\begin{array}{cccc} & & & + \\ . & \cdot & \cdot & + \\ 1 & \cdot & + & + \\ \cdot & \cdot & \cdot & + \\ \cdot & \cdot & \cdot & + \\ + & \cdot & + & \cdot \\ \cdot & \cdot & \cdot & + \\ . & \cdot & . & +\end{array}$


Number of relevé (Zaporedna številka popisa)

PS Physoplexido comosae-Saxifragion petraeae

Saxifraga hostii

Silene hayekiana

PC Potentilletalia caulescentis

Campanula cochleariifolia

Festuca stenantha

Campanula cespitosa

Rhamnus pumilus

Primula auricula

Festuca alpina

Erysimum sylvestre

AT Asplenietea trichomanis

Asplenium viride

Asplenium ruta-muraria

Moehringia muscosa

Asplenium trichomanes

Polypodium vulgare

Gypsophila repens

Kernera saxatilis

ML Mosses and lichens (Mahovi in lišaji)

Tortella tortuosa

Ctenidium molluscum

Schistidium sp.

Fissidens dubius

Dicranum scoparium

Rhytidiadelphus triquetrus

Hylocomium splendens

Homalothecium lutescens

Letharia vulpina

Homalothecium philippeanum

Scleropodium purum

Polytrichum formosum

Cladonia pyxidata

Peltigera canina

Plagiomnium undulatum

Usnea sp.

Peltigera leucophlebia

Plagiothecium undulatum

Hypogymnia physodes

Isothecium alopecuroides

Bartramia pomiformis

\begin{tabular}{l|llll|llll} 
& 1 & 2 & 3 & 4 & 5 & 6 & 7 & 8
\end{tabular}

E1

E1

E1

E1

E1

E1

E1

E1

E1

E1

E1

E1

E1

E1

E1

E1

E0

E0

E0

E0

E0

E0

E0

E0

E3c

E0

E0

E0

E0

E0

E0

E3c

E0

E0

E3c

E0

E0

\section{Legend - Legenda}

ID Igor Dakskobler

MČ Miran Čas

AS Andrej Seliškar

A Limestone - apnenec

D Dolomite-dolomit

Gr Gravel - grušč
Re Rendzina - rendzina

JA Julian Alps - Julijske Alpe

KSA Kamnik-Savinja Alps - Kamniško-Savinjske Alpe

K Karavanke - Karavanke

Pr. Presence (number of relevés in which the species is presented) - število popisov, v katerih se pojavlja vrsta

Fr. Frequency in $\%-$ frekvenca $v \%$ 


\begin{tabular}{|c|c|c|c|c|c|c|c|c|c|c|c|c|c|c|}
\hline 9 & 10 & 11 & 12 & 13 & 14 & 15 & 16 & 17 & 18 & 19 & 20 & 21 & Pr. & $\mathrm{Fr}$. \\
\hline . & . & . & . & & & . & . & . & . & . & . & . & 1 & 5 \\
\hline . & . & . & . & . & . & . & . & . & . & . & + & . & 1 & 5 \\
\hline . & & . & . & . & . & + & . & + & . & + & . & . & 5 & 24 \\
\hline . & . & . & 1 & . & + & . & + & . & . & . & + & . & 5 & 24 \\
\hline . & + & 1 & . & . & . & + & . & + & . & . & + & . & 5 & 24 \\
\hline . & . & . & + & . & . & . & $\mathrm{r}$ & + & . & . & + & . & 4 & 19 \\
\hline . & $\mathrm{r}$ & . & . & . & . & . & . & . & . & . & + & . & 3 & 14 \\
\hline . & & . & . & . & . & + & . & . & . & . & . & . & 1 & 5 \\
\hline . & . & . & . & & & . & . & . & . & . & + & . & 1 & 5 \\
\hline+ & + & . & + & . & + & + & . & 1 & . & + & . & . & 13 & 62 \\
\hline . & + & . & + & & + & + & + & + & + & + & . & . & 12 & 57 \\
\hline . & + & . & + & . & . & . & . & . & . & + & . & . & 9 & 43 \\
\hline . & + & . & . & . & . & + & . & + & + & + & . & . & 8 & 38 \\
\hline . & . & . & . & . & . & . & . & . & . & . & . & . & 1 & 5 \\
\hline . & . & . & . & . & . & . & . & . & . & . & . & . & 1 & 5 \\
\hline . & & . & . & . & + & . & . & . & . & . & . & . & 1 & 5 \\
\hline+ & + & . & + & + & + & + & 1 & + & + & + & + & + & 19 & 90 \\
\hline+ & + & . & + & + & + & + & + & + & . & + & . & . & 14 & 67 \\
\hline . & + & . & + & & + & . & + & . & + & + & . & . & 10 & 48 \\
\hline+ & + & . & . & . & . & + & . & . & . & . & . & . & 6 & 29 \\
\hline+ & . & . & . & . & . & + & . & + & . & . & . & . & 6 & 29 \\
\hline . & . & . & . & . & . & + & . & + & . & . & . & + & 4 & 19 \\
\hline . & . & . & . & . & . & . & . & . & . & . & + & 1 & 4 & 19 \\
\hline . & + & . & . & . & . & . & . & . & . & . & . & . & 3 & 14 \\
\hline . & . & . & . & . & . & . & . & + & . & . & . & . & 2 & 10 \\
\hline . & . & . & + & . & . & . & . & . & . & . & . & . & 2 & 10 \\
\hline . & . & . & . & . & . & . & . & . & . & . & + & 1 & 2 & 10 \\
\hline & . & . & . & & & . & . & . & . & . & . & . & 1 & 5 \\
\hline . & . & . & . & . & . & . & . & . & . & . & . & . & 1 & 5 \\
\hline . & . & . & . & . & . & . & . & . & . & . & . & . & 1 & 5 \\
\hline . & . & . & . & . & . & . & . & . & . & . & . & . & 1 & 5 \\
\hline . & & . & . & . & . & . & . & . & . & . & . & . & 1 & 5 \\
\hline . & . & . & . & . & . & . & . & . & . & . & . & . & 1 & 5 \\
\hline . & . & . & + & . & . & . & . & . & . & . & . & . & 1 & 5 \\
\hline . & . & . & . & . & . & + & . & . & . & . & . & . & 1 & 5 \\
\hline . & . & . & . & . & . & . & . & + & . & . & . & . & 1 & 5 \\
\hline . & . & . & . & & & . & . & . & . & . & . & + & 1 & 5 \\
\hline
\end{tabular}




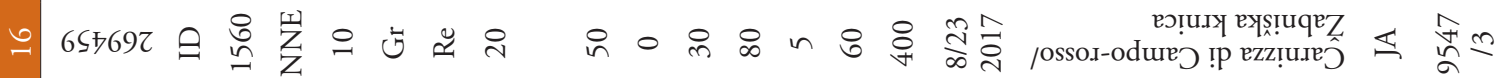

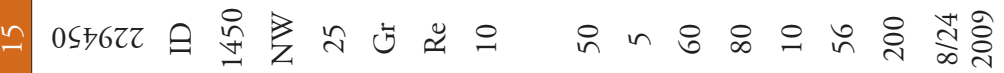

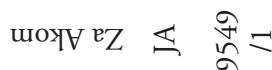

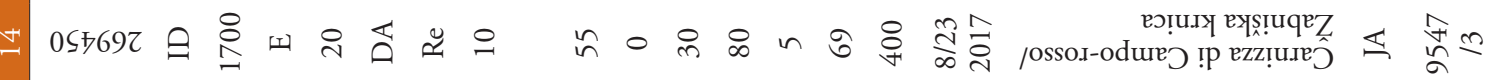

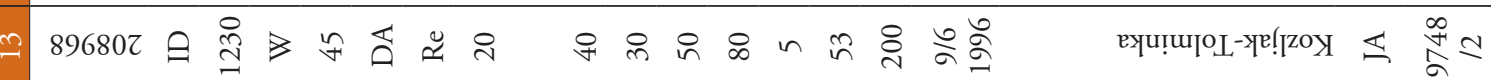

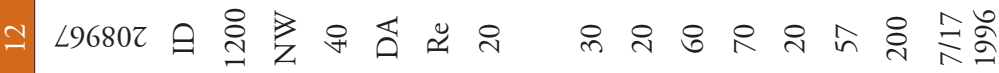

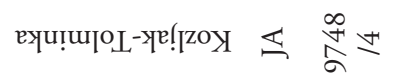

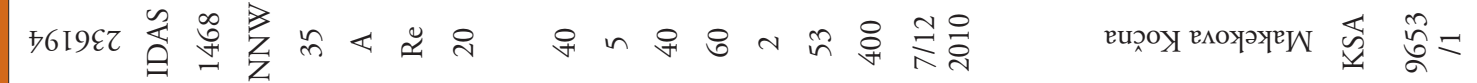

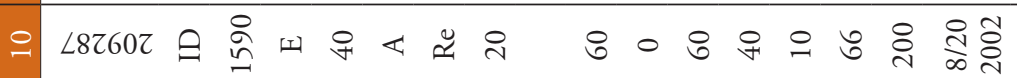

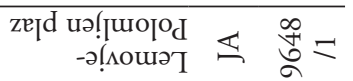

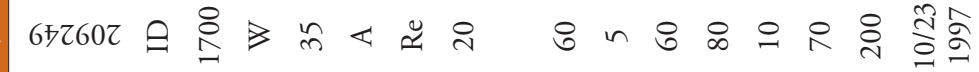

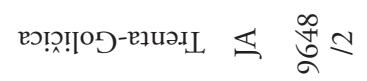
682607 은

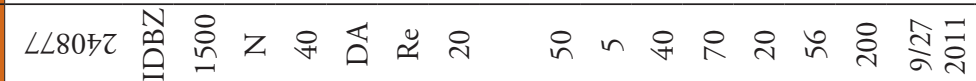

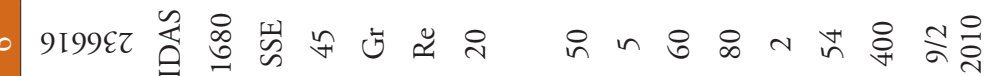

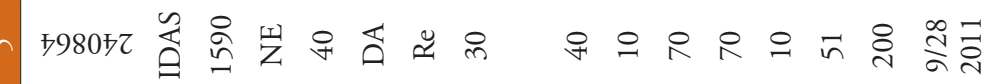

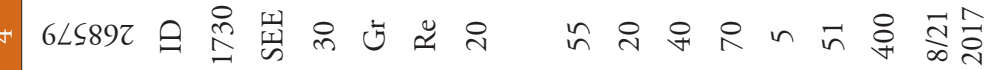

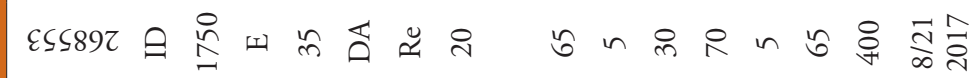

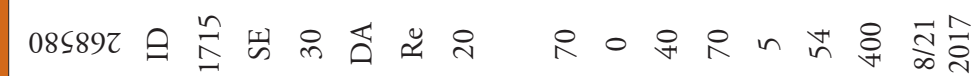

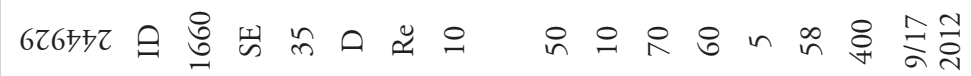

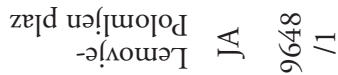

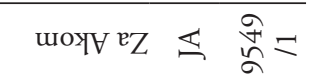

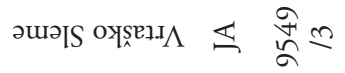

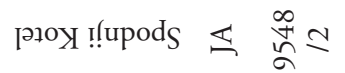

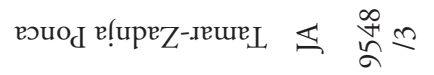

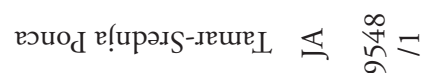

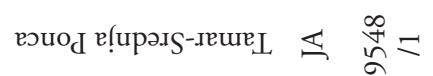

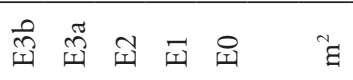

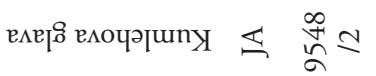



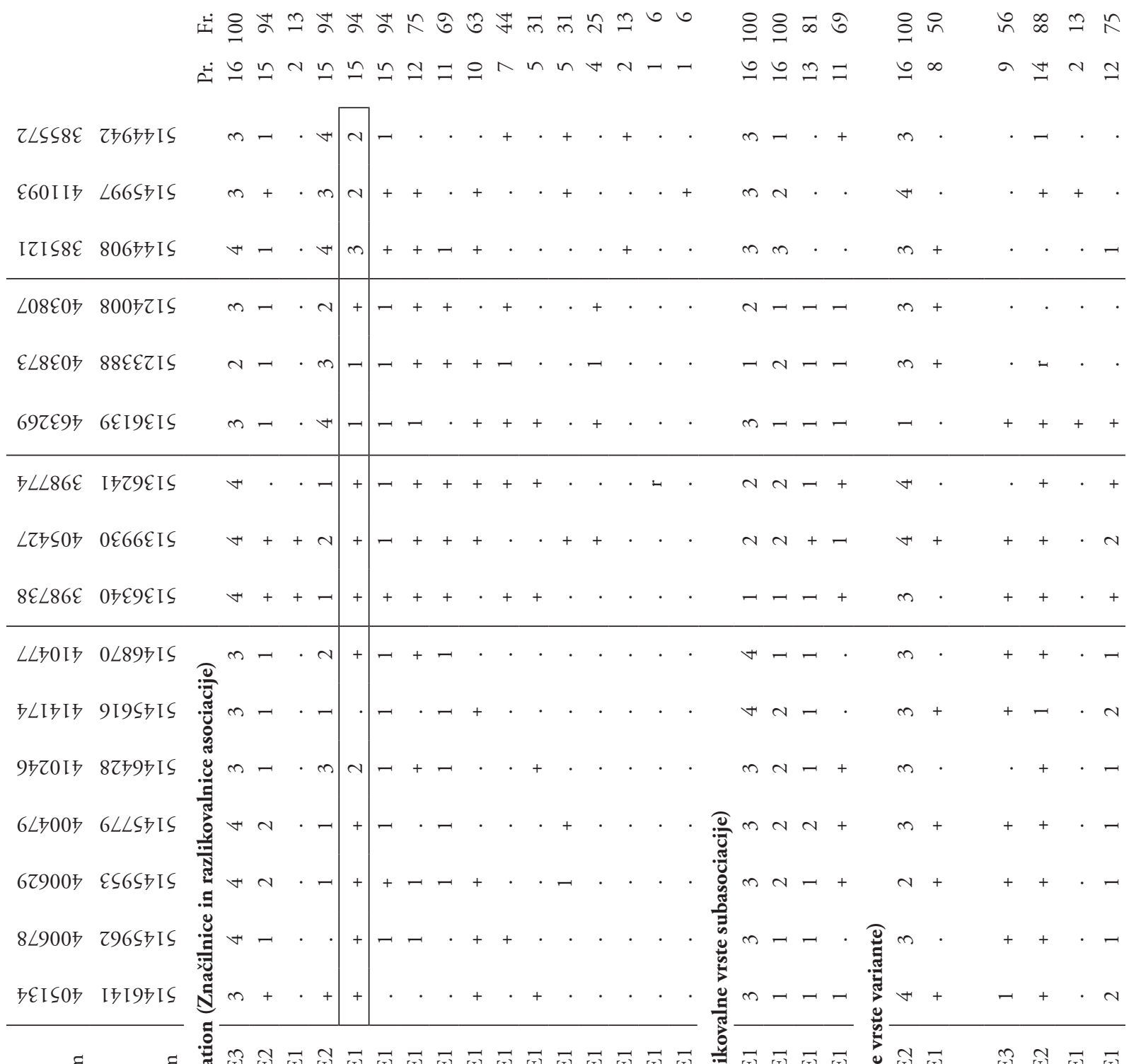

घ

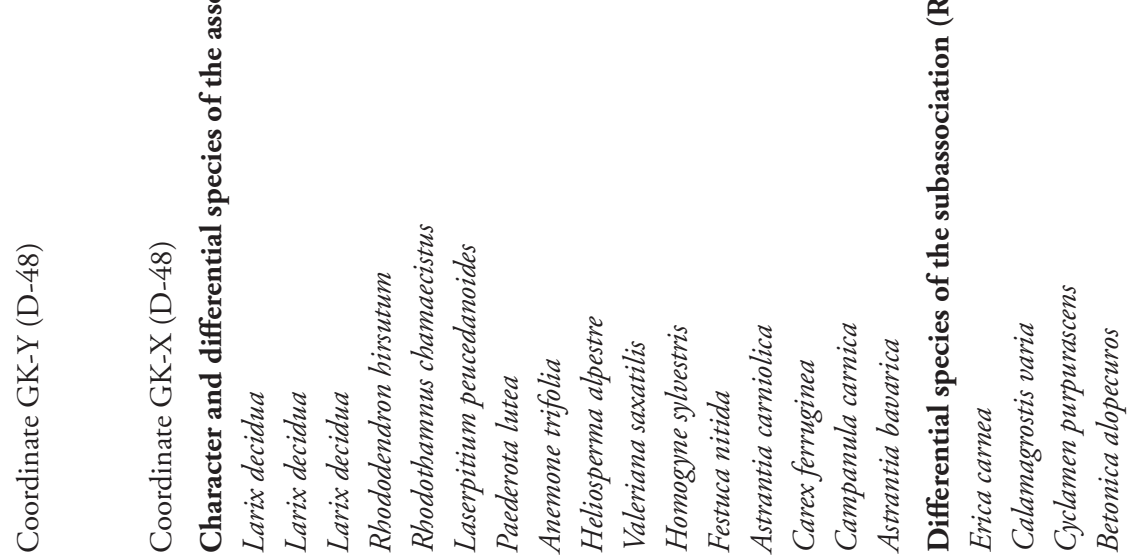

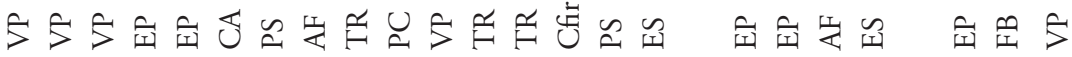




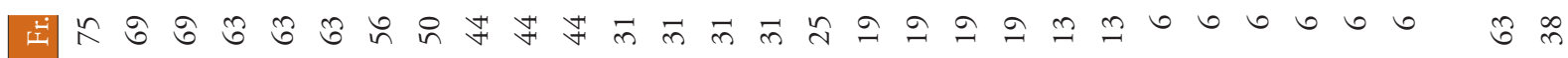

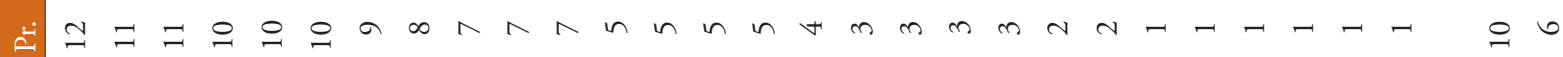

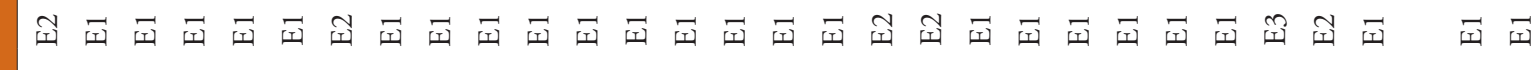

h +4

$+$

. . . . + + +

$+4+-\tau+-+\cdot-$

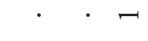

$+\neg \cdot+$

$\checkmark-$

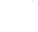

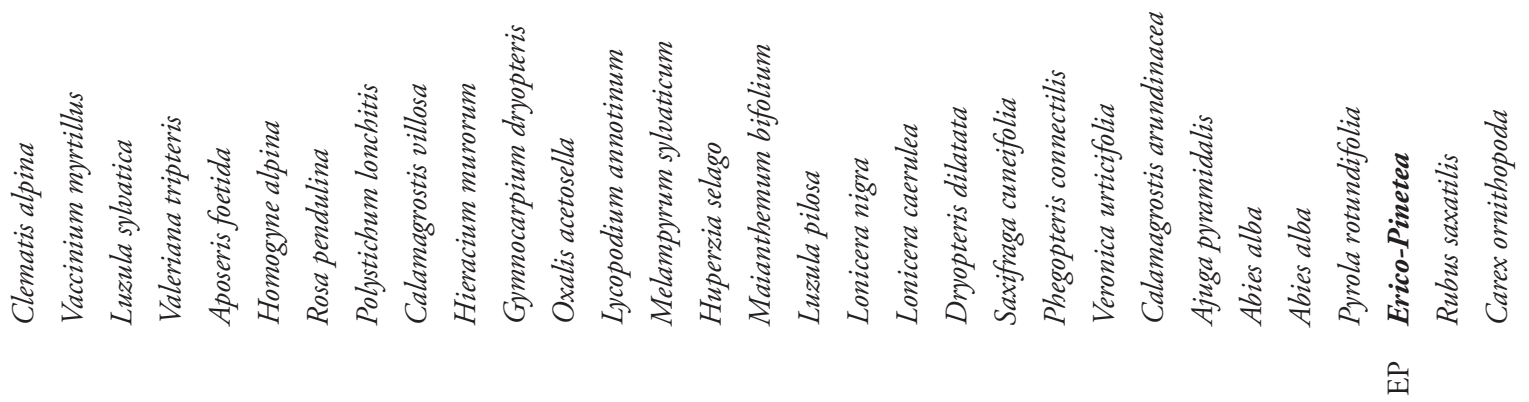




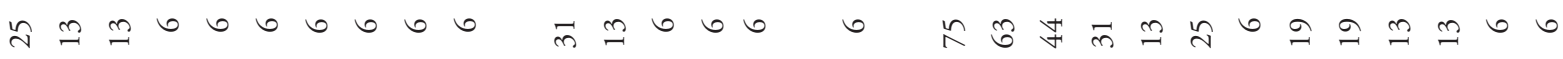

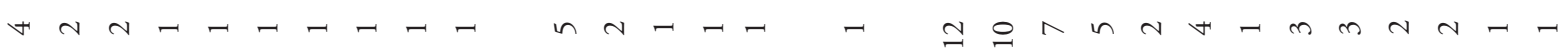
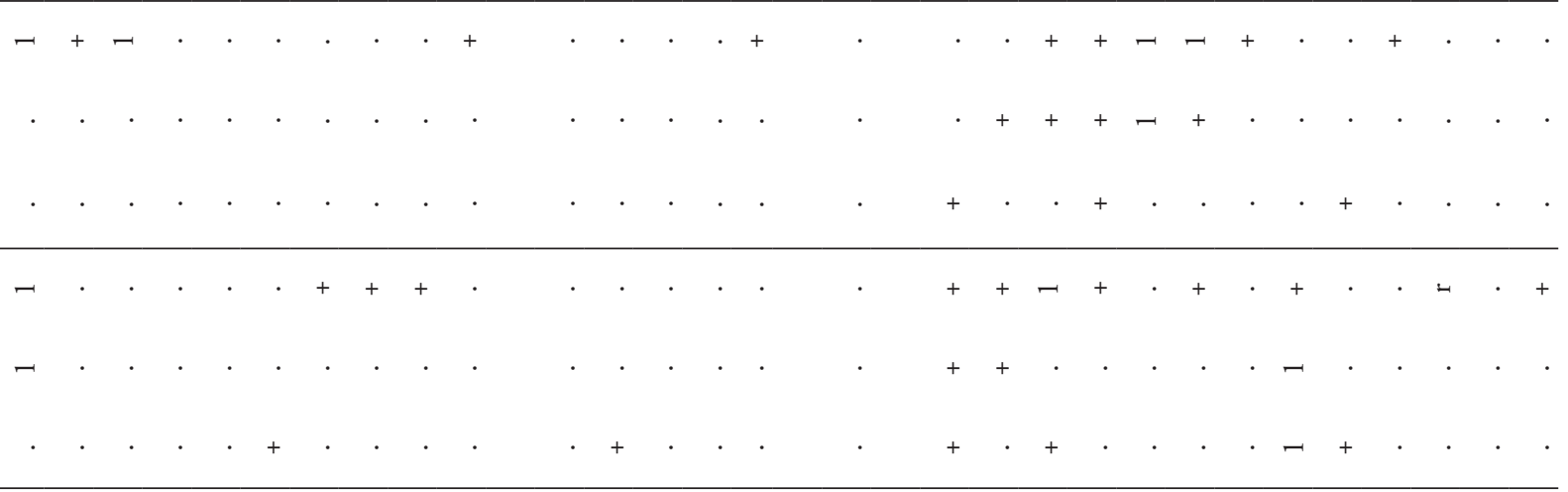

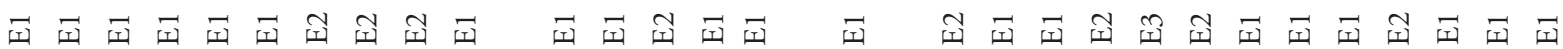

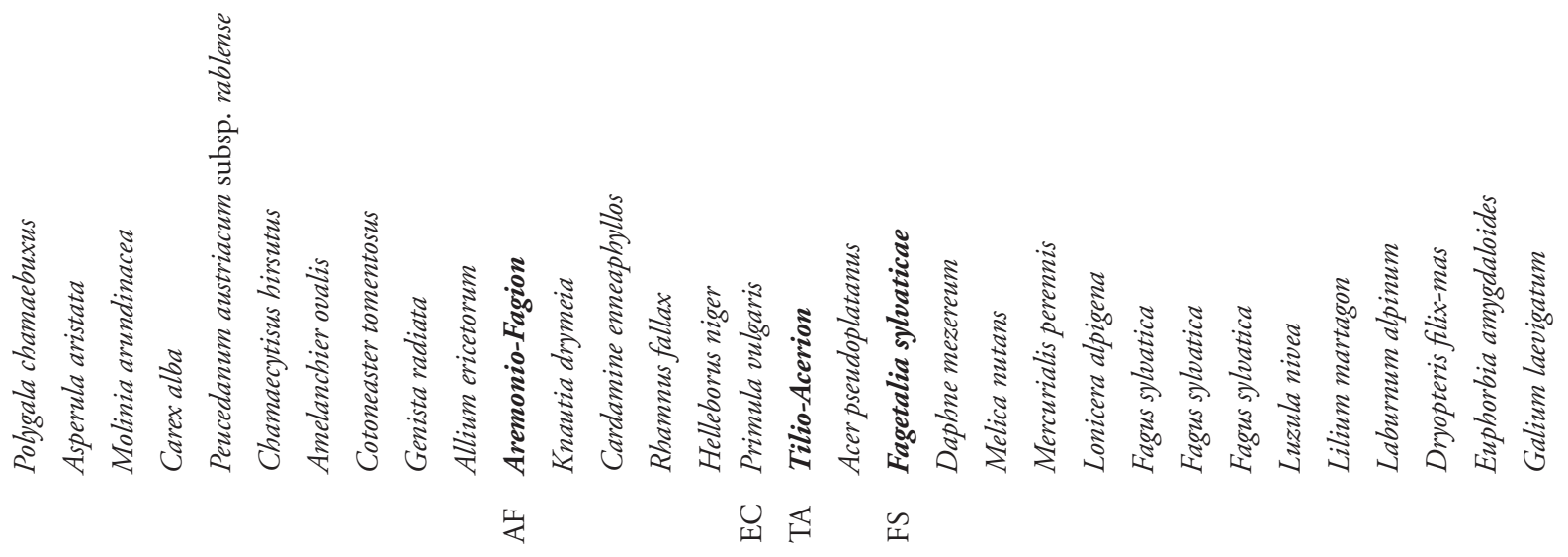




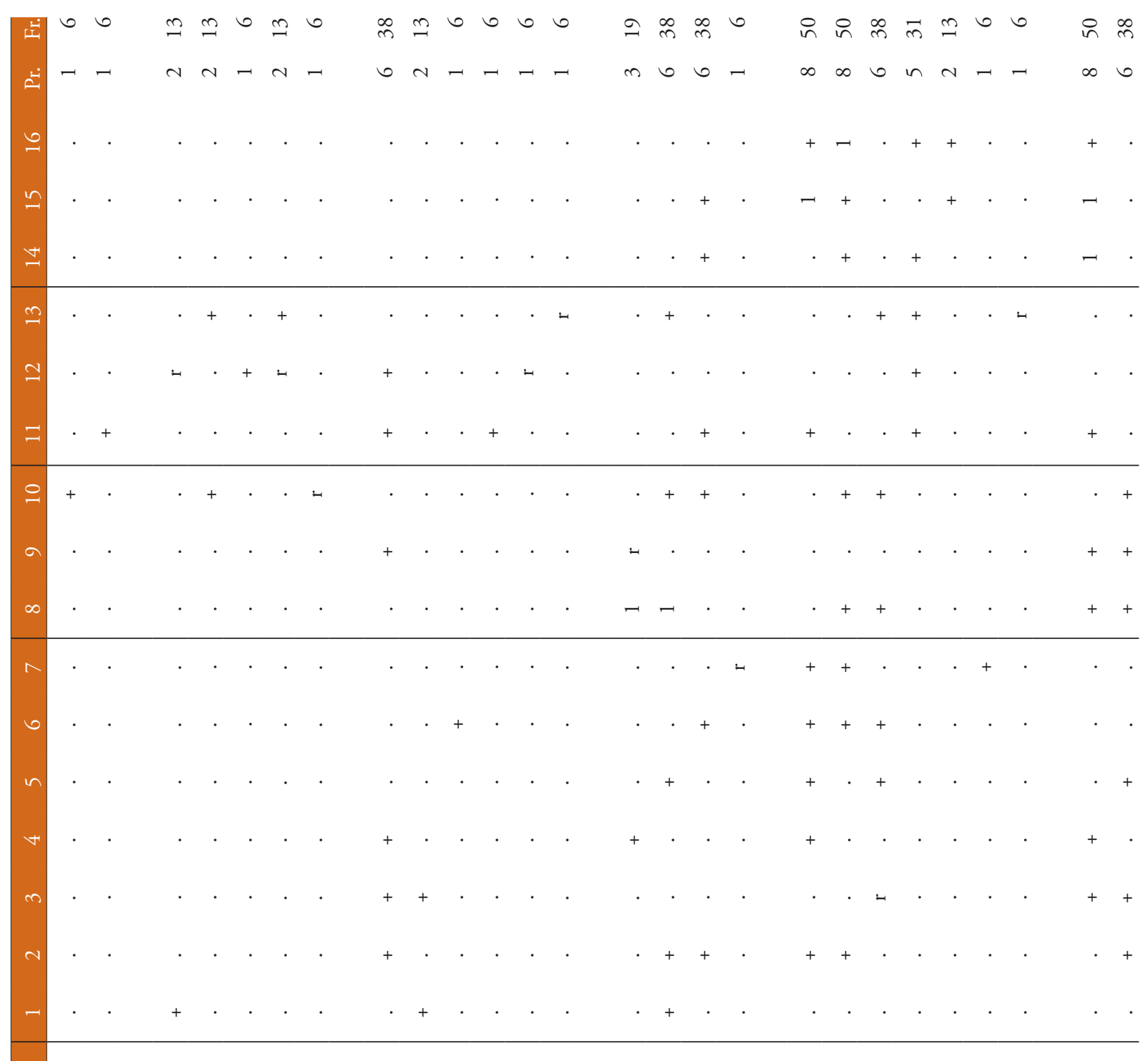

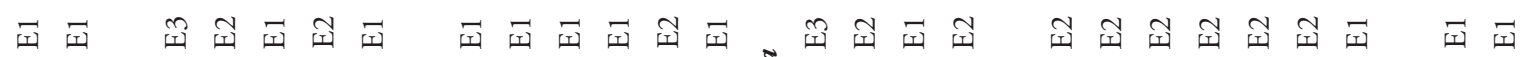




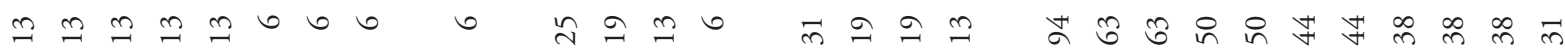

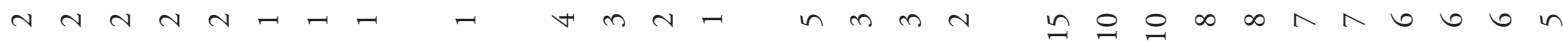

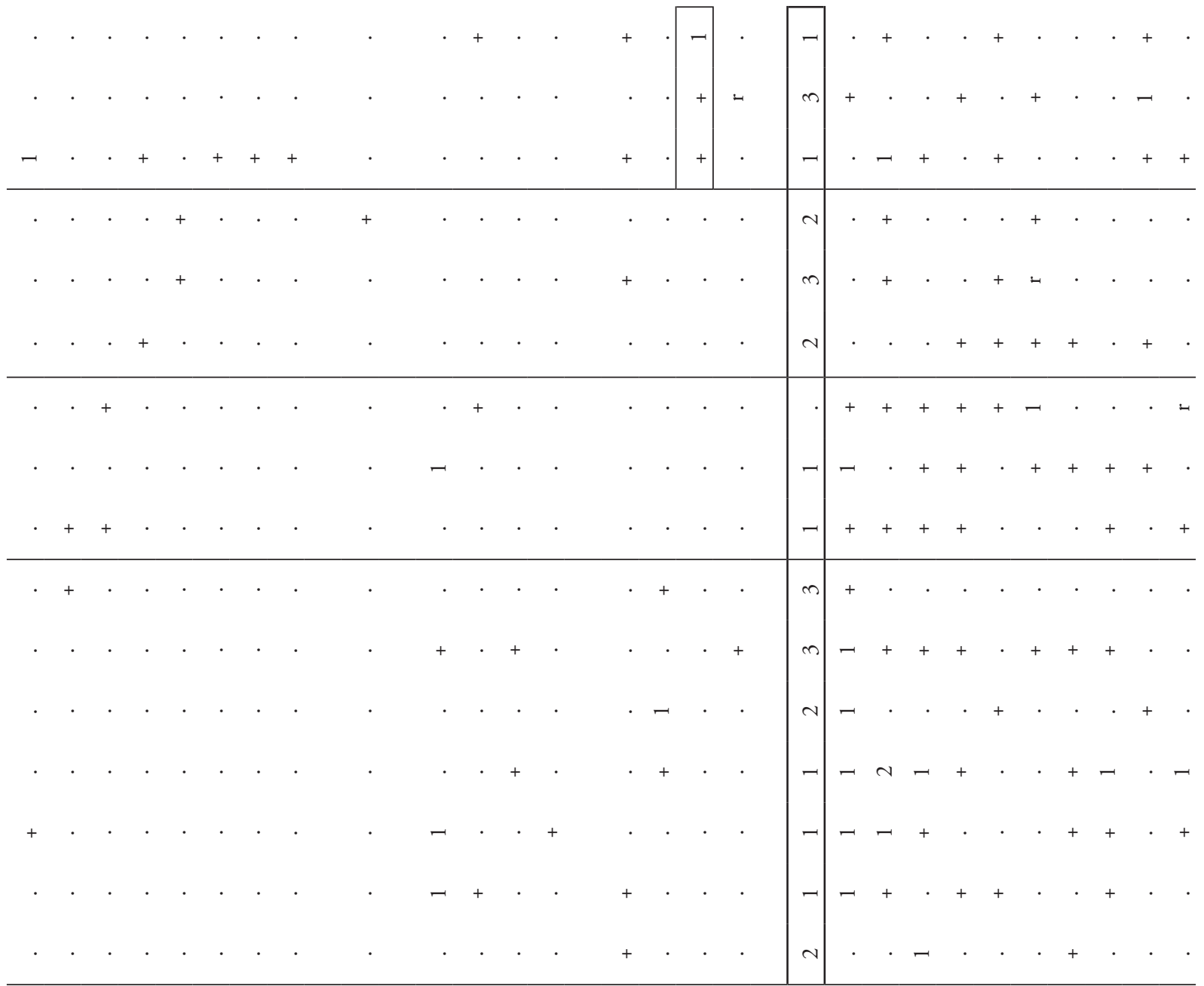

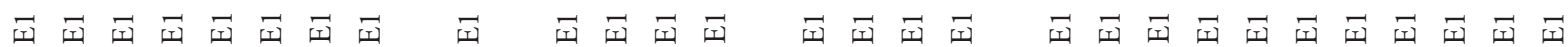
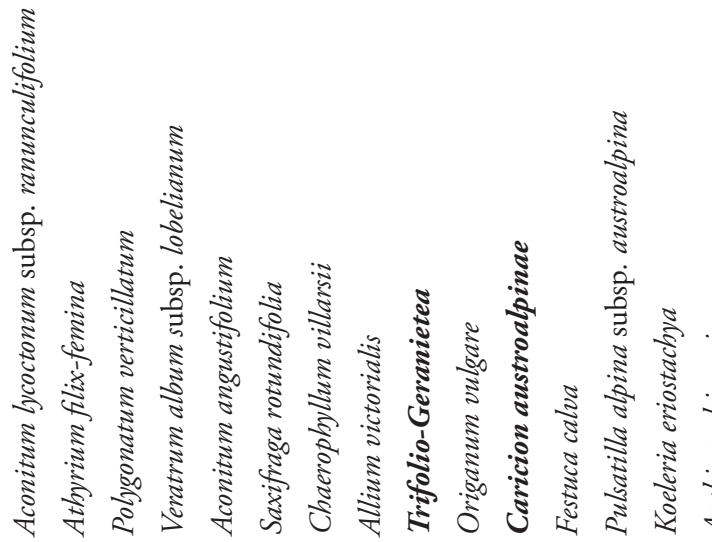

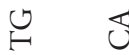

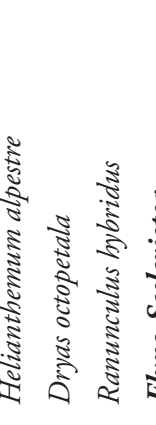

$\frac{\sqrt{2}}{\frac{2}{5}}$

कू

के

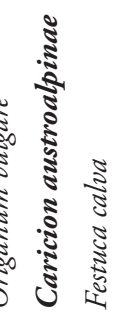

$\frac{2}{3}$ है

苛

盗 


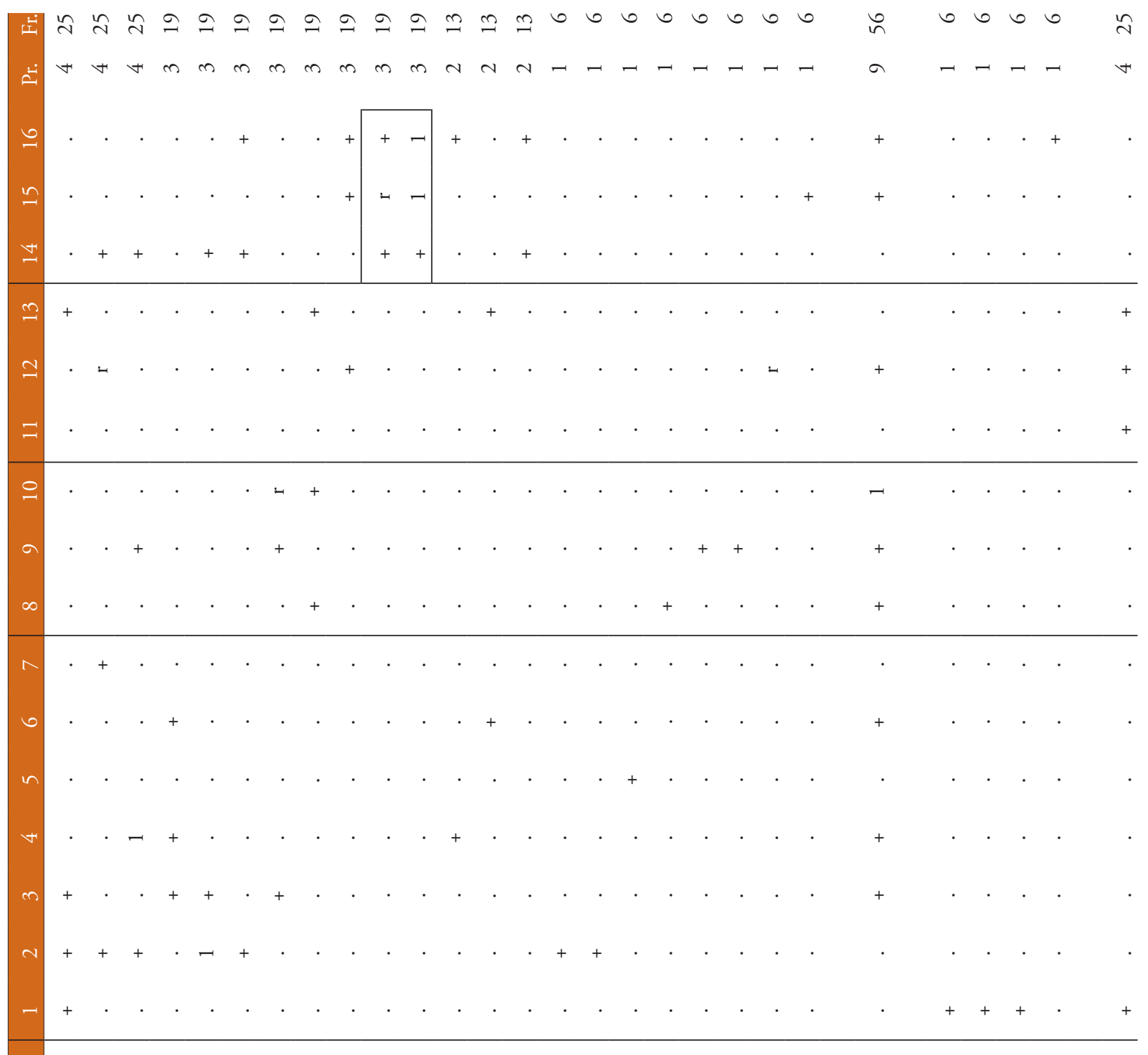

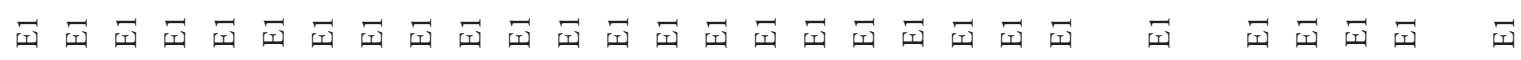



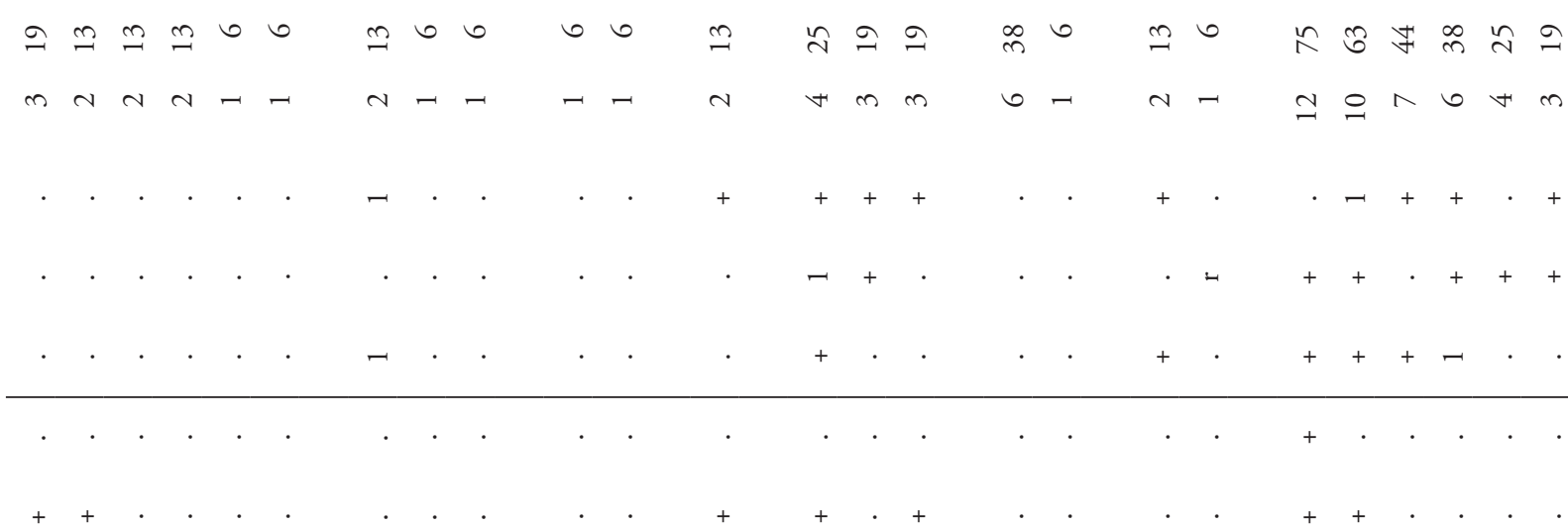

$+\quad+\quad+\quad \cdot \quad \cdot \quad \cdot \quad \cdot$

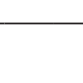

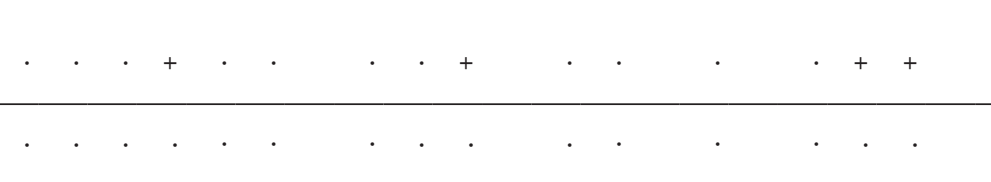

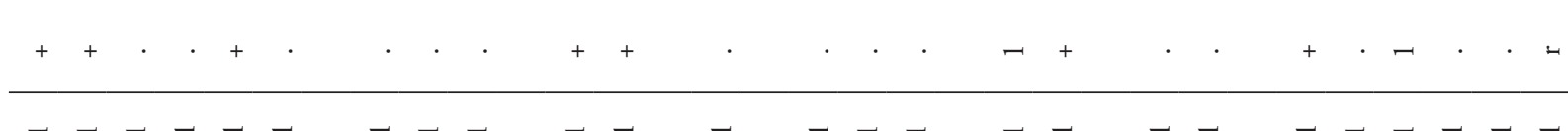

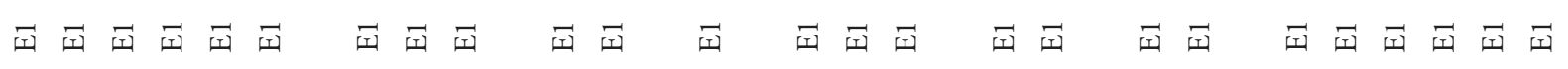

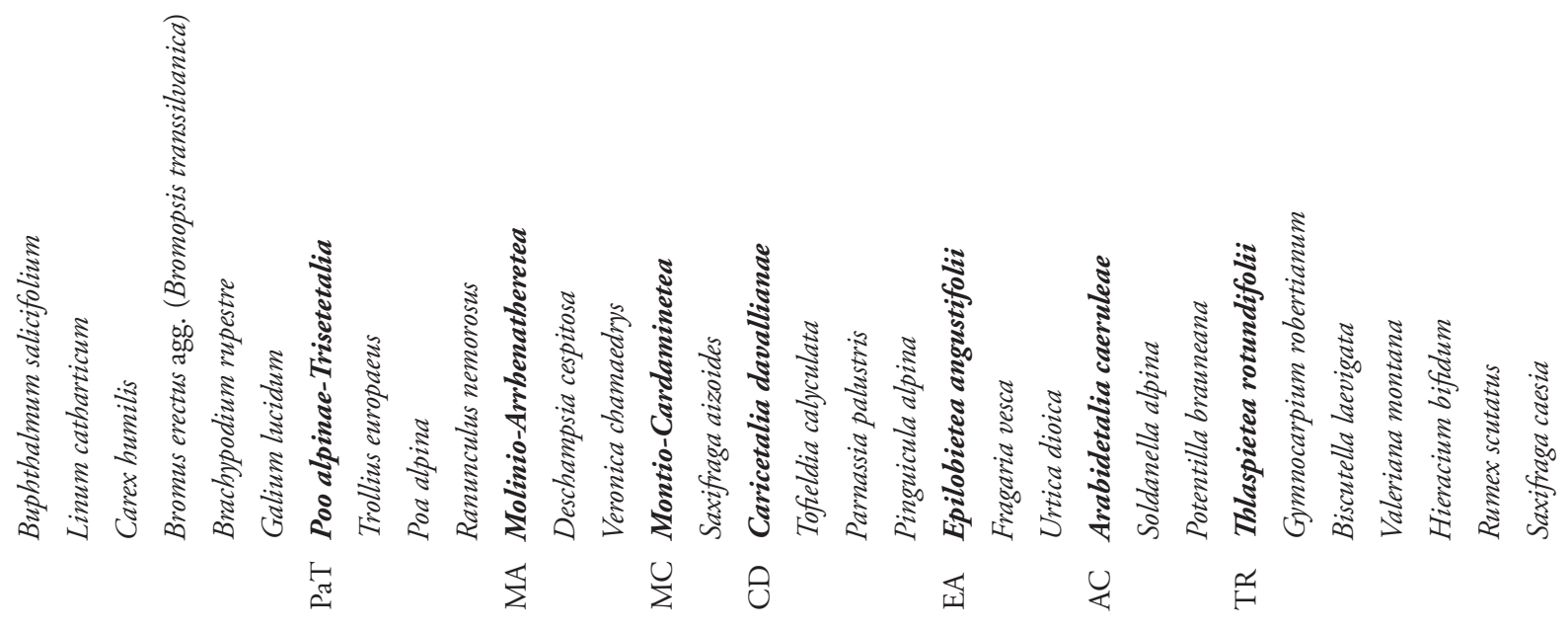




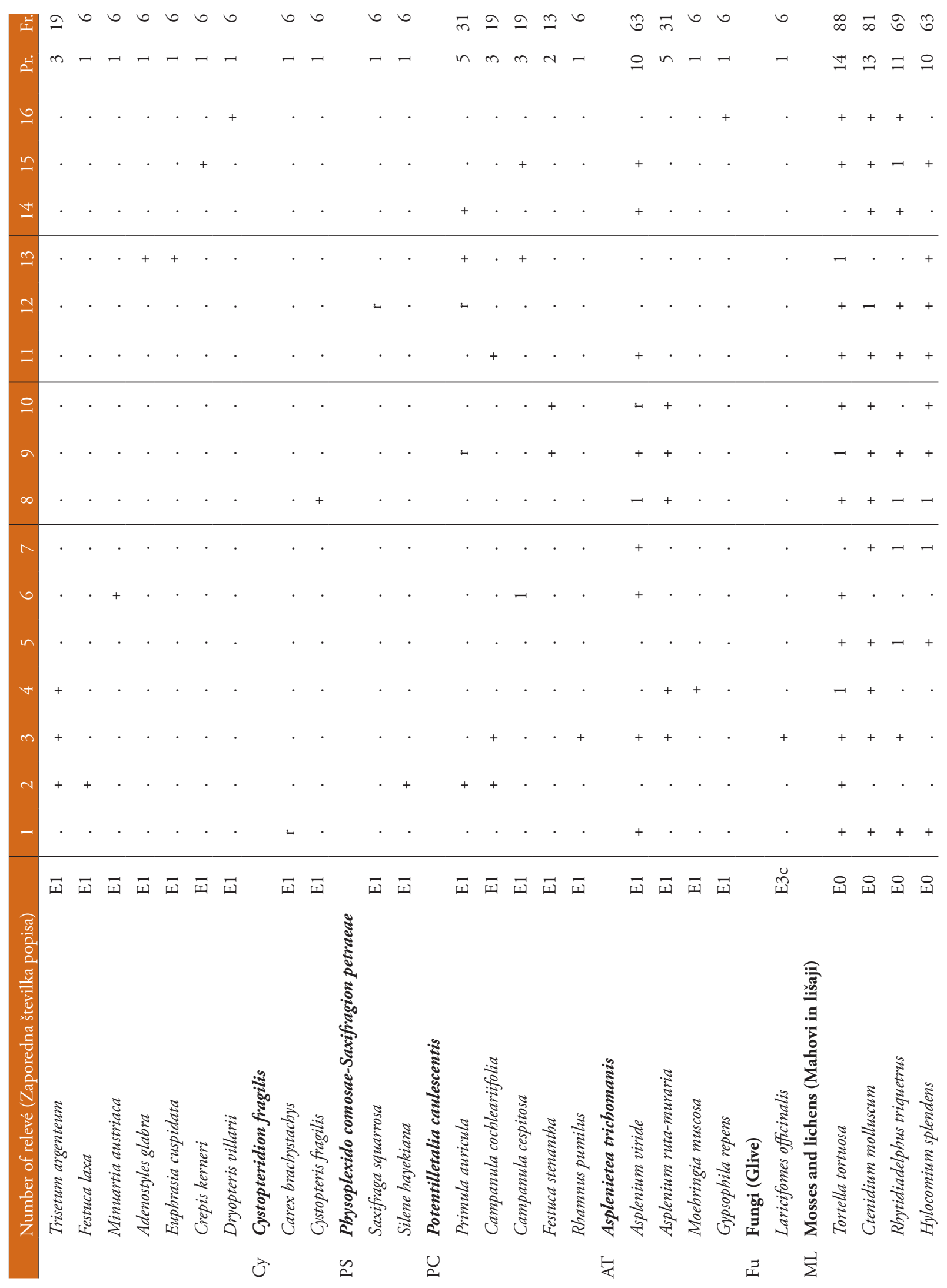




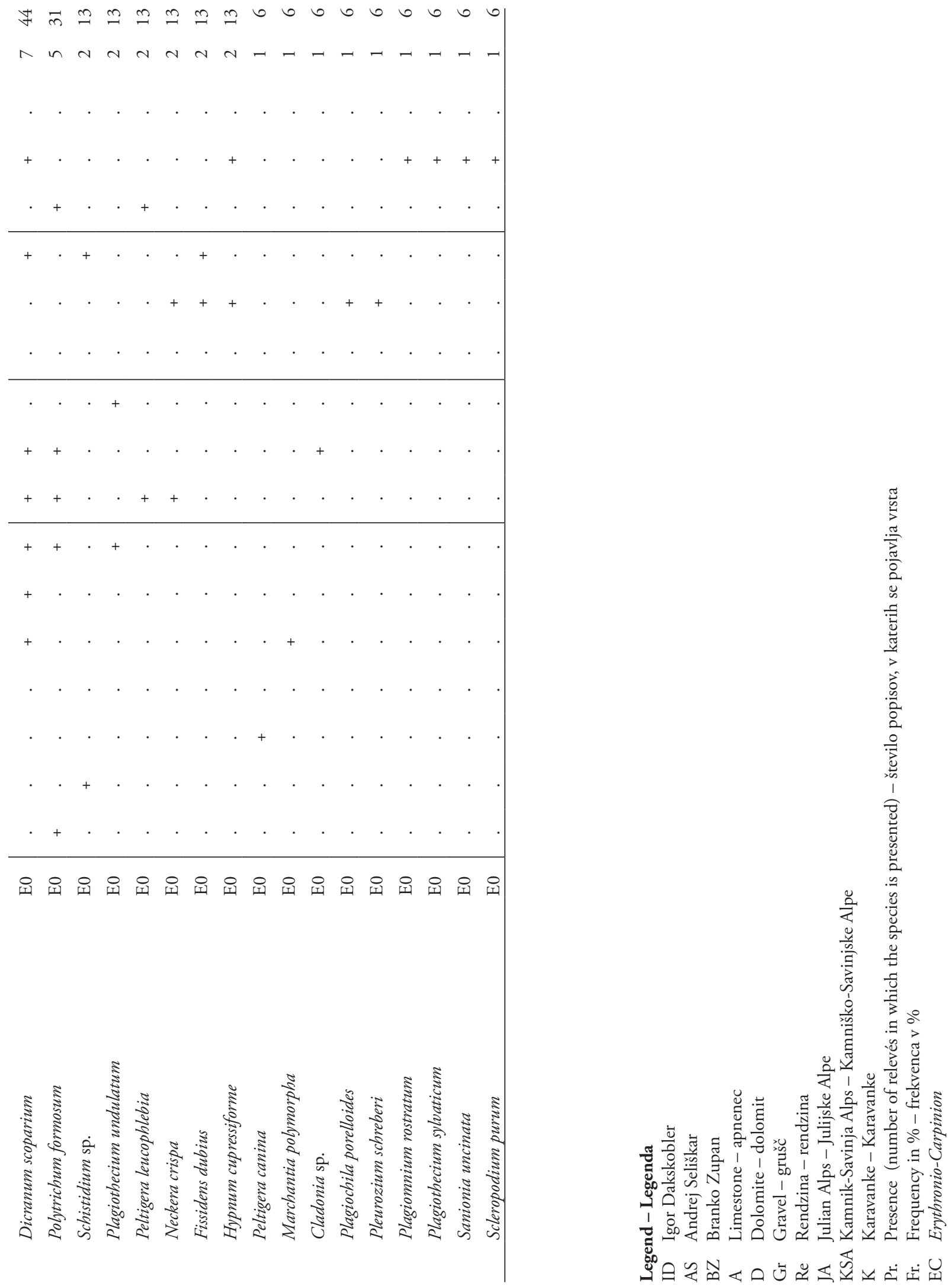


亲

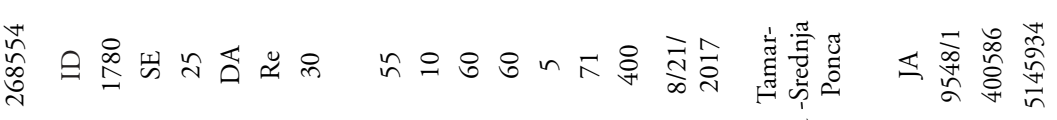
栾

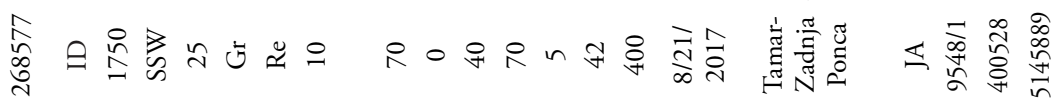

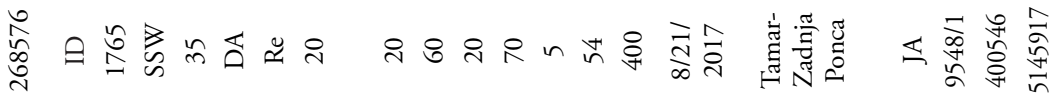
离

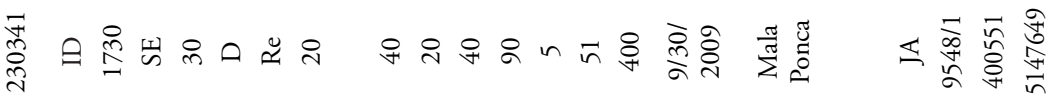

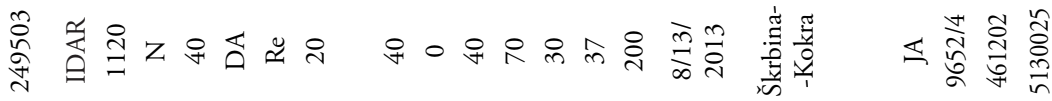

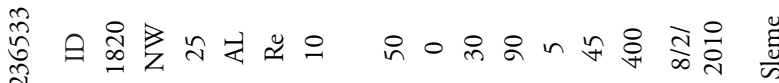

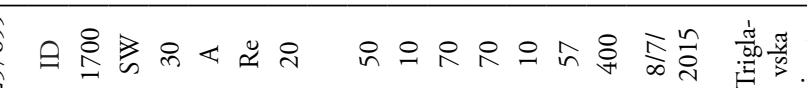

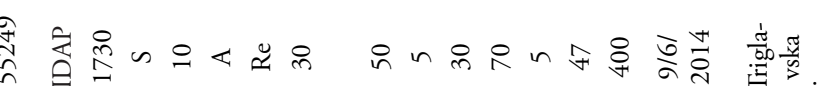
तै

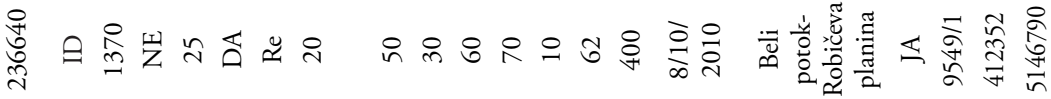

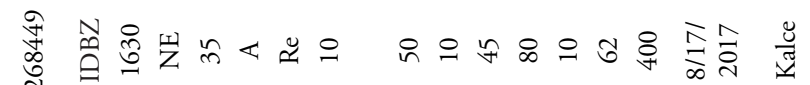

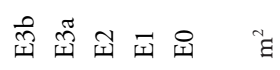

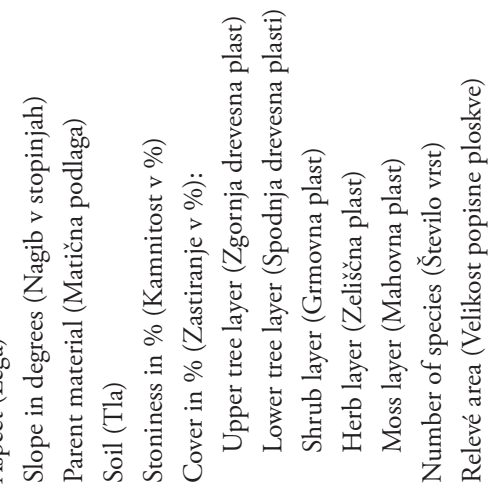
敢

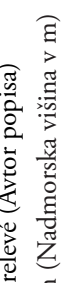

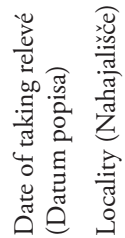

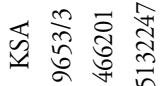
घ $\Xi$

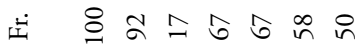
$\dot{I} \simeq=N \infty \infty \wedge 0$

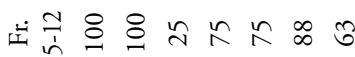
मี

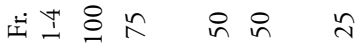

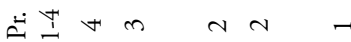

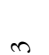




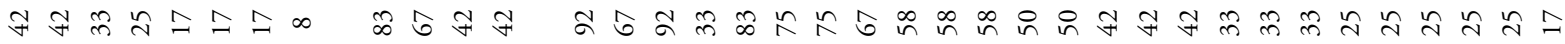

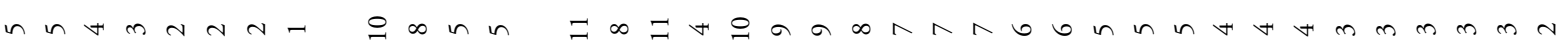

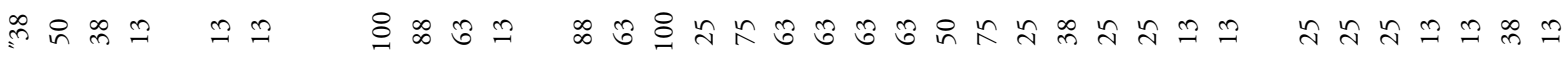

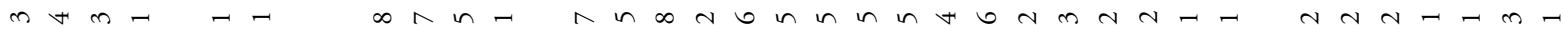

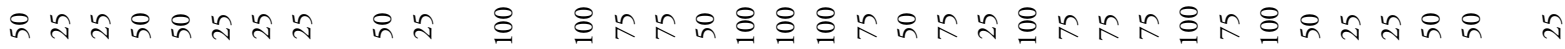

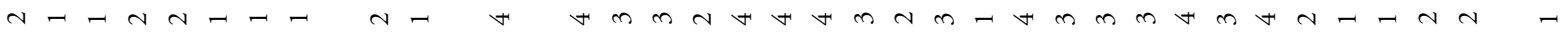

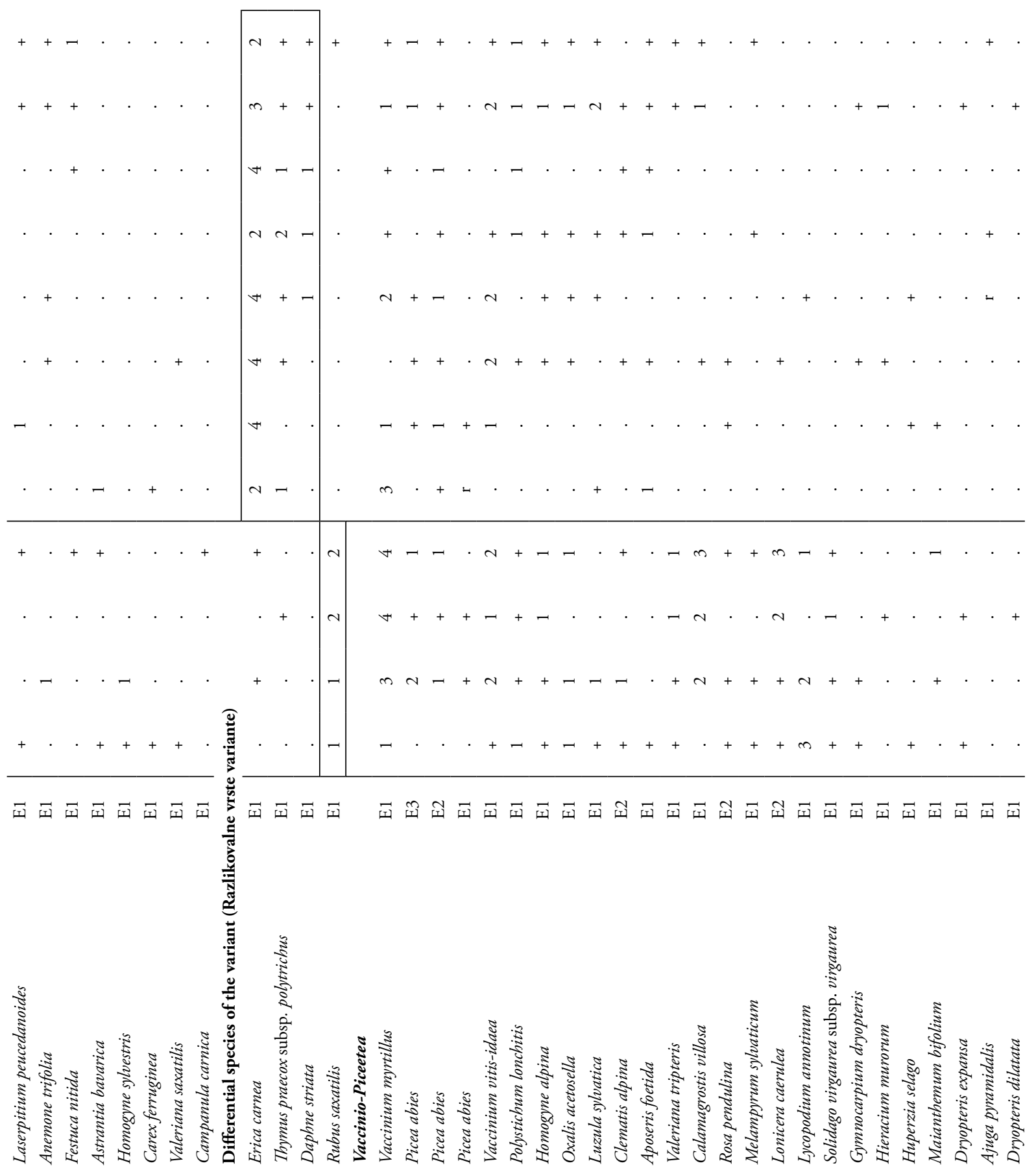

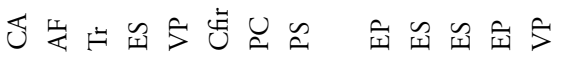




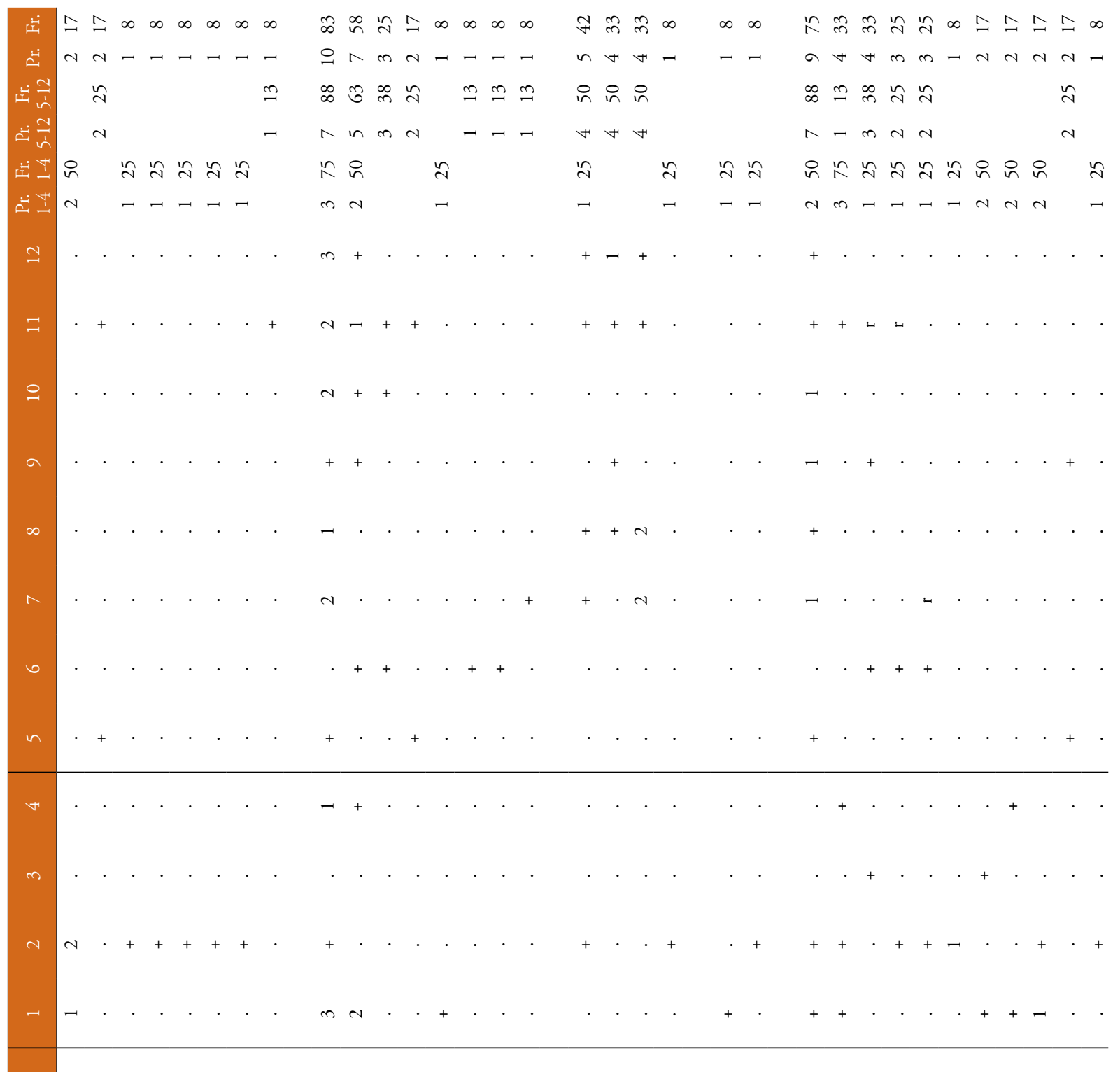

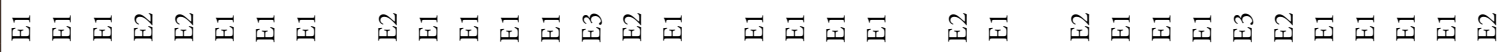

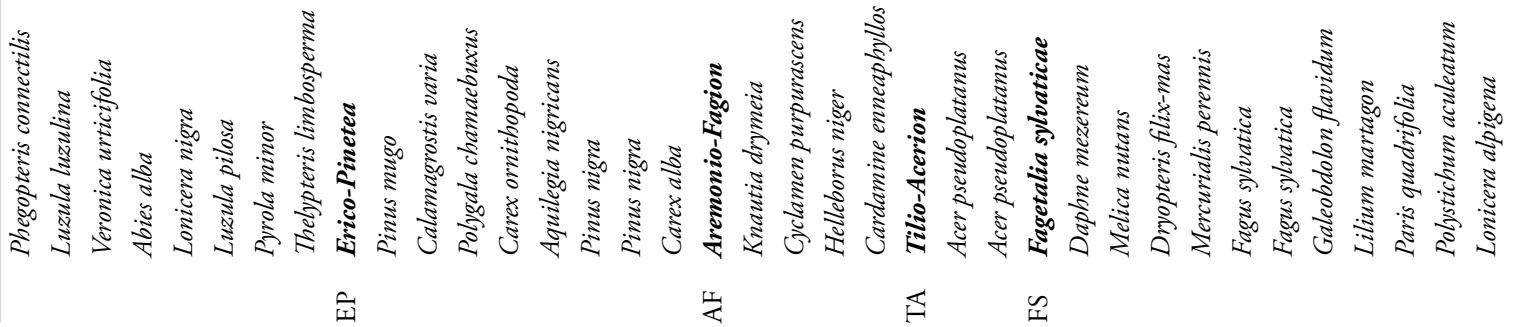




\begin{tabular}{|c|c|c|c|c|c|c|c|c|c|c|c|c|c|c|c|c|c|c|c|c|c|c|c|c|c|c|c|c|c|}
\hline$\infty$ & $\underset{f}{\sim}$ & 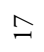 & $\triangleq$ & 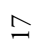 & $\infty$ & & $\approx$ & $\stackrel{\infty}{n}$ & $\approx$ & $\approx$ & $\triangleq$ & $\infty$ & $\infty$ & $\infty$ & $\infty$ & $\Re$ & $\stackrel{\infty}{n}$ & $\underset{\forall}{\sim}$ & $\tilde{m}$ & $\approx$ & $\approx$ & $\triangleq$ & 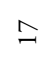 & 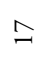 & $\infty$ & $\infty$ & $\infty$ & $\infty$ & $\infty$ \\
\hline- & in & $N$ & $\sim$ & N & - & $\sim$ & $n$ & $\Lambda$ & $n$ & $m$ & $\sim$ & - & - & - & - & $a$ & $\wedge$ & $n$ & 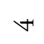 & $m$ & $m$ & $\sim$ & $N$ & $N$ & - & - & - & - & - \\
\hline$\stackrel{\approx}{\tilde{I}}$ & in & 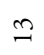 & $\dddot{m}$ & $\approx$ & & $\stackrel{n}{\sim}$ & $\approx$ & $\infty$ & & $\stackrel{m}{n}$ & & & & & & $\Re$ & $\infty$ & $\tilde{\sigma}$ & & $\stackrel{m}{\sim}$ & $\approx$ & & & & & & & & \\
\hline- & 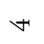 & - & - & $\sim$ & & - & $N$ & $m$ & & - & & & & & & ○ & $m$ & in & & - & $\sim$ & & & & & & & & \\
\hline & $\approx$ & $\approx$ & $\approx$ & & $\approx$ & $\approx$ & $\approx$ & 8 & 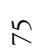 & in & ํㅜ & $\approx$ & $\approx$ & $\approx$ & $\approx$ & $\Re$ & 8 & & 8 & ำ & $\approx$ & in & in & $\stackrel{\circ}{n}$ & $\approx$ & $\approx$ & $\approx$ & $\approx$ & $\approx$ \\
\hline & - & - & $\neg$ & & - & $\neg$ & - & $\psi$ & $n$ & $N$ & $N$ & - & - & - & - & $n$ & $\psi$ & & 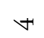 & N & - & $N$ & $N$ & $N$ & - & - & - & - & - \\
\hline
\end{tabular}

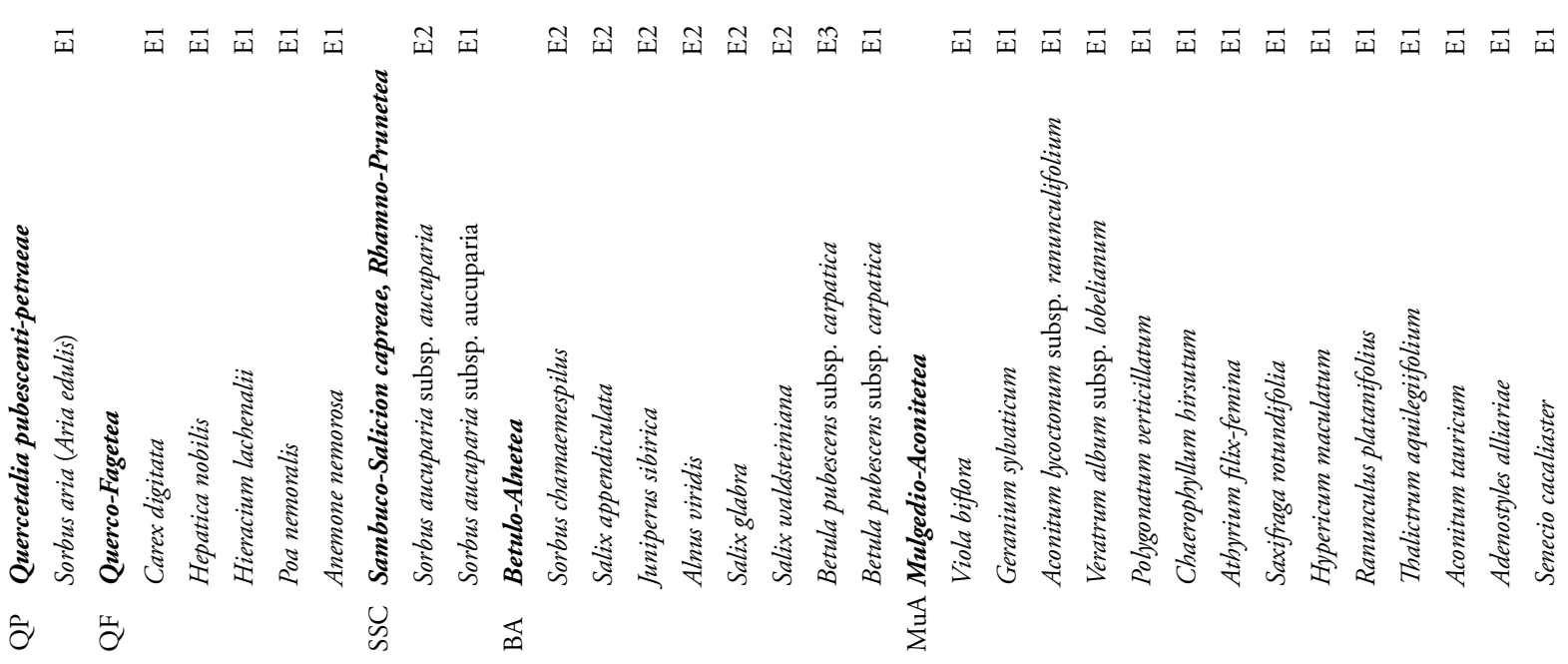




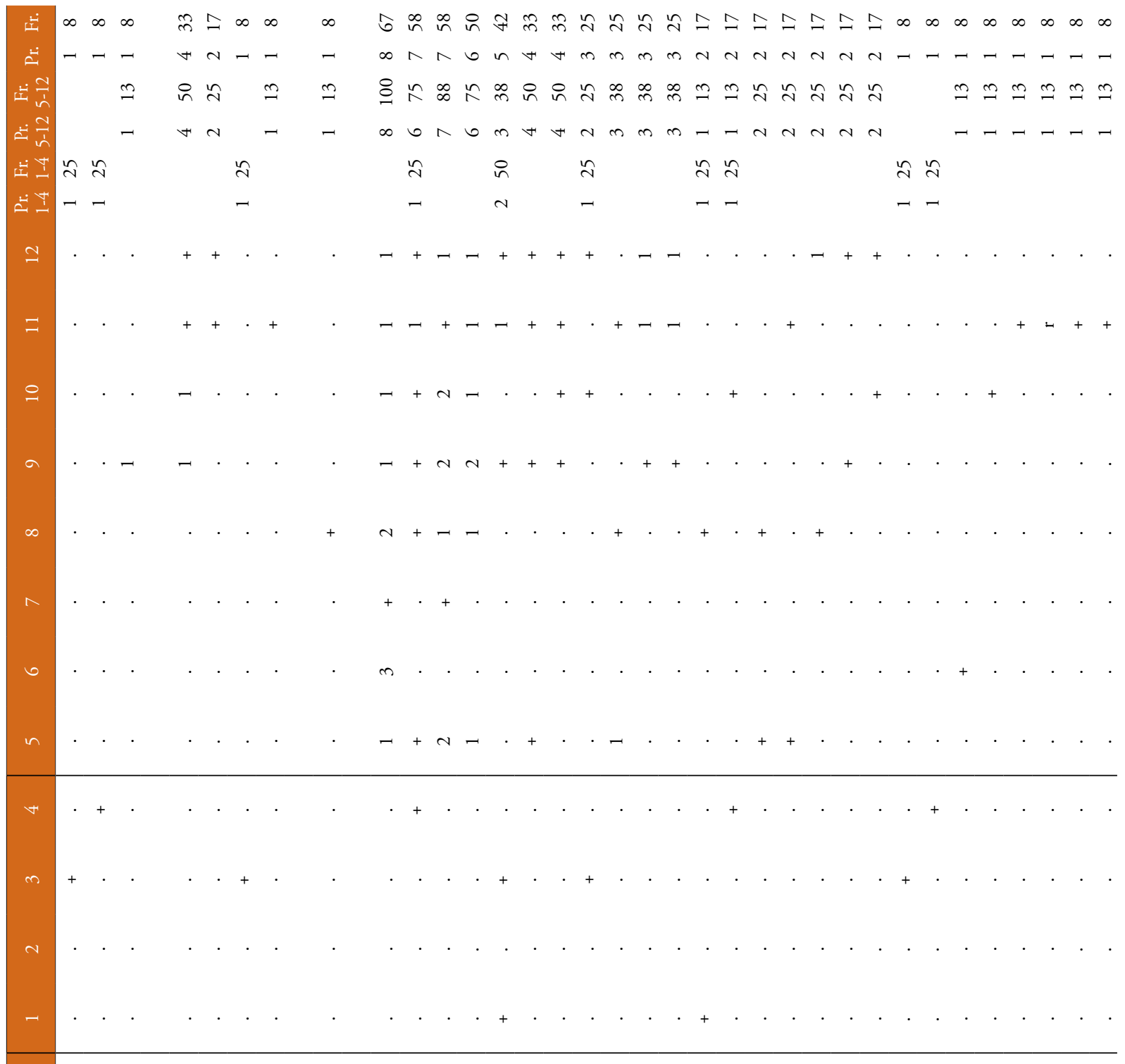

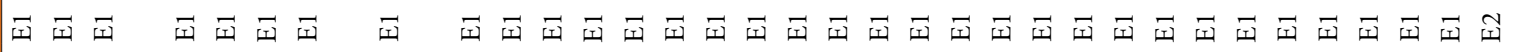

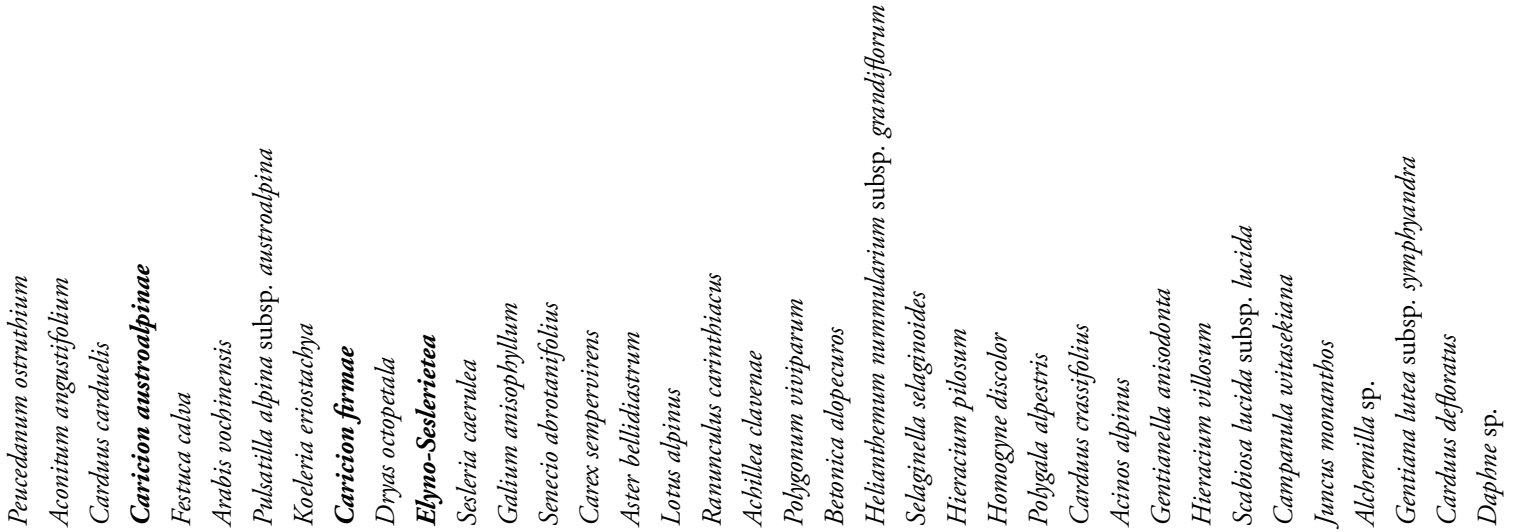
उ 


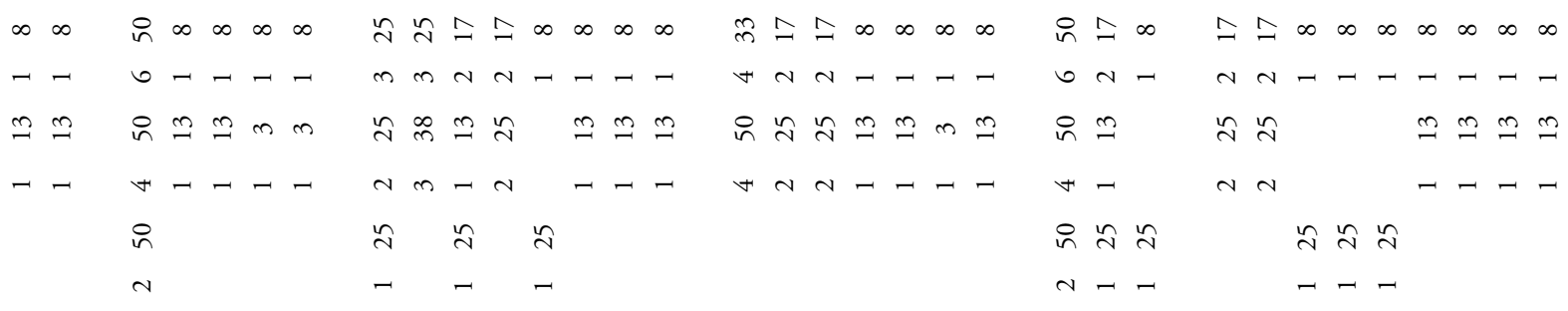

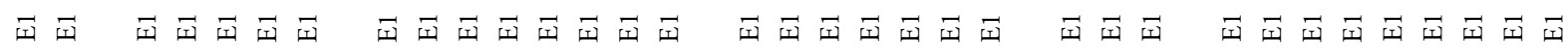

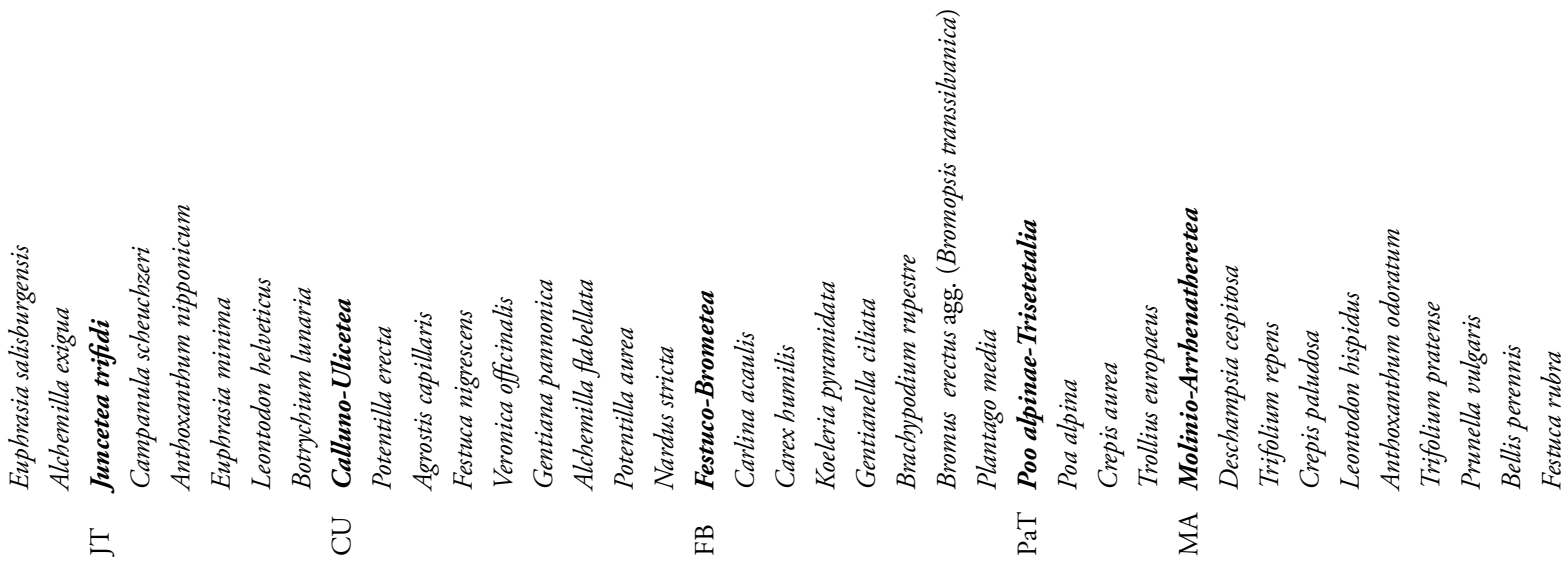




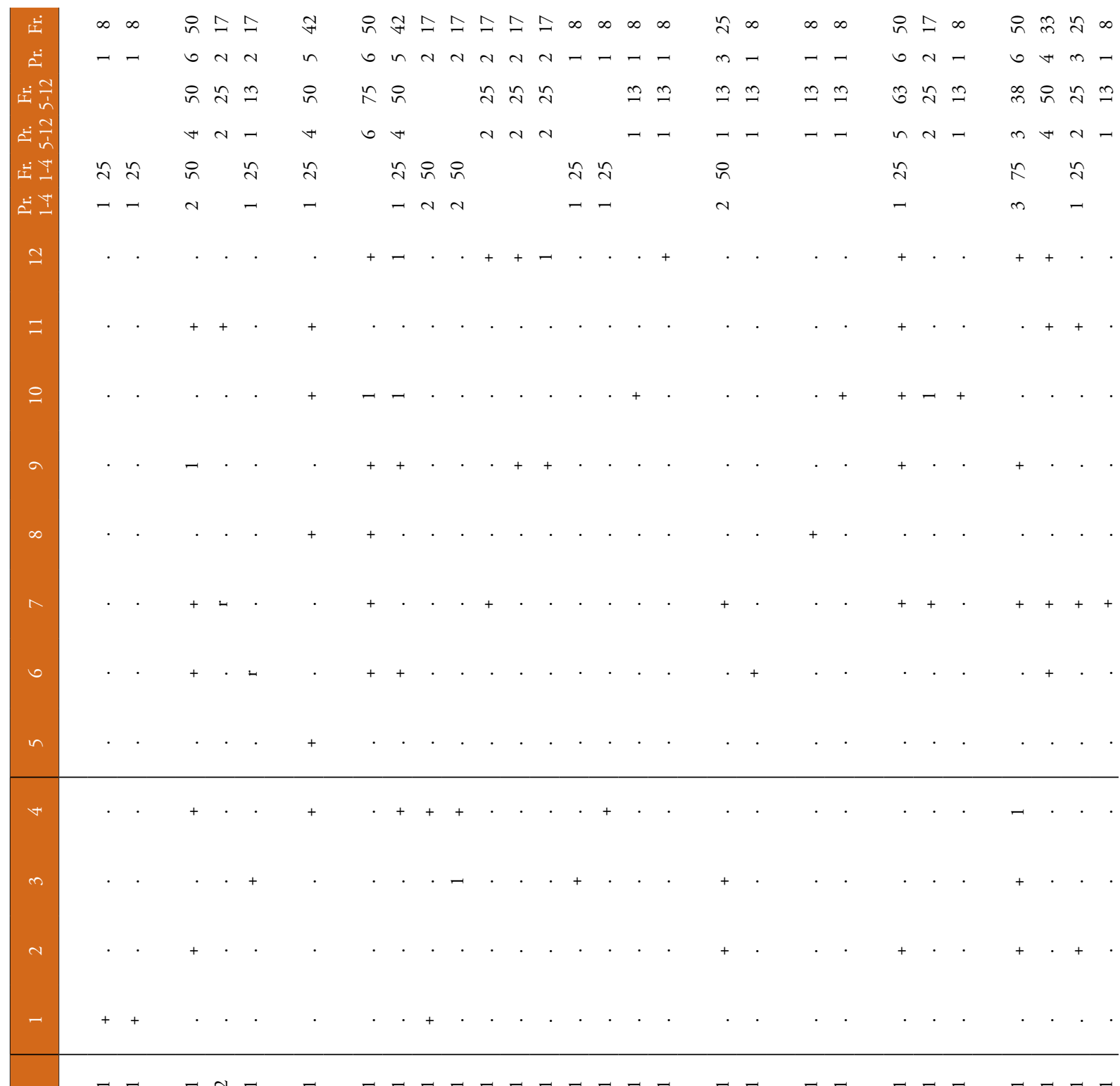

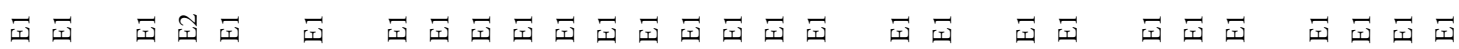
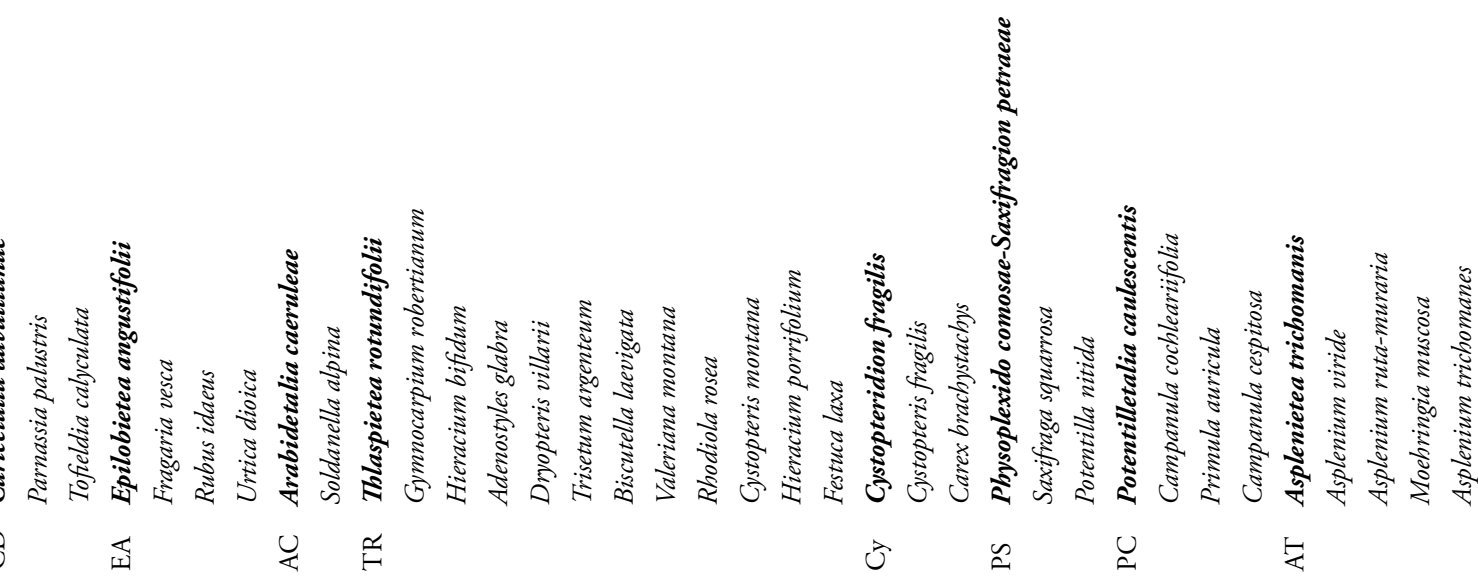

$\cup$

安 


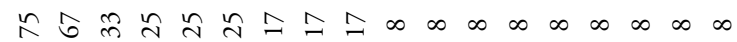

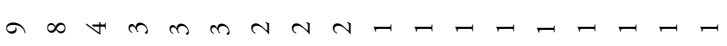

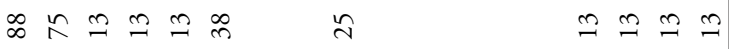
$n \in-n-m \quad-r-$

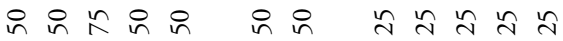
$\sim \sim m \sim ⿻-1,-$

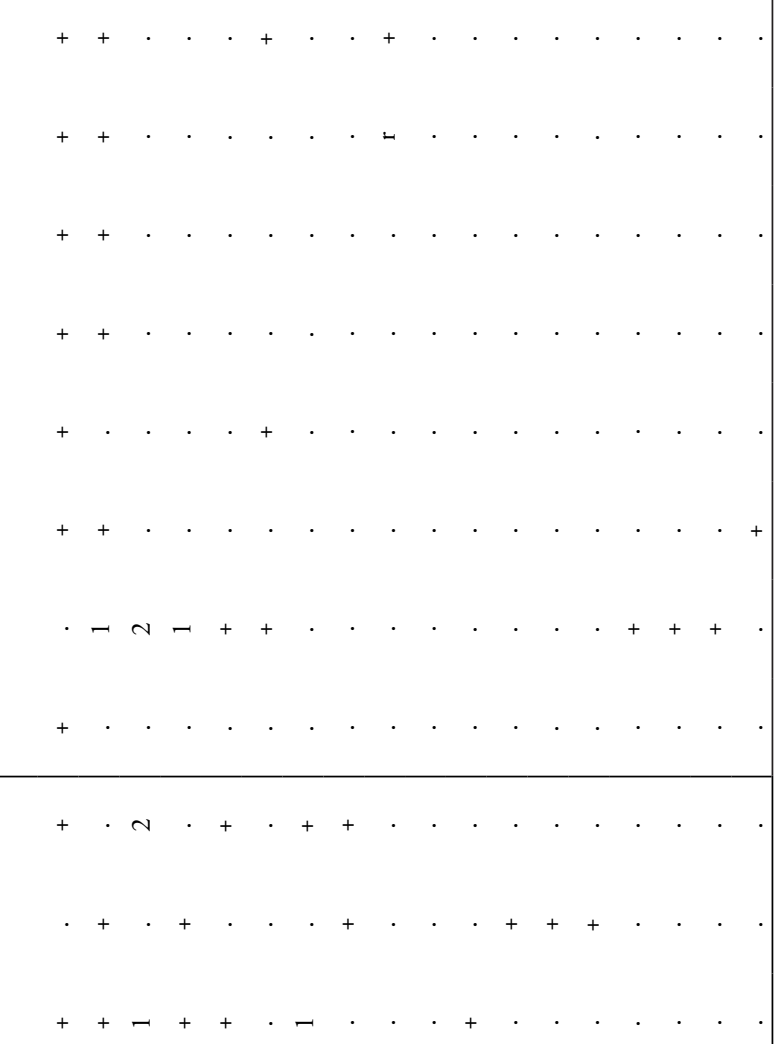

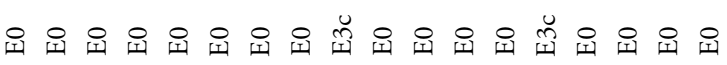

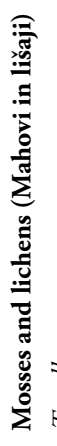

$\vec{\Sigma}$

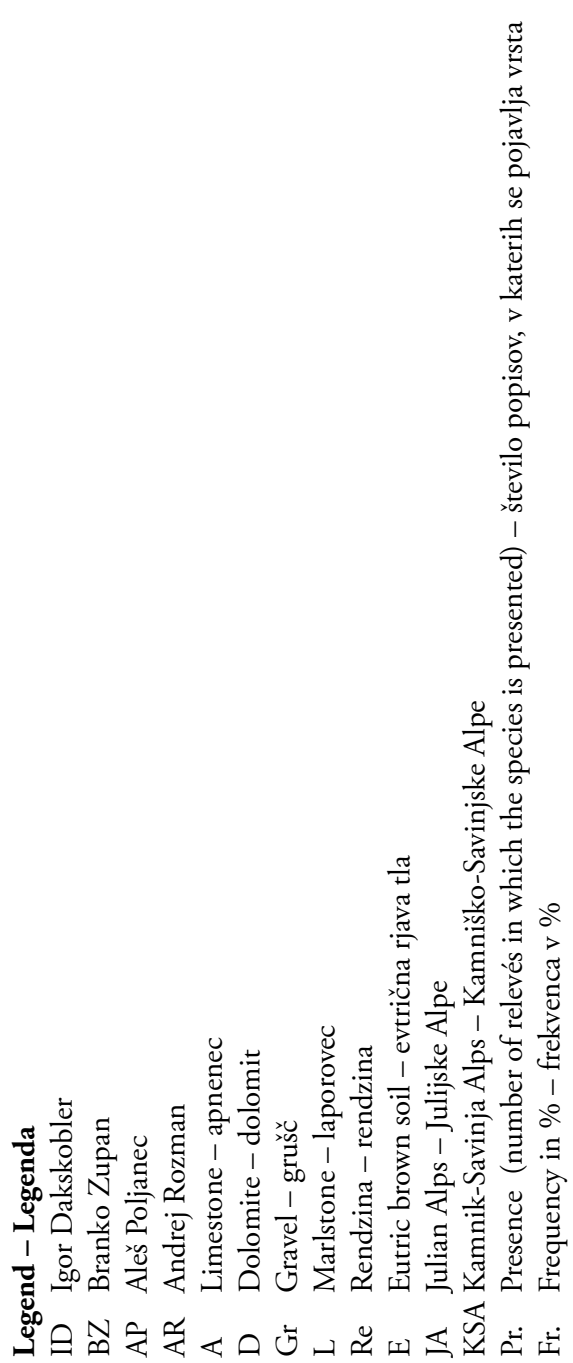




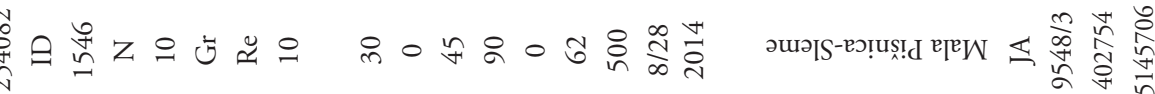

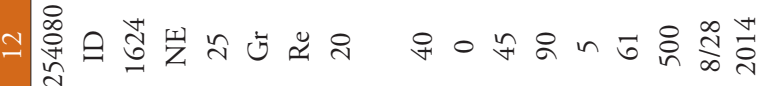

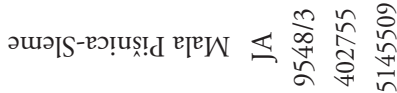

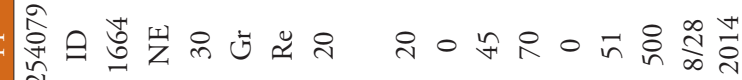

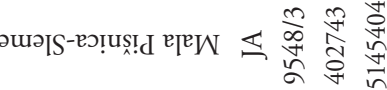

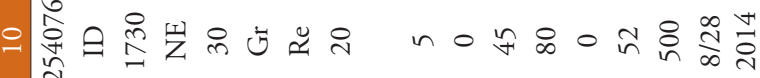

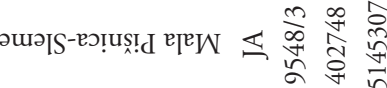

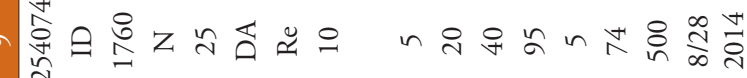

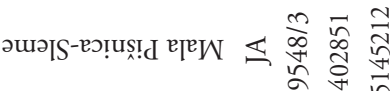

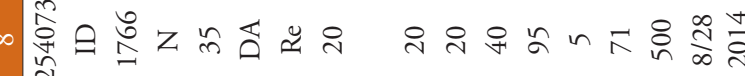

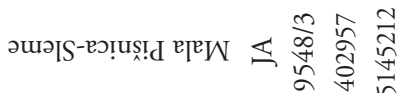

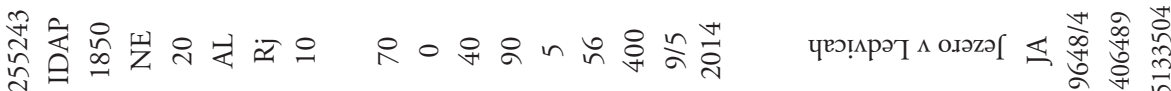

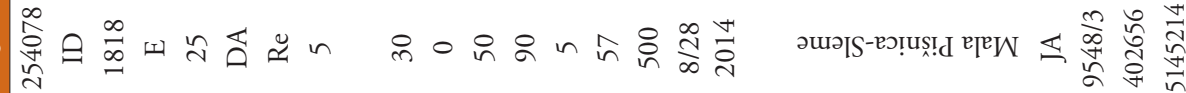
速 8 \& तुำ

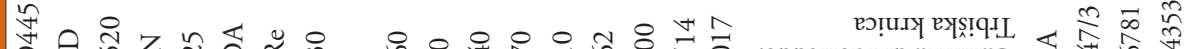

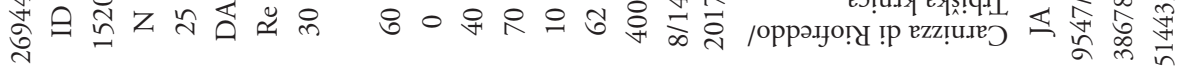

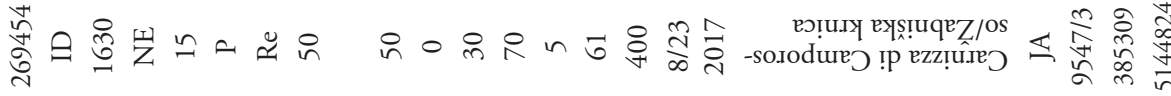
疋央 荇

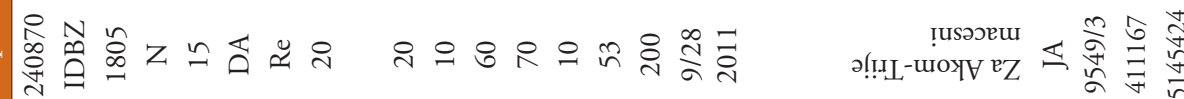

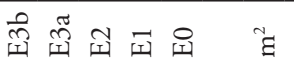

$\Xi \leftleftarrows$

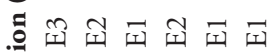
要

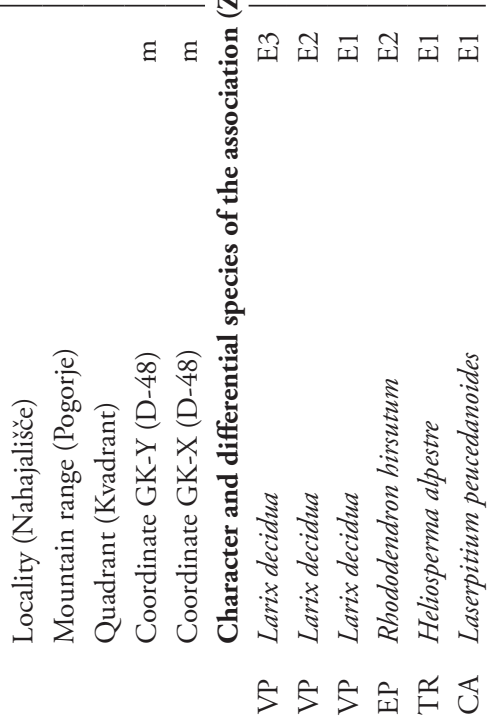




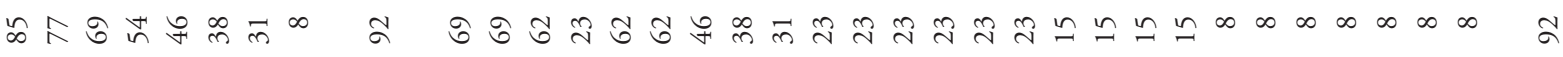

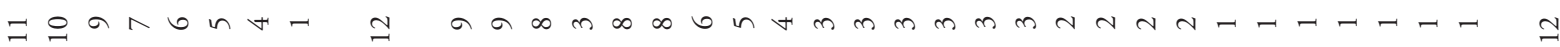
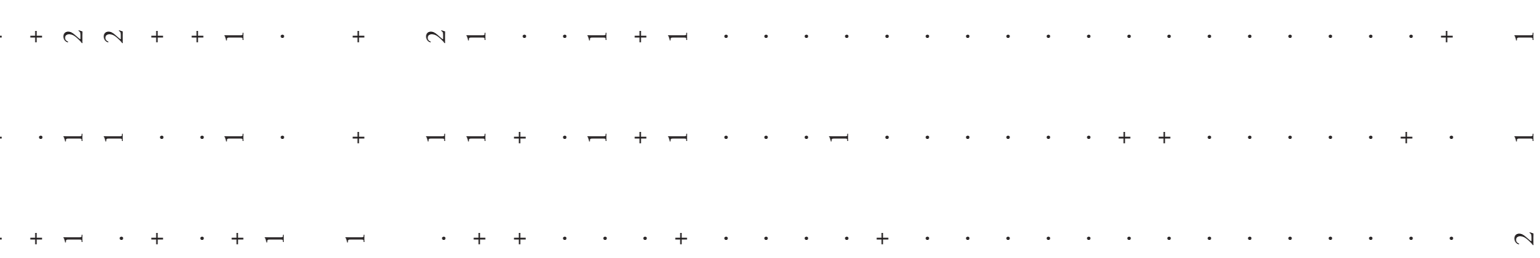

$\cdot-$
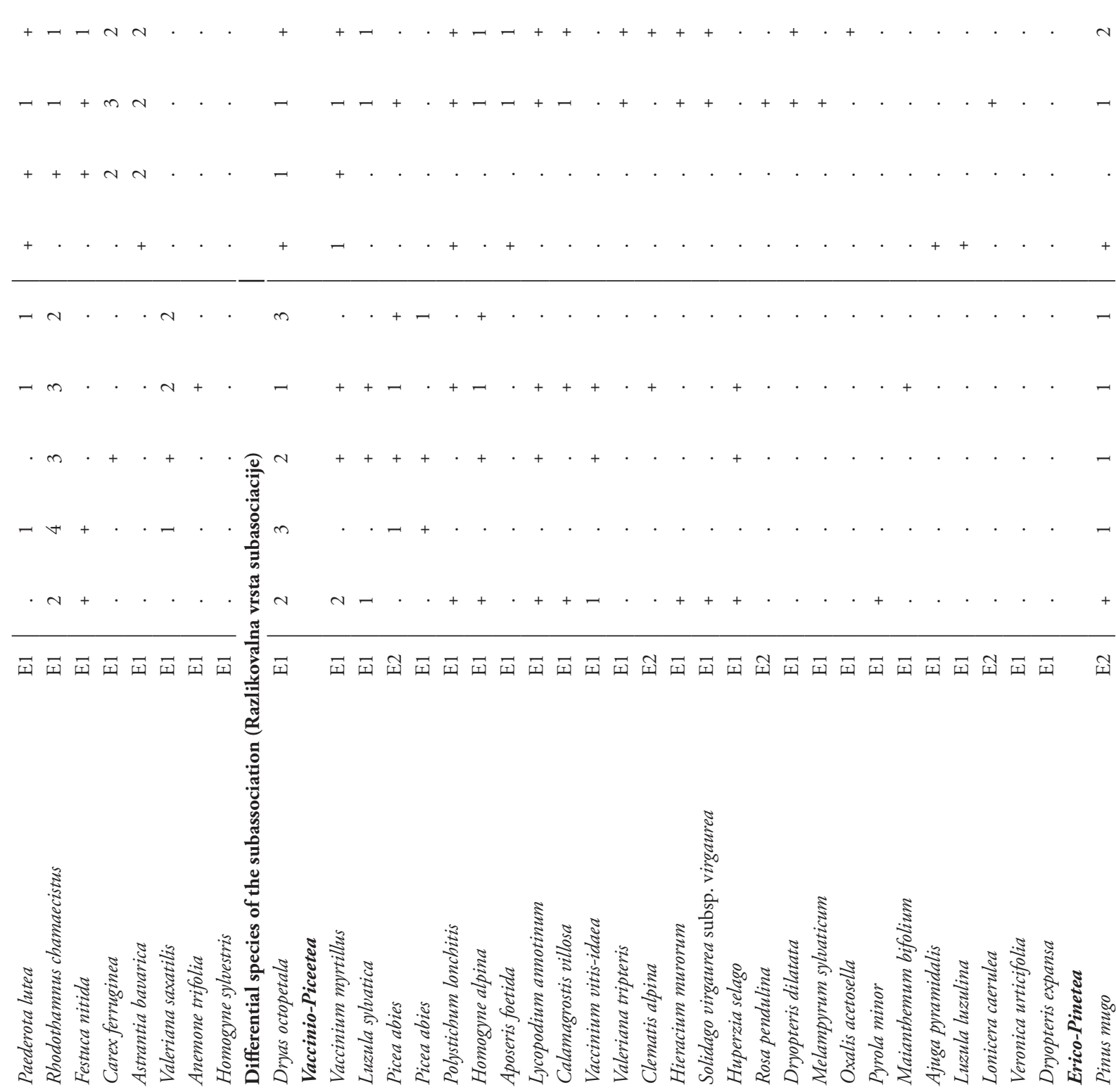

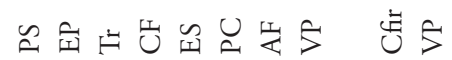




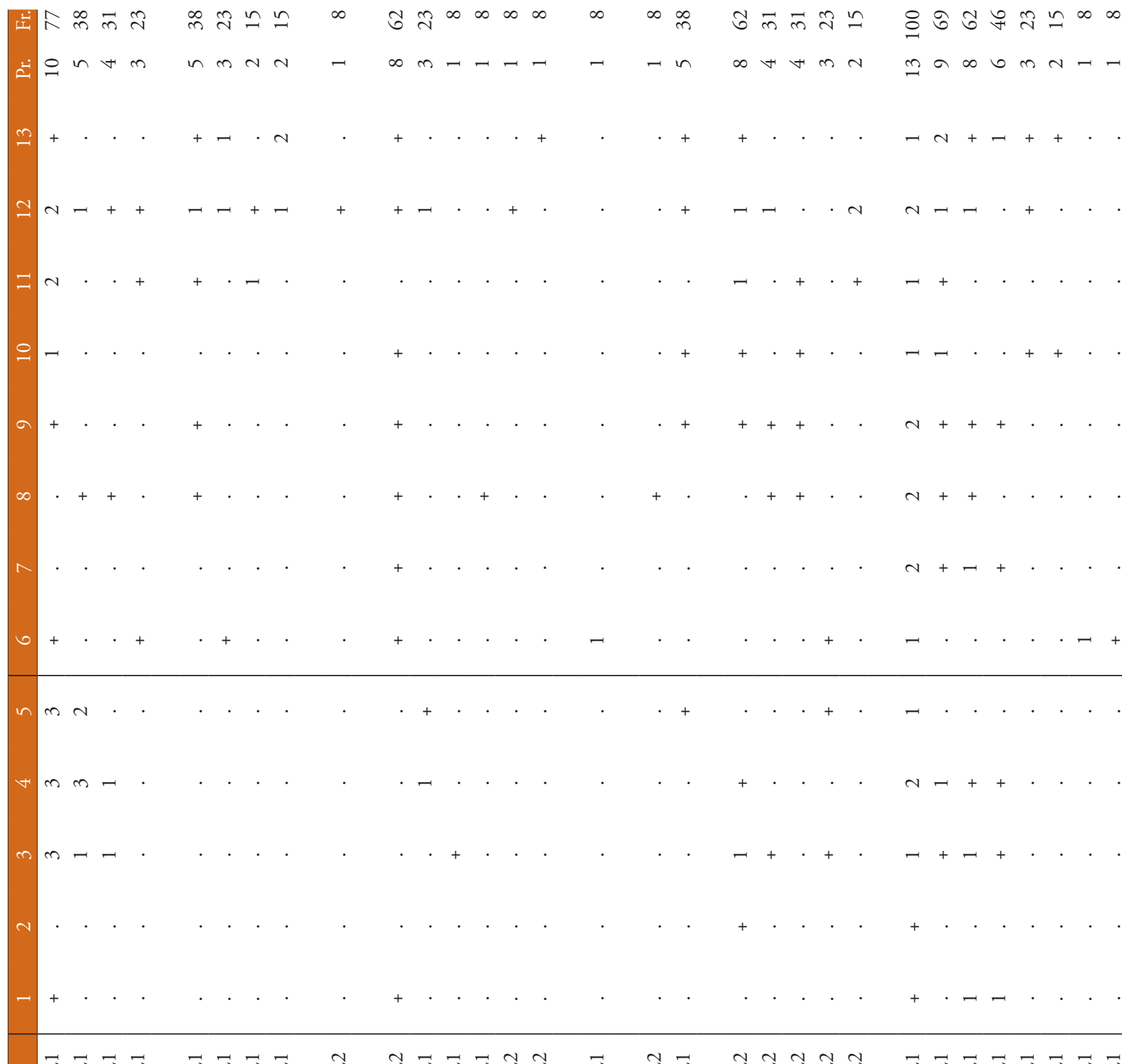

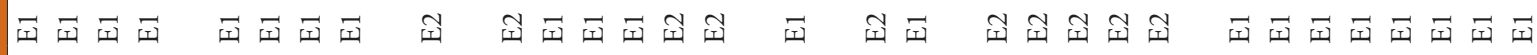

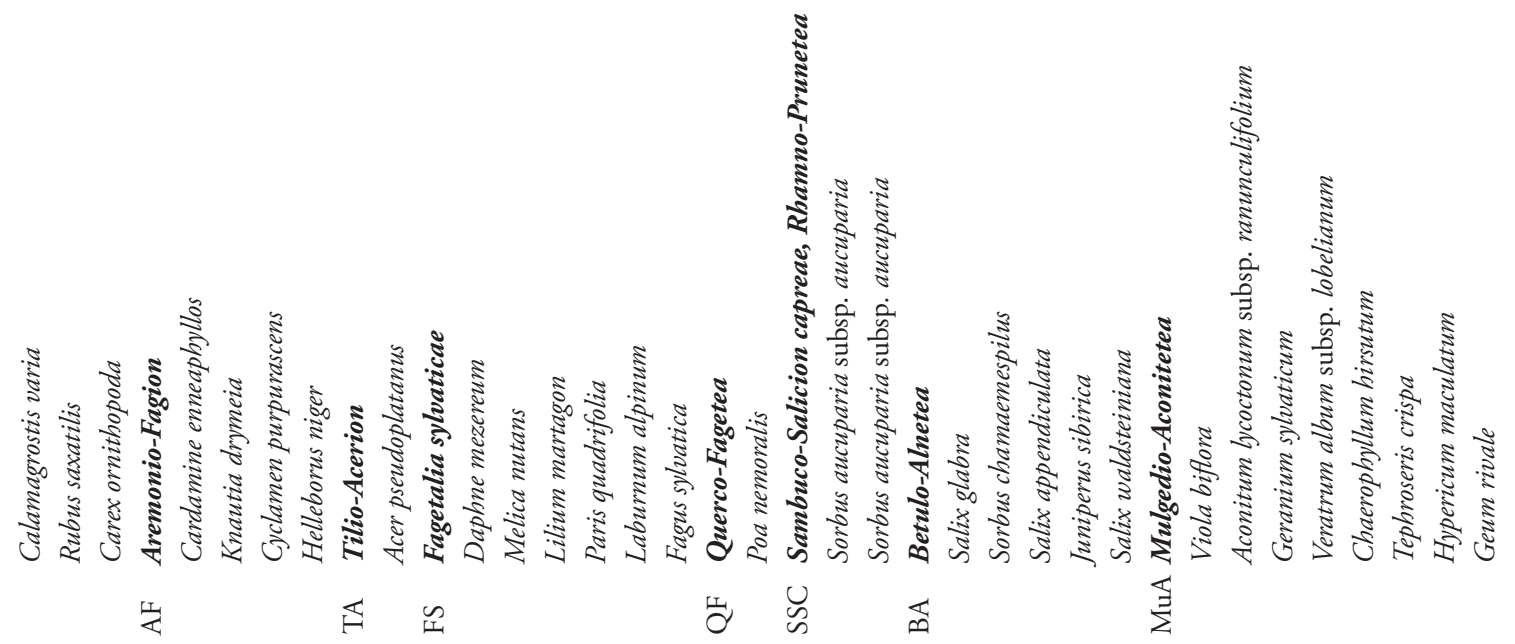




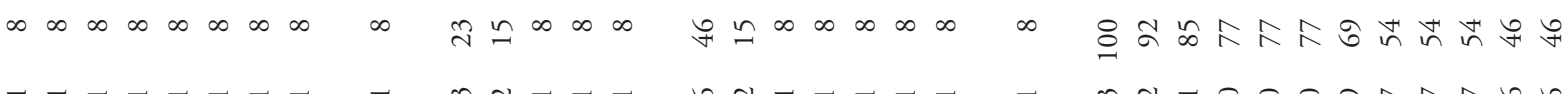

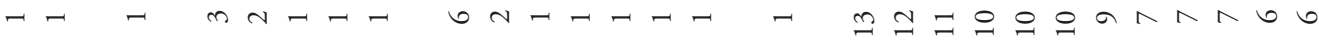

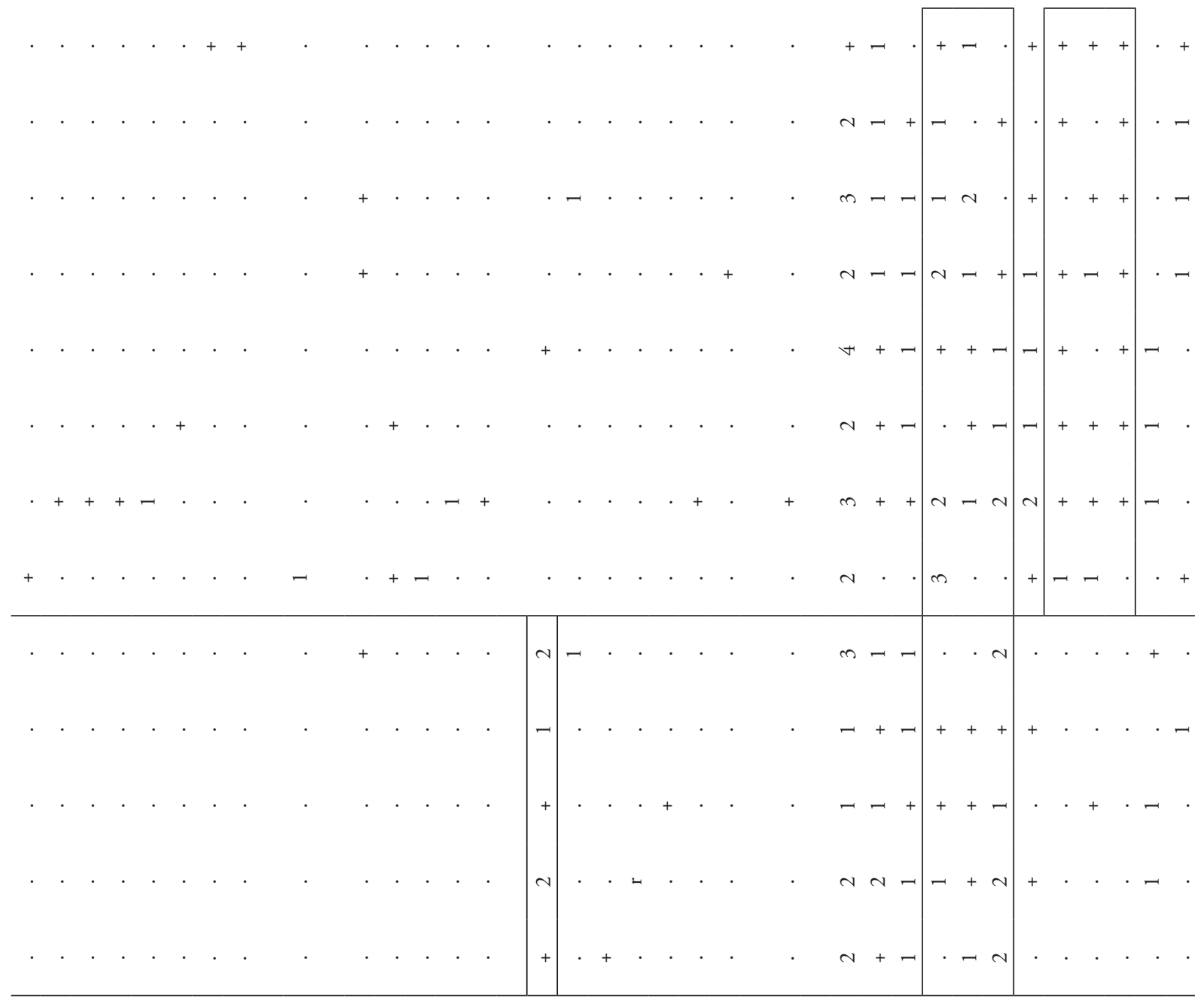

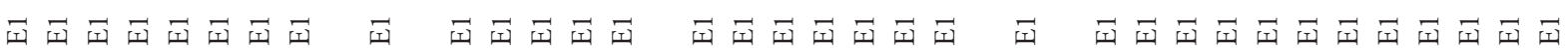

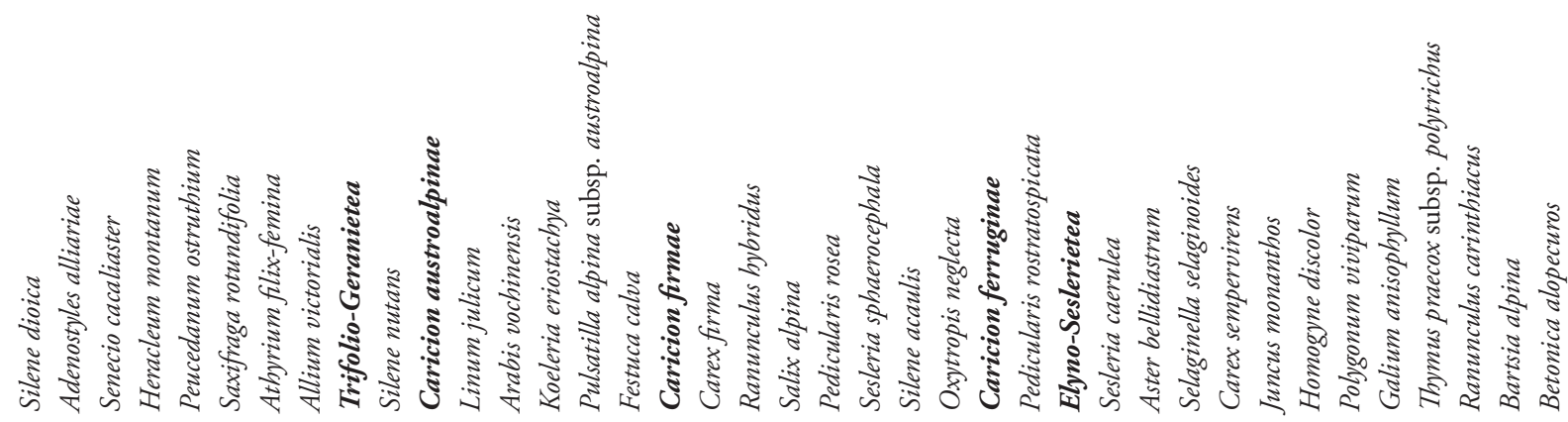
ษ
岂
岂嵒 

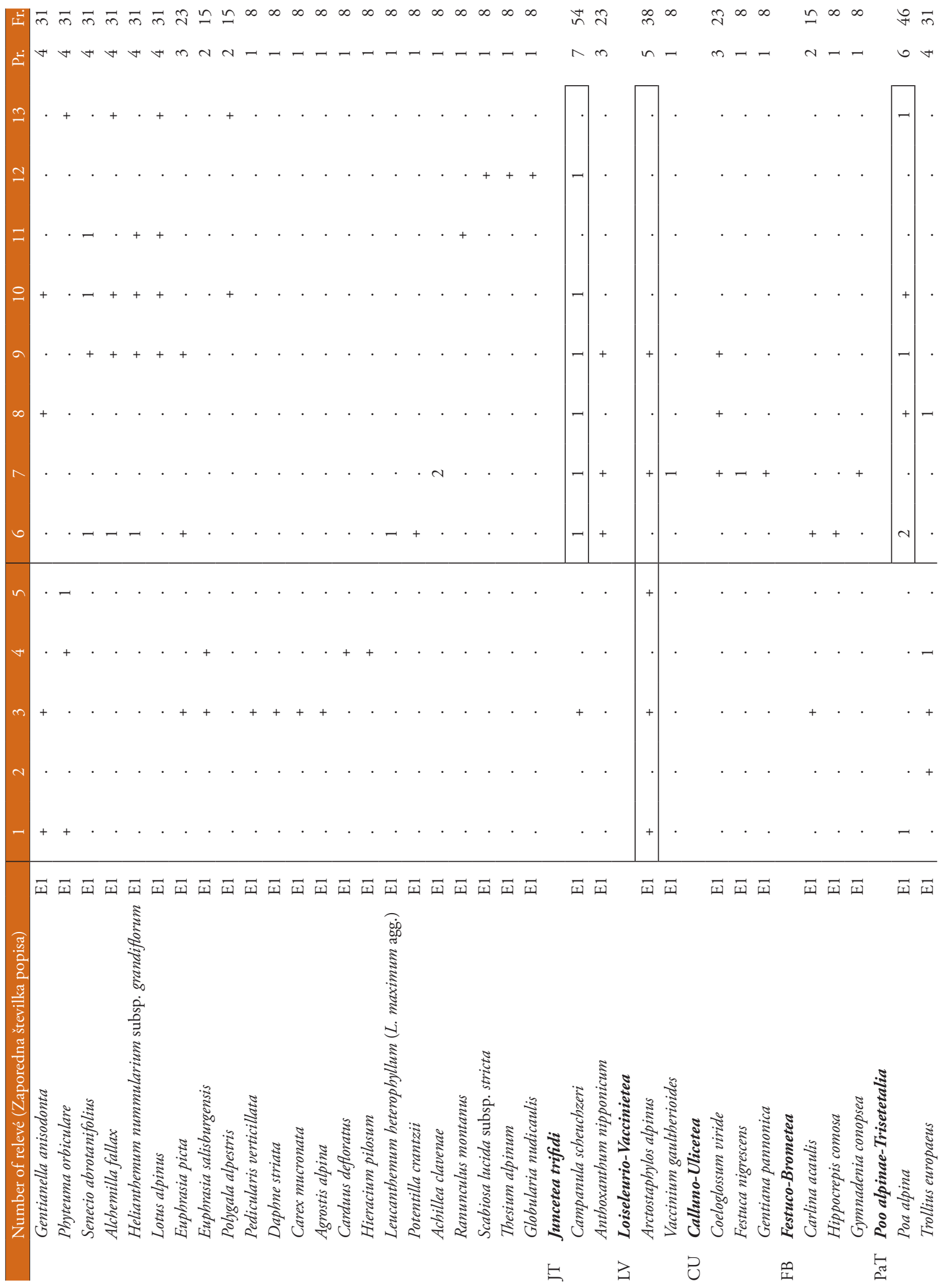


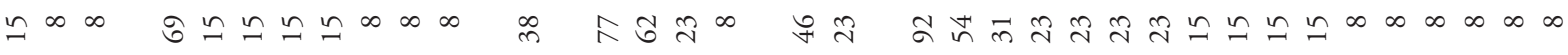

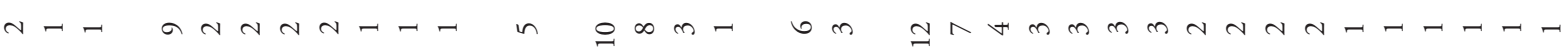

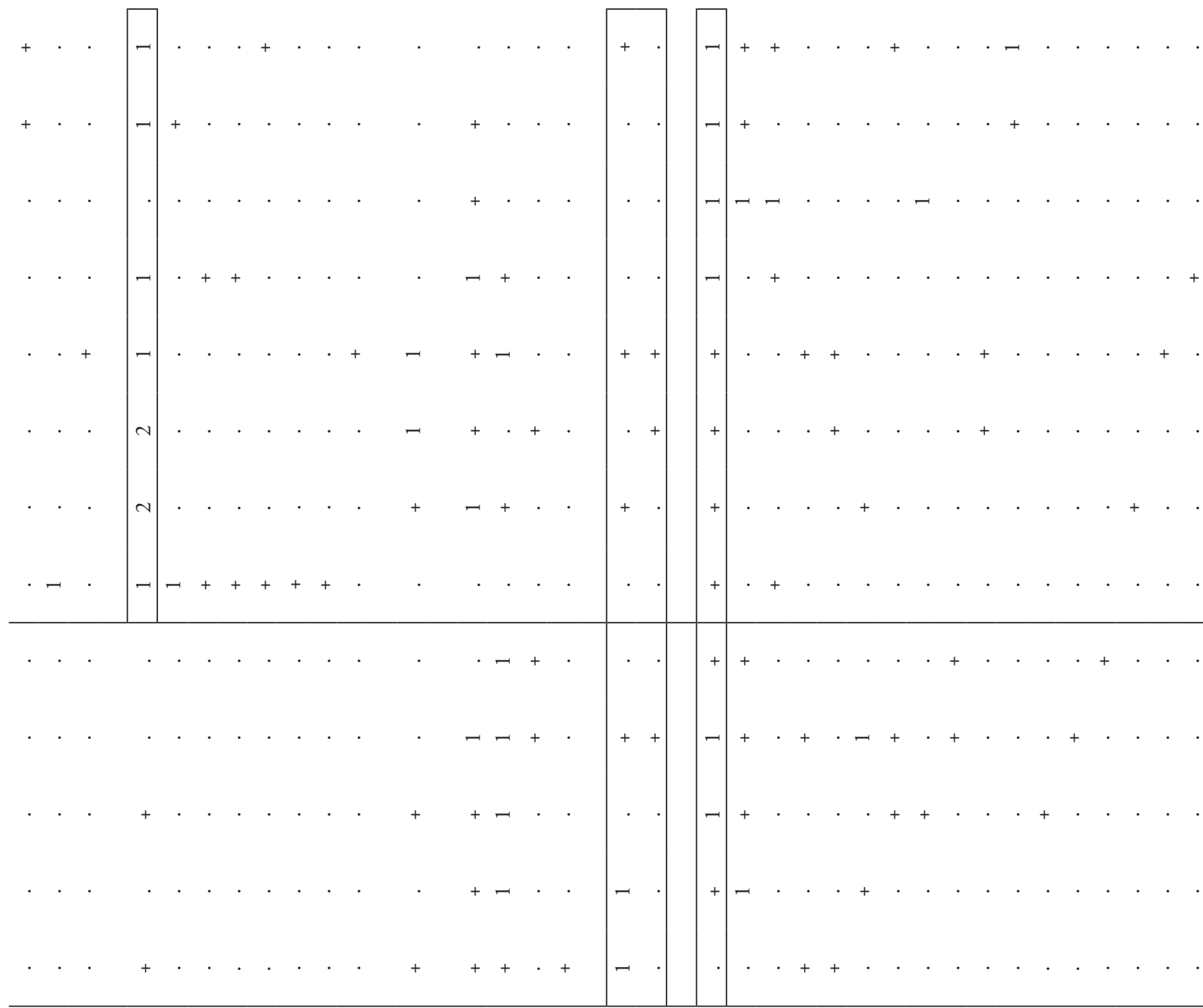

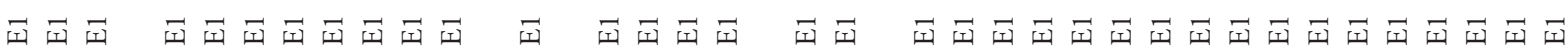

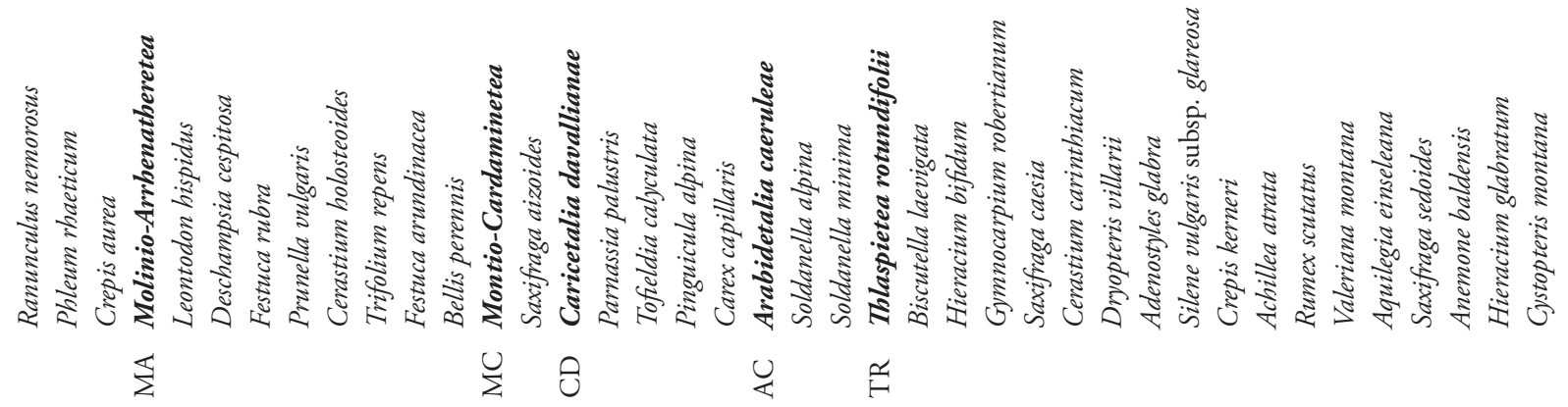



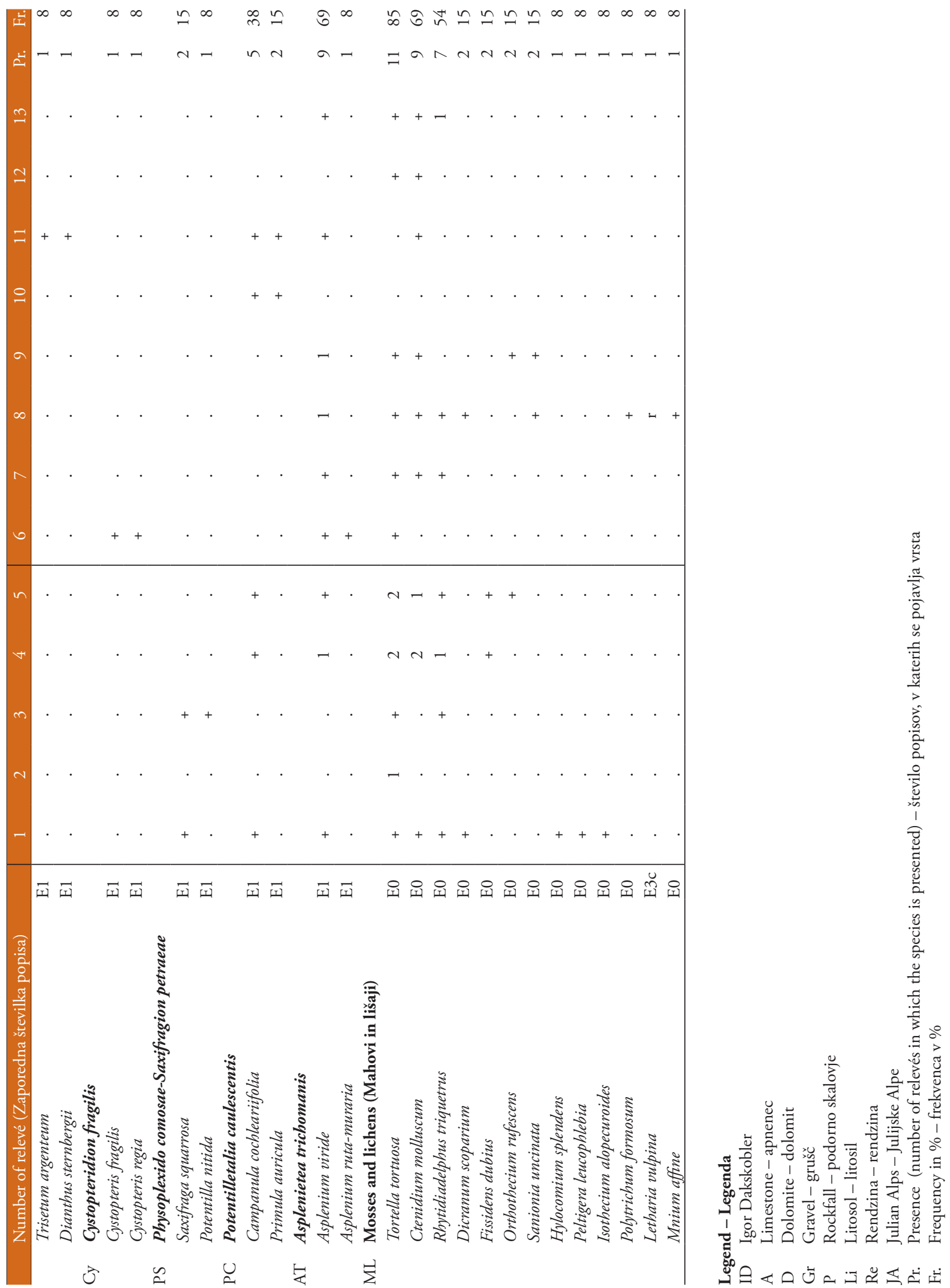
Table 21 (Tabela 21): Rhodothamno-Laricetum dryadetosum octoptelae var. Juniperus sibirica

\begin{tabular}{|c|c|c|c|c|c|c|c|c|c|c|c|c|c|}
\hline & Number of relevé (Zaporedna številka popisa) & & 1 & 2 & 3 & 4 & 5 & 6 & 7 & 8 & 9 & Pr. & Fr. \\
\hline & $\begin{array}{l}\text { Database number of relevé } \\
\text { (Delovna številka popisa) }\end{array}$ & & 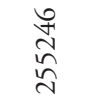 & 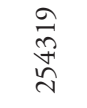 & \begin{tabular}{l}
$\stackrel{\Downarrow}{\sim}$ \\
\multirow{n}{n}{} \\
$\stackrel{n}{v}$
\end{tabular} & 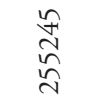 & 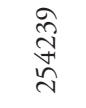 & $\begin{array}{l}\underset{7}{7} \\
\stackrel{+}{\checkmark}\end{array}$ & 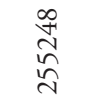 & 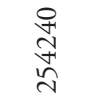 & 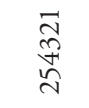 & & \\
\hline & Author of the relevé (Avtor popisa) & & IDAP & IDAP & IDAP & IDAP & IDAP & IDAP & IDAP & IDAP & IDAP & & \\
\hline & Elevation in $\mathrm{m}$ (Nadmorska višina $\mathrm{v} \mathrm{m})$ & & 1760 & 1860 & 1771 & 1760 & 1905 & 1870 & 1760 & 1875 & 1780 & & \\
\hline & Aspect (Lega) & & NEE & SW & NW & SSE & SW & 0 & SE & W & E & & \\
\hline & Slope in degrees (Nagib v stopinjah) & & 15 & 5 & 25 & 10 & 5 & 5 & 15 & 10 & 22 & & \\
\hline & Parent material (Matična podlaga) & & A & A & $\mathrm{AL}$ & A & $\mathrm{AL}$ & A & A & A & A & & \\
\hline & Soil (Tla) & & $\operatorname{Re}$ & $\operatorname{Re}$ & $\operatorname{Re}$ & $\operatorname{Re}$ & $\operatorname{Re}$ & $\operatorname{Re}$ & $\operatorname{Re}$ & $\operatorname{Re}$ & $\operatorname{Re}$ & & \\
\hline & Stoniness in \% (Kamnitost v \%) & & 30 & 30 & 20 & 30 & 30 & 60 & 40 & 30 & 20 & & \\
\hline & \multicolumn{13}{|l|}{ Cover in \% (Zastiranje v \%): } \\
\hline & Upper tree layer (Zgornja drevesna plast) & $\mathrm{E} 3 \mathrm{~b}$ & 40 & 20 & 40 & 50 & 50 & 30 & 40 & 60 & 40 & & \\
\hline & Lower tree layer (Spodnja drevesna plasti) & E3a & 5 & 30 & 10 & 10 & 5 & 30 & 5 & 30 & 10 & & \\
\hline & Shrub layer (Grmovna plast) & E2 & 30 & 60 & 30 & 30 & 35 & 35 & 45 & 35 & 55 & & \\
\hline & Herb layer (Zeliščna plast) & E1 & 70 & 70 & 90 & 70 & 80 & 60 & 60 & 80 & 90 & & \\
\hline & Moss layer (Mahovna plast) & E0 & 5 & 5 & 5 & 5 & 5 & 10 & 5 & 10 & 5 & & \\
\hline & Number of species (Število vrst) & & 58 & 56 & 52 & 66 & 82 & 70 & 51 & 73 & 71 & & \\
\hline & Relevé area (Velikost popisne ploskve) & $\mathrm{m}^{2}$ & 400 & 400 & 400 & 400 & 400 & 400 & 400 & 400 & 400 & & \\
\hline & Date of taking relevé (Datum popisa) & & $\begin{array}{l}9 / 6 \\
2014\end{array}$ & $\begin{array}{c}9 / 5 \\
2014\end{array}$ & $\begin{array}{c}9 / 6 \\
2014\end{array}$ & $\begin{array}{c}9 / 6 \\
2014\end{array}$ & $\begin{array}{c}9 / 5 \\
2014\end{array}$ & $\begin{array}{c}9 / 5 \\
2014\end{array}$ & $\begin{array}{c}9 / 6 \\
2014\end{array}$ & $\begin{array}{c}9 / 5 \\
2014\end{array}$ & $\begin{array}{c}9 / 6 \\
2014\end{array}$ & & \\
\hline & Locality (Nahajališče) & & 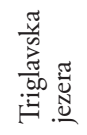 & 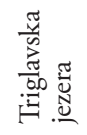 & 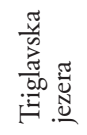 & 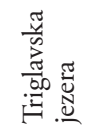 & 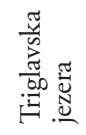 & 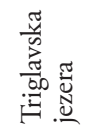 & 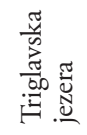 & 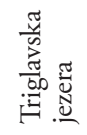 & 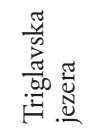 & & \\
\hline & Mountain range (Pogorje) & & JA & JA & JA & JA & JA & JA & JA & JA & JA & & \\
\hline & Quadrant (Kvadrant) & & $\begin{array}{c}9648 \\
14\end{array}$ & $\begin{array}{c}9648 \\
14\end{array}$ & $\begin{array}{c}9648 \\
14\end{array}$ & $\begin{array}{c}9648 \\
14\end{array}$ & $\begin{array}{c}9648 \\
14\end{array}$ & $\begin{array}{c}9648 \\
14\end{array}$ & $\begin{array}{c}9648 \\
14\end{array}$ & $\begin{array}{c}9648 \\
14\end{array}$ & $\begin{array}{c}9648 \\
14\end{array}$ & & \\
\hline & Coordinate GK-Y (D-48) & $\mathrm{m}$ & 节 & $\begin{array}{l}\text { ᄋ } \\
\text { స్ర } \\
\stackrel{+}{+}\end{array}$ & \begin{tabular}{l}
0 \\
\multirow{2}{0}{} \\
$\stackrel{+}{1}$
\end{tabular} & 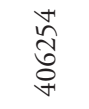 & 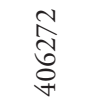 & 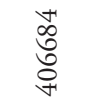 & 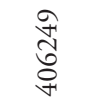 & 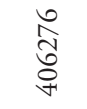 & 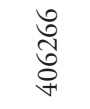 & & \\
\hline & Coordinate GK-X (D-48) & $\mathrm{m}$ & 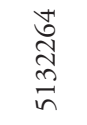 & 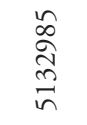 & $\begin{array}{l}\vec{a} \\
\underset{\sim}{n} \\
\tilde{n}\end{array}$ & $\begin{array}{l}\tilde{n} \\
\approx \\
\tilde{n} \\
\text { हn }\end{array}$ & $\begin{array}{l}\tilde{n} \\
\hat{n} \\
\tilde{n} \\
\tilde{n}\end{array}$ & $\begin{array}{l}\infty \\
\infty \\
\infty \\
m \\
\stackrel{n}{n}\end{array}$ & $\begin{array}{l}8 \\
\stackrel{0}{2} \\
m \\
i n\end{array}$ & $\begin{array}{l}\stackrel{\sim}{\sim} \\
\underset{n}{n}\end{array}$ & $\begin{array}{l}\stackrel{2}{d} \\
\underset{\hbar}{n}\end{array}$ & & \\
\hline & \multicolumn{11}{|c|}{ Character and differential species of the association (Značilnice in razlikovalnice asociacije) } & Pr. & Fr. \\
\hline VP & Larix decidua & E3 & 2 & 3 & 3 & 3 & 3 & 4 & 3 & 5 & 3 & 9 & 100 \\
\hline VP & Larix decidua & E2 & 1 & 1 & 1 & 1 & 1 & 1 & 1 & 1 & 2 & 9 & 100 \\
\hline VP & Larix decidua & E1 & . & . & + & . & . & . & . & . & . & 1 & 11 \\
\hline $\mathrm{EP}$ & Rhododendron hirsutum & E2 & 4 & 4 & 4 & 3 & 4 & 4 & 3 & 4 & 4 & 9 & 100 \\
\hline $\mathrm{EP}$ & Rhodothamnus chamaecistus & E1 & 1 & 2 & 2 & 1 & + & 1 & 1 & 1 & 1 & 9 & 100 \\
\hline CA & Laserpitium peucedanoides & E1 & 2 & 1 & 2 & 1 & 1 & 1 & 1 & 1 & 1 & 9 & 100 \\
\hline PS & Paederota lutea & E1 & + & 1 & + & + & + & + & + & 1 & + & 9 & 100 \\
\hline ES & Astrantia bavarica & E1 & 2 & 2 & 2 & . & 1 & + & 1 & 1 & 2 & 8 & 89 \\
\hline TR & Festuca nitida & E1 & 1 & + & . & 1 & + & . & 1 & + & 1 & 7 & 78 \\
\hline TR & Heliosperma alpestre & E1 & . & + & + & . & . & + & 1 & + & + & 6 & 67 \\
\hline $\mathrm{CF}$ & Carex ferruginea & E1 & + & . & . & 1 & . & 1 & . & 1 & 1 & 5 & 56 \\
\hline PC & Valeriana saxatilis & E1 & . & . & + & . & . & . & . & . & . & 1 & 11 \\
\hline \multicolumn{14}{|c|}{ Differential species of the subassociation (Razlikovalna vrsta subasociacije) } \\
\hline Cfir & Dryas octopetala & E1 & 1 & + & 1 & . & . & + & + & + & . & 6 & 67 \\
\hline \multicolumn{14}{|c|}{ Differential species of the variant (Razlikovalna vrsta variante) } \\
\hline BA & Juniperus sibirica & E2 & 1 & 1 & 2 & 2 & + & + & 2 & 1 & 1 & 9 & 100 \\
\hline VP & Vaccinio-Piceetea & & & & & & & & & & & & \\
\hline
\end{tabular}




\begin{tabular}{|c|c|c|c|c|c|c|c|c|c|c|c|c|c|}
\hline & Number of relevé (Zaporedna številka popisa) & & 1 & 2 & 3 & 4 & 5 & 6 & 7 & 8 & 9 & Pr. & Fr. \\
\hline & Vaccinium myrtillus & E1 & + & . & + & + & 3 & 1 & 1 & 1 & 1 & 8 & 89 \\
\hline & Polystichum lonchitis & E1 & + & + & . & + & 1 & 1 & + & + & + & 8 & 89 \\
\hline & Homogyne alpina & E1 & . & + & + & + & + & 1 & + & + & 1 & 8 & 89 \\
\hline & Clematis alpina & E2 & . & + & + & + & 1 & 1 & 1 & . & + & 7 & 78 \\
\hline & Solidago virgaurea subsp. virgaurea & E1 & 1 & . & . & 1 & 1 & + & . & 1 & 1 & 6 & 67 \\
\hline & Vaccinium vitis-idaea & E1 & + & . & . & 1 & 1 & 1 & . & + & 1 & 6 & 67 \\
\hline & Rosa pendulina & $\mathrm{E} 2$ & . & 1 & . & 1 & 1 & . & 1 & + & . & 5 & 56 \\
\hline & Valeriana tripteris & E1 & . & . & . & + & + & . & 1 & + & + & 5 & 56 \\
\hline & Calamagrostis villosa & E1 & . & . & . & + & . & + & 1 & + & + & 5 & 56 \\
\hline & Lonicera caerulea & E2 & + & . & . & + & 1 & 1 & . & . & . & 4 & 44 \\
\hline & Aposeris foetida & E1 & . & + & . & . & . & . & + & + & 1 & 4 & 44 \\
\hline & Lycopodium annotinum & E1 & . & . & + & . & . & . & + & + & + & 4 & 44 \\
\hline & Luzula sylvatica & E1 & . & . & . & . & + & 2 & . & + & 1 & 4 & 44 \\
\hline & Hieracium murorum & E1 & . & . & + & . & . & . & . & + & + & 3 & 33 \\
\hline & Huperzia selago & E1 & . & . & + & . & . & . & + & . & . & 2 & 22 \\
\hline & Dryopteris dilatata & E1 & . & . & . & . & . & . & . & + & + & 2 & 22 \\
\hline & Picea abies & E3 & . & . & . & . & + & . & . & . & . & 1 & 11 \\
\hline & Picea abies & E2 & . & . & . & . & + & . & . & . & . & 1 & 11 \\
\hline & Picea abies & E1 & . & . & + & . & . & . & . & . & . & 1 & 11 \\
\hline & Dryopteris expansa & E1 & . & . & . & + & . & . & . & . & . & 1 & 11 \\
\hline & Abies alba & $\mathrm{E} 2$ & . & . & . & + & . & . & . & . & . & 1 & 11 \\
\hline & Luzula luzulina & E1 & . & . & . & + & . & . & . & . & . & 1 & 11 \\
\hline & Melampyrum sylvaticum & E1 & . & . & . & . & + & . & . & . & . & 1 & 11 \\
\hline & Maianthemum bifolium & E1 & . & . & . & . & + & . & . & . & . & 1 & 11 \\
\hline & Gentiana asclepiadea & E1 & . & . & . & . & + & . & . & . & . & 1 & 11 \\
\hline \multirow[t]{6}{*}{ EP } & Erico-Pinetea & & & & & & & & & & & & \\
\hline & Pinus mugo & E2 & . & 3 & 2 & + & 2 & 2 & 3 & 2 & 4 & 8 & 89 \\
\hline & Erica carnea & E1 & . & 4 & 2 & + & 2 & . & 2 & . & 1 & 6 & 67 \\
\hline & Rubus saxatilis & E1 & 1 & + & . & 1 & + & . & 2 & . & 1 & 6 & 67 \\
\hline & Calamagrostis varia & E1 & . & . & . & + & . & . & . & + & . & 2 & 22 \\
\hline & Molinia arundinacea & E1 & . & + & . & . & . & . & . & . & . & 1 & 11 \\
\hline \multirow[t]{2}{*}{ TA } & Tilio-Acerion & & & & & & & & & & & & \\
\hline & Acer pseudoplatanus & E2 & + & . & . & + & . & . & . & . & . & 2 & 22 \\
\hline \multirow[t]{6}{*}{ FS } & Fagetalia sylvaticae & & & & & & & & & & & & \\
\hline & Daphne mezereum & E2 & + & . & + & . & . & 1 & + & + & + & 6 & 67 \\
\hline & Lilium martagon & E1 & . & . & . & + & . & . & . & 1 & . & 2 & 22 \\
\hline & Dryopteris filix-mas & E1 & . & . & . & . & + & . & . & . & + & 2 & 22 \\
\hline & Melica nutans & E1 & . & . & . & . & . & . & 1 & . & 1 & 2 & 22 \\
\hline & Galeobdolon flavidum & E1 & . & . & . & . & . & . & . & . & + & 1 & 11 \\
\hline \multirow[t]{3}{*}{ QF } & Querco-Fagetea & & & & & & & & & & & & \\
\hline & Hieracium lachenalii & E1 & . & . & . & . & + & . & . & . & + & 2 & 22 \\
\hline & Poa nemoralis & E1 & . & . & . & . & + & . & . & . & . & 1 & 11 \\
\hline \multirow[t]{2}{*}{ SSC } & Sambuco-Salicion capreae, Rhamno-Prunetea & & & & & & & & & & & & \\
\hline & Sorbus aucuparia subsp. aucuparia & E2 & . & . & . & + & + & . & . & . & . & 2 & 22 \\
\hline \multirow[t]{5}{*}{ BA } & Betulo-Alnetea & & & & & & & & & & & & \\
\hline & Sorbus chamaemespilus & E2 & + & . & + & + & + & . & 1 & 1 & 2 & 7 & 78 \\
\hline & Salix appendiculata & E2 & + & . & . & + & + & . & + & . & + & 5 & 56 \\
\hline & Salix waldsteiniana & E2 & . & . & . & 1 & . & . & . & . & + & 2 & 22 \\
\hline & Salix glabra & E2 & . & . & . & . & + & . & + & . & . & 2 & 22 \\
\hline \multicolumn{14}{|c|}{ MuA Mulgedio-Aconitetea } \\
\hline & Veratrum album subsp. lobelianum & E1 & 1 & 1 & + & + & + & 1 & 1 & 1 & 1 & 9 & 100 \\
\hline & Viola biflora & E1 & + & . & 1 & 1 & + & 2 & 1 & 1 & 2 & 8 & 89 \\
\hline
\end{tabular}




\begin{tabular}{|c|c|c|c|c|c|c|c|c|c|c|c|c|c|}
\hline & \multicolumn{2}{|l|}{ Number of relevé (Zaporedna številka popisa) } & \multirow{2}{*}{$\begin{array}{l}1 \\
1\end{array}$} & \multirow{2}{*}{$\frac{2}{1}$} & \multirow{2}{*}{$\begin{array}{l}3 \\
.\end{array}$} & \multirow{2}{*}{$\frac{4}{1}$} & \multirow{2}{*}{$\frac{5}{+}$} & \multirow{2}{*}{$\frac{6}{+}$} & \multirow{2}{*}{7} & \multirow{2}{*}{$\begin{array}{l}8 \\
1\end{array}$} & \multirow{2}{*}{$\frac{9}{1}$} & \multirow{2}{*}{$\frac{\text { Pr. }}{7}$} & \multirow{2}{*}{$\begin{array}{l}\text { Fr. } \\
78\end{array}$} \\
\hline & Aconitum lycoctonum subsp. ranunculifolium & E1 & & & & & & & & & & & \\
\hline & Adenostyles alliariae & E1 & + & . & . & 2 & + & 2 & + & 2 & 2 & 7 & 78 \\
\hline & Senecio cacaliaster & E1 & + & . & + & 1 & + & + & + & . & + & 7 & 78 \\
\hline & Peucedanum ostruthium & E1 & 1 & . & . & 1 & 2 & 2 & . & 2 & 2 & 6 & 67 \\
\hline & Geranium sylvaticum & E1 & . & . & . & 1 & 1 & 2 & 1 & 1 & + & 6 & 67 \\
\hline & Polygonatum verticillatum & E1 & . & + & . & + & + & . & + & . & . & 4 & 44 \\
\hline & Saxifraga rotundifolia & E1 & . & . & . & . & + & . & . & + & . & 2 & 22 \\
\hline & Alchemilla xanthochlora & E1 & + & . & . & . & . & . & . & . & + & 2 & 22 \\
\hline & Carduus carduelis & E1 & . & 1 & . & + & . & . & . & . & . & 2 & 22 \\
\hline & Rumex arifolius & E1 & . & . & + & . & . & . & . & + & . & 2 & 22 \\
\hline & Silene vulgaris subsp. antelopum & E1 & . & . & . & . & . & + & . & + & . & 2 & 22 \\
\hline & Hieracium valdepilosum & E1 & + & . & . & . & . & . & . & . & . & 1 & 11 \\
\hline & Geum rivale & E1 & . & . & . & . & + & . & . & . & . & 1 & 11 \\
\hline & Poa hybrida & E1 & . & . & . & . & + & . & . & . & . & 1 & 11 \\
\hline & Allium victorialis & E1 & . & . & . & . & + & . & . & . & . & 1 & 11 \\
\hline & Chaerophyllum villarsii & E1 & . & . & . & . & . & . & . & 1 & . & 1 & 11 \\
\hline & Athyrium filix-femina & E1 & . & . & . & . & . & . & . & . & + & 1 & 11 \\
\hline & Hypericum maculatum & E1 & . & . & . & . & . & . & . & . & + & 1 & 11 \\
\hline \multirow[t]{2}{*}{ TG } & Trifolio-Geranietea & & & & & & & & & & & & \\
\hline & Silene nutans & E1 & . & . & . & . & . & . & . & + & . & 1 & 11 \\
\hline \multirow[t]{7}{*}{ CA } & Caricion austroalpinae & & & & & & & & & & & & \\
\hline & Pulsatilla alpina subsp. austroalpina & E1 & + & + & . & + & + & + & 1 & 1 & 1 & 8 & 89 \\
\hline & Heracleum austriacum subsp. siifolium & E1 & + & + & 1 & . & . & . & + & + & + & 6 & 67 \\
\hline & Arabis vochinensis & E1 & + & . & . & . & + & + & . & + & . & 4 & 44 \\
\hline & Festuca calva & E1 & . & . & . & . & + & + & . & . & . & 2 & 22 \\
\hline & Koeleria eriostachya & E1 & . & . & . & . & + & + & . & . & . & 2 & 22 \\
\hline & Linum julicum & E1 & . & . & + & . & . & . & . & . & . & 1 & 11 \\
\hline \multirow[t]{3}{*}{ Cfir } & Caricion firmae & & & & & & & & & & & & \\
\hline & Helianthemum alpestre & E1 & . & . & + & . & . & . & . & . & . & 1 & 11 \\
\hline & Ranunculus hybridus & E1 & . & . & + & . & . & . & . & . & . & 1 & 11 \\
\hline \multirow[t]{4}{*}{ CF } & Caricion ferrugineae & & & & & & & & & & & & \\
\hline & Pedicularis rostratospicata & E1 & . & . & + & . & . & . & . & . & . & 1 & 11 \\
\hline & Allium schoenoprasum subsp. alpinum & E1 & . & . & . & . & . & + & . & . & . & 1 & 11 \\
\hline & Knautia longifolia & E1 & . & . & . & . & . & . & . & 1 & . & 1 & 11 \\
\hline ES & Elyno-Seslerietea & & & & & & & & & & & & \\
\hline & Polygonum viviparum & E1 & 1 & + & 1 & + & + & 1 & + & 1 & 1 & 9 & 100 \\
\hline & Helianthemum nummularium subsp. grandiflorum & E1 & 2 & 1 & 2 & + & + & + & 1 & 1 & 1 & 9 & 100 \\
\hline & Thymus praecox subsp. polytrichus & E1 & 1 & + & + & . & + & + & . & + & + & 7 & 78 \\
\hline & Lotus alpinus & E1 & . & + & + & + & + & . & + & + & + & 7 & 78 \\
\hline & Hieracium villosum & E1 & + & + & . & + & + & 1 & . & + & . & 6 & 67 \\
\hline & Leucanthemum heterophyllum (L.maximum agg.) & E1 & + & 1 & + & . & . & . & + & . & + & 5 & 56 \\
\hline & Aster bellidiastrum & E1 & + & . & + & 1 & . & 1 & + & . & . & 5 & 56 \\
\hline & Sesleria caerulea & E1 & . & 1 & 2 & + & 2 & 2 & . & . & . & 5 & 56 \\
\hline & Galium anisophyllum & E1 & . & + & 1 & . & . & + & . & + & + & 5 & 56 \\
\hline & Bartsia alpina & E1 & . & . & + & + & . & + & . & + & + & 5 & 56 \\
\hline & Betonica alopecuros & E1 & 1 & + & . & . & + & . & . & . & 1 & 4 & 44 \\
\hline & Phyteuma orbiculare & E1 & + & . & + & . & . & . & . & + & + & 4 & 44 \\
\hline & Homogyne discolor & E1 & + & . & + & . & . & + & . & + & . & 4 & 44 \\
\hline & Scabiosa lucida subsp. stricta & E1 & . & + & . & . & + & . & . & + & 1 & 4 & 44 \\
\hline & Myosotis alpestris & E1 & . & + & . & . & + & + & . & 1 & . & 4 & 44 \\
\hline & Achillea clavenae & E1 & 1 & + & . & . & + & . & . & . & . & 3 & 33 \\
\hline & Selaginella selaginoides & E1 & + & . & . & . & . & . & + & + & . & 3 & 33 \\
\hline
\end{tabular}




\begin{tabular}{|c|c|c|c|c|c|c|c|c|c|c|c|c|c|}
\hline & Number of relevé (Zaporedna številka popisa) & & 1 & 2 & 3 & 4 & 5 & 6 & 7 & 8 & 9 & Pr. & Fr. \\
\hline & Senecio abrotanifolius & E1 & . & + & . & . & . & . & . & + & + & 3 & 33 \\
\hline & Anthyllis vulneraria subsp. alpestris & E1 & . & + & + & . & . & . & . & . & . & 2 & 22 \\
\hline & Hieracium pilosum & E1 & + & . & . & . & . & . & . & . & . & 1 & 11 \\
\hline & Carduus crassifolius & E1 & . & 1 & . & . & . & . & . & . & . & 1 & 11 \\
\hline & Helictotrichon parlatorei & $\mathrm{E} 1$ & . & 1 & . & . & . & . & . & . & . & 1 & 11 \\
\hline & Polygala alpestris & E1 & . & + & . & . & . & . & . & . & . & 1 & 11 \\
\hline & Juncus monanthos & E1 & . & . & + & . & . & . & . & . & . & 1 & 11 \\
\hline & Pedicularis verticillata & E1 & . & . & . & . & . & + & . & . & . & 1 & 11 \\
\hline \multirow[t]{5}{*}{ JT } & Juncetea trifidi & & & & & & & & & & & & \\
\hline & Campanula scheuchzeri & E1 & 1 & 1 & 1 & + & + & 1 & . & + & 1 & 8 & 89 \\
\hline & Anthoxanthum nipponicum & E1 & + & . & . & . & . & + & . & . & + & 3 & 33 \\
\hline & Centaurea nervosa & E1 & . & . & + & . & . & . & . & . & . & 1 & 11 \\
\hline & Euphrasia minima & E1 & . & . & . & . & + & . & . & . & . & 1 & 11 \\
\hline \multirow[t]{3}{*}{ LV } & Loiseleurio-Vaccinietea & & & & & & & & & & & & \\
\hline & Vaccinium gaultherioides & E1 & + & . & . & + & + & 1 & . & . & . & 4 & 44 \\
\hline & Empetrum hermaphroditum & E1 & . & . & . & . & . & + & . & . & . & 1 & 11 \\
\hline \multirow[t]{3}{*}{$\mathrm{CU}$} & Calluno-Ulicetea & & & & & & & & & & & & \\
\hline & Festuca nigrescens & E1 & 3 & 1 & 3 & 1 & 3 & 1 & 1 & 2 & 1 & 9 & 100 \\
\hline & Gentiana pannonica & E1 & . & . & . & . & + & + & . & 1 & . & 3 & 33 \\
\hline \multirow[t]{5}{*}{ FB } & Festuco-Brometea & & & & & & & & & & & & \\
\hline & Cirsium erisithales & E1 & . & . & . & 1 & . & . & + & . & . & 2 & 22 \\
\hline & Buphthalmum salicifolium & E1 & . & 1 & . & . & . & . & . & . & . & 1 & 11 \\
\hline & Carlina acaulis & E1 & . & + & . & . & . & . & . & . & . & 1 & 11 \\
\hline & Gymnadenia conopsea & E1 & . & + & . & . & . & . & . & . & . & 1 & 11 \\
\hline \multirow[t]{5}{*}{$\mathrm{PaT}$} & Poo alpinae-Trisetetalia & & & & & & & & & & & & \\
\hline & Poa alpina & E1 & + & . & . & 1 & 1 & 1 & + & 1 & + & 7 & 78 \\
\hline & Trollius europaeus & E1 & . & . & . & . & . & . & . & + & + & 2 & 22 \\
\hline & Crepis aurea & E1 & . & . & . & . & . & . & . & 1 & . & 1 & 11 \\
\hline & Ranunculus nemorosus & E1 & . & . & . & . & . & . & . & + & . & 1 & 11 \\
\hline \multirow[t]{5}{*}{ MA } & Molinio-Arrbenatheretea & & & & & & & & & & & & \\
\hline & Leontodon hispidus & E1 & + & . & . & + & + & + & 1 & 1 & 1 & 7 & 78 \\
\hline & Deschampsia cespitosa & E1 & . & . & . & . & + & . & . & . & . & 1 & 11 \\
\hline & Trifolium repens & E1 & . & . & . & . & + & . & . & . & . & 1 & 11 \\
\hline & Trifolium pratense & $\mathrm{E} 1$ & . & . & . & . & . & . & . & + & . & 1 & 11 \\
\hline \multirow[t]{2}{*}{$\mathrm{CD}$} & Caricetalia davallianae & & & & & & & & & & & & \\
\hline & Carex capillaris & E1 & . & . & . & + & . & . & . & . & . & 1 & 11 \\
\hline \multirow[t]{5}{*}{$\mathrm{AC}$} & Arabidetalia caeruleae & & & & & & & & & & & & \\
\hline & Soldanella alpina & E1 & . & + & + & . & + & . & . & . & . & 3 & 33 \\
\hline & Carex atrata & E1 & + & . & . & . & . & . & . & . & . & 1 & 11 \\
\hline & Carex parviflora & E1 & . & . & . & . & . & + & . & . & . & 1 & 11 \\
\hline & Gnaphalium hoppeanum & E1 & . & . & . & . & . & . & . & + & . & 1 & 11 \\
\hline \multirow[t]{10}{*}{ TR } & Thlaspietea rotundifolii & & & & & & & & & & & & \\
\hline & Dryopteris villarii & E1 & + & + & . & 1 & 1 & 2 & + & 1 & + & 8 & 89 \\
\hline & Hieracium bifidum & E1 & + & + & . & + & + & + & . & . & + & 6 & 67 \\
\hline & Adenostyles glabra & E1 & + & 2 & . & . & + & + & + & . & . & 5 & 56 \\
\hline & Cirsium spinosissimum & E1 & . & . & + & . & + & + & . & + & . & 4 & 44 \\
\hline & Rhodiola rosea & E1 & . & . & . & + & 1 & + & . & 1 & . & 4 & 44 \\
\hline & Hieracium glabratum & E1 & + & + & . & . & . & + & . & . & . & 3 & 33 \\
\hline & Valeriana montana & E1 & . & . & . & . & + & 1 & . & . & . & 2 & 22 \\
\hline & Cirsium $x$ flavescens & E1 & + & . & . & . & . & . & . & . & . & 1 & 11 \\
\hline & Biscutella laevigata & E1 & . & + & . & . & . & . & . & . & . & 1 & 11 \\
\hline
\end{tabular}




\begin{tabular}{|c|c|c|c|c|c|c|c|c|c|c|c|c|c|}
\hline & Number of relevé (Zaporedna številka popisa) & & 1 & 2 & 3 & 4 & 5 & 6 & 7 & 8 & 9 & Pr. & Fr. \\
\hline & Dianthus sternbergii & E1 & . & . & + & . & . & . & . & . & . & 1 & 11 \\
\hline & Cerastium carinthiacum & E1 & . & . & . & . & . & + & . & . & . & 1 & 11 \\
\hline \multirow[t]{2}{*}{ Сy } & Cystopteridion fragilis & & & & & & & & & & & & \\
\hline & Cystopteris regia & E1 & . & . & . & . & . & + & . & . & . & 1 & 11 \\
\hline \multirow[t]{2}{*}{ PS } & Physoplexido comosae-Saxifragion petraeae & & & & & & & & & & & & \\
\hline & Saxifraga hostii & E1 & . & . & . & . & + & . & . & . & . & 1 & 11 \\
\hline \multirow[t]{2}{*}{ PC } & Potentilletalia caulescentis & & & & & & & & & & & & \\
\hline & Campanula cochleariifolia & E1 & . & . & . & . & + & . & . & . & . & 1 & 11 \\
\hline \multirow[t]{6}{*}{ AT } & Asplenietea trichomanis & & & & & & & & & & & & \\
\hline & Asplenium viride & E1 & + & + & . & . & + & 1 & + & + & + & 7 & 78 \\
\hline & Asplenium ruta-muraria & E1 & . & . & . & + & . & + & . & . & . & 2 & 22 \\
\hline & Polypodium vulgare & E1 & . & . & . & . & + & . & . & . & . & 1 & 11 \\
\hline & Saxifraga paniculata & E1 & . & . & . & . & . & + & . & . & . & 1 & 11 \\
\hline & Festuca sp. & E1 & . & . & . & . & . & . & . & . & + & 1 & 11 \\
\hline \multirow[t]{14}{*}{ ML } & Mosses and lichens (Mahovi in lišaji) & & & & & & & & & & & & \\
\hline & Tortella tortuosa & E0 & + & . & + & + & . & + & + & + & + & 7 & 78 \\
\hline & Dicranum scoparium & E0 & + & + & + & + & + & + & . & . & + & 7 & 78 \\
\hline & Ctenidium molluscum & E0 & . & + & . & + & + & . & + & . & + & 5 & 56 \\
\hline & Rhytidiadelphus triquetrus & E0 & . & . & . & + & . & . & . & . & 1 & 2 & 22 \\
\hline & Fissidens dubius & E0 & . & . & + & . & . & . & . & . & . & 1 & 11 \\
\hline & Hylocomium splendens & E0 & . & . & . & + & . & . & . & . & . & 1 & 11 \\
\hline & Rhytidiadelphus loreus & E0 & . & . & . & + & . & . & . & . & . & 1 & 11 \\
\hline & Cladonia pyxidata & E0 & . & . & . & + & . & . & . & . & . & 1 & 11 \\
\hline & Homalothecium philippeanum & E0 & . & . & . & . & + & . & . & . & . & 1 & 11 \\
\hline & Pseudoleskea incurvata & E0 & . & . & . & . & + & . & . & . & . & 1 & 11 \\
\hline & Mnium sp. & E0 & . & . & . & . & . & + & . & . & . & 1 & 11 \\
\hline & Dicranodontium sp. & E0 & . & . & . & . & . & + & . & . & . & 1 & 11 \\
\hline & Letharia vulpina & $\mathrm{E} 3 \mathrm{c}$ & . & . & . & . & . & . & . & + & . & 1 & 11 \\
\hline
\end{tabular}

\section{Legend - Legenda}

ID Igor Dakskobler

AP Aleš Poljanec

A Limestone-apnenec

L Marlstone - laporovec

Re Rendzina - rendzina

JA Julian Alps - Julijske Alpe

Pr. Presence (number of relevés in which the species is presented) - število popisov, v katerih se pojavlja vrsta

Fr. Frequency in $\%$ - frekvenca v \% 


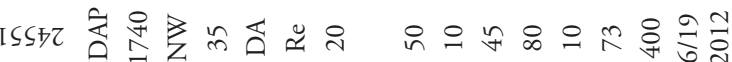

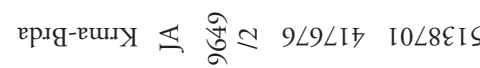

$m n+v$

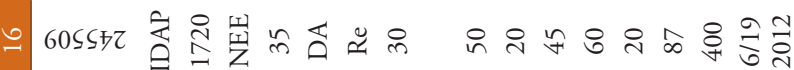

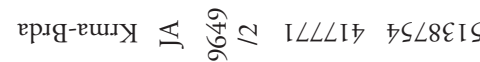

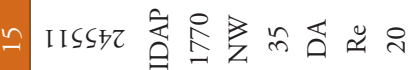
우옹요 \&

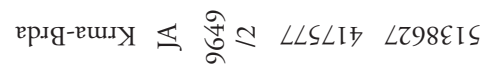

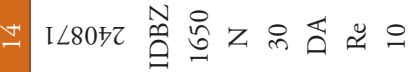
৪요 \& ๙

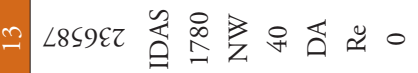

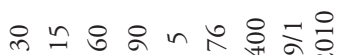

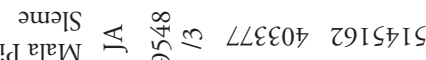

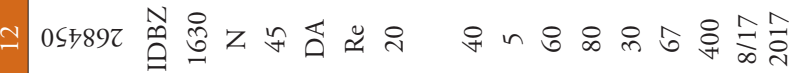
ग्गएप्र

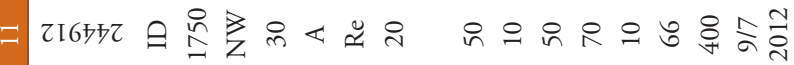

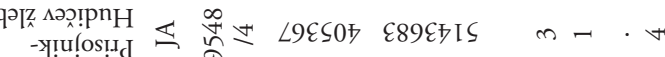

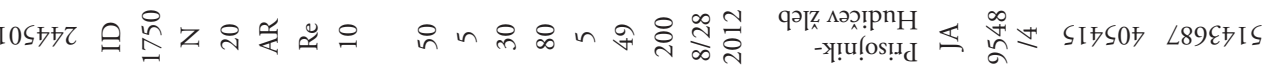

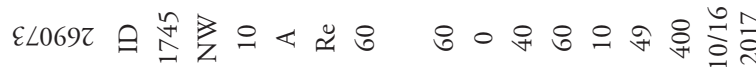

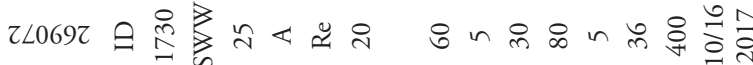

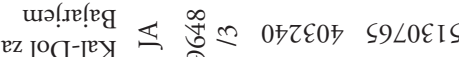
$m n+c$

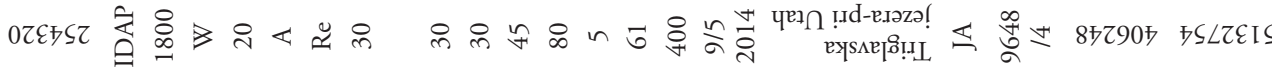

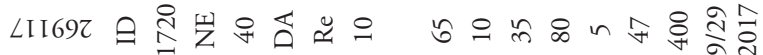

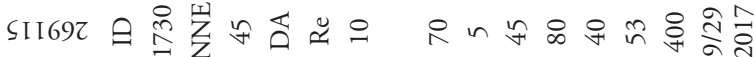

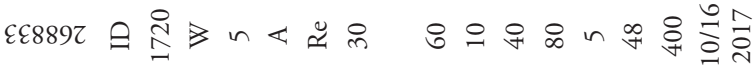

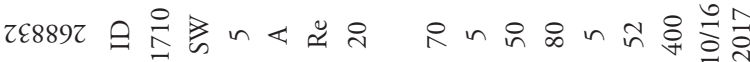

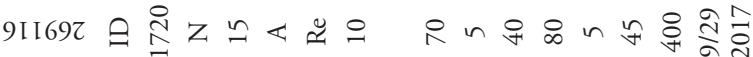

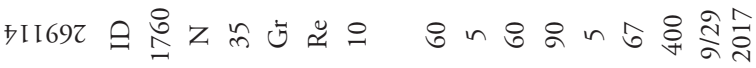

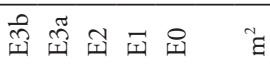

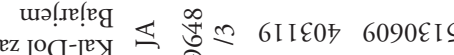

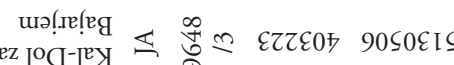

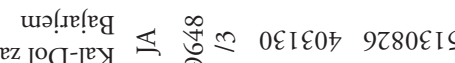

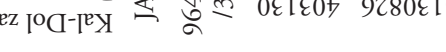

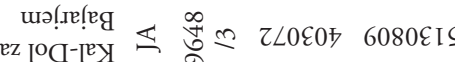

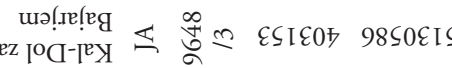

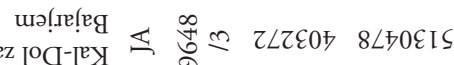
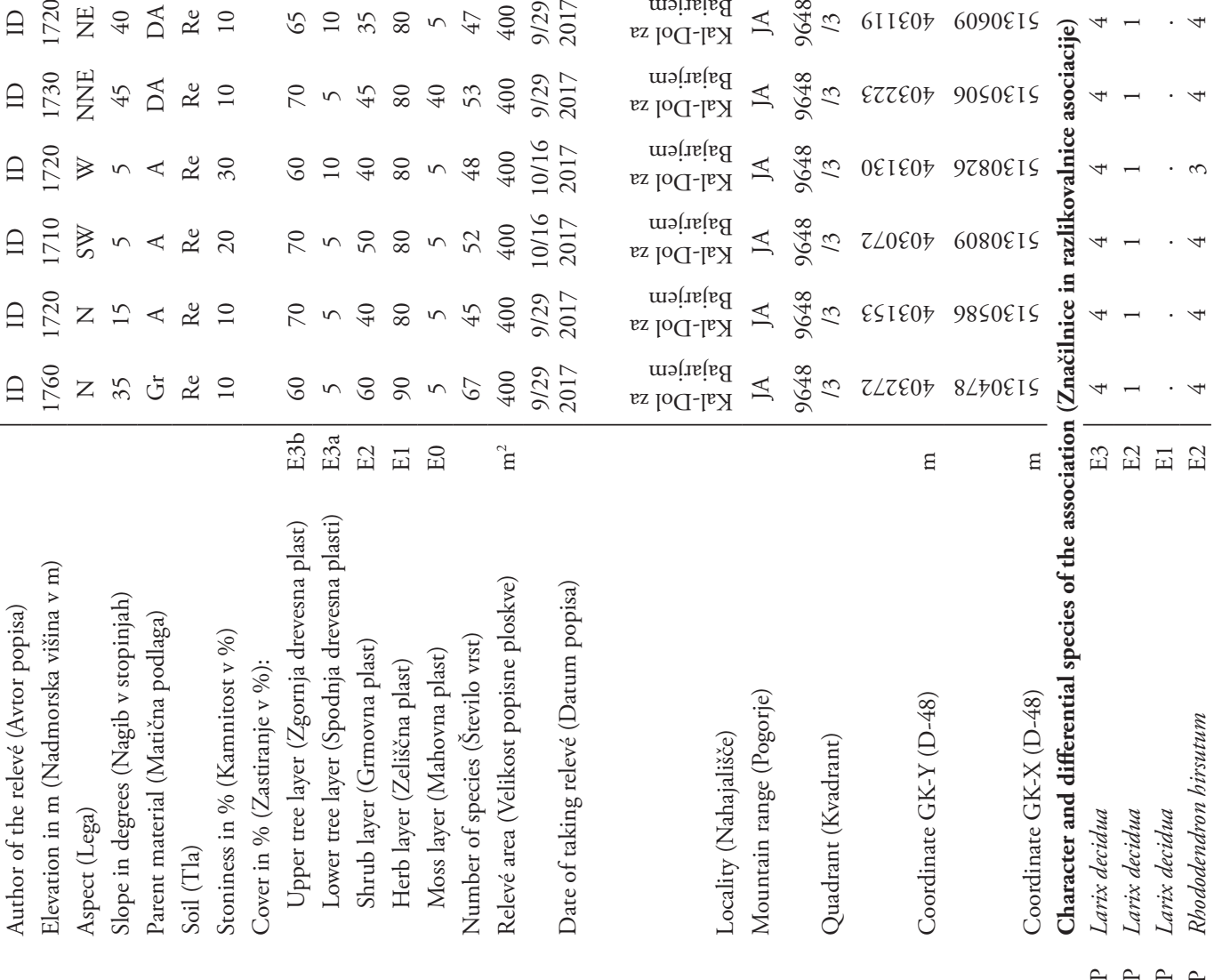


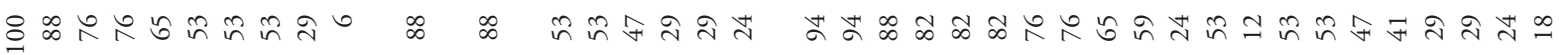

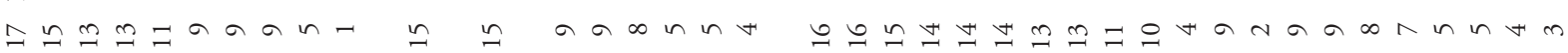
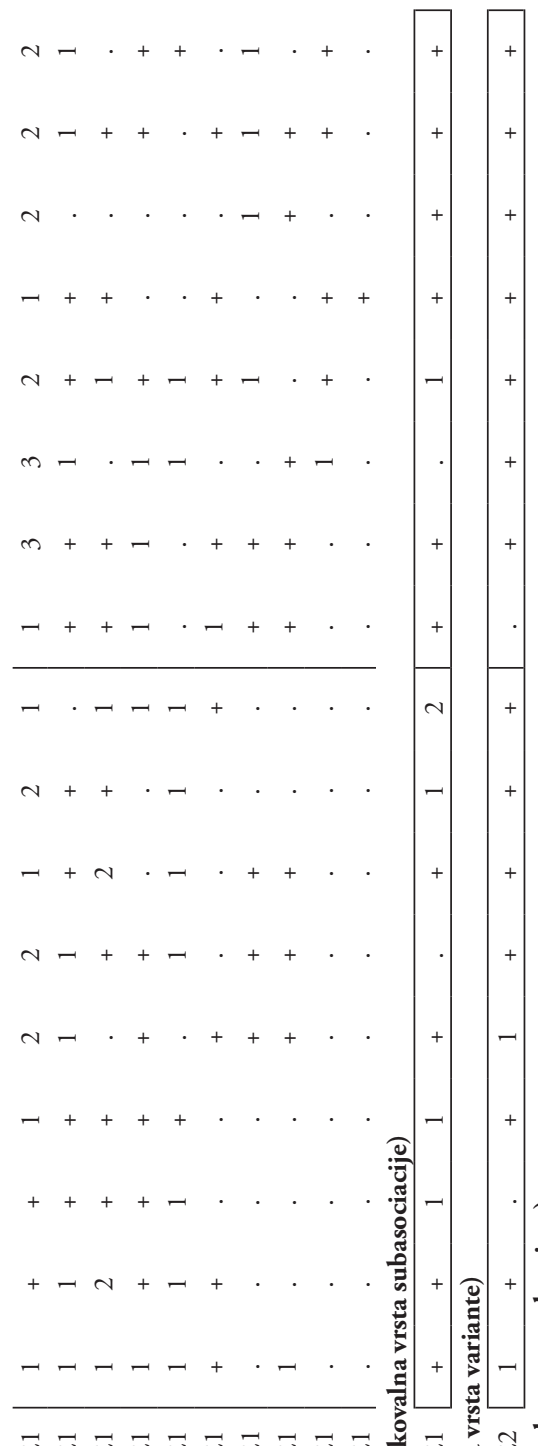

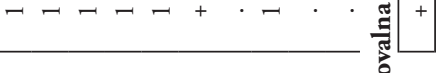

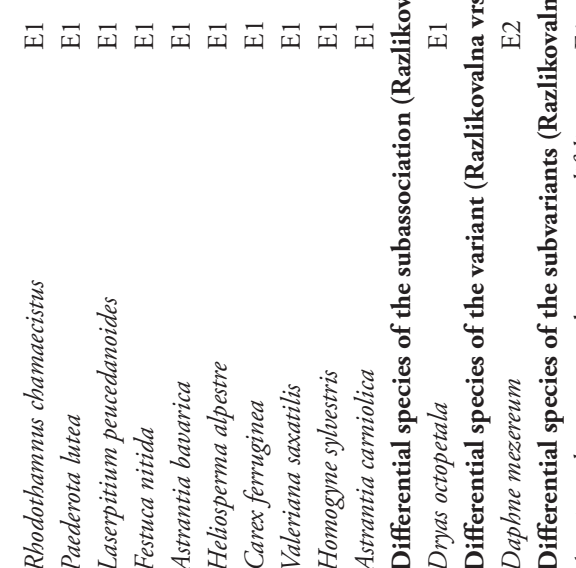
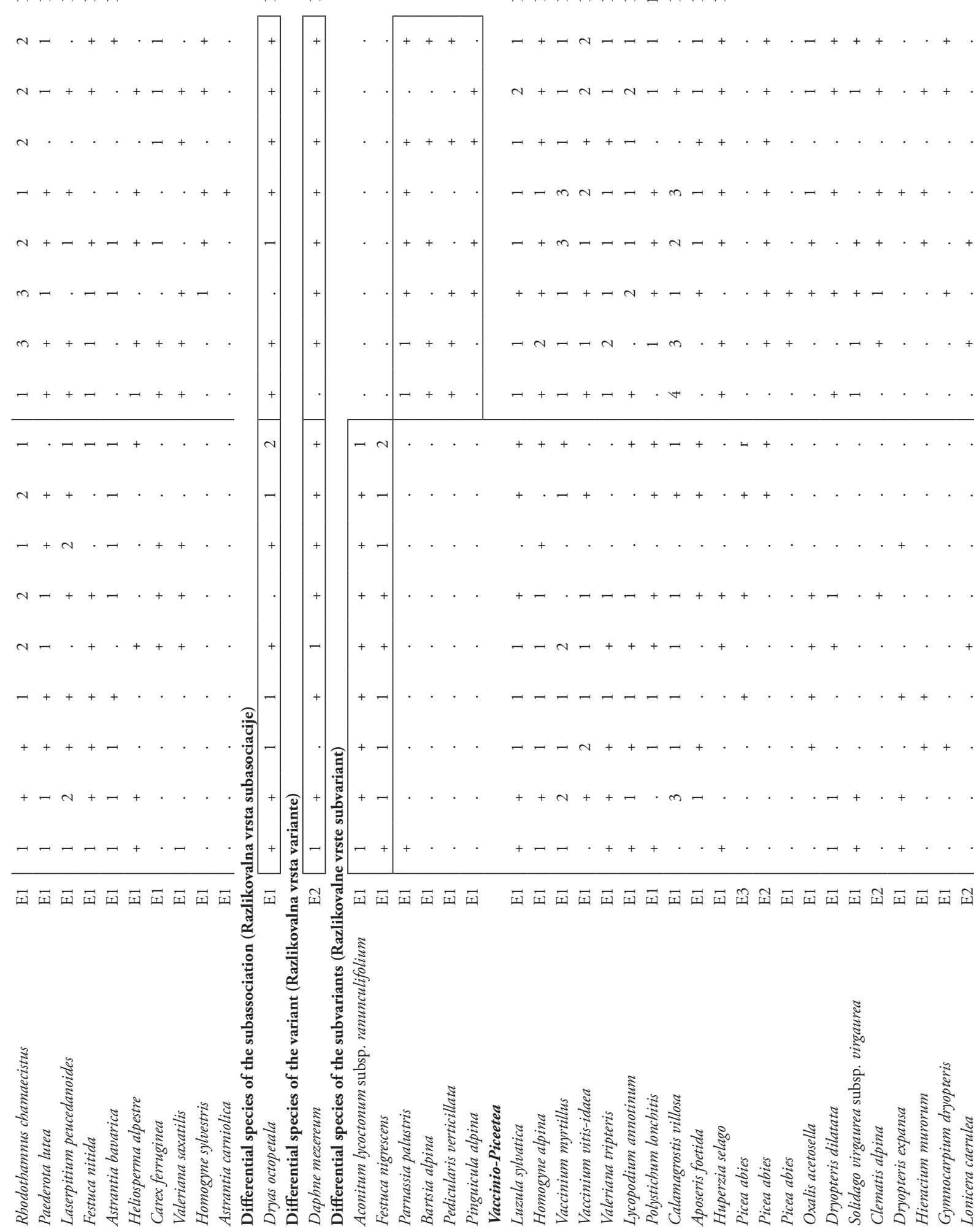

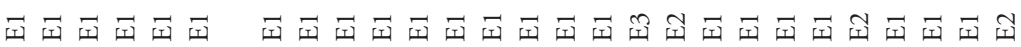

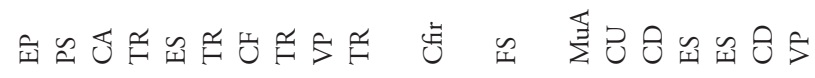




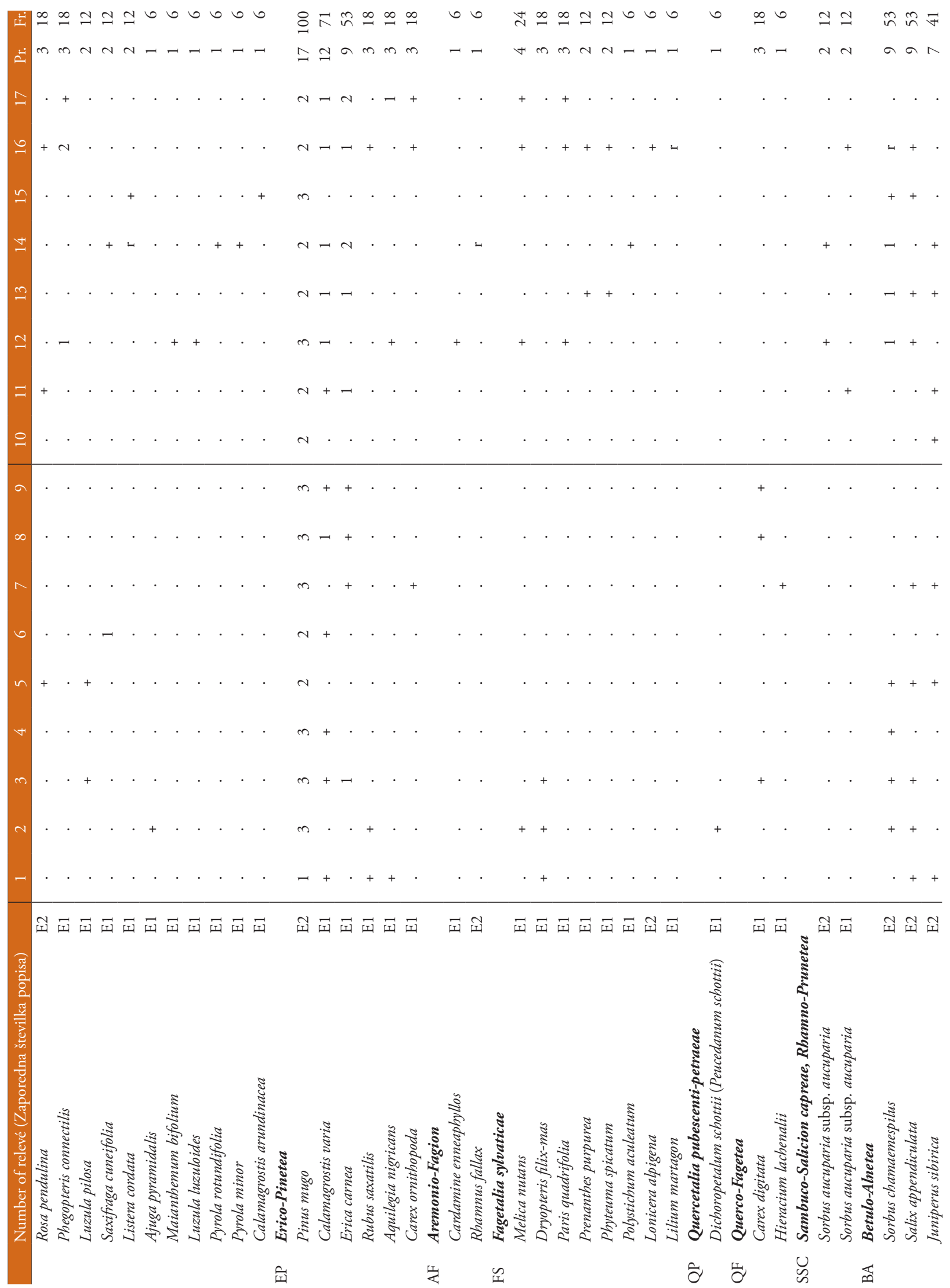


๙ิฟ

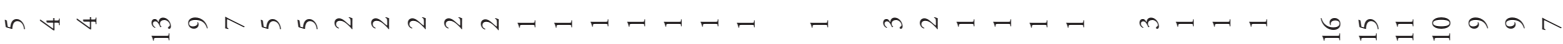

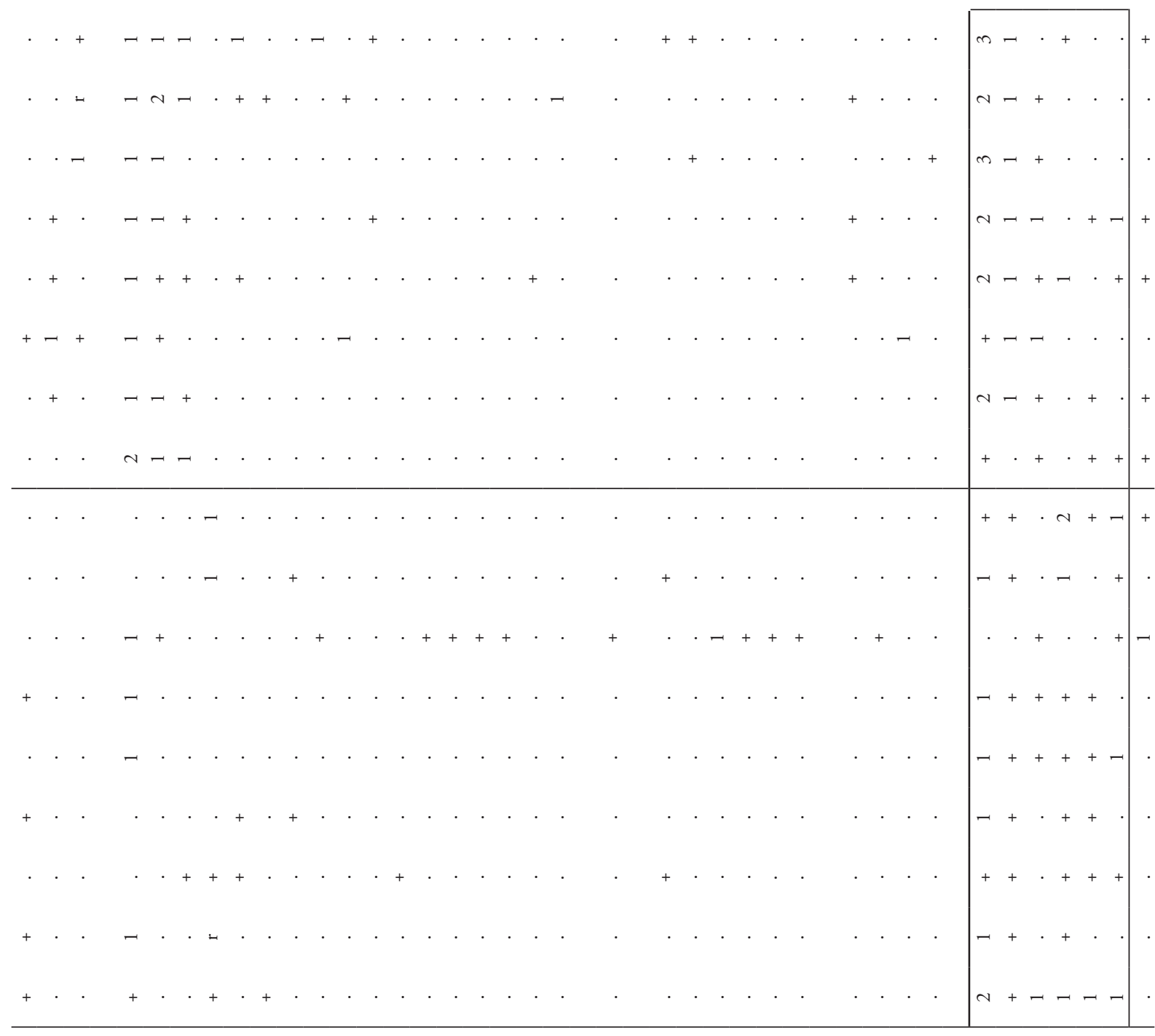

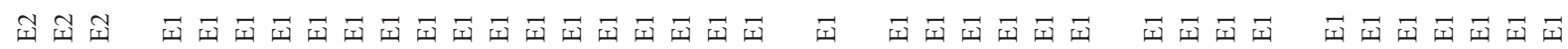

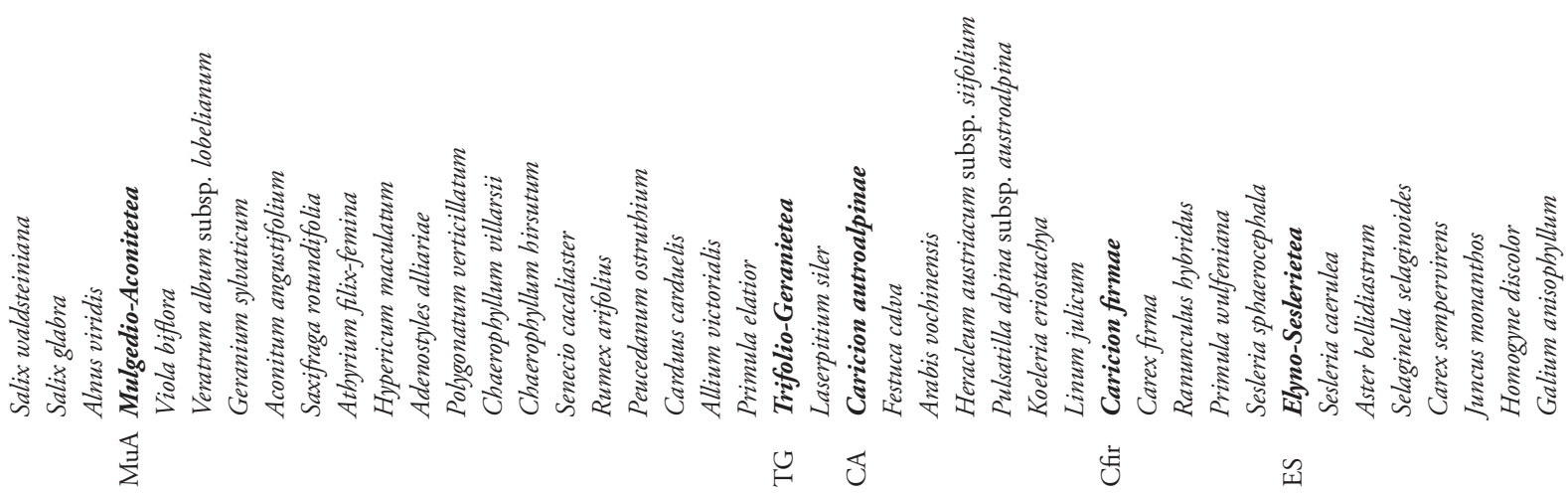



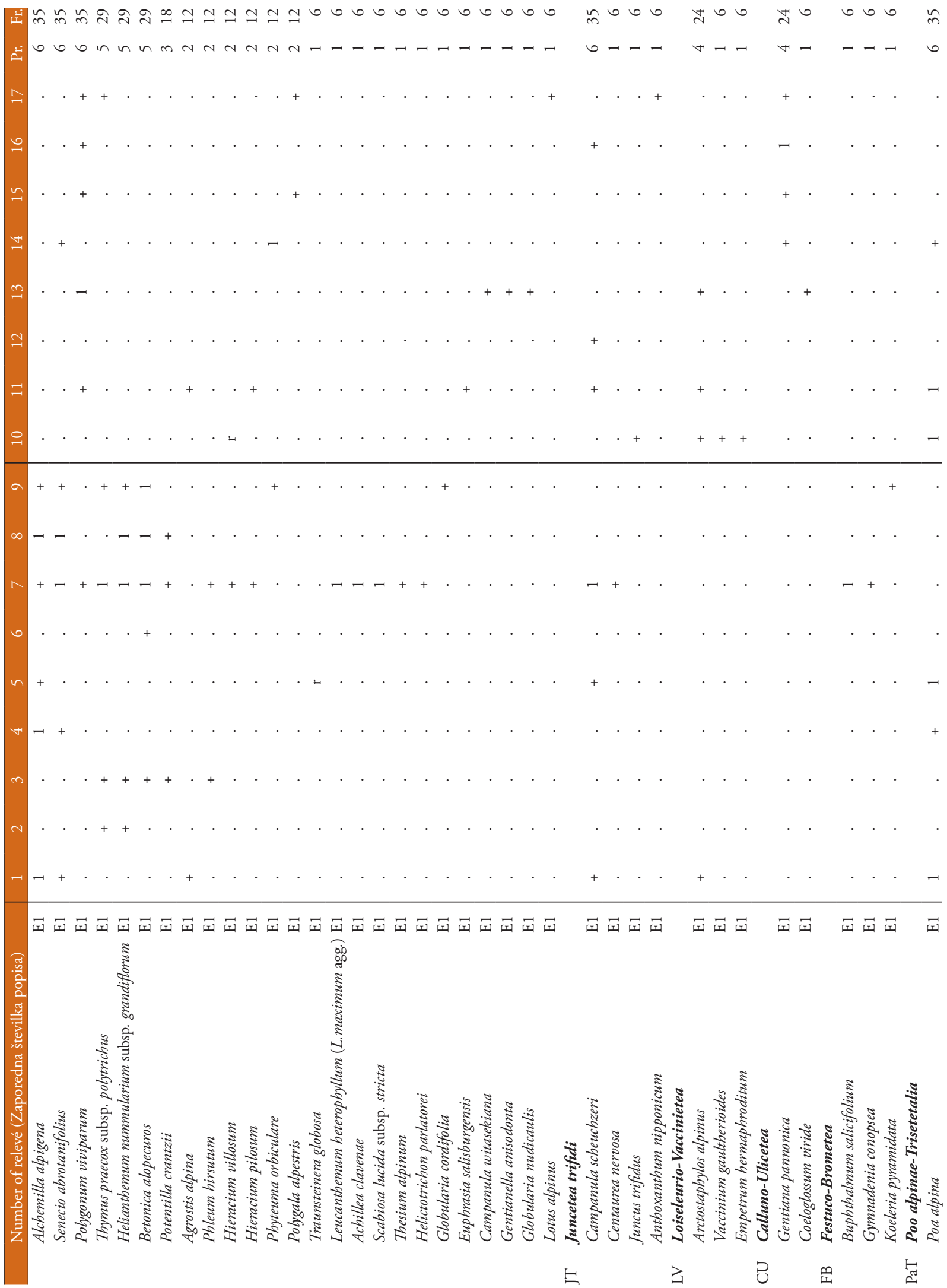


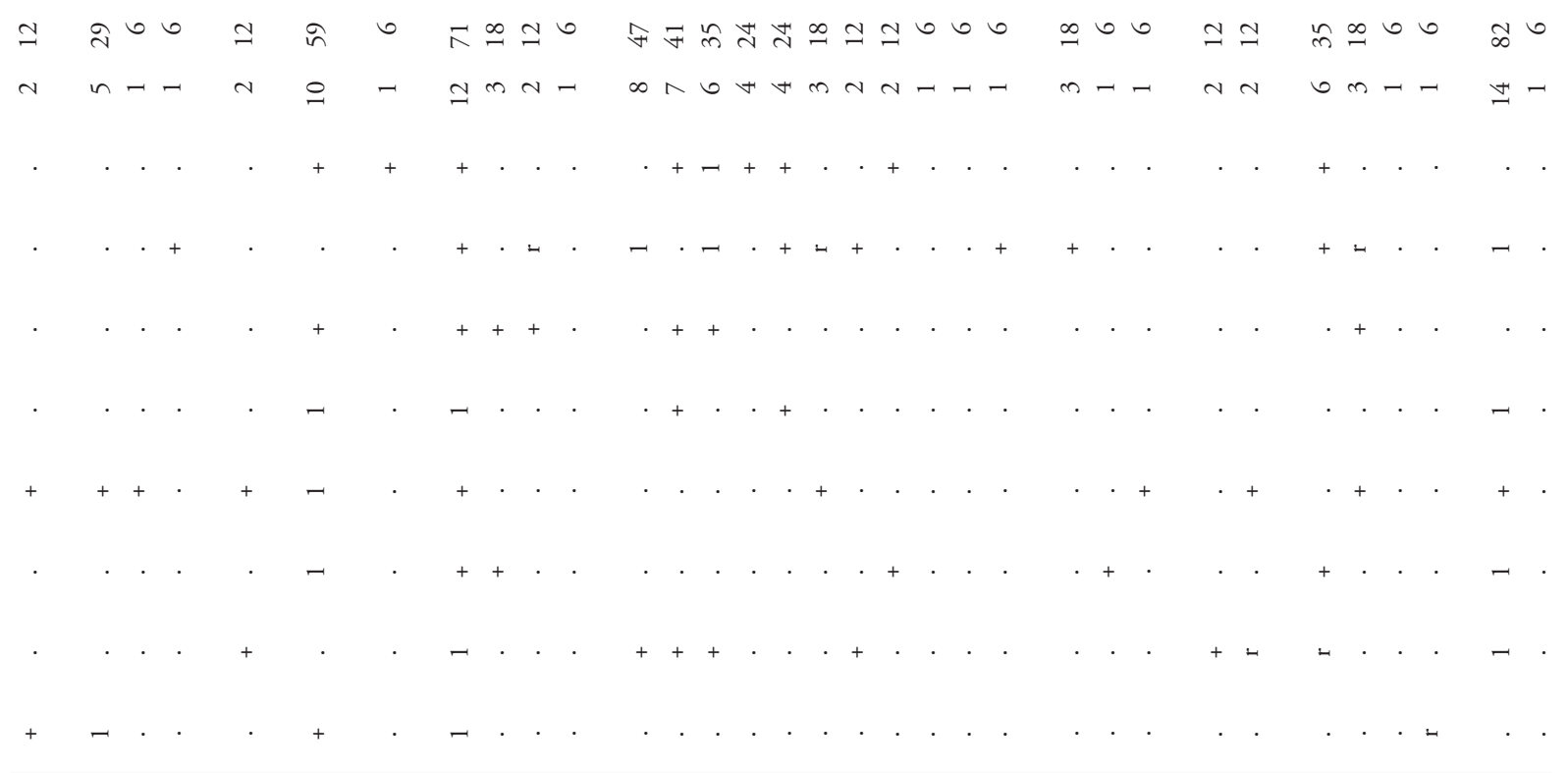

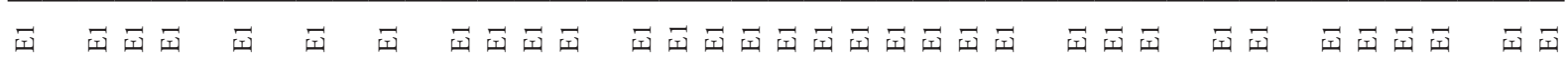

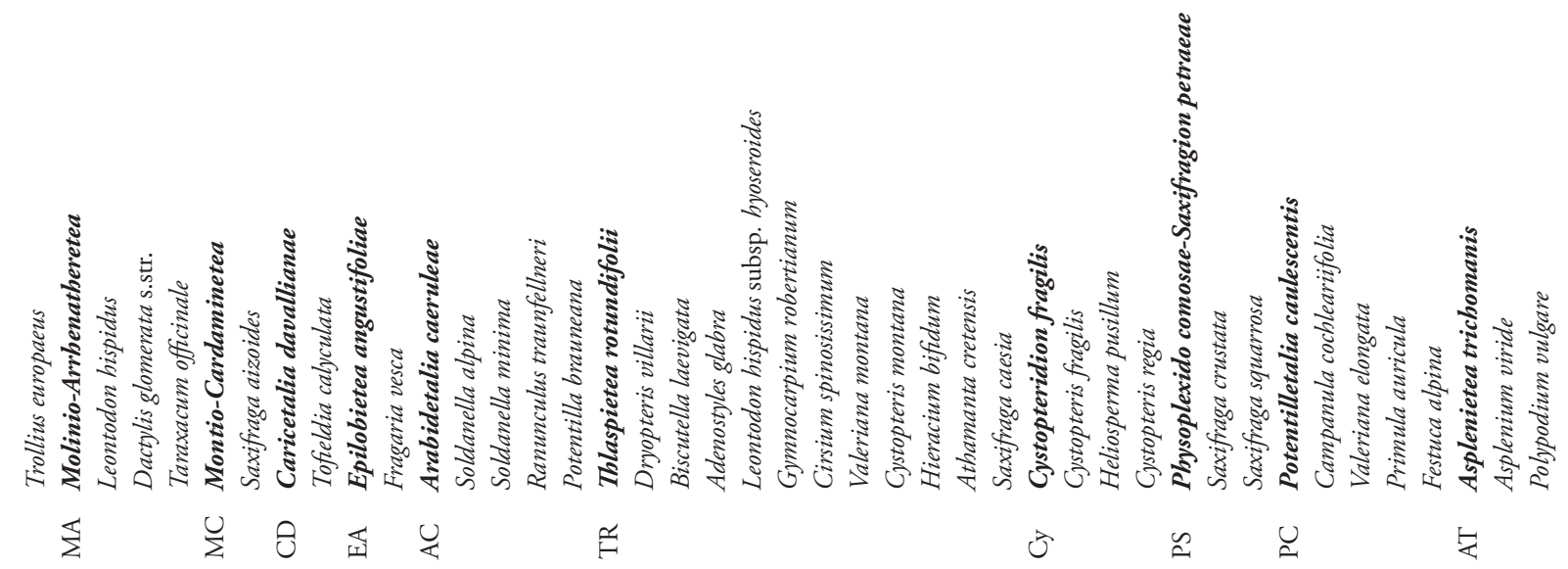



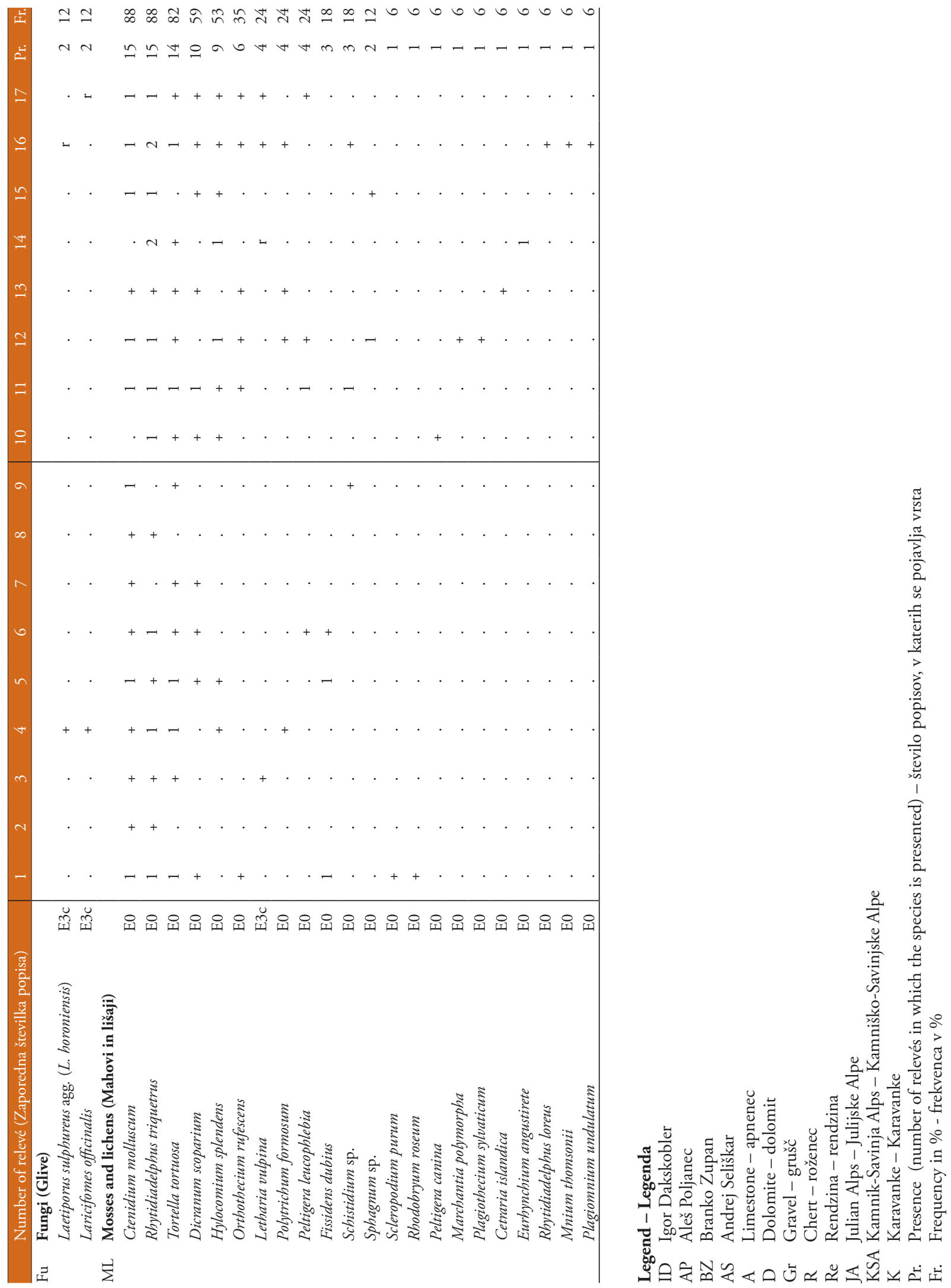
Table 23 (Tabela 23): Rhodothamno-Laricetum adoxetosum moschatellinae

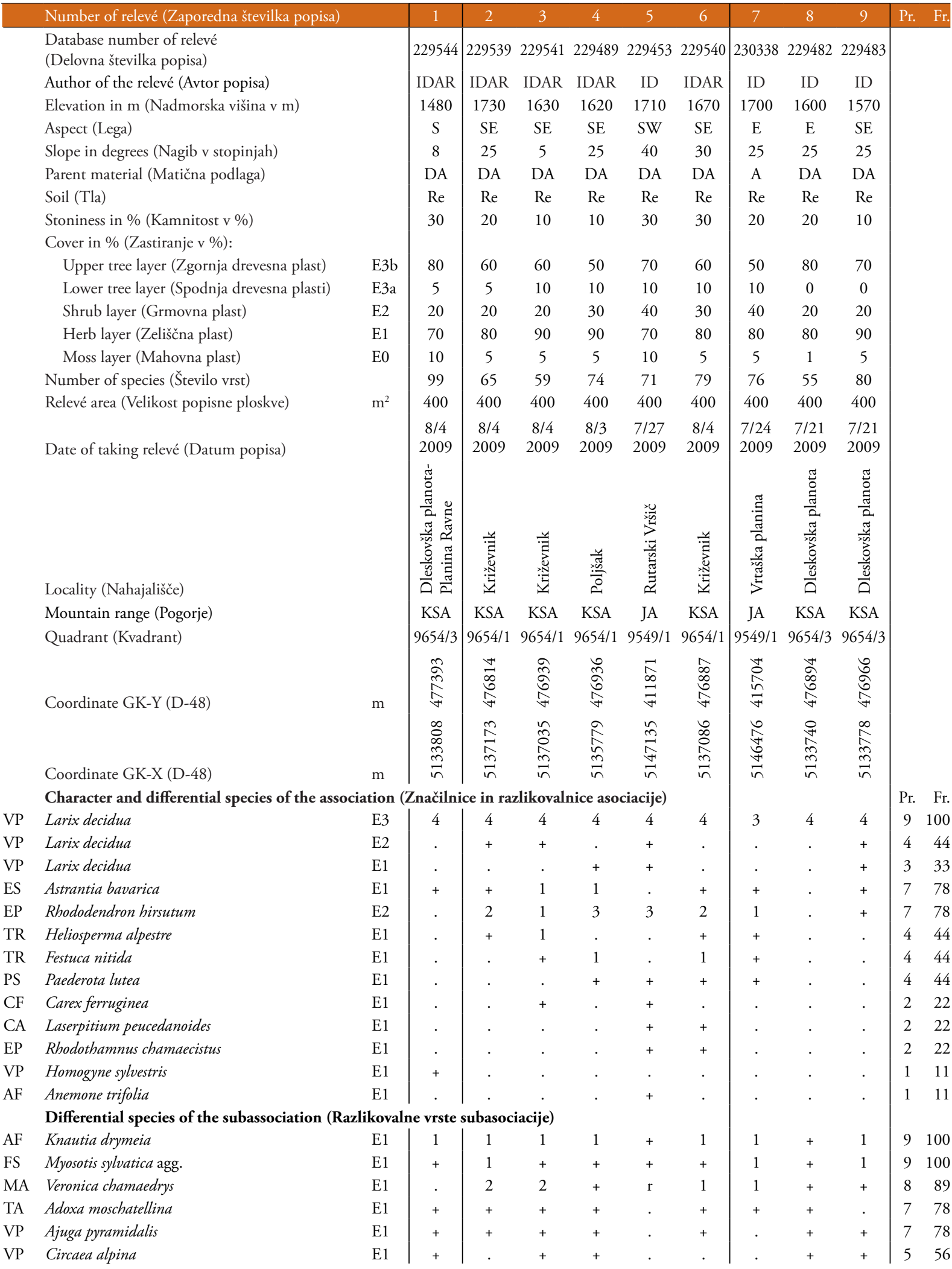




\begin{tabular}{|c|c|c|c|c|c|c|c|c|c|c|c|c|c|}
\hline & Number of relevé (Zaporedna številka popisa) & & 1 & 2 & 3 & 4 & 5 & 6 & 7 & 8 & 9 & Pr. & Fr. \\
\hline \multirow[t]{33}{*}{ VP } & Vaccinio-Piceetea & & & & & & & & & & & & \\
\hline & Vaccinium myrtillus & E1 & 1 & 1 & 1 & 3 & 1 & 1 & 1 & 2 & 1 & 9 & 100 \\
\hline & Vaccinium vitis-idaea & E1 & + & 1 & + & + & 2 & 1 & 1 & + & + & 9 & 100 \\
\hline & Luzula sylvatica & E1 & + & 1 & 3 & 1 & 1 & + & 1 & 1 & 1 & 9 & 100 \\
\hline & Picea abies & E3 & 2 & + & + & + & + & + & + & + & 1 & 9 & 100 \\
\hline & Picea abies & E2 & 1 & + & + & + & 1 & + & . & . & + & 7 & 78 \\
\hline & Picea abies & E1 & + & . & . & . & . & . & . & . & . & 1 & 11 \\
\hline & Aposeris foetida & E1 & 1 & 1 & 1 & 1 & 1 & 1 & 1 & . & + & 8 & 89 \\
\hline & Oxalis acetosella & E1 & 1 & 1 & 1 & + & + & + & . & + & . & 7 & 78 \\
\hline & Dryopteris dilatata & E1 & + & + & + & + & . & + & 1 & 1 & . & 7 & 78 \\
\hline & Luzula luzuloides & E1 & 1 & 1 & . & 1 & . & 1 & . & + & 1 & 6 & 67 \\
\hline & Polystichum lonchitis & E1 & . & + & . & . & 1 & + & + & + & + & 6 & 67 \\
\hline & Clematis alpina & E2 & + & + & . & + & 1 & + & . & . & + & 6 & 67 \\
\hline & Gymnocarpium dryopteris & E1 & + & + & . & . & + & + & . & + & + & 6 & 67 \\
\hline & Homogyne alpina & E1 & + & . & 1 & . & 1 & . & + & . & + & 5 & 56 \\
\hline & Valeriana tripteris & E1 & + & . & . & + & 1 & + & . & . & + & 5 & 56 \\
\hline & Hieracium murorum & E1 & + & . & . & + & 1 & + & . & . & + & 5 & 56 \\
\hline & Melampyrum sylvaticum & E1 & + & . & + & + & + & . & . & . & . & 4 & 44 \\
\hline & Calamagrostis villosa & E1 & . & + & + & . & + & . & . & . & + & 4 & 44 \\
\hline & Saxifraga cuneifolia & E1 & 2 & . & . & . & + & . & . & . & + & 3 & 33 \\
\hline & Luzula luzulina & E1 & + & + & . & . & . & . & + & . & . & 3 & 33 \\
\hline & Maianthemum bifolium & E1 & + & . & . & . & . & . & + & . & . & 2 & 22 \\
\hline & Dryopteris expansa & E1 & + & . & . & . & . & . & + & . & . & 2 & 22 \\
\hline & Abies alba & E3 & . & . & $\mathrm{r}$ & . & . & . & $\mathrm{r}$ & . & . & 2 & 22 \\
\hline & Abies alba & E2 & + & . & . & . & . & . & . & . & . & 1 & 11 \\
\hline & Rosa pendulina & E2 & + & . & . & . & . & . & . & . & . & 1 & 11 \\
\hline & Solidago virgaurea subsp. virgaurea & E1 & + & . & . & . & . & . & . & . & . & 1 & 11 \\
\hline & Gentiana asclepiadea & E1 & + & . & . & . & . & . & . & . & . & 1 & 11 \\
\hline & Calamagrostis arundinacea & E1 & . & . & . & + & . & . & . & . & . & 1 & 11 \\
\hline & Lycopodium annotinum & E1 & . & . & . & . & + & . & . & . & . & 1 & 11 \\
\hline & Phegopteris connectilis & E1 & . & . & . & . & + & . & . & . & . & 1 & 11 \\
\hline & Veronica urticifolia & E1 & . & . & . & . & + & . & . & . & . & 1 & 11 \\
\hline & Luzula pilosa & E1 & . & . & . & . & + & . & . & . & . & 1 & 11 \\
\hline \multirow[t]{6}{*}{ EP } & Erico-Pinetea & & & & & & & & & & & & \\
\hline & Erica carnea & E1 & 3 & 1 & + & 1 & 3 & 2 & 1 & + & + & 9 & 100 \\
\hline & Pinus mugo & E2 & . & + & + & + & + & 2 & + & 1 & 1 & 8 & 89 \\
\hline & Rubus saxatilis & E1 & 1 & . & . & . & . & + & . & + & . & 3 & 33 \\
\hline & Calamagrostis varia & E1 & . & + & . & . & + & + & . & . & . & 3 & 33 \\
\hline & Carex ornithopoda & E1 & . & . & . & . & . & + & . & . & . & 1 & 11 \\
\hline \multirow[t]{7}{*}{$\mathrm{AF}$} & Aremonio-Fagion & & & & & & & & & & & & \\
\hline & Helleborus niger & E1 & 2 & 2 & 2 & 1 & 1 & 1 & 1 & 1 & 1 & 9 & 100 \\
\hline & Cardamine enneaphyllos & E1 & + & + & + & + & . & + & 1 & . & 1 & 7 & 78 \\
\hline & Aremonia agrimonoides & E1 & + & . & . & . & + & . & . & . & + & 3 & 33 \\
\hline & Cyclamen purpurascens & E1 & . & . & . & 1 & + & . & . & . & . & 2 & 22 \\
\hline & Rhamnus fallax & E2 & . & . & . & . & + & + & . & . & . & 2 & 22 \\
\hline & Cardamine trifolia & E1 & 1 & . & . & . & . & . & . & . & . & 1 & 11 \\
\hline \multirow[t]{2}{*}{ TA } & Tilio-Acerion & & & & & & & & & & & & \\
\hline & Acer pseudoplatanus & E2 & . & . & . & . & . & . & . & + & + & 2 & 22 \\
\hline \multirow[t]{7}{*}{ FS } & Fagetalia sylvaticae & & & & & & & & & & & & \\
\hline & Melica nutans & E1 & + & 2 & 1 & + & + & 1 & + & + & + & 9 & 100 \\
\hline & Daphne mezereum & E2 & + & 1 & 1 & + & 1 & 1 & . & . & + & 7 & 78 \\
\hline & Galeobdolon flavidum & E1 & + & . & + & + & . & + & 1 & 1 & + & 7 & 78 \\
\hline & Epilobium montanum & E1 & 1 & + & . & + & . & + & + & + & 1 & 7 & 78 \\
\hline & Euphorbia amygdaloides & E1 & + & . & + & 1 & . & 1 & 1 & . & + & 6 & 67 \\
\hline & Dryopteris flix-mas & E1 & + & . & . & + & . & . & + & 1 & 1 & 5 & 56 \\
\hline
\end{tabular}




\begin{tabular}{|c|c|c|c|c|c|c|c|c|c|c|c|c|c|}
\hline & Number of relevé (Zaporedna številka popisa) & & 1 & 2 & 3 & 4 & 5 & 6 & 7 & 8 & 9 & Pr. & Fr. \\
\hline & Mycelis muralis & E1 & 1 & . & . & . & + & + & . & . & + & 4 & 44 \\
\hline & Paris quadrifolia & E1 & 1 & . & . & . & . & . & . & . & + & 2 & 22 \\
\hline & Lilium martagon & E1 & + & . & . & . & . & . & . & . & + & 2 & 22 \\
\hline & Polystichum aculeatum & E1 & + & . & . & . & + & . & . & . & . & 2 & 22 \\
\hline & Mercurialis perennis & E1 & . & . & . & . & . & . & . & + & + & 2 & 22 \\
\hline & Fagus sylvatica & E3 & . & . & . & . & $\mathrm{r}$ & . & . & . & . & 1 & 11 \\
\hline & Fagus sylvatica & $\mathrm{E} 2$ & + & . & . & . & . & . & . & . & . & 1 & 11 \\
\hline & Petasites albus & E1 & + & . & . & . & . & . & . & . & . & 1 & 11 \\
\hline & Ranunculus lanuginosus & E1 & + & . & . & . & . & . & . & . & . & 1 & 11 \\
\hline & Carex sylvatica & E1 & + & . & . & . & . & . & . & . & . & 1 & 11 \\
\hline & Cardamine impatiens & E1 & . & . & . & . & . & . & . & + & . & 1 & 11 \\
\hline & Actaea spicata & E1 & . & . & . & . & . & . & . & . & + & 1 & 11 \\
\hline \multirow[t]{12}{*}{ QF } & Querco-Fagetea & & & & & & & & & & & & \\
\hline & Poa nemoralis & E1 & + & 2 & + & + & + & 1 & . & + & . & 7 & 78 \\
\hline & Hepatica nobilis & E1 & 1 & . & + & + & . & + & . & + & + & 6 & 67 \\
\hline & Anemone nemorosa & E1 & + & + & . & 1 & . & + & 1 & . & + & 6 & 67 \\
\hline & Carex digitata & E1 & + & . & . & + & + & . & . & . & . & 3 & 33 \\
\hline & Ranunculus auricomus agg. & $\mathrm{E} 1$ & + & . & . & . & . & . & . & + & + & 3 & 33 \\
\hline & Moehringia trinervia & $\mathrm{E} 1$ & . & . & . & + & . & + & . & . & . & 2 & 22 \\
\hline & Festuca heterophylla & E1 & . & . & . & . & . & . & . & + & + & 2 & 22 \\
\hline & Galium schultesii & E1 & . & . & . & . & . & . & . & + & + & 2 & 22 \\
\hline & Platanthera bifolia & E1 & + & . & . & . & . & . & . & . & . & 1 & 11 \\
\hline & Viola riviniana & E1 & . & . & . & . & + & . & . & . & . & 1 & 11 \\
\hline & Hieracium lachenalii & E1 & . & . & . & . & . & . & . & . & + & 1 & 11 \\
\hline \multirow[t]{3}{*}{ SSC } & Sambuco-Salicion capreae, Rhamno-Prunetea & & & & & & & & & & & & \\
\hline & Sorbus aucuparia subsp. aucuparia & E2 & + & . & . & . & . & . & . & . & . & 1 & 11 \\
\hline & Sorbus aucuparia subsp. aucuparia & E1 & . & . & . & . & + & + & . & . & . & 2 & 22 \\
\hline \multirow[t]{5}{*}{$\mathrm{BA}$} & Betulo-Alnetea & & & & & & & & & & & & \\
\hline & Sorbus chamaemespilus & E2 & + & . & . & . & + & . & 2 & . & + & 4 & 44 \\
\hline & Salix appendiculata & E2 & . & . & . & . & . & + & . & . & . & 1 & 11 \\
\hline & Juniperus sibirica & E2 & . & . & . & . & . & . & . & . & + & 1 & 11 \\
\hline & Ribes alpinum & E2 & . & . & . & . & . & . & . & . & + & 1 & 11 \\
\hline \multicolumn{14}{|c|}{ MuA Mulgedio-Aconitetea } \\
\hline & Athyrium filix-femina & E1 & + & + & + & 1 & + & . & 1 & + & + & 8 & 89 \\
\hline & Hypericum maculatum & E1 & 1 & + & + & + & . & + & 1 & 1 & + & 8 & 89 \\
\hline & Polygonatum verticillatum & $\mathrm{E} 1$ & 1 & + & . & + & . & + & + & + & . & 6 & 67 \\
\hline & Saxifraga rotundifolia & E1 & 1 & + & + & + & . & + & 1 & . & . & 6 & 67 \\
\hline & Senecio cacaliaster & E1 & 2 & . & . & + & . & . & + & + & 1 & 5 & 56 \\
\hline & Chaerophyllum hirsutum & E1 & 1 & . & 1 & 1 & . & . & + & . & + & 5 & 56 \\
\hline & Veratrum album subsp. album & E1 & + & . & + & 1 & . & . & . & . & + & 4 & 44 \\
\hline & Veratrum album subsp. lobelianum & E1 & . & . & . & . & + & . & 1 & + & 1 & 4 & 44 \\
\hline & Geum rivale & E1 & + & . & . & + & + & . & . & . & . & 3 & 33 \\
\hline & Senecio ovatus & E1 & + & . & . & + & . & . & . & 1 & . & 3 & 33 \\
\hline & Milium effusum & E1 & + & . & . & . & . & . & . & 1 & 1 & 3 & 33 \\
\hline & Viola biflora & E1 & . & . & . & + & 1 & . & 1 & . & . & 3 & 33 \\
\hline & Alchemilla xanthochlora & E1 & . & . & . & . & . & . & + & + & + & 3 & 33 \\
\hline & Silene dioica & $\mathrm{E} 1$ & + & . & . & . & . & . & . & . & + & 2 & 22 \\
\hline & Geranium sylvaticum & E1 & . & . & . & . & + & . & 1 & . & . & 2 & 22 \\
\hline & Epilobium alpestre & E1 & . & . & . & . & . & . & 1 & + & . & 2 & 22 \\
\hline & Adenostyles alliariae & E1 & + & . & . & . & . & . & . & . & . & 1 & 11 \\
\hline & Ranunculus platanifolius & E1 & + & . & . & . & . & . & . & . & . & 1 & 11 \\
\hline & Thalictrum aquilegiifolium & E1 & + & . & . & . & . & . & . & . & . & 1 & 11 \\
\hline & Aconitum lobelianum & E1 & + & . & . & . & . & . & . & . & . & 1 & 11 \\
\hline & Rumex arifolius & E1 & . & . & . & . & . & . & 1 & . & . & 1 & 11 \\
\hline & Primula elatior & E1 & . & . & . & . & . & . & + & . & . & 1 & 11 \\
\hline
\end{tabular}




\begin{tabular}{|c|c|c|c|c|c|c|c|c|c|c|c|c|c|}
\hline & \multicolumn{2}{|l|}{ Number of relevé (Zaporedna številka popisa) } & 1 & 2 & 3 & 4 & 5 & 6 & 7 & 8 & 9 & Pr. & Fr. \\
\hline & Stellaria nemorum & E1 & . & . & . & . & . & . & + & . & . & 1 & 11 \\
\hline & Scrophularia scopolii & E1 & . & . & . & . & . & . & . & . & + & 1 & 11 \\
\hline & Aconitum napellus & E1 & . & . & . & . & . & . & . & . & + & 1 & 11 \\
\hline \multirow[t]{3}{*}{ TG } & Trifolio-Geranietea & & & & & & & & & & & & \\
\hline & Digitalis grandiflora & E1 & + & . & . & . & . & . & . & . & + & 2 & 22 \\
\hline & Dianthus barbatus & E1 & . & . & . & . & . & . & + & . & . & 1 & 11 \\
\hline \multirow[t]{3}{*}{$\mathrm{CA}$} & Caricion austroalpinae & & & & & & & & & & & & \\
\hline & Heracleum austriacum subsp. siifolium & E1 & . & . & . & . & . & . & + & . & . & 1 & 11 \\
\hline & Koeleria eriostachya & E1 & . & . & . & . & . & . & . & + & . & 1 & 11 \\
\hline \multirow[t]{20}{*}{ ES } & Elyno-Seslerietea & & & & & & & & & & & & \\
\hline & Senecio abrotanifolius & E1 & + & + & + & 1 & 1 & 2 & + & . & + & 8 & 89 \\
\hline & Galium anisophyllum & E1 & + & + & 1 & + & . & + & + & . & . & 6 & 67 \\
\hline & Campanula witasekiana & E1 & + & + & + & . & . & + & . & + & . & 5 & 56 \\
\hline & Phyteuma orbiculare & E1 & . & 1 & + & + & . & . & + & . & . & 4 & 44 \\
\hline & Alchemilla sp. & E1 & . & + & + & + & . & . & + & . & . & 4 & 44 \\
\hline & Thymus praecox subsp. polytrichus & E1 & . & + & + & . & + & + & . & . & . & 4 & 44 \\
\hline & Helianthemum nummularium subsp. grandiflorum & E1 & . & + & . & + & . & + & + & . & . & 4 & 44 \\
\hline & Ranunculus montanus & E1 & + & . & . & . & . & . & . & + & + & 3 & 33 \\
\hline & Lotus alpinus & E1 & . & . & . & + & . & + & + & . & . & 3 & 33 \\
\hline & Euphrasia picta & E1 & . & + & . & . & . & + & . & . & . & 2 & 22 \\
\hline & Polygala alpestris & E1 & . & . & . & . & . & + & + & . & . & 2 & 22 \\
\hline & Homogyne discolor & E1 & . & . & + & . & . & . & . & . & . & 1 & 11 \\
\hline & Betonica alopecuros & E1 & . & . & . & + & . & . & . & . & . & 1 & 11 \\
\hline & Polygonum viviparum & E1 & . & . & . & + & . & . & . & . & . & 1 & 11 \\
\hline & Gentianella anisodonta & E1 & . & . & . & + & . & . & . & . & . & 1 & 11 \\
\hline & Aster bellidiastrum & E1 & . & . & . & . & + & . & . & . & . & 1 & 11 \\
\hline & Hieracium pilosum & E1 & . & . & . & . & + & . & . & . & . & 1 & 11 \\
\hline & Juncus monanthos & E1 & . & . & . & . & . & + & . & . & . & 1 & 11 \\
\hline & Ranunculus carinthiacus & E1 & . & . & . & . & . & . & + & . & . & 1 & 11 \\
\hline \multirow[t]{3}{*}{$\mathrm{JT}$} & Juncetea trifidi & & & & & & & & & & & & \\
\hline & Campanula scheuchzeri & E1 & 1 & + & 1 & + & 1 & . & + & . & + & 7 & 78 \\
\hline & Anthoxanthum nipponicum & E1 & . & . & + & . & . & . & . & . & . & 1 & 11 \\
\hline \multirow[t]{5}{*}{$\mathrm{CU}$} & Calluno-Ulicetea & & & & & & & & & & & & \\
\hline & Festuca nigrescens & E1 & + & + & + & + & . & + & 1 & . & + & 7 & 78 \\
\hline & Veronica officinalis & E1 & + & . & . & + & + & . & . & + & + & 5 & 56 \\
\hline & Potentilla aurea & E1 & . & + & + & . & . & . & + & . & . & 3 & 33 \\
\hline & Agrostis capillaris & E1 & . & . & . & + & . & . & . & . & . & 1 & 11 \\
\hline \multirow[t]{3}{*}{ FB } & Festuco-Brometea & & & & & & & & & & & & \\
\hline & Euphorbia cyparissias & E1 & + & . & . & . & . & . & . & + & + & 3 & 33 \\
\hline & Cirsium erisithales & E1 & + & . & . & . & . & . & . & . & . & 1 & 11 \\
\hline \multirow[t]{5}{*}{$\mathrm{PaT}$} & Poo alpinae-Trisetetalia & & & & & & & & & & & & \\
\hline & Poa alpina & E1 & . & + & . & . & . & + & + & . & . & 3 & 33 \\
\hline & Astrantia major & E1 & . & . & . & . & . & . & + & . & + & 2 & 22 \\
\hline & Ranunculus nemorosus & E1 & . & . & . & . & . & . & + & . & . & 1 & 11 \\
\hline & Phleum rhaeticum & E1 & . & . & . & . & . & . & + & . & . & 1 & 11 \\
\hline \multirow[t]{10}{*}{ MA } & Molinio-Arrhenatheretea & & & & & & & & & & & & \\
\hline & Festuca rubra & E1 & + & 2 & 2 & + & . & . & . & . & . & 4 & 44 \\
\hline & Ajuga reptans & E1 & + & . & . & . & . & . & . & + & + & 3 & 33 \\
\hline & Cerastium fontanum & E1 & + & . & . & + & . & . & . & . & . & 2 & 22 \\
\hline & Trifolium pratense & E1 & . & . & + & . & . & . & + & . & . & 2 & 22 \\
\hline & Poa pratensis & E1 & . & + & . & . & . & . & . & . & . & 1 & 11 \\
\hline & Achillea millefolium & E1 & . & . & + & . & . & . & . & . & . & 1 & 11 \\
\hline & Prunella vulgaris & E1 & . & . & . & + & . & . & . & . & . & 1 & 11 \\
\hline & Vicia sepium & E1 & . & . & . & . & . & . & . & + & . & 1 & 11 \\
\hline & Deschampsia cespitosa & E1 & . & . & . & . & . & . & . & . & + & 1 & 11 \\
\hline
\end{tabular}




\begin{tabular}{|c|c|c|c|c|c|c|c|c|c|c|c|c|c|}
\hline & Number of relevé (Zaporedna številka popisa) & & 1 & 2 & 3 & 4 & 5 & 6 & 7 & 8 & 9 & Pr. & Fr. \\
\hline & Galium mollugo & E1 & . & . & . & . & . & . & . & . & + & 1 & 11 \\
\hline \multirow[t]{3}{*}{ MC } & Montio-Cardaminetea & & & & & & & & & & & & \\
\hline & Cardamine flexuosa & E1 & . & . & + & . & . & . & . & + & . & 2 & 22 \\
\hline & Epilobium alsinifolium & E1 & . & . & . & + & . & . & . & . & . & 1 & 11 \\
\hline \multirow[t]{7}{*}{ EA } & Epilobietea angustifolii & & & & & & & & & & & & \\
\hline & Fragaria vesca & E1 & 1 & + & + & + & 1 & 1 & + & 1 & + & 9 & 100 \\
\hline & Rubus idaeus & E2 & + & . & . & + & . & . & 2 & 1 & 1 & 5 & 56 \\
\hline & Urtica dioica & E1 & . & . & . & . & + & + & + & 1 & + & 5 & 56 \\
\hline & Chamaenerion angustifolium & E1 & . & . & . & + & . & . & + & + & + & 4 & 44 \\
\hline & Myosotis arvensis & E1 & . & + & . & . & . & . & . & . & . & 1 & 11 \\
\hline & Cirsium eriophorum & E1 & . & . & . & . & . & . & . & . & + & 1 & 11 \\
\hline \multirow[t]{2}{*}{ AC } & Arabidetalia caeruleae & & & & & & & & & & & & \\
\hline & Soldanella alpina & E1 & . & + & + & . & . & . & . & . & . & 2 & 22 \\
\hline \multirow[t]{6}{*}{ TR } & Thlaspietea rotundifolii & & & & & & & & & & & & \\
\hline & Gymnocarpium robertianum & E1 & + & + & . & + & + & + & . & + & . & 6 & 67 \\
\hline & Trisetum argenteum & E1 & . & + & . & . & 1 & 1 & . & . & . & 3 & 33 \\
\hline & Adenostyles glabra & E1 & + & . & . & + & . & . & . & . & . & 2 & 22 \\
\hline & Arabis alpina & E1 & . & . & . & + & . & + & . & . & . & 2 & 22 \\
\hline & Minuartia austriaca & E1 & . & + & . & . & . & . & . & . & . & 1 & 11 \\
\hline \multirow[t]{3}{*}{ Сy } & Cystopteridion fragilis & & & & & & & & & & & & \\
\hline & Cystopteris fragilis & E1 & + & + & . & + & + & + & + & . & + & 7 & 78 \\
\hline & Sedum hispanicum & E1 & . & . & . & . & . & + & . & . & . & 1 & 11 \\
\hline \multirow[t]{3}{*}{ PC } & Potentilletalia caulescentis & & & & & & & & & & & & \\
\hline & Campanula cochleariifolia & E1 & . & + & + & . & + & + & . & . & . & 4 & 44 \\
\hline & Festuca stenantha & E1 & . & . & . & . & . & + & . & . & . & 1 & 11 \\
\hline \multirow[t]{8}{*}{ AT } & Asplenietea trichomanis & & & & & & & & & & & & \\
\hline & Asplenium viride & E1 & + & + & . & . & 1 & + & + & + & + & 7 & 78 \\
\hline & Asplenium ruta-muraria & E1 & + & . & . & . & + & + & + & . & . & 4 & 44 \\
\hline & Moehringia muscosa & E1 & . & + & . & . & + & + & + & . & . & 4 & 44 \\
\hline & Asplenium trichomanes & E1 & + & . & . & . & . & + & . & . & . & 2 & 22 \\
\hline & Polypodium vulgare & E1 & + & . & . & . & . & . & . & . & . & 1 & 11 \\
\hline & Kernera saxatilis & E1 & . & . & . & . & + & . & . & . & . & 1 & 11 \\
\hline & Silene saxifraga & E1 & . & . & . & . & . & + & . & . & . & 1 & 11 \\
\hline \multirow[t]{10}{*}{ ML } & Mosses and lichens (Mahovi in lišaji) & & & & & & & & & & & & \\
\hline & Ctenidium molluscum & E0 & 1 & + & + & . & + & + & + & . & + & 7 & 78 \\
\hline & Tortella tortuosa & E0 & + & + & + & . & + & + & . & . & + & 6 & 67 \\
\hline & Schistidium sp. & E0 & + & . & + & . & + & + & . & . & . & 4 & 44 \\
\hline & Peltigera canina & E0 & . & . & . & . & . & + & + & . & + & 3 & 33 \\
\hline & Cladonia pyxidata & E0 & + & . & . & . & . & + & . & . & . & 2 & 22 \\
\hline & Dicranum scoparium & E0 & . & + & . & . & . & . & + & . & . & 2 & 22 \\
\hline & Polytrichum formosum & E0 & . & + & . & . & . & . & . & . & . & 1 & 11 \\
\hline & Fissidens dubius & E0 & . & + & . & . & . & . & . & . & . & 1 & 11 \\
\hline & Plagiochila porelloides & E0 & . & . & . & . & + & . & . & . & . & 1 & 11 \\
\hline
\end{tabular}

\section{Legend - Legenda}

ID Igor Dakskobler

AR Andrej Rozman

A Limestone - apnenec

D Dolomite - dolomit

Re Rendzina - rendzina

JA Julian Alps - Julijske Alpe

KSA Kamnik-Savinja Alps - Kamniško-Savinjske Alpe

Pr. (number of relevés in which the species is presented) - število popisov, $v$ katerih se pojavlja vrsta

Fr. Frequency in $\%$ - frekvenca $v \%$ 


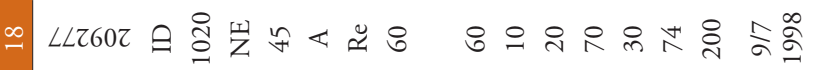

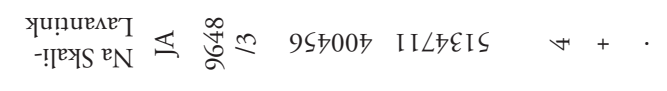

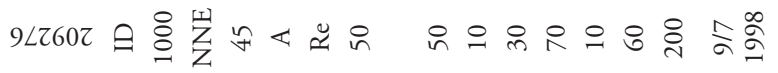

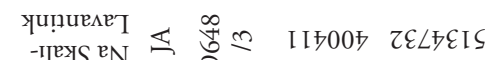

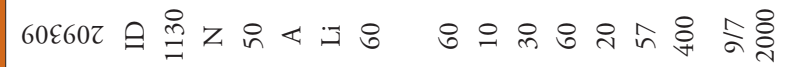

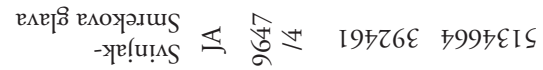

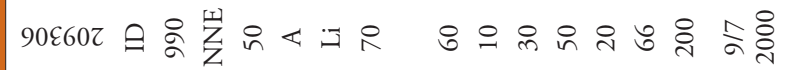

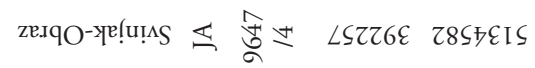

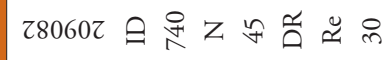

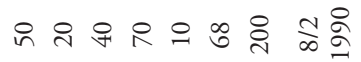

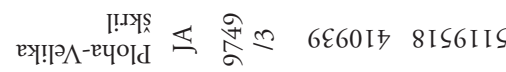

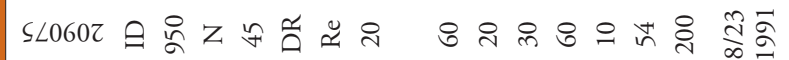

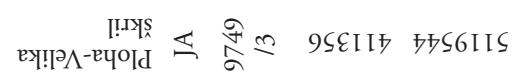

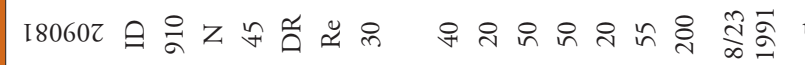

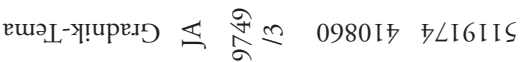

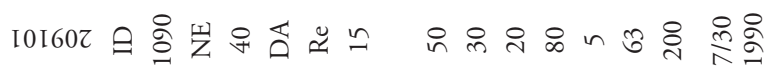
001602 요의

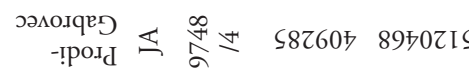

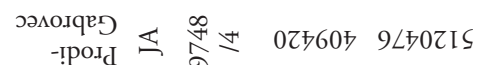

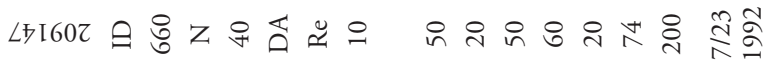

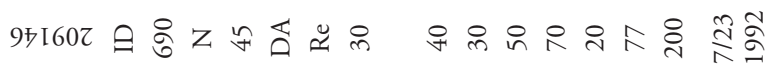

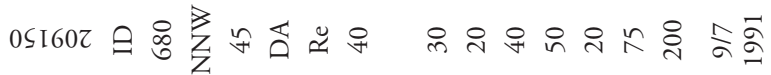

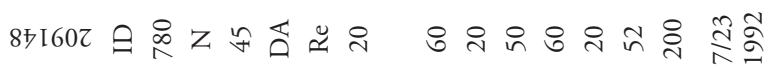

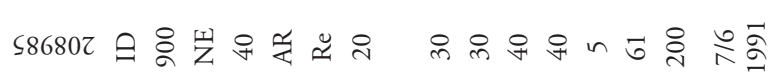

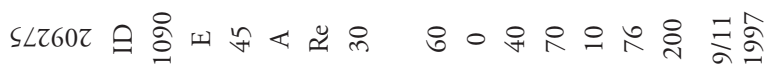

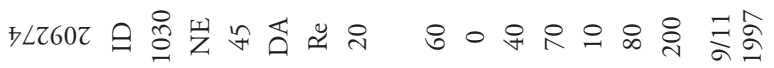

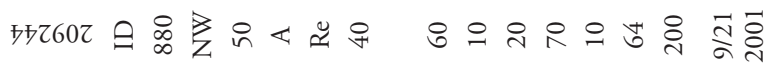

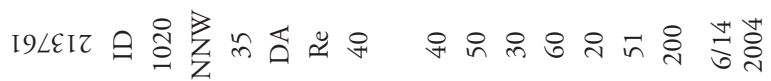

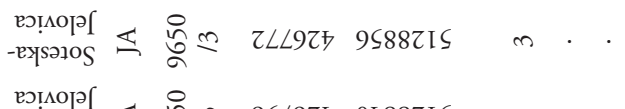

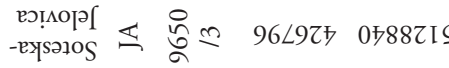

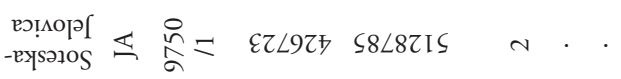

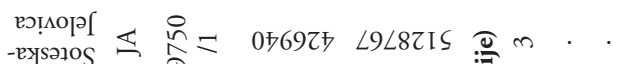

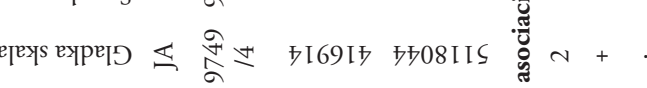

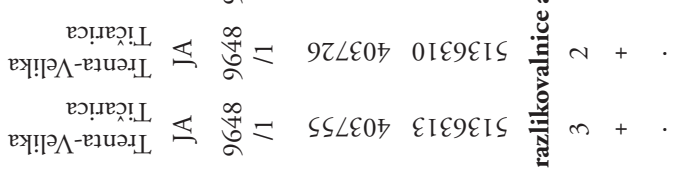

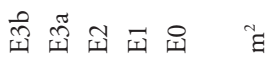

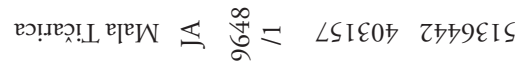

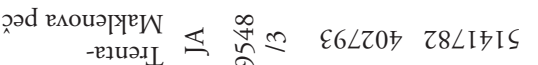
\& $n$ 


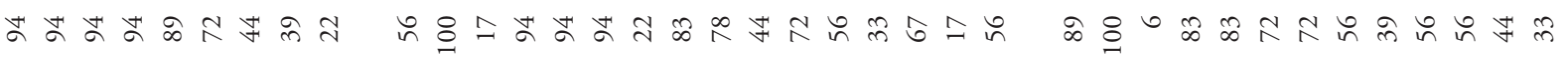

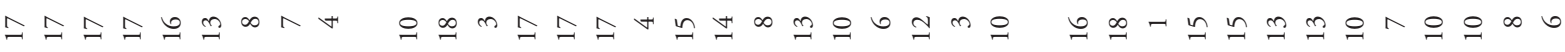

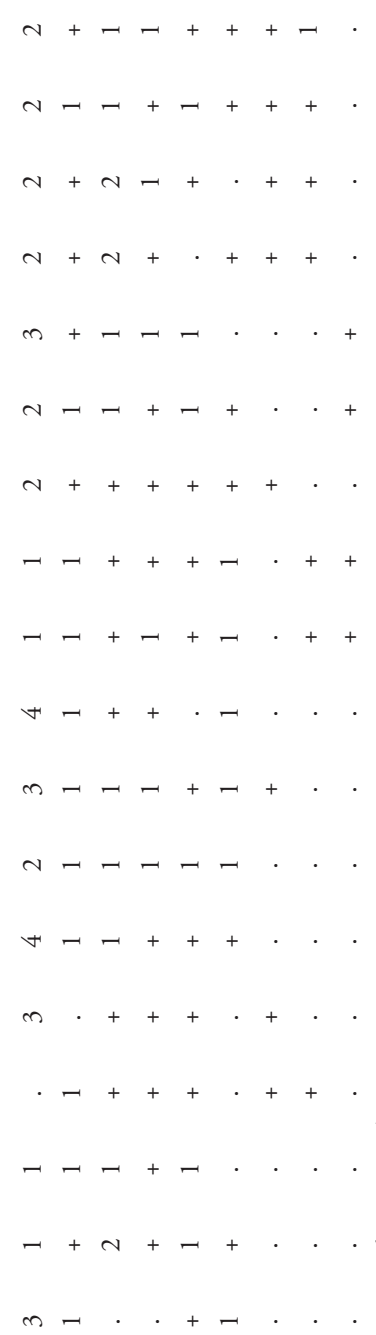

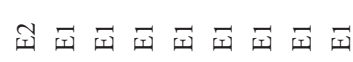

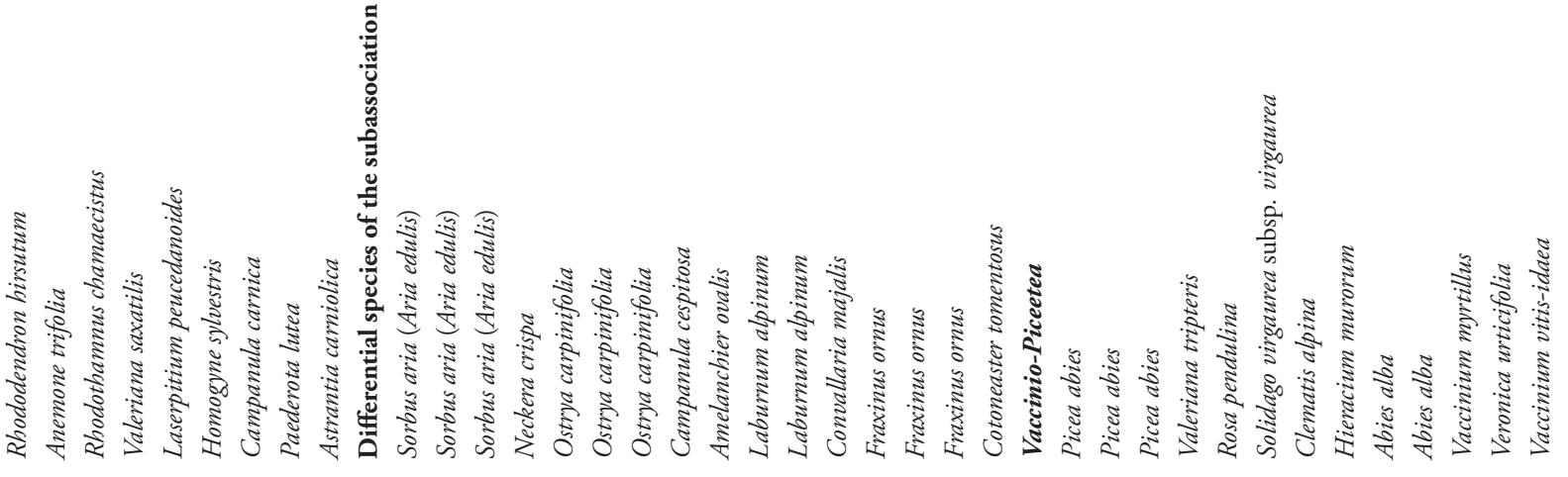

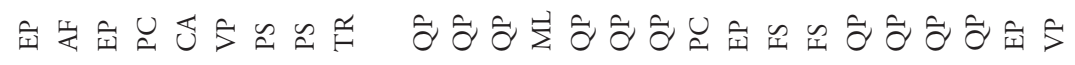


$\stackrel{\infty}{\sim} \stackrel{\infty}{\sim} \Xi z=z=\bullet \circ$

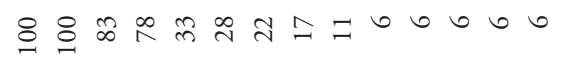

8 ป $=0$

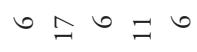

$\operatorname{Nin}$

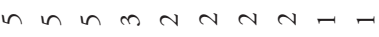

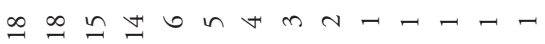

$\stackrel{\sim}{\infty} \sim$

$-m-n-$

$2=$
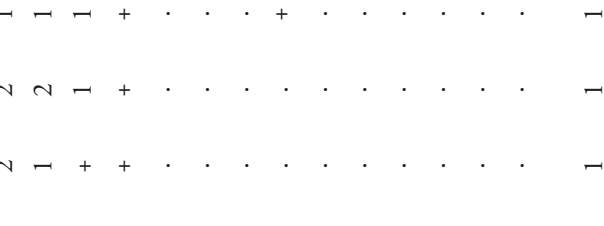

N $n-+$

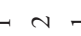

ข -+

$\mathrm{N}+$

$\cos (-10)$

$m \sim$

$2+$

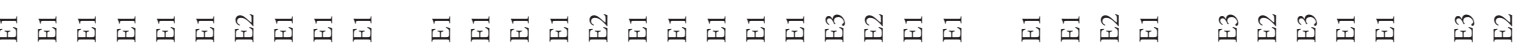




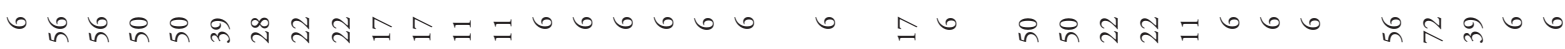

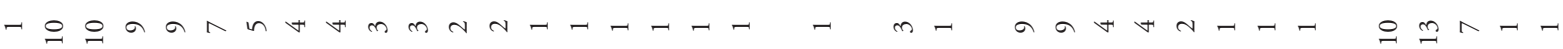

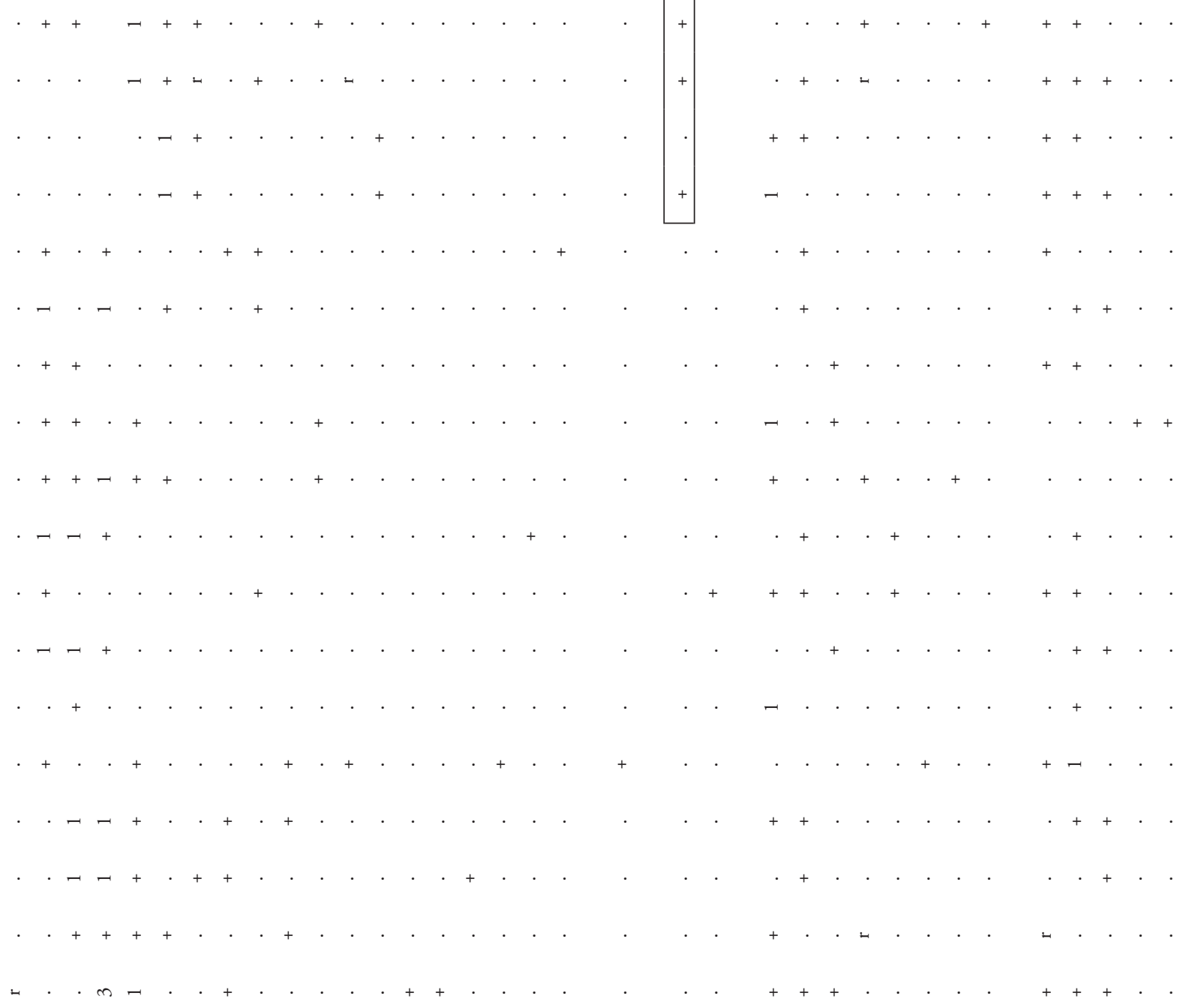

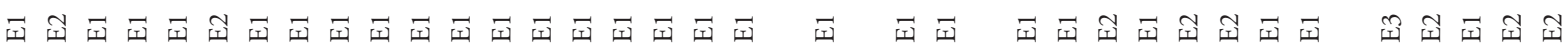

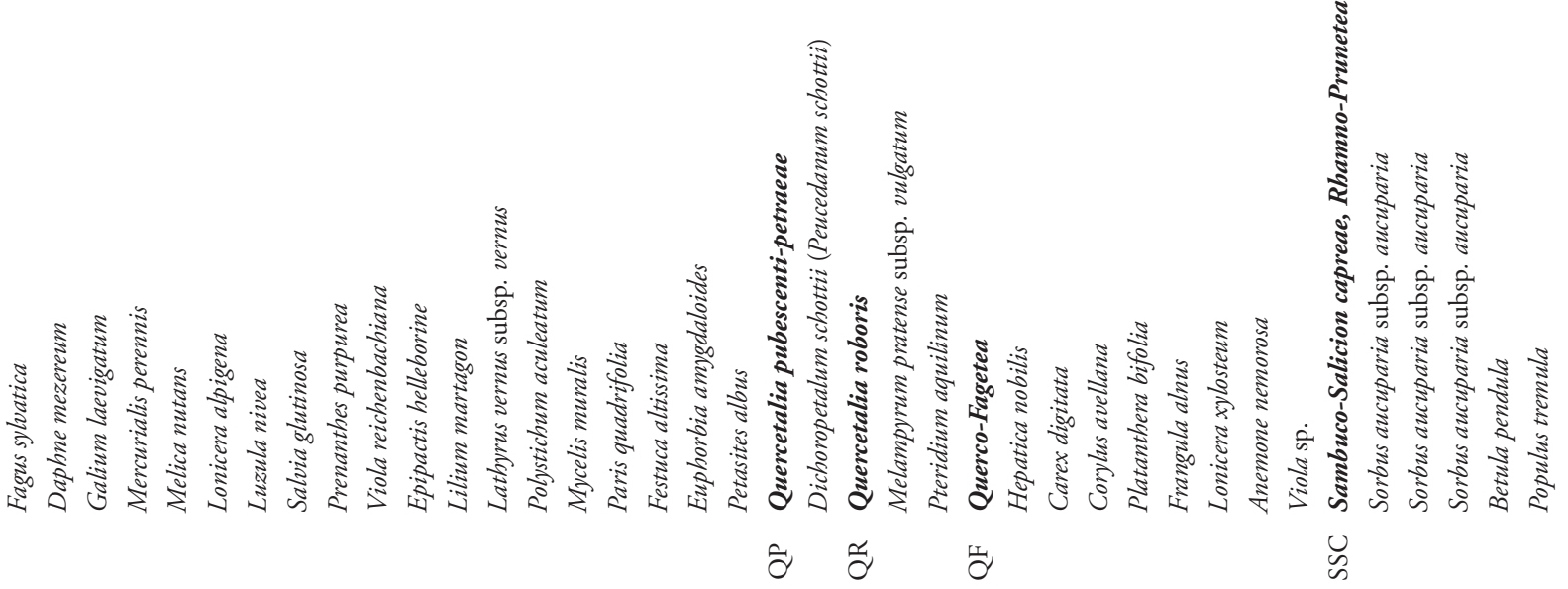




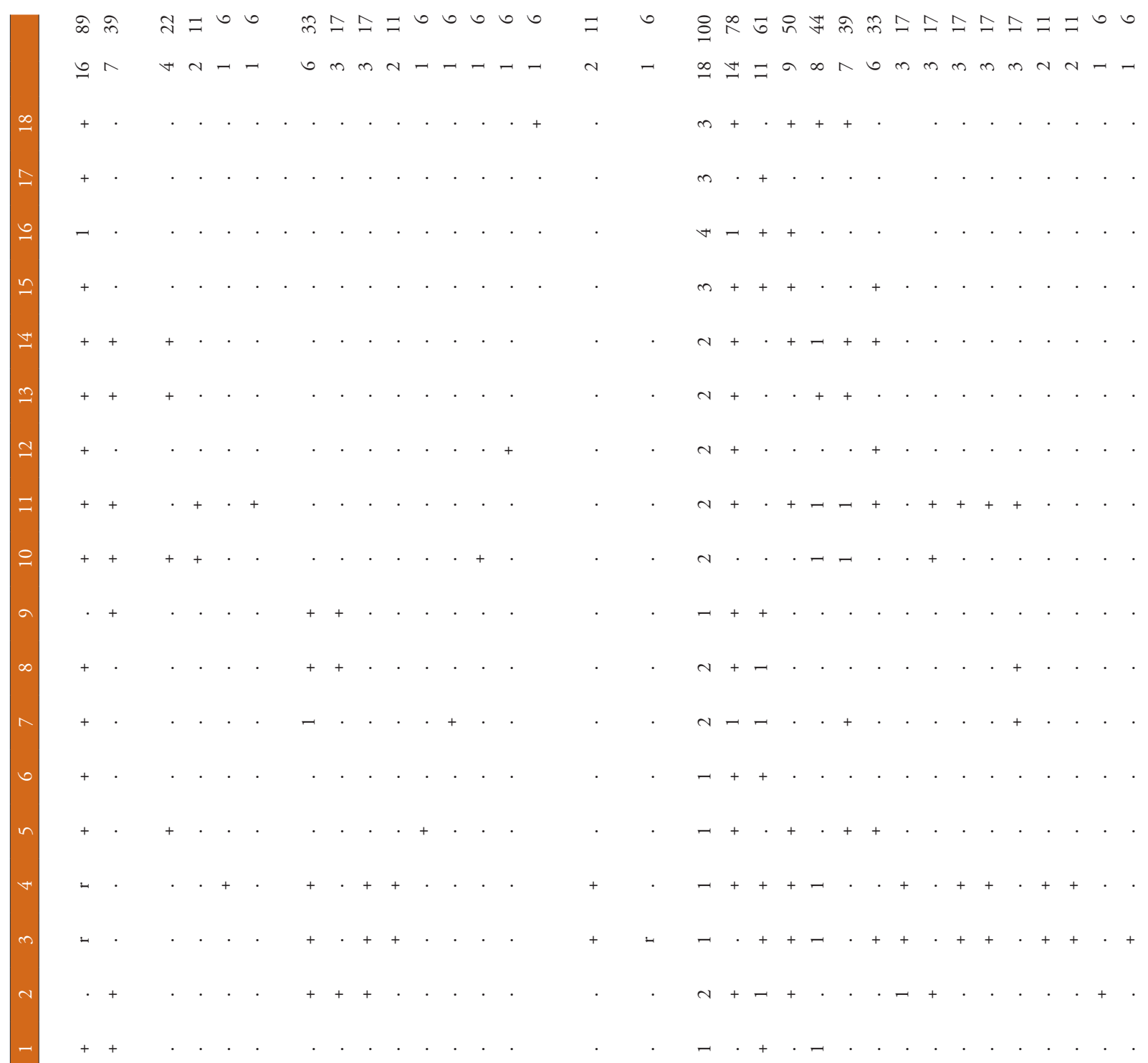

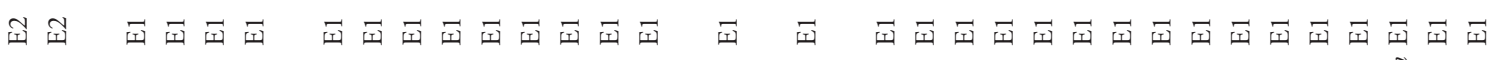

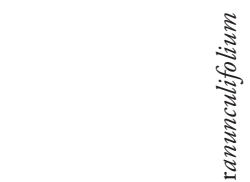

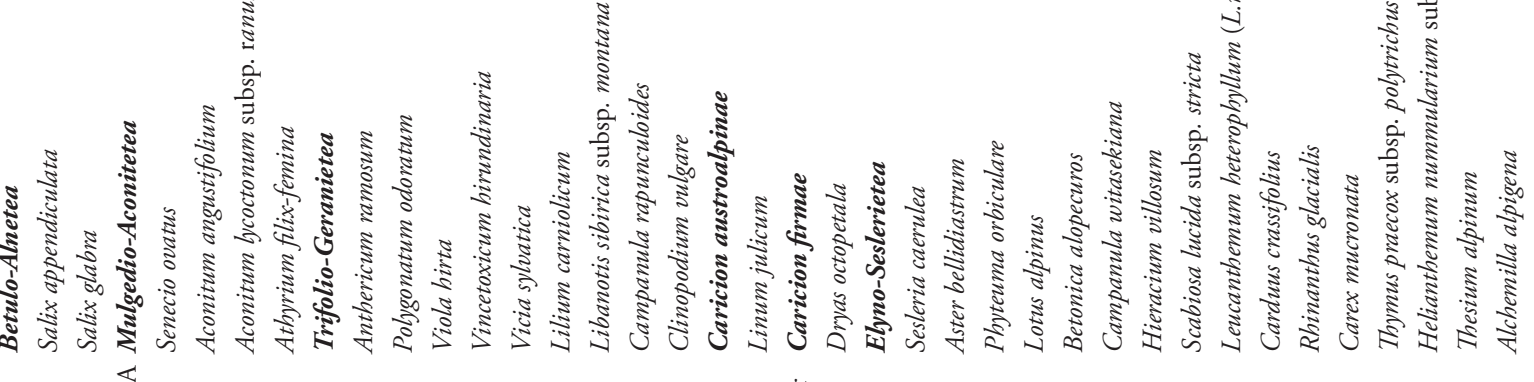

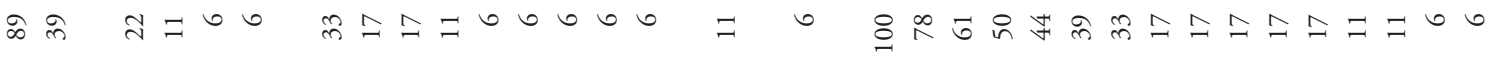
$\overleftrightarrow{\infty}$ 


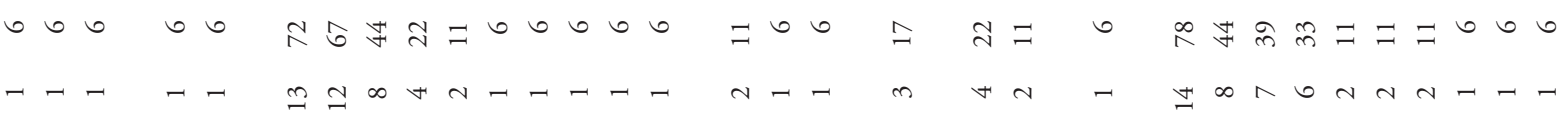

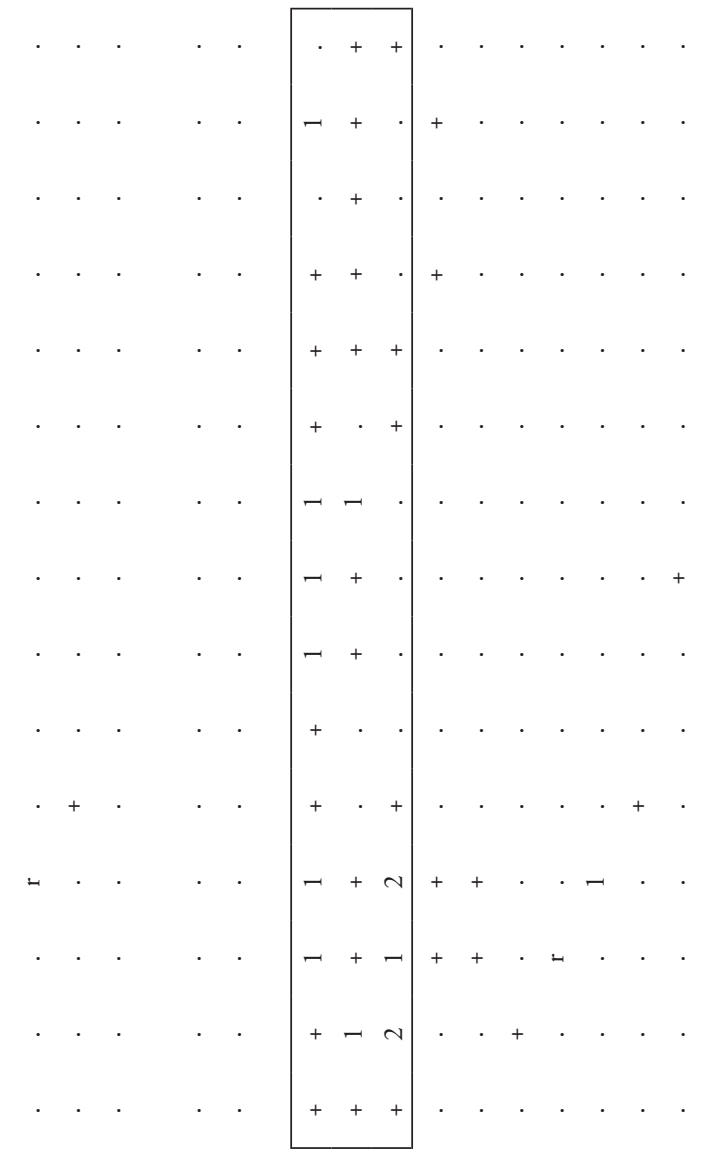

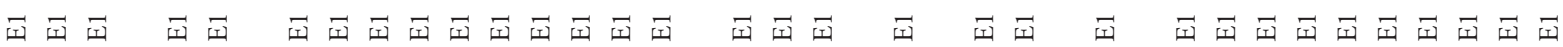

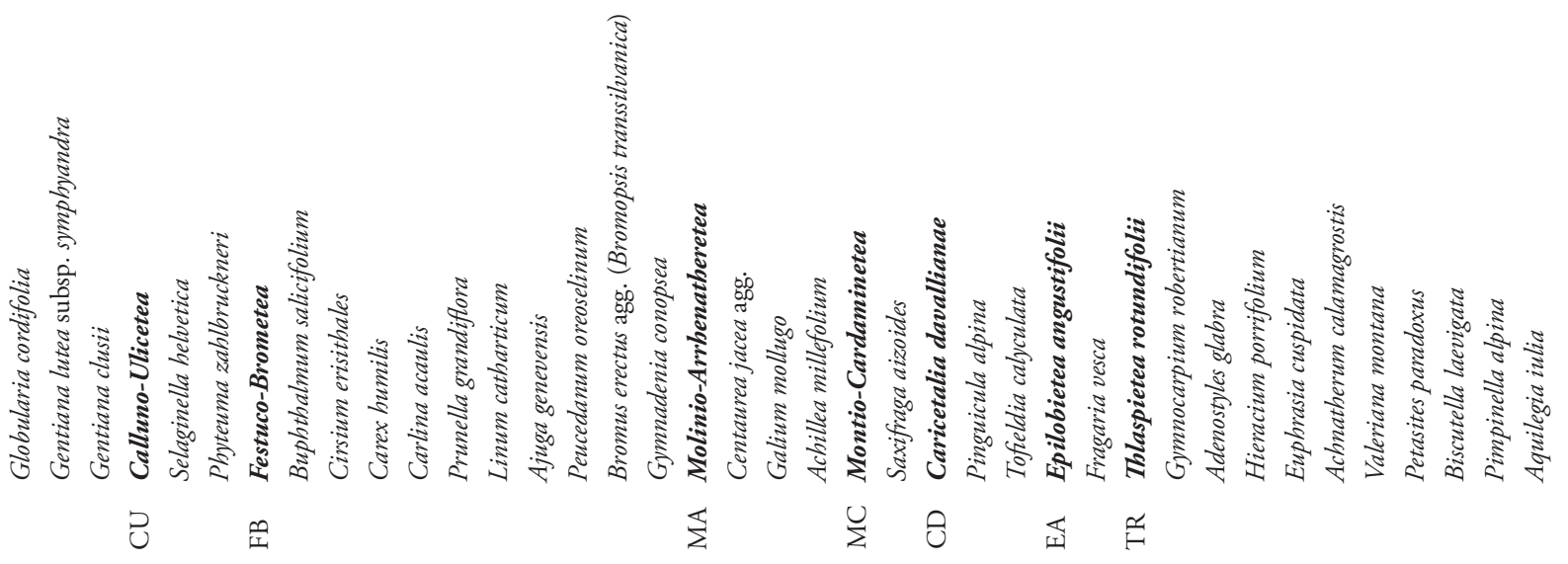




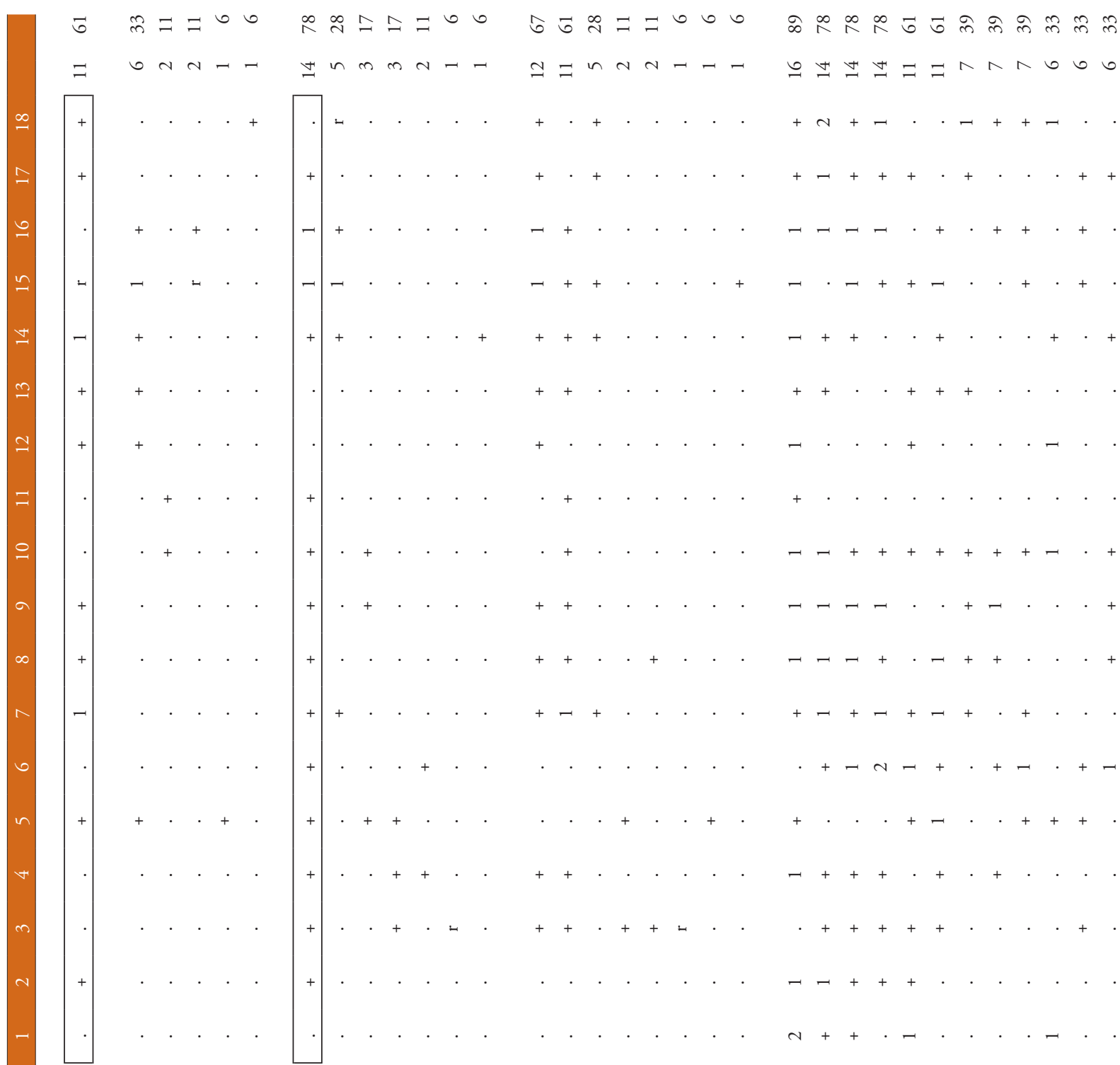

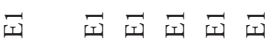

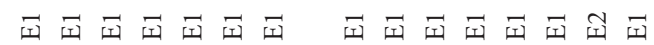

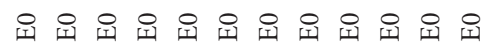

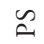
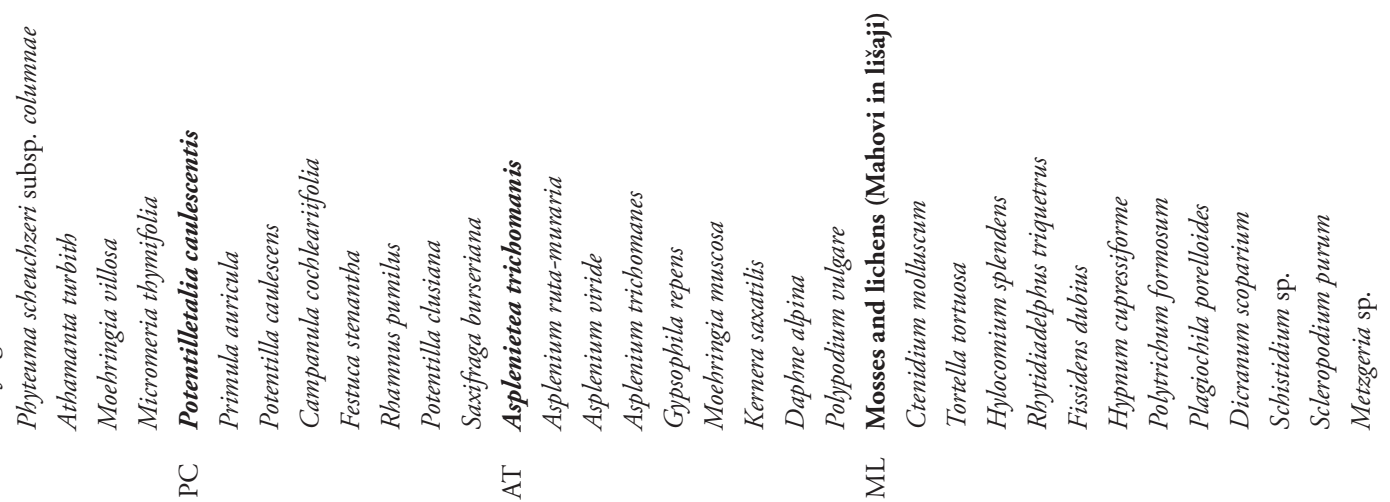


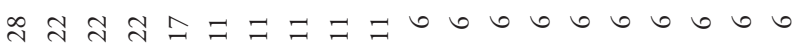

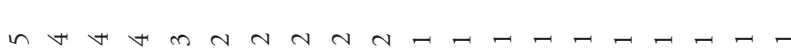

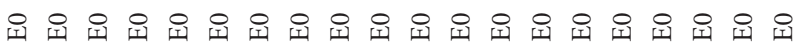

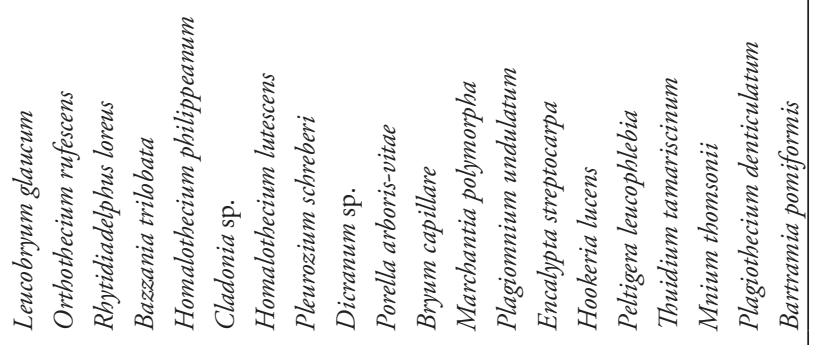

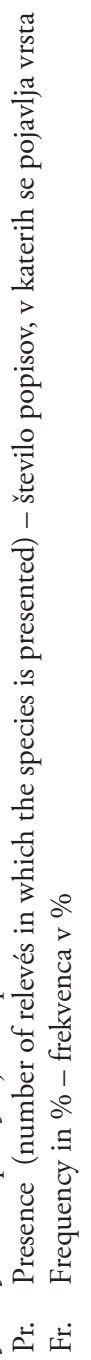


Table 25 (Tabela 25): Rhodothamno-Laricetum sorbetosum ariae

\begin{tabular}{|c|c|c|c|c|c|c|c|c|c|}
\hline \multicolumn{2}{|l|}{ Number of relevé (Zaporedna štev. popisa) } & 1 & 2 & 3 & 4 & 5 & 6 & 7 & 8 \\
\hline \multicolumn{2}{|l|}{$\begin{array}{l}\text { Database number of relevé } \\
\text { (Delovna številka popisa) }\end{array}$} & 236642 & 223130 & 209098 & 209099 & 209068 & 209079 & 208966 & 223134 \\
\hline \multicolumn{2}{|l|}{ Author of the relevé (Avtor popisa) } & ID & ID & ID & ID & ID & ID & ID & ID \\
\hline \multicolumn{2}{|l|}{ Elevation in $\mathrm{m}$ (Nadmorska višina $\mathrm{v} \mathrm{m})$} & 1420 & 1360 & 1370 & 1240 & 1190 & 1190 & 1420 & 1530 \\
\hline \multicolumn{2}{|l|}{ Aspect (Lega) } & $\mathrm{N}$ & $\mathrm{N}$ & NW & $\mathrm{N}$ & NW & $\mathrm{N}$ & NW & $\mathrm{N}$ \\
\hline \multicolumn{2}{|l|}{ Slope in degrees (Nagib v stopinjah) } & 45 & 35 & 45 & 40 & 45 & 40 & 35 & 45 \\
\hline \multicolumn{2}{|l|}{ Parent material (Matična podlaga) } & DA & DA & DA & DA & ALR & DR & DA & DA \\
\hline \multicolumn{2}{|l|}{ Soil (Tla) } & $\operatorname{Re}$ & $\operatorname{Re}$ & $\operatorname{Re}$ & $\operatorname{Re}$ & $\operatorname{Re}$ & $\operatorname{Re}$ & $\operatorname{Re}$ & $\operatorname{Re}$ \\
\hline \multicolumn{2}{|l|}{ Stoniness in \% (Kamnitost v \%) } & 20 & 30 & 10 & 20 & 40 & 20 & 30 & 20 \\
\hline \multicolumn{10}{|l|}{ Cover in \% (Zastiranje v \%): } \\
\hline Upper tree layer (Zgor. drevesna plast) & $\mathrm{E} 3 \mathrm{~b}$ & 70 & 60 & 60 & 40 & 70 & 60 & 20 & 60 \\
\hline Lower tree layer (Spod. drevesna plast) & E3a & 10 & 20 & 0 & 0 & 10 & 20 & 20 & 0 \\
\hline Shrub layer (Grmovna plast) & E2 & 30 & 40 & 40 & 80 & 40 & 60 & 70 & 70 \\
\hline Herb layer (Zeliščna plast) & E1 & 80 & 50 & 80 & 60 & 40 & 70 & 40 & 30 \\
\hline Moss layer (Mahovna plast) & E0 & 10 & 10 & 5 & 10 & 30 & 20 & 20 & 10 \\
\hline Number of species (Število vrst) & & 63 & 57 & 49 & 55 & 69 & 83 & 55 & 44 \\
\hline Relevé area (Velikost popisne ploskve) & $\mathrm{m}^{2}$ & 400 & 200 & 200 & 200 & 200 & 200 & 200 & 100 \\
\hline Date of taking relevé (Datum popisa) & & $\begin{array}{l}6 / 11 \\
2010\end{array}$ & $\begin{array}{c}8 / 8 \\
1991\end{array}$ & $\begin{array}{l}7 / 18 \\
1995\end{array}$ & $\begin{array}{l}6 / 19 \\
1993\end{array}$ & $\begin{array}{l}5 / 31 \\
2002\end{array}$ & $\begin{array}{c}8 / 3 \\
1990\end{array}$ & $\begin{array}{l}7 / 17 \\
1996\end{array}$ & $\begin{array}{l}7 / 23 \\
1993\end{array}$ \\
\hline Locality (Nahajališče) & & 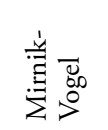 & 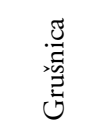 & 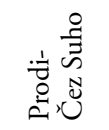 & 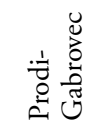 & $\frac{\pi}{0}$ & 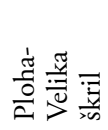 & 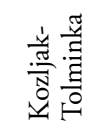 & 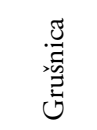 \\
\hline Mountain range (Pogorje) & & JA & JA & JA & JA & JA & JA & JA & JA \\
\hline Quadrant (Kvadrant) & & $9749 / 1$ & $9748 / 4$ & $9749 / 3$ & $9748 / 4$ & $9749 / 3$ & $9749 / 3$ & $9748 / 4$ & $9748 / 4$ \\
\hline Coordinate GK-Y (D-48) & $\mathrm{m}$ & 410999 & 403849 & 410822 & 409071 & 411871 & 411919 & 404157 & 403920 \\
\hline Coordinate GK-X (D-48) & $\mathrm{m}$ & 5125152 & 5122742 & 5121275 & 5120480 & 5119464 & 5119488 & 5123354 & 5122514 \\
\hline
\end{tabular}

inate GK-X (D-48)

association (Značilnice in razlikovalnice asociacije)

Pr. Fr.

$\begin{array}{ll}\text { VP } & \text { Larix decidua } \\ \text { VP } & \text { Larix decidua } \\ \text { EP } & \text { Rhododendron hirsutum } \\ \text { PS } & \text { Paederota lutea } \\ \text { PC } & \text { Valeriana saxatilis } \\ \text { EP } & \text { Rhodothamnus chamaecistus } \\ \text { CA } & \text { Laserpitium peucedanoides } \\ \text { TR } & \text { Astrantia carniolica } \\ \text { PS } & \text { Campanula carnica } \\ \text { AF } & \text { Anemone trifolia } \\ \text { CF } & \text { Carex ferruginea }\end{array}$

E3
E2
E2
E1
E1
E1
E1
E1
E1
E1
E1

Differential species of the subassociation (Razlikovalnice subasociacije)

SSC Sorbus aucuparia subsp. aucuparia

SSC Sorbus aucuparia subsp. aucuparia

$\begin{array}{rrrrrrrrrrr}\text { E3 } & + & . & + & + & + & 1 & + & 1 & 7 & 88 \\ \text { E2 } & 1 & + & 1 & 1 & . & 1 & + & 1 & 7 & 88 \\ \text { E1 } & + & . & . & 1 & + & . & \text { r } & . & 4 & 50 \\ \text { E3 } & \cdot & . & . & . & \text { r } & + & . & . & 2 & 25 \\ \text { E2 } & + & + & . & + & + & + & + & + & 7 & 88 \\ \text { E2 } & 1 & . & . & \text { r } & . & . & + & + & 4 & 50 \\ & & & & & & & & & & \\ \text { E1 } & 1 & + & + & + & + & + & + & 1 & 8 & 100 \\ \text { E1 } & + & + & 1 & + & + & 1 & + & + & 8 & 100 \\ \text { E2 } & 2 & + & 1 & 1 & + & + & 1 & 1 & 8 & 100 \\ \text { E1 } & + & 1 & + & + & + & + & 1 & 1 & 8 & 100 \\ \text { E1 } & \cdot & + & 1 & + & 2 & 1 & 1 & + & 7 & 88 \\ \text { E3 } & . & . & + & . & . & . & . & . & 1 & 13\end{array}$




\begin{tabular}{|c|c|c|c|c|c|c|c|c|c|c|c|c|}
\hline & \multicolumn{2}{|c|}{ Number of relevé (Zaporedna štev. popisa) } & 1 & 2 & 3 & 4 & 5 & 6 & 7 & 8 & Pr. & Fr. \\
\hline & Picea abies & E2 & . & + & + & + & $\mathrm{r}$ & + & $\mathrm{r}$ & + & 7 & 88 \\
\hline & Clematis alpina & E2 & 1 & . & 1 & + & 1 & 2 & 1 & 1 & 7 & 88 \\
\hline & Calamagrostis arundinacea & E1 & . & 2 & 2 & + & 1 & 1 & 2 & 1 & 7 & 88 \\
\hline & Lycopodium annotinum & E1 & . & + & + & + & + & + & + & . & 6 & 75 \\
\hline & Homogyne sylvestris & E1 & 1 & + & + & + & . & . & 1 & + & 6 & 75 \\
\hline & Gymnocarpium dryopteris & E1 & + & + & . & . & + & + & + & + & 6 & 75 \\
\hline & Huperzia selago & E1 & + & . & . & + & r & + & + & . & 5 & 63 \\
\hline & Solidago virgaurea subsp. virgaurea & E1 & . & . & + & + & . & 1 & 1 & + & 5 & 63 \\
\hline & Polystichum lonchitis & E1 & . & + & + & . & r & + & . & . & 4 & 50 \\
\hline & Oxalis acetosella & E1 & . & 1 & . & . & + & 1 & + & . & 4 & 50 \\
\hline & Calamagrostis villosa & E1 & 1 & . & . & + & . & . & . & 1 & 3 & 38 \\
\hline & Abies alba & E3 & + & . & . & . & + & 1 & . & . & 3 & 38 \\
\hline & Abies alba & E2 & + & . & + & . & . & + & . & . & 3 & 38 \\
\hline & Abies alba & E1 & . & . & . & . & + & . & . & . & 1 & 13 \\
\hline & Gentiana asclepiadea & E1 & + & + & . & . & . & + & . & . & 3 & 38 \\
\hline & Lonicera nigra & E2 & + & . & . & . & 1 & 1 & . & . & 3 & 38 \\
\hline & Veronica urticifolia & E1 & + & . & . & $\mathrm{r}$ & . & + & . & . & 3 & 38 \\
\hline & Dryopteris dilatata & E1 & . & . & . & + & + & + & . & . & 3 & 38 \\
\hline & Homogyne alpina & E1 & . & . & . & + & . & . & + & + & 3 & 38 \\
\hline & Aposeris foetida & E1 & + & + & . & . & . & . & . & . & 2 & 25 \\
\hline & Luzula sylvatica & E1 & + & . & . & . & . & . & + & . & 2 & 25 \\
\hline & Maianthemum bifolium & E1 & . & 1 & . & . & . & . & + & . & 2 & 25 \\
\hline & Saxifraga cuneifolia & E1 & . & . & + & . & . & . & . & + & 2 & 25 \\
\hline & Lonicera caerulea & E2 & . & . & . & . & . & 1 & . & 1 & 2 & 25 \\
\hline & Hieracium murorum & E1 & + & . & . & . & . & . & . & . & 1 & 13 \\
\hline & Melampyrum sylvaticum & E1 & . & . & + & . & . & . & . & . & 1 & 13 \\
\hline EP & Erico-Pinetea & & & & & & & & & & & \\
\hline & Pinus mugo & E2 & + & 1 & 2 & 2 & 2 & 3 & 4 & 4 & 8 & 100 \\
\hline & Erica carnea & E1 & 2 & 1 & 1 & 2 & + & + & + & + & 8 & 100 \\
\hline & Rubus saxatilis & E1 & 1 & 1 & 1 & 1 & . & + & + & 1 & 7 & 88 \\
\hline & Calamagrostis varia & E1 & + & + & + & + & + & 1 & + & . & 7 & 88 \\
\hline & Carex alba & E1 & . & . & + & + & . & . & . & . & 2 & 25 \\
\hline & Aquilegia nigricans & E1 & . & . & + & . & . & . & . & . & 1 & 13 \\
\hline & Amelanchier ovalis & E2 & . & . & . & + & . & . & . & . & 1 & 13 \\
\hline & Carex ornithopoda & E1 & . & . & . & . & + & . & . & . & 1 & 13 \\
\hline $\mathrm{AF}$ & Aremonio-Fagion & & & & & & & & & & & \\
\hline & Cyclamen purpurascens & E1 & 1 & 1 & 1 & 1 & . & . & + & . & 5 & 63 \\
\hline & Cardamine enneaphyllos & E1 & + & 1 & 1 & . & . & . & + & . & 4 & 50 \\
\hline TA & Tilio-Acerion & & & & & & & & & & & \\
\hline & Acer pseudoplatanus & E3 & . & . & . & . & $\mathrm{r}$ & + & . & . & 2 & 25 \\
\hline & Acer pseudoplatanus & E2 & + & . & . & . & . & . & . & . & 1 & 13 \\
\hline & Acer pseudoplatanus & E1 & . & . & . & . & + & . & . & . & 1 & 13 \\
\hline & Aruncus dioicus & E1 & + & . & . & . & . & . & . & . & 1 & 13 \\
\hline FS & Fagetalia sylvaticae & & & & & & & & & & & \\
\hline & Fagus sylvatica & E3 & 3 & 2 & + & + & . & + & 1 & + & 7 & 88 \\
\hline & Fagus sylvatica & $\mathrm{E} 2$ & 1 & 1 & . & + & $\mathrm{r}$ & . & + & . & 5 & 63 \\
\hline & Fagus sylvatica & E1 & + & . & . & . & . & . & . & . & 1 & 13 \\
\hline & Lonicera alpigena & E2 & + & 1 & . & 1 & + & 1 & 1 & . & 6 & 75 \\
\hline & Mercurialis perennis & E1 & + & 1 & 1 & . & + & + & . & . & 5 & 63 \\
\hline & Prenanthes purpurea & E1 & 1 & . & . & + & + & + & . & . & 4 & 50 \\
\hline & Galium laevigatum & E1 & + & + & + & . & . & + & . & . & 4 & 50 \\
\hline & Laburnum alpinum & E3 & + & . & . & . & + & + & . & . & 3 & 38 \\
\hline & Laburnum alpinum & E2 & 1 & . & . & . & . & + & . & . & 2 & 25 \\
\hline & Melica nutans & E1 & . & + & 1 & . & . & . & $\mathrm{r}$ & . & 3 & 38 \\
\hline
\end{tabular}




\begin{tabular}{|c|c|c|c|c|c|c|c|c|c|c|c|c|}
\hline & \multicolumn{2}{|c|}{ Number of relevé (Zaporedna štev. popisa) } & 1 & 2 & 3 & 4 & 5 & 6 & 7 & 8 & Pr. & Fr. \\
\hline & Daphne mezereum & E2 & . & . & + & . & . & + & . & . & 2 & 25 \\
\hline & Polystichum aculeatum & E1 & . & . & . & . & $\mathrm{r}$ & + & . & . & 2 & 25 \\
\hline & Lilium martagon & E1 & + & . & . & . & . & . & . & . & 1 & 13 \\
\hline & Dryopteris filix-mas & E1 & . & . & . & . & + & . & . & . & 1 & 13 \\
\hline & Lathyrus vernus subsp. flaccidus & E1 & . & . & . & . & . & + & . & . & 1 & 13 \\
\hline \multirow[t]{5}{*}{ QP } & Quercetalia pubescenti-petraeae & & & & & & & & & & & \\
\hline & Convallaria majalis & E1 & + & + & . & + & . & . & . & + & 4 & 50 \\
\hline & Ostrya carpinifolia & E3 & . & . & . & . & $\mathrm{r}$ & . & . & . & 1 & 13 \\
\hline & Ostrya carpinifolia & E2 & . & . & . & . & $\mathrm{r}$ & + & . & . & 2 & 25 \\
\hline & Arabis turrita & E1 & . & . & . & . & + & . & . & . & 1 & 13 \\
\hline \multirow[t]{4}{*}{ QF } & Querco-Fagetea & & & & & & & & & & & \\
\hline & Anemone nemorosa & E1 & 1 & . & 1 & 1 & . & + & + & . & 5 & 63 \\
\hline & Hepatica nobilis & E1 & . & 1 & + & + & . & + & . & . & 4 & 50 \\
\hline & Carex digitata & E1 & + & . & + & . & . & . & . & . & 2 & 25 \\
\hline \multirow[t]{6}{*}{ BA } & Betulo-Alnetea & & & & & & & & & & & \\
\hline & Salix appendiculata & E2 & . & + & 1 & 1 & 1 & 2 & $\mathrm{r}$ & 1 & 7 & 88 \\
\hline & Salix glabra & E2 & . & + & + & 1 & . & . & + & 1 & 5 & 63 \\
\hline & Sorbus austriaca s.lat. & E3 & . & . & + & . & . & . & . & . & 1 & 13 \\
\hline & Sorbus austriaca s.lat. & E2 & . & . & + & . & . & . & . & . & 1 & 13 \\
\hline & Alnus viridis & E2 & . & . & . & . & . & + & . & . & 1 & 13 \\
\hline \multicolumn{13}{|c|}{ MuA Mulgedio-Aconietetea } \\
\hline & Athyrium filix-femina & E1 & . & . & + & . & $\mathrm{r}$ & + & $\mathrm{r}$ & . & 4 & 50 \\
\hline & Polygonatum verticillatum & E1 & + & + & . & . & . & . & . & . & 2 & 25 \\
\hline & Senecio ovatus & E1 & . & . & . & . & + & + & . & . & 2 & 25 \\
\hline & Veratrum album subsp. lobelianum & E1 & 1 & . & . & . & . & . & . & . & 1 & 13 \\
\hline & Phyteuma ovatum & E1 & + & . & . & . & . & . & . & . & 1 & 13 \\
\hline & Viola biflora & E1 & . & . & . & . & . & . & + & . & 1 & 13 \\
\hline \multirow[t]{2}{*}{ TG } & Trifolio-Geranietea & & & & & & & & & & & \\
\hline & Verbascum lanatum & E1 & . & . & . & . & . & + & . & . & 1 & 13 \\
\hline \multirow[t]{2}{*}{ Cfir } & Caricion firmae & & & & & & & & & & & \\
\hline & Carex firma & E1 & . & . & . & . & . & . & . & + & 1 & 13 \\
\hline \multirow[t]{10}{*}{ ES } & Elyno-Seslerietea & & & & & & & & & & & \\
\hline & Sesleria caerulea & E1 & + & 2 & 2 & 3 & + & 1 & 1 & 1 & 8 & 100 \\
\hline & Betonica alopecuros & E1 & + & + & + & . & . & + & . & . & 4 & 50 \\
\hline & Aster bellidiastrum & E1 & + & . & + & . & + & . & . & . & 3 & 38 \\
\hline & Campanula witasekiana & E1 & . & . & . & . & + & + & . & + & 3 & 38 \\
\hline & Polygonum viviparum & E1 & . & + & . & + & . & . & . & . & 2 & 25 \\
\hline & Phyteuma orbiculare & E1 & + & . & . & . & . & . & . & . & 1 & 13 \\
\hline & Selaginella selaginoides & E1 & . & + & . & . & . & . & . & . & 1 & 13 \\
\hline & Carex sempervirens & E1 & . & + & . & . & . & . & . & . & 1 & 13 \\
\hline & Carex mucronata & E1 & . & . & . & + & . & . & . & . & 1 & 13 \\
\hline \multirow[t]{3}{*}{ JT } & Juncetea trifidi & & & & & & & & & & & \\
\hline & Campanula scheuchzeri & E1 & + & 1 & . & . & . & . & . & . & 2 & 25 \\
\hline & Solidago virgaurea subsp. minuta & E1 & . & . & . & + & . & . & . & . & 1 & 13 \\
\hline \multirow[t]{4}{*}{ FB } & Festuco-Brometea & & & & & & & & & & & \\
\hline & Cirsium erisithales & E1 & + & . & . & . & + & + & . & . & 3 & 38 \\
\hline & Carex humilis & E1 & . & . & . & + & . & . & . & . & 1 & 13 \\
\hline & Carlina acaulis & E1 & . & . & . & . & $\mathrm{r}$ & . & . & . & 1 & 13 \\
\hline \multirow[t]{2}{*}{ MA } & Molinio-Arrhenatheretea & & & & & & & & & & & \\
\hline & Achillea millefolium & E1 & . & . & . & . & + & . & . & . & 1 & 13 \\
\hline \multirow[t]{2}{*}{ MC } & Montio-Cardaminetea & & & & & & & & & & & \\
\hline & Saxifraga aizoides & E1 & . & . & . & . & $\mathrm{r}$ & + & . & . & 2 & 25 \\
\hline \multirow[t]{2}{*}{$\mathrm{CD}$} & Caricetalia davallianae & & & & & & & & & & & \\
\hline & Parnassia palustris & E1 & . & . & . & . & . & + & . & . & 1 & 13 \\
\hline \multirow[t]{3}{*}{ EA } & Epilobietea angustifolii & & & & & & & & & & & \\
\hline & Rubus idaeus & E2 & . & . & . & . & + & 1 & . & . & 2 & 25 \\
\hline & Urtica dioica & E1 & . & . & . & . & . & + & . & . & 1 & 13 \\
\hline
\end{tabular}


$\begin{array}{lllllllllll}\text { Number of relevé (Zaporedna štev. popisa) } & 1 & 2 & 3 & 4 & 5 & 6 & 7 & 8 & \text { Pr. } & \text { Fr. }\end{array}$

TR Thlasopietea rotundifolii

Adenostyles glabra

Gymnocarpium robertianum

Aquilegia einseleana

Trisetum argenteum

Saxifraga caesia

Biscutella laevigata

Dryopteris villarii

Pimpinella alpina

Cy Cystopteridion fragilis

Cystopteris fragilis

Carex brachystachys

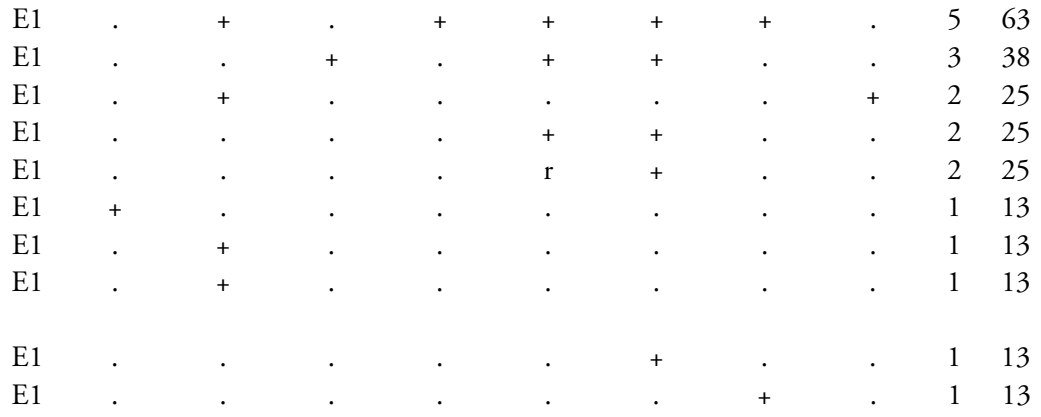

PS Physoplexido comosae-Saxifragion petraeae

Saxifraga crustata

E1

PC Potentilletalia caulescentis

Primula auricula

Campanula cochleariifolia

AT Asplenietea trichomanis

Asplenium viride

Asplenium ruta-muraria

E1-20.

ML Mosses and lichens (Mahovi in lišaji)

Ctenidium molluscum

Dicranum scoparium

Tortella tortuosa

Rhytidiadelphus triquetrus

Fissidens dubius

Orthothecium rufescens

Hylocomium splendens

Plagiochila porelloides

Polytrichum formosum

Neckera crispa

Hypnum cupressiforme

Marchantia polymorpha

Mnium thomsonii

Dicranum sp.

Thuidium tamariscinum

Cladonia pyxidata

Cladonia sp.

Dicranella heteromalla

Bryum capillare

E1

E1

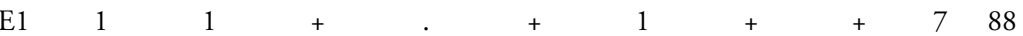

Musci spp.

Plagiothecium denticulatum

Cladonia fimbriata

$\mathrm{E} 1 . \quad . \quad . \quad+\quad . \quad . \quad . \quad 2525$

E0

E0

E0

E0

$\mathrm{E} 0$

E0

E0

E0

0

E0

E0

E0

E0

0

0

0

E0

E0

E0

E0

E0

E0

\section{Legend - Legenda}

ID Igor Dakskobler

A Limestone-apnenec

D Dolomite - dolomit

L Marlstone - laporovec

R Chert-roženec

Re Rendzina - rendzina

JA Julian Alps - Julijske Alpe

Pr. Presence (number of relevés in which the species is presented) - število popisov, v katerih se pojavlja vrsta

Fr. Frequency in $\%$ - frekvenca $\mathrm{\%} \%$

CA Caricion austroalpinae

SSC Sambuco-Salicion capreae 


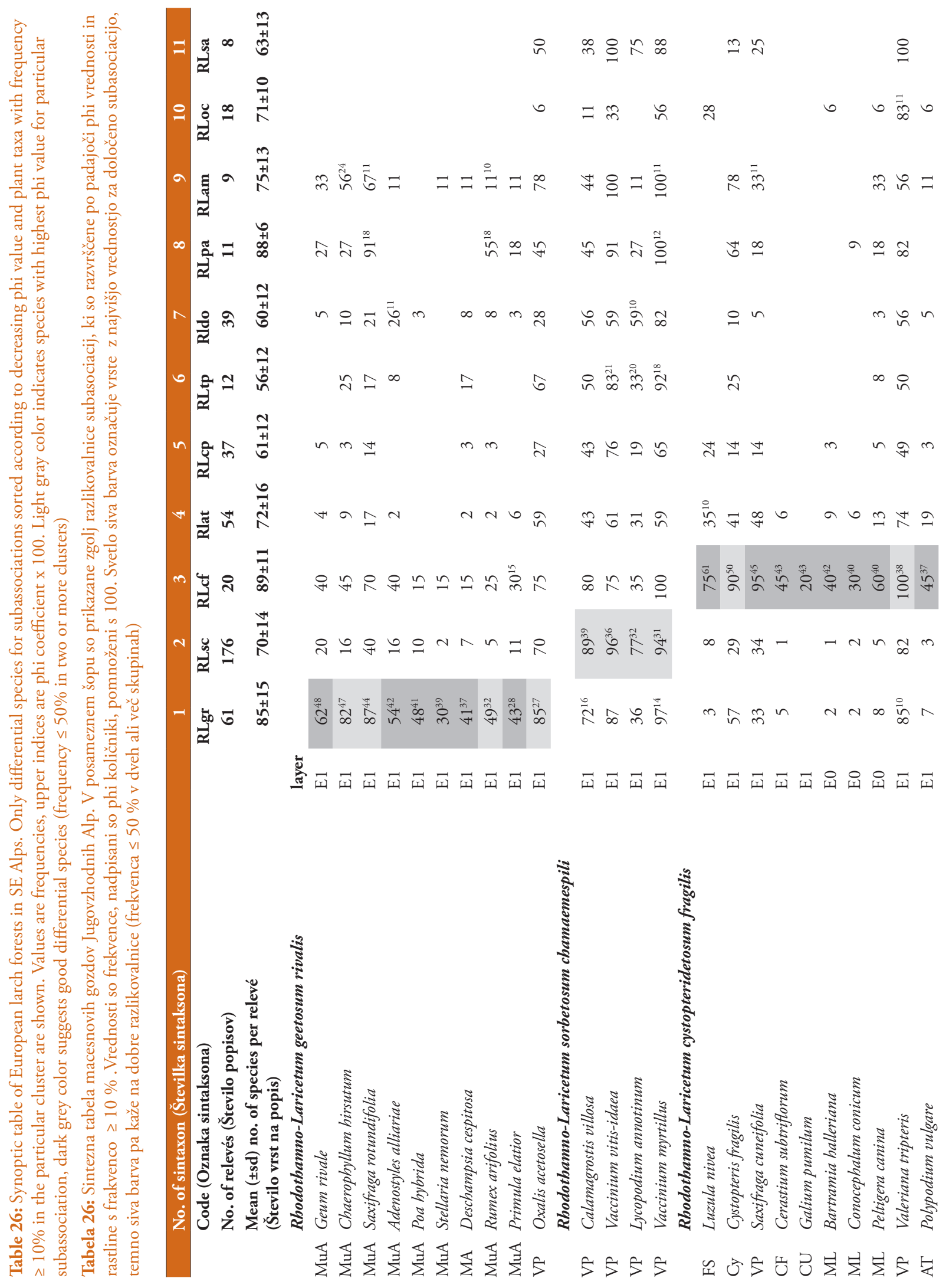




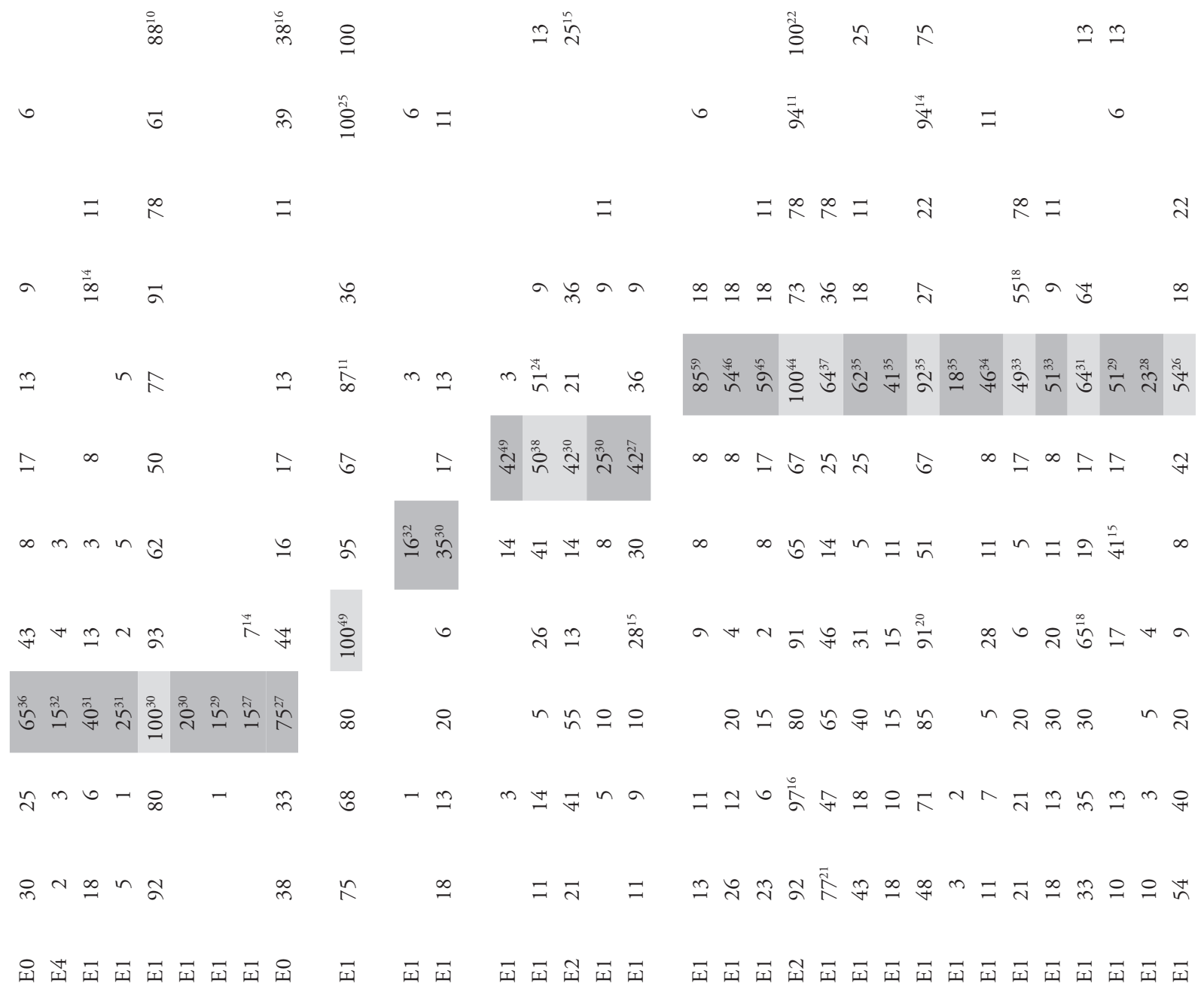

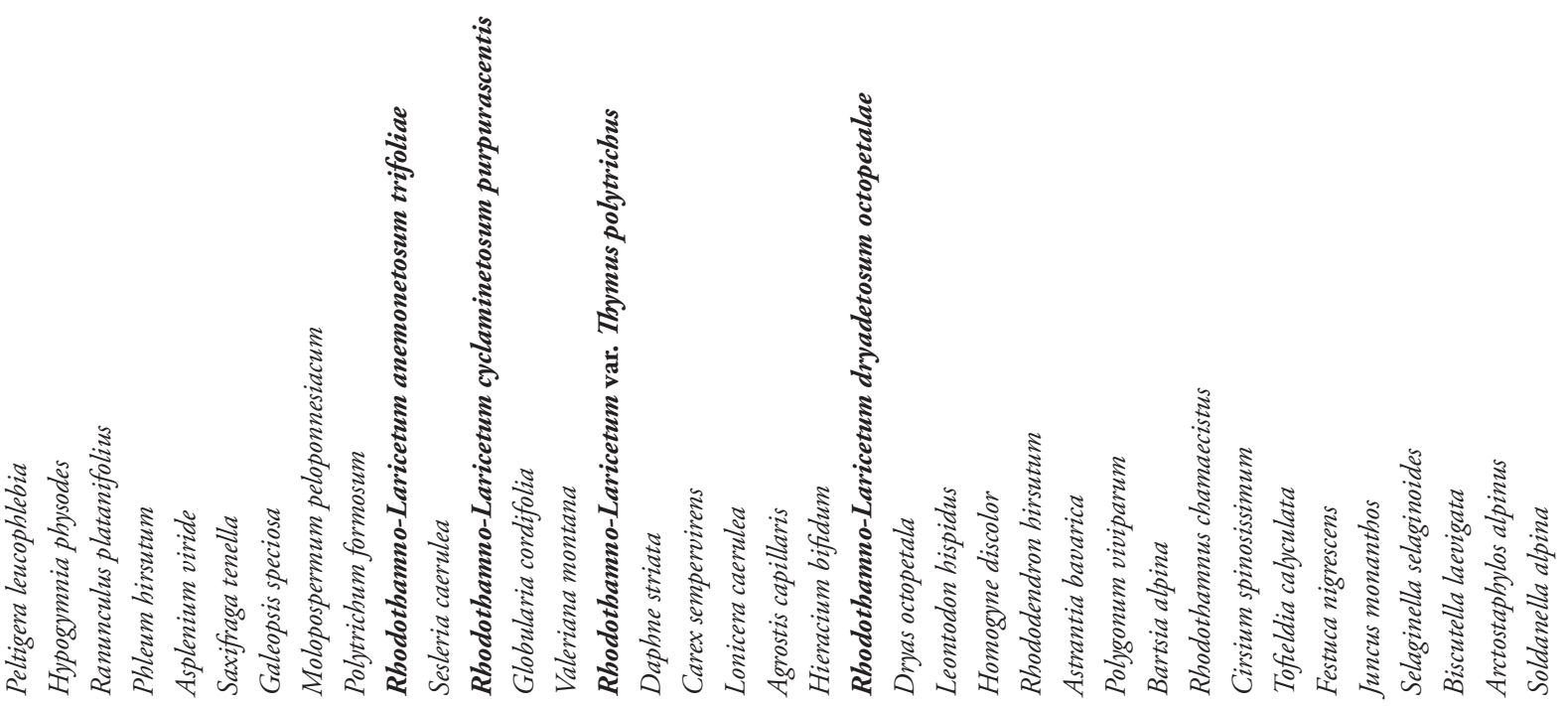

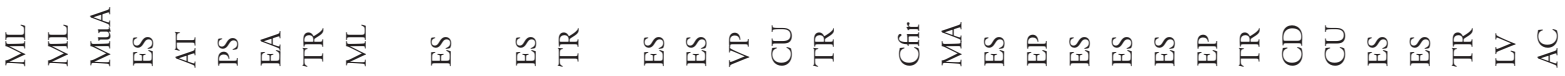




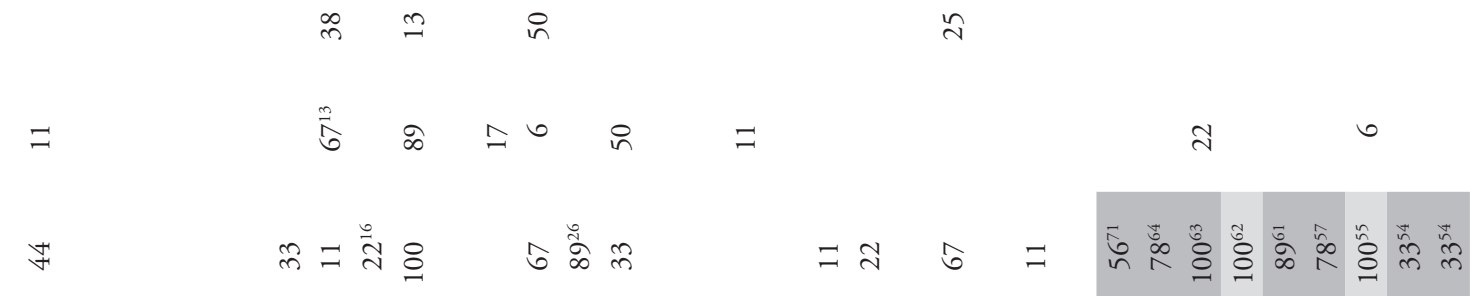

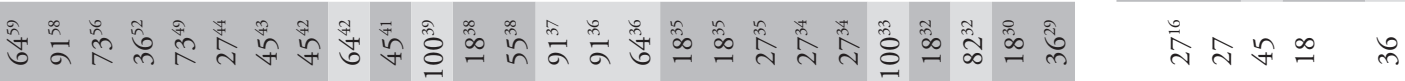

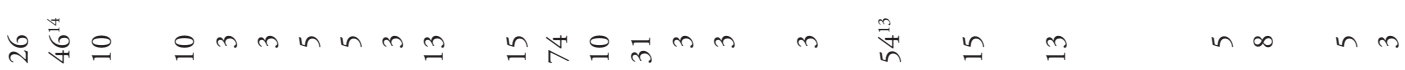

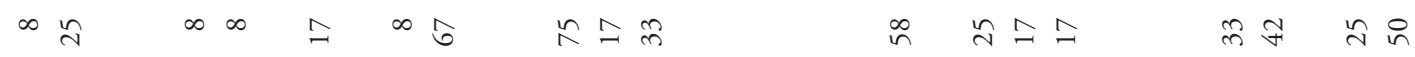

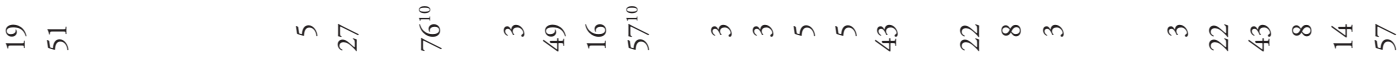

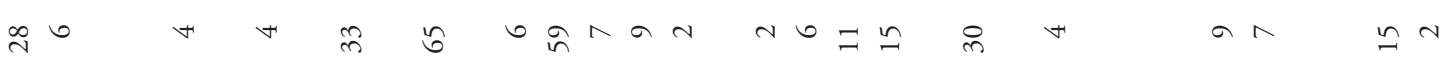

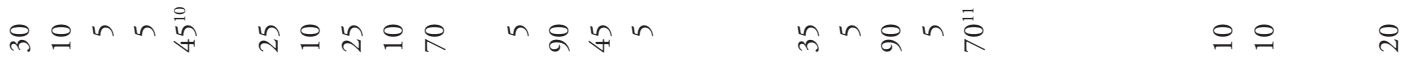

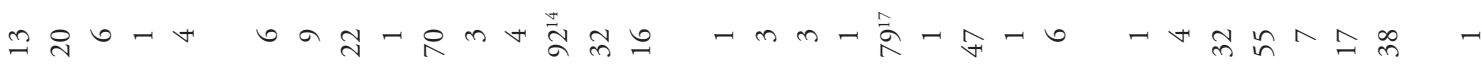

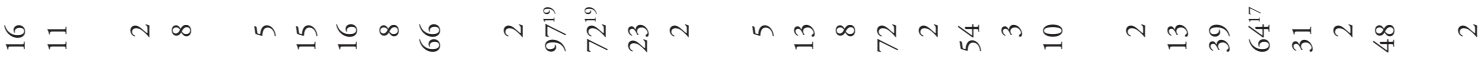

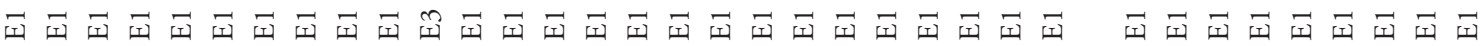
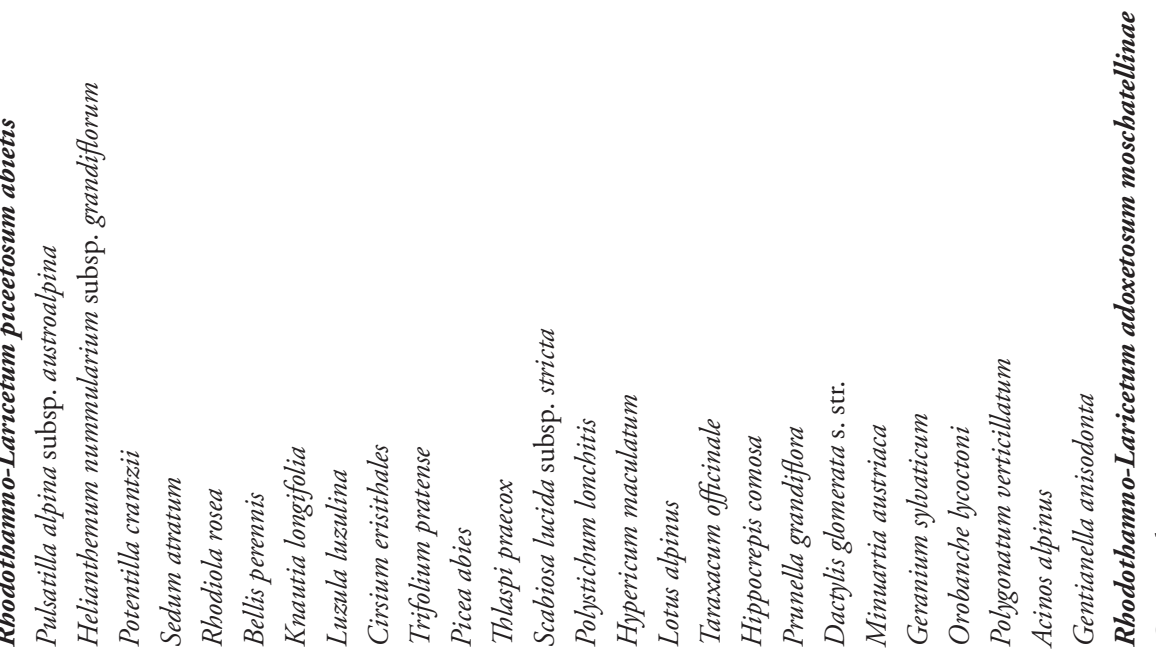

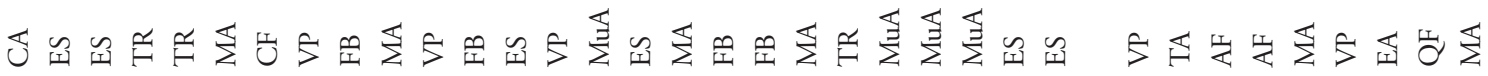




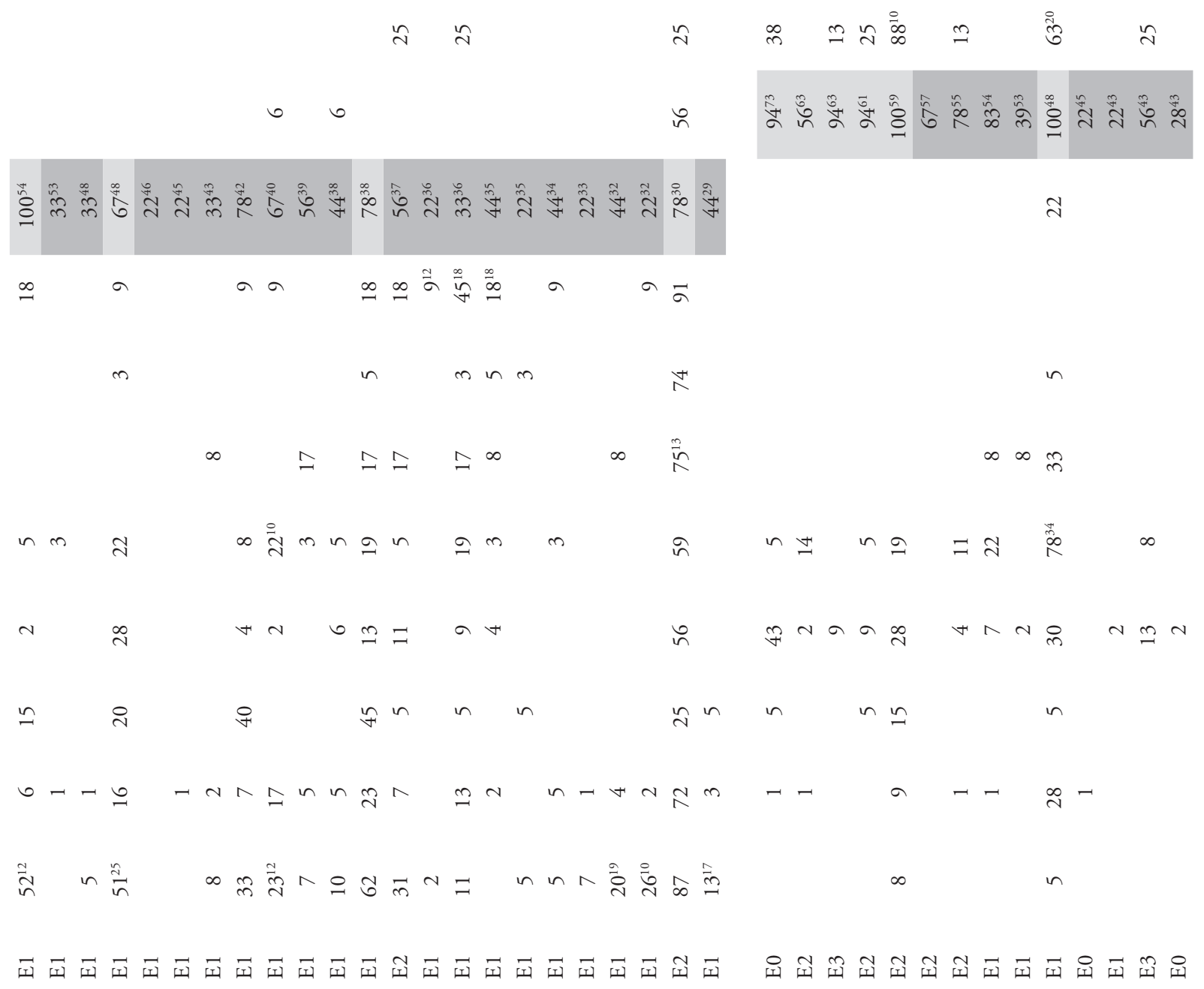

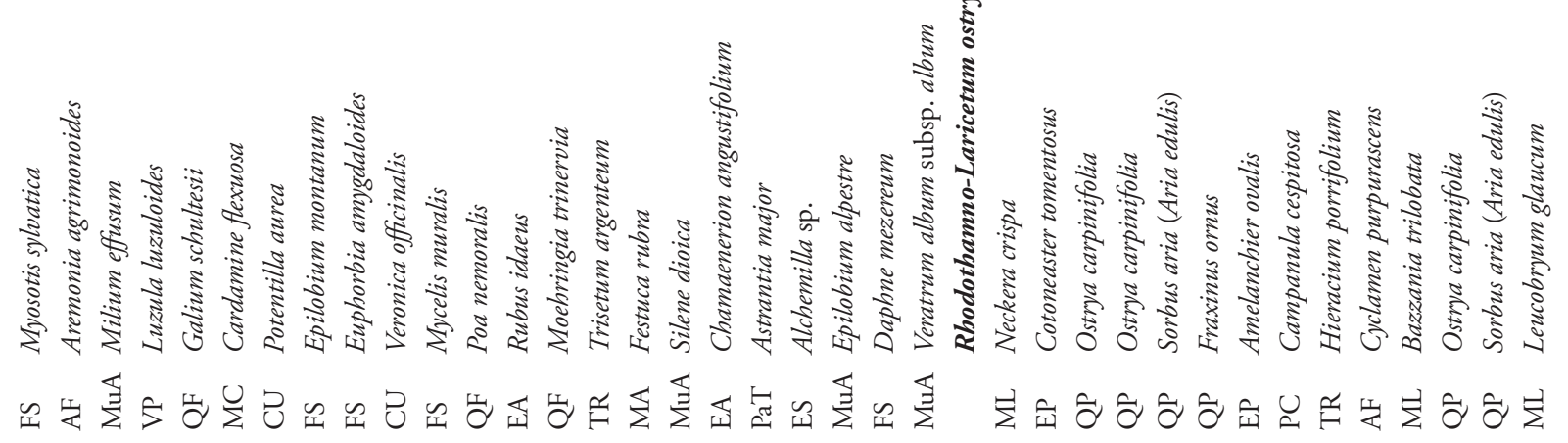




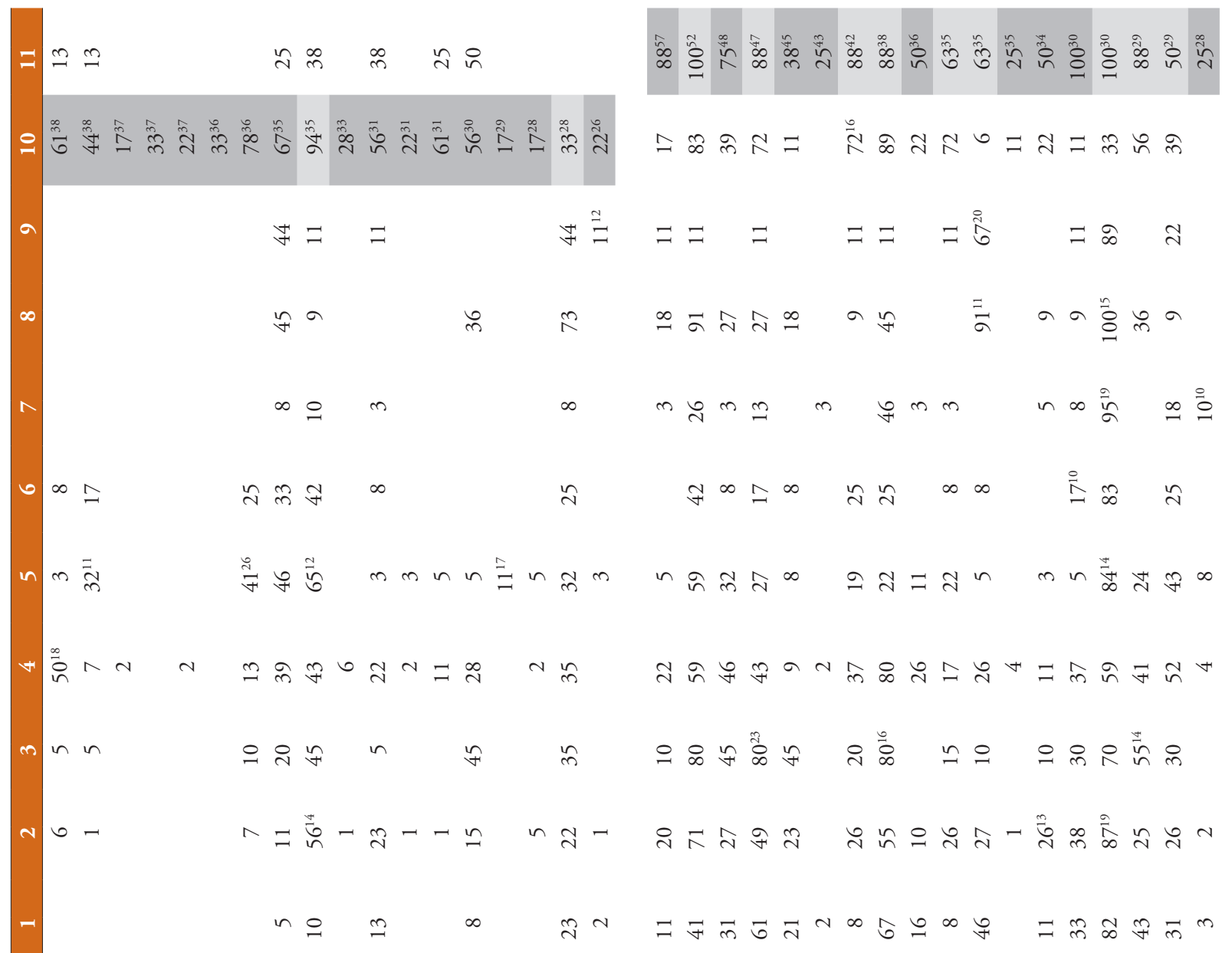

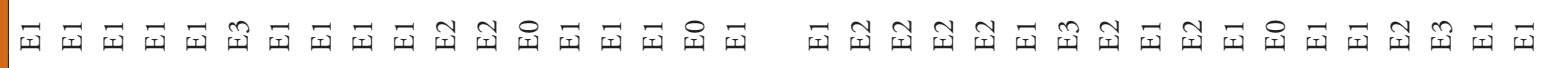

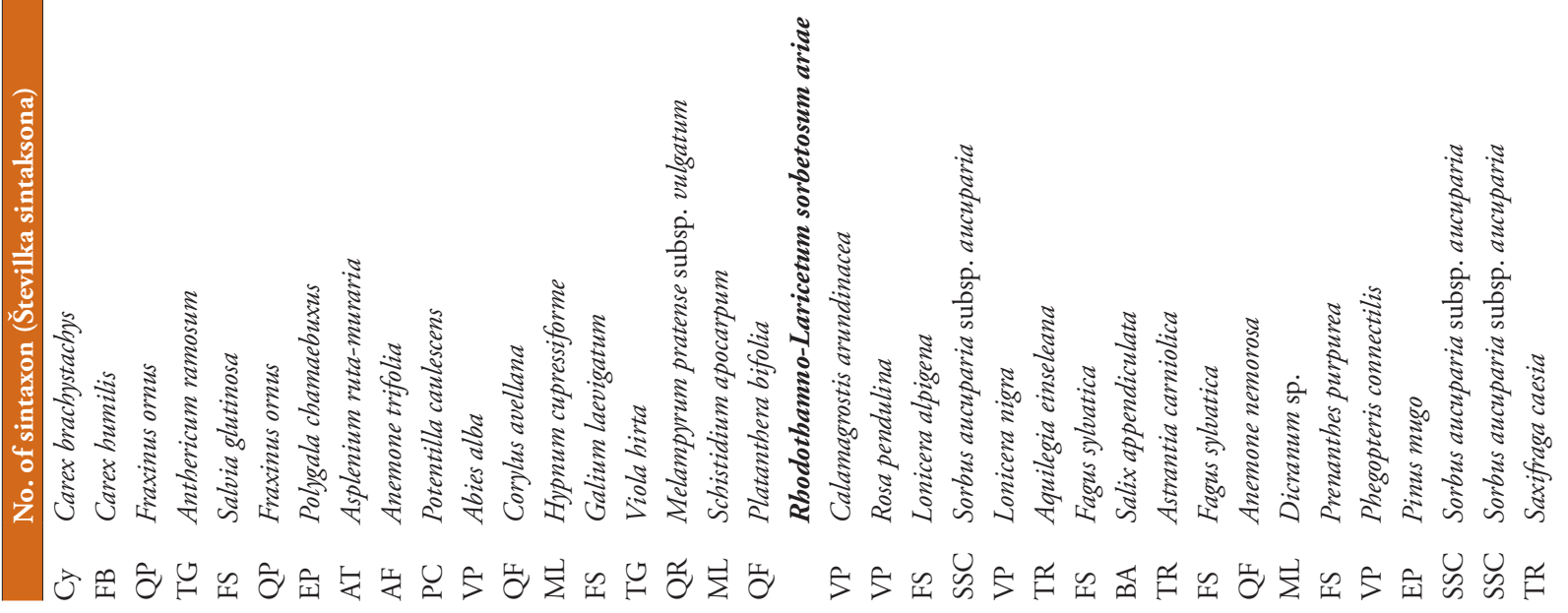




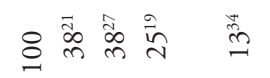

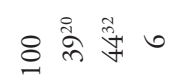

$\stackrel{\square}{\text { ส }}$

$\stackrel{\stackrel{n}{\infty}}{\stackrel{-}{\leftrightarrows}}$

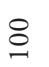

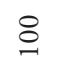

$\stackrel{\circ}{\circ} n n n n$

$\Xi \succcurlyeq$

$\stackrel{\circ}{\circ} n n$

$\stackrel{m}{8}$ ㄴำ

$\stackrel{n}{\circ}=n n$

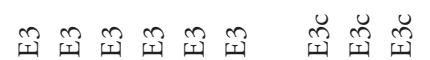

n o

$\infty n$ $\infty \infty_{\infty}^{\infty} \bar{a}_{\infty}$ in

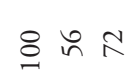

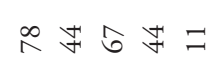

$\alpha=\sqrt[n]{2}$

$\stackrel{2}{2}=$

ส

$4=$

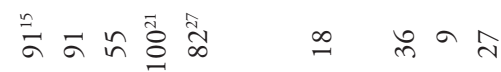

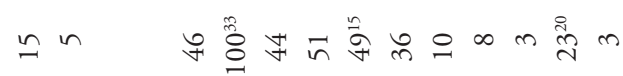

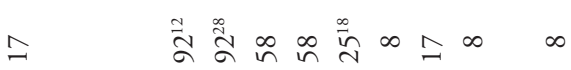

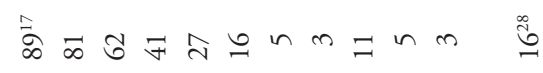

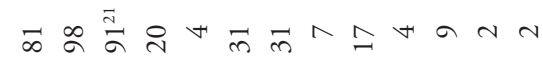

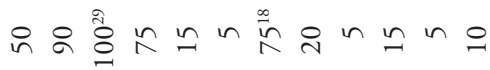

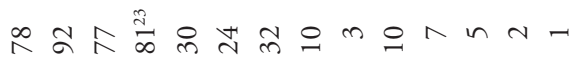

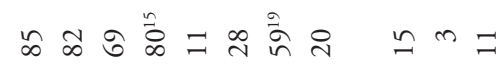

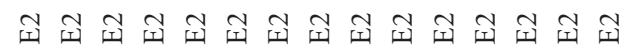

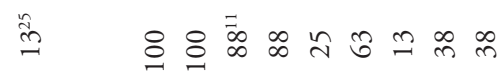
$\stackrel{2}{=} \infty \infty \stackrel{\circ}{\circ} \infty \stackrel{\circ}{\circ}$

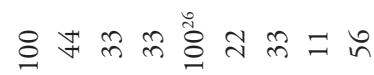

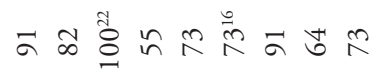

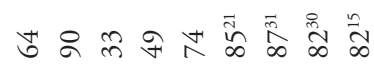

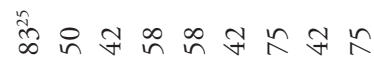

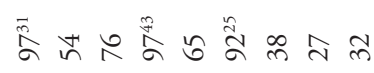

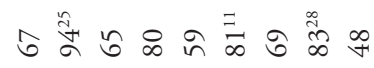

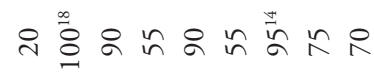

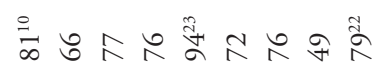
舟

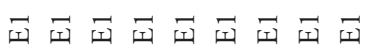

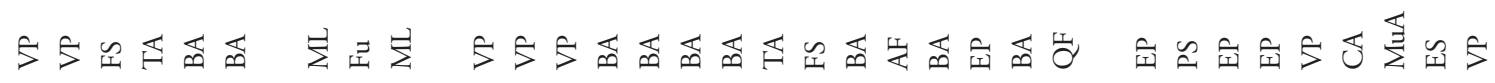




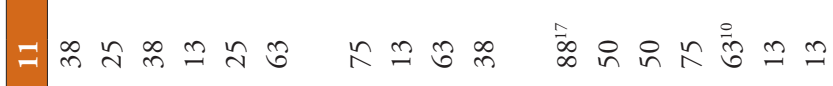

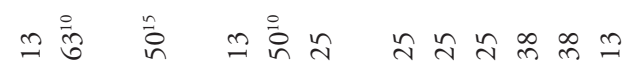

은 范

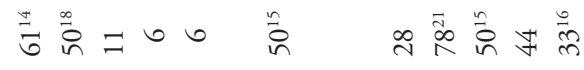

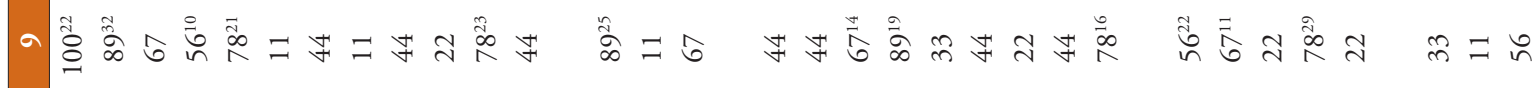

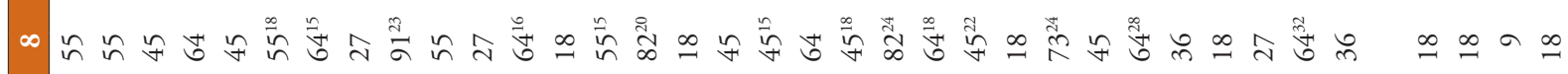

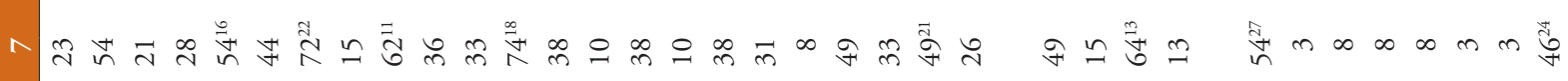

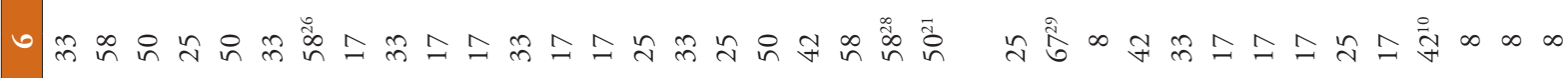

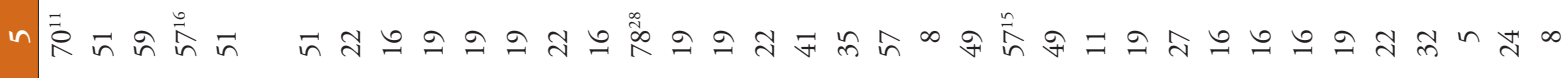

H

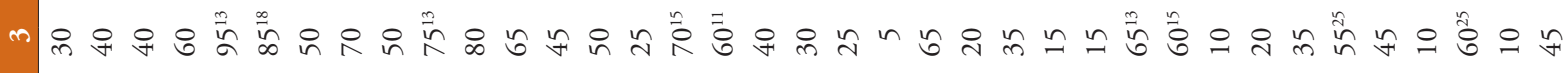

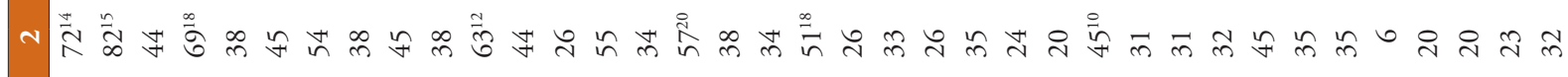

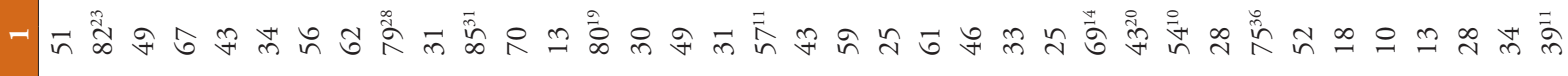

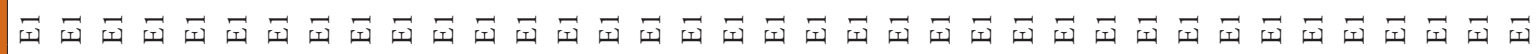

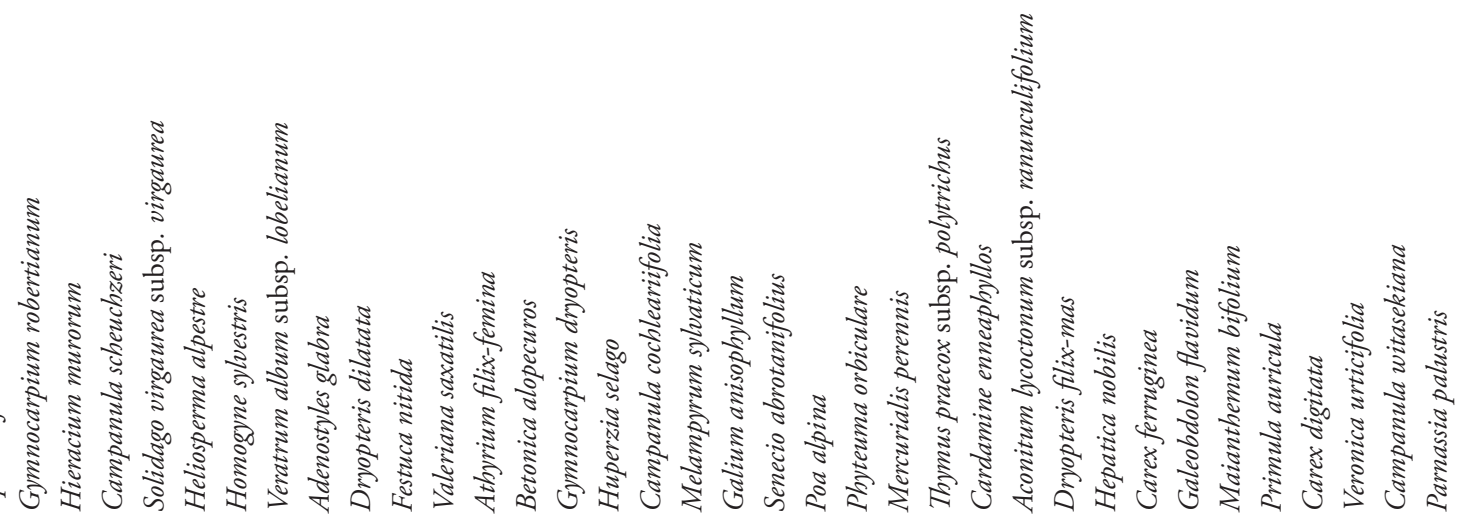

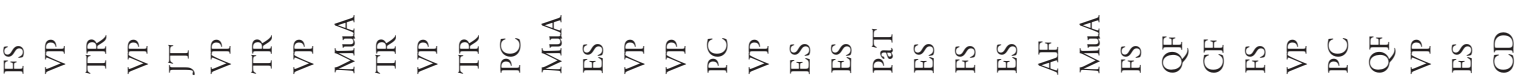




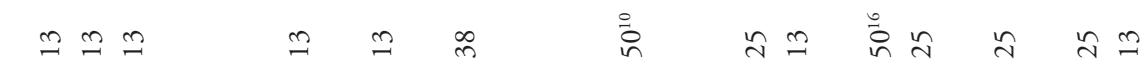

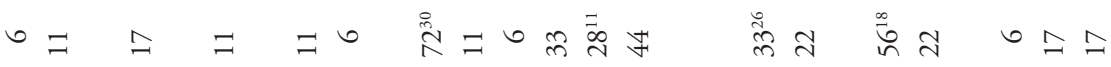

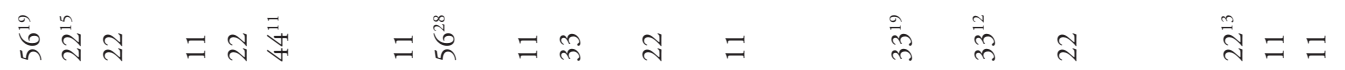

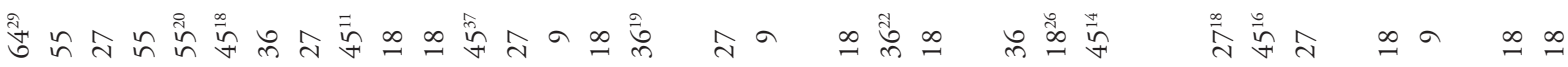

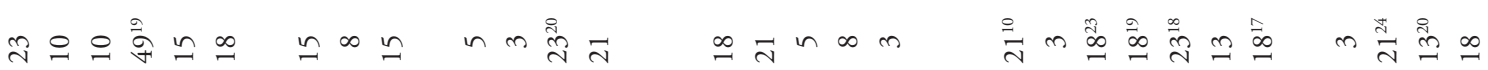

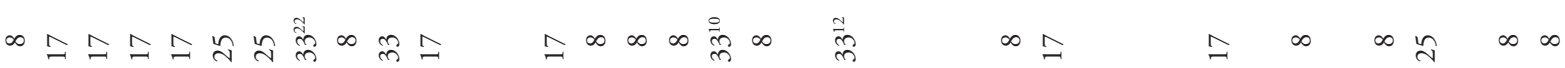

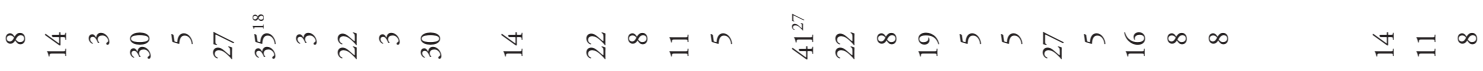
小

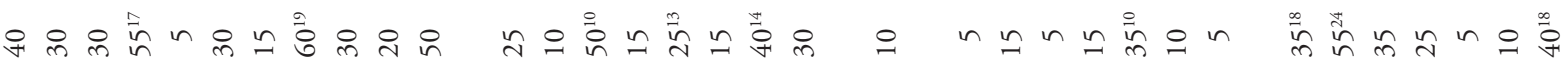

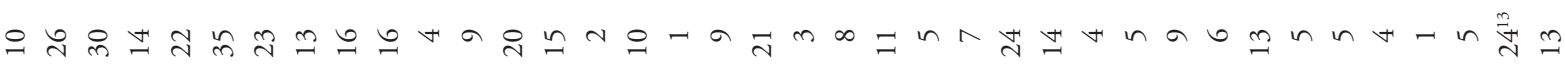

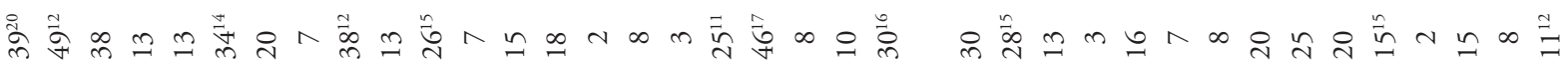

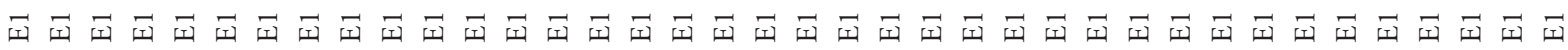

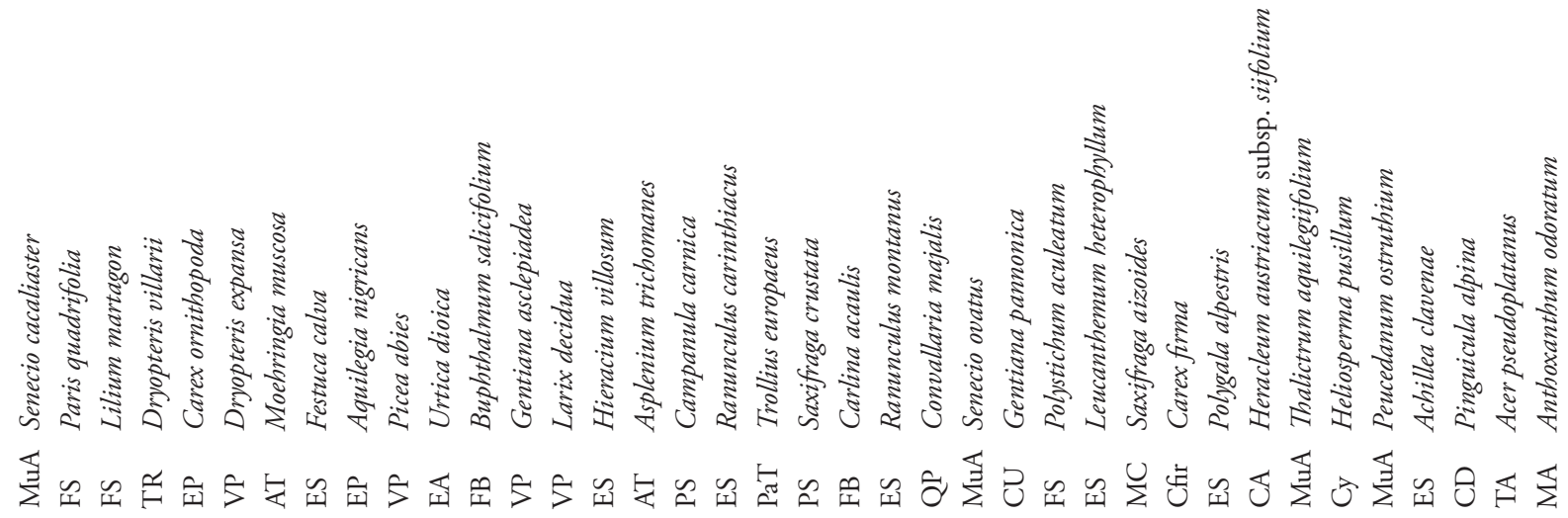




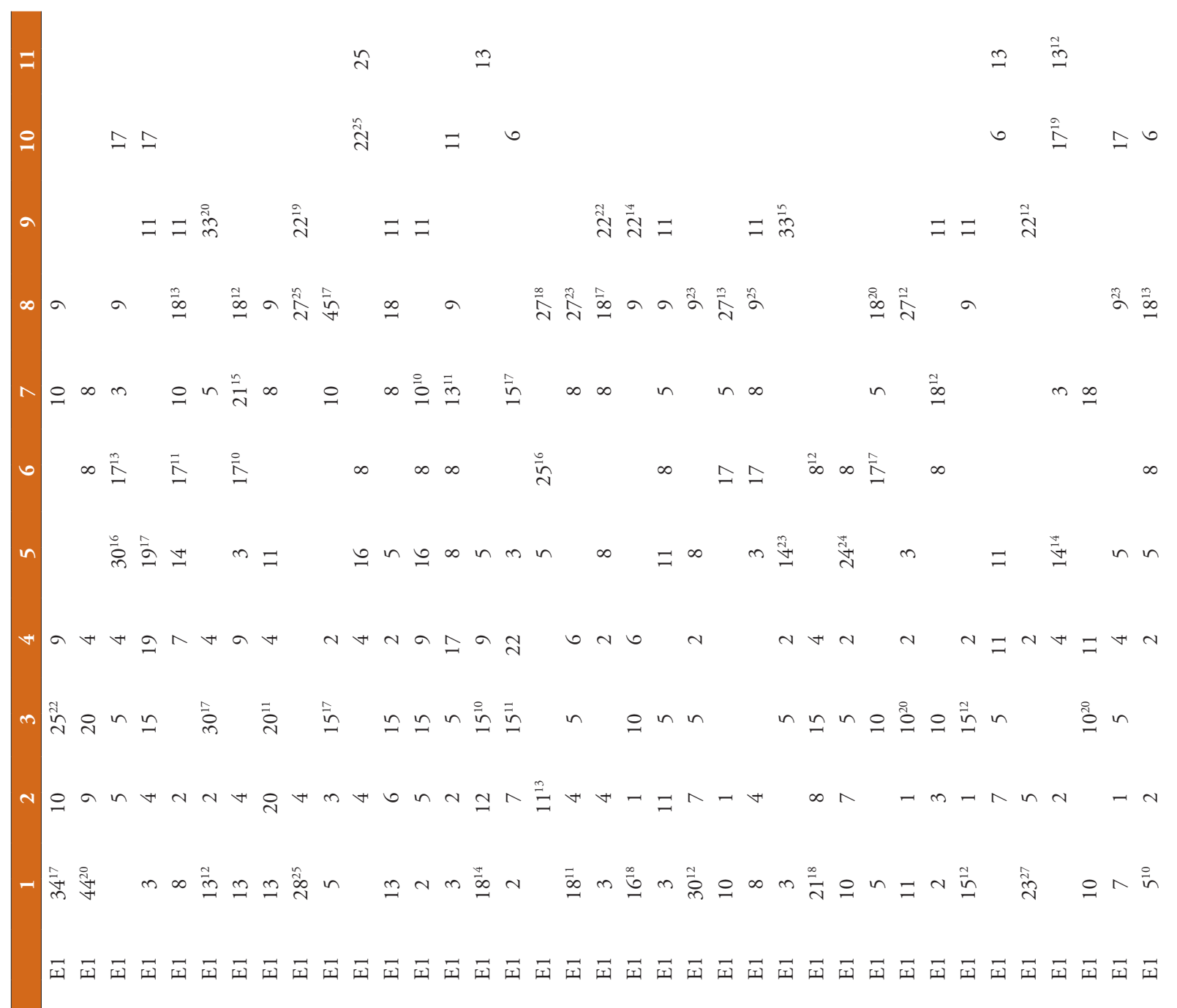

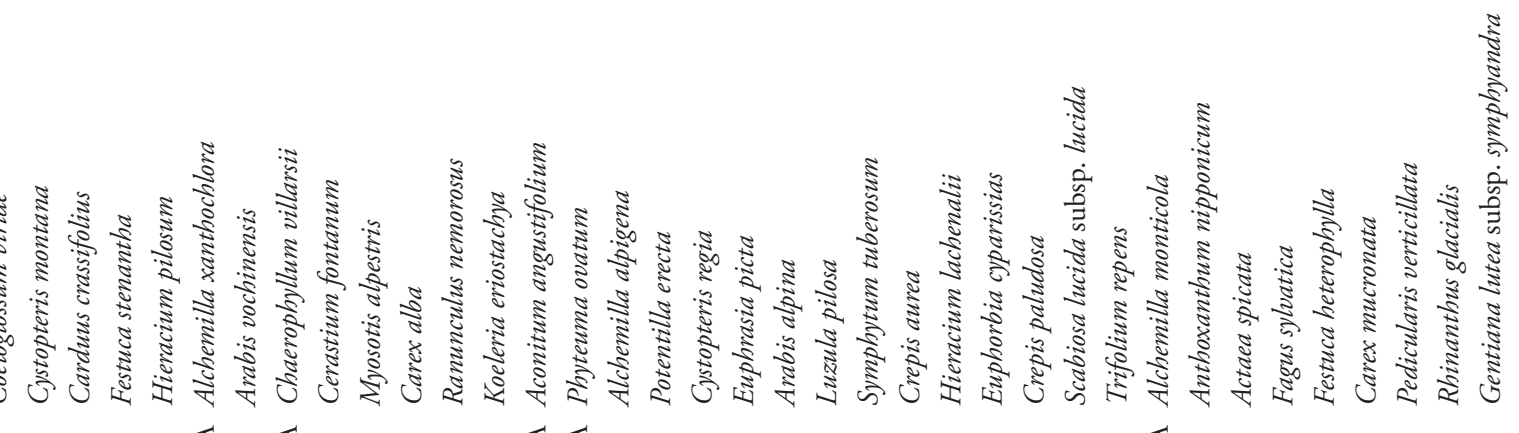

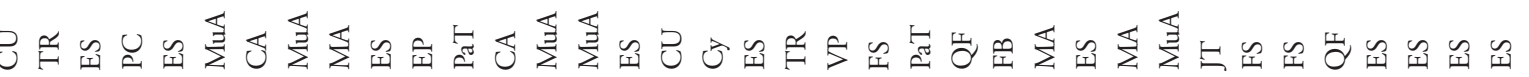


- Hacquetia $17 / 2 \cdot 2018,247-519$

I. Dakskobler, A. Seliškar \& A. Rozman

Phytosociological analysis of European larch forests in the Southeastern Alps

$$
\begin{aligned}
& \stackrel{\infty}{\stackrel{m}{m}} \\
& \text { i }
\end{aligned}
$$

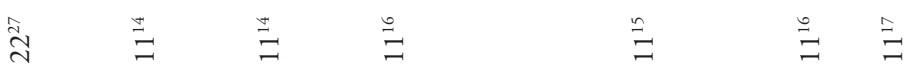

$$
\begin{aligned}
& \stackrel{20}{\stackrel{2}{2} 0} \\
& 6 \\
& \stackrel{\Xi}{\exists}
\end{aligned}
$$

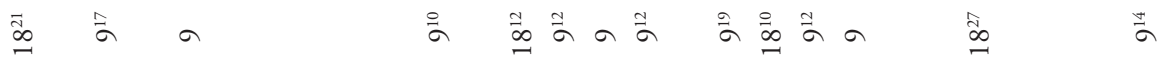

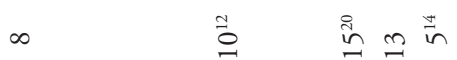

$$
\begin{aligned}
& \stackrel{\infty}{\infty} \infty \stackrel{\circ}{\infty} \infty \\
& \stackrel{\circ}{2}
\end{aligned}
$$

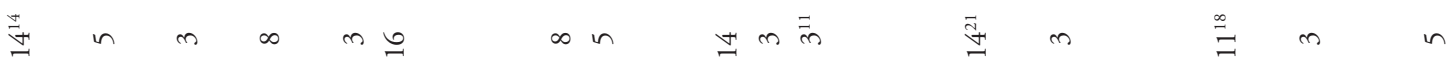

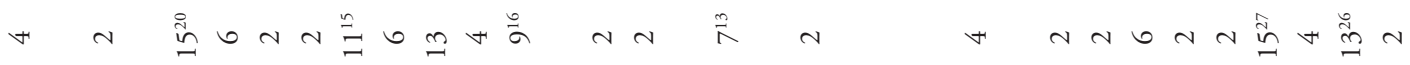

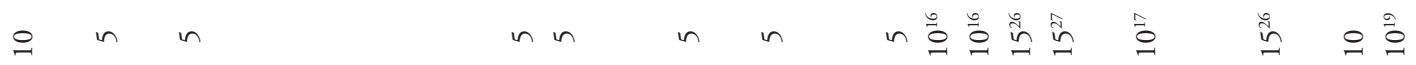

$-n-$

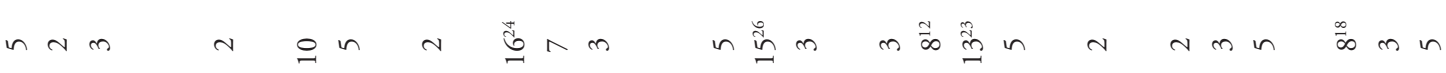

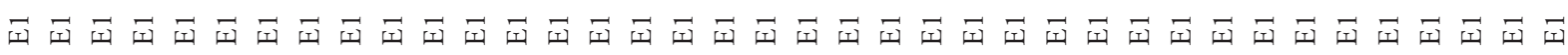

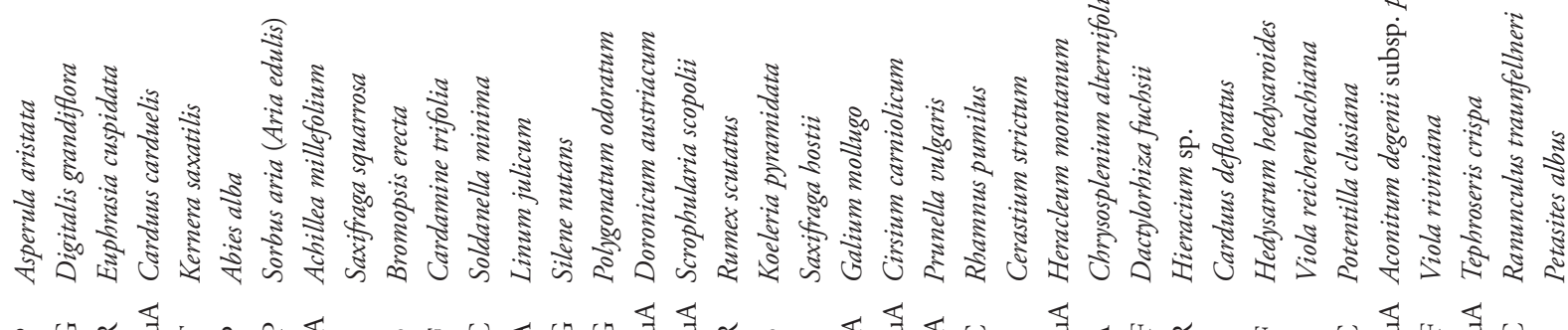

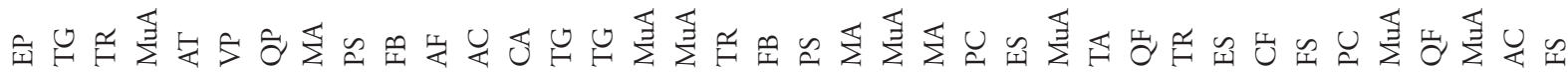

515 
- Hacquetia $17 / 2 \cdot 2018,247-519$

I. Dakskobler, A. Seliškar \& A. Rozman

Phytosociological analysis of European larch forests in the Southeastern Alps

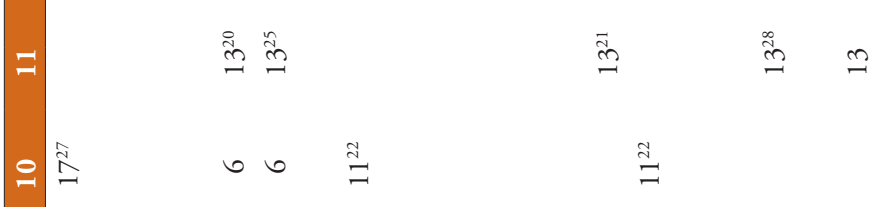

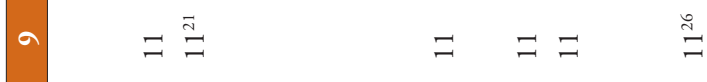

$\infty$

$\wedge$ के in

$\ln \quad$ g $n \infty \quad n n$

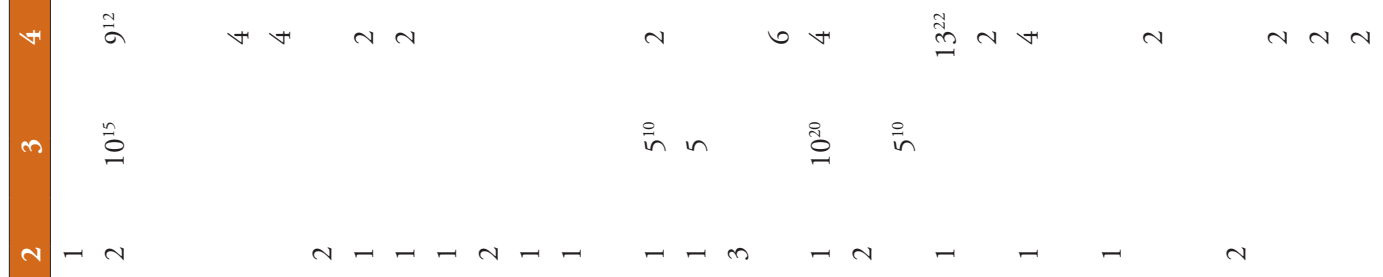

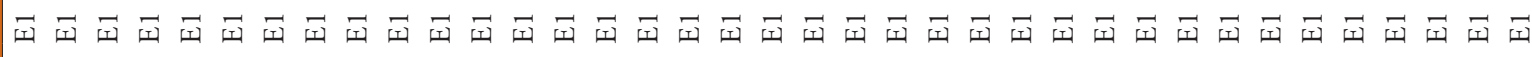

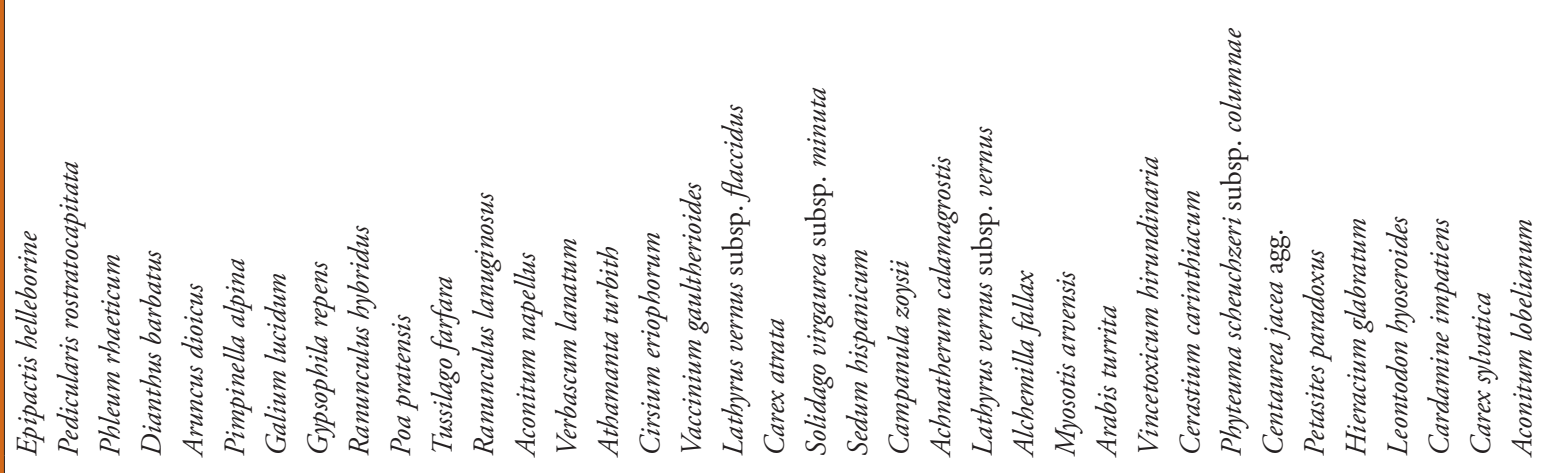

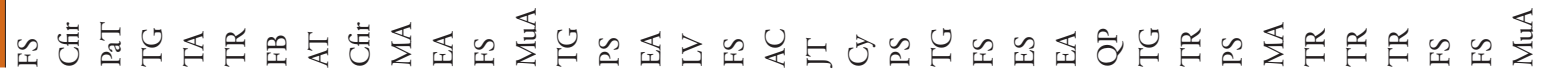

516 


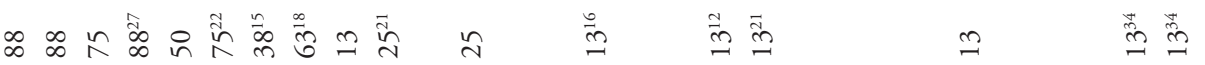

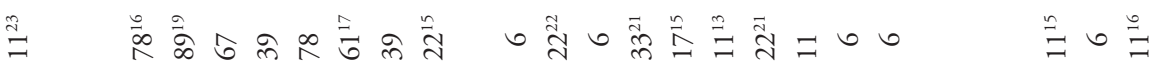

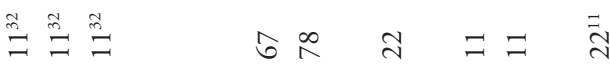

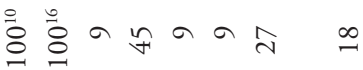

疋

$n n$

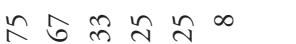

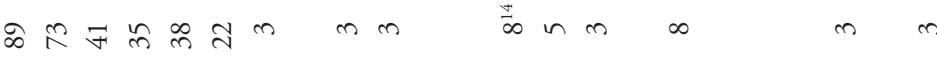

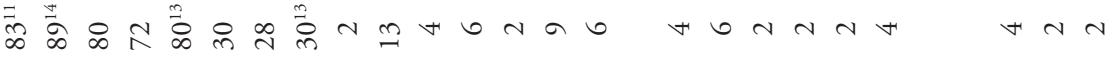

응 \&

n

豖

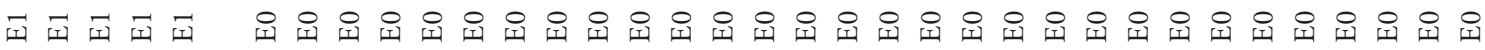

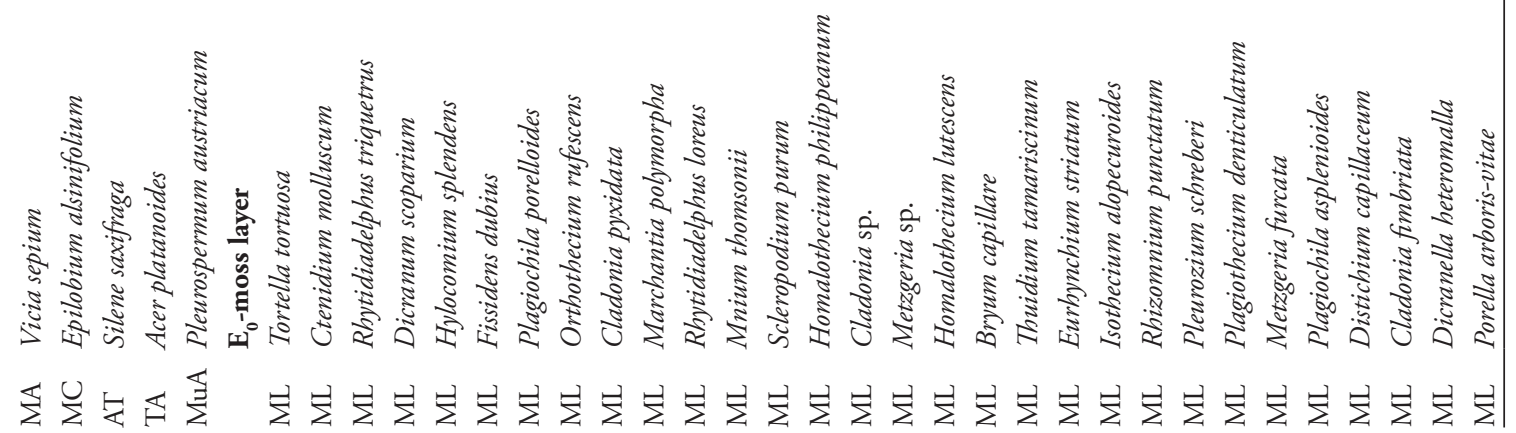




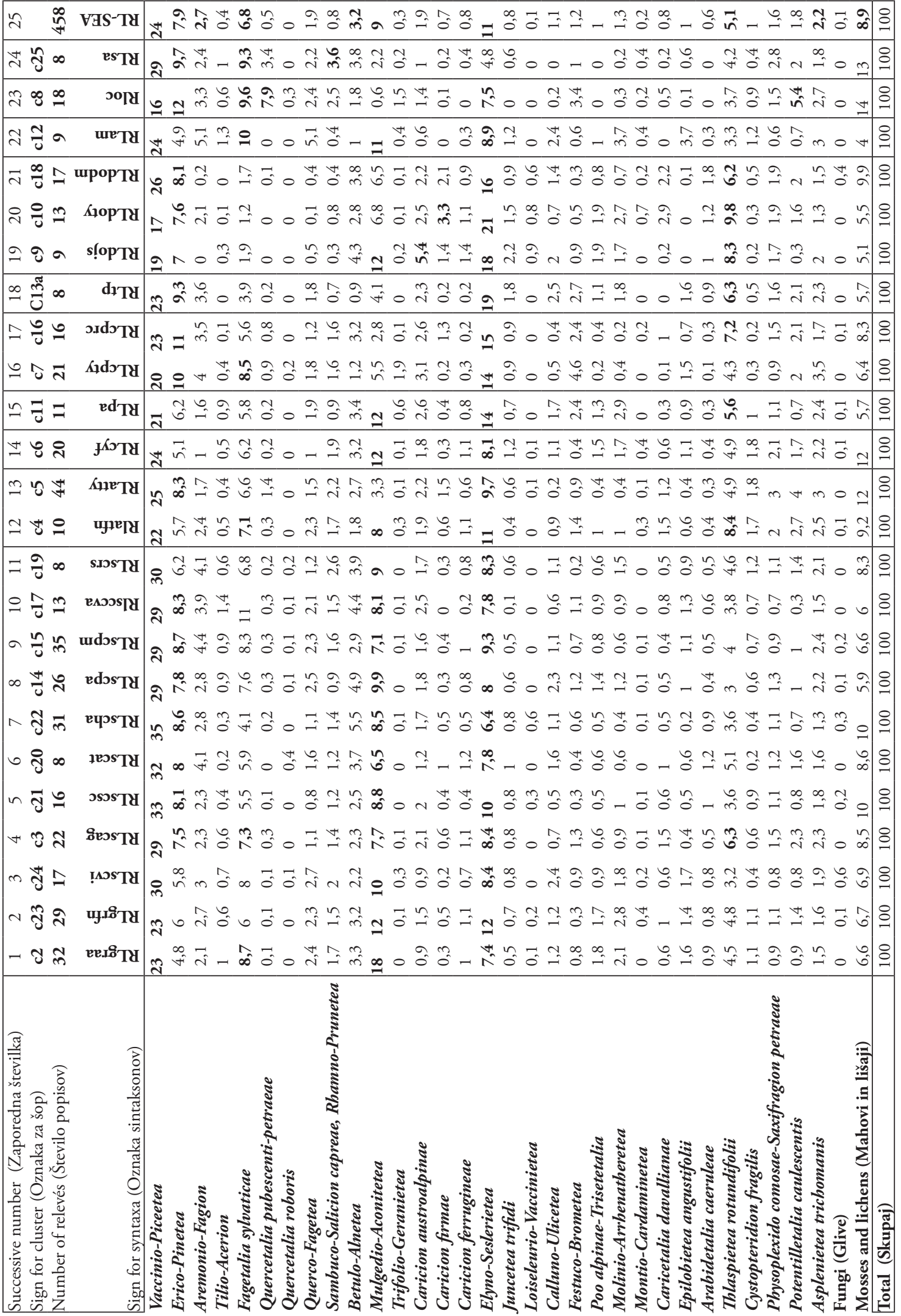


Table 28: Characteristic species combination in the stands of the association Rhodothamnno-Laricetum in the Southeastern Alps (species with constancy $40 \%$ and more)

Tabela 28: Značilna (stanovitna) kombinacija vrst v asociaciji Rhodothamno-Laricetum v Jugovzhodnih Alpah (vrste $s$ stalnostjo 40 in več \%)

\begin{tabular}{|c|c|c|c|c|c|c|}
\hline $\mathrm{VP}$ & Larix decidua & E3 & 100 & Hieracium murorum & E1 & 57 \\
\hline EP & Rhododendron hirsutum & E2 & 90 & Melica nutans & E1 & 57 \\
\hline VP & Vaccinium myrtillus & E1 & 85 & Aster bellidiastrum & E1 & 56 \\
\hline AT & Asplenium viride & E1 & 81 & Heliosperma alpestre & E1 & 52 \\
\hline VP & Vaccinium vitis-idaea & E1 & 81 & Lycopodium annotinum & E1 & 50 \\
\hline EP & Pinus mugo & E2 & 80 & Dryopteris dilatata & E1 & 49 \\
\hline VP & Luzula sylvatica & E1 & 79 & Gymnocarpium robertianum & E1 & 49 \\
\hline $\mathrm{VP}$ & Picea abies & E3 & 78 & Homogyne sylvestris & E1 & 47 \\
\hline VP & Polystichum lonchitis & E1 & 78 & Astrantia bavarica & E1 & 47 \\
\hline ES & Sesleria caerulea & E1 & 78 & SSC Sorbus aucuparia subsp. aucuparia & E3 & 46 \\
\hline VP & Valeriana tripteris & E1 & 76 & MuA Veratrum album subsp. lobelianum & E1 & 46 \\
\hline VP & Clematis alpina & E2 & 73 & TR Festuca nitida & E1 & 45 \\
\hline EP & Erica carnea & E1 & 72 & Solidago virgaurea subsp. virgaurea & E1 & 45 \\
\hline EP & Calamagrostis varia & E1 & 71 & MuA Athyrium filix-femina & E1 & 44 \\
\hline PS & Paederota lutea & E1 & 71 & JT Campanula scheuchzeri & E1 & 44 \\
\hline EP & Rubus saxatilis & E1 & 70 & Gymnocarpium dryopteris & E1 & 43 \\
\hline EP & Rhodothamnus chamaecistus & E1 & 70 & Anemone trifolia & E1 & 43 \\
\hline $\mathrm{Mu}$ & Viola biflora & E1 & 70 & TR Adenostyles glabra & E1 & 41 \\
\hline CA & Laserpitium peucedanoides & E1 & 69 & $\mathrm{VP}$ Melampyrum sylvaticum & E1 & 40 \\
\hline FS & Daphne mezereum & E2 & 68 & MuA Polygonatum verticillatum & E1 & 40 \\
\hline VP & Calamagrostis villosa & E1 & 65 & AF Knautia drymeia & E1 & 40 \\
\hline VP & Aposeris foetida & E1 & 62 & CF Carex ferruginea & E1 & 40 \\
\hline BA & Sorbus chamaemespilus & $\mathrm{E} 2$ & 62 & MuA Saxifraga rotundifolia & E1 & 40 \\
\hline VP & Homogyne alpina & E1 & 61 & ML Tortella tortuosa & E0 & 84 \\
\hline VP & Oxalis acetosella & E1 & 60 & ML Ctenidium molluscum & E0 & 72 \\
\hline & Rosa pendulina & E2 & 60 & ML Rhytidiadelphus triquetrus & E0 & 66 \\
\hline $\mathrm{Mu}$ & Geranium sylvaticum & E1 & 59 & ML Dicranum scoparium & E0 & 54 \\
\hline & Salix appendiculata & E2 & 58 & ML Hylocomium splendens & E0 & 48 \\
\hline
\end{tabular}

Legend (Legenda) - see Appendix Table 2, Column 25 (glej Tabelo 2 v dodatku, stolpec 25) 